\title{
Hanford Site Near-Facility Environmental Monitoring Annual Report, Calendar Year 1997
}

C. J. Perkins

A. R. Johnson

B. M. Markes

S. M. McKinney

R. M. Mitchell

Waste Management Federal Services, Inc., Northwest Operations

Date Published

July 1998

Prepared for the U.S. Department of Energy

FLUOR DANUEL HANFORD, INC.

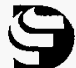

P.O. Box 1000

Richland, Washington

Hanford Management and integration Contractor for the

U.S. Department of Energy under Contract DE-AC06-96RL.13200

Copyright License By acceptance of this article, the publisher and/or recipient acknowledges the

U.S. Government's right to retain a nonexclusive, royalty-tree license in and to any copyright covering this paper. 


\section{LEOAL DISCLAIMEA}

This report was prepored as an account of work sponsored by an agency of the United States Government. Neither the United States Government nor any agency thereol, nor any of their employees, nor any of thair contractors. subcontractors or their employees, makes any warranty. express or implied, or assumes any legal liability or responsibility for the accuracy, completeness, or any third party's use or the results of such use of any information, apparatus, product, of process disciosed, of represe nts that its use would not infringe privatoly owned rights. Reference herein to any specilic commercial product, process, or service by trade name, trademark. manufacturer, or otherwise, does not necessarily constitute or imply its endorsement, recommendation, or favoring by the United States Government or any agency thereof or its contractors of subcontractors. The views and opinions of authors expressed herein do not necessarily state of reflect those of the United States Government of any egency thereof.

This report has been reproduced from the best available copy.

Available in paper copy and microfiche.

Avaitable to the U.S. Department of Energy and its contractors from

U.S. Department of Energy

Office of Scientific and Technical Information (OST! P.O. Box 62

Oak Ridge, TN 37831

(615) 576-B401

Available to the public from the U.S. Department of Commerce

National Technical Information Service (NTIS) 5285 Port Royal Road

Springfield, VA 22161

(703) 487.4650

Printed in the United Stotes of Ammice 
Document Title: Hanford Site Near-Facility Environmental Monitoring Annual Report, Calendar Year 1997

Prepared by:

Reviewed and Approved by:

Approved by:

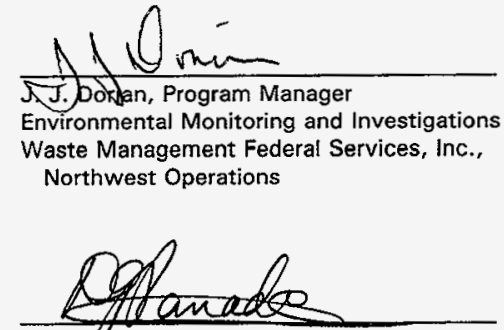

D. G. Banade, Technical Representative Effluent and Environmental Monitoring Program Fiuor Daniel Hanford, Inc.

C. F. Perkins, Senior Engineer Environmental Monitoring and Investigations Waste Management Federal Services, Inc., Northwest Operations

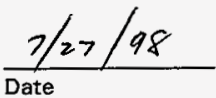

Date $\frac{7 / 27 / 98}{\text { Date }}$ 
HNF-EP-0573-6

This page intentionally left blank. 


\section{EXECUTIVE SUMMARY}

\section{NEAR-FACILITY ENVIRONMENTAL MONITORING}

Near-facility environmental monitoring provides a means to measure the impacts of operations, waste management, and remediation activities on the environment adjacent to facilities and ensure compliance with local, state, and federal environmental regulations.

Specifically, near-facility environmental monitoring monitors new and existing sites, processes, and facilities for potential impacts and releases; fugitive emissions and diffuse sources associated with contaminated areas, facilities (both active and those undergoing surveillance and maintenance), and environmental restoration activities. External radiation, ambient air particulates, ground and surface water, soil, sediment, and biota (plants and animals) are sampled or monitored. Parameters include, as appropriate, radionuclides; radiation fields; chemical or physical constituents, such as nitrates; $\mathrm{pH}$; and water temperature.

All ambient air results were below the U.S. Department of Energy (DOE) Derived Concentration Guides (DCGs). Groundwater concentrations at the two wells at the 107-N Facility were below both the DOE DCG and U.S. Environmental Protection Agency Interim Drinking Water Standards for gammaemitting radionuciides. Soil and vegetation results were generally within historic ranges and mostly below the Accessible Soil Concentration limits (included in HNF-PRO-454, Inactive Waste Sites) with the exception of one soil sampling location at $100 \mathrm{~N}$ Area. External radiation fields continued an overall downward trend. Surface water disposal unit samples (water, sediment, and aquatic vegetation) showed radionuclide concentrations below their respective DCG and Accessible Soil Concentration limits. The $100 \mathrm{~N}$ Area Columbia river shoreline springs results were below DCGs with the exception of one ${ }^{90} \mathrm{Sr}$ concentration. More than 4,600 ha $(11,300$ acres $)$ of radiologically controlled areas were surveyed in 1997, approximately the same as in 1996. 
While the 1997 analytical results showed a high degree of variability, in general, the samples collected from media located on or directly adjacent to waste disposal units and other facilities had significantly higher concentrations than those farther away. As expected, certain radionuclides were found in higher concentrations within different operational areas. Generally speaking, the predominant radionuclides were activation (e.g., ${ }^{60} \mathrm{Co}$ ) and fission products (e.g., ${ }^{90} \mathrm{Sr},{ }^{137} \mathrm{Cs}$ ) in the 100 Areas; fission products in the $200 / 600$ Areas; and uranium in the 300 Area.

Air Monitoring. Radioactivity in air was sampled by a network of continuously operated samplers at 62 locations near nuclear facilities: 4 in the $100 \mathrm{~N}$ Area, 4 in the $100 \mathrm{~K}$ Area, 37 in the 200 Areas, 3 at the Environmental Restoration Disposal Facility, 4 in the 100 D/DR Area, 3 in the 100 B/C Area, 3 at the 300-FF-1 project, 2 at the 3732 Building ( 300 Area), 1 near the 300 Area Treated Effluent Disposal Facility, and 1 station collocated with the Surface Environmental Surveillance Project (Pacific Northwest National Laboratory) and the Washington State Department of Health at the Wye Barricade. Air samplers were primarily located at or near sites and/or facilities having the potential for, or history of, release, with an emphasis on the prevailing downwind directions. Of the radionuclide analyses performed, ${ }^{90} \mathrm{Sr},{ }^{137} \mathrm{Cs},{ }^{239,240} \mathrm{Pu}$, and uranium were consistently detectable in the 200 Areas, and ${ }^{60} \mathrm{Co}$ was consistently detectable in the $100 \mathrm{~N}$ Area. Air concentrations for these radionuclides were elevated near facilities when compared to the concentrations measured offsite by the Surface Environmental Surveillance Project, but were well below the applicable derived concentration guides used to indicate performance.

Groundwater Monitoring. The groundwater monitoring performed and reported through this near-facility monitoring activity is limited to two wells at the $100 \mathrm{~N}$ Area. These wells are located near the 107-N Fuel Storage Basin Recirculation Facility and are sampled to provide an indication of the integrity of the building sumps and underground piping system.

Soil and Vegetation Monitoring. Soil and vegetation samples were collected on, or adjacent to, waste disposal units, from locations downwind and within the operating environment of facilities, and 
environmental restoration activities. Special samples were collected where physical or biological transport problems were identified. Soil and vegetation sample concentrations for some radionuclides were elevated near facilities when compared to the concentrations measured offsite. The concentrations show a large degree of variability; in general, samples collected on, or directly adjacent to, waste disposal facilities had significantly higher concentrations than those collected farther away.

External Radiation. External radiation fields were surveyed near facilities and waste handling, storage, and disposal sites, and environmental restoration activities to measure, assess, and control the effects of operations.

A hand-held meter (to measure low-level radiation fields) was used in the $100 \mathrm{~N}$ Area to survey points near and in the $\mathrm{N}$ Springs area. The levels continued to decline in 1997, reflecting the continuing decay of the radionuclide inventory in the nearby 1301-N Liquid Waste Disposal Facility.

Radiation levels measured with thermoluminescent dosimeters at the $100 \mathrm{~N}$ Area were highest near facilities that had contained or received liquid effluent from $N$ Reactor, primarily the $1325-\mathrm{N}$ and the 1301-N Liquid Waste Disposal Facilities. The average annual dose rate in 1997 for these two facilities decreased approximately $17 \%$ from 1996.

Radiation levels measured in 1997 at three Environmental Restoration Contractor remediation sites (100 B,C Area, 100 D,DR Area and the Environmental Restoration Disposal Facility) were comparable to offsite, ambient background levels.

The highest dose rates measured at $100 \mathrm{~K}$ Area were at locations nearest radioactive waste storage areas and/or stored radioactive rail equipment. The average annual dose rate for 1997 in the $100 \mathrm{~K}$ Area decreased approximately $2 \%$ from 1996. 
The highest dose rates measured in the 200/600 Areas were near waste handling facilities, such as tank farms. The average annual dose rate for 1997 in the 200/600 Areas decreased approximately $8 \%$ from 1996 .

The highest dose rates in the 300 Area were measured near the 340 Waste Handling and Isolation Facility. The average annual dose rate measured in the 300 Area in 1997 decreased approximately $8 \%$ from 1996 . The average annual dose rate at the 300 Area Treated Effluent Disposal Facility decreased approximately $4 \%$ from 1996.

The average annual dose rate for 1997 in the 400 Area increased approximately $3 \%$ from 1996.

Monitoring of Surface-Water Disposal Units and Seeps. Sampling of surface-water disposal units included water, sediment, and aquatic vegetation. Samples collected at river shoreline seeps consisted of water only. Radiological analysis included total alpha, total beta, ${ }^{3} \mathrm{H},{ }^{90} \mathrm{Sr},{ }^{239,240} \mathrm{Pu}$, and gammaemitting radionuclides. Nonradiological analyses performed on water samples included $\mathrm{pH}$, temperature, and nitrates.

Radionuclide concentrations in surface-water disposal units were below the applicable derived concentration guides used as indices of performance and, in most cases, were at or below the analytical detection limit. Although some slightly elevated levels were seen in both aquatic vegetation and sediment, in all cases the radiological analytical results were well below the standards for radiological control. The results for $\mathrm{pH}$ were well within the range of 2.0 to 12.5 , the standard for liquid effluent discharges required by the Resource Conservation and Recovery Act of 1976 . The analytical results for nitrates were all below the $45 \mathrm{mg} / \mathrm{L}$ Drinking Water Standard.

Groundwater seeps along the $100 \mathrm{~N}$ Area shoreline were sampled to verify the reported radionuclide releases to the Columbia River from past operations of the $N$ Reactor. By characterizing 
the radionuclide concentrations in the seeps along the shoreline, the results can be compared to the concentrations measured in the facility effluent monitoring well. In 1997, the concentrations detected in the seep samples were highest in those seeps nearest the facility effluent monitoring well, although the seep concentrations were considerably lower than those measured in the well.

Radiological Surveys. In 1997 , there were approximately 3,990 ha $\{9,859$ acres $\}$ of posted outdoor contamination areas and 615 ha $(1,517$ acres $)$ of posted underground radioactive materials areas at the Hanford Site. These areas were typically associated with cribs, ponds, trenches, burial grounds, tank farms, and covered ditches. The posted contamination areas vary between years because of an ongoing effort to clean, stabilize, and remediate areas of known contamination. During this time, new areas of contamination were also identified. It was estimated that the external dose rate at $80 \%$ of the identified outdoor contamination areas was less than $1 \mathrm{mrem} / \mathrm{h}$ measured at $1 \mathrm{~m}(3.28 \mathrm{ft})$ although isolated, direct dose rate readings from radioactive specks (a diameter less than $0.6 \mathrm{~cm}[0.25 \mathrm{in}$.$] )$ could have been considerably higher. Contamination levels of this magnitude did not significantly add to dose rates for the public or Hanford Site workers in 1997.

\section{Investigative Sampling}

Investigative sampling was conducted in the operations areas to help resolve questions about the radiological status of the facilities and waste sites. Special sampies were collected where radionuclide transport was suspected or identified to be caused by physical (e.g., wind erosion) or biotic vectors (e.g., animal burrowing or tumbleweed uptake) and included sludge, soil, vegetation (e.g., grass, tumbleweeds, rabbitbrush, and sagebrush), insects (darkling beetles), reptiles (sagebrush lizards), a bird nest (starling), mammal feces (e.g., mouse, rabbit, and coyote), and mammals (e.g., deer mouse, Great Basin pocket mouse, and cottontail rabbit). There were 30 samples analyzed for radionuclides in 1997 and 27 showed measurable levels of contamination. Another 115 contamination incidents were reported and samples disposed of without isotopic analyses lalthough field instrument readings were recorded) during operation and cleanup activities. Predominant radionuclides identified 
were activation products and ${ }^{90} \mathrm{Sr}$ in the 100 Areas, and fission products in the 200 and 600 Areas. No investigative samples were collected from the $300,400,1100$, or 3000 Areas in 1997. Maximum concentrations of all measured radionuclides (i.e., ${ }^{60} \mathrm{Co},{ }^{90} \mathrm{Sr},{ }^{137} \mathrm{Cs},{ }^{154} \mathrm{Eu},{ }^{155} \mathrm{Eu},{ }^{238} \mathrm{Pu},{ }^{239 / 240} \mathrm{Pu}$, and ${ }^{\text {Total }} \mathrm{U}$ ) in 1997 were in mouse feces collected along railroad tracks east of the 218-W-4B Burial Ground in the 200 West Area. Additionally, a sample of three house mice from the 244-AR Vault in the 200 East Area had an equally high concentration of ${ }^{30} \mathrm{Sr}$. 


\section{SUMMARY}

\section{NEAR-FACILITY ENVIRONMENTAL MONITORING}

Several types of environmental media are sampled near nuclear facilities to monitor waste management and restoration activities, and the effectiveness of effluent treatment and control practices. These media include air, surface water and springs, groundwater, surface contamination, soil, vegetation, sediments, biota, and external radiation. Sampling and analysis information, and analytical results for 1997 for each of these media are summarized in the following section.

\section{Near-Facility Environmental Monitoring at the Hanford Site}

Near-facility environmental monitoring is defined as monitoring near facilities that have potential to discharge or have discharged, stored, or disposed of radioactive or hazardous materials. Monitoring locations are associated mostly with major nuclear facilities, such as the Plutonium Uranium Extraction (PUREX) Plant and N Reactor, environmental restoration activities, and waste storage or disposal facilities, such as burial grounds, tank farms, ponds, cribs, trenches, and ditches.

Much of the monitoring consists of collecting and analyzing environmental samples and methodically surveying areas near facilities releasing effluents and waste streams. The program also evaluates acquired analytical data, determines the effectiveness of facility effluent monitoring and controls, measures the adequacy of containment at waste disposal units, and detects and monitors unusual conditions. The monitoring implements applicable portions of U.S. Department of Energy (DOE) Orders 5400.1 (DOE 1988a), 5400.5 (DOE 1990), and 5820.2A (DOE 1988b); Washington Administrative Code (WAC) 246-247; Title 40, Code of Federal Regulations (CFR) Part 61, Subpart $\mathrm{H}$; and $10 \mathrm{CFR} 835$.

Routine sampling and monitoring includes ambient air, water from surface water disposal units, external radiation, soil, sediment, vegetation, and animals. Some of the parameters typically monitored are $\mathrm{pH}$, radionuclide concentrations, and radiation fields. Samples are collected from known or expected effluent pathways. These pathways are generaily downwind of potential or actual airborne releases and down gradient of liquid discharges. Table S-1 shows the type, quantity, and location of routine near-facility monitoring samples collected in 1997.

Waste disposal sites and the terrain surrounding them are surveyed to detect and characterize any radioactive surface contamination. Routine survey locations include cribs, trenches, retention basin perimeters, pond perimeters, ditch banks, perimeters of active solid waste disposal sites, unplanned release sites, tank farm perimeters, stabilized waste disposal sites, roads, and firebreaks in and around the Site operational areas.

\section{AIR MONITORING}

Near-facility air sampling monitors the effectiveness of waste management and environmental remediation controls and effluent treatment systems in reducing effluents and emissions; these air samplers also monitor diffuse source emissions. 
Table S-1. Near-Facility Routine Environmental Samples and Locations, 1997.

\begin{tabular}{|l|c|c|c|c|c|c|c|c|c|}
\hline \multirow{2}{*}{ Sample type } & \multirow{2}{*}{$\begin{array}{c}\text { Number of } \\
\text { sample locations }\end{array}$} & \multicolumn{9}{|c|}{ Operational area } \\
\cline { 3 - 11 } & & $100 \mathrm{~B} / \mathrm{C}$ & $100 \mathrm{D}$ & $100 \mathrm{~K}$ & $100 \mathrm{~N}$ & ERDF & $200 / 600$ & $300 / 400$ & TWRS \\
\hline Air & 62 & 3 & 4 & 4 & 4 & 3 & $38^{(\mathrm{s})}$ & 6 & 0 \\
\hline Water & 13 & 0 & 0 & 0 & 11 & 0 & 2 & 0 & 0 \\
\hline External radiation & 165 & 4 & 5 & 11 & $48^{(0)}$ & 3 & 63 & 21 & 10 \\
\hline Soil & 80 & 0 & 2 & 0 & 7 & 1 & 55 & 15 & 0 \\
\hline Vegetation & 66 & 0 & 0 & 0 & 10 & 0 & 41 & 15 & 0 \\
\hline
\end{tabular}

ERDF = Environmental Restoration Disposal Facility.

TWRS $=$ Tank Waste Remediation System.

Includes one station at the Wye Barricade.

22 thermoluminescent dosimeters and 26 survey points.

\section{Sample Collection and Analysis}

Radioactivity in air was sampled by a network of continuously operating samplers at 62 locations near nuclear facilities: 4 in the $100 \mathrm{~N}$ Area, 4 the $100 \mathrm{~K}$ Area, 37 in the 200 Areas, 3 at the Environmental Restoration Disposal Facility (ERDF), 4 at the 100 D,DR Area, 3 at the $100 \mathrm{~B}, \mathrm{C}$ Area, 3 at the $300-\mathrm{FF}-1$ project (north of the 300 Area), 2 at the 3732 Building (300 Area), 1 near the 300 Area Treated Effluent Disposal Facility (TEDF), and 1 collocated with samplers operated by the Pacific Northwest National Laboratory (PNNL) and the Washington State Department of Health (WDOH) at the Wye Barricade in the 600 Area. To avoid duplication of sampling, the near-facility environmental monitoring program also used existing PNNL air samplers in the 300 and 400 Areas. Air samplers were located primarily at or near (within approximately $500 \mathrm{~m}[1,500 \mathrm{ft}]$ ) sites and/or facilities having the potential for, or history of, environmental releases, with an emphasis on the prevailing downwind direction.

Samples were collected according to a schedule established before the monitoring year. Airborne particles were sampled at each of these stations by drawing air through a glass-fiber filter. The filters were collected biweekly, field surveyed for gross radioactivity, held for at least seven days, and then analyzed for total alpha and beta activity. The seven-day holding period was necessary to allow for the decay of naturally occurring radionuclides that would otherwise obscure detection of longer-lived radionuclides associated with emissions from nuclear facilities. The total radioactivity measurements were used to indicate changes in trends in the near-facility environment.

For most radionuclides, the amount of radioactive material collected on a single filter during a two week sampling period was too small to be measured accurately. The accuracy of the sample analysis was increased by compositing the samples into biannual samples for each location. Composite samples were analyzed for site specific radionuclides including ${ }^{90} \mathrm{Sr},{ }^{234} \mathrm{U},{ }^{235} \mathrm{U},{ }^{238} \mathrm{U}$, ${ }^{238} \mathrm{Pu},{ }^{239,240} \mathrm{Pu},{ }^{241} \mathrm{Pu},{ }^{241} \mathrm{Am}$, and gamma-emitting radionuclides (e.g., $\left.{ }^{137} \mathrm{Cs},{ }^{60} \mathrm{Co}\right)$. A more detailed discussion is available in Section 2.0 .

\section{Results}

Of the radionuclide analyses performed, ${ }^{137} \mathrm{Cs},{ }^{239,240} \mathrm{Pu},{ }^{90} \mathrm{Sr}$, and uranium were consistently detectable in the $100 \mathrm{~N}$ and 200 Areas. ${ }^{60} \mathrm{Co}$ was consistently detectable in the $100 \mathrm{~N}$ Area. Air concentrations for these radionuclides were elevated near facilities compared to the concentrations 
measured offsite. Figure S-1 shows average values for 1997 and the preceding five years for selected radionuclides compared to DOE derived concentration guides (DCGs) and the background air concentration as measured by the PNNL in distant communities. The DOE DCGs (DOE Order 5400.5) are reference values that are used as indexes of performance (Appendix C, Table C.5). The data indicate a large degree of variability. In general, air samples collected from samplers located at, or directly adjacent to, nuclear facilities had higher concentrations than did those samples collected farther away. The data also show that concentrations of certain radionuclides were higher within different operational areas. Generally, the predominant radionuclides are activation and fission products in the 100 Areas and fission products in the 200 Areas.

100 B,C Area. Near-facility air sampling was conducted at the $100 \mathrm{~B}, \mathrm{C}$ remediation site through a network of three continuous air samplers. Monitoring began in July 1996, and the analytical results indicated that the concentrations were much less than the DOE DCGs and were comparable to or only slightly greater than levels measured offsite.

100 D,DR Area. Near-facility air sampling was conducted at the $100 \mathrm{D}, \mathrm{DR}$ remediation site through a network of four continuous air samplers. Monitoring began in November 1996, and the analytical results indicated that the concentrations were much less than the DOE DCGs and were comparable to or only slightly greater than levels measured offsite.

$100 \mathrm{~K}$ Area. Analytical results from $100 \mathrm{~K}$ Area ambient air samples show quantities of ${ }^{90} \mathrm{Sr},{ }^{137} \mathrm{Cs}$, ${ }^{239,240} \mathrm{Pu},{ }^{241} \mathrm{Pu}$, and ${ }^{241} \mathrm{Am}$ that were above detection levels. These levels were much less than the DOE DCGs; however, they were greater than levels measured offsite. Facility emissions decreased substantially in 1996, and radionuclide concentrations seen in the ambient air samples were near detection limits. The 1997 results did not differ significantly from those measured in 1996.

$100 \mathrm{~N}$ Area. Analytical results from ambient air samples taken in the $100 \mathrm{~N}$ Area continued to be at or near Hanford Site background concentrations for most radionuclides as a result of facility shutdowns and improved effluent controls and waste management practices. Concentrations were much less than the DOE DCGs; however, they were slightly higher than levels measured offsite.

200 Areas. Analytical results from ambient air samples taken in the 200 Areas were at or near Hanford Site background concentrations for most radionuclides as a result of faciiity shutdowns, better effluent controls, and improved waste management practices. Although levels were much less than the DOE DCGs, they were greater than those measured offsite. Levels of ${ }^{90} \mathrm{Sr},{ }^{239,240} \mathrm{Pu}$, and uranium were comparable to those measured in the $100 \mathrm{~N}$ Area.

Environmental Restoration Disposal Facility. Near-facility air sampling was conducted at the ERDF remediation site through a network of continuous air samplers. This network utilized two existing Hanford Site monitors for upwind monitoring and was supplemented by three additional air monitors that provided downwind monitoring. Monitoring began in June 1996, and the analytical results indicated that the concentrations were much less than the DOE DCGs and only slightly greater than levels measured offsite.

300-FF-1 Project. Near-facility air sampling was conducted at the 300-FF-1 remediation site through a network of continuous air samplers. This network utilized one existing Hanford Site monitor for upwind monitoring and was supplemented by three additional air monitors that provided downwind monitoring. Monitoring began in May 1997, and the analytical results indicated that the concentrations were much less than the DOE DCGs and only slightiy greater than levels measured offsite. 
Figure S-1. Average concentrations ( \pm 2 standard error of the mean) of selected radionuclides in near-facility air samples compared to those in distant communities, 1992 through 1997. As a result of figure scale, some uncertainties (error bars) are concealed by point symbols. ${ }^{60} \mathrm{Co}$ was not detected in the $100 \mathrm{~K}$ and 200 Areas in 1997.
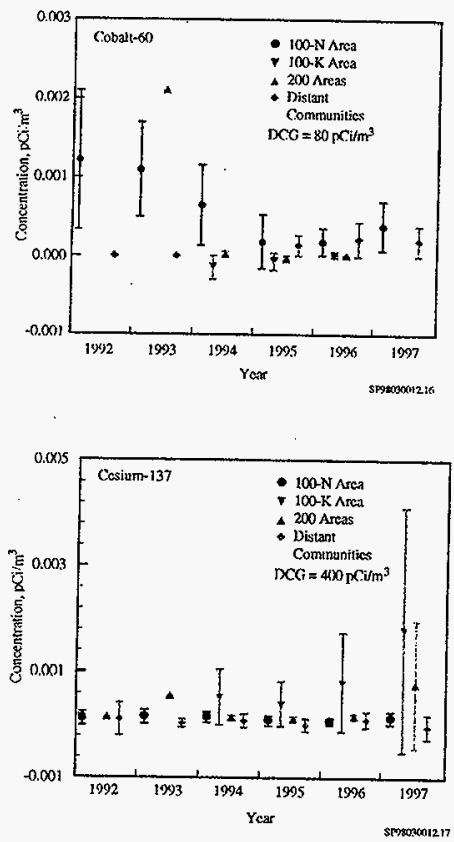
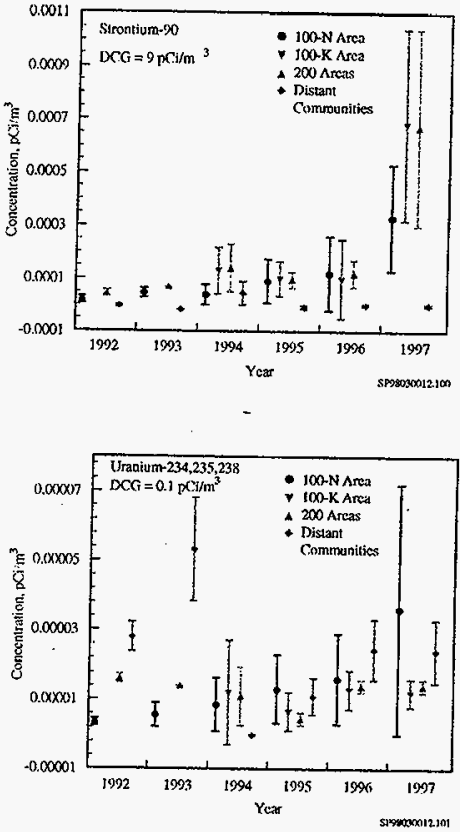

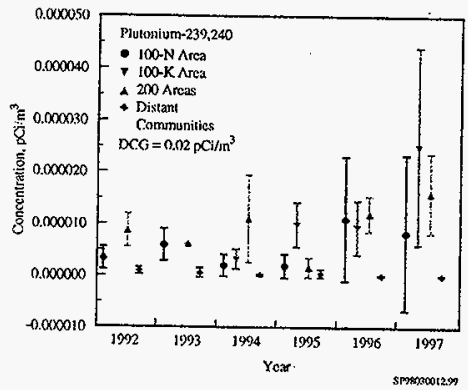


3732 Building ( 300 Area). Near-facility air sampling was conducted during the demolition of the 3732 Building in the 300 Area through the use of two continuous air samplers. These stations provided downwind monitoring during the two week demolition period during September 1997. and the analytical results indicated that the concentrations were much less than the DOE DCGs and only slightly greater than levels measured offsite.

\section{GROUNDWATER MONITORING}

Currently, the only groundwater wells that are monitored and reported through the near-facility monitoring program are wells $\mathrm{N}-47$ and $\mathrm{N}-48$ located in the $100 \mathrm{~N}$ Area. Samples are collected monthly and analyzed for gamma-emitting radionuclides. The analytical results for all samples collected from these wells indicate that concentrations of gamma-emitting radionuclides are below DOE DCGs and U.S. Environmental Protection Agency (EPA) Interim Drinking Water Standards.

\section{SOIL AND VEGETATION SAMPLING}

Soil and vegetation samples were collected on or adjacent to waste disposal units, and from locations downwind and near or within the boundaries of the operating facilities. Samples were collected to detect potential migration and deposition of facility effluents. Special samples were also taken where physical or biological transport problems were identified. Migration of radionuclides can occur as the result of resuspension from radioactively contaminated surface areas, absorption by the roots of vegetation growing on or near underground and surface water disposal units, or intrusion by animals. The results of the 1997 sampling effort are discussed below.

\section{Collection of Soil and Vegetation Samples and Analytes of Interest}

Sampling methods and locations are discussed in detail in Waste Management Federal Services, Inc., Northwest Operations Operational Environmental Monitoring, WMNW-CM-004. Radiological analyses of soil and vegetation samples included ${ }^{90} \mathrm{Sr},{ }^{239.240} \mathrm{Pu}$, uranium, and gamma-emitting radionuclides.

\section{Radiological Results for Soil Samples}

Of the radionuclide analyses performed, ${ }^{60} \mathrm{Co},{ }^{90} \mathrm{Sr},{ }^{137} \mathrm{Cs},{ }^{239,240} \mathrm{Pu}$, and uranium were consistently detectable. Soil concentrations for these radionuclides were elevated near and within facility boundaries when compared to concentrations measured offsite in 1997. Figure S-2 shows average soil values for 1997 and the preceding five years. The concentrations show a large degree of variability. In general, concentrations in samples collected on or directly adjacent to waste disposal facilities were higher than concentrations in samples collected farther away and significantly higher than historical concentrations measured offsite. The data also show, as expected, that concentrations of certain radionuclides were higher within different operational areas when compared to concentrations measured in distant communities. Generally, the predominant radionuclides were activation products and ${ }^{90} \mathrm{~S} r$ in the $100 \mathrm{~N}$ Area, fission products in the 200 Areas, and uranium in the 300 Area. 
Figure S-2. Average concentrations ( \pm 2 standard error of the mean) of selected radionuclides in near-facility soil samples compared to those in samples distant communities, 1992 through 1997. As a result of figure scale, some uncertainties (error bars) are concealed by point symbols. The 1994, 1995, 1996, and 1997 100 Areas data include the $100 \mathrm{~N}$ Area only.
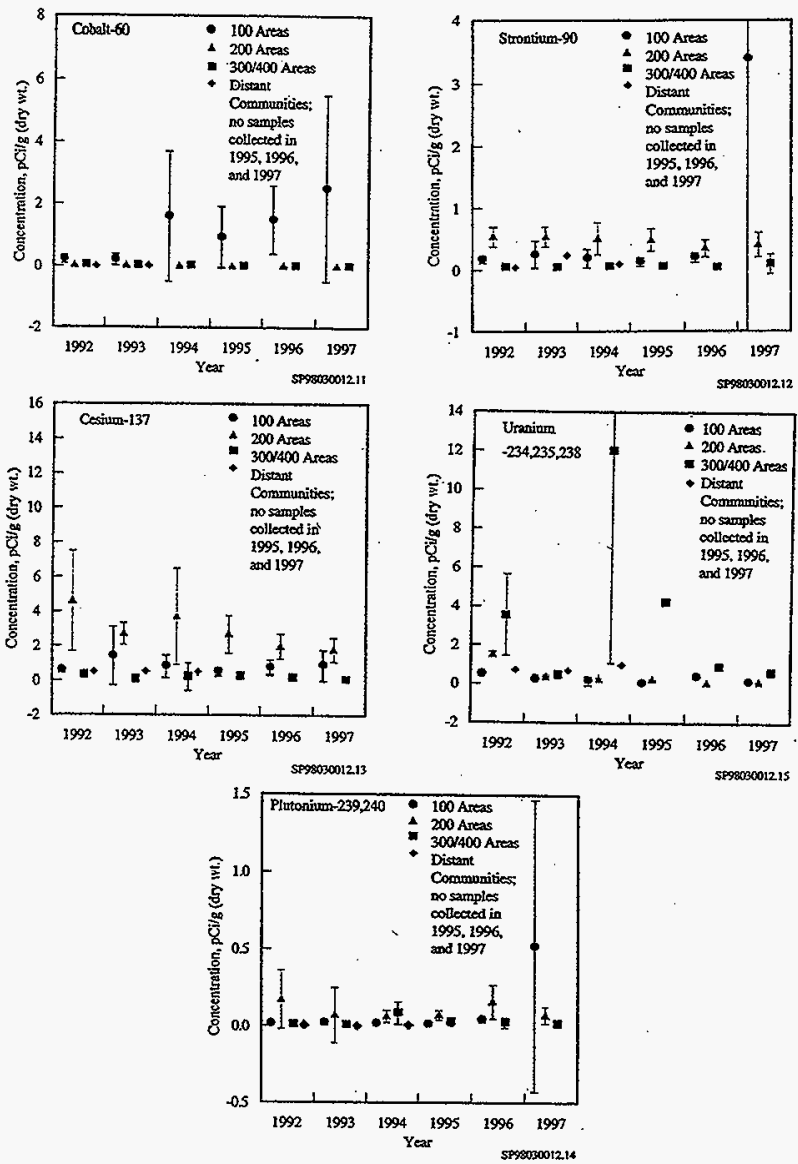
$100 \mathrm{~N}$ Area. The analytical results from soil samples collected in the $100 \mathrm{~N}$ Area in 1997 generally exhibited concentrations at or near historical onsite levels. However, concentrations of ${ }^{50} \mathrm{Co},{ }^{90} \mathrm{Sr}$, ${ }^{238} \mathrm{Pu}$, and ${ }^{239,240} \mathrm{Pu}$ were noticeably elevated at a sampling location near the retired $1301-\mathrm{N}$ liquid waste disposal facility (LWDF). With the exception of uranium, contamination levels were greater than those measured offsite. The average concentrations of ${ }^{60} \mathrm{Co}$ and ${ }^{239,240} \mathrm{Pu}$ were greater than those measured in the 200 and $300 / 400$ Areas. The ${ }^{60} \mathrm{Co},{ }^{90} \mathrm{Sr}$, and ${ }^{239,240} \mathrm{Pu}$ concentrations in the $100 \mathrm{~N}$ Area soils resulted from past discharges to waste disposal structures, primarily the 1301-N LWDF.

200 Areas. Analytical results from soil samples taken in the 200 Areas were on a downward trend for most radionuclides as a result of facility shutdowns, better effluent controls, and improved waste management practices. However, for ${ }^{90} \mathrm{Sr},{ }^{137} \mathrm{Cs}$, and ${ }^{239.240} \mathrm{Pu}$, the results were greater than those measured offsite and in the $300 / 400$ Areas. The average concentrations of ${ }^{90} \mathrm{Sr}$ and ${ }^{137} \mathrm{Cs}$ were higher than those measured in the $100 \mathrm{~N}$ Area.

$300 / 400$ Areas. Analytical results from soil samples taken in the 300/400 Areas were compared to results for other operational areas and to those measured offsite. Uranium levels for the $300 / 400$ Areas were higher than those measured offsite, from the 100 and 200 Areas, and slightly lower than levels measured at the same locations in 1996. Uranium was expected in these samples because it was used during past fuel fabrication operations in the 300 Area.

\section{Vegetation Results}

Of the radionuclide analyses performed, ${ }^{60} \mathrm{Co},{ }^{90} \mathrm{Sr},{ }^{137} \mathrm{Cs},{ }^{239,240} \mathrm{Pu}$, and uranium were consistently detectable. Concentrations of these radionuclides in vegetation were elevated near and within facility boundaries compared to the concentrations measured offsite in 1997. Figure S-3 shows average vegetation values for 1997 and the preceding five years. The concentrations show a large degree of variability. In general, concentrations in samples collected on or directly adjacent to the waste disposal facilities were higher than concentrations in samples collected farther away. As with the soil samples, the data show that certain radionuclides were found in higher concentrations in vegetation within different operational areas when compared to concentrations measured in distant communities in 1997. The predominant radionuclides are generally activation and fission products in the 100 Areas, fission products in the 200 Areas, and uranium in the 300 Area.

$100 \mathrm{~N}$ Area. Analytical results from vegetation samples collected in the $100 \mathrm{~N}$ Area in 1997 were comparable to those seen in the past. The values observed for ${ }^{90} \mathrm{Sr}$ in samples collected near the $\mathrm{N}$ Springs were typically higher than those seen at other locations in the $100 \mathrm{~N}$ Area. Generally, 1997 radionuclide levels in $100 \mathrm{~N}$ Area vegetation were greater than those measured offsite and levels for ${ }^{60} \mathrm{Co},{ }^{90} \mathrm{Sr}$, and ${ }^{137} \mathrm{Cs}$ were higher compared to the concentrations measured in the 200 and $300 / 400$ Areas.

200 Areas. Analytical results from vegetation samples taken in 1997 in the 200 Areas were comparable to those seen in 1996. Radionuclide concentrations were greater than those measured offsite and were higher for ${ }^{239.240} \mathrm{Pu}$ compared to the 100 and $300 / 400$ Areas. 
Figure S-3. Average concentrations ( \pm standard error of the mean) of selected radionuclides in near-facility vegetation samples compared to those in samples in distant communities, 1992 through 1997. As a result of figure scale, some uncertainties (error bars) are concealed by point symbols. The 1994, 1995, 1996, and 1997100 Areas data include the $100 \mathrm{~N}$ Area only. The $1997{ }^{137} \mathrm{Cs}$ data point for the $300 / 400$ Areas is less than 0 and cannot be plotted on a log scale.
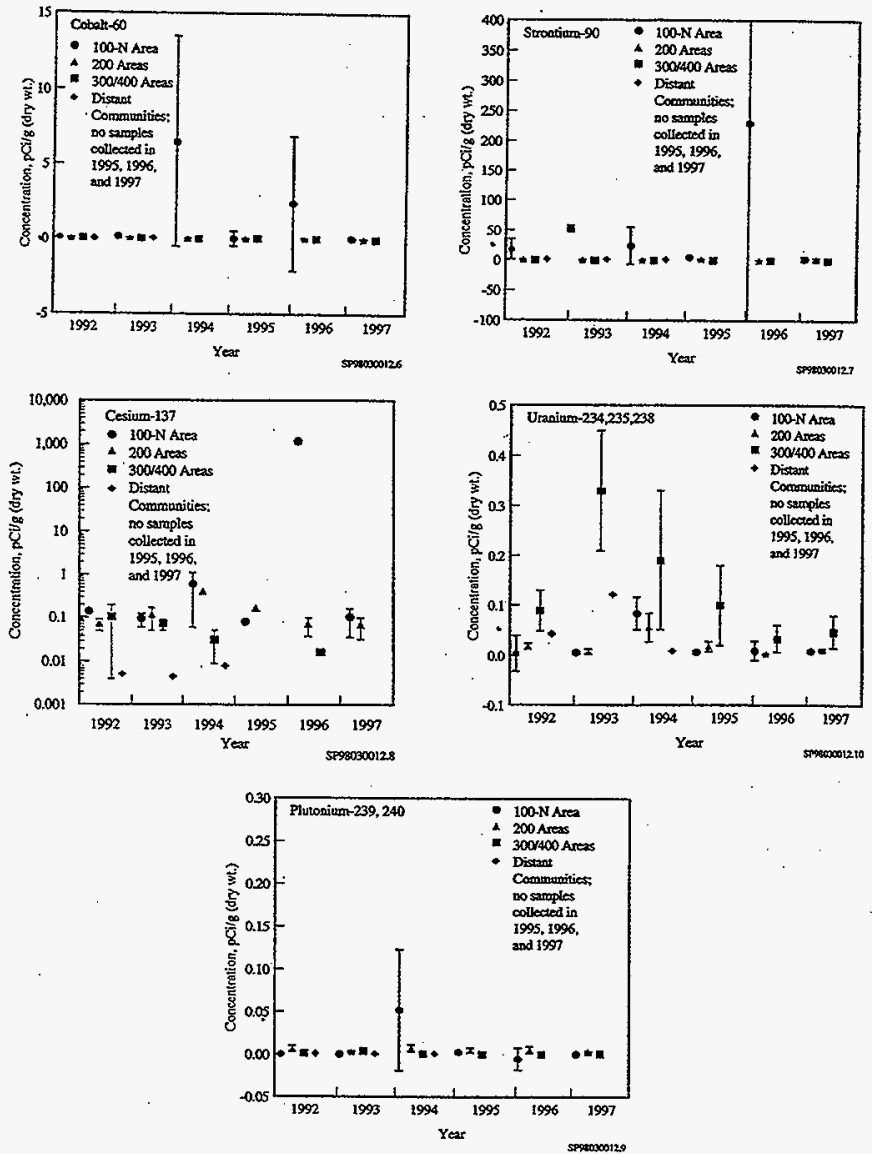
300/400 Areas. Generally, the levels of most radionuclides measured in the 300 Area were greater than those measured offsite, and uranium levels were higher than levels measured in the 100 and 200 Areas. The higher uranium levels were expected because uranium was released during past fuel fabrication operations in the 300 Area. The levels recorded in the 400 Area were at or near those measured offsite.

\section{EXTERNAL RADIATION}

External radiation fields were monitored near facilities and waste handling, storage, and disposal sites to measure, assess, and control the impacts of operations.

\section{Radiological Field Measurements}

Two methods are used for measuring external radiation fields. Hand-held meters are used at individual points of interest to give real-time assessments. Thermoluminescent dosimeters (TLDs) are used at numerous fixed locations to gather dose rate information over longer periods of time. TLD results can be used individually or averaged to determine dose rates in a given area for a particular sampling period. Specific information about external radiation sampling methods and locations can be found in WMNW-CM-004. A summary of the results of the TLD readings is presented in Table S-2.

Table S-2. Thermoluminescent Dosimeter Results for Nuclear Facilities in the Operations Areas 1996 and 1997 (mrem/yr, based on $24 \mathrm{~h} / \mathrm{day}$ ).

\begin{tabular}{|c|c|c|c|c|c|c|}
\hline \multirow[t]{2}{*}{ Area } & \multirow{2}{*}{$\begin{array}{c}\text { Number of } \\
\text { locations, } 1997\end{array}$} & \multicolumn{2}{|c|}{1996} & \multicolumn{3}{|c|}{1997} \\
\hline & & Maximum & Mean & Maximum & Mean & $\%$ change $^{(a)}$ \\
\hline $100 B, C^{(b)}$ & 4 & NA & NA & 96 & 93 & NA \\
\hline $100 \mathrm{D}, \mathrm{DR}$ & 5 & 92 & 88 & 91 & 88 & 0 \\
\hline $100 \mathrm{~K}$ & 11 & 2,250 & 480 & 2,250 & 470 & -2 \\
\hline $100 \mathrm{~N}$ & 22 & 9,200 & 1,500 & 7,700 & 1,250 & -15 \\
\hline $200 / 600$ & 63 & 500 & 120 & 350 & 110 & -8 \\
\hline TWRS $^{(b)}$ & 10 & NA & NA & 81 & 78 & NA \\
\hline ERDF & 3 & 100 & 100 & 100 & 95 & -7 \\
\hline 300 & 8 & 240 & 120 & 200 & 110 & -8 \\
\hline 300 TEDF & 6 & 87 & 85 & 87 & 82 & -4 \\
\hline 400 & 7 & 92 & 83 & 88 & 86 & 3 \\
\hline
\end{tabular}

EROF = Environmental Restoration Disposal Facility.

NA $=$ Not applicable.

TEDF = Treated Effluent Disposal Facility.

TWRS $=$ Tank Waste Remediation System.

${ }^{2}$ Numbers indicate a decrease (-) or increase from the 1996 mean.

'Thermoluminescent dosimeter network was established during the fourth quarter of 1997. 
Results of Radiological Field Measurements

Radiation Surveys. A hand-held micro-rem meter was used to survey points along the $100 \mathrm{~N}$ Area shoreline (N Springs). Radiation measurements were taken at a height of approximately $1 \mathrm{~m}$ $(3.28 \mathrm{ft})$. Figure $\mathrm{S}-4$ shows the overall shape of the curve for 1997 , which indicates that $\mathrm{N}$-Springs shoreline areas with the highest exposure rate are, as in the past, juxtapositional with the 1301-N LWDF.

\section{Thermoluminescent Dosimeters}

100 B,C Area. Four new TLD monitoring sites were established in the 100 B,C Area during the fourth quarter of 1997 to evaluate environmental restoration activities. Because only 27 days of data were collected at these sites during 1997, the TLD results were extrapolated to one year, resulting in an average of $93 \mathrm{mrem} / \mathrm{yr}$, which is comparable to offsite ambient background.

100 D,DR Area. This is the second year that TLDs have been placed in the 100 D,DR Area to evaluate environmental restoration activities. Average dose rates measured at these locations in 1997 were approximately $88 \mathrm{mrem} / \mathrm{yr}$, comparable to the 1996 results and offsite ambient background levels.

$100 \mathrm{~K}$ Area. This is the fifth year that TLDs have been placed in the $100 \mathrm{~K}$ Area surrounding the $K$ Basins and adjacent reactor buildings. Three of the TLDs have, as expected, shown consistently elevated readings (ranging from 3.5 to 30 times greater than the overall $100 \mathrm{~K}$ Area average) because of their proximity to radioactive waste storage areas or stored radioactive rail equipment.

$100 \mathrm{~N}$ Area. The 1997 TLD results indicate that direct radiation levels are highest near facilities that had contained or received liquid effluent from $N$ Reactor. These facilities primarily include the 1301-N and 1325-N LWDFs. While the results for these two facilities were noticeably higher than those for other $100 \mathrm{~N}$ Area TLD locations, they were approximately $17 \%$ lower than exposure levels measured at these locations in 1996. Overall, dose rates measured at all locations in the $100 \mathrm{~N}$ Area in 1997 were approximately $15 \%$ lower than those measured in 1996.

200 Areas. The highest dose rates were measured near waste handling facilities such as tank farms. The highest dose rate was measured at the 241-A Tank Farm complex located in the 200 East Area. The average annual dose rate measured in $1997,110 \mathrm{mrem} / \mathrm{yr}$, was $8 \%$ lower than the 1996 measurement.

Tank Waste Remediation System Phase I Demonstration Project. Ten new TLD locations were established around the perimeter of the TWRS Phase I Demonstration Project Site during the fourth quarter of 1997 to collect preconstruction characterization monitoring data. Because only 67 days of data were collected at these sites during 1997, the TLD results were extrapolated to one year, resulting in an average of $78 \mathrm{mrem} / \mathrm{yr}$, which is comparable to offsite ambient background levels.

Environmental Restoration Disposal Facility. This is the second year that TLDs have been placed in the ERDF to evaluate environmental restoration disposal activities. Dose rates measured at these locations were slightly lower than the results of 1996 TLD analyses with an average of $95 \mathrm{mrem} / \mathrm{yr}$, which is comparable to offsite ambient background levels 
Figure S-4. Radiation Survey Measurement Locations at the $100 \mathrm{~N}$ Area Shoreline.

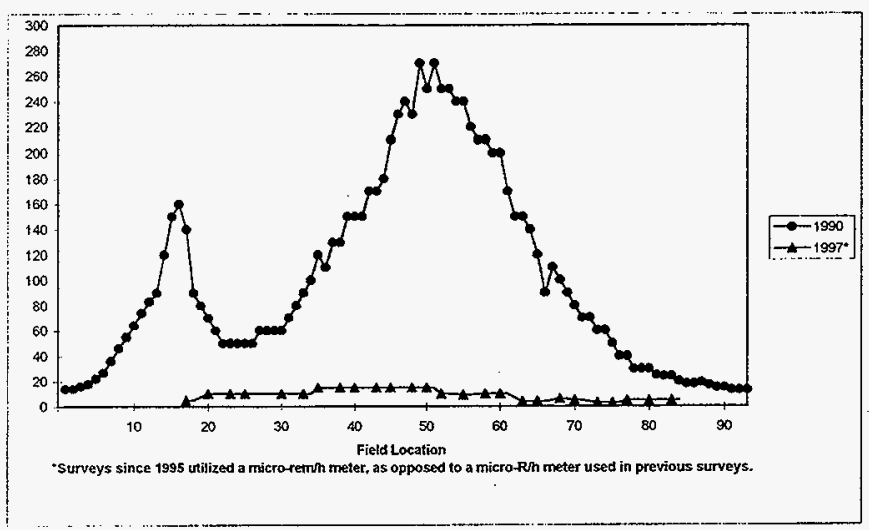

Columbia River

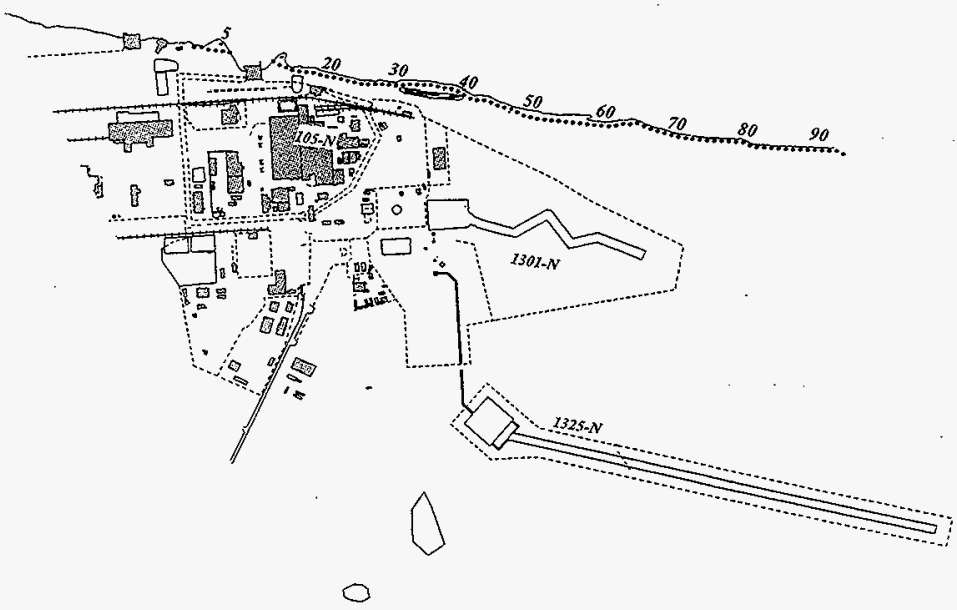


300/300 Treated Effluent Disposal Facility/400 Areas. The highest dose rates in the 300 Area were measured near the 340 Waste Handling Facility. The average annual dose rate measured in the 300 Area in 1997 was $110 \mathrm{mrem} / \mathrm{yr}$, which is a decrease of $8 \%$ compared to the average dose rate of $120 \mathrm{mrem} / \mathrm{yr}$ measured in 1996. The average annual dose rate at the 300 Area TEDF in 1997 was $82 \mathrm{mrem} / \mathrm{yr}$, which is an decrease of $4 \%$ compared to the average dose rate of $85 \mathrm{mrem} / \mathrm{yr}$ measured in 1996. The average annual dose rate measured in the 400 Area in 1997 was $86 \mathrm{mrem} / \mathrm{yr}$, which is an increase of $3 \%$ compared to the average dose rate of $83 \mathrm{mrem} / \mathrm{yr}$ measured in 1996.

\section{SURFACE-WATER DISPOSAL UNITS AND RIVERBANK SPRINGS MONITORING}

Surface water disposal units (open ponds and ditches) used by the operating facilities and springs along the $100 \mathrm{~N}$ Area Columbia River shoreline were monitored to assess the effectiveness of effluent and contamination controls. Two surface water disposal units in the 200 Areas were sampled during 1997: the 200 East Area Powerhouse Ditch and the 216-B-3C Expansion Pond in 200 East Area.

\section{Sample Collection and Analysis}

Samples collected from surface water disposal units included water, sediment, and aquatic vegetation. Only water samples were taken at river shoreline springs. The sampling methods are discussed in detail in WMNW-CM-004. To avoid duplication of sampling, the near-facility environmental monitoring program used surface water sample data collected by the PNNL for the 400 Area. Results for the 400 Area sampling are reported in Section 4.2, "Surface Water and Sediment Surveillance," of the Hanford Site Environmental Report for Calendar Year 1997 (Dirkes and Hanf 1998).

Radiological analyses of water samples from surface water disposal units were performed onsite at the Waste Sampling and Characterization Facility (WSCF) (near the 200 West Area) in 1997. Analyses included uranium, tritium, ${ }^{90} \mathrm{Sr},{ }^{238} \mathrm{Pu},{ }^{239,240} \mathrm{Pu}$, and gamma-emitting radionuclides. Radiological analyses of sediment and aquatic vegetation samples were performed for uranium, ${ }^{90} \mathrm{Sr},{ }^{239}, 240 \mathrm{Pu}$, and gamma-emitting radionuclides. Nonradiological analyses were performed for $\mathrm{pH}$, temperature, and nitrates. Analytes of interest were selected based on their presence in effluent discharges and their importance in verifying effluent control and determining compliance with applicable effluent discharge standards. Surface water disposal units that received potentially radioactively contaminated effluents were within posted radiological control areas. A more detailed discussion is available in Section 6.0.

\section{Radiological Results}

Surface Water Disposal Units. Radiological results for liquid samples from surface water disposal units (ponds and ditches) located in the 200 Areas are summarized in Table S-3. In all cases, radionuclide concentrations in surface-water disposal units were less than the DOE DCGs.

Radiological results for aquatic vegetation and sediment samples taken from surface water disposal units located in the 200 Areas are summarized in Tables S.4 and S-5, respectively. Although there were some elevated levels in both aquatic vegetation and sediment, in all cases the results were much less than the standards cited in the Hanford Site Radiological Control Manual (HSRCM-1). 
Table S-3. Radiological Results for Liquid Samples from Surface Water Disposal Units, 200 Areas, 1997 (pCi/L).

\begin{tabular}{|c|c|c|c|c|c|c|c|c|}
\hline $\begin{array}{l}\text { Sample } \\
\text { location }^{(a)}\end{array}$ & $\begin{array}{c}\text { Number } \\
\text { of } \\
\text { samples }\end{array}$ & & ${ }^{238} \mathrm{Pu}$ & ${ }^{239 / 240} \mathrm{Pu}$ & rotal U & ${ }^{3} \mathrm{H}^{(0)}$ & ${ }^{90} \mathrm{Sr}$ & ${ }^{137} \mathrm{Cs}$ \\
\hline \multirow{2}{*}{$\begin{array}{l}200 \text { East } \\
\text { Area Ditch }\end{array}$} & \multirow[t]{2}{*}{12} & Mean & $6.4 \mathrm{E}-03$ & $1.4 \mathrm{E}-\mathrm{0} 2$ & $4.7 \mathrm{E}-01$ & ND & 3.0 E-01 & ND \\
\hline & & Max & $3.6 \mathrm{E}-\mathrm{O} 2$ & 7.3 E-02 & $5.8 \mathrm{E}-01$ & ND & $3.0 \mathrm{E}+00$ & ND \\
\hline \multirow{3}{*}{$\begin{array}{l}200 \text { East } \\
\text { Area Pond }\end{array}$} & \multirow[t]{3}{*}{9} & Mean & ND & ND & 6.5 E-01 & ND & ND & ND \\
\hline & & Max & ND & ND & 9.9 E-01 & ND & ND & ND \\
\hline & & $D C G$ & $4.0 E+01$ & $3.0 E+01$ & $5.0 E+02^{(c)}$ & $2.0 E+06$ & $1.0 \mathrm{E}+03$ & $3.0 E+03$ \\
\hline
\end{tabular}

DCG $=$ Derived concentration guide.

ND $=$ Not detected.

200 East Area Pond: $216-8-3 \mathrm{C}$ Pond.

${ }^{0}$ The detection limit for ${ }^{3} \mathrm{H}$ is $300 \mathrm{pCi} / \mathrm{L}$.

Using ${ }^{234} \mathrm{U}$ as the most limiting $\mathrm{DCG}$.

Table S-4. Radiological Results for Aquatic Vegetation Samples from Surface Water Disposal Units (pCi/g, dry wt), 200 Areas, 1997.

\begin{tabular}{|l|c|c|c|c|c|c|c|}
\hline Sample location $^{(a)}$ & $\begin{array}{c}\text { Number of } \\
\text { samples }\end{array}$ & ${ }^{90} \mathrm{Sr}$ & ${ }^{137} \mathrm{Cs}$ & ${ }^{239.240} \mathrm{Pu}$ & ${ }^{234} \mathrm{U}$ & ${ }^{235} \mathrm{U}$ & ${ }^{238} \mathrm{U}$ \\
\hline 200 East Area Pond & 1 & $3.4 \mathrm{E}-01$ & $3.8 \mathrm{E}-01$ & $3.8 \mathrm{E}-03$ & $1.7 \mathrm{E}-02$ & $8.0 \mathrm{E}-03$ & $8.5 \mathrm{E}-03$ \\
\hline 200 East Area Ditch & 1 & $3.7 \mathrm{E}-01$ & $\mathrm{ND}$ & ND & $1.3 \mathrm{E}-02$ & $9.9 \mathrm{E}-03$ & $7.8 \mathrm{E}-03$ \\
\hline
\end{tabular}

ND $=$ Not detected.

200 East Area Pond - 216-B-3C Pond.

200 East Area Ditch - Powerhouse Ditch.

Table S-5. Radiological Results for Sediment Samples from Surface Water Disposal Units (pCi/g, dry wt), 200 Areas, 1997.

\begin{tabular}{|l|c|c|c|c|c|c|c|}
\hline Sample location & $\begin{array}{c}\text { Number of } \\
\text { samples }\end{array}$ & ${ }^{90} \mathrm{Sr}$ & ${ }^{137} \mathrm{Cs}$ & ${ }^{239.240} \mathrm{Pu}$ & ${ }^{234} \mathrm{U}$ & ${ }^{235} \mathrm{U}$ & ${ }^{238} \mathrm{U}$ \\
\hline 200 East Area Pond & 1 & $7.1 \mathrm{E}-01$ & $1.1 \mathrm{E}+01$ & $2.0 \mathrm{E}-01$ & $3.3 \mathrm{E}-01$ & $6.0 \mathrm{E}-\mathrm{O} 2$ & $2.9 \mathrm{E}-01$ \\
\hline 200 East Area Ditch & 1 & $\mathrm{ND}$ & $\mathrm{ND}$ & $4.9 \mathrm{E}-\mathrm{O} 3$ & $3.8 \mathrm{E}-01$ & $2.2 \mathrm{E}-\mathrm{O} 2$ & $3.9 \mathrm{E}-\mathrm{O} 1$ \\
\hline
\end{tabular}

ND $=$ Not detected.

200 East Area Pond - 216-B-3C Pond.

200 East Area Ditch - Powerhouse Ditch 
Riverbank Springs. In the past, radioactive effluent streams sent to the $1301-\mathrm{N}$ and $1325-\mathrm{N}$ LWDFs in the $100 \mathrm{~N}$ Area contributed to the release of radionuclides to the Columbia River through their migration with the groundwater. Radionuclides enter the Columbia River along the riverbank region known as $\mathrm{N}$ Springs. Releases into the river at $\mathrm{N}$ Springs are calculated based on analysis of monthly samples collected from monitoring well 199-N-46 located near the shoreline. A more detailed discussion of the release calculations may be found in Environmental Releases for Calendar Year 1997 (Gleckler 1998).

Groundwater springs along the $100 \mathrm{~N}$ Area shoreline are sampled annually to verify that the reported radionuclide releases to the Columbia River are conservative (i.e., not under reported). To verify releases, conservatively high radionuclide concentrations in samples collected from well 199-N-46 are used and multiplied by the estimated groundwater discharge into the river. The N Springs groundwater flow rate was estimated using a computer model developed by Gilmore et al. (1992). The estimated groundwater flow rate used to calculate 1997 releases from N Springs was $43 \mathrm{~L} / \mathrm{min}(11 \mathrm{gal} / \mathrm{min})$. The results of characterizing the radionuclide concentrations in the springs along the shoreline can then be compared to the concentrations measured in well 199-N-46 to ensure that the well is located in the groundwater migration route that has the highest concentrations of radionuclides.

In 1997, the concentrations of tritium and ${ }^{90} \mathrm{Sr}$ detected in samples from shoreline springs were highest in springs nearest well $199-\mathrm{N}-46$. The highest ${ }^{60} \mathrm{Co}$ concentrations, although very low, were from a location approximately $200 \mathrm{~m}$ down-river (northeast) of 199-N-46. All of the concentrations were lower than those measured in the well. The data from shoreline springs sampling are summarized in Table S-6.

Table S-6. Radiological Results for Water Samples from $100 \mathrm{~N}$ Area Columbia River Shoreline Springs, $1997(\mathrm{pCi} / \mathrm{L})$.

\begin{tabular}{|c|c|c|c|c|}
\hline \multirow{2}{*}{ Radionuclide } & \multirow{2}{*}{$\begin{array}{c}\text { Facility effluent } \\
\text { monitoring well } \\
\end{array}$} & \multicolumn{3}{|c|}{ Shoreline springs } \\
\cline { 3 - 5 } & $(09 / 97)$ & Maximum & Mean & DCG \\
\hline${ }^{3} \mathrm{H}$ & $1.6 \mathrm{E}+04$ & $3.0 \mathrm{E}+03$ & $2.1 \mathrm{E}+02$ & $2.0 \mathrm{E}+06$ \\
\hline${ }^{90} \mathrm{Sr}$ & $1.1 \mathrm{E}+04$ & $3.2 \mathrm{E}+03$ & $3.5 \mathrm{E}+02$ & $1.0 \mathrm{E}+03$ \\
\hline
\end{tabular}

DCG $=$ Derived concentration guide.

\section{Nonradiological Results for Surface-Water Disposal Units}

Nonradiological analytical results for water samples collected from surface water disposal units located in the 200 Areas are shown in Table S-7. The results for $\mathrm{pH}$ were well within the Resource Conservation and Recovery Act of 1976 corrosivity designation limits of 2.0 to 12.5 . The analytical results for nitrates were all less than the $45 \mathrm{mg} / \mathrm{L}$ Drinking Water Standard for public water supplies. 
Table S-7. Nonradiological Results for Water Samples from Surface Water Disposal Units, 200 Areas, 1997.

\begin{tabular}{|c|c|c|c|c|c|c|c|}
\hline \multirow{2}{*}{$\begin{array}{c}\text { Sample } \\
\text { locations }^{(a)}\end{array}$} & \multirow{2}{*}{$\begin{array}{c}\text { Number of } \\
\text { samples }\end{array}$} & \multicolumn{3}{|c|}{$\mathrm{pH}$} & \multirow{2}{*}{$\begin{array}{c}\text { Number of } \\
\text { samples }\end{array}$} & \multicolumn{2}{|c|}{ Nitrate $\left(\mathrm{NO}_{3}\right), \mathrm{mg} / \mathrm{L}$} \\
\hline & & Mean & $\operatorname{Max}$ & Min. & & Mean & Max \\
\hline 200 East Area Ditch & 52 & 8.8 & 9.3 & 8.0 & 4 & $1.7 \mathrm{E}-01$ & 2.4 E-01 \\
\hline 200 East Area Pond & 36 & 8.4 & 8.9 & 7.9 & 3 & $2.2 \mathrm{E}-01$ & $3.7 E-01$ \\
\hline
\end{tabular}

"200 East Area Ditch - Powerhouse Ditch.

200 East Area Pond - 216-B-32.

\section{RADIOLOGICAL SURVEYS}

Radiological surveys are used to monitor and detect radiological contamination on the Hanford Site. The two main types of posted radiological controlied areas are underground radioactive materials (URMs) and contamination areas. Contamination areas include contamination areas, soil contamination areas, and high contamination areas.

URM areas are posted areas with contamination contained below the soil surface. These areas are typically "stabilized" cribs, burial grounds, and covered ponds, trenches, and ditches. Barriers over the contamination sources are used to inhibit radionuclide transport to the surface environs. These areas are surveyed at least annually to document the current radiological status.

Contamination/soil contamination areas may or may not be associated with a URM structure. A breech in the barrier of a URM area may result in the growth of contaminated vegetation. Insects or animals may burrow into a URM area and bring contamination to the surface. Vent pipes or risers from an underground structure may be a source of speck contamination. Fallout from stacks or unplanned releases from previously operating facilities may cause an area of contamination that is not related to a subsurface structure. All types of contamination areas may be susceptible to contamination migration. All known contamination areas were surveyed at least annually to document the current radiological status.

In 1997, the Hanford Site had approximately 3,990 ha $(9,859$ acres $)$ of posted outdoor contamination areas and 614 ha $\{1,517$ acres $)$ of posted URM areas not including active facilities. The number of hectares (acres) of contamination areas is approximately six times larger than the URM areas. This is primarily because of the BC Cribs Controlled Area located south of the 200 East Area. This area was initially posted as a Radiologically Controlled Area in 1958 because of widespread speck contamination and encompassed approximately 1,000 ha $(2,500$ acres $)$. Additional investigative surveys were conducted adjacent to the BC Controlled Area in 1996 and the area was enlarged to 3,832 ha $(9,469$ acres). Table S- 8 lists the acreage for contamination areas and URM areas in 1997. A global positioning system was used in 1996 and 1997 to measure the surface areas more accurately than in previous years. Area measurements are entered into the Hanford Geographical Information System (HGIS), a computer database maintained by the Environmental Restoration Contractor (ERC). 
Table S-8. Outdoor Contamination Status, 1997 (approximate surface area in hectares [acres]).

\begin{tabular}{|c|c|c|}
\hline Hanford Site area & Contamination area ${ }^{(\mathrm{b})}$ & $\begin{array}{l}\text { Underground radioactive } \\
\text { material }^{(t)}\end{array}$ \\
\hline $100 \mathrm{~B}, \mathrm{C}$ & $8(20)$ & $39(96)$ \\
\hline $100 \mathrm{D}, \mathrm{DR}$ & $0.1(0.2)$ & $39(96)$ \\
\hline $100 \mathrm{~F}$ & $0.7(1.7)$ & $33(82)$ \\
\hline $100 \mathrm{H}$ & $0.1(0.2)$ & $14(35)$ \\
\hline $100 \mathrm{~K}$ & $9(22)$ & $62(153)$ \\
\hline $100 \mathrm{~N}$ & $29(73)$ & $0.2(0.5)$ \\
\hline 200 East $^{(\mathrm{c})}$ & $62(153)$ & $139(343)$ \\
\hline 200 West $^{(\mathrm{c})}$ & $30(74)$ & $221(546)$ \\
\hline 300 & $19(47)$ & $13(32)$ \\
\hline 400 & 0 & 0 \\
\hline $600^{(d)}$ & $3,832(9,469)$ & $54(133)$ \\
\hline Total & $3,990(9,859)$ & $614(1,517)$ \\
\hline \multicolumn{3}{|c|}{$\begin{array}{l}\text { "Includes areas posted as "contamination/soil contamination" or as "Radiologically } \\
\text { Controlled" and areas that had both underground and contamination/soil contamination. } \\
\text { "Includes areas with only underground contamination. Does not include areas that had } \\
\text { contamination/soil contamination as well as underground radioactive material. } \\
\text { "Includes tank farms. } \\
\text { "Includes redesignated BC Controlled Area (historicaliy included in } 200 \text { Area acreage } \\
\text { estimates), waste disposal facilities outside the } 200 \text { East Area boundary that received waste } \\
\text { from } 200 \text { East Area facilities (e.g., 216-A-25, } 216-8-3) \text {; and waste disposal facilities outside the } \\
200 \text { West Area boundary that received waste from } 200 \text { West Area facilities (e.g., 216-9-19, } \\
216-U-11 \text { ). }\end{array}$} \\
\hline
\end{tabular}

The posted contamination areas vary in number and size between years because of an ongoing effort to clean, stabilize, and remediate areas of known contamination. During this time, new areas of contamination are also being identified. Table S-9 indicates the changes resulting from stabilization activities during 1997. Approximately 13.4 ha (33.1 acres) were reclassified from contamination/soil contamination areas to URM areas, 1.9 ha (4.7 acres) were posted as soil contamination areas, and 5.2 ha (12.8 acres) were changed from no posting to URM areas. Newly identified areas may have resulted from contaminant migration or an increased effort to investigate outdoor areas for radiological contamination. Vehicles equipped with radiation detection devices and an ultrasonic ranging and data system have identified areas of contamination that were previously undetected.

It was estimated that the external dose rate at $80 \%$ of the identified outdoor contamination areas was estimated at less than $1 \mathrm{mrem} / \mathrm{h}$, although direct dose rate readings from isolated radioactive specks (a diameter less than $0.6 \mathrm{~cm}[0.25 \mathrm{in}$.) could have been considerably higher. Contamination levels of this magnitude did not significantly add to dose rates for the public or Hanford Site workers in 1997. 
Table S-9. Zone Status Change of Posted Contamination Areas, 1997.

\begin{tabular}{|l|c|c|}
\hline \multicolumn{1}{|c|}{ Location } & Zone change & $\begin{array}{c}\text { Area } \\
\text { ha (acres) }\end{array}$ \\
\hline 100 Areas & CA to URM & $1.7(4.2)$ \\
\hline 200 East Area & CA to URM & $5.6(13.8)$ \\
\hline 200 West Area & CA to URM & $4.1(10.1)$ \\
\hline 300 Area & CA to URM & 0 \\
\hline 400 Area & CA to URM & 0 \\
\hline 600 Area & CA to URM & $2.0(5.0)$ \\
\hline 600 Area & NP to CA & $1.9(4.7)$ \\
\hline 600 Area & NP to URM & $5.2(12.8)$ \\
\hline
\end{tabular}

$\mathrm{CA}=$ Contamination/soil contamination area.

NP $=$ No posting.

$\mathrm{URM}=$ Underground radioactive materials area.

\section{INVESTIGATIVE SAMPLING}

Investigative sampling was conducted in the operations areas to confirm the absence or presence of radioactive and/or hazardous contaminants. Investigative sampling took place near facilities such as storage and disposal sites for at least one of the following reasons:

- To follow up radiological surface surveys that had indicated radioactive contamination was present.

- To conduct preoperational surveys that quantify the radiological/hazardous conditions at a site before facility construction or operation.

- To quantify the radiological condition of a site before remediation.

- To determine if biotic intrusion (e.g., animal burrows or deep-rooted vegetation) had created a potential for contaminants to spread.

- To determine the integrity of waste containment systems.

The maximum concentrations of radioactive isotopes from samples collected during these investigations are included in this summary. Complete results, including counting errors, for these investigations, including field instrument and dose readings, where appropriate, are provided in Section 8.0 of this report.

Generally, the predominant radionuclides discovered during these efforts were activation and fission products and ${ }^{90} \mathrm{Sr}$ in the 100 Areas, fission products in the 200 Areas, and uranium in the 300 Area. Hazardous chemicals generally have not been identified above background levels in preoperational environmental monitoring samples and no special characterization samples were collected in 1997. 


\section{Collection of Investigative Samples and Analytes of Interest}

Investigative samples collected in 1997 included sludge, soil, vegetation (e.g., grass, tumbleweeds, rabbitbrush, and sagebrush), insects (darkling beetles), reptiles (sagebrush lizards), a bird nest, mammal feces (e.g., mouse, rabbit, and coyote), and mammals (e.g., deer mouse, Great Basin pocket mouse, and cottontail rabbit).

Methods for collecting or otherwise obtaining investigative samples are described in WMNW-CM-004. Field monitoring was conducted to detect radioactivity in samples before they were submitted for analysis. Field monitoring results are expressed as disintegrations per minute per 100 square centimeters $\left(\mathrm{dpm} / 100 \mathrm{~cm}^{2}\right)$ when a Geiger-Müeller detector is used or as millirad per hour when an ion chamber is used. Laboratory sample analysis results are generally expressed in picocuries per gram $(\mathrm{pCi} / \mathrm{g})$, except for extremely small samples and then in $\mathrm{pCi}$ per sample (pCi/sample).

\section{Radiological Results for Investigative Samples}

Investigative samples (e.g., soil, vegetation, and animals) were collected where known or suspected radioactive contamination was present or to verify radiological conditions at project sites. In 1997, 30 samples were analyzed for radionuclides and 27 showed measurable levels of contamination. Another 115 environmental contamination incidents were reported during cleanup operations and the material was disposed of without isotopic analysis, although field instrument readings were recorded. Analytical results are provided in Table S-10. Complete results are provided in Section 8.0 and Appendix 1.

Table S-10. Maximum Radionuclide Concentrations in Investigative Samples Collected from the Operational Areas, 1997. (2 sheets total)

\begin{tabular}{|c|c|c|c|}
\hline Sample type & $\begin{array}{c}\text { Collection area } \\
\text { (number of samples) }\end{array}$ & Radionuclides & $\begin{array}{l}\text { Maximum concentration } \\
\mathrm{pCi} / \mathrm{g}\end{array}$ \\
\hline Dried sludge & 200 East Area (2) & $\begin{array}{l}{ }^{60} \mathrm{Co} \\
{ }^{90} \mathrm{Sr} \\
{ }^{137} \mathrm{Cs} \\
{ }^{152} \mathrm{Eu} \\
{ }^{154} \mathrm{Eu} \\
{ }^{155} \mathrm{Eu} \\
{ }^{238} \mathrm{Pu} \\
{ }^{239 / 240} \mathrm{Pu} \\
{ }^{T o t 8 t} \mathrm{U}\end{array}$ & Not detectable \\
\hline Grass & 200 East Area (1) & $\begin{array}{c}{ }^{60} \mathrm{Co} \\
{ }^{90} \mathrm{Sr} \\
{ }^{137} \mathrm{Cs} \\
{ }^{152} \mathrm{Eu} \\
{ }^{154} \mathrm{Eu} \\
{ }^{155} \mathrm{Eu} \\
{ }^{238} \mathrm{Pu} \\
{ }^{239 / 240} \mathrm{Pu} \\
{ }^{70 t e l} \mathrm{U}\end{array}$ & Not detectable \\
\hline Rabbitbrush & 200 West Area & $\begin{array}{l}{ }^{90} \mathrm{Sr} \\
{ }^{137} \mathrm{Cs}\end{array}$ & $\begin{array}{l}1.1 \mathrm{E}+03 \\
3.1 \mathrm{E}+02 \\
\end{array}$ \\
\hline Tumbleweeds & 200 East Area (2) & $\begin{array}{l}{ }^{90} \mathrm{Sr} \\
{ }^{137} \mathrm{Cs}\end{array}$ & $\begin{array}{l}2.8 E+01 \\
1.5 E+02\end{array}$ \\
\hline
\end{tabular}


Table S-10. Maximum Radionuclide Concentrations in Investigative Samples Collected from the Operational Areas, 1997. (2 sheets total)

\begin{tabular}{|c|c|c|c|}
\hline Sample type & $\begin{array}{c}\text { Collection area } \\
\text { (number of samples) }\end{array}$ & Radionuclides & $\begin{array}{c}\text { Maximum concentration } \\
\mathrm{pCi} / \mathrm{g}\end{array}$ \\
\hline Tumbleweeds & 200 West Area (2) & $\begin{array}{l}{ }^{90} \mathrm{Sr} \\
{ }^{137} \mathrm{Cs} \\
\end{array}$ & $\begin{array}{l}2.5 E+05 \\
1.8 E+06 \\
\end{array}$ \\
\hline Darking beetles & 200 West Area (1) & ${ }^{90} \mathrm{Sr}$ & $1.8 \mathrm{E}+02$ \\
\hline Sagebrush lizards & 200 East Area (1) & ${ }^{90} \mathrm{Sr}$ & $\begin{array}{l}6.8 E+01 \\
3.3 E+01 \\
\end{array}$ \\
\hline Bird nest & 200 West Area (1) & $\begin{array}{l}{ }^{90} \mathrm{Sr} \\
{ }^{137} \mathrm{Cs}\end{array}$ & $\begin{array}{l}2.9 E+01 \\
3: 7 E+02 \\
\end{array}$ \\
\hline Mouse feces & 200 West Area (1) & $\begin{array}{c}{ }^{60} \mathrm{Co} \\
{ }^{90} \mathrm{Sr} \\
{ }^{137} \mathrm{Cs} \\
{ }^{154} \mathrm{Eu} \\
{ }^{155} \mathrm{Eu} \\
{ }^{238} \mathrm{Pu} \\
{ }^{239 / 240} \mathrm{Pu} \\
{ }^{\text {Totel }} \mathrm{U} \\
\end{array}$ & $\begin{array}{l}2.8 E+04 \\
1.7 E+05 \\
1.3 E+05 \\
2.6 E+04 \\
1.1 E+04 \\
8.1 E+03 \\
3.3 E+04 \\
1.6 E+05 \\
\end{array}$ \\
\hline Mouse nest & 200 East Area (2) & $\begin{array}{l}{ }^{90} \mathrm{Sr} \\
{ }^{137} \mathrm{Cs} \\
\end{array}$ & $\begin{array}{l}9.2 \mathrm{E}+03 \\
8.6 \mathrm{E}+\mathrm{O} 2 \\
\end{array}$ \\
\hline House mouse & 200 East Area (1) & $\begin{array}{l}{ }^{90} \mathrm{Sr} \\
{ }^{137} \mathrm{Cs} \\
\end{array}$ & $\begin{array}{l}1.6 E+05 \\
4.6 E+03 \\
\end{array}$ \\
\hline Deer mouse & 200 East Area (3) & $\begin{array}{l}{ }^{90} \mathrm{Sr} \\
{ }^{137} \mathrm{Cs} \\
\end{array}$ & $\begin{array}{l}5.9 E+02 \\
1.0 E+03 \\
\end{array}$ \\
\hline Deer mouse & 200 West Area (3) & ${ }^{90} \mathrm{Sr}$ & $\begin{array}{l}9.2 \mathrm{E}+04 \\
1.2 \mathrm{E}+04 \\
\end{array}$ \\
\hline $\begin{array}{l}\text { Great Basin pocket } \\
\text { mouse }\end{array}$ & $100 \mathrm{~N}$ Area (1) & $\begin{array}{l}{ }^{60} \mathrm{Co} \\
{ }^{90} \mathrm{Sr} \\
{ }^{137} \mathrm{Cs} \\
\end{array}$ & $\begin{array}{l}4.3 E+01 \\
1.5 E+02 \\
1.0 E+01 \\
\end{array}$ \\
\hline $\begin{array}{l}\text { Great Basin pocket } \\
\text { mouse }\end{array}$ & 200 East Area (2) & $\begin{array}{l}90 \mathrm{Sr} \\
{ }^{137} \mathrm{Cs} \\
\end{array}$ & $\begin{array}{l}1.9 E+04 \\
2.6 E+02 \\
\end{array}$ \\
\hline $\begin{array}{l}\text { Great Basin pocket } \\
\text { mouse }\end{array}$ & 600 Area $\{t\}$ & $\begin{array}{l}{ }^{90} \mathrm{Sr} \\
{ }^{137} \mathrm{CS} \\
\end{array}$ & $\begin{array}{l}2.2 E+02 \\
1.7 E+01 \\
\end{array}$ \\
\hline Cottontail rabbit feces & 200 West Area (1) & $\begin{array}{r}{ }^{90} \mathrm{Sr} \\
{ }^{137} \mathrm{Cs} \\
\end{array}$ & $\begin{array}{l}4.4 E+03 \\
1.2 E+02 \\
\end{array}$ \\
\hline Cottontail rabbit & 200 East Area (1) & $\begin{array}{l}{ }^{90} \mathrm{Sr} \\
{ }^{137} \mathrm{Cs} \\
{ }^{\mathrm{T} o t a 1} \mathrm{U}\end{array}$ & $\begin{array}{l}7.2 E+03 \\
2.6 E+03 \\
1.2 E+03 \\
\end{array}$ \\
\hline Cottontail rabbit & 200 West Area (2) & $\begin{array}{l}{ }^{90} \mathrm{Sr} \\
{ }^{137} \mathrm{Cs} \\
\end{array}$ & $\begin{array}{l}5.0 E+03 \\
1.9 E+02 \\
\end{array}$ \\
\hline Coyote feces & 200 West Area (1) & $\begin{array}{l}{ }^{90} \mathrm{Sr} \\
{ }^{137} \mathrm{Cs} \\
\end{array}$ & $\begin{array}{l}4.6 E+01 \\
7.1 E+02 \\
\end{array}$ \\
\hline
\end{tabular}


Sludge. In 1997, two samples of dried sludge were collected from the contaminated C-5 Tank in the 200 East Area to determine if flaking and subsequent blowing might require the surface to be stabilized. The analytical results from these samples showed that all radionuclide concentrations were below analytical detection limits.

Soil. In 1997, no investigative soil samples were collected for radioisotopic analysis. There were 51 incidents of contaminated soil or specks found during cleanup operations which were disposed of in low-level burial grounds without analysis. External radioactivity ranged from slightly above background (approximately $1,000 \mathrm{dpm} / 100 \mathrm{~cm}^{2}$ ) to 38 millirads per hour (mrads/h). The contaminated areas were posted or cleaned up.

In 1997, the number of contamination incidents, the range of radiation dose levels, and radionuclide concentrations generally were within historical ranges. Areas of special soil sampling that were outside radiological control areas and had radiation levels greater than radiological control limits were posted as surface contamination areas.

Vegetation. In 1997, four tumbleweed samples, one rabbitbrush sample, and one grass sample were analyzed for radionuclide concentrations. The maximum radionuclide concentrations were in a tumbleweed sample from near $221-\mathrm{U}$ Building in the 200 West Area and consisted primarily of ${ }^{90} \mathrm{~S}$ : $\left(2.5 \times 10^{5} \mathrm{pCi} / \mathrm{g}\right)$ and ${ }^{137} \mathrm{Cs}\left(1.8 \times 10^{6} \mathrm{pCi} / \mathrm{g}\right)$. The rabbitbrush sample from near $241-\mathrm{T}$ Tank Farm in the 200 West Area contained primarily ${ }^{90} \mathrm{Sr}\left(1.1 \times 10^{3} \mathrm{pCi} / \mathrm{g}\right)$ and ${ }^{137} \mathrm{Cs}\left(3.1 \times 10^{2} \mathrm{pCi} / \mathrm{g}\right)$. In addition, 40 instances of contaminated vegetation (e.g., grass, tumbleweeds, rabbitbrush, and sagebrush) were recorded in the operational areas in 1997. This vegetation was discovered during remedial operations, surveyed with field instruments, and disposed of in low-level burial grounds. The field instrument readings for the vegetation ranged from approximately 1,000 to $1,000,000 \mathrm{dpm} / \mathrm{cm}^{2}$. During 1997 , the numbers of contaminated vegetation (both samples and those disposed without analysis) exceeded those of the previous year by a factor of four. It is believed that this was primarily because of climatological conditions (i.e., increased frequency and quantity of precipitation) making the vegetation control program on the waste sites considerably less effective, and resuiting in more tumbleweed growth. The radioactivity levels, and range of radionuclide concentrations were all within historical ranges. Historically, the greatest number of contaminated vegetation samples (42) were submitted for analysis in 1978 (Johnson et al. 1994), but it is not recorded how many contaminated vegetation were disposed of without analysis that year.

Wildlife. Wildlife is collected either as part of an integrated pest management program designed to limit the exposure to and potential contamination of animals with radioactive material, or as a result of finding radiologically contaminated wildlife-related material (e.g., feces, nests, etc.). Animals were collected directly from, or near, facilities to identify potential problems with preventive measures designed to deter animal intrusion. Surveys were performed after collection to determine whether an animal was radioactively contaminated. If a live animal was found to be free of contamination, it was taken to an area of suitable habitat, still in a controlled area, and released. If an animal was contaminated, a decision was made based on the level of contamination, sampling facility, and frequency of occurrence either to collect the animal as a sample or to dispose of the animal in a low-level burial ground.

In 1997, 22 wildlife and wildlife-related samples were submitted for analysis. All 22 of the special animal samples analyzed showed detectable levels of contamination. This compares to 37 contaminated samples (of 41 collected) that were analyzed in 1996, 22 contaminated samples (of 25 collected) that were analyzed in 1995, and 16 contaminated samples (of 27 collected) in 1994. The numbers of samples submitted depended on opportunity (i.e., resulting from the pest control activities at facilities) rather than exact numbers submitted from established sampling points. The maximum radionuclide concentrations in 1997 were all in mouse feces collected along railroad tracks east of $218-W-4 \mathrm{~B}$ Burial Ground in the 200 West Area. The concentrations included ${ }^{60} \mathrm{Co}$ $\left(2.8 \times 10^{4} \mathrm{pCi} / \mathrm{g}\right) ;{ }^{90} \mathrm{Sr}\left(1.7 \times 10^{5} \mathrm{pCi} / \mathrm{g}\right) ;{ }^{137} \mathrm{Cs}\left(1.3 \times 10^{5} \mathrm{pCi} / \mathrm{g}\right) ;{ }^{154} \mathrm{Eu}\left(2.6 \times 10^{4} \mathrm{pCi} / \mathrm{g}\right) ;{ }^{155} \mathrm{Eu}$ 
$\left(1.1 \times 10^{4} \mathrm{pCi} / \mathrm{g}\right) ;{ }^{238} \mathrm{Pu}\left(8.1 \times 10^{3} \mathrm{pCi} / \mathrm{g}\right) ;{ }^{239 / 240} \mathrm{Pu}\left(3.3 \times 10^{4} \mathrm{pCi} / \mathrm{g}\right)$, and total uranium $\left(1.6 \times 10^{5}\right.$ $\mathrm{pCi} / \mathrm{g})$. A sample of three house mice from 244-AR Vault in the 200 East Area had $1.6 \times 10^{5} \mathrm{pCi} / \mathrm{g}$ of ${ }^{90} \mathrm{Sr}$.

Contaminated animal samples, which were somewhat atypical for the special sample program, included a sample of darkling beetles found inside 272-S in the 200 West Area with elevated ${ }^{90} \mathrm{Sr}\left(1.8 \times 10^{2} \mathrm{pCi} / \mathrm{g}\right)$; and a sample of 12 dead sagebrush lizards found in a contaminated cabinet outside PUREX in the 200 East Area with low levels of ${ }^{90} \mathrm{Sr}\left(6.8 \times 10^{1} \mathrm{pCi} / \mathrm{g}\right)$ and ${ }^{137} \mathrm{Cs}(3.3$ $\times 10^{1} \mathrm{pCi} / \mathrm{g}$ ).

Additionally, there were eight cases of contaminated wildlife or related samples (e.g., nests or feces) found during cleanup operations that were not analyzed. The numbers of animals found to be contaminated with radioactivity, the radioactivity levels, and the range of radionuclide concentrations were within historical ranges (Johnson et al. 1994). 
HNF-EP-0573-6

This page intentionally left biank. 
CONTENTS

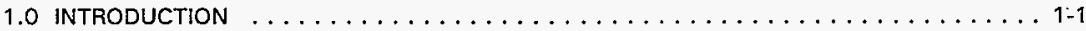

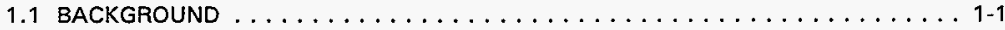

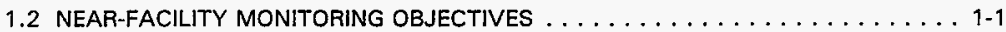

1.3 NEAR-FACILITY TRANSPORT PATHWAYS $\ldots \ldots \ldots \ldots \ldots \ldots \ldots \ldots \ldots$

1.3.1 Environmental Transport Sources . . . . . . . . . . . . 1-8

1.3.2 General Types of Facilities . . . . . . . . . . . . . .

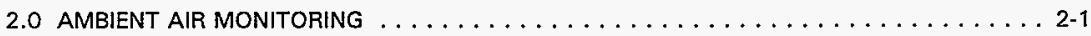

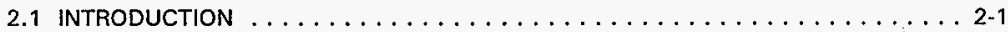

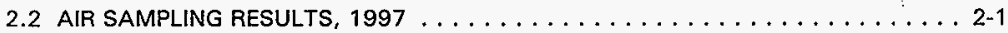

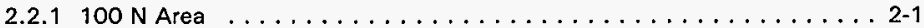

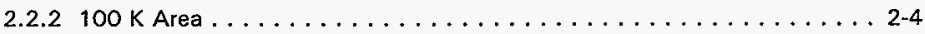

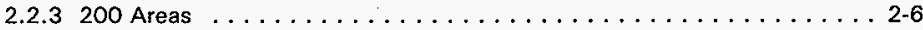

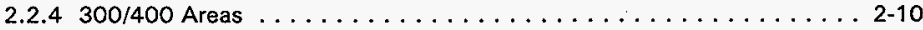

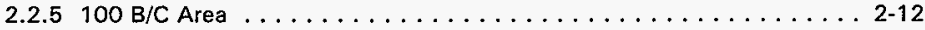

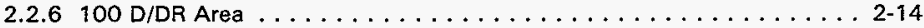

2.2.7 Environmental Restoration Disposal Facility . . . . . . . . . . 2-16

2.2.8 300-FF-1 Project . . . . . . . . . . . . . . . . 2-18

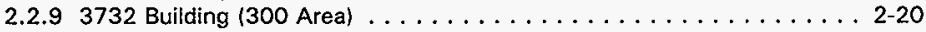

2.3 CONCLUSIONS . . . . . . . . . . . . . . . . . . . . 2-20

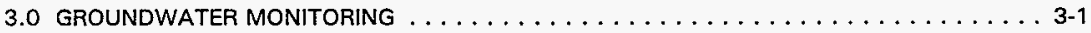

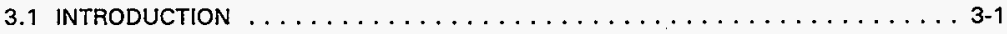

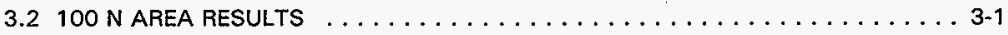

4.0 SOIL AND VEGETATION MONITORING $\ldots \ldots \ldots \ldots \ldots \ldots \ldots \ldots \ldots \ldots$

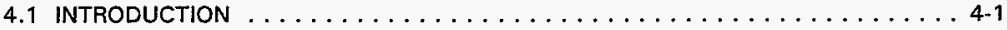

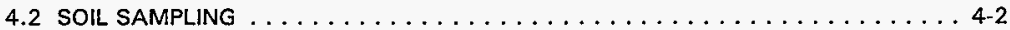

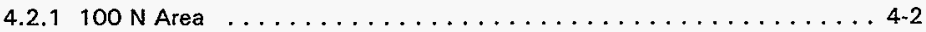

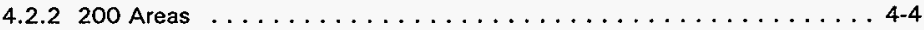

$4.2 .3300 / 400$ Areas . . . . . . . . . . . . . . . . . . . 4-5

4.2.4 Environmental Restoration Contractor Compliance Sampling . . . . . . 4-6

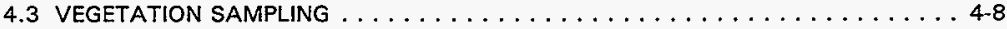

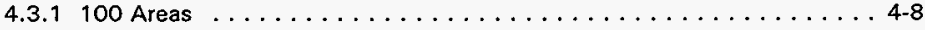

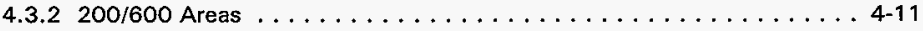

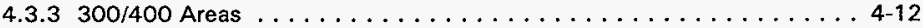

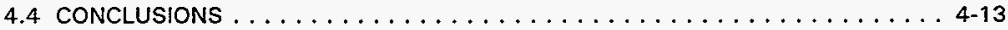

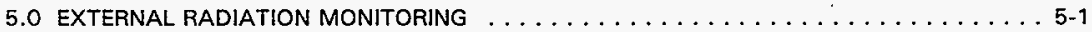

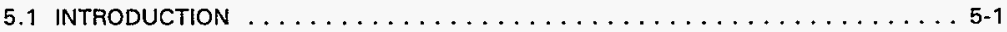

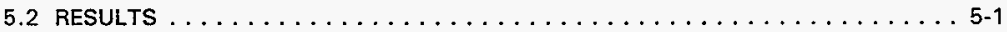

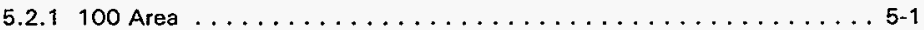

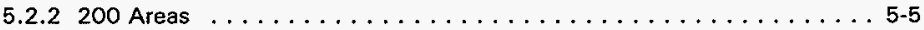

5.2.3 Tank Waste Remediation System Phase I Demonstration Project . . . . 5-7

5.2 .4 Environmental Restoration Disposal Facility $\ldots \ldots \ldots \ldots \ldots \ldots \ldots . . .7$

$5.2 .5300 / 300$ TEDF/400 Areas . . . . . . . . . . . . . . . 5 
CONTENTS (cont)

6.0 SURFACE WATER DISPOSAL UNITS AND SEEPAGES $\ldots \ldots \ldots \ldots \ldots \ldots \ldots \ldots$

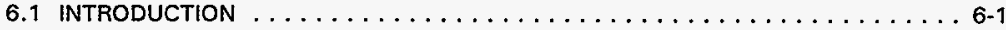

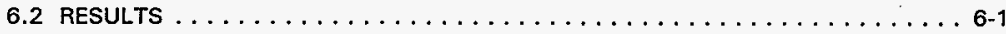

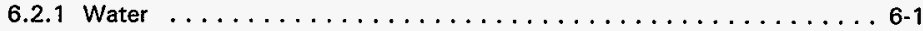

6.2 .2 Nonradiological Parameters . . . . . . . . . . . . . . 6-4

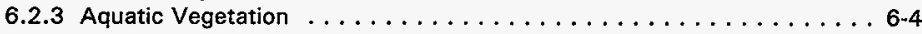

6.2 .4 Sediment (Surface Samples) . . . . . . . . . . . . . . . 6-4

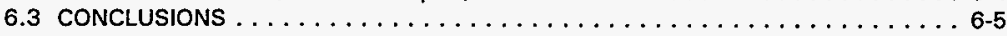

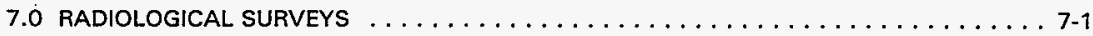

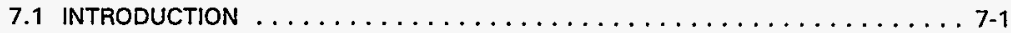

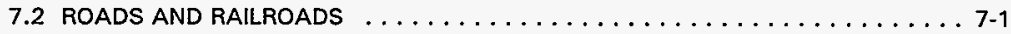

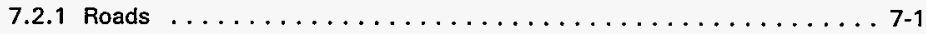

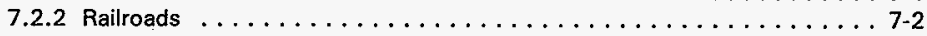

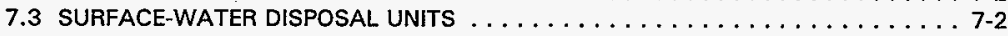

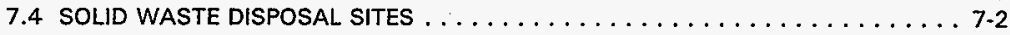

7.5200 AREAS LOW-LEVEL LIOUID WASTE DISPOSAL SITES $\ldots \ldots \ldots \ldots \ldots \ldots \ldots .7-3$

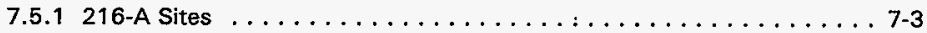

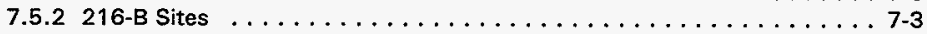

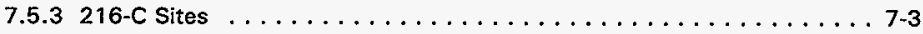

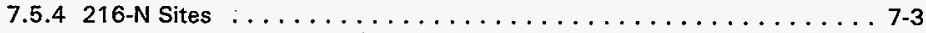

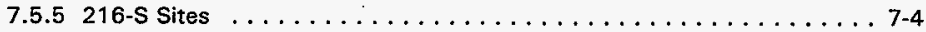

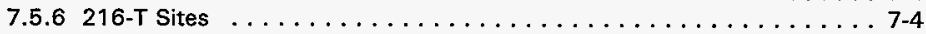

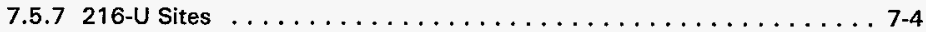

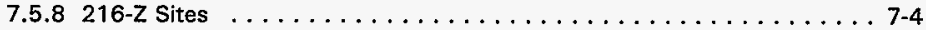

7.5 .9 Unplanned Release Sites . . . . . . . . . . . . . . . 7-4

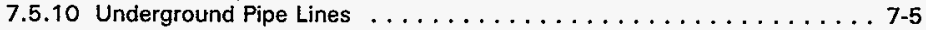

7.6 TANK FARM PERIMETERS $\ldots \ldots \ldots \ldots \ldots \ldots \ldots \ldots \ldots \ldots \ldots \ldots \ldots$

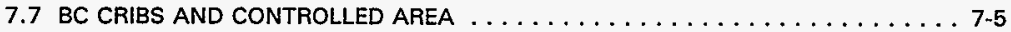

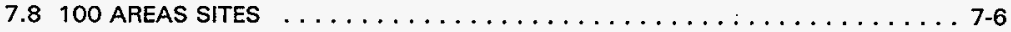

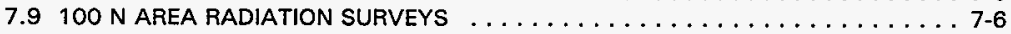

7.10 COLUMBIA RIVER SHORELINE SURVEY $\ldots \ldots \ldots \ldots \ldots \ldots \ldots \ldots \ldots$

7.11 Retired 100 Area Waste Disposal Facilities $\ldots \ldots \ldots \ldots \ldots \ldots \ldots \ldots$

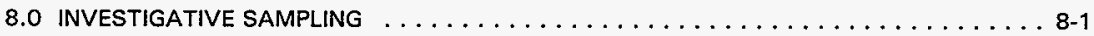

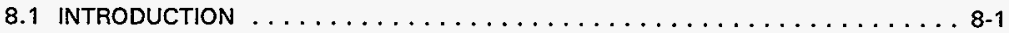

8.2 COLLECTION AND ANALYSES OF INVESTIGATIVE SAMPLES $\ldots \ldots \ldots \ldots \ldots \ldots . .8 .1$

8.3 RADIOLOGICAL RESULTS FOR INVESTIGATIVE SAMPLES $\ldots \ldots \ldots \ldots \ldots \ldots \ldots$. $\ldots \ldots$

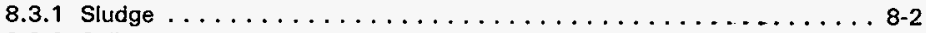

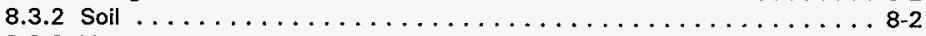

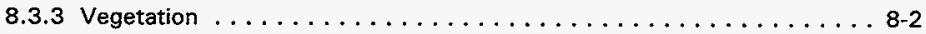

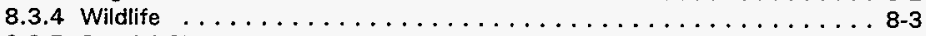

8.3.5 Special Characterization Sampling $\ldots \ldots \ldots \ldots \ldots \ldots \ldots \ldots$ 8-3

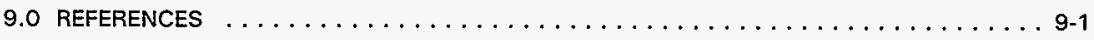


HNF-EP-0573-6

APPENDICES

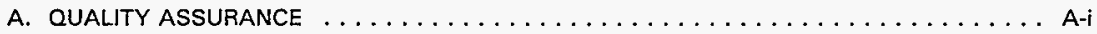

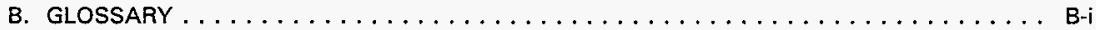

C. AMBIENT AIR MONITORING FIGURES AND TABLES $\ldots \ldots \ldots \ldots \ldots \ldots \ldots \ldots$ Ci

D. GROUNDWATER MONITORING FIGURES AND TABLES $\ldots \ldots \ldots \ldots \ldots \ldots \ldots \ldots \ldots$ Di

E. SOIL AND VEGETATION MONITORING FIGURES AND TABLES $\ldots \ldots \ldots \ldots \ldots \ldots \ldots$ E-i

F. EXTERNAL RADIATION MONITORING FIGURES AND TABLES $\ldots \ldots \ldots \ldots \ldots \ldots \ldots \ldots$ F- $\mathrm{j}$

G. SURFACE WATER DISPOSAL UNIT (POND AND DITCH) MONITORING FIGURES AND

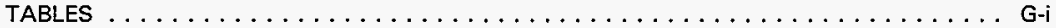

H. $100 \mathrm{~N}$ AREA SHORELINE RADIATION SURVEYS $\ldots \ldots \ldots \ldots \ldots \ldots \ldots \ldots \ldots \ldots$

I. INVESTIGATIVE SPECIAL SAMPLE TABLES $\ldots \ldots \ldots \ldots \ldots \ldots \ldots \ldots \ldots \ldots \ldots$

J. CONCENTRATION GUIDES $\ldots \ldots \ldots \ldots \ldots \ldots \ldots \ldots \ldots \ldots \ldots \ldots \ldots \ldots \ldots \ldots \ldots$

K. DATA SUMMARY METHODS $\ldots \ldots \ldots \ldots \ldots \ldots \ldots \ldots \ldots \ldots \ldots \ldots \ldots$

XXXV 


\section{LIST OF FIGURES}

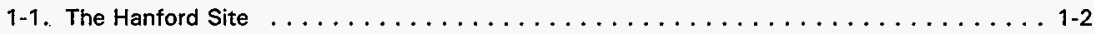

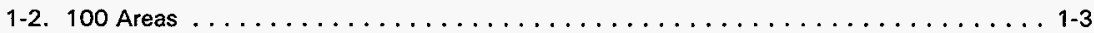

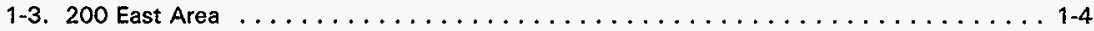

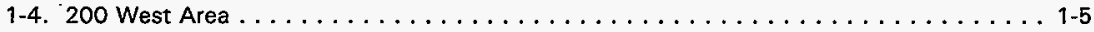

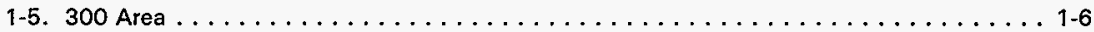

1-6. 400 Area . . . . . . . . . . . . . . . . . . . . . . . . . 1-7

2-1. Average Concentrations of ${ }^{60} \mathrm{Co}$ Detected in $100 \mathrm{~N}$ Area Ambient Air from 1982 to $19972-2$

2-2. Annual Average ${ }^{137} \mathrm{Cs}$ Concentrations in Air for the $200 / 600$ Areas . . . . . . . . . . . 2-7

2-3. Annual Average ${ }^{90} \mathrm{Sr}$ Concentrations in Air for the $200 / 600$ Areas . . . . . . . . . . . . 2-7

2-4. Annual Average ${ }^{239,240} \mathrm{Pu}$ Concentrations in Air for the $200 / 600$ Areas . . . . . . . . . . 2-8

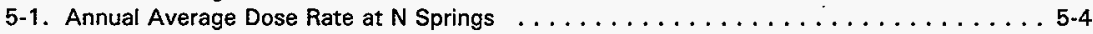

5-2. Comparison of Thermoluminescent Dosimeter Results at $100 \mathrm{~N}$ Area . . . . . . . . . . . 5-4

5-3. Comparison of Thermoluminescent Dosimeter Results at the 200 Areas . . . . . . . . 5-5

5-4. Comparison of Thermoluminescent Dosimeter Results at the $300 / 300$ TEDF/400

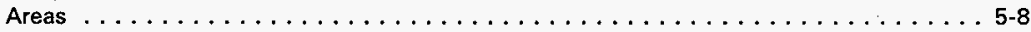

7-1. $100 \mathrm{~N}$ Shoreline Survey Results. . . . . . . . . . . . . . . . . 7-7 
HNF-EP-0573-6

\section{LIST OF TABLES}

1-1. Hanford Site Surface and Underground Contamination . . . . . . . . . . . 1-11

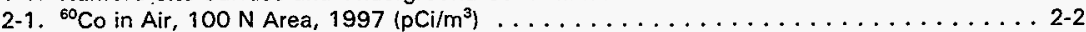

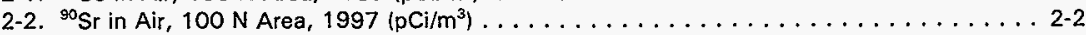

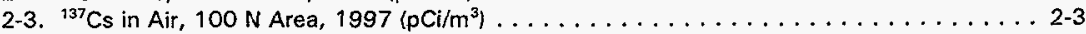

2-4. ${ }^{234} \mathrm{U}$ in $\mathrm{Air}, 100 \mathrm{~N}$ Area, $1997\left(\mathrm{pCi} / \mathrm{m}^{3}\right) \ldots \ldots \ldots \ldots \ldots \ldots \ldots \ldots \ldots \ldots . . \ldots \ldots$

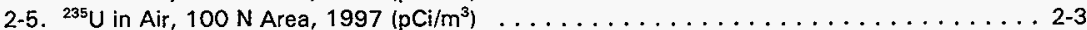

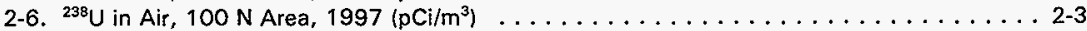

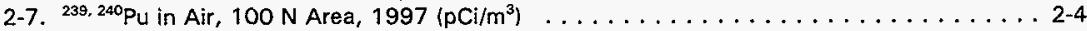

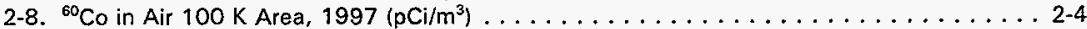

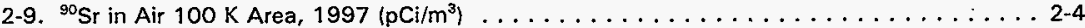

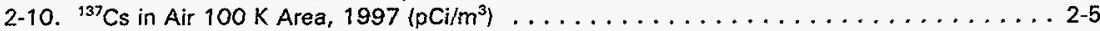

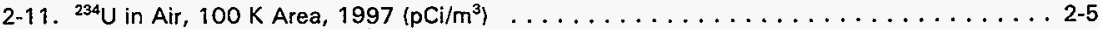

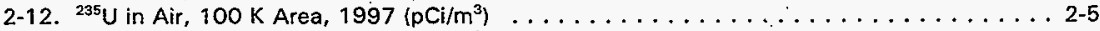

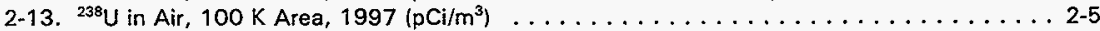

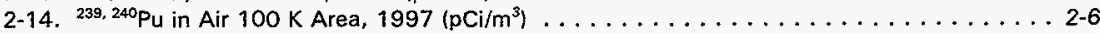

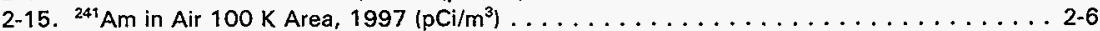

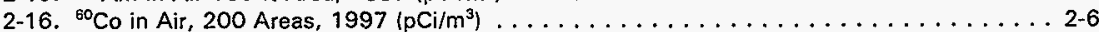

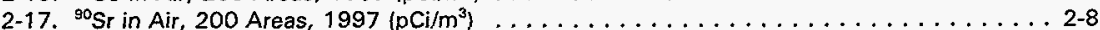

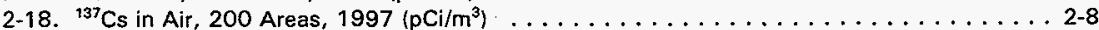

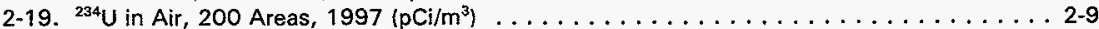

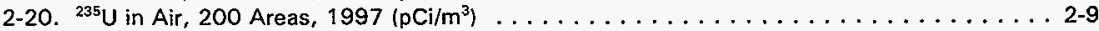

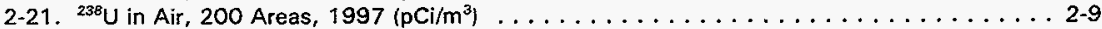

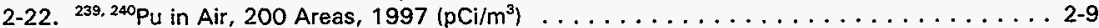

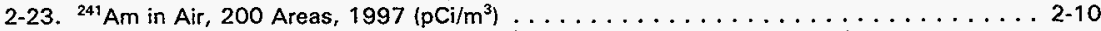

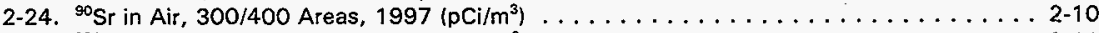

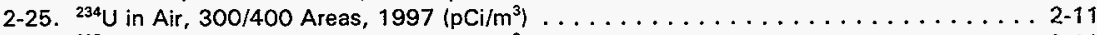

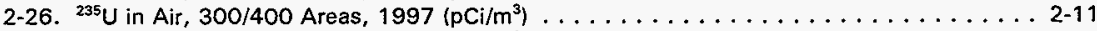

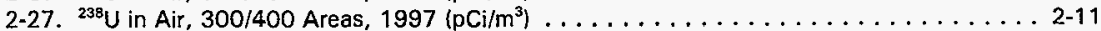

2-28. ${ }^{239.240} \mathrm{Pu}$ in Air, $300 / 400$ Areas, $1997\left(\mathrm{pCi} / \mathrm{m}^{3}\right) \ldots \ldots \ldots \ldots \ldots \ldots \ldots \ldots . \ldots \ldots 2$

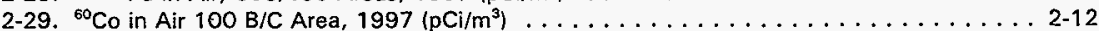

2-30. ${ }^{90} \mathrm{Sr}$ in Air $100 \mathrm{~B} / \mathrm{C}$ Area, $1997\left(\mathrm{pCi} / \mathrm{m}^{3}\right) \ldots \ldots \ldots \ldots \ldots \ldots \ldots \ldots \ldots \ldots \ldots \ldots \ldots \ldots \ldots .12$

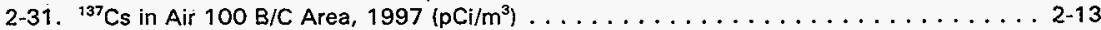

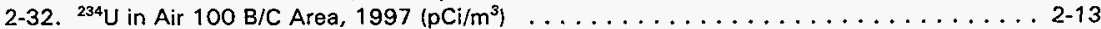

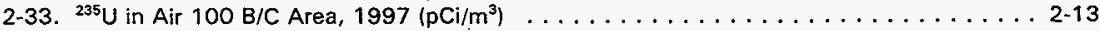

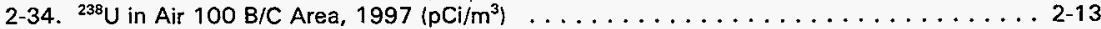

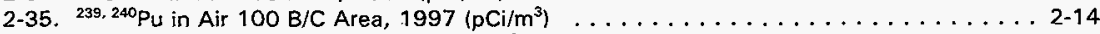

2-36. ${ }^{60} \mathrm{Co}$ in Air $100 \mathrm{D} / \mathrm{DR}$ Area, $1997\left(\mathrm{pCi} / \mathrm{m}^{3}\right) \ldots \ldots \ldots \ldots \ldots \ldots \ldots \ldots \ldots \ldots \ldots \ldots \ldots .14$

2-37. ${ }^{90} \mathrm{Sr}$ in Air $100 \mathrm{D} / \mathrm{DR}$ Area, $1997\left(\mathrm{pCi} / \mathrm{m}^{3}\right) \ldots \ldots \ldots \ldots \ldots \ldots \ldots \ldots \ldots \ldots .14$

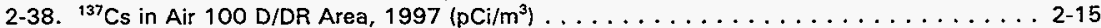

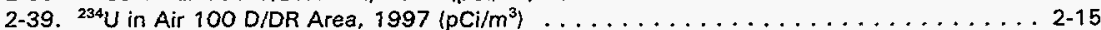

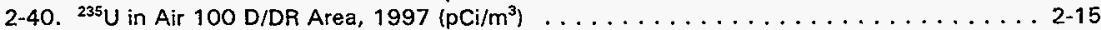

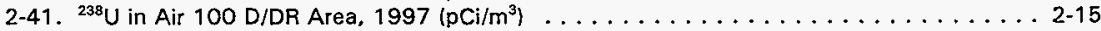

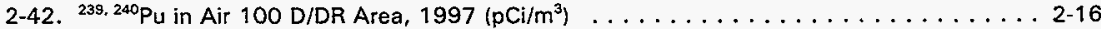

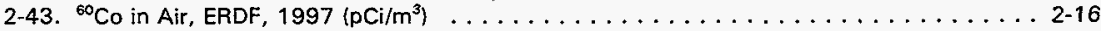

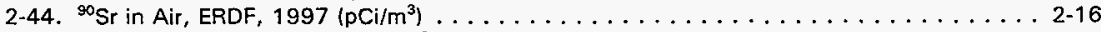

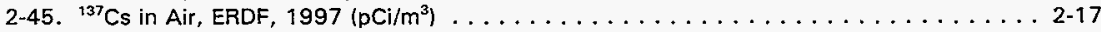

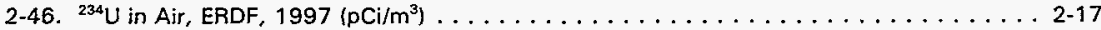

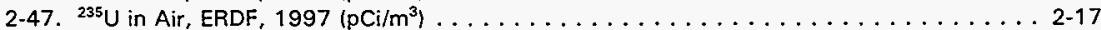

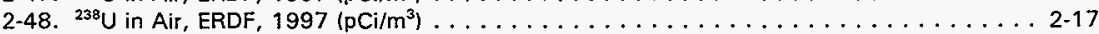

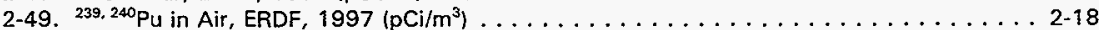

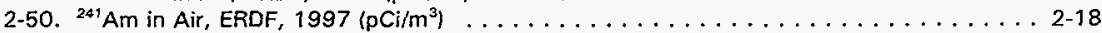




\section{LIST OF TABLES (cont)}

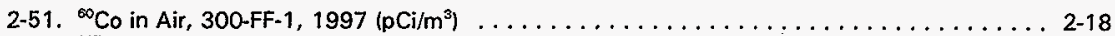

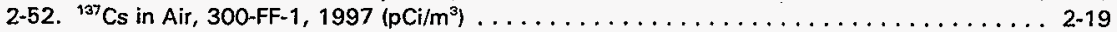

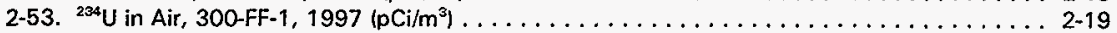

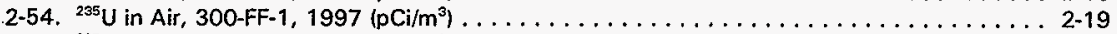

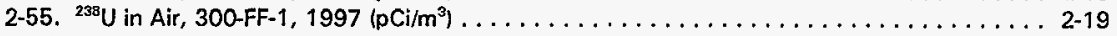

4-1. Average Radionuclide Concentrations Detected in Soil Samples Near the 1301-N Liquid Waste Disposal Facility from 1980 through $1997(\mathrm{pCi} / \mathrm{g}) \ldots \ldots \ldots \ldots \ldots \ldots \ldots$. . . . . .

4-2. Average Radionuclide Concentrations Detected in $100 \mathrm{~N}$ Area Surface Soil Samples from

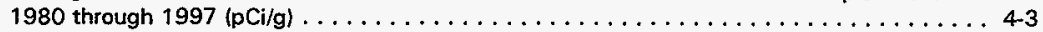

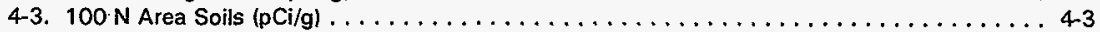

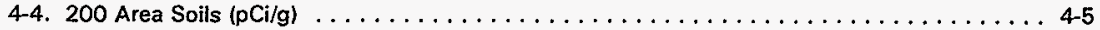

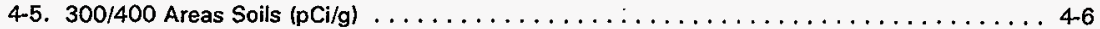

4-6. Comparative Radionuclide Concentrations in 100 D,DR and ERDF Soil (pCi/gm) ... . . . 447

4-7. Average Radionuclide Concentrations Detected in Vegetation Samples Near the 1301- $\mathrm{N}$ Liquid Waste Disposal. Facility from 1980 to $1997(\mathrm{pCi} / \mathrm{g}) \ldots \ldots \ldots \ldots \ldots \ldots \ldots$. . . . . . . . .

4-8. Average Radionuclide Concentrations Detected in $100 \mathrm{~N}$ Vegetation Samples from 1980

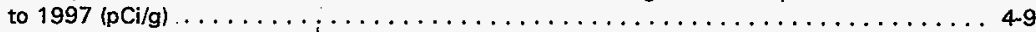

4-9. Average Radionuclide Concentrations Detected in N Springs Vegetation Samples from

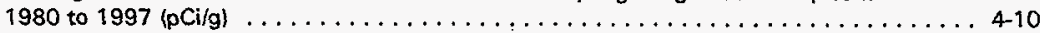

4-10. Radionuclide Concentrations in $100 \mathrm{~N}$ Area Vegetation $(\mathrm{pCi} / \mathrm{g}) \ldots \ldots \ldots \ldots \ldots \ldots \ldots . . .440$

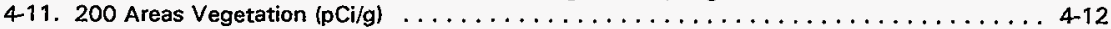

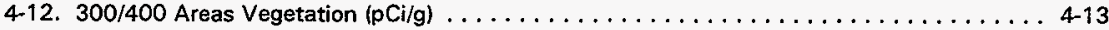

5-1. $100 \mathrm{D} / \mathrm{DR}$ Area Thermoluminescent Dosimeter Results (mrem/yr) $\ldots \ldots \ldots \ldots \ldots \ldots . .5$.2

5-2. $100 \mathrm{~N}$ and $100 \mathrm{KE} / \mathrm{KW}$ Areas Thermoluminescent Dosimeter Results $(\mathrm{mrem} / \mathrm{yr}) \ldots \ldots \ldots .5$

5-3. 200 Areas Thermoluminescent Dosimeter Results $(\mathrm{mrem} / \mathrm{yr}) \ldots \ldots \ldots \ldots \ldots \ldots . . . . .56$

5-4. Tank Waste Remediation System Phase I Demonstration Project Thermoluminescent

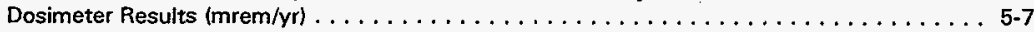

5-5. Environmental Restoration Disposal Facility Thermoluminescent Dosimeter Results

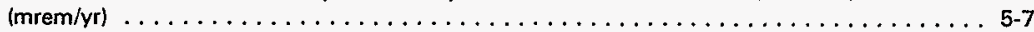

5-6. $300 / 300$ TEDF/400 Areas Thermoluminescent Dosimeter Results (mrem/yr) . . . . . . . . . 5-9

6-1. N Springs Shoreline Tritium Concentrations, 1987 to $1997(\mathrm{pCi} / \mathrm{L}) \ldots \ldots \ldots \ldots$. . . . . . . . 6-2

6-2. N Springs Shoreline ${ }^{90} \mathrm{Sr}$ Concentrations, 1987 to $1997 \mathrm{(pCi/L)} \mathrm{.} \mathrm{.} \mathrm{.} \mathrm{.} \mathrm{.} \mathrm{.} \mathrm{.} \mathrm{.} \mathrm{.} \mathrm{.} \mathrm{.} \mathrm{.} \mathrm{.} \mathrm{.} \mathrm{.} \mathrm{.} \mathrm{.} \mathrm{6-3}$

7-1. Samples with Contamination Above Detectable Levels . . . . . . . . . . . . . . 7-8

7-2. Sites Remediated or Radiologically Reposted in 1997 [approximate surface area in hectares (acres)] 
HNF-EP-0573-6

LIST OF TERMS

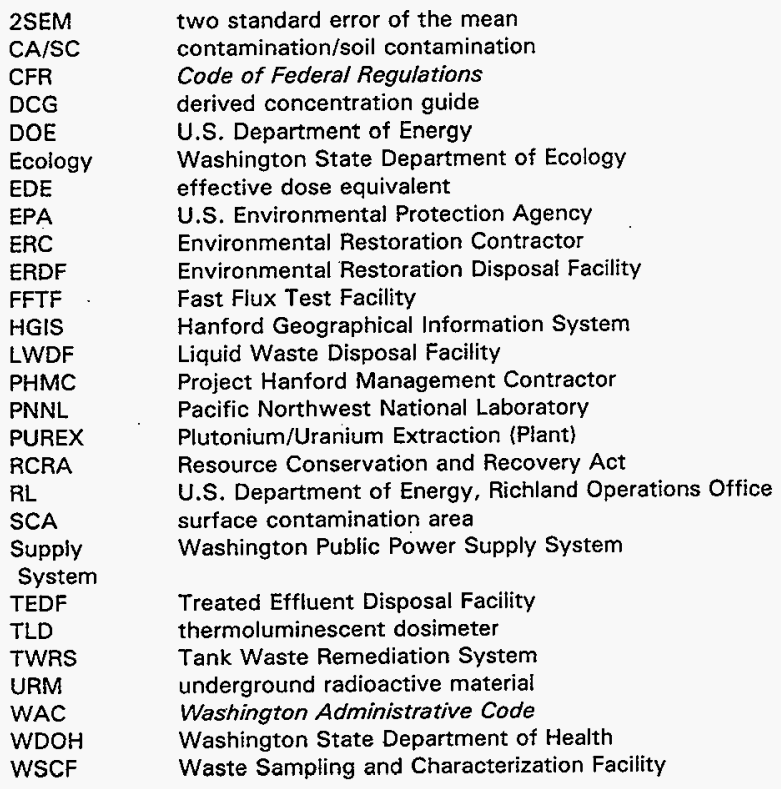

xxxix 
HNF-EP-0573-6

This page intentionally left blank. 
HNF-EP-0573-6

\section{HANFORD SITE NEAR-FACILITY ENVIRONMENTAL \\ MONITORING ANNUAL REPORT, CALENDAR YEAR 1997}

\subsection{INTRODUCTION}

\subsection{BACKGROUND}

The Hanford Site, consisting of $1,450 \mathrm{~km}^{2}\left(560 \mathrm{mi}^{2}\right)$, is located within the Pasco Basin in south-central Washington State, approximately $270 \mathrm{~km}(170 \mathrm{mi})$ southeast of Seattle and $200 \mathrm{~km}$ $(120 \mathrm{mi})$ southwest of Spokane. As shown in Figure 1-1, the 100 Areas are located in the north-central part of the Site, along the southern (right) bank of the Columbia River. The locations of facilities within the 100 Areas are shown in Figure 1-2. The 200 Areas are almost in the center of the Site, $11 \mathrm{~km}(7 \mathrm{mi})$ south of the Columbia River. The locations of facilities, tank farms, solid waste burial grounds, and liquid waste disposal sites in the 200 Areas are shown in Figures 1-3 and 1-4. The 300 and 400 Areas are located in the southwestern part of the Site as shown in Figure $1-5$ and $1-6$, respectively. The areas not associated with any of the listed areas are considered the 600 Area.

Near-facility environmental monitaring is conducted in the $100,200,300,400$, and 600 Areas to monitor diffuse sources and the impact of effluent releases and waste management practices at and near facilities, waste disposal sites, and environmental restoration activities.

\subsection{NEAR-FACILITY MONITORING OBJECTIVES}

The objectives of the near-facility monitoring are to evaluate the following:

- $\quad$ Compliance with the U.S. Department of Energy (DOE), U.S. Environmental Protection Agency (EPA), Washington State Department of Ecology (Ecology), Washington State Department of Health (WDOH), and internal environmental protection requirements and guidelines.

- Performance of radioactive waste confinement systems.

- Trends of radioactive materials in the environment at and adjacent to facilities, waste disposal units, and restoration units so that appropriate control actions can be taken in a timely manner.

This report presents and interprets the results of the near-facility monitoring activities performed at the Hanford Site during 1997.

\subsection{NEAR-FACILITY TRANSPORT PATHWAYS}

The Hanford Site consists of semi-arid shrub-steppe land, of which approximately $6 \%$ has been disturbed and actively used for processing nuclear material. This disturbed land, about $82.9 \mathrm{~km}^{2}\left(32 \mathrm{mi}^{2}\right)$, is divided into large operational areas: the $100,200,300,400$, and 1100 Areas. Radioactive emissions from the Site are grouped into three broad categories for regulatory purposes: point, diffuse, and fugitive source emissions. Point sources are easily defined and recognized as facilities with effluent emission structures, such as stacks, and are typically equipped with filtering and monitoring systems. Diffuse sources are extremely varied, do not or 
Figure 1-1. The Hanford Site.

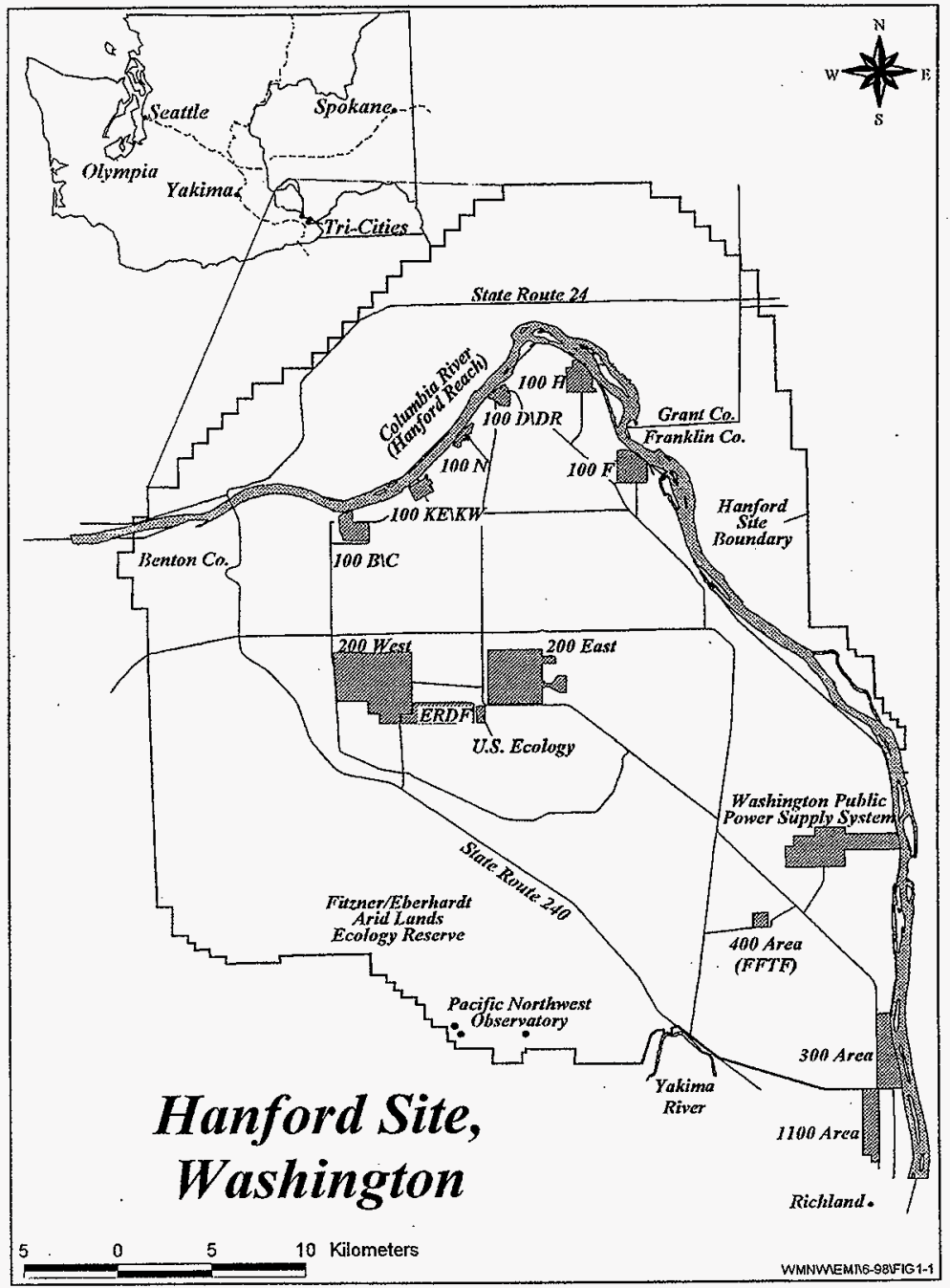


Figure 1-2. 100 Areas.

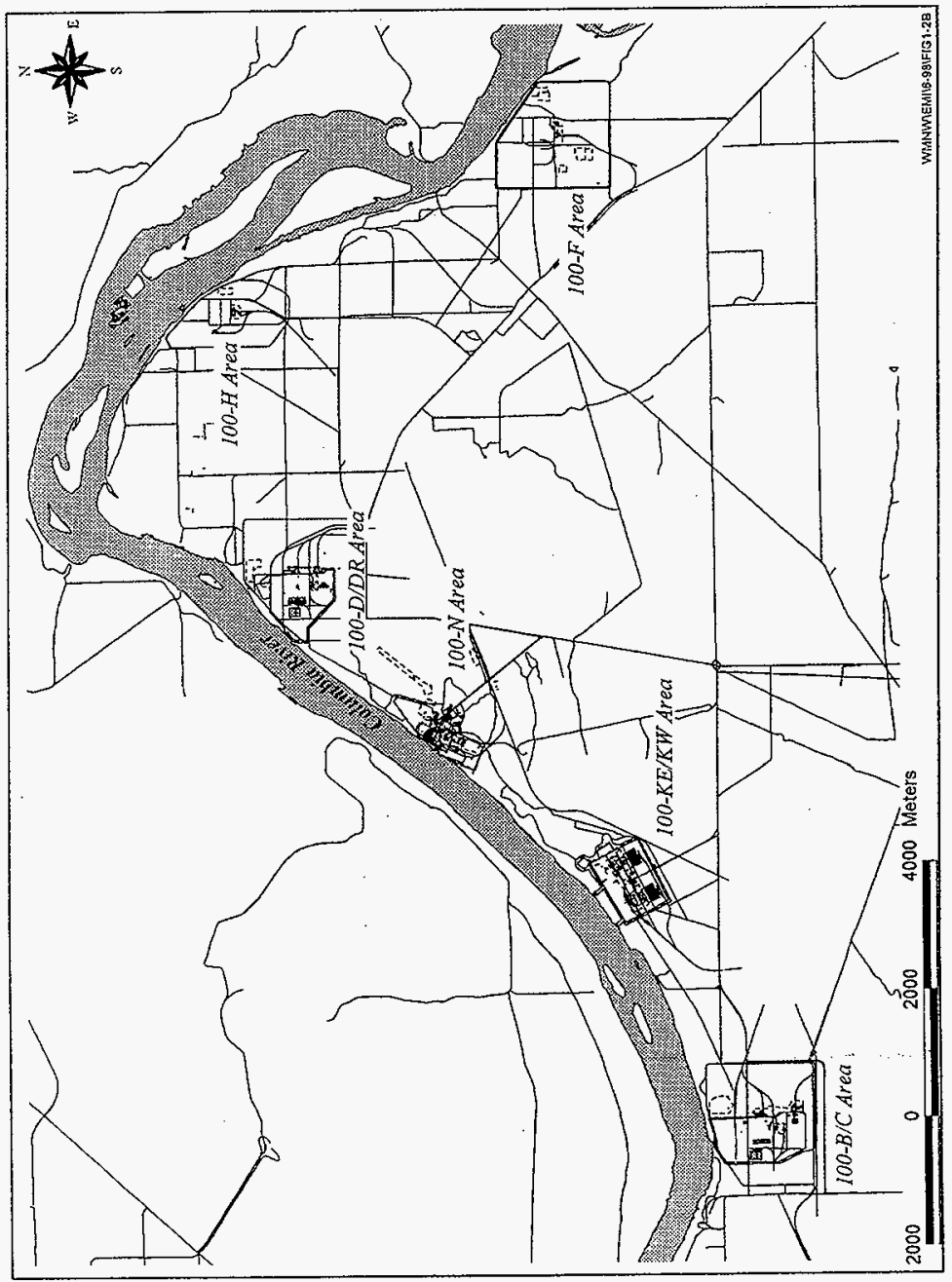


HNF-EP-0573-6

Figure 1-3. 200 East Area.

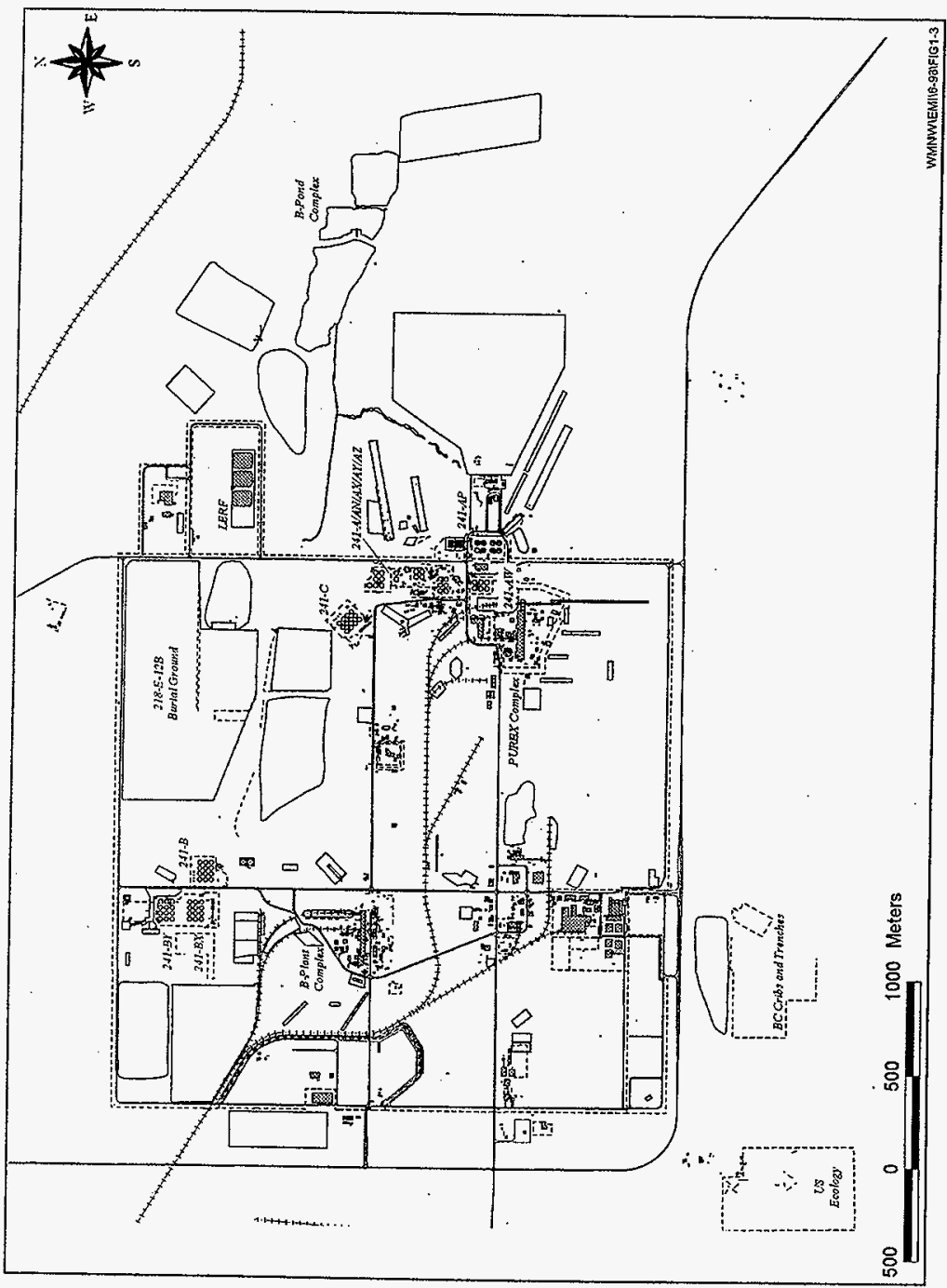


HNF-EP-0573-6

Figure 1.4. 200 West Area.

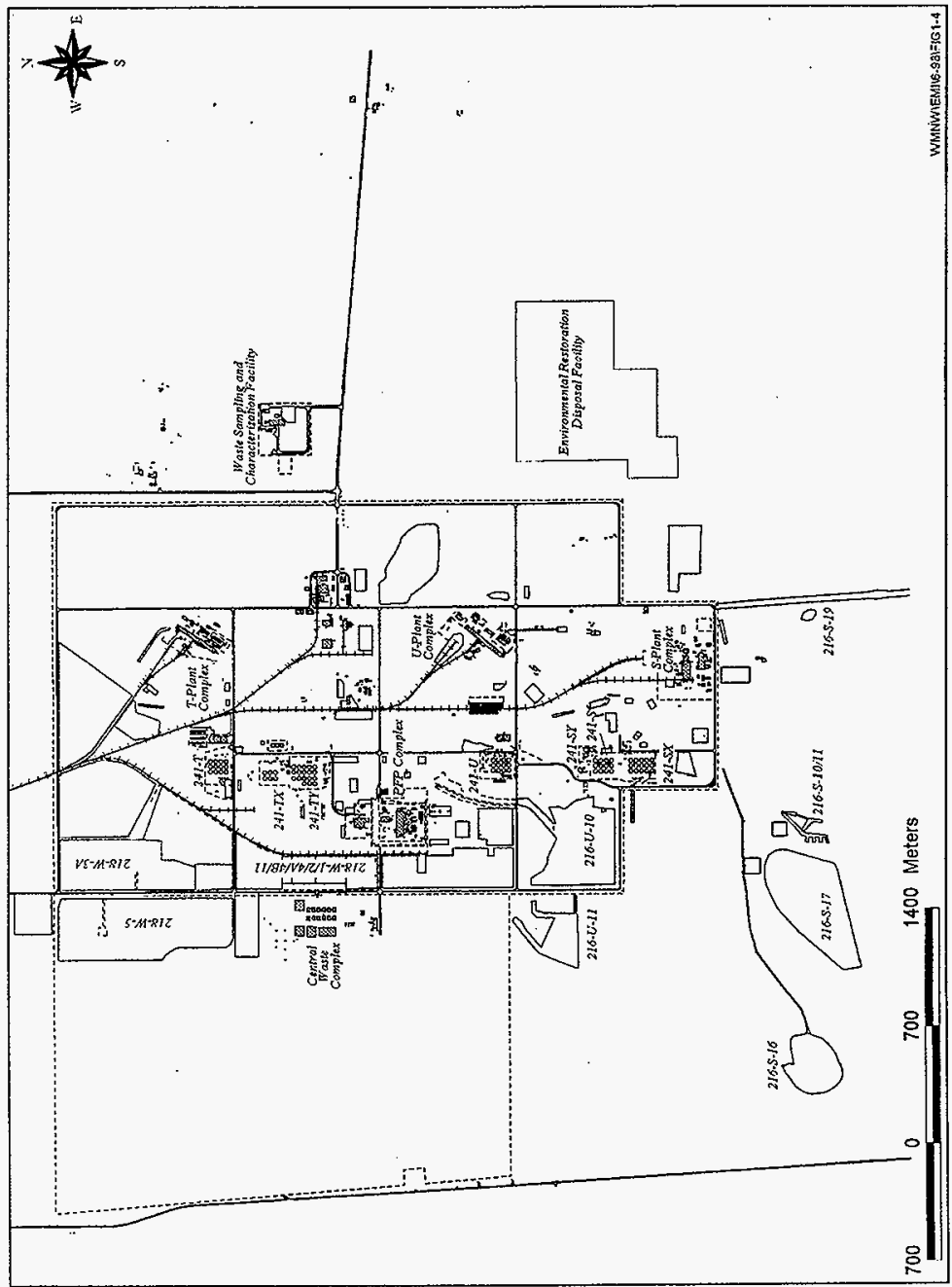


Figure 1-5. 300 Area.

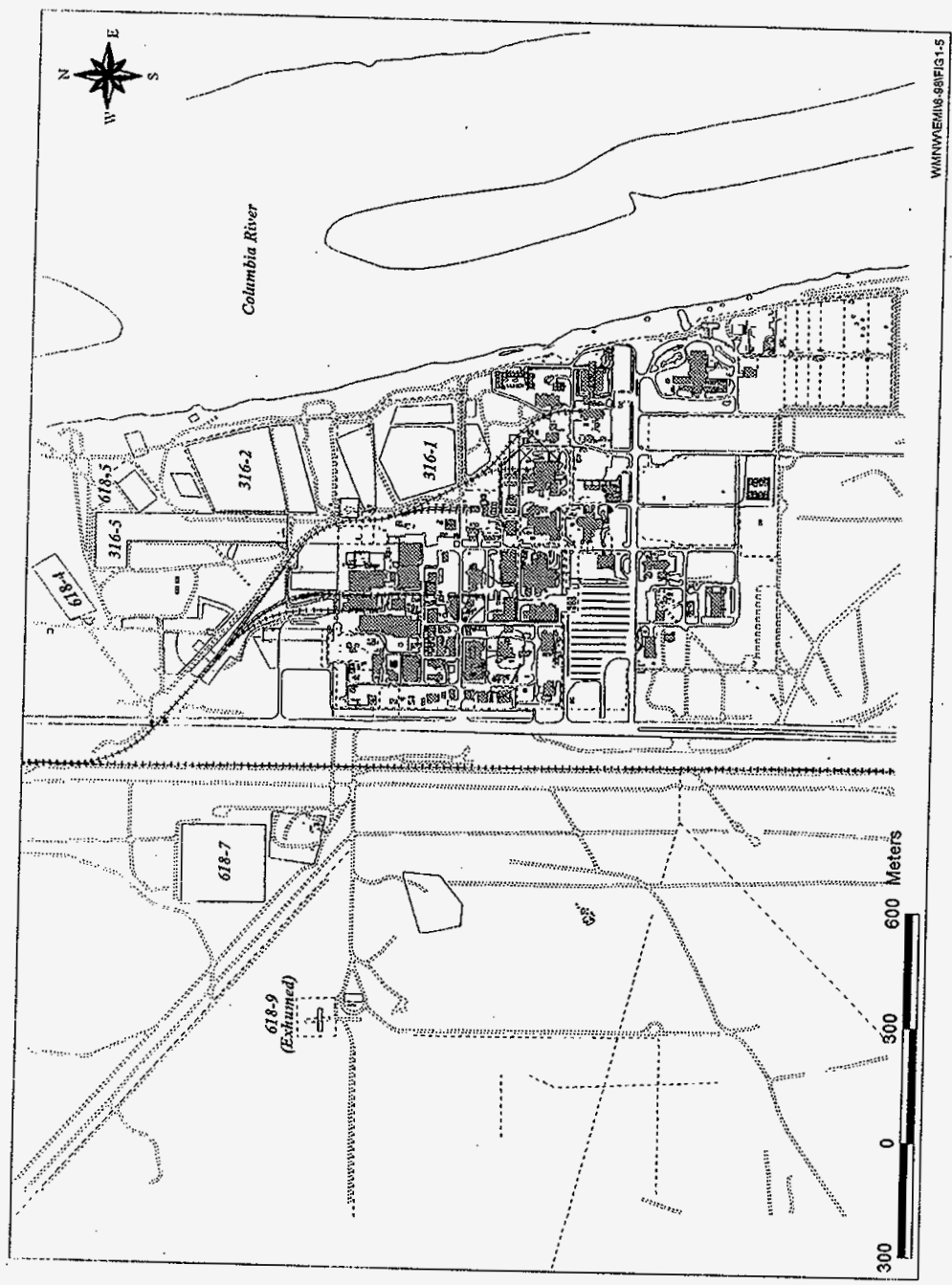


Figure 1-6. 400 Area.

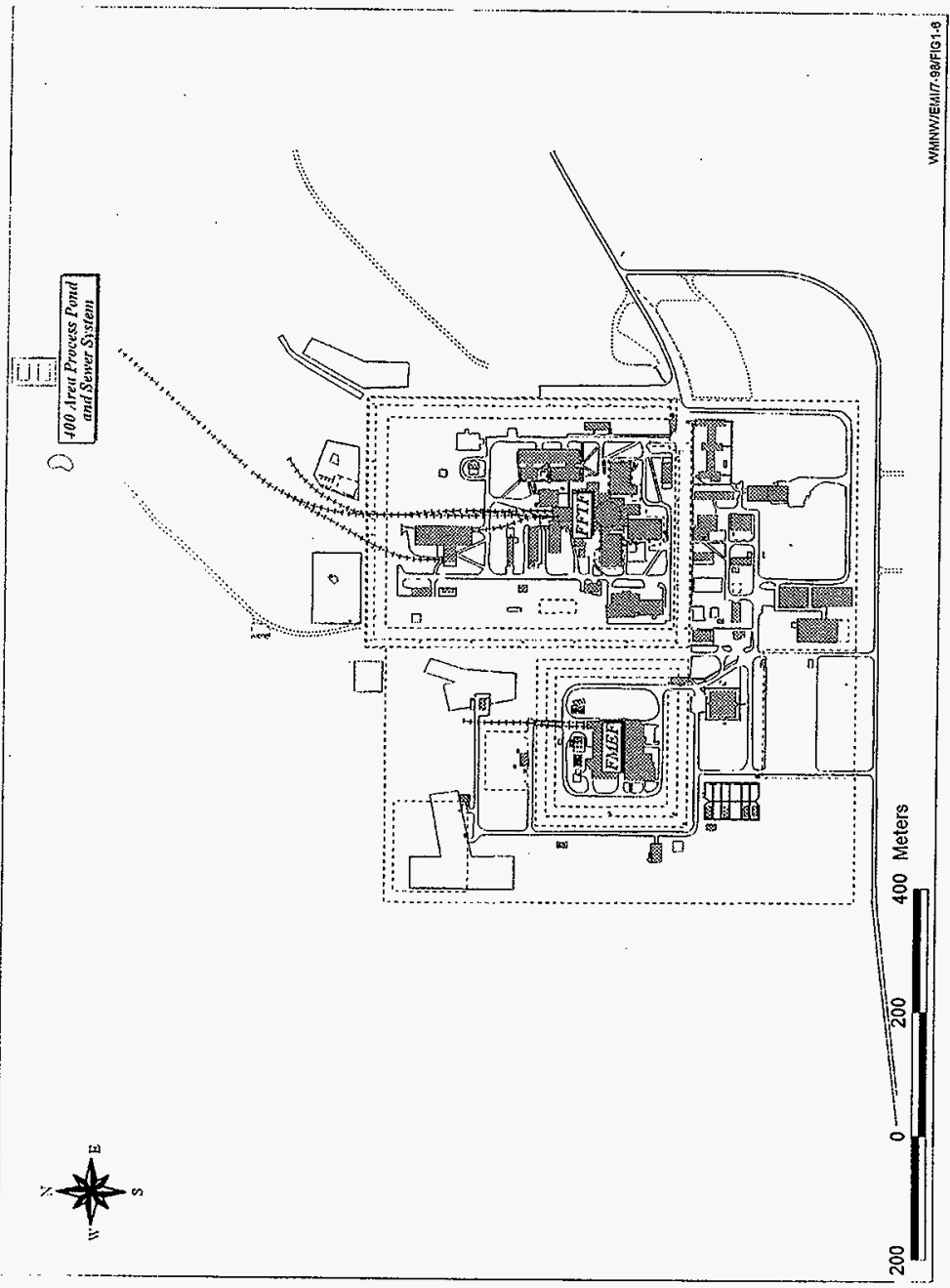


cannot have direct monitoring systems, and are measured through the near-facility environmental monitoring systems. Operating facilities and facilities that are on standby or inactive could also contribute diffuse and fugitive emissions. Deactivation, decontamination, and decommissioning of facilities and cleanup of inactive waste sites are other possible contributors of diffuse and fugitive emissions. Fugitive emissions may occur from doors, vents, or seams in facilities. A facility may be considered a point source and still contribute to fugitive emissions. The 600 Area designates the Site land outside the operational areas. Almost all sources of radionuclide emissions are within the five operational areas; three minor point sources are in the 600 Area. Emissions from point sources, such as stacks and vents, are measured directly or calculated from process knowledge. Diffuse emissions are measured through a network of monitoring systems near and/or adjacent to active and inactive waste sites.

The Hanford Site was acquired in 1943 and dedicated to producing plutonium for national defense and managing the resulting production wastes. Restoring the Hanford Site environment is the current mission that has largely supplanted the previous operational objectives. The environmental restoration effort will entail activities such as decontaminating and decommissioning more than 100 facilities, and cleaning up and restoring more than 2,000 waste sites.

Environmental restoration activities are not anticipated to cause a significant increase in the potential air emissions. Proper planning and controls (e.g., application of dust suppressants) will be utilized to minimize emissions from environmental restoration activities. Until the restoration and cleanup work is completed, diffuse and fugitive radioactive emissions potentially may be released from hundreds of sources besides the 125 point sources (e.g., stacks and vents) registered with the WDOH. Emissions and offsite doses to the public are reported annually to the EPA and WDOH (RL 1997).

\subsubsection{Environmental Transport Sources}

The presently identified actual or potential sources of diffuse and fugitive radionuclide emissions and biotic transport to the environment at the Hanford Site are described in this section. Among the sources that could contribute to biotic transport and diffuse radionuclide emissions are several kinds of waste handling and disposal facilities, such as cribs, ponds, ditches, trenches, retention basins, valve pits, French drains, reverse wells, tanks, and burial grounds. Operating facilities or facilities on standby, that are inactive, or that are being remediated also could contribute diffuse emissions. Each site or facility usually has one or more unique features or characteristics that may influence the contribution to diffuse and fugitive emissions. Features may include structures such as passive vents, risers, equipment access doors, and personnel access doors. Exhausters on facilities are point sources that are monitored at the stack or main point of effluent release in accordance with state and federal regulations. Characteristics may include an undetected leak, unburied waste, or an absence of intrusion barriers.

Various environmental conditions, such as changing atmospheric pressures, wind speed, erosion, evaporation, percolation, biotic intrusion, and/or resuspension, influence rates of diffuse and fugitive emissions.

\subsubsection{General Types of Facilities}

Sections 1.3.2.1 through 1.3.2.12 briefly describe general types of sites and facilities, and the potential primary sources of diffuse emissions and environmental transport pathways from each. 
1.3.2.1 Crib. Low-level liquid waste is discharged to the ground via structures called cribs. These are generaliy subsurface systems, similar to sanitary drain fields, that allow the liquid component of the waste to percolate into the soil. The adsorptive properties of the soil are used to remove radioactive material from the effluent through filtration, ion exchange, precipitation reactions, absorption, and adsorption.

Many cribs are vented to the atmosphere through vents and pipe risers. These engineered structures promote downward flow of liquids disposed of in the cribs, but also provide a pathway to the surface and atmosphere. Secondary routes for diffuse emissions include erosion, biotic uptake, and intrusion followed by wind-caused resuspension.

1.3.2.2 Pond. Ponds were used to manage the large quantities of water (i.e., cooling water and chemical-sewer waste water) associated with chemical processing operations. Normally, these liquid effluents are nonradioactive. The ponds allow percolation of the liquid effluent into the soil column. Diffuse emissions from ponds are primarily by wind-caused resuspension.

1.3.2.3 Ditch. A ditch is an open, unlined excavation that was used for disposing of liquid effluents or for transporting liquid effluents to ponds for disposal. Diffuse emissions from ditches are materials resuspended by the wind.

1.3.2.4 Trench. Early disposal practices included disposing of liquid effluents into unlined, open trenches, then filling the trenches with soil over time. These trenches were replaced with cribs (such as the $B C$ cribs where both cribs and trenches can be found) or trenches with concrete covers (100 N Area). Diffuse emissions from trenches are primarily the result of erosion, biotic uptake, or intrusion, followed by wind-caused resuspension.

1.3.2.5 Retention Basin. Similar to trenches, retention basins generally were lined with concrete and used to hoid liquids before they were routed to ditches or ponds. Diffuse and fugitive emissions from retention basins are primarily wind-caused resuspension.

1.3.2.6 Diversion Box. A diversion box is usually an underground, concrete structure formed around a junction of transfer lines carrying liquid effluent. When diversion boxes are accessed for operation or maintenance, radioactively contaminated material may be released in the form of diffuse and/or fugitive emissions.

1.3.2.7 Valve Pit. A valve pit is similar in structure to a diversion box, but contains piping valves. When valve pits are accessed for operations or maintenance, radioactively contaminated material may be released in the form of diffuse and/or fugitive emissions.

1.3.2.8 French Drain and Reverse or Injection Well. A French drain is a rock-filled encasement inserted into the ground. A reverse or injection well is an ordinary well used for mixing liquid waste with groundwater. These subsurface systems manage potentially contaminated liquid waste by promoting percolation into the soil. The natural filtration properties of the soil remove radionuclide material from effluent water. Diffuse airborne emissions from French drains and reverse wells could occur from erosion, biotic uptake, or intrusion, followed by wind-caused resuspension.

1.3.2.9 Tank. A tank generally is a large, reinforced, metal structure that receives liquid effluent for storage. Examples are the double-shell tanks and single-shell tanks in the 200 Areas, which typically are arrayed in clusters called tank farms. Sources of diffuse and fugitive emissions from tanks include vents and exhausters open to the atmosphere plus deposition and resuspension from surface soil contamination. 
1.3.2.10 Burial Ground. Burial grounds are trenches in which contaminated solid waste is buried. This waste is generated by various activities on the Hanford Site. Waste packaging procedures and burial practices depend on the type of waste. Diffuse and fugitive emissions occur at burial grounds through direct release to the atmosphere before the waste is buried, erosion, biotic uptake and intrusion, and wind-caused resuspension.

1.3.2.11 Deactivation, Decontamination, and Decommissioning Activities. Deactivation, decontamination, and decommissioning activities are being conducted to minimize the potential release or spread of contamination from facilities and equipment. Deactivation activities are intended to remove facility systems and/or areas from operational service with the intent of being ready for facility transition to either convert the facility for another use or move to permanent shutdown. Activities could include removal of fuel, draining, and/or de-energizing of systems, removal of accessible stored radioactive and hazardous material and other actions to place the facility systems and/or areas in a safe and stable condition so that a surveillance and maintenance program will be able to most cost-effectively prevent any unacceptable risk to the public or the environment until ultimate disposition of the facility.

Decontamination consists of either physically removing contaminants or "fixing" contaminants in place to prevent mobility during demolition. Methods might include washing with water, scraping, scabbling, sandblasting, or "fixing" the contamination in place by painting, applying asphalt, etc. Demolition involves destroying and removing the structure and might include excavating any foundation. In some cases, contaminated material might be exposed to the atmosphere, but proper planning and controls minimize these exposures. In addition, monitors around demolition sites confirm that controls are effective. To date, no significant increases at demolition sites have been observed.

1.3.2.12 Waste Site Cleanup Activities. Cleanup activities are being conducted to minimize the potential release or spread of contamination from inactive waste sites. Contaminated soils and structures are being excavated and transported to the Environmental Restoration Disposal Facility (ERDF) for disposal. Contaminated materials are exposed to the atmosphere during excavation and disposal activities. Proper planning and controls such as tarps, water sprays, and fixatives are used to minimize the potential for airborne emissions. The waste sites are backfilled after excavation and the disposed material is covered with soil.

1.3.2.13 Radioactively Contaminated Surface Areas. All radioactive burial grounds, cribs, trenches, retention basins, and unplanned release sites are surveyed routinely. The surveys are performed at least annually, but may be done more frequently when needed. The number of surface-contaminated acres varies. The number is not fixed because a continuing effort to clean, stabilize, or remediate known contaminated areas is under way, while new areas of contamination continue to be identified. Newly identified contamination may be caused by resuspension or biological intrusion to previously uncontaminated areas or because the radiological criteria have become more stringent. Approximately $80 \%$ of all areas of surface contamination are estimated to have dose rates of less than $1 \mathrm{mrem} / \mathrm{h}$.

The radiologically contaminated areas have been reposted to meet the new requirements outlined in HSRCM-1, Hanford Site Radiological Control Manual. The postings include "contamination," "high contamination" (activity of $>100,000 \mathrm{dpm} / 100 \mathrm{~cm}^{2}$ beta/gamma or $>10,000 \mathrm{dpm} / 100 \mathrm{~cm}^{2}$ alpha), "soil contamination," "underground radioactive material," "radiological buffer," and "radiation/high radiation" areas. For continuity between annual reports issued in 1994 and 1997, the use of "surface contamination area" (SCA) in this report includes contamination, high contamination, and soil contamination areas. If an area has surface and underground contamination, such as the surface above a crib, it will have both postings. Table 1- 1 shows the general location (by area), the approximate area of surface contamination, and the underground contamination. Sources of diffuse emissions from surface-contamination areas inciude erosion, biotic uptake, or intrusion followed by wind-caused resuspension. 
A more detailed description of specific sites and facilities can be found in Radionuclide Air Emissions Report for the Hanford Site, Calendar Year 1997 (RL 1998), and in the facility effluent monitoring plans for major facilities, which are referenced in this report.

Table 1-1. Hanford Site Surface and Underground Contamination.

\begin{tabular}{|l|c|c|}
\hline \multicolumn{1}{|c|}{ Hanford Site area } & $\begin{array}{c}\text { Surface contamination } \\
\text { ha (acres) }\end{array}$ & $\begin{array}{c}\text { Underground radioactive material } \\
\text { ha (acres) }\end{array}$ \\
\hline 100 Areas & $47(116)$ & $187(462)$ \\
\hline 200 Areas $^{\circ}$ & $92(227)$ & $361(892)$ \\
\hline 300 Area (north) & $19(47)$ & $13(32)$ \\
\hline 600 Area & (n) & $54(133)$ \\
\hline Total $^{\circ}$ & $3,832(9,469)$ & $615(1,519)$ \\
\hline
\end{tabular}

"Includes areas posted as "contamination/soil contamination" or as "radiologically controlled" and areas that had both underground and surface/soil contamination.

Includes areas with only underground contamination. Does not include areas that had surface and underground radioactive material.

includes tank farms.

Includes redesignated BC Controlled Area (historically included in 200 Area acreage estimates) and waste disposal facilities outside the 200 East Area boundary that received waste from 200 East Area facilities (e.g., 216-A-25, 216-B-3) and waste disposal facilities outside the 200 West Area boundary that received waste from 200 West Area facilities (e.g., 216-S-19, 216-U-11). The Environmental Restoration Disposal Facility was added to the 600 Area waste sites in 1997. 
HNF-EP-0573-6

This page intentionally left blank. 
HNF-EP-0573-6

\subsection{AMBIENT AIR MONITORING}

\subsection{INTRODUCTION}

Ambient air monitoring is conducted to determine baseline concentrations of radionuclides in the operations areas, to assess the impact of operations on the local environment, and to monitor diffuse and fugitive emissions from sources located within the operations area. These measurements also provide an indication of the Project Hanford Management Contract (PHMC)- and environmental restoration contractor (ERC)-managed facilities' performance and are used to demonstrate compliance with environmental protection criteria. Location-specific maps and monitoring results are located in Appendix $\mathrm{C}$ of this report. Only those radionuclide concentrations above analytical detection limits are provided in this report.

Air monitoring within the 300 and 400 Areas is performed by Pacific Northwest National Laboratory (PNNL) as part of the Site Surface Environmental Surveillance Project. A more detailed discussion of these can be found in the Hanford Site Environmental Report for Calendar Year 1997 (Dirkes and Hanf 1998).

The air monitoring network takes into consideration potential source terms as well as prevailing wind direction. Meteorological conditions are monitored continuously by PNNL meteorology stations, which are strategically positioned in and around the Hanford Site.

Hanford Site air samplers operate at a flow rate of $0.056 \mathrm{~m}^{3} / \mathrm{min}\left(2 \mathrm{ft}^{3} / \mathrm{min}\right)$, drawing a sample through a $47-\mathrm{mm}(2$-in.), open-faced filter about $2 \mathrm{~m}(6 \mathrm{ft})$ aboveground. All sample filters are exchanged biweekly, held one week (to allow for decay of the short-lived natural radioactivity), and then sent to the analytical laboratory for initial analysis of total alpha and total beta activity. These initial analyses serve as an indicator of potential environmental problems.

The filters are stored until the end of the six-month sampling period, then segregated and composited by sample location (or as deemed appropriate) for specific radionuclide analysis. Segregating and compositing air fitters by site provides a larger sample size and, thus, a more sensitive and accurate measurement of the concentration of airborne radionuclides.

To help assess the impact of Site operations, monitoring results are compared to DOE derived concentration guides (DCGs) and to the results obtained from the distant communities of Yakima and Sunnyside as reported by the PNNL. Site Environmental Surveillance Program. The data acquired from distant station N-981 (Appendix C, Figure C-6) is used to compare to the data from samples collected in the operating areas and to data from samples collected by PNNL, the WDOH, and Washington Public Power Supply System (Supply System). A split sampling site is used for comparability and precision.

\subsection{AIR SAMPLING RESULTS, 1997}

\subsection{1 $100 \mathrm{~N}$ Area}

The concentrations of airborne radionuclides measured at the $100 \mathrm{~N}$ Area were many times less than the applicable DOE DCGs. The 1997 air sampling results were comparable to the 1996 results and are shown in Appendix $\mathrm{C}$, Table $\mathrm{C}-1$. The annual average concentrations of ${ }^{60} \mathrm{Co}$ detected in the $100 \mathrm{~N}$ Area ambient air samples since 1982 are shown in Figure 2-1. Sampling locations are shown in Appendix C, Figure C-1. 
Figure 2-1. Average Concentrations of ${ }^{60} \mathrm{Co}$ Detected in 100 N Area Ambient Air from 1982 to 1997.

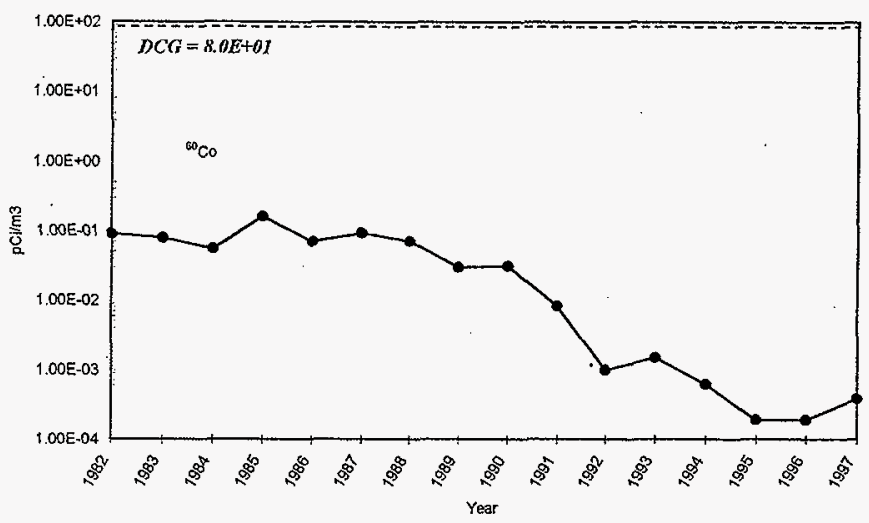

2.2.1.1 ${ }^{60} \mathrm{Co}$. The maximum annual result was at site $\mathrm{N}-106$ (Table 2-1).

Table 2-1. ${ }^{60} \mathrm{Co}$ in Air, $100 \mathrm{~N}$ Area, $1997\left(\mathrm{pCi} / \mathrm{m}^{3}\right)$.

\begin{tabular}{|c|c|c|c|}
\hline $\begin{array}{c}\text { Maximum result } \pm \\
\text { overall error (\%) }\end{array}$ & $\begin{array}{c}\text { Annual average } \\
\pm 2 \text { SEM (\%) }\end{array}$ & DCG & $\begin{array}{c}\text { Distant community } \\
\pm 2 S E M(\%)\end{array}$ \\
\hline $8.3 \mathrm{E}-04 \pm 11 \%$ & $3.9 \mathrm{E}-04 \pm 79 \%$ & $8.0 \mathrm{E}+01$ & $<2.0 \mathrm{E}-04 \pm 97 \%$ \\
\hline
\end{tabular}

2SEM = Two standard error of the mean.

DCG $=$ Derived concentration guide.

2.2.1.2 ${ }^{90} \mathrm{Sr}$. The maximum annual result was at site $\mathrm{N}-106$ (Table 2-2).

Table 2-2. ${ }^{90} \mathrm{Sr}$ in Air, $100 \mathrm{~N}$ Area, $1997\left(\mathrm{pCi} / \mathrm{m}^{3}\right)$.

\begin{tabular}{|c|c|c|c|}
\hline $\begin{array}{c}\text { Maximum result } \pm \\
\text { overall error }(\%)\end{array}$ & $\begin{array}{c}\text { Annual average } \\
\pm 2 \text { SEM }(\%)\end{array}$ & DCG & $\begin{array}{c}\text { Distant community } \\
\pm 2 S E M(\%)\end{array}$ \\
\hline $3.9 \mathrm{E}-04 \pm 30 \%$ & $3.3 \mathrm{E}-04 \pm 59 \%$ & $9.0 \mathrm{E}+00$ & $<-6.3 \mathrm{E}-06 \pm 81 \%$ \\
\hline
\end{tabular}

2SEM = Two standard error of the mean.

DCG $=$ Derived concentration guide. 
2.2.1.3 ${ }^{137} \mathrm{Cs}$. The maximum annual result was at site $\mathrm{N}-105$ (Table 2-3).

Table 2-3. ${ }^{137} \mathrm{Cs}$ in Air, $100 \mathrm{~N}$ Area, $1997\left(\mathrm{pCi} / \mathrm{m}^{3}\right)$.

\begin{tabular}{|c|c|c|c|}
\hline $\begin{array}{c}\text { Maximum result } \pm \\
\text { overail error }(\%)\end{array}$ & $\begin{array}{c}\text { Annual average } \\
\pm 2 \text { SEM }(\%)\end{array}$ & DCG & $\begin{array}{c}\text { Distant community } \\
\pm 2 S E M(\%)\end{array}$ \\
\hline $1.8 \mathrm{E}-\mathrm{O} 4 \pm 45 \%$ & $1.4 \mathrm{E}-04 \pm 88 \%$ & $4.0 \mathrm{E}+02$ & $<2.7 \mathrm{E}-05 \pm 850 \%$ \\
\hline
\end{tabular}

2SEM = Two standard error of the mean.

DCG $=$ Derived concentration guide.

2.2.1.4 ${ }^{234} \mathrm{U}$. The maximum annual result was at site $\mathrm{N}-106$ (Table 2-4).

Table 2-4, ${ }^{234} \mathrm{U}$ in Air, $100 \mathrm{~N}$ Area, $1997\left(\mathrm{pCi} / \mathrm{m}^{3}\right)$.

\begin{tabular}{|c|c|c|c|}
\hline $\begin{array}{c}\text { Maximum result } \pm \\
\text { overall error }(\%)\end{array}$ & $\begin{array}{c}\text { Annual average } \\
\pm 2 \text { SEM (\%) }\end{array}$ & DCG & $\begin{array}{c}\text { Distant community } \\
\pm 2 \text { SEM }(\%)\end{array}$ \\
\hline 1.2 E-O4 $\pm 24 \%$ & 4.0 E-O5 $\pm 81 \%$ & 9.0 E-O2 & 2.1 E-O5 $\pm 3.3 \%$ \\
\hline
\end{tabular}

2SEM = Two standard error of the mean.

$D C G=$ Derived concentration guide.

2.2.1.5 ${ }^{235} \mathrm{U}$. The maximum annual result was at site $\mathrm{N}-106$ (Table 2-5).

Table 2-5. ${ }^{235} \mathrm{U}$ in Air, $100 \mathrm{~N}$ Area, $1997\left(\mathrm{pCi} / \mathrm{m}^{3}\right)$.

\begin{tabular}{|c|c|c|c|}
\hline $\begin{array}{c}\text { Maximum result } \pm \\
\text { overall error }(\%)\end{array}$ & $\begin{array}{c}\text { Annual average } \\
\pm 2 \text { SEM }(\%)\end{array}$ & DCG & $\begin{array}{c}\text { Distant community } \\
\pm 2 \text { SEM }(\%)\end{array}$ \\
\hline 1.0 E-O4 $\pm 26 \%$ & 3.4 E-O5 $\pm 142 \%$ & 1.0 E-01 & $<1.5 \mathrm{E}-07 \pm 230 \%$ \\
\hline
\end{tabular}

2SEM = Two standard error of the mean.

DCG $=$ Derived concentration guide.

2.2.1.6 ${ }^{238} \mathrm{U}$. The maximum annual result was at site $\mathrm{N}-106$ (Table 2-6).

Table 2-6. ${ }^{238} \mathrm{U}$ in Air, $100 \mathrm{~N}$ Area, $1997\left(\mathrm{pCi} / \mathrm{m}^{3}\right)$.

\begin{tabular}{|c|c|c|c|}
\hline $\begin{array}{c}\text { Maximum result } \pm \\
\text { overall error }(\%)\end{array}$ & $\begin{array}{c}\text { Annual average } \\
\pm 2 \text { SEM }(\%)\end{array}$ & DCG & $\begin{array}{c}\text { Distant community } \\
\pm 2 \text { SEM (\%) }\end{array}$ \\
\hline 8.3 E-O5 $\pm 26 \%$ & 3.5 E-05 $\pm 77 \%$ & 1.0 E-O 1 & 1.7 E-05 $050.6 \%$ \\
\hline
\end{tabular}

2SEM = Two standard error of the mean.

$\mathrm{DCG}=$ Derived concentration guide. 
2.2.1.7 ${ }^{239 .}{ }^{240} \mathrm{Pu}$. The maximum annual result was at site $\mathrm{N}-103$ (Table 2-7).

Table 2-7. ${ }^{239,}{ }^{240} \mathrm{Pu}$ in Air, $100 \mathrm{~N}$ Area, 1997. $\left(\mathrm{pCi} / \mathrm{m}^{3}\right)$.

\begin{tabular}{|c|c|c|c|}
\hline $\begin{array}{c}\text { Maximum result } \pm \\
\text { overall error }(\%)\end{array}$ & $\begin{array}{c}\text { Annual average } \\
\pm 2 \mathrm{SEM}(\%)\end{array}$ & DCG & $\begin{array}{c}\text { Distant community } \\
\pm 2 \mathrm{SEM}(\%)\end{array}$ \\
\hline $1.3 \mathrm{E}-05 \pm 49 \%$ & $8.7 \mathrm{E}-06 \pm 180 \%$ & $2.0 \mathrm{E}-02$ & $<2.2 \mathrm{E}-07 \pm 73 \%$ \\
\hline
\end{tabular}

2SEM = Two standard error of the mean.

$\mathrm{DCG}=$ Derived concentration guide.

\subsection{2 $100 \mathrm{~K}$ Area}

Sampling at $100 \mathrm{~K}$ Area is conducted in an area surrounding the $105 \mathrm{KE}$ Basin. This is the preferred sampling area since the stack emissions from that facility are the largest emissions source in the $100 \mathrm{~K}$ Area. (Stack emissions from the $105 \mathrm{KW}$ Basin are approximately one order of magnitude less than $105 \mathrm{KE}$ Basin.) In general, the concentrations of airborne radionuclides measured at the $100 \mathrm{~K}$ Area were many times less than the DOE DCGs. Concentrations of many of . the isotopes were negative or had large standard errors of the mean, indicating that the result was at or near background levels of radioactivity. The 1997 results are shown in Appendix C, Table C-2. Sampling locations are shown in Appendix C, Figure C-2.

2.2.2.1 ${ }^{60} \mathrm{Co}$. ${ }^{60} \mathrm{Co}$ concentrations were less than $(<)$ detection limits in 1997 (Table 2-8).

Table 2-8. ${ }^{60} \mathrm{Co}$ in Air $100 \mathrm{~K}$ Area, $1997\left(\mathrm{pCi} / \mathrm{m}^{3}\right)$.

\begin{tabular}{|c|c|c|c|}
\hline $\begin{array}{c}\text { Maximum result } \pm \\
\text { overall error }(\%)\end{array}$ & $\begin{array}{c}\text { Annual average } \\
\pm 2 \text { SEM }(\%)\end{array}$ & DCG & $\begin{array}{c}\text { Distant community } \\
\pm 2 \text { SEM }(\%)\end{array}$ \\
\hline$<4.0$ E-05 $\pm 92 \%$ & Not applicable & $8.0 E+01$ & $<2.0 E-04 \pm 97 \%$ \\
\hline
\end{tabular}

2SEM = Two standard error of the mean.

DCG = Derived concentration guide.

2.2.2.2 ${ }^{90} \mathrm{Sr}$. The maximum annual result was at site $\mathrm{N}-404$ (Table 2-9).

Table 2-9. ${ }^{90} \mathrm{Sr}$ in Air $100 \mathrm{~K}$ Area, $1997\left(\mathrm{pCi} / \mathrm{m}^{3}\right)$.

\begin{tabular}{|c|c|c|c|}
\hline $\begin{array}{c}\text { Maximum result } \pm \\
\text { overall error }(\%)\end{array}$ & $\begin{array}{c}\text { Annual average } \\
\pm 2 \text { SEM (\%) }\end{array}$ & DCG & $\begin{array}{c}\text { Distant community } \\
\pm 2 \text { SEM (\%) }\end{array}$ \\
\hline $1.6 \mathrm{E}-03 \pm 16 \%$ & $6.8 \mathrm{E}-04 \pm 54 \%$ & $9.0 \mathrm{E}+00$ & $<-5.3 \mathrm{E}-06 \pm 81 \%$ \\
\hline
\end{tabular}

2SEM = Two standard error of the mean.

$D C G=$ Derived concentration guide . 
2.2.2.3 ${ }^{137} \mathrm{Cs}$. The maximum annual result was at site $\mathrm{N}-401$ (Table 2-10).

Table 2-10. ${ }^{137} \mathrm{Cs}$ in Air $100 \mathrm{~K}$ Area, $1997\left(\mathrm{pCi} / \mathrm{m}^{3}\right)$.

\begin{tabular}{|c|c|c|c|}
\hline $\begin{array}{c}\text { Maximum result } \pm \\
\text { overall error }(\%)\end{array}$ & $\begin{array}{c}\text { Annual average } \\
\pm 2 \text { SEM }(\%)\end{array}$ & DCG & $\begin{array}{c}\text { Distant Community } \\
\pm 2 \text { SEM }(\%)\end{array}$ \\
\hline $9.0 \mathrm{E}-\mathrm{O} 3 \pm 14 \%$ & $1.8 \mathrm{E}-03 \pm 124 \%$ & $4.0 \mathrm{E}+02$ & $<2.7 \mathrm{E}-05 \pm 850 \%$ \\
\hline
\end{tabular}

2SEM = Two standard error of the mean.

DCG $=$ Derived concentration guide.

2.2.2.4 ${ }^{234} \mathrm{U}$. The maximum annual result was at site $\mathrm{N}-401$ (Table 2-11).

Table 2-11. ${ }^{234} \mathrm{U}$ in Air, $100 \mathrm{~K}$ Area, $1997\left(\mathrm{pCi} / \mathrm{m}^{3}\right)$.

\begin{tabular}{|c|c|c|c|}
\hline $\begin{array}{c}\text { Maximum result } \pm \\
\text { overall error }(\%)\end{array}$ & $\begin{array}{c}\text { Annual average } \\
\pm 2 \text { SEM (\%) }\end{array}$ & DCG & $\begin{array}{c}\text { Distant community } \\
\pm 2 \text { SEM }(\%)\end{array}$ \\
\hline $1.8 \mathrm{E}-05 \pm 43 \%$ & $1.5 \mathrm{E}-05 \pm 31 \%$ & $9.0 \mathrm{E}-02$ & $2.1 \mathrm{E}-05 \pm 3.3 \%$ \\
\hline
\end{tabular}

2SEM = Two standard error of the mean.

DCG $=$ Derived concentration guide.

2.2.2.5 ${ }^{235} \mathrm{U}$. The maximum annual result was at site $\mathrm{N}-404$ (Table 2-12).

Table 2-12. ${ }^{235} \mathrm{U}$ in Air, $100 \mathrm{~K}$ Area, $1997\left(\mathrm{pCi} / \mathrm{m}^{3}\right)$.

\begin{tabular}{|c|c|c|c|}
\hline $\begin{array}{c}\text { Maximum result } \pm \\
\text { overall error }(\%)\end{array}$ & $\begin{array}{c}\text { Annual average } \\
\pm 2 \text { SEM }(\%)\end{array}$ & DCG & $\begin{array}{c}\text { Distant community } \\
\pm 2 \text { SEM (\%) }\end{array}$ \\
\hline $9.5 \mathrm{E}-\mathrm{O} 6 \mathrm{6} \pm 54 \%$ & $6.6 \mathrm{E}-06 \pm 42 \%$ & $1.0 \mathrm{E}-01$ & $<1.5 \mathrm{E}-07 \pm 230 \%$ \\
\hline
\end{tabular}

2SEM = Two standard error of the mean.

$\mathrm{DCG}=$ Derived concentration guide.

2.2.2.6 ${ }^{238} \mathrm{U}$. The maximum annual result was at site $\mathrm{N}-404$ (Table 2-13).

Table 2-13. ${ }^{238} \mathrm{U}$ in Air, $100 \mathrm{~K}$ Area, $1997\left(\mathrm{pCi} / \mathrm{m}^{3}\right)$.

\begin{tabular}{|c|c|c|c|}
\hline $\begin{array}{c}\text { Maximum result } \pm \\
\text { overall error }(\%)\end{array}$ & $\begin{array}{c}\text { Annual average } \\
\pm 2 \text { SEM }(\%)\end{array}$ & DCG & $\begin{array}{c}\text { Distant community } \\
\pm 2 \text { SEM (\%) }\end{array}$ \\
\hline $2.0 \mathrm{E}-05 \pm 47 \%$ & $1.3 \mathrm{E}-05 \pm 36 \%$ & $1.0 \mathrm{E}-01$ & $1.7 \mathrm{E}-05 \pm 0.6 \%$ \\
\hline
\end{tabular}

2SEM = Two standard error of the mean.

DCG $=$ Derived concentration guide. 
2.2.2.7 ${ }^{239,}{ }^{240} \mathrm{Pu} .{ }^{239,240} \mathrm{Pu}$ was detected in only one sample (N-401) in 1997 (Table 2-14).

Table 2-14. ${ }^{239,}{ }^{240} \mathrm{Pu}$ in Air $100 \mathrm{~K}$ Area, $1997\left(\mathrm{pCi} / \mathrm{m}^{3}\right)$.

\begin{tabular}{|c|c|c|c|}
\hline $\begin{array}{c}\text { Maximum result } \pm \\
\text { overall error }(\%)\end{array}$ & $\begin{array}{c}\text { Annual average } \\
\pm 2 \text { SEM (\%) }\end{array}$ & DCG & $\begin{array}{c}\text { Distant community } \\
\pm 2 \text { SEM }(\%)\end{array}$ \\
\hline $2.5 \mathrm{E}-05 \pm 74 \%$ & Not applicable & $2.0 \mathrm{E}-02$ & $<2.2 \mathrm{E}-07 \pm 73 \%$ \\
\hline
\end{tabular}

2SEM = Two standard error of the mean.

DCG $=$ Derived concentration guide.

2.2.2.8 ${ }^{241} \mathrm{Am}$. The maximum annual result was at site $\mathrm{N}-401$ (Table 2-15).

Table 2-15. ${ }^{241} \mathrm{Am}$ in Air $100 \mathrm{~K}$ Area, $1997\left(\mathrm{pCi} / \mathrm{m}^{3}\right)$.

\begin{tabular}{|c|c|c|c|}
\hline $\begin{array}{c}\text { Maximum result } \pm \\
\text { overall error }(\%)\end{array}$ & $\begin{array}{c}\text { Annual average } \\
\pm 2 \text { SEM (\%) }\end{array}$ & DCG & $\begin{array}{c}\text { Distant community } \\
\pm 2 S E M(\%)\end{array}$ \\
\hline 6.3 E-O5 $\pm 31 \%$ & 3.6 E-O5 $\pm 36 \%$ & 2.0 E-02 & Not reported \\
\hline
\end{tabular}

2SEM = Two standard error of the mean.

DCG $=$ Derived concentration guide.

\subsubsection{Areas}

The concentrations of airborne radionuclides measured in 1997 in the 200 Areas were many times less than the DOE DCGs. The 1997 air sampling results for the 200 Areas are shown in Appendix C, Table C-3. The annual average concentrations since 1979 are illustrated in Figures 2-2 through 2-4. Radionuclide concentrations detected in the ambient air in 1997 are comparable to those observed in 1996. Increasing trends seen in the past are associated with the operation of nuclear facilities, such as the Plutonium Uranium Extraction (PUREX) Plant. Sampling locations are shown in Appendix C, Figures $\mathrm{C}-3$ and $\mathrm{C}-4$.

2.2.3.1 ${ }^{60} \mathrm{Co}$. ${ }^{60} \mathrm{Co}$ concentrations were less than $(<)$ detection limits in 1997 (Table 2-16).

Table 2-16. ${ }^{60} \mathrm{Co}$ in Air, 200 Areas, $1997\left(\mathrm{pCi} / \mathrm{m}^{3}\right)$.

\begin{tabular}{|c|c|c|c|}
\hline $\begin{array}{c}\text { Maximum result } \pm \\
\text { overall error }(\%)\end{array}$ & $\begin{array}{c}\text { Annual average } \\
\pm 2 \text { SEM (\%) }\end{array}$ & DCG & $\begin{array}{c}\text { Distant community } \\
\pm 2 S E M(\%)\end{array}$ \\
\hline$<3.3 \mathrm{E}-04 \pm 180 \%$ & Not applicable & $8.0 \mathrm{E}+01$ & $<2.0 \mathrm{E}-04 \pm 97 \%$ \\
\hline
\end{tabular}

2SEM $=$ Two standard error of the mean.

$\mathrm{DCG}=$ Derived concentration guide. 
Figure 2-2. Annual Average ${ }^{137} \mathrm{Cs}$ Concentrations in Air for the 200/600 Areas.

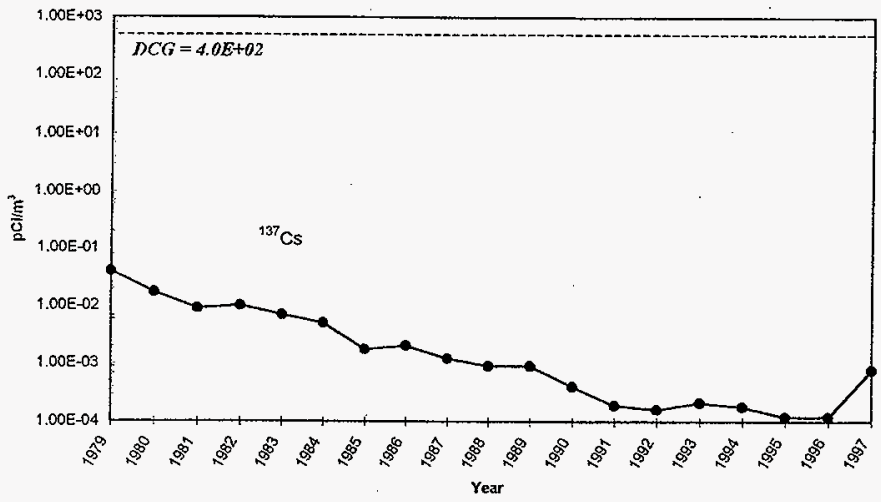

Figure 2-3. Annual Average ${ }^{90} \mathrm{Sr}$ Concentrations in Air for the $200 / 600$ Areas.

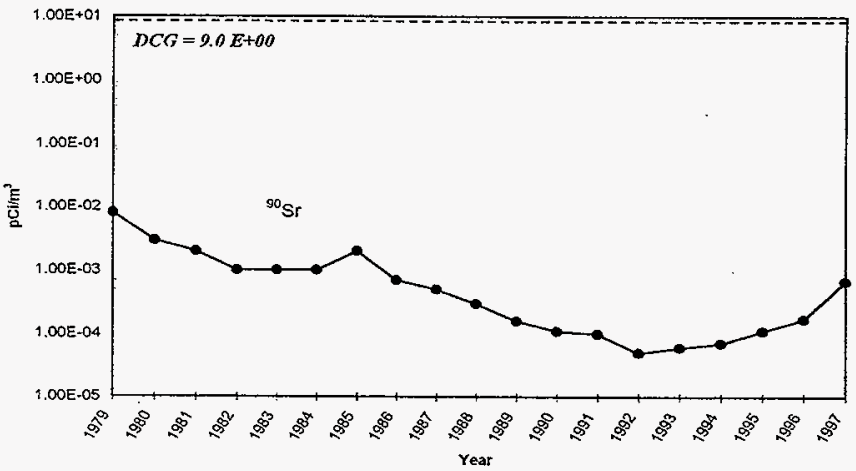


Figure 2-4. Annual Average ${ }^{239,240} \mathrm{Pu}$ Concentrations in Air for the $200 / 600$ Areas.

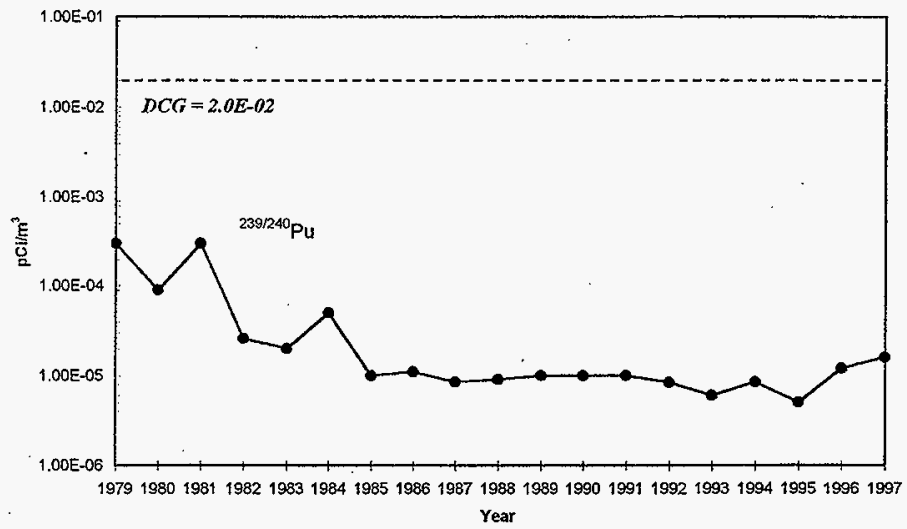

2.2.3.2 ${ }^{90} \mathrm{Sr}$. The maximum annual result was at site $\mathrm{N}-019$, near the B Plant waste disposal complex in the 200 East Area (Table 2-17).

Table 2-17. ${ }^{90} \mathrm{Sr}$ in Air, 200 Areas, $1997\left(\mathrm{pCi} / \mathrm{m}^{3}\right)$.

\begin{tabular}{|c|c|c|c|}
\hline $\begin{array}{c}\text { Maximum result } \pm \\
\text { overall error }(\%)\end{array}$ & $\begin{array}{c}\text { Annual average } \\
\pm 2 \mathrm{SEM}(\%)\end{array}$ & DCG & $\begin{array}{c}\text { Distant community } \\
\pm 2 \mathrm{SEM}(\%)\end{array}$ \\
\hline $9.8 \mathrm{E}-03 \pm 12 \%$ & $6.7 \mathrm{E}-04 \pm 56 \%$ & $9.0 \mathrm{E}+00$ & $<-5.3 \mathrm{E}-06 \pm 81 \%$ \\
\hline
\end{tabular}

2SEM = Two standard error of the mean.

$D C G=$ Derived concentration guide.

2.2.3.3 ${ }^{137} \mathrm{Cs}$. The maximum annual result was at site $\mathrm{N}-019$, near the $\mathrm{B}$ Plant waste disposal complex in the 200 East Area (Table 2-18).

Table 2-18. ${ }^{137} \mathrm{Cs}$ in Air, 200 Areas, $1997\left(\mathrm{pCi} / \mathrm{m}^{3}\right)$.

\begin{tabular}{|c|c|c|c|}
\hline $\begin{array}{c}\text { Maximum result } \pm \\
\text { overall error }(\%)\end{array}$ & $\begin{array}{c}\text { Annual average } \\
\pm 2 \text { SEM }(\%)\end{array}$ & DCG & $\begin{array}{c}\text { Distant community } \\
\pm 2 S E M(\%)\end{array}$ \\
\hline $1.9 \mathrm{E}-02 \pm 18 \%$ & $7.7 \mathrm{E}-04 \pm 152 \%$ & $4.0 \mathrm{E}+02$ & $<2.7 \mathrm{E}-05 \pm 850 \%$ \\
\hline
\end{tabular}

2SEM = Two standard error of the mean.

DCG $=$ Derived concentration guide. 
2.2.3.4 ${ }^{234} \mathrm{U}$. The maximum annual result was at site $\mathrm{N}-998$, near the $218-\mathrm{E}-12 \mathrm{~B}$ burial ground in the 200 East Area (Table 2-19).

Table 2-19. ${ }^{234} \mathrm{U}$ in Air, 200 Areas, $1997\left(\mathrm{pCi} / \mathrm{m}^{3}\right)$.

\begin{tabular}{|c|c|c|c|}
\hline $\begin{array}{c}\text { Maximum resuit } \pm \\
\text { overall error }(\%)\end{array}$ & $\begin{array}{c}\text { Annual average } \\
\pm 2 \text { SEM }(\%)\end{array}$ & DCG & $\begin{array}{c}\text { Distant community } \\
\pm 2 \text { SEM }(\%)\end{array}$ \\
\hline $7.2 \mathrm{E}-05 \pm 31 \%$ & $1.8 \mathrm{E}-05 \pm 31 \%$ & $9.0 \mathrm{E}-\mathrm{O} 2$ & $2.1 \mathrm{E}-05 \pm 3.3 \%$ \\
\hline
\end{tabular}

2SEM = Two standard error of the mean.

$\mathrm{DCG}=$ Derived concentration guide.

2.2.3.5 ${ }^{235} \mathrm{U}$. The maximum annual result was at site $\mathrm{N}-998$, near the $218-\mathrm{E}-12 \mathrm{~B}$ burial ground in the 200 East Area (Table 2-20).

Table 2-20. ${ }^{235} \mathrm{U}$ in Air, 200 Areas, $1997\left(\mathrm{pCi} / \mathrm{m}^{3}\right)$.

\begin{tabular}{|c|c|c|c|}
\hline $\begin{array}{c}\text { Maximum result } \pm \\
\text { overall error }(\%)\end{array}$ & $\begin{array}{c}\text { Annual average } \\
\pm 2 \text { SEM }(\%)\end{array}$ & DCG & $\begin{array}{c}\text { Distant community } \\
\pm 2 \text { SEM }(\%)\end{array}$ \\
\hline 2.8 E-O5 $\pm 56 \%$ & $9.5 \mathrm{E}-06 \pm 13 \%$ & $1.0 \mathrm{E}-01$ & $<1.5 \mathrm{E}-07 \pm 230 \%$ \\
\hline
\end{tabular}

2SEM = Two standard error of the mean.

$D C G=$ Derived concentration guide.

2.2.3.6 ${ }^{238} \mathrm{U}$. The maximum annual result was at site $\mathrm{N}-998$, near the $218-\mathrm{E}-12 \mathrm{~B}$ burial ground in the 200 East Area (Table 2-21).

Table 2-21. ${ }^{238} \mathrm{U}$ in Air, 200 Areas, $1997\left(\mathrm{pCi} / \mathrm{m}^{3}\right)$.

\begin{tabular}{|c|c|c|c|}
\hline $\begin{array}{c}\text { Maximum result } \pm \\
\text { overall error }(\%)\end{array}$ & $\begin{array}{c}\text { Annual average } \\
\pm 2 \text { SEM }(\%)\end{array}$ & DCG & $\begin{array}{c}\text { Distant community } \\
\pm 2 \text { SEM }(\%)\end{array}$ \\
\hline 7.4 E-O5 $\pm 31 \%$ & 1.4 E-OS $\pm 16 \%$ & $1.0 \mathrm{E}-01$ & 1.7 E-05 $050.6 \%$ \\
\hline
\end{tabular}

2SEM = Two standard error of the mean.

$\mathrm{DCG}=$ Derived concentration guide.

2.2.3.7 ${ }^{239 .}{ }^{240} \mathrm{Pu}$. The maximum annual result was at site $\mathrm{N}-165$, near the $216-\mathrm{Z}$ - $\mathrm{Tg}$ Ditcin in the 200 West Area (Table 2-22).

Table 2-22. ${ }^{239,}{ }^{240} \mathrm{Pu}$ in Air, 200 Areas, $1997\left(\mathrm{pCi} / \mathrm{m}^{3}\right)$.

\begin{tabular}{|c|c|c|c|}
\hline $\begin{array}{c}\text { Maximum result } \pm \\
\text { overall error }(\%)\end{array}$ & $\begin{array}{c}\text { Annual average } \\
\pm 2 \mathrm{SEM}(\%)\end{array}$ & DCG & $\begin{array}{c}\text { Distant community } \\
\pm 2 \mathrm{SEM}(\%)\end{array}$ \\
\hline $1.3 \mathrm{E}-04 \pm 32 \%$ & $1.6 \mathrm{E}-05 \pm 48 \%$ & $2.0 \mathrm{E}-02$ & $<2.2 \mathrm{E}-07 \pm 73 \%$ \\
\hline
\end{tabular}

2SEM = Two standard error of the mean.

DCG $=$ Derived concentration guide. 
2.2.3.8 ${ }^{241} \mathrm{Am}$. The maximum annual result was at site $\mathrm{N}-165$, near the $216-\mathrm{Z}-19$ Ditch in the 200 West Area (Table 2-23).

Table 2-23. ${ }^{241} \mathrm{Am}$ in Air, 200 Areas, $1997\left(\mathrm{pCi} / \mathrm{m}^{3}\right)$.

\begin{tabular}{|c|c|c|c|}
\hline $\begin{array}{c}\text { Maximum result } \pm \\
\text { overall error }(\%)\end{array}$ & $\begin{array}{c}\text { Annual average } \\
\pm 2 \text { SEM (\%) }\end{array}$ & DCG & $\begin{array}{c}\text { Distant community } \\
\pm 2 \text { 2SEM (\%) }\end{array}$ \\
\hline $6.1 \mathrm{E}-05 \pm 39 \%$ & $3.3 \mathrm{E}-05 \pm 48 \%$ & $2.0 \mathrm{E}-02$ & Not reported \\
\hline
\end{tabular}

2SEM = Two standard error of the mean.

DCG $=$ Derived concentration guide.

\subsection{4 $300 / 400$ Areas}

The concentrations of airborne radionuclides measured during. 1997 in the 300 and 400 Areas were many times less than the DOE DCGs, and the results are provided in Appendix C, Tables C-4 and C-5, respectively. Air monitoring near the operating facilities in the 300 (300 Area and 300 Area NE) and 400 Areas is provided by PNNL and the data are transferred to PHMC after review and validation. For a more detailed discussion regarding these monitors, refer to the Hanford. Site Environmental Report for Calendar Year 1997 (Dirkes and Hanf 1998). One ambient air sampler, located at the 300 Area Treated Effluent Disposal Facility (TEDF), is PHMC-operated and the data included in this report. Sampling locations are shown in Appendix C, Figure C-5.

2.2.4.1 ${ }^{90} \mathrm{Sr}$. The maximum annual average result in the $300 / 400$ Areas was at the TEDF site, N-130 (Table 2-24).

Table 2-24. ${ }^{90} \mathrm{Sr}$ in Air, 300/400 Areas, $1997\left(\mathrm{pCi} / \mathrm{m}^{3}\right)$.

\begin{tabular}{|l|c|c|c|}
\hline \multicolumn{1}{|c|}{ Area } & $\begin{array}{c}\text { Annual average } \pm \text { overall } \\
\text { analytical error }(\%)\end{array}$ & DCG & $\begin{array}{c}\text { Distant Community } \\
\pm 2 \text { 2SM (\%) }\end{array}$ \\
\hline 300 (PNNL) & $3.1 \mathrm{E}-05 \pm 41 \%$ & & \\
\cline { 1 - 2 } 300 NE (PNNL) & $1.3 \mathrm{E}-05 \pm 74 \%$ & \multirow{2}{*}{$9.0 \mathrm{E}+00$} & $<-5.3 \mathrm{E}-06 \pm 81 \%$ \\
\cline { 1 - 2 } 300 Area TEDF N-130 & $3.9 \mathrm{E}-04 \pm 30 \%$ & & \\
\hline 400 & $1.1 \mathrm{E}-04 \pm 26 \%$ & & \\
\hline
\end{tabular}

2SEM = Two standard error of the mean.

DCG $=$ Derived concentration guide.

PNNL = Pacific Northwest National Laboratory.

TEDF = Treated Effluent Disposal Facility.

2.2.4.2 ${ }^{234} \mathrm{U}$. The maximum annual average result in the 300 Area was at the $300 \mathrm{NE}$ composite group site. This anaiysis was not performed in the 400 Area (Table 2-25). 
Table 2-25. ${ }^{234} \mathrm{U}$ in Air, 300/400 Areas, $1997\left(\mathrm{pCi} / \mathrm{m}^{3}\right)$.

\begin{tabular}{|c|c|c|c|}
\hline Area & $\begin{array}{c}\text { Annual average } \pm \text { overall } \\
\text { analytical error }(\%)\end{array}$ & DCG & $\begin{array}{c}\text { Distant community } \\
\pm 2 \operatorname{SEM}(\%)\end{array}$ \\
\hline 300 (PNNL) & $1.9 E-05 \pm 21 \%$ & \multirow{3}{*}{9.0 E-02 } & \multirow{3}{*}{$2.1 \mathrm{E}-05 \pm 3.3 \%$} \\
\hline 300 NE (PNNL) & $3.3 E-05 \pm 22 \%$ & & \\
\hline 300 Area TEDF $N-130$ & $1.8 \mathrm{E}-05 \pm 5.4 \%{ }^{(\mathrm{a})}$ & & \\
\hline
\end{tabular}

2SEM = Two standard error of the mean.

DCG = Derived concentration guide.

PNNL = Pacific Northwest National Laboratory.

TEDF $=$ Treated Effluent Disposal Facility.

22SEM value reported.

2.2.4.3 ${ }^{235} \mathrm{U}$. The maximum annual average result in the 300 Area was at the TEDF site, $\mathrm{N}-130$. This analysis was not performed in the 400 Area (Tabie 2-26).

Table 2-26. ${ }^{235} \mathrm{U}$ in Air, $300 / 400$ Areas, $1997\left(\mathrm{pCi} / \mathrm{m}^{3}\right)$.

\begin{tabular}{|c|c|c|c|}
\hline Area & $\begin{array}{c}\text { Annual average } \pm \text { overall } \\
\text { analytical error }(\%)\end{array}$ & $\mathrm{DCG}$ & $\begin{array}{l}\text { Distant community } \\
\pm 2 \text { SEM (\%) }\end{array}$ \\
\hline 300 (PNNL) & $4.2 E-07 \pm 178 \%$ & \multirow{3}{*}{$1.0 \mathrm{E}-01$} & \multirow{3}{*}{$<1.5 \mathrm{E}-\mathrm{O} 7 \pm 230 \%$} \\
\hline 300 NE (PNNL) & $9.4 E-07 \pm 160 \%$ & & \\
\hline 300 Area TEDF $N-130$ & $1.2 \mathrm{E}-05 \pm 50 \%$ & & \\
\hline
\end{tabular}

2SEM = Two standard error of the mean.

DCG = Derived concentration guide.

PNNL = Pacific Northwest National Laboratory.

TEDF = Treated Effluent Disposal Facility.

2.2.4.4 ${ }^{238} \mathrm{U}$. The maximum annual average result in the 300 Area was at the $300 \mathrm{NE}$ composite group site. This analysis was not performed in the 400 Area (Table 2-27).

Table 2-27. ${ }^{238} \mathrm{U}$ in Air, 300/400 Areas, $1997\left(\mathrm{pCi} / \mathrm{m}^{3}\right)$.

\begin{tabular}{|c|c|c|c|}
\hline Area & $\begin{array}{c}\text { Annual average } \pm \text { overall } \\
\text { analytical error }(\%)\end{array}$ & DCG & $\begin{array}{c}\text { Distant community } \\
\pm 2 \operatorname{SEM}(\%)\end{array}$ \\
\hline 300 (PNNL) & $1.4 E-05 \pm 23 \%$ & \multirow{3}{*}{1.0 E-O1 } & \multirow{3}{*}{$1.7 \mathrm{E}-05 \pm 0.6 \%$} \\
\hline 300 NE (PNNL) & $2.3 \mathrm{E}-05 \pm 25 \%$ & & \\
\hline 300 Area TEDF $N-130$ & $1.7 \mathrm{E}-05 \pm 17 \%^{(8)}$ & & \\
\hline
\end{tabular}

2SEM $=$ Two standard error of the mean.

DCG $=$ Derived concentration guide.

PNNL = Pacific Northwest National Laboratory.

TEDF = Treated Effluent Disposal Facility

2SEM value reported. 
2.2.4.5 ${ }^{239,}{ }^{240} \mathrm{Pu}$. The maximum annual average result in the $300 / 400$ Areas was at the 300 composite group site (Table 2-28).

Table 2-28. ${ }^{239,240} \mathrm{Pu}$ in Air, 300/400 Areas, $1997\left(\mathrm{pCi} / \mathrm{m}^{3}\right)$.

\begin{tabular}{|l|c|c|c|}
\hline \multicolumn{1}{|c|}{ Area } & $\begin{array}{c}\text { Annual average } \pm \text { overall } \\
\text { analytical error (\%) }\end{array}$ & DCG & $\begin{array}{c}\text { Distant community } \\
\pm 2 \text { 2SEM (\%) }\end{array}$ \\
\hline 300 (PNNL) & $3.1 \mathrm{E}-07 \pm 124 \%$ & & \\
\cline { 1 - 2 } 300 NE (PNNL) & $9.0 \mathrm{E}-08 \pm 245 \%$ & \multirow{2}{*}{$2.0 \mathrm{E}-02$} & $<2.2 \mathrm{E}-07 \pm 73 \%$ \\
\cline { 1 - 2 } 300 Area TEDF $\mathrm{N}-130$ & $<3.1 \mathrm{E}-06 \pm 120 \%{ }^{(3)}$ & & \\
\hline 400 & $1.5 \mathrm{E}-07 \pm 117 \%$ & \\
\hline
\end{tabular}

2SEM = Two standard error of the mean.

DCG $=$ Derived concentration guide.

PNNL $=$ Pacific Northwest National Laboratory.

TEDF = Treated Effluent Disposal Facility.

'2SEM value reported.

\subsection{5 $100 \mathrm{~B} / \mathrm{C}$ Area}

Near-facility air sampling was conducted for the ERC at the $100 \mathrm{~B} / \mathrm{C}$ remediation site through a network of three continuous air samplers. Monitoring began in July 1996. The analytical results indicated that the concentrations were much less than the DOE DCGs and comparable to levels measured at other locations onsite. Appendix C, Table C-6 provides the 1997 air sampling results. Sampling locations are shown in Appendix C, Figure C-7.

2.2.5. $1{ }^{60} \mathrm{Co} .{ }^{60} \mathrm{Co}$ concentrations were less than $(<)$ detection limits in 1997 (Table 2-29).

Table 2-29. ${ }^{60} \mathrm{Co}$ in Air $100 \mathrm{~B} / \mathrm{C}$ Area, $1997\left(\mathrm{pCi} / \mathrm{m}^{3}\right)$.

\begin{tabular}{|c|c|c|c|}
\hline $\begin{array}{c}\text { Maximum result } \pm \\
\text { overall error }(\%)\end{array}$ & $\begin{array}{c}\text { Annual average } \\
\pm 2 \text { SEM }(\%)\end{array}$ & DCG & $\begin{array}{c}\text { Distant community } \\
\pm 2 \text { SEM }(\%)\end{array}$ \\
\hline$<6.1 \mathrm{E}-05 \pm 94 \%$ & Not applicable & $8.0 \mathrm{E}+01$ & $<2.0 \mathrm{E}-04 \pm 97 \%$ \\
\hline
\end{tabular}

2SEM = Two standard error of the mean.

DCG $=$ Derived concentration guide.

2.2.5.2 ${ }^{90} \mathrm{Sr}$. The maximum annual result was at site $\mathrm{N}-464$ (Table 2-30).

Table 2-30. ${ }^{90} \mathrm{Sr}$ in Air $100 \mathrm{~B} / \mathrm{C}$ Area, $1997\left(\mathrm{pCi} / \mathrm{m}^{3}\right)$.

\begin{tabular}{|c|c|c|c|}
\hline $\begin{array}{c}\text { Maximum result } \pm \\
\text { overall error }(\%)\end{array}$ & $\begin{array}{c}\text { Annual average } \\
\pm 2 \text { SEM }(\%)\end{array}$ & DCG & $\begin{array}{c}\text { Distant community } \\
\pm 2 \text { SEM (\%) }\end{array}$ \\
\hline $1.1 \mathrm{E}-\mathrm{OS} \pm 17 \%$ & $7.9 \mathrm{E}-04 \pm 80 \%$ & $9.0 \mathrm{E}+00$ & $<-5.3 \mathrm{E}-06 \pm 81 \%$ \\
\hline
\end{tabular}

2SEM = Two standard error of the mean.

$\mathrm{DCG}=$ Derived concentration guide. 
2.2.5.3 ${ }^{137}$ Cs. The maximum annual result was at site $\mathrm{N}-466$ (Table 2-31).

Table 2-31. ${ }^{137} \mathrm{Cs}$ in Air $100 \mathrm{~B} / \mathrm{C}$ Area, $1997\left(\mathrm{pCi} / \mathrm{m}^{3}\right)$.

\begin{tabular}{|c|c|c|c|}
\hline $\begin{array}{c}\text { Maximum result } \pm \\
\text { overall error }(\%)\end{array}$ & $\begin{array}{c}\text { Annual average } \\
\pm 2 \mathrm{SEM}(\%)\end{array}$ & DCG & $\begin{array}{c}\text { Distant community } \\
\pm 2 \mathrm{SEM}(\%)\end{array}$ \\
\hline $3.7 \mathrm{E}-04 \pm 25 \%$ & $1.8 \mathrm{E}-04 \pm 73 \%$ & $4.0 \mathrm{E}+02$ & $<2.7 \mathrm{E}-05 \pm 850 \%$ \\
\hline
\end{tabular}

2SEM = Two standard error of the mean.

DCG = Derived concentration guide.

2.2.5.4 ${ }^{234} \mathrm{U}$. The maximum annual result was at site $\mathrm{N}-465$ (Table 2-32).

Table 2-32. ${ }^{234} \mathrm{U}$ in Air $100 \mathrm{~B} / \mathrm{C}$ Area, $1997\left(\mathrm{pCi} / \mathrm{m}^{3}\right)$.

\begin{tabular}{|c|c|c|c|}
\hline $\begin{array}{c}\text { Maximum result } \pm \\
\text { overall error }(\%)\end{array}$ & $\begin{array}{c}\text { Annual average } \\
\pm 2 \text { SEM }(\%)\end{array}$ & DCG & $\begin{array}{c}\text { Distant community } \\
\pm 2 S E M(\%)\end{array}$ \\
\hline $5.4 \mathrm{E}-05 \pm 23 \%$ & $2.0 \mathrm{E}-05 \pm 77 \%$ & $9.0 \mathrm{E}-\mathrm{O} 2 \mathrm{~S}$ & $2.1 \mathrm{E}-05 \pm 3.3 \%$ \\
\hline
\end{tabular}

2SEM = Two standard error of the mean.

DCG $=$ Derived concentration guide.

2.2.5.5 ${ }^{235} \mathrm{U}$. The maximum annual result was at site $\mathrm{N}-465$ (Table 2-33).

Table 2-33. ${ }^{235} \mathrm{U}$ in Air $100 \mathrm{~B} / \mathrm{C}$ Area, $1997\left(\mathrm{pCi} / \mathrm{m}^{3}\right)$.

\begin{tabular}{|c|c|c|c|}
\hline $\begin{array}{c}\text { Maximum result } \pm \\
\text { overall error }(\%)\end{array}$ & $\begin{array}{c}\text { Annual average } \\
\pm 2 \text { SEM }(\%)\end{array}$ & DCG & $\begin{array}{c}\text { Distant community } \\
\pm 2 \text { SEM }(\%)\end{array}$ \\
\hline $3.2 \mathrm{E}-05 \pm 28 \%$. & $1.7 \mathrm{E}-05 \pm 89 \%$ & $1.0 \mathrm{E}-01$ & $<1.5 \mathrm{E}-07 \pm 230 \%$ \\
\hline
\end{tabular}

2SEM = Two standard error of the mean.

$\mathrm{DCG}=$ Derived concentration guide.

2.2.5.6 ${ }^{238} \mathrm{U}$. The maximum annual result was at site $\mathrm{N}-466$ (Table 2-34).

Table 2-34. ${ }^{238} \mathrm{U}$ in Air $100 \mathrm{~B} / \mathrm{C}$ Area, $1997\left(\mathrm{pCi} / \mathrm{m}^{3}\right)$.

\begin{tabular}{|c|c|c|c|}
\hline $\begin{array}{c}\text { Maximum result } \pm \\
\text { overall errot }(\%)\end{array}$ & $\begin{array}{c}\text { Annual average } \\
\pm 2 \text { SEM }(\%)\end{array}$ & DCG & $\begin{array}{c}\text { Distant community } \\
\pm 2 \text { SEM }(\%)\end{array}$ \\
\hline 1.4 E-O5 $\pm 56 \%$ & 9.6 E-06 $\pm 47 \%$ & 1.0 E-01 & 1.7 E-05 $\pm 0.6 \%$ \\
\hline
\end{tabular}

2SEM = Two standard error of the mean.

DCG $=$ Derived concentration guide. 
HNF-EP-0573-6

2.2.5.7 ${ }^{239}{ }^{240} \mathrm{Pu}$. The maximum annual result was at site $\mathrm{N}-465$ (Table 2-35).

Table 2-35. ${ }^{239,}{ }^{240} \mathrm{Pu}$ in Air $100 \mathrm{~B} / \mathrm{C}$ Area, $1997\left(\mathrm{pCi} / \mathrm{m}^{3}\right)$.

\begin{tabular}{|c|c|c|c|}
\hline $\begin{array}{c}\text { Maximum result } \pm \\
\text { overall error (\%) }\end{array}$ & $\begin{array}{c}\text { Annual average } \\
\pm 2 \text { SEM (\%) }\end{array}$ & DCG & $\begin{array}{c}\text { Distant community } \\
\pm 2 \text { SEM }(\%)\end{array}$ \\
\hline $1.4 \mathrm{E}-05 \pm 54 \%$ & $8.4 \mathrm{E}-06 \pm 64 \%$ & $2.0 \mathrm{E}-02$ & $<2.2 \mathrm{E}-07 \pm 73 \%$ \\
\hline
\end{tabular}

2SEM = Two standard error of the mean.

$\mathrm{DCG}=$ Derived concentration guide.

\subsubsection{D/DR Area}

Near-facility air sampling was conducted for the ERC at the $100 \mathrm{D} / \mathrm{DR}$ remediation site through a network of four continuous air samplers. Monitoring began in November 1996. The analytical results indicated that the concentrations were much less than the DOE DCGs and comparable to levels measured at other locations onsite. Appendix C, Table C-7, provides the 1997 air sampling results. Sampling locations are shown in Appendix C, Figure C-8.

2.2.6.1 ${ }^{60}$ Co. ${ }^{60} \mathrm{Co}$ concentrations were less than $(<)$ detection limits in 1997 (Table 2-36).

Table 2-36. ${ }^{60} \mathrm{Co}$ in Air $100 \mathrm{D} / \mathrm{DR}$ Area, $1997\left(\mathrm{pCi} / \mathrm{m}^{3}\right)$.

\begin{tabular}{|c|c|c|c|}
\hline $\begin{array}{c}\text { Maximum result } \pm \\
\text { overall error }(\%)\end{array}$ & $\begin{array}{c}\text { Annual average } \\
\pm 2 \text { SEM }(\%)\end{array}$ & DCG & $\begin{array}{c}\text { Distant community } \\
\pm 2 S E M(\%)\end{array}$ \\
\hline$<1.5 \mathrm{E}-05 \pm 320 \%$ & Not applicable & $8.0 \mathrm{E}+01$ & $<2.0 \mathrm{E}-04 \pm 97 \%$ \\
\hline
\end{tabular}

2SEM = Two standard error of the mean.

$\mathrm{DCG}=$ Derived concentration guide.

2.2.6.2 ${ }^{90} \mathrm{Sr}$. The maximum annual result was at site $\mathrm{N}-470$ (Table 2-37).

Table 2-37. ${ }^{90} \mathrm{Sr}$ in Air 100 D/DR Area, $1997\left(\mathrm{pCi} / \mathrm{m}^{3}\right)$.

\begin{tabular}{|c|c|c|c|}
\hline $\begin{array}{c}\text { Maximum result } \pm \\
\text { overall error }(\%)\end{array}$ & $\begin{array}{c}\text { Annual average } \\
\pm 2 \text { SEM }(\%)\end{array}$ & DCG & $\begin{array}{c}\text { Distant community } \\
\pm 2 \text { SEM }(\%)\end{array}$ \\
\hline 8.4 E-04 $\pm 25 \%$ & 5.7 E-O $04 \pm 75 \%$ & $9.0 E+00$ & $<-5.3 E-06 \pm 81 \%$ \\
\hline
\end{tabular}

2SEM = Two standard error of the mean.

DCG $=$ Derived concentration guide. 
2.2.6.3 ${ }^{137} \mathrm{Cs}$. The maximum annual result was at site $\mathrm{N}-468$ (Table $2-38$ ).

Table 2-38. ${ }^{137} \mathrm{Cs}$ in Air $100 \mathrm{D} / \mathrm{DR}$ Area, $1997\left(\mathrm{pCi} / \mathrm{m}^{3}\right)$.

\begin{tabular}{|c|c|c|c|}
\hline $\begin{array}{c}\text { Maximum result } \pm \\
\text { overall error }(\%)\end{array}$ & $\begin{array}{c}\text { Annual average } \\
\pm 2 \text { SEM }(\%)\end{array}$ & DCG & $\begin{array}{c}\text { Distant community } \\
\pm 2 \text { SEM }(\%)\end{array}$ \\
\hline $1.2 \mathrm{E}-\mathrm{O} 4 \pm 35 \%$ & $1.1 \mathrm{E}-\mathrm{O} 4 \pm 84 \%$ & $4.0 \mathrm{E}+02$ & $<2.7 \mathrm{E}-05 \pm 850 \%$ \\
\hline
\end{tabular}

2SEM = Two standard error of the mean.

$\mathrm{DCG}=$ Derived concentration guide.

2.2.6.4 ${ }^{234} \mathrm{U}$. The maximum annual result was at site $\mathrm{N}-468$ (Table 2-39).

Table 2-39, ${ }^{234} \mathrm{U}$ in Air $100 \mathrm{D} / \mathrm{DR}$ Area, $1997\left(\mathrm{pCi} / \mathrm{m}^{3}\right)$.

\begin{tabular}{|c|c|c|c|}
\hline $\begin{array}{c}\text { Maximum result } \pm \\
\text { overall error }(\%)\end{array}$ & $\begin{array}{c}\text { Annual average } \\
\pm 2 \text { 2SEM (\%) }\end{array}$ & DCG & $\begin{array}{c}\text { Distant community } \\
\pm 2 \text { SEM (\%) }\end{array}$ \\
\hline 2.0 E-05 $\pm 47 \%$ & $8.1 \mathrm{E}-06 \pm 43 \%$ & $9.0 \mathrm{E}-02$ & $2.1 \mathrm{E}-05 \pm 3.3 \%$ \\
\hline
\end{tabular}

2SEM = Two standard error of the mean.

DCG $=$ Derived concentration guide.

2.2.6.5 ${ }^{235} \mathrm{U}$. The maximum annual result was at site $\mathrm{N}-467$ (Table 2-40).

Table 2-40. ${ }^{235} \mathrm{U}$ in Air $100 \mathrm{D} / \mathrm{DR}$ Area, $1997\left(\mathrm{pCi} / \mathrm{m}^{3}\right)$.

\begin{tabular}{|c|c|c|c|}
\hline $\begin{array}{c}\text { Maximum result } \pm \\
\text { overall error }(\%)\end{array}$ & $\begin{array}{c}\text { Annual average } \\
\pm 2 \text { SEM }(\%)\end{array}$ & DCG & $\begin{array}{c}\text { Distant community } \\
\pm 2 S \mathrm{SM}(\%)\end{array}$ \\
\hline $1.4 \mathrm{E}-\mathrm{O} 5 \pm 47 \%$ & $8.1 \mathrm{E}-\mathrm{O}=6 \pm 43 \%$ & $1.0 \mathrm{E}-01$ & $<1.5 \mathrm{E}-07 \pm 230 \%$ \\
\hline
\end{tabular}

2SEM = Two standard error of the mean.

$D C G=$ Derived concentration guide.

2.2.6.6 ${ }^{238} \mathrm{U}$. The maximum annual result was at site $\mathrm{N}-468$ (Table 2-41).

Table 2-41. ${ }^{238} \mathrm{U}$ in Air $100 \mathrm{D} / \mathrm{DR}$ Area, $1997\left(\mathrm{pCi} / \mathrm{m}^{3}\right)$.

\begin{tabular}{|c|c|c|c|}
\hline $\begin{array}{c}\text { Maximum result } \pm \\
\text { overall error }(\%)\end{array}$ & $\begin{array}{c}\text { Annual average } \\
\pm 2 \text { SEM }(\%)\end{array}$ & DCG & $\begin{array}{c}\text { Distant community } \\
\pm 2 S E M(\%)\end{array}$ \\
\hline $1.0 \mathrm{E}-05 \pm 65 \%$ & $8.1 \mathrm{E}-06 \pm 34 \%$ & $1.0 \mathrm{E}-01$ & $1.7 \mathrm{E}-05 \pm 0.6 \%$ \\
\hline
\end{tabular}

2SEM = Two standard error of the mean.

DCG $=$ Derived concentration guide. 
2.2.6.7 ${ }^{239 .}{ }^{240} \mathrm{Pu}$. The maximum annual result was at site $\mathrm{N}-467$ (Table 2-42).

Table 2-42. ${ }^{239,}{ }^{240} \mathrm{Pu}$ in Air $100 \mathrm{D} / \mathrm{DR}$ Area, $1997\left(\mathrm{pCi} / \mathrm{m}^{3}\right)$.

\begin{tabular}{|c|c|c|c|}
\hline $\begin{array}{c}\text { Maximum result } \pm \\
\text { overall error }(\%)\end{array}$ & $\begin{array}{c}\text { Annual average } \\
\pm 2 \mathrm{SEM}(\%)\end{array}$ & DCG & $\begin{array}{c}\text { Distant community } \\
\pm 2 \mathrm{SEM}(\%)\end{array}$ \\
\hline $8.7 \mathrm{E}-06 \pm 60 \%$ & $7.5 \mathrm{E}-06 \pm 86 \%$ & $2.0 \mathrm{E}-02$ & $<2.2 \mathrm{E}-07 \pm 73 \%$ \\
\hline
\end{tabular}

2SEM = Two standard error of the mean.

DCG $=$ Derived concentration guide.

\subsubsection{Environmental Restoration Disposal Facility}

Near-facility air sampling was conducted for the ERC at the ERDF site through a network of five continuous air samplers. This network utilized two existing Hanford Site air monitors for upwind sampling and was supplemented by three additional samplers which provided downwind monitoring. The upwind monitors were $\mathrm{N}-963$ and PNL 200 West, Southeast. Interestingly, several of the "maximum" concentrations observed among the five sampling stations were at the upwind sites signifying generally low radionuclide concentrations for all sampiing locations. The individual data results are shown in Appendix C, Table C-8. Monitoring began in June 1996. The analytical results indicated that the concentrations were much less than the DOE DCGs and comparable to levels measured at other locations onsite. Sampling locations are shown in Appendix C, Figure C-4.

2.2.7.1 ${ }^{60} \mathrm{Co}$. The maximum annual result was at site PNL-200 (Table 2-43).

Table 2-43. ${ }^{60} \mathrm{Co}$ in Air, ERDF, $1997\left(\mathrm{pCi} / \mathrm{m}^{3}\right)$.

\begin{tabular}{|c|c|c|c|}
\hline $\begin{array}{c}\text { Maximum result } \pm \\
\text { overall error }(\%)\end{array}$ & $\begin{array}{c}\text { Annual average } \\
\pm 2 \text { SEM (\%) }\end{array}$ & DCG & $\begin{array}{c}\text { Distant community } \\
\pm 2 \text { SEM (\%) }\end{array}$ \\
\hline $4.1 \mathrm{E}-04 \pm 78 \%$ & $2.7 \mathrm{E}-04 \pm 175 \%$ & $8.0 \mathrm{E}+01$ & $<2.0 \mathrm{E}-04 \pm 97 \%$ \\
\hline
\end{tabular}

2SEM = Two standard error of the mean.

$\mathrm{DCG}=$ Derived concentration guide.

ERDF = Environmental Restoration Disposal Facility.

2.2.7.2 ${ }^{90} \mathrm{Sr}$. The maximum annual result was at site $\mathrm{N}-963$ (Table 2-44).

Table 2-44. ${ }^{90} \mathrm{Sr}$ in Air, ERDF, $1997\left(\mathrm{pCi} / \mathrm{m}^{3}\right)$.

\begin{tabular}{|c|c|c|c|}
\hline $\begin{array}{c}\text { Maximum result } \pm \\
\text { overall error }(\%)\end{array}$ & $\begin{array}{c}\text { Annual average } \\
\pm 2 \text { SEM }(\%)\end{array}$ & DCG & $\begin{array}{c}\text { Distant community } \\
\pm 2 \text { SEM }(\%)\end{array}$ \\
\hline $2.8 \mathrm{E}-04 \pm 40 \%$ & $2.4 \mathrm{E}-04 \pm 51 \%$ & $9.0 \mathrm{E}+00$ & $\leq-5.3 \mathrm{E}-06 \pm 81 \%$ \\
\hline
\end{tabular}

2SEM = Two standard error of the mean.

$\mathrm{DCG}=$ Derived concentration guide.

ERDF = Environmental Restoration Disposal Facility. 
2.2.7.3 ${ }^{137} \mathrm{Cs}$. The maximum annual result was at site PNL-200 (Table 2-45).

Table 2-45, ${ }^{137} \mathrm{Cs}$ in Air, ERDF, $1997\left(\mathrm{pCi} / \mathrm{m}^{3}\right)$.

\begin{tabular}{|c|c|c|c|}
\hline $\begin{array}{c}\text { Maximum result } \pm \\
\text { overall error }(\%)\end{array}$ & $\begin{array}{c}\text { Annual average } \\
\pm 2 \text { SEM }(\%)\end{array}$ & DCG & $\begin{array}{c}\text { Distant community } \\
\pm 2 \text { SEM }(\%)\end{array}$ \\
\hline $2.8 \mathrm{E}-04 \pm 93 \%$ & $1.5 \mathrm{E}-\mathrm{O} 4 \pm 67 \%$ & $4.0 \mathrm{E}+02$ & $<2.7 \mathrm{E}-05 \pm 850 \%$ \\
\hline
\end{tabular}

2SEM = Two standard error of the mean.

DCG $=$ Derived concentration guide.

ERDF = Environmental Restoration Disposal Facility,

2.2.7.4 ${ }^{234} \mathrm{U}$. The maximum annual result was at site PNL-200 (Table 2-46).

Table 2-46. ${ }^{234} \mathrm{U}$ in Air, ERDF, $1997\left(\mathrm{pCi} / \mathrm{m}^{3}\right)$.

\begin{tabular}{|c|c|c|c|}
\hline $\begin{array}{c}\text { Maximum result } \pm \\
\text { overall error }(\%)\end{array}$ & $\begin{array}{c}\text { Annual average } \\
\pm 2 \text { SEM (\%) }\end{array}$ & DCG & $\begin{array}{c}\text { Distant community } \\
\pm 2 \text { 2SEM (\%) }\end{array}$ \\
\hline 2.8 E-05 $\pm 18 \%$ & 2.0 E-05 $\pm 43 \%$ & $9.0 E-02$ & 2.1 E-O $\pm 5.3 \%$ \\
\hline
\end{tabular}

2SEM = Two standard error of the mean.

$\mathrm{DCG}=$ Derived concentration guide.

ERDF = Environmental Restoration Disposal Facility.

2.2.7.5 ${ }^{235} \mathrm{U}$. The maximum annual result was at site $\mathrm{N}-963$ (Table $2-47$ ).

Table 2-47. ${ }^{235} \mathrm{U}$ in Air, ERDF, $1997\left(\mathrm{pCi} / \mathrm{m}^{3}\right)$.

\begin{tabular}{|c|c|c|c|}
\hline $\begin{array}{c}\text { Maximum result } \pm \\
\text { overall error }(\%)\end{array}$ & $\begin{array}{c}\text { Annual average } \\
\pm 2 \text { SEM (\%) }\end{array}$ & DCG & $\begin{array}{c}\text { Distant community } \\
\pm 2 \text { SEM }(\%)\end{array}$ \\
\hline 1.6 E-05 $\pm 44 \%$ & 1.1 E-OS $\pm 58 \%$ & 1.0 E-01 & $<1.5$ E-O $07 \pm 230 \%$ \\
\hline
\end{tabular}

2SEM = Two standard error of the mean.

$\mathrm{DCG}=$ Derived concentration guide.

ERDF = Environmental Restoration Disposal Facility.

2.2.7.6 ${ }^{238} \mathrm{U}$. The maximum annual result was at site PNL-200 (Table 2-48).

Table 2-48. ${ }^{238} \mathrm{U}$ in Air, ERDF, $1997\left(\mathrm{pCl} / \mathrm{m}^{3}\right)$.

\begin{tabular}{|c|c|c|c|}
\hline $\begin{array}{c}\text { Maximum result } \pm \\
\text { overall error }(\%)\end{array}$ & $\begin{array}{c}\text { Annual average } \\
\pm 2 \text { SEM }(\%)\end{array}$ & DCG & $\begin{array}{c}\text { Distant community } \\
\pm 2 \text { SEM }(\%)\end{array}$ \\
\hline $2.4 \mathrm{E}-05 \pm 19 \%$ & $1.6 \mathrm{E}-05 \pm 45 \%$ & $1.0 \mathrm{E}-01$ & $1.7 \mathrm{E}-05 \pm 0.6 \%$ \\
\hline
\end{tabular}

2SEM = Two standard error of the mean.

$\mathrm{DCG}=$ Derived concentration guide.

ERDF = Environmental Restoration Disposal Facility. 
2.2.7.7 ${ }^{239,}{ }^{240} \mathrm{Pu}$. The maximum annual result was at site $\mathrm{N}-484$ (Table 2-49).

Table 2-49. ${ }^{239},{ }^{240} \mathrm{Pu}$ in Air, ERDF, $1997\left(\mathrm{pCi} / \mathrm{m}^{3}\right)$.

\begin{tabular}{|c|c|c|c|}
\hline $\begin{array}{c}\text { Maximum result } \pm \\
\text { overall error }(\%)\end{array}$ & $\begin{array}{c}\text { Annual average } \\
\pm 2 \mathrm{SEM}(\%)\end{array}$ & DCG & $\begin{array}{c}\text { Distant community } \\
\pm 2 \mathrm{SEM}(\%)\end{array}$ \\
\hline $1.5 \mathrm{E}-05 \pm 51 \%$ & $8.9 \mathrm{E}-06 \pm 50 \%$ & $2.0 \mathrm{E}-02$ & $<2.2 \mathrm{E}-07 \pm 73 \%$ \\
\hline
\end{tabular}

2SEM = Two standard error of the mean.

DCG $=$ Derived concentration guide.

ERDF = Environmental Restoration Disposal Facility.

2.2.7.8 ${ }^{241} \mathrm{Am} .{ }^{241} \mathrm{Am}$ concentrations were less than $(<)$ detection limits in 1997 (Table 2-50).

Table 2-50. ${ }^{241} \mathrm{Am}$ in Air, ERDF, $1997\left(\mathrm{pCi} / \mathrm{m}^{3}\right)$.

\begin{tabular}{|c|c|c|c|}
\hline $\begin{array}{c}\text { Maximum result } \pm \\
\text { overall error }(\%)\end{array}$ & $\begin{array}{c}\text { Annual average } \\
\pm 2 \text { SEM }(\%)\end{array}$ & DCG & $\begin{array}{c}\text { Distant community } \\
\pm 2 \text { SEM }(\%)\end{array}$ \\
\hline$<1.7 \mathrm{E}-05 \pm 110 \%$ & Not applicable & $2.0 \mathrm{E}-02$ & Not reported \\
\hline
\end{tabular}

2SEM = Two standard error of the mean.

DCG $=$ Derived concentration guide.

ERDF = Environmental Restoration Disposal Facility.

\subsubsection{0-FF-1 Project}

Near-facility air sampling was conducted at the 300-FF-1 remediation site through a network of continuous air samplers. This network utilized one existing Hanford Site monitor for upwind monitoring $(\mathrm{N}-130)$ and was supplemented by three additional air monitors that provided downwind monitoring. Monitoring began in May 1997; therefore, the data provided in this report reflect only one set of composite samples. Samples were analyzed for gamma emitting radionuclides and isotopic uranium. The individual data results are shown in Appendix $\mathrm{C}$, Table C-9. The analytical results indicated that the concentrations were much less than the DOE DCGs and comparable to levels measured at other locations onsite. Sampling locations are shown in Appendix C, Figure C-5.

2.2.8.1 ${ }^{60} \mathrm{Co}$. ${ }^{60} \mathrm{Co}$ concentrations were less than $(<)$ detection limits in 1997 (Table 2-51).

Table 2-51. ${ }^{60} \mathrm{Co}$ in Air, 300-FF-1, $1997\left(\mathrm{pCi} / \mathrm{m}^{3}\right)$.

\begin{tabular}{|c|c|c|c|}
\hline $\begin{array}{c}\text { Maximum result } \pm \\
\text { overall error (\%) }\end{array}$ & $\begin{array}{c}\text { Annual average } \\
\pm 2 \mathrm{SEM}(\%)\end{array}$ & DCG & $\begin{array}{c}\text { Distant community } \\
\pm 2 \text { SEM }(\%)\end{array}$ \\
\hline$<2.2 \mathrm{E}-05 \pm 210 \%$ & Not applicable & $8.0 \mathrm{E}+01$ & $<2.0 \mathrm{E}-04 \pm 97 \%$ \\
\hline
\end{tabular}

2SEM = Two standard error of the mean.

DCG $=$ Derived concentration guide 
2.2.8.2 ${ }^{137} \mathrm{Cs}$. The maximum annual result was at site $\mathrm{N}-130$ (Table 2-52).

Table 2-52. ${ }^{137} \mathrm{Cs}$ in Air, 300-FF-1, $1997\left(\mathrm{pCi} / \mathrm{m}^{3}\right)$.

\begin{tabular}{|c|c|c|c|}
\hline $\begin{array}{c}\text { Maximum result } \pm \\
\text { overall error }(\%)\end{array}$ & $\begin{array}{c}\text { Annual average } \\
\pm 2 \text { SEM }(\%)\end{array}$ & DCG & $\begin{array}{c}\text { Distant community } \\
\pm 2 S E M(\%)\end{array}$ \\
\hline $1.1 \mathrm{E}-\mathrm{O} 4 \pm 62 \%$ & $9.6 \mathrm{E}-05 \pm 28 \%$ & $4.0 \mathrm{E}+02$ & $<2.7 \mathrm{E}-05 \pm 850 \%$ \\
\hline
\end{tabular}

2SEM = Two standard error of the mean.

DCG $=$ Derived concentration guide .

2.2.8.3 ${ }^{234} \mathrm{U}$. The maximum annual result was at site $\mathrm{N}-487$ (Table $2-53$ ).

Table 2-53. ${ }^{234} \mathrm{U}$ in Air, 300-FF-1, $1997\left(\mathrm{pCi} / \mathrm{m}^{3}\right)$.

\begin{tabular}{|c|c|c|c|}
\hline $\begin{array}{c}\text { Maximum result } \pm \\
\text { overall error }(\%)\end{array}$ & $\begin{array}{c}\text { Annual average } \\
\pm 2 \text { SEM }(\%)\end{array}$ & DCG & $\begin{array}{c}\text { Distant community } \\
\pm 2 \text { SEM }(\%)\end{array}$ \\
\hline 6.5 E-05 $\pm 27 \%$ & 3.2 E-O5 $\pm 35 \%$ & 9.0 E-02 & 2.1 E-0.5 $\pm 3.3 \%$ \\
\hline
\end{tabular}

2SEM = Two standard error of the mean.

DCG $=$ Derived concentration guide.

2.2.8.4 ${ }^{235} \mathrm{U}$. The maximum annual result was at site $\mathrm{N}-486$ (Table 2-54).

Table 2-54. ${ }^{235} \mathrm{U}$ in Air, 300-FF-1, $1997\left(\mathrm{pCi} / \mathrm{m}^{3}\right)$.

\begin{tabular}{|c|c|c|c|}
\hline $\begin{array}{c}\text { Maximum result } \pm \\
\text { overall error }(\%)\end{array}$ & $\begin{array}{c}\text { Annual average } \\
\pm 2 \text { SEM }(\%)\end{array}$ & DCG & $\begin{array}{c}\text { Distant community } \\
\pm 2 \text { SEM }(\%)\end{array}$ \\
\hline 2.0 E-05 $\pm 41 \%$ & 2.2 E-05 $\pm 62 \%$ & 1.0 E-01 & $\leq 1.5 \mathrm{E}-07 \pm 230 \%$ \\
\hline
\end{tabular}

2SEM = Two standard error of the mean.

DCG $=$ Derived concentration guide.

2.2.8.5 ${ }^{238} \mathrm{U}$. The maximum annual result was at site $\mathrm{N}-487$ (Table 2-55).

Table 2-55. ${ }^{238} \mathrm{U}$ in Air, 300-FF-1, $1997\left(\mathrm{pCi} / \mathrm{m}^{3}\right)$.

\begin{tabular}{|c|c|c|c|}
\hline $\begin{array}{c}\text { Maximum result } \pm \\
\text { overall error (\%) }\end{array}$ & $\begin{array}{c}\text { Annual average } \\
\pm 2 \text { SEM (\%) }\end{array}$ & DCG & $\begin{array}{c}\text { Distant community } \\
\pm 2 S E M(\%)\end{array}$ \\
\hline 6.6 E-O5 $\pm 27 \%$ & 3.5 E-05 $\pm 35 \%$ & 1.0 E-O & 1.7 E-05 $\pm 0.6 \%$ \\
\hline
\end{tabular}

2SEM = Two standard error of the mean.

$\mathrm{DCG}=$ Derived concentration guide. 


\subsubsection{Building (300 Area)}

Near-facility air sampling was conducted during the demolition of the 3732 Building in the 300 Area through the use of two continuous air samplers. These stations provided downwind monitoring during the two week demolition period during September 1997. Samples were analyzed for gamma emitting radionuclides only. The analytical results indicated that the concentrations were below detection limits and much less than the DOE DCGs. The results are therefore not summarized in this report. Sampling locations are shown in Appendix C, Figure C-5.

\subsection{CONCLUSIONS}

Operational activities in the $100,200 / 600$, and $300 / 400$ Areas contributed to average air radionuclide concentrations that were above background. Over the past 15 years, the average ambient air concentrations generally have decreased because of overall improvement in the operational environmental controls and reduced Site operations. All airborne radionuclide concentrations in air were below the DOE DCGs in 1997. 


\subsection{GROUNDWATER MONITORING}

\subsection{INTRODUCTION}

Currently the only groundwater wells that are monitored and reported through the near-facility monitoring effort are wells $\mathrm{N}-47$ and $\mathrm{N}-48$, located in the $100 \mathrm{~N}$ Area. Samples are collected monthly and are analyzed for gamma-emitting radionuclides. Trend analyses provide indications of the integrity of the building sumps and underground piping associated with the 107-N facility. Wells $\mathrm{N}-47$ and $\mathrm{N}-48$ are located adjacent to the 107-N Fuel Storage Basin Recirculation Facility. Radionuclide concentrations detected in the groundwater samples are consistent with values observed in previous years. Only those radionuclide concentrations above analytical detection limits are provided in this report.

Groundwater monitoring at the Hanford Site is an integral part of the Hanford Site Groundwater Protection Management Plan (RL 1994b). This plan integrates monitoring at active waste disposal facilities to comply with monitoring requirements of the Resources Conservation and Recovery Act of 1976 (RCRA) and Washington State regulations, as well as requirements for operational monitoring around reactor and chemical processing facilities, and environmental surveillance monitoring. PNNL manages these monitoring efforts through the Groundwater Monitoring Project. This project is responsible for assessing the distribution and movement of existing groundwater contamination, identifying potential and emerging groundwater contamination problems, and integrating the various groundwater projects to minimize redundancy. Information on contaminant distribution and transport are integrated into a site-wide evaluation of groundwater quality, which is documented in an annual groundwater monitoring report (Hartman and Dresel 1997). Groundwater monitoring is also carried out during cleanup investigations under the Comprehensive Environmental Response, Compensation, and Liability Act of 1980 (CERCLA). These investigations are managed by the ERC.

\section{$3.2100 \mathrm{~N}$ AREA RESULTS}

Samples of groundwater (1.0 L) were collected from wells $\mathrm{N}-47$ and $\mathrm{N}-48$ by ERC personnel and submitted to Quanterra Environmental Services laboratory for radioanalysis. Though the collection frequency was planned to be monthly, due to mechanical problems in the sampling equipment in each well, the January collection was missed.

The analytical results for all samples collected from these wells indicate that concentrations of gamma-emitting radionuclides are below DOE DCGs and EPA Interim Drinking Water Standards. Well locations and analysis results are provided in Appendix D of this report. 
HNF-EP-0573-6

This page intentionally left blank. 
HNF-EP-0573-6

\subsection{SOIL AND VEGETATION MONITORING}

\subsection{INTRODUCTION}

The radionuclide content of soil and vegetation was measured to evaluate long-term trends in environmental accumulation of radioactivity in the 100,200/600, and 300/400 Areas. Soil and vegetation samples were collected on or near facilities that store, handle, or dispose of radioactive waste. Eighty soil samples $(7$ in the $100 \mathrm{~N}$ Area, 55 in the $200 / 600$ Areas, 15 in the $300 / 400$ Areas, 1 at the ERDF, and 2 in the 100 D,DR Area) and 76 vegetation samples (10 in the $100 \mathrm{~N}$ Area, 41 in the 200/600 Areas, and 15 in the 300/400 Areas) were collected from locations shown in Appendix E, Figures $E-1$ through E-13. The analytical results of the data obtained from the samples collected are presented in Tables E-1 through E-7 (Appendix E). Onty those radionuclide concentrations above analytical detection limits are provided in this report.

The number of locations for soil and vegetation sampling in the $100 \mathrm{~N}$ environs was reduced in 1996. Analyses of the data collected at sites not associated with the 1301-N and 1325-N facilities indicated decreasing trends for contaminant migration and prompted a determination that sampling at these focations was not cost-effective. For these same reasons, some $\mathrm{N}$-Springs sample locations were also deleted. Locations of all current $100 \mathrm{~N}$ sample points are provided in Figures E-1 and E-7 (Appendix E).

Each soil sample represents a composite of five plugs of soil $2.5 \mathrm{~cm}(1 \mathrm{in}$.) deep and $10 \mathrm{~cm}$ (4 in.) in diameter collected from each site. Each vegetation sample consists of new-growth leaf cuttings taken from the available species of interest at each sample location. Often, the vegetation sample consisted of a composite of several like members of the sampling site community to avoid decimation of any individual plant through over-harvesting.

Early in the summer of each year, soil and vegetation samples are collected and submitted for radioanalyses. The analyses include those for radionuclides expected to be found in the areas sampled (i.e., gamma-emitting radionuclides, strontium isotopes, uranium isotopes, and/or plutonium isotopes). The results are then compared to levels found at various offsite sample locations in Yakima, and Benton and Franklin counties. These levels are derived from data reported from these locations by the PNNL surveillance program to determine the difference between contributions from Site operations and contributions from natural causes and worldwide fallout. Special sampling for selected radionuclides in soil and vegetation were collected from Franklin County by PNNL during 1997. For more detail, see Section 4.6, "Soil and Vegetation Surveillance," Hanford Site Environmental Report for Calendar Year 1997 (Dirkes and Hanf 1998).

The results are also compared to the soil concentration limits included in HNF-PRO-454, Inactive Waste Sites (Appendix J), developed for use at the Hanford Site. Radioactive soil concentration limits were established to ensure that effective dose equivalents (EDE) to the public do not exceed the established limits for any reasonable scenario (such as direct exposure, inadvertent ingestion, inhalation, and ingestion of food crops, including animal products). Conservatism inherent in pathway programming ensures that the required degrees of protection are in place. The concentration limits specified apply to the Hanford Site with respect to onsite disposal operations, stabilization and cleanup, decontamination and decommissioning operations.

Special investigative soil and biota (plant and animal) samples are collected for Site-specific monitoring, or whenever radiological contamination is known or suspected to have occurred. These special samples are discussed in Section 8.0. 


\subsection{SOIL SAMPLING}

\subsection{1 $100 \mathrm{~N}$ Area}

Surface soil samples collected near the 1301-N liquid waste disposal facility (LWDF) contained radionuclides that were typically present in past effluent stream discharges. Generally, the samples collected near this facility exhibited relatively higher radionuclide concentrations than those collected at the other soil sampling locations at $100 \mathrm{~N}$. Radionuclide concentrations from sampling site $\mathrm{Y} 602$, located on the east side of the retired 1301-N LWDF, exhibited significantly elevated levels of ${ }^{60} \mathrm{Co},{ }^{90} \mathrm{Sr},{ }^{137} \mathrm{Cs},{ }^{238} \mathrm{Pu}$, and ${ }^{239,240} \mathrm{Pu}$. It is likely that these increased levels are due to resuspension of contaminated material from the LWDF itself as the vegetation in the immediate vicinity of $Y 602$ (i.e., Y702 vegetation sampling site), does not exhibit a corresponding pattern of elevated radionuclide concentrations. Average radionuclide concentrations detected in the surface soil samples near the 1301-N LWDF from 1980 through 1997 are presented in Table 4-1. Average radionuclide concentrations detected in the surface soil samples collected in the $100 \mathrm{~N}$ Area from 1980 through 1997 are presented in Table 4-2. The 1997 maximum, average, the offsite average, and the accessible soil concentration limits are compared in Table 4-3. Offsite averages for ${ }^{137} \mathrm{Cs},{ }^{90} \mathrm{Sr}$, and isotopic uranium are from the Hanford Site Environmental Report for Calendar Year 1997 (Dirkes and Hanf 1998) and offsite values for ${ }^{239,240} \mathrm{Pu}$ are contained in the Hanford Site Environmental Report for Calendar Year 1994 (Dirkes and Hanf 1995). Radionuclide concentrations are summarized in Appendix $E$, Table E- 1 .

Table 4- 3 . Average Radionuclide Concentrations Detected in Soil Samples Near the 1301-N Liquid Waste Disposal Facility from 1980 through 1997 (pCi/g).

\begin{tabular}{|c|c|c|c|c|c|}
\hline Year & ${ }^{60} \mathrm{CO}$ & ${ }^{90} \mathrm{Sr}$ & ${ }^{137} \mathrm{Cs}$ & ${ }^{238} \mathrm{Pu}$ & ${ }^{239.240} \mathrm{Pu}$ \\
\hline 1980 & $1.3 \mathrm{E}+01$ & $3.5 \mathrm{E}-01$ & $4.1 \mathrm{E}+00$ & $\mathrm{NR}$ & $2.5 \mathrm{E}-02$ \\
\hline 1981 & $4.0 \mathrm{E}+00$ & $7.0 \mathrm{E}-01$ & $6.1 \mathrm{E}+00$ & $\mathrm{NR}$ & $4.4 \mathrm{E}-02$ \\
\hline 1982 & $6.3 \mathrm{E}+00$ & $2.7 \mathrm{E}-01$ & $2.7 \mathrm{E}+00$ & $\mathrm{NR}$ & $1.8 \mathrm{E}-02$ \\
\hline 1983 & $5.4 \mathrm{E}+00$ & $1.3 \mathrm{E}+00$ & $3.8 \mathrm{E}+00$ & $\mathrm{NR}$ & $4.3 \mathrm{E}-02$ \\
\hline 1984 & $2.8 \mathrm{E}+00$ & $2.1 \mathrm{E}-01$ & $1.1 \mathrm{E}+00$ & $\mathrm{NR}$ & $1.7 \mathrm{E}-02$ \\
\hline 1985 & $1.3 \mathrm{E}+01$ & $6.5 \mathrm{E}-01$ & $3.9 \mathrm{E}+00$ & $\mathrm{NR}$ & $3.2 \mathrm{E}-02$ \\
\hline 1986 & $4.5 \mathrm{E}+00$ & $2.2 \mathrm{E}-01$ & $2.5 \mathrm{E}+00$ & $\mathrm{NR}$ & $1.7 \mathrm{E}-02$ \\
\hline 1987 & $5.1 \mathrm{E}+00$ & $3.4 \mathrm{E}-01$ & $1.6 \mathrm{E}+00$ & $5.4 \mathrm{E}-03$ & $2.2 \mathrm{E}-02$ \\
\hline 1988 & $7.8 \mathrm{E}+00$ & $3.5 \mathrm{E}-01$ & $2.0 \mathrm{E}+00$ & $2.3 \mathrm{E}-03$ & $1.7 \mathrm{E}-02$ \\
\hline 1989 & $2.3 \mathrm{E}+00$ & $1.5 \mathrm{E}-01$ & $5.0 \mathrm{E}-01$ & $6.4 \mathrm{E}-03$ & $4.0 \mathrm{E}-02$ \\
\hline 1990 & $4.7 \mathrm{E}+00$ & $3.2 \mathrm{E}-01$ & $1.7 \mathrm{E}+00$ & $4.6 \mathrm{E}-03$ & $3.5 \mathrm{E}-02$ \\
\hline 1991 & $1.3 \mathrm{E}+01$ & $2.7 \mathrm{E}-01$ & $1.3 \mathrm{E}+00$ & $7.7 \mathrm{E}-03$ & $5.3 \mathrm{E}-02$ \\
\hline 1992 & $6.7 \mathrm{E}+00$ & $2.2 \mathrm{E}-01$ & $1.1 \mathrm{E}+00$ & $3.5 \mathrm{E}-03$ & $2.3 \mathrm{E}-02$ \\
\hline 1993 & $1.0 \mathrm{E}+00$ & $8.7 \mathrm{E}-01$ & $6.2 \mathrm{E}+00$ & $1.3 \mathrm{E}-02$ & $6.9 \mathrm{E}-02$ \\
\hline 1994 & $3.7 \mathrm{E}+00$ & $3.3 \mathrm{E}-01$ & $1.5 \mathrm{E}+00$ & $9.3 \mathrm{E}-03$ & $2.8 \mathrm{E}-02$ \\
\hline 1995 & $2.1 \mathrm{E}+00$ & $1.5 \mathrm{E}-01$ & $7.7 \mathrm{E}-01$ & $2.0 \mathrm{E}-03$ & $1.0 \mathrm{E}-02$ \\
\hline 1996 & $2.5 \mathrm{E}+00$ & $2.3 \mathrm{E}-01$ & $9.8 \mathrm{E}-01$ & $5.4 \mathrm{E}-02$ & $4.8 \mathrm{E}-02$ \\
\hline 1997 & $4.3 \mathrm{E}+00$ & $5.8 \mathrm{E}+00$ & $1.5 \mathrm{E}+00$ & $2.2 \mathrm{E}-01$ & $9.8 \mathrm{E}-01$ \\
\hline
\end{tabular}

$N R=$ Not Reported. 
Table 4-2. Average Radionuclide Concentrations Detected in $100 \mathrm{~N}$ Area Surface Soil Samples from 1980 through 1997 (pCi/g).

\begin{tabular}{|c|c|c|c|c|c|}
\hline Year & ${ }^{60} \mathrm{Co}$ & ${ }^{90} \mathrm{Sr}$ & ${ }^{137} \mathrm{Cs}$ & ${ }^{238} \mathrm{Pu}$ & ${ }^{239,240} \mathrm{Pu}$ \\
\hline 1980 & 8.5 E-01 & $1.8 \mathrm{E}-01$ & $5.0 \mathrm{E}-01$ & NR & $1.8 \mathrm{E}-\mathrm{O} 2$ \\
\hline 1981 & $1.3 E+00$ & $2.1 \mathrm{E}-01$ & $1.0 \mathrm{E}+00$ & NR & $1.1 \mathrm{E}-\mathrm{O} 2$ \\
\hline 1982 & $1.6 E+00$ & $9.9 \mathrm{E}-\mathrm{O} 2$ & $3.4 \mathrm{E}-01$ & NR & $5.0 \mathrm{E}-03$ \\
\hline 1983 & $2.7 E+\infty 0$ & 2.9 E-01 & 4.4 E-01 & NR & $8.5 \mathrm{E}-03$ \\
\hline 1984 & $8.8 \mathrm{E}-01$ & $2.8 \mathrm{E}-01$ & 6.2 E-01 & NR & 1.4 E-O2 \\
\hline 1985 & $1.2 \mathrm{E}+00$ & $1.3 \mathrm{E}-01$ & 5.2 E-O? & NR & $1.3 \mathrm{E}-\mathrm{O} 2$ \\
\hline 1986 & $4.1 \mathrm{E}-01$ & 8.3 E-02 & $5.0 \mathrm{E}-01$ & NR & 8.2 E-03 \\
\hline 1987 & 4.1 E-O1 & 1.1 E-01 & 3.9 E-01 & $1.1 \mathrm{E}-\mathrm{O} 3$ & 6.7 E-03 \\
\hline 1988 & $3.4 \mathrm{E}-01$ & $1.6 \mathrm{E}-01$ & 3.9 E-OT & 4.5 E-04 & 9.5 E-03 \\
\hline 1989 & $1.4 \mathrm{E}-01$ & $2.1 \mathrm{E}-\mathrm{O}$ ? & 1.3 E-O1 & $1.1 \mathrm{E}-03$ & $1.3 \mathrm{E}-\mathrm{O} 2$ \\
\hline 1990 & $3.0 \mathrm{E}-01$ & $1.2 \mathrm{E}-01$ & 4.4 E-01 & $6.7 \mathrm{E}-04$ & $1.0 \mathrm{E}-\mathrm{O} 2$ \\
\hline 1991 & 4.3 E-O1 & $1.1 \mathrm{E}-01$ & $4.5 \mathrm{E}-01$ & 6.2 E-04 & $7.8 \mathrm{E}-03$ \\
\hline 1992 & 8.6 E-O2 & 9.5 E-02 & 3.6 E-01 & $5.1 \mathrm{E}-04$ & $7.6 \mathrm{E}-03$ \\
\hline 1993 & $3.0 \mathrm{E}-\mathrm{O} 2$ & $1.2 \mathrm{E}-01$ & 1.6 E-01 & 1.8 E-04 & $3.4 \mathrm{E}-03$ \\
\hline 1994 & $1.6 \mathrm{E}+00$ & $1.9 \mathrm{E}-\mathrm{O} 1$ & 8.1 E-O1 & $4.2 \mathrm{E}-03$ & $1.6 \mathrm{E}-02$ \\
\hline 1995 & 9.4 E-01 & 1.3 E-01 & 5.7 E-01 & $1.9 \mathrm{E}-03$ & $1.4 \mathrm{E}-\mathrm{O} 2$ \\
\hline 1996 & $1.5 E+00$ & 2.0 E-01 & $7.7 \mathrm{E}-01$ & $1.2 \mathrm{E}-\mathrm{O} 2$ & 4.3 E-O2 \\
\hline 1997 & $2.5 E+00$ & $3.9 E+00$ & 8.9 E-01 & 7.8 E-01 & 9.1 E-01 \\
\hline
\end{tabular}

NR $=$ Not Reported

Table 4-3. $100 \mathrm{~N}$ Area Soils (pCi/g).

\begin{tabular}{|c|c|c|c|c|c|c|c|}
\hline & ${ }^{60} \mathrm{Co}$ & ${ }^{90} \mathrm{Sr}$ & ${ }^{137} \mathrm{Cs}$ & ${ }^{234} \mathrm{U}$ & ${ }^{235} \mathrm{U}$ & ${ }^{238} \mathrm{U}$ & $239,240 \mathrm{Pu}$ \\
\hline & Site Y602 & Site Y602 & Site Y602 & Site Y605 & Site Y605 & Site Y605 & Site Y602 \\
\hline $\begin{array}{l}\text { Maximum } \pm \text { counting } \\
\text { error }(\%)\end{array}$ & $\begin{array}{c}1.2 E+01 \\
\pm 10 \% \\
\end{array}$ & $\begin{array}{c}2.2 E+01 \\
\pm 14 \% \\
\end{array}$ & $\begin{array}{c}3.6 E+00 \\
\pm 13 \% \\
\end{array}$ & $\begin{array}{c}3.2 \mathrm{E}-\mathrm{O} 1 \\
\pm 19 \% \\
\end{array}$ & $\begin{array}{c}2.5 \mathrm{E}-02 \\
\pm 47 \% \\
\end{array}$ & $\begin{array}{c}3.0 \text { E-O1 } \\
\pm 20 \% \\
\end{array}$ & $\begin{array}{c}3.6 E+00 \\
\pm 16 \% \\
\end{array}$ \\
\hline Average $\pm 2 \operatorname{SEM}(\%)$ & $\begin{array}{c}2.5 E+00 \\
\pm 12 \% \\
\end{array}$ & $\begin{array}{l}3.9 \mathrm{E}-01 \\
\pm 167 \% \\
\end{array}$ & $\begin{array}{r}8.9 \mathrm{E}-01 \\
\pm 101 \% \\
\end{array}$ & $\begin{array}{r}2.1 \mathrm{E}-01 \\
\pm 18 \% \\
\end{array}$ & $\begin{array}{c}2.0 \mathrm{E}-\mathrm{O} 2 \\
\pm 10 \% \\
\end{array}$ & $\begin{array}{c}2.1 \mathrm{E}-01 \\
\pm 16 \% \\
\end{array}$ & $\begin{array}{l}9.1 \mathrm{E}-01 \\
\pm 170 \% \\
\end{array}$ \\
\hline $\begin{array}{l}\text { PNNL offsite average } \\
\pm 2 \text { SEM }(\%)^{\circ}\end{array}$ & NR & $\begin{array}{c}6.2 \mathrm{E}-\mathrm{O} 2 \\
\pm 84 \% \\
\end{array}$ & $\begin{array}{r}3.0 \mathrm{E}-01 \\
\pm 100 \% \\
\end{array}$ & $\begin{array}{c}2.4 E-01 \\
\pm 38 \% \\
\end{array}$ & $\begin{array}{l}1.1 \mathrm{E}-02 \\
\pm 36 \% \\
\end{array}$ & $\begin{array}{c}2.5 \mathrm{E}-01 \\
\pm 40 \% \\
\end{array}$ & $\begin{array}{c}1.0 \mathrm{E}-\mathrm{O} 2 \\
\pm 09 \% \\
\end{array}$ \\
\hline $\begin{array}{l}\text { Accessible soil } \\
\text { concentration limits }\end{array}$ & $7.1 E+00$ & $2.8 E+03$ & $3.0 E+01$ & $6.3 E+02$ & $1.7 E+02$ & $3.7 E+02$ & $1.9 E+02$ \\
\hline
\end{tabular}

2SEM = Two standard error of the mean.

NR $=$ Not reported.

PNNL = Pacific Northwest National Laboratory

'Dirkes and Hanf 1998, and Dirkes and Hanf 1995. 
HNF-EP-0573-6

4.2.1.1 ${ }^{60} \mathrm{Co}$. The highest ${ }^{60} \mathrm{Co}$ resuit was found at Site Y602 located near the 1301-N LWDF. The ${ }^{60} \mathrm{Co}$ concentration was $12.0 \mathrm{E}+00 \pm 8 \% \mathrm{pCi} / \mathrm{g}$. The offsite average for ${ }^{60} \mathrm{Co}$ is not reported by PNNL. The average concentration of ${ }^{60} \mathrm{Co}$ in solls in the $100 \mathrm{~N}$ Area was $2.5 \mathrm{E}+00 \pm 120 \%$ $\mathrm{pCi} / \mathrm{g}$.

4.2.1.2 ${ }^{137} \mathrm{Cs}$. The highest ${ }^{137} \mathrm{Cs}$ result was found at Site $Y 602$, located near the $1301-\mathrm{N}$ LWDF. The ${ }^{137} \mathrm{Cs}$ concentration was $3.6 \mathrm{E}+00 \pm 13 \% \mathrm{pCi} / \mathrm{g}$, or 12 times the offsite measured concentration of $3.0 \mathrm{E}-01 \pm 100 \% \mathrm{pCi} / \mathrm{g}$. The average concentration of ${ }^{137} \mathrm{Cs}$ in soils in the $100 \mathrm{~N}$ Area was $8.9 \mathrm{E}-01 \pm 101 \% \mathrm{pCi} / \mathrm{g}$.

4.2.1.3 ${ }^{90} \mathrm{Sr}$. The highest ${ }^{90} \mathrm{Sr}$ resuit was found at Site $\mathrm{Y} 602$, located near the 1301-N LWDF. The ${ }^{90} \mathrm{Sr}$ concentration was $2.2 \mathrm{E}+01 \pm 14 \% \mathrm{pCi} / \mathrm{g}$, or 360 times the offsite measured concentration of $6.2 \mathrm{E}-02 \pm 84 \% \mathrm{pCi} / \mathrm{g}$. The average concentration of ${ }^{90} \mathrm{Sr}$ in soil in the $100 \mathrm{~N}$ Area was $3.9 \mathrm{E}-01 \pm 167 \% \mathrm{pCi} / \mathrm{g}$.

4.2.1.4 ${ }^{239,}{ }^{240} \mathrm{Pu}$. The highest ${ }^{239,}{ }^{240} \mathrm{Pu}$ result was found at Site $\mathrm{Y} 602$, located near the 1301-N LWDF. The ${ }^{239,}{ }^{240} \mathrm{Pu}$ concentration was $3.6 \mathrm{E}+00 \pm 16 \% \mathrm{pCi} / \mathrm{g}$, or 330 times the offsite measured concentration of $1.1 \mathrm{E}-02 \pm 9 \% \mathrm{pCi} / \mathrm{g}$. The average concentration of ${ }^{239}, 240 \mathrm{Pu}$ in soil in the $100 \mathrm{~N}$ Area was $9.1 \mathrm{E}-01 \pm 170 \% \mathrm{pCi} / \mathrm{g}$.

4.2.1.5. Uranium. The highest value for total uranium was found at Site $Y 605$ located near the 1301-N LWDF. The uranium concentrations were $3.2 \mathrm{E}-01 \pm 19 \% \mathrm{pCi} / \mathrm{g}$ for ${ }^{234} \mathrm{U}$, $2.5 \mathrm{E}-02 \pm 47 \% \mathrm{pCi} / \mathrm{g}$ for ${ }^{235} \mathrm{U}$, and $3.0 \mathrm{E}-01 \pm 20 \% \mathrm{pCi} / \mathrm{g}$ for ${ }^{238} \mathrm{U}$, or $6.5 \mathrm{E}-01 \mathrm{pCi} / \mathrm{g}$ total uranium. The total uranium concentration was slightly higher than the offsite concentration of $5.0 \mathrm{E}-01 \pm 38 \% \mathrm{pCi} / \mathrm{g}$. The average concentration for total uranium in soil in the $100 \mathrm{~N}$ Area was $1.5 \mathrm{E}-01 \pm 15 \% \mathrm{pCi} / \mathrm{g}$.

\subsubsection{Areas}

Soil samples from 55 of 111 sample locations were collected in 1997. A new sample location (D146) was included this year from the south end of the ERDF facility (see Figure E-3). It will be sampled on an annual basis. Radionuclide concentrations are summarized in Appendix $E$, Table E-2. The 1997 maximum, average, the offsite average, and the accessible soil concentration limits are compared in Table 4-4. Offsite averages for ${ }^{137} \mathrm{Cs}$, ${ }^{90} \mathrm{Sr}$, and isotopic uranium are from the . Hanford Site Environmental Report for Calendar Year 1997 (Dirkes and Hanf 1998) and offsite values for ${ }^{239,240} \mathrm{Pu}$ are contained in the Hanford Site Environmental Report for Calendar Year 1994 (Dirkes and Hanf 1995). Radionuclide concentrations are summarized in Appendix E, Table E-2.

4.2.2.1 ${ }^{60} \mathrm{Co}$. The highest ${ }^{60} \mathrm{Co}$ result was found at Site D021, located northwest of T Plant. The ${ }^{60} \mathrm{Co}$ concentration was $5.8 \mathrm{E}-02 \pm 12 \% \mathrm{pCi} / \mathrm{g}$. The offsite average for ${ }^{60} \mathrm{Co}$ is not reported by PNNL. The average concentration of ${ }^{60} \mathrm{Co}$ in soils in the 200 Areas was $3.0 \mathrm{E}-02 \pm 76 \% \mathrm{pCi} / \mathrm{g}$.

4.2.2.2 ${ }^{137} \mathrm{Cs}$. The highest ${ }^{137} \mathrm{Cs}$ result was found at Site $\mathrm{D} 033$, located east of the $241-U$ Tank Farm. The ${ }^{137} \mathrm{Cs}$ concentration was $1.2 \mathrm{E}+01 \pm 16 \% \mathrm{pCi} / \mathrm{g}$, or 40 times the offsite measured concentration of $3.0 \mathrm{E}-01 \pm 100 \% \mathrm{pCi} / \mathrm{g}$. The average concentration of ${ }^{137} \mathrm{Cs}$ in soils in the 200 Areas was $1.8 \mathrm{E}+00 \pm 38 \% \mathrm{pCi} / \mathrm{g}$. 
Table 4-4. 200 Area Soils (pCi/g).

\begin{tabular}{|c|c|c|c|c|c|c|c|}
\hline & ${ }^{60} \mathrm{Co}$ & ${ }^{90} \mathrm{Sr}$ & ${ }^{137} \mathrm{Cs}$ & ${ }^{234} \mathrm{U}$ & ${ }^{235} \mathrm{U}$ & ${ }^{238} \mathrm{U}$ & $239,240 \mathrm{Pu}$ \\
\hline & Site D021 & Site D035 & Site D033 & Site D047 & Site D105 & Site D047 & Site D039 \\
\hline $\begin{array}{l}\text { Maximum } \pm \text { counting } \\
\text { error }(\%)\end{array}$ & $\begin{array}{c}5.8 \mathrm{E}-02 \\
\pm 12 \% \\
\end{array}$ & $\begin{array}{c}3.4 E+\infty 0 \\
\pm 15 \% \\
\end{array}$ & $\begin{array}{c}1.2 E+01 \\
\pm 16 \%\end{array}$ & $\begin{array}{c}3.8 \mathrm{E}-01 \\
\pm 18 \% \\
\end{array}$ & $\begin{array}{c}3.1 \mathrm{E}-\mathrm{O} 2 \\
\pm 39 \% \\
\end{array}$ & $\begin{array}{c}3.8 \mathrm{E}-01 \\
\pm 18 \% \\
\end{array}$ & $\begin{array}{c}1.4 E+00 \\
\pm 16 \%\end{array}$ \\
\hline Average \pm 2 SEM $(\%)$ & $\begin{array}{c}3.0 \text { E-O2 } \\
\pm 76 \%\end{array}$ & $\begin{array}{l}6.7 \mathrm{E}-01 \\
\pm 34 \%\end{array}$ & $\begin{array}{c}1.8 \mathrm{E}+00 \\
\pm 38 \%\end{array}$ & $\begin{array}{l}2.0 \mathrm{E}-01 \\
\pm 07 \%\end{array}$ & $\begin{array}{c}1.4 \mathrm{E}-\mathrm{O} 2 \\
\pm 10 \%\end{array}$ & $\begin{array}{l}2.0 \text { E-01 } \\
\pm 06 \%\end{array}$ & $\begin{array}{c}1.0 \text { E-O } 1 \\
\pm 74 \%\end{array}$ \\
\hline $\begin{array}{l}\text { PNNL offsite average } \\
\pm 2 \operatorname{SEM}(\%)^{\circ}\end{array}$ & NR & $\begin{array}{c}6.2 \mathrm{E}-02 \\
\pm 84 \% \\
\end{array}$ & $\begin{array}{l}3.0 \mathrm{E}-01 \\
\pm 100 \%\end{array}$ & $\begin{array}{c}2.4 \mathrm{E}-01 \\
\pm 38 \%\end{array}$ & $\begin{array}{c}1.1 E-01 \\
\pm 36 \%\end{array}$ & $\begin{array}{c}2.5 \mathrm{E}-01 \\
\pm 40 \%\end{array}$ & $\begin{array}{l}1.1 \mathrm{E}-02 \\
\pm 09 \%\end{array}$ \\
\hline $\begin{array}{l}\text { Accessible soil } \\
\text { concentration limits }\end{array}$ & $7.1 E+\infty 0$ & $2.8 E+03$ & $3.0 E+01$ & $6.3 E+02$ & $1.7 E \div 02$ & $3.7 E+02$ & $1.9 E+02$ \\
\hline
\end{tabular}

2SEM $=2$ standard error of the mean.

NR $=$ Not reported.

-Dirkes and Hanf 1998, and Dirkes and Hanf 1995..

4.2.2.3 ${ }^{90} \mathrm{Sr}$. The highest ${ }^{90} \mathrm{Sr}$ result was found at Site D035, located southeast of $241-T$ Tank Farm. The ${ }^{90} \mathrm{Sr}$ concentration was $3.4 \mathrm{E}+00 \pm 15 \% \mathrm{pCi} / \mathrm{g}$, or 54 times the offsite measured concentration of $6.2 \mathrm{E}-02 \pm 84 \% \mathrm{pCi} / \mathrm{g}$. The average concentration of ${ }^{90} \mathrm{Sr}$ in soil in the 200 Areas was $6.7 \mathrm{E}-01 \pm 34 \% \mathrm{pCi} / \mathrm{g}$.

4.2.2.4 ${ }^{239,}{ }^{240} \mathrm{Pu}$. The highest ${ }^{239,240} \mathrm{Pu}$ result was found at Site D039, located northeast of REDOX. The ${ }^{239}, 240 \mathrm{Pu}$ concentration was $1.4 \mathrm{E}+00 \pm 16 \% \mathrm{pCi} / \mathrm{g}$, or 140 times the offsite measured concentration of $1.0 \mathrm{E}-02 \pm 9 \% \mathrm{pCi} / \mathrm{g}$. The average concentration of ${ }^{239,}{ }^{240} \mathrm{Pu}$ in soil in the 200 Areas was $1.0 \mathrm{E}-01 \pm 74 \% \mathrm{pCi} / \mathrm{g}$.

4.2.2.5 Uranium. The highest total uranium was found at Site D047, located north of 216-U-17 Crib. The uranium concentrations were $3.8 \mathrm{E}-01 \pm 18 \% \mathrm{pCi} / \mathrm{g}$ for ${ }^{234} \mathrm{U}, 3.1 \mathrm{E}-02 \pm 39 \% \mathrm{pCi} / \mathrm{g}$ for ${ }^{235} \mathrm{U}$, and $3.8 \mathrm{E}-01 \pm 18 \% \mathrm{pCi} / \mathrm{g}$ for ${ }^{238} \mathrm{U}$, or $7.9 \mathrm{E}-01 \mathrm{pCi} / \mathrm{g}$ total uranium. The total uranium concentration was less than the offsite concentration of $5.0 \mathrm{E}-01 \mathrm{pCi} / \mathrm{g}$. The average concentration for total uranium in soil in the 200 Areas was $4.1 \mathrm{E}-01 \pm 8 \% \mathrm{pCi} / \mathrm{g}$.

\subsubsection{0/400 Areas}

Soil samples from 15 sample locations were collected in 1997; 14 from the 300 Area and 1 from the 400 Area. Radionuclide concentrations are listed in Appendix E, Table E-3. The 1997 maximum, average, the offsite average, and the accessible soil concentration limits are compared in Table 4-5. Offsite averages for ${ }^{137} \mathrm{Cs},{ }^{90} \mathrm{Sr}$, and isotopic uranium are from the Hanford Site Environmental Report for Calendar Year 1997 (Dirkes and Hanf 1998) and offsite values for ${ }^{239,240} \mathrm{Pu}$ are contained in the Hanford Site Environmental Report for Calendar Year 1994 (Dirkes and Hanf 1995).

4.2.3.1. ${ }^{60} \mathrm{Co}$. There were no ${ }^{60} \mathrm{Co}$ results above detection limits in 1997 . The offsite average for ${ }^{60} \mathrm{Co}$ is not reported by PNNL. 
HNF-EP-0573-6

Table 4-5. 300/400 Areas Soils (pCi/g).

\begin{tabular}{|c|c|c|c|c|c|c|c|}
\hline & ${ }^{60} \mathrm{Co}$ & ${ }^{90} \mathrm{Sr}$ & ${ }^{137} \mathrm{Cs}$ & ${ }^{234} \mathrm{U}$ & ${ }^{235} \mathrm{U}$ & ${ }^{238} \mathrm{U}$ & ${ }^{239,}{ }^{240} \mathrm{Pu}$ \\
\hline & & Site 0119 & Site 0119 & Site D119 & Site D119. & Site D119 & Site D119 \\
\hline $\begin{array}{l}\text { Maximum } \pm \text { counting } \\
\text { error }(\%)\end{array}$ & ND & $\begin{array}{c}8.0 \mathrm{E}-01 \\
\pm 25 \%\end{array}$ & $\begin{array}{c}2.0 \mathrm{E}-\mathrm{O} 1 \\
\pm 15 \%\end{array}$ & $\begin{array}{c}7.9 \mathrm{E}+00 \\
\pm 8 \%\end{array}$ & $\begin{array}{c}4.7 \mathrm{E}-01 \\
\pm 5 \% \\
\end{array}$ & $\begin{array}{c}7.8 \mathrm{E}+00 \\
\pm 8 \%\end{array}$ & $\begin{array}{c}1.7 \mathrm{E}-01 \\
\pm 22 \% \\
\end{array}$ \\
\hline Average \pm 2 SEM $(\%)$ & ND & $\begin{array}{c}4.5 \mathrm{E}-01 \\
\pm 42 \% \\
\end{array}$ & $\begin{array}{c}6.8 \mathrm{E}-02 \\
\pm 46 \% \\
\end{array}$ & $\begin{array}{c}9.0 \mathrm{E}- \\
01 \pm 08 \% \\
\end{array}$ & $\begin{array}{c}6.2 \mathrm{E}-02 \\
\pm 92 \% \\
\end{array}$ & $\begin{array}{c}8.8 \mathrm{E}-01 \pm \\
109 \%\end{array}$ & $\begin{array}{l}3.8 \mathrm{E}-02 \\
\pm 129 \% \\
\end{array}$ \\
\hline $\begin{array}{l}\text { PNNL offsite average } \\
\pm 2 \text { SEM }(\%)^{*}\end{array}$ & NR & $\begin{array}{c}6.2 \mathrm{E}-\mathrm{O} 2 \\
\pm 84 \%\end{array}$ & $\begin{array}{l}3.0 \text { E-01 } \\
\pm 100 \%\end{array}$ & $\begin{array}{c}2.4 \mathrm{E}-01 \\
\pm 38 \%\end{array}$ & $\begin{array}{c}1.1 \mathrm{E}-01 \\
\pm 36 \%\end{array}$ & $\begin{array}{l}2.5 E-01 \\
\pm 40 \%\end{array}$ & $\begin{array}{c}1.0 \mathrm{E}-02 \\
\pm 9 \%\end{array}$ \\
\hline $\begin{array}{l}\text { Accessible soil } \\
\text { concentration limits }\end{array}$ & $7.1 E+00$ & $2.8 E+03$ & $3.0 E+01$ & $6.3 \mathrm{E}+\mathrm{O} 2$ & $1.7 E+02$ & $3.7 E+02$ & $1.9 E+02$ \\
\hline
\end{tabular}

2SEM $=2$ standard error of the mean.

ND $=$ Not detected.

NR $=$ Not reported.

'Dirkes and Hanf 1998 , and Dirkes and Hanf 1995.

4.2.3.2 ${ }^{137} \mathrm{Cs}$. The highest ${ }^{137} \mathrm{Cs}$ result was found at-Site $\mathrm{D} 119$, located northeast of the $316-2$ Process Pond. The ${ }^{137} \mathrm{Cs}$ concentration was $2.0 \mathrm{E}-01 \pm 15 \% \mathrm{pCi} / \mathrm{g}$, and was less than the offsite measured concentration of $3.0 \mathrm{E}-01 \pm 100 \% \mathrm{pCi} / \mathrm{g}$. The average concentration of ${ }^{137} \mathrm{Cs}$ in soil in the $300 / 400$ Areas was $6.8 \mathrm{E}-02 \pm 46 \% \mathrm{pCi} / \mathrm{g}$.

4.2.3.3 ${ }^{90} \mathrm{~S} r$. The highest ${ }^{90} \mathrm{Sr}$ result was collected at Site $\mathrm{D} 119$, located northeast of the $316-2$ Process Pond. The ${ }^{90} \mathrm{Sr}$ concentration was $8.0 \mathrm{E}-01 \pm 25 \% \mathrm{pCi} / \mathrm{g}$, and was 13 times the offsite measured concentration of $6.2 \mathrm{E}-02 \pm 84 \% \mathrm{pCi} / \mathrm{g}$. The average concentration of ${ }^{90} \mathrm{Sr}$ in soil in the $300 / 400$ Areas was $4.5 \mathrm{E}-01 \pm 42 \% \mathrm{pCi} / \mathrm{g}$.

4.2.3.4 ${ }^{239 .}{ }^{240} \mathrm{Pu}$. The highest ${ }^{239,}{ }^{240} \mathrm{Pu}$ result was found at Site D119, located northeast of the 316-2 Process Pond. The ${ }^{239,}{ }^{240} \mathrm{Pu}$ concentration was $1.7 \mathrm{E}-01 \pm 22 \% \mathrm{pCi} / \mathrm{g}$, or 17 times the offsite measured concentration of $1.0 \mathrm{E}-02 \pm 9 \% \mathrm{pCi} / \mathrm{g}$. The average concentration of ${ }^{239,}{ }^{240} \mathrm{Pu}$ in soil in the $300 / 400$ Areas was $3.8 \mathrm{E}-02 \pm 129 \% \mathrm{pCi} / \mathrm{g}$.

4.2.3.5 Uranium. The highest uranium result was found at Site $D 119$, located northeast of the 316-2 Process Pond. The uranium concentrations were $7.9 \mathrm{E}+00 \pm 8 \% \mathrm{pCi} / \mathrm{g}$ for ${ }^{234} \mathrm{U}, 4.7 \mathrm{E}-01$ $\pm 15 \% \mathrm{pCi} / \mathrm{g}$ for ${ }^{235} \mathrm{U}$, and $7.8 \mathrm{E}+00 \pm 8 \% \mathrm{pCi} / \mathrm{g}$ for ${ }^{238} \mathrm{U}$, or $1.6 \mathrm{E}+00 \mathrm{pCi} / \mathrm{g}$ total uranium. The total uranium concentration was 3 times the offsite concentration of $5.0 \mathrm{E}-01 \pm 38 \% \mathrm{pCi} / \mathrm{g}$. The average concentration of total uranium in soil in the $300 / 400$ Areas was $1.8 \mathrm{E}+00 \pm 67 \% \mathrm{pCi} / \mathrm{g}$.

\subsubsection{Environmental Restoration Contractor Compliance Sampling}

In accordance with WDOH regulatory requirements, soil samples were collected at the $100 \mathrm{D}$ Area and the ERDF remediation projects managed and operated by the ERC to determine the effectiveness of contamination controls. The sample collected at the ERDF site in 1997 represents the initial (baseline) sample to be used for future comparison. At $100 \mathrm{D}$ Area, the 1997 sample was a follow-up to the 1996 sample collected from the same location. The sampling results indicate that at both sites, radionuclide concentrations were comparable to those measured offsite. At $100 \mathrm{D}$, the 1997 results were higher than the 1996 results although within the historical ranges 
observed in soil samples collected in the 100 Areas during the time period from 1981 through 1990. The apparent increase in soil sample concentrations does not correlate with the results observed in the nearby air samplers and most likely is due to the relatively small data set being used for comparison. These samples will be continue to be collected annually at ERDF, periodically at $100 \mathrm{D}$, and the results carefully monitored to determine any trends. Radionuclide concentrations are shown in Appendix E, Table E-4. A summarized comparison of the results to offsite averages and accessible soil concentration limits is provided in Table 4-6.

At $100 \mathrm{BC}$, initial soil sampling was performed by the ERC in 1997. Soil samples will continue to be taken periodically during the remedial action efforts at $100 \mathrm{BC}$. The results will be monitored for trends.

Table 4-6. Comparative Radionuclide Concentrations in 100 D,DR and ERDF Soil (pCi/gm).

\begin{tabular}{|c|c|c|c|c|c|c|c|c|}
\hline Sample & Site & ${ }^{90} \mathrm{Sr}$ & ${ }^{137} \mathrm{Cs}$ & ${ }^{234} \mathrm{U}$ & ${ }^{235} \mathrm{U}$ & ${ }^{238} \mathrm{U}$ & ${ }^{238} \mathrm{Pu}$ & ${ }^{239,240} \mathrm{Pu}$ \\
\hline D-146 & $\begin{array}{l}\text { ERDF, } \\
1997\end{array}$ & ND & $\begin{array}{l}2.9 \mathrm{E}-01 \\
\pm 14 \%\end{array}$ & $\begin{array}{l}2.2 \text { E- } 01 \\
\pm 14 \%\end{array}$ & $\begin{array}{c}2.2 \mathrm{E}-\mathrm{O} 2 \\
\pm 44 \%\end{array}$ & $\begin{array}{l}2.0 \mathrm{E}-01 \\
\pm 15 \%\end{array}$ & ND & $\begin{array}{c}1.7 \text { E-O2 } \\
\pm 63 \%\end{array}$ \\
\hline D-147 & $\begin{array}{l}100-D \\
1997\end{array}$ & ND & $\begin{array}{l}1.1 \mathrm{E}-01 \\
\pm 18 \%\end{array}$ & $\begin{array}{c}1.6 \mathrm{E}-01 \\
\pm 15 \%\end{array}$ & $\begin{array}{c}2.1 \mathrm{E}-02 \\
\pm 42 \%\end{array}$ & $\begin{array}{c}1.6 \mathrm{E}-01 \\
\pm 15 \%\end{array}$ & ND & ND \\
\hline D-148 & $\begin{array}{l}100 \mathrm{D} \\
1996\end{array}$ & $\begin{array}{l}1.4 \mathrm{E}-01 \\
\pm 20 \% \\
\end{array}$ & $\begin{array}{l}4.4 \mathrm{E}-01 \\
\pm 11 \%\end{array}$ & $\begin{array}{c}5.2 \mathrm{E}-02 \\
\pm 19 \%\end{array}$ & $\begin{array}{c}2.6 \mathrm{E}-03 \\
\pm 72 \%\end{array}$ & $\begin{array}{c}5.6 \mathrm{E}-02 \\
\pm 18 \%\end{array}$ & $\begin{array}{c}2.8 \mathrm{E}-03 \\
\pm 32 \%\end{array}$ & $\begin{array}{c}1.0 \mathrm{E}-02 \\
\pm 20 \%\end{array}$ \\
\hline D-148 & $\begin{array}{l}100 \mathrm{D} \\
1997\end{array}$ & ND & $\begin{array}{c}6.9 \mathrm{E}-01 \\
\pm 13 \%\end{array}$ & $\begin{array}{l}2.0 \mathrm{E}-01 \\
\pm 50 \%\end{array}$ & $\begin{array}{c}7.3 \mathrm{E}-02 \\
\pm 29 \%\end{array}$ & $\begin{array}{c}2.0 \mathrm{E}-\mathrm{O} 1 \\
\pm 21 \%\end{array}$ & ND & $\begin{array}{c}2.8 \mathrm{E}-\mathrm{O} 2 \\
\pm 58 \%\end{array}$ \\
\hline \multicolumn{2}{|c|}{$\begin{array}{l}\text { PNNL Offsite Average } \\
\pm 2 \text { SEM }(\%)^{3}\end{array}$} & $\begin{array}{c}6.2 \mathrm{E}-02 \\
\pm 84 \%\end{array}$ & $\begin{array}{l}3.0 \mathrm{E}-01 \\
\pm 100 \%\end{array}$ & $\begin{array}{c}2.4 \mathrm{E}-01 \\
\pm 38 \%\end{array}$ & $\begin{array}{c}1.1 \mathrm{E}-01 \\
\pm 36 \%\end{array}$ & $\begin{array}{l}2.5 \mathrm{E}-01 \\
\pm 40 \%\end{array}$ & NR & $\begin{array}{c}1.0 \mathrm{E}-02 \\
\pm 09 \%\end{array}$ \\
\hline \multicolumn{2}{|c|}{$\begin{array}{l}\text { Accessible Soil } \\
\text { Concentration Limit }\end{array}$} & $2.8 E+03$ & $3.0 E+01$ & $6.3 E+02$ & $1.7 E+02$ & $3.7 E+02$ & $2.1 E+02$ & $1.9 E+02$ \\
\hline
\end{tabular}

2SEM = Two standard error of the mean.

ND $=$ Not Detected.

NR $=$ Not Reported.

'Dirkes and Hanf 1998, and Dirkes and Hanf 1995.

4.2.4.1 ${ }^{60} \mathrm{Co}$. There were no ${ }^{60} \mathrm{Co}$ results above detection limits in 1997. The offsite average for ${ }^{60} \mathrm{Co}$ is not reported by PNNL.

4.2.4.2 ${ }^{137} \mathrm{Cs}$. The highest ${ }^{137} \mathrm{Cs}$ result was found at Site D-148, located at $100 \mathrm{D}, \mathrm{DR}$ Area. The ${ }^{137} \mathrm{Cs}$ concentration was $6.9 \mathrm{E}-01 \pm 13 \% \mathrm{pCi} / \mathrm{g}$, or 2 times the offsite measured concentration of $3.0 \mathrm{E}-01 \pm 100 \% \mathrm{pCi} / \mathrm{g}$.

4.2.4.3 ${ }^{90} \mathrm{Sr}$. There were no ${ }^{90} \mathrm{Sr}$ results above detection limits in 1997 . The offsite average for ${ }^{90} \mathrm{Sr}$ in 1997 was $6.2 \mathrm{E}-02 \pm 84 \% \mathrm{pCi} / \mathrm{g}$.

4.2.4.4 ${ }^{239 .}{ }^{240} \mathrm{Pu}$. The highest ${ }^{239 .}{ }^{240} \mathrm{Pu}$ result was found at Site $\mathrm{D}-148$, located at $100 \mathrm{D}, \mathrm{DR}$ Area. The ${ }^{239 .}{ }^{240} \mathrm{Pu}$ concentration was $2.8 \mathrm{E}-02 \pm 58 \% \mathrm{pCi} / \mathrm{g}$, or 3 times the 1994 offsite average of $1.0 \mathrm{E}-\mathrm{O} 2 \pm 9 \% \mathrm{pCi} / \mathrm{g}$. 
4.2.4.5. Uranium. The highest value for total uranium was found at Site $D-148$, located at $100 \mathrm{D}, \mathrm{DR}$ Area. The uranium concentrations were $2.0 \mathrm{E}-01 \pm 50 \% \mathrm{pCi} / \mathrm{g}$ for ${ }^{234} \mathrm{U}, 7.3$ $\mathrm{E}-02 \pm 29 \% \mathrm{pCi} / \mathrm{g}$ for ${ }^{235} \mathrm{U}$, and $2.0 \mathrm{E}-01 \pm 21 \% \mathrm{pCi} / \mathrm{g}$ for ${ }^{238} \mathrm{U}$. The total uranium concentration was $4.7 \mathrm{E}-01 \mathrm{pCi} / \mathrm{g}$, slightly lower than the offsite concentration of $5.0 \mathrm{E}-01 \pm 38 \% \mathrm{pCi} / \mathrm{g}$.

\subsection{VEGETATION SAMPLING}

\subsubsection{Areas}

Vegetation samples collected near the 1301-N and 1325-N LWDFs contain radionuclides that were typically present in past effluent stream discharges. The levels of contaminant concentrations near the 1301-N Facility were at or near historic levels. Average radionuclide concentrations detected in the vegetation samples near the 1301-N LWDF from 1980 through 1997 are presented in Table 4-7. Average radionuclide concentrations detected in the vegetation samples collected in the $100 \mathrm{~N}$ Area from 1980 through 1997 are presented in Table 4-8.

Table 4-7. Average Radionuclide Concentrations Detected in Vegetation Samples Near the 1301-N Liquid Waste Disposal Facility from 1980 to 1997 (pCi/g).

\begin{tabular}{|l|c|c|c|c|c|}
\hline \multicolumn{1}{|c|}{ Year } & ${ }^{60} \mathrm{Co}$ & ${ }^{90} \mathrm{Sr}$ & ${ }^{137} \mathrm{Cs}$ & ${ }^{238} \mathrm{Pu}$ & ${ }^{239,240} \mathrm{Pu}$ \\
\hline 1980 & $4.0 \mathrm{E}+00$ & $\mathrm{NR}$ & $1.1 \mathrm{E}+00$ & $\mathrm{NR}$ & $\mathrm{NR}$ \\
\hline 1981 & $1.2 \mathrm{E}+01$ & $1.8 \mathrm{E}+00$ & $1.8 \mathrm{E}+00$ & $\mathrm{NR}$ & $7.1 \mathrm{E}-03$ \\
\hline 1982 & $1.6 \mathrm{E}+00$ & $1.2 \mathrm{E}-01$ & $2.6 \mathrm{E}-01$ & $\mathrm{NR}$ & $2.6 \mathrm{E}-03$ \\
\hline 1983 & $1.9 \mathrm{E}+00$ & $6.0 \mathrm{E}-01$ & $3.9 \mathrm{E}-01$ & $\mathrm{NR}$ & $3.2 \mathrm{E}-03$ \\
\hline 1984 & $1.0 \mathrm{E}+00$ & $1.2 \mathrm{E}-01$ & $8.3 \mathrm{E}-02$ & $\mathrm{NR}$ & $8.5 \mathrm{E}-04$ \\
\hline 1985 & $1.7 \mathrm{E}+00$ & $1.9 \mathrm{E}+00$ & $1.0 \mathrm{E}-01$ & $\mathrm{NR}$ & $1.5 \mathrm{E}-03$ \\
\hline 1986 & $3.5 \mathrm{E}+00$ & $7.3 \mathrm{E}-02$ & $6.5 \mathrm{E}-01$ & $\mathrm{NR}$ & $2.6 \mathrm{E}-03$ \\
\hline 1987 & $2.8 \mathrm{E}+00$ & $6.3 \mathrm{E}-02$ & $2.0 \mathrm{E}-01$ & $1.2 \mathrm{E}-03$ & $5.6 \mathrm{E}-03$ \\
\hline 1988 & $2.0 \mathrm{E}+00$ & $1.2 \mathrm{E}-01$ & $1.3 \mathrm{E}-01$ & $4.3 \mathrm{E}-04$ & $1.7 \mathrm{E}-03$ \\
\hline 1989 & $1.3 \mathrm{E}+00$ & $3.8 \mathrm{E}-02$ & $1.5 \mathrm{E}-01$ & $2.8 \mathrm{E}-04$ & $2.0 \mathrm{E}-03$ \\
\hline 1990 & $1.3 \mathrm{E}+00$ & $3.1 \mathrm{E}-02$ & $1.2 \mathrm{E}-01$ & $2.7 \mathrm{E}-04$ & $1.1 \mathrm{E}-03$ \\
\hline 1991 & $6.3 \mathrm{E}-01$ & $1.5 \mathrm{E}-02$ & $6.0 \mathrm{E}-01$ & $8.1 \mathrm{E}-05$ & $1.2 \mathrm{E}-03$ \\
\hline 1992 & $2.0 \mathrm{E}-01$ & $6.5 \mathrm{E}-03$ & $2.0 \mathrm{E}-01$ & $-2.8 \mathrm{E}-05$ & $1.6 \mathrm{E}-04$ \\
\hline 1993 & $2.2 \mathrm{E}-01$ & $5.7 \mathrm{E}-02$ & $2.1 \mathrm{E}-01$ & $-7.8 \mathrm{E}-04$ & $4.1 \mathrm{E}-04$ \\
\hline 1994 & $2.5 \mathrm{E}+01$ & $4.8 \mathrm{E}+00$ & $1.8 \mathrm{E}+00$ & $3.3 \mathrm{E}-02$ & $2.0 \mathrm{E}-01$ \\
\hline 1995 & $5.4 \mathrm{E}-02$ & $6.4 \mathrm{E}-02$ & $1.2 \mathrm{E}-01$ & $1.3 \mathrm{E}-03$ & $8.6 \mathrm{E}-03$ \\
\hline 1996 & $6.1 \mathrm{E}+01$ & $5.8 \mathrm{E}+02$ & $2.8 \mathrm{E}+03$ & $-1.8 \mathrm{E}-02$ & $-9.6 \mathrm{E}-03$ \\
\hline 1997 & $4.2 \mathrm{E}-01$ & $4.9 \mathrm{E}-01$ & $1.4 \mathrm{E}-01$ & $8.3 \mathrm{E}-03$ & ND \\
\hline
\end{tabular}

ND $=$ Not detected.

$\mathrm{NR}=$ Not reported.

Negative value indicates results at or below background levels of radioactivity. 
Table 4-8. Average Radionuclide Concentrations Detected in $100 \mathrm{~N}$ Vegetation Samples from 1980 to $1997(\mathrm{pCi} / \mathrm{g})$.

\begin{tabular}{|l|c|c|c|c|c|}
\hline \multicolumn{1}{|c|}{ Year } & ${ }^{60} \mathrm{Co}$ & ${ }^{90} \mathrm{Sr}$ & ${ }^{137} \mathrm{Cs}$ & ${ }^{238} \mathrm{PU}$ & ${ }^{239,240} \mathrm{PU}$ \\
\hline 1980 & $1.0 \mathrm{E}+00$ & $\mathrm{NR}$ & $2.8 \mathrm{E}-01$ & $\mathrm{NR}$ & $\mathrm{NR}$ \\
\hline 1981 & $2.5 \mathrm{E}+01$ & $5.8 \mathrm{E}-01$ & $7.1 \mathrm{E}-01$ & $\mathrm{NR}$ & $2.1 \mathrm{E}-02$ \\
\hline 1982 & $1.5 \mathrm{E}+00$ & $2.0 \mathrm{E}-01$ & $1.3 \mathrm{E}-01$ & $\mathrm{NR}$ & $7.8 \mathrm{E}-03$ \\
\hline 1983 & $1.0 \mathrm{E}+00$ & $2.9 \mathrm{E}-01$ & $9.0 \mathrm{E}-02$ & $\mathrm{NR}$ & $8.6 \mathrm{E}-03$ \\
\hline 1984 & $4.6 \mathrm{E}-01$ & $8.1 \mathrm{E}-02$ & $9.0 \mathrm{E}-02$ & $\mathrm{NR}$ & $1.3 \mathrm{E}-03$ \\
\hline 1985 & $1.4 \mathrm{E}+00$ & $5.1 \mathrm{E}-02$ & $1.6 \mathrm{E}-01$ & $\mathrm{NR}$ & $8.7 \mathrm{E}-04$ \\
\hline 1986 & $9.5 \mathrm{E}-01$ & $2.2 \mathrm{E}-01$ & $7.9 \mathrm{E}-01$ & $\mathrm{NR}$ & $1.1 \mathrm{E}-03$ \\
\hline 1987 & $7.0 \mathrm{E}-01$ & $2.6 \mathrm{E}-01$ & $9.4 \mathrm{E}-02$ & $1.3 \mathrm{E}-04$ & $5.7 \mathrm{E}-04$ \\
\hline 1988 & $8.0 \mathrm{E}-01$ & $2.5 \mathrm{E}-01$ & $1.6 \mathrm{E}-01$ & $1.7 \mathrm{E}-04$ & $6.6 \mathrm{E}-04$ \\
\hline 1989 & $3.2 \mathrm{E}-01$ & $6.8 \mathrm{E}-02$ & $1.5 \mathrm{E}-01$ & $1.1 \mathrm{E}-04$ & $8.7 \mathrm{E}-04$ \\
\hline 1990 & $1.1 \mathrm{E}-01$ & $9.3 \mathrm{E}-03$ & $3.6 \mathrm{E}-02$ & $-9.6 \mathrm{E}-05$ & $1.7 \mathrm{E}-04$ \\
\hline 1991 & $1.3 \mathrm{E}-01$ & $9.4 \mathrm{E}-03$ & $3.4 \mathrm{E}-03$ & $1.6 \mathrm{E}-04$ & $2.5 \mathrm{E}-03$ \\
\hline 1992 & $5.6 \mathrm{E}-02$ & $5.6 \mathrm{E}-02$ & $7.8 \mathrm{E}-02$ & $-5.2 \mathrm{E}-06$ & $1.3 \mathrm{E}-04$ \\
\hline 1993 & $1.0 \mathrm{E}-01$ & $3.6 \mathrm{E}-02$ & $6.5 \mathrm{E}-02$ & $-5.2 \mathrm{E}-05$ & $3.3 \mathrm{E}-04$ \\
\hline 1994 & $6.5 \mathrm{E}+00$ & $2.5 \mathrm{E}+01$ & $5.8 \mathrm{E}-01$ & $8.6 \mathrm{E}-03$ & $5.3 \mathrm{E}-02$ \\
\hline 1995 & $3.0 \mathrm{E}-02$ & $5.4 \mathrm{E}+00$ & $8.1 \mathrm{E}-02$ & $6.8 \mathrm{E}-04$ & $3.3 \mathrm{E}-03$ \\
\hline 1996 & $2.4 \mathrm{E}+00$ & $2.3 \mathrm{E}+02$ & $1.1 \mathrm{E}+03$ & $-7.6 \mathrm{E}-03$ & $-5.1 \mathrm{E}-03$ \\
\hline 1997 & $4.2 \mathrm{E}-01$ & $3.6 \mathrm{E}+00$ & $1.6 \mathrm{E}-01$ & $4.8 \mathrm{E}-03$ & $\mathrm{ND}$ \\
\hline
\end{tabular}

ND $=$ Not detected.

$\mathrm{NR}=$ Not reported.

Negative value indicates results at or below background levels of radioactivity.

Vegetation samples collected along the $100 \mathrm{~N}$ Area shoreline ( $\mathrm{N}$ Springs) contain radionuclides that were not completely retained in the soil columns beneath the $1301-\mathrm{N} / 1325-\mathrm{N}$ LWDFs. Biotransport via root uptake of ${ }^{60} \mathrm{Co},{ }^{90} \mathrm{Sr}$, and ${ }^{137} \mathrm{Cs}$ was evident in the reed canary grass samples collected from this region. Most notable were the average levels of ${ }^{90} \mathrm{Sr}$ and ${ }^{137} \mathrm{Cs}$ which exhibited concentrations orders of magnitude higher than the offsite averages. Average radionuclide concentrations detected in the vegetation samples collected along the N Springs from 1980 through 1997 are presented in Table 4-9.

The 1997 maximum, average, the offsite average, and the accessible soil concentration limits are compared in Table 4-10. Offsite averages for ${ }^{90} \mathrm{Sr}$, and isotopic uranium are from the Hanford Site Environmental Report for Calendar Year 1997 (Dirkes and Hanf 1998) and offsite values for ${ }^{137} \mathrm{Cs}$ and ${ }^{239,240} \mathrm{Pu}$ are contained in the Hanford Site Environmental Report for Calendar Year 1994 (Dirkes and Hanf 1995). Radionuclide concentrations are summarized in Appendix E, Table E-5.

4.3.1.1 ${ }^{60} \mathrm{Co}$. The highest ${ }^{60} \mathrm{Co}$ result was from the only sample above detection limits at Site $\mathrm{Y} 702$, located near the $1325-\mathrm{N}$ LWDF. The ${ }^{60} \mathrm{Co}$ concentration was $4.2 \mathrm{E}-01 \pm 12 \% \mathrm{pCi} / \mathrm{g}$. The offsite average for ${ }^{60} \mathrm{Co}$ is not reported by PNNL. 
Table 4-9. Average Radionuclide Concentrations Detected in N Springs Vegetation Samples from 1980 to $1997(\mathrm{pCi} / \mathrm{g})$.

\begin{tabular}{|c|c|c|c|c|c|}
\hline Year & ${ }^{60} \mathrm{Co}$ & ${ }^{90} \mathrm{Sr}$ & ${ }^{137} \mathrm{Cs}$ & ${ }^{238} \mathrm{Pu}$ & ${ }^{239.240} \mathrm{Pu}$ \\
\hline 1980 & $5.6 E+00$ & NR & $4.4 E-01$ & NR & NR \\
\hline 1981 & $3.3 \mathrm{E}+00$ & $2.0 \mathrm{E}+02$ & NR & NR & $3.7 \mathrm{E}-03$ \\
\hline 1982 & $2.8 E+00$ & $4.8 E+02$ & NR & NR & 8.3 E-O3 \\
\hline 1983 & $3.0 E+00$ & $3.3 \mathrm{E}+02$ & 4.0 E-02 & NR & $8.0 \mathrm{E}-03$ \\
\hline 1984 & NS & NS & NS & NS & NS \\
\hline 1985 & $1.2 \varepsilon+00$ & $4.2 \mathrm{E}+\mathrm{O} 2$ & 1.7 E-01 & NR & 4.4 E-04 \\
\hline 1986 & $1.1 E+00$ & $2.2 \mathrm{E}+\mathrm{O} 2$ & $2.1 \mathrm{E}-01$ & NR & 4.2 E-O4 \\
\hline 1987 & 9.0 E-01 & $2.9 E+02$ & 1.1 E-01 & -1.3 E-04 & $7.6 \mathrm{E}-04$ \\
\hline 1988 & $1.4 E+00$ & $1.2 \mathrm{E}+02$ & 2.0 E-O1 & $8.5 \mathrm{E}-05$ & $2.0 \mathrm{E}-04$ \\
\hline 1989 & 4.3 E-01 & $8.0 \mathrm{E}+01$ & $1.5 \mathrm{E}-01$ & $1.1 \mathrm{E}-03$ & 4.5 E-04 \\
\hline 1990 & NS & NS & NS & NS & NS \\
\hline 1991 & 1.5 E-01 & $3.1 E+01$ & $1.6 \mathrm{E}-01$ & 1.2 E-04 & $2.9 \mathrm{E}-\mathrm{O} 3$ \\
\hline 1992 & 4.2 E-02 & $7.8 \mathrm{E}+01$ & $3.0 \mathrm{E}-01$ & 1.5 E-04 & $2.9 \mathrm{E}-04$ \\
\hline 1993 & 4.5 E-01 & $2.6 E+02$ & 2.0 E-01 & 2.3 E-03 & -8.5 E-04 \\
\hline 1994 & $1.4 \mathrm{E}-01$ & $6.0 \mathrm{E}+01$ & 1.5 E-01 & 3.6 E-04 & 2.0 E-03 \\
\hline 1995 & 1.4 E-02 & $1.3 \mathrm{E}+01$ & 9.4 E-02 & $5.9 \mathrm{E}-04$ & $2.8 E-03$ \\
\hline 1996 & $1.0 \mathrm{E}-\mathrm{O} 2$ & $1.8 E+01$ & $3.8 E-02$ & $1.7 \mathrm{E}-03$ & -1.5 E-03 \\
\hline 1997 & ND & $6.2 \mathrm{E}+00$ & $1.8 \mathrm{E}-01$ & $4.8 \mathrm{E}-03$ & ND \\
\hline
\end{tabular}

ND $=$ Not detected.

NR $=$ Not reported.

NS $=$ No sample results.

Negative value indicates results at or below background levels of radioactivity.

Table 4-10. Radionuclide Concentrations in $100 \mathrm{~N}$ Area Vegetation (pCi/g).

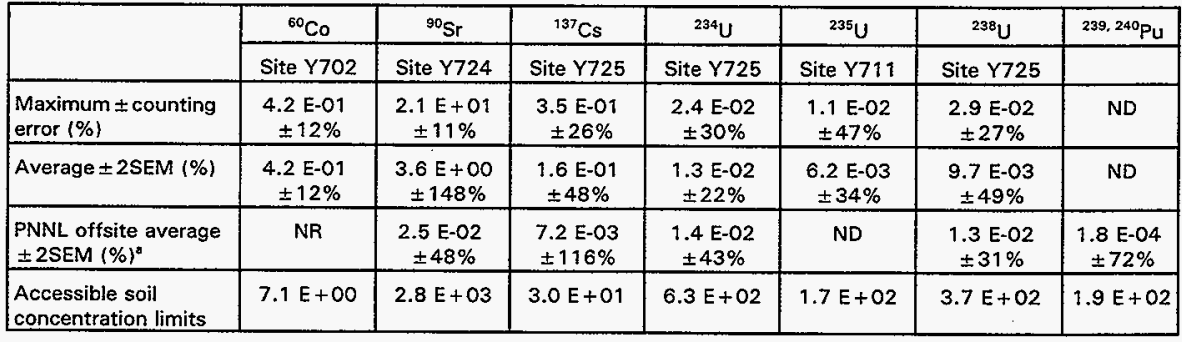

2SEM = Two standard error of the mean.

$N D=$ Not detected

NR = Not reported.

'Dirkes and Hanf 1998, and Dirkes and Hanf 1995. 
4.3.1.2 ${ }^{137} \mathrm{Cs}$. The highest ${ }^{137} \mathrm{Cs}$ result was found at Site $Y 725$, located along the $\mathrm{N}$ Springs shoreline area. The ${ }^{137} \mathrm{Cs}$ concentration was $3.5 \mathrm{E}-01 \pm 26 \% \mathrm{pCi} / \mathrm{g}$ or 49 times the offsite average concentration of $7.2 \mathrm{E}-03 \pm 116 \% \mathrm{pCi} / \mathrm{g}$. The average concentration of ${ }^{137} \mathrm{Cs}$ in vegetation in the $100 \mathrm{~N}$ Area was $1.6 \mathrm{E}-01 \pm 48 \% \mathrm{pCi} / \mathrm{g}$.

4.3.1.3 ${ }^{90} \mathrm{Sr}$. The highest ${ }^{90} \mathrm{Sr}$ result was found at Site $Y 724$, located along the $\mathrm{N}$ Springs shoreline area. The ${ }^{90} \mathrm{Sr}$ concentration was $2.1 \mathrm{E}+01 \pm 11 \% \mathrm{pCi} / \mathrm{g}$, or 840 times the offsite measured concentration of $2.5 \mathrm{E}-02 \pm 48 \% \mathrm{pCi} / \mathrm{g}$. The average concentration of ${ }^{90} \mathrm{Sr}$ in vegetation in the $100 \mathrm{~N}$ Area was $3.6 \mathrm{E}+00 \pm 148 \% \mathrm{pCi} / \mathrm{g}$.

4.3.1.4 ${ }^{239,}{ }^{240} \mathrm{Pu}$. There were no ${ }^{239,}{ }^{240} \mathrm{Pu}$ results above the detection limits in 1997 . The offsite measured concentration was $1.8 \mathrm{E}-04 \pm 72 \% \mathrm{pCi} / \mathrm{g}$.

4.3.1.5 Uranium. The highest total uranium was found at Site $Y 725$, located along the $N$ Springs shoreline area. The uranium concentrations were $2.4 \mathrm{E}-02 \pm 30 \% \mathrm{pCi} / \mathrm{g}$ for ${ }^{234} \mathrm{U}, 1.1 \mathrm{E}-02$ $\pm 47 \% \mathrm{pCi} / \mathrm{g}$ for ${ }^{235} \mathrm{U}$, and $2.9 \mathrm{E}-02 \pm 27 \% \mathrm{pCi} / \mathrm{g}$ for ${ }^{238} \mathrm{U}$, or $6.4 \mathrm{E}-02 \mathrm{pCi} / \mathrm{g}$ uranium total. The concentration of total uranium was 2 times the offsite concentration of $2.7 \mathrm{E}-02 \pm 37 \% \mathrm{pCi} / \mathrm{g}$. The average concentration for total uranium in vegetation in the $100 \mathrm{~N}$ Area was $2.9 \mathrm{E}-02$ $\pm 35 \% \mathrm{pCi} / \mathrm{g}$.

\subsubsection{0/600 Areas}

In 1997, 41 vegetation samples were collected. The 1997 maximum, average, the offsite average, and the accessible soil concentration limits are compared in Table 4-11. Offsite averages for ${ }^{90} \mathrm{Sr}$, and isotopic uranium are from the Hanford Site Environmental Report for Calendar Year 1997 (Dirkes and Hanf 1998) and offsite values for ${ }^{137} \mathrm{Cs}$ and ${ }^{239.240} \mathrm{Pu}$ are contained in the Hanford Site Environmental Report for Calendar Year 1994 (Dirkes and Hanf 1995). Radionuclide concentrations are summarized in Appendix E, Table E-6.

4.3.2.1. ${ }^{60} \mathrm{Co}$. There were no ${ }^{60} \mathrm{Co}$ results above detection limits in 1997 . The offsite average for ${ }^{60} \mathrm{Co}$ is not reported by PNNL.

4.3.2.2 ${ }^{137} \mathrm{Cs}$. The highest ${ }^{137} \mathrm{Cs}$ result was found at Site V033, located east of the $241-U$ Tank Farm. The ${ }^{137} \mathrm{Cs}$ concentration was $5.9 \mathrm{E}-01 \pm 18 \% \mathrm{pCi} / \mathrm{g}$, or 82 times the offsite concentration of $7.2 \mathrm{E}-03 \pm 117 \%$. The average concentration of ${ }^{137} \mathrm{Cs}$ in vegetation in the 200 Areas was $1.3 \mathrm{E}-01 \pm 44 \% \mathrm{pCi} / \mathrm{g}$.

4.3.2.3. ${ }^{90} \mathrm{Sr}$. The highest ${ }^{90} \mathrm{Sr}$ result was found at Site V015, located south of the $24^{2} \mathrm{r}-\mathrm{T}$ Tank Farm. The ${ }^{90} \mathrm{Sr}$ concentration was $2.7 \mathrm{E}+01 \pm 11 \% \mathrm{pCi} / \mathrm{g}$, or 1,100 times the offsite measured concentration of $2.5 \mathrm{E}-02 \pm 48 \% \mathrm{pCi} / \mathrm{g}$. The average concentration of ${ }^{90} \mathrm{Sr}$ in vegetation in the 200 Areas was $2.9 \mathrm{E}+00 \pm 87 \% \mathrm{pCi} / \mathrm{g}$.

4.3.2.4 ${ }^{239,}{ }^{240} \mathrm{Pu}$. The highest ${ }^{239,}{ }^{240} \mathrm{Pu}$ result was found at Site VO25, located north of T Plant. The ${ }^{239 .}{ }^{240} \mathrm{Pu}$ concentration was $9.7 \mathrm{E}-03 \pm 52 \% \mathrm{pCi} / \mathrm{g}$, or 54 times the offsite measured concentration of $1.8 \mathrm{E}-04 \pm 72 \% \mathrm{pCi} / \mathrm{g}$. The average concentration of ${ }^{239.240} \mathrm{Pu}$ in vegetation in the 200 Areas was $6.6 \mathrm{E}-03 \pm 16 \% \mathrm{pCi} / \mathrm{g}$. 
Table 4-11. 200 Areas Vegetation (pCi/g).

\begin{tabular}{|c|c|c|c|c|c|c|c|}
\hline & ${ }^{60} \mathrm{Co}$ & ${ }^{90} \mathrm{Sr}$ & ${ }^{137} \mathrm{Cs}$ & ${ }^{234} \mathrm{U}$ & ${ }^{235} \mathrm{U}$ & ${ }^{238} \mathrm{U}$ & ${ }^{239,240} \mathrm{Pu}$ \\
\hline & & Site V015 & Site V033 & Site V091 & Site V091 & Site V035 & Site V025 \\
\hline $\begin{array}{l}\text { Maximum } \pm \text { counting } \\
\text { error }(\%)\end{array}$ & ND & $\begin{array}{c}2.7 \mathrm{E}+01 \\
\pm 11 \% \\
\end{array}$ & $\begin{array}{c}5.9 \mathrm{E}-01 \\
\pm 18 \% \\
\end{array}$ & $\begin{array}{c}3.4 \mathrm{E}-02 \\
\pm 26 \% \\
\end{array}$ & $\begin{array}{c}1.9 \mathrm{E}-02 \\
\pm 36 \% \\
\end{array}$ & $\begin{array}{c}2.5 \mathrm{E}-02 \\
\pm 34 \% \\
\end{array}$ & $\begin{array}{c}9.7 \mathrm{E}-03 \\
\pm 52 \% \\
\end{array}$ \\
\hline Average \pm 2 SEM $(\%)$ & ND & $\begin{array}{c}2.9 \mathrm{E}+00 \\
\pm 87 \% \\
\end{array}$ & $\begin{array}{c}1.3 \mathrm{E}-01 \\
\pm 44 \% \\
\end{array}$ & $\begin{array}{c}1.5 \mathrm{E}-02 \\
\pm 16 \% \\
\end{array}$ & $\begin{array}{l}9.7 \mathrm{E}-03 \\
\pm 20 \% \\
\end{array}$ & $\begin{array}{c}1.1 \mathrm{E}-02 \\
\pm 19 \% \\
\end{array}$ & $\begin{array}{c}6.6 \mathrm{E}-03 \\
\pm 16 \% \\
\end{array}$ \\
\hline $\begin{array}{l}\text { PNNL offsite average } \\
\pm 2 \text { SEM }(\%)^{\circ}\end{array}$ & NR & $\begin{array}{c}2.5 \mathrm{E}-02 \\
\pm 48 \% \\
\end{array}$ & $\begin{array}{l}7.2 \mathrm{E}-03 \\
\pm 116 \% \\
\end{array}$ & $\begin{array}{c}1.4 \mathrm{E}-02 \\
\pm 43 \% \\
\end{array}$ & ND & $\begin{array}{c}1.3 \mathrm{E}-02 \\
\pm 31 \% \\
\end{array}$ & $\begin{array}{c}1.8 \mathrm{E}-04 \\
\pm 72 \% \\
\end{array}$ \\
\hline $\begin{array}{l}\text { Accessible soil } \\
\text { concentration limits }\end{array}$ & $7.1 E+00$ & $2.8 E+03$ & $3.0 \mathrm{E}+01$ & $6.3 E+02$ & $1.7 E+02$ & $3.7 E+02$ & $1.9 E+02$ \\
\hline
\end{tabular}

2SEM = Two standard error of the mean.
ND $=$ Not detected.
NR $=$ Not reported.
'Dirkes and Hanf 1998, and Dirkes and Hanf 1995.

4.3.2.5 Uranium. The highest total uranium result was found at Site V091, located at the west end of 216-A-24 Crib. The uranium concentrations were $3.4 \mathrm{E}-02 \pm 26 \% \mathrm{pCi} / \mathrm{g}$ for ${ }^{234} \mathrm{U}, 1.9 \mathrm{E}-02$ $\pm 36 \% \mathrm{pCi} / \mathrm{g}$ for ${ }^{235} \mathrm{U}$, and $2.5 \mathrm{E}-02 \pm 34 \% \mathrm{pCi} / \mathrm{g}$ for ${ }^{238} \mathrm{U}$, or $7.8 \mathrm{E}-02 \mathrm{pCi} / \mathrm{g}$ total uranium. The total isotopic uranium concentration was 9 times the offsite measured concentration of $2.7 \mathrm{E}-02 \pm$ $37 \% \mathrm{pCi} / \mathrm{g}$. The average concentration of total uranium in vegetation in the 200 Areas was $3.6 \mathrm{E}-02 \pm 18 \% \mathrm{pCi} / \mathrm{g}$.

\subsection{3 $300 / 400$ Areas}

This was the seventh year of sampling from locations established to more directly monitor facilities and active/inactive waste sites. In 1997, 15 vegetation samples were collected from 14 locations in the 300 Area and one in the 400 Area. The 1997 maximum, average, the offsite average and the accessible soil concentration limits are compared in Table 4-12. Offsite averages for ${ }^{90} \mathrm{Sr}$, and isotopic uranium are from the Hanford Site Environmental Report for Calendar Year 1997 (Dirkes and Hanf 1998) and offsite values for ${ }^{137} \mathrm{Cs}$ and ${ }^{239,240} \mathrm{Pu}$ are contained in the Hanford Site Environmental Report for Calendar Year 1994 (Dirkes and Hanf 1995). Radionuclide concentrations for all sites sampled are listed in Appendix E, Table E-7.

4.3.3.1. ${ }^{60} \mathrm{Co}$. There were no ${ }^{60} \mathrm{Co}$ results above detection limits in 1997 . The offsite average for ${ }^{60} \mathrm{Co}$ is not reported in by PNNL.

4.3.3.2 ${ }^{137} \mathrm{Cs}$. There were no ${ }^{137} \mathrm{Cs}$ results above detection limits in 1997. The offsite measured concentration for ${ }^{137} \mathrm{Cs}$ was $7.2 \mathrm{E}-03 \pm 117 \%$.

4.3.3.3 ${ }^{90} \mathrm{Sr}$. The highest ${ }^{90} \mathrm{Sr}$ result was found at Site $\mathrm{V} 130$, located near the 400 Area Process Pond. The ${ }^{90} \mathrm{Sr}$ concentration was $9.6 \mathrm{E}-01 \pm 25 \% \mathrm{pCi} / \mathrm{g}$, or 38 times the offsite measured concentration of $2.5 \mathrm{E}-02 \pm 48 \% \mathrm{pCi} / \mathrm{g}$. The average concentration of ${ }^{90} \mathrm{Sr}$ in vegetation in the $300 / 400$ Areas was $6.6 \mathrm{E}-01 \pm 59 \% \mathrm{pCi} / \mathrm{g}$. 
Table 4-12. 300/400 Areas Vegetation (pCi/g).

\begin{tabular}{|c|c|c|c|c|c|c|c|}
\hline & ${ }^{60} \mathrm{Co}$ & ${ }^{90} \mathrm{Sr}$ & ${ }^{137} \mathrm{Cs}$ & ${ }^{234} \mathrm{U}$ & ${ }^{235} \mathrm{U}$ & ${ }^{238} \mathrm{U}$ & ${ }^{239 .}{ }^{240} \mathrm{Pu}$ \\
\hline & & Site $V 130$ & & Site V120 & Site V119 & Site V120 & Site $V_{123}$ \\
\hline $\begin{array}{l}\text { Maximum } \pm \text { counting } \\
\text { error }(\%)\end{array}$ & ND & $\begin{array}{l}9.6 \mathrm{E}-01 \\
\pm 25 \% \\
\end{array}$ & ND & $\begin{array}{c}3.6 \mathrm{E}-01 \\
\pm 10 \% \\
\end{array}$ & $\begin{array}{c}2.2 \mathrm{E}-02 \\
\pm 36 \% \\
\end{array}$ & $\begin{array}{c}3.3 \mathrm{E}-01 \\
\pm 10 \% \\
\end{array}$ & $\begin{array}{c}4.4 \mathrm{E}-03 \\
\pm 65 \% \\
\end{array}$ \\
\hline Average \pm 2 SEM $(\%)$ & ND & $\begin{array}{r}6.6 \mathrm{E}-01 \\
\pm 59 \% \\
\end{array}$ & ND & $\begin{array}{c}6.9 \mathrm{E}-02 \\
\pm 70 \% \\
\end{array}$ & $\begin{array}{c}1.0 \mathrm{E}-02 \pm \\
33 \%\end{array}$ & $\begin{array}{c}6.2 \mathrm{E}-02 \pm \\
73 \% \\
\end{array}$ & $\begin{array}{c}4.4 \mathrm{E}-03 \pm \\
65 \%\end{array}$ \\
\hline $\begin{array}{l}\text { PNNL offsite average } \\
\pm 2 \text { SEM }(\%)^{a}\end{array}$ & NR & $\begin{array}{c}2.5 \mathrm{E}-\mathrm{O} 2 \\
\pm 48 \%\end{array}$ & $\begin{array}{l}7.2 \mathrm{E}-\mathrm{O} 3 \\
\pm 116 \%\end{array}$ & $\begin{array}{c}1.4 \mathrm{E}-02 \\
\pm 43 \%\end{array}$ & ND & $\begin{array}{c}1.3 \mathrm{E}-02 \\
\pm 31 \%\end{array}$ & $\begin{array}{c}1.8 \text { E-04 } \\
\pm 72 \%\end{array}$ \\
\hline $\begin{array}{l}\text { Accessible soil } \\
\text { concentration limits }\end{array}$ & $7.1 E+\infty$ & $2.8 E+03$ & $3.0 E+01$ & $6.3 E+02$ & $1.7 E+02$ & $3.7 E+02$ & $1.9 \mathrm{E}+\mathrm{O} 2$ \\
\hline
\end{tabular}

2SEM = Two standard error of the mean.

$\mathrm{ND}=$ Not detected.

NR $=$ Not reported.

'Dirkes and Hanf 1998, and Dirkes and Hanf 1995.

4.3.3.4 ${ }^{239,}{ }^{240} \mathrm{Pu}$. The only detectable ${ }^{239},{ }^{240} \mathrm{Pu}$ result was found at Site $\mathrm{V} 123$, located near the 316-3 Building. The ${ }^{239,}{ }^{240} \mathrm{Pu}$ concentration was $4.4 \mathrm{E}-\mathrm{O} 3 \pm 65 \% \mathrm{pCi} / \mathrm{g}$, or 24 times the offsite measured concentration of $1.8 \mathrm{E}-04 \pm 72 \% \mathrm{pCi} / \mathrm{g}$.

4.3.3.5 Uranium. The highest uranium result was found at Site $V 120$, located south of the 316-2 Process Pond. The uranium concentrations were $3.6 \mathrm{E}-01 \pm 10 \% \mathrm{pCi} / \mathrm{g}$ of ${ }^{234} \mathrm{U}, 2.2 \mathrm{E}-02$ $\pm 36 \% \mathrm{pCi} / \mathrm{g}$ of ${ }^{235} \mathrm{U}$, and $3.3 \mathrm{E}-01 \pm 10 \% \mathrm{pCi} / \mathrm{g}$ of ${ }^{238} \mathrm{U}$, or $7.1 \mathrm{E}-01 \mathrm{pCi} / \mathrm{g}$ total isotopic uranium. The total isotopic uranium concentration was 12 times the offsite measured concentration of 2.7 $\mathrm{E}-02 \pm 37 \% \mathrm{pCi} / \mathrm{g}$. The average concentration of total uranium in vegetation in the $300 / 400$ Areas was $1.4 \mathrm{E}-01 \pm 59 \% \mathrm{pCi} / \mathrm{g}$.

\subsection{CONCLUSIONS}

The impact of past and current operations on the near-facility environment, although above the concentrations in distant communities reported by PNNL, were below the accessible soil concentration limits (Appendix $\mathrm{J}$, Table $\mathrm{J}-4$ ). Trending of the average concentrations, contained in the summary (Figures S-2 and S-3), indicate the decrease in concentrations of certain radionuclides.

As the data indicate, the concentrations have a large degree of variance. In general, the samples collected on, or directly adjacent to, the waste disposal facilities had higher concentrations of radionuclides than those further away. The data also show, as expected, that certain radionuclides had higher concentrations within different operating areas. Generally speaking, the predominant radionuclides are: activation and fission products in the 100 Areas, fission products and plutonium in the 200 Areas, and uranium in the 300 Areas. Facility monitoring sites are selected to maximize detection of radionuclides in the near-facility environment and the results do not necessarily represent the entire operating area. 


\section{HNF-EP-0573-6}

This page intentionally left blank.

4-14 


\subsection{EXTERNAL RADIATION MONITORING}

\subsection{INTRODUCTION}

A network of thermoluminescent dosimeters (TLDS) were positioned in and around the 100 , 200 , and $300 / 300$ TEDF/400 Areas to monitor dose rates from external radiation sources (gamma rays). The TLD measurements were taken to determine dose rates in the operations area environment. From these data, the contribution of Hanford Site activities to the dose rates in these areas can be discerned. Figures showing these sites and the TLD results are in Appendix F.

The environmental TLDs measure dose rates from all types of external radiation sources. These include cosmic radiation, naturally occurring radioactivity in air and soil, and fallout from nuclear weapons testing, as well as any contribution from Hanford Site activities. These outside radiation sources cause an estimated $\pm 20 \%$ deviation in TLD analyses. The results are reported in units of millirems per year (mrem/yr).

The Hanford Site uses the Harshaw TLD system which includes the Harshaw 8807 dosimeter and the Harshaw 8800 TLD reader. The TLD reader has a better signal-to-noise ratio than those used in the past. The TLD packaging, which uses an "O ring" seal, protects the TLDs from light, heat, moisture, and dirt. The TLDs were placed $1 \mathrm{~m}(3 \mathrm{ft})$ aboveground at each location. The TLDs were placed near active and inactive surface-water disposal sites and near facilities (tank farms, active cribs, and the facility fence line), and near environmental restoration activities. Changing conditions in the vicinity of the TLD sample locations, such as remediation activities, removal, or storage of radioactive material, and tank farm operations may also cause fluctuations in TLD analyses over time. The TLDs were exchanged and analyzed each calendar quarter. The PNNL Radiation Calibration Laboratory has calibrated the response of the chips; results were reported in terms of external dose (mrem).

\subsection{RESULTS}

\subsubsection{Area}

5.2.1.1 $100 \mathrm{~B} / \mathrm{C}$. Four new TLD monitoring sites were established in the $100 \mathrm{~B} / \mathrm{C}$ Area during the fourth quarter of 1997 to evaluate environmental restoration activities. Because only 27 days of data were collected at these sites during 1997, the TLD results were extrapolated to one year, resuiting in an average of $93 \mathrm{mrem} / \mathrm{yr}$, which is comparable to offsite ambient background levels.

5.2.1.2 100 D/DR. This is the second year that thermoluminescent dosimeters have been placed in the $100 \mathrm{D} / \mathrm{DR}$ Area to evaluate environmental restoration activities. Dose rates measured at these locations in 1997 were similar to the 1996 results, with an average of $88 \mathrm{mrem} / \mathrm{yr}$, which is comparable to offsite ambient background levels. Comparative data from 1997 and 1996 are provided in Table 5-1. 
Table 5-1. $100 \mathrm{D} / \mathrm{DR}$ Area Thermoluminescent

Dosimeter Rẹults (mrem/yr).

\begin{tabular}{|l|c|c|c|}
\hline \multicolumn{1}{|c|}{ Site I.D. } & 1996 average & 1997 average & $\begin{array}{c}97 \text { vs } 96 \\
\% \text { change }\end{array}$ \\
\hline 100-D TLD 001 & $9.0 E+01$ & $8.7 E+01$ & -4 \\
\hline 100-D TLD 002 & $8.3 E+01$ & $8.1 E+01$ & -2 \\
\hline 100-D TLD 003 & $8.7 E+01$ & $9.2 E+01$ & 6 \\
\hline 100-D TLD 004 & $9.2 E+01$ & $9.1 E+01$ & -1 \\
\hline 100-D TLD 005 & $8.9 E+01$ & $9.0 E+01$ & 1 \\
\hline Average & $8.8 E+01$ & $8.8 E+01$ & 0 \\
\hline
\end{tabular}

5.2.1.3 100 K Area. This is the fifth year of external radiation monitoring at the $100 \mathrm{~K}$ Area. Dose rates observed were generally at or near typical Hanford Site background levels with a few exceptions. The largest increase was at site K-8 at $105 \mathrm{~K}$ East. Low-level, radioactive wastes awaiting shipment were intermittently staged near this site during 1997. Comparative data from 1997 and 1996 are provided in Table 5-2.

5.2.1.4 $100 \mathrm{~N}$. The $1997 \mathrm{TLD}$ results indicate that the DOE exposure guidelines for nonradiation area workers (100 mrem/yr) were not exceeded in any nonradiologically controlled area except near the N Springs shoreline. Reasons for this elevated radiation level are discussed later in this section. .The overall trend is that dose rates at most locations continue to decrease.

Direct radiation levels were predictably higher near facilities that had contained or received liquid effluents from $\mathrm{N}$ Reactor or where mixed (radiological and hazardous) waste was stored. These facilities included the 1301-N LWDF, the 1325-N LWDF, the 1304-N emergency dump tank, and 116-N-8 (the 163-N Mixed Waste and Hazardous Waste Container Storage Pad).

Elevated readings were observed at TLD Sites $14,18,28$, and 29 which were near locations where radioactive waste were temporarily stored.

Readings were markedly decreased at TLD Site 17 near the 1330-N Waste Storage Pad where approximately 100 boxes of radioactive waste were removed during 1997.

The 1997 average environmental dose rates measured near the 1301-N LWDF and the $1325-N$ LWDF were approximately $17 \%$ and $16 \%$ lower, respectively, than those measured in 1996. This decline represents a trend of decreased dose rates at these facilities attributable to the continuing decay of ${ }^{60} \mathrm{Co}(5.3$-year half life).

Dose rates were measured at the $\mathrm{N}$ Springs shoreline to determine potential external radiation doses to the public as well as to a Hanford Site worker. Because of the "skyshine" effect from the 1301-N LWDF, dose rates at the $\mathrm{N}$ Springs were elevated above $100 \mathrm{mrem} / \mathrm{yr}$, the DOE annual external dose limit to members of the public. However, neither a member of the public nor a Hanford worker would conceivably spend an entire year at the N Springs; therefore, the values shown in Figure 5-1 are for comparison only. N Springs dose reduction measures are currently being studied.

The TLD measurements from the $100 \mathrm{~N}$ Area for 1987 through 1997 are shown in Figure 5-2. Comparative data from 1997 and 1996 are provided in Table 5-2. 
Table 5-2. $100 \mathrm{~N}$ and $100 \mathrm{KE} / \mathrm{KW}$ Areas Thermoluminescent Dosimeter Results (mrem/yr).

\begin{tabular}{|c|c|c|c|}
\hline $\begin{array}{c}100 \text { N Area } \\
\text { site I.D. }\end{array}$ & $\begin{array}{c}1996 \\
\text { average }\end{array}$ & $\begin{array}{c}1997 \\
\text { average }\end{array}$ & $\begin{array}{l}97 \text { vs } 96 \\
\% \text { change }\end{array}$ \\
\hline 1 & $8.3 E+02$ & $7.2 \mathrm{E}+02$ & -13 \\
\hline 2 & $8.3 E+02$ & $7.2 \mathrm{E}+02$ & -13 \\
\hline 3 & $3.7 E+02$ & $3.3 E+02$ & -11 \\
\hline 4 & $1.7 E+03$ & $1.2 \mathrm{E}+03$ & -29 \\
\hline 5 & $5.6 \mathrm{E}+02$ & $4.4 \mathrm{E}+\mathrm{O} 2$ & -21 \\
\hline 6 & $2.3 E+02$ & $2.0 \mathrm{E}+02$ & -13 \\
\hline 7 & $4.0 E+02$ & $3.4 \mathrm{E}+02$ & -15 \\
\hline 14 & $3.6 E+02$ & $5.5 \mathbf{E}+02$ & 54 \\
\hline 16 & $2.9 E+02$ & $3.0 E+02$ & 2 \\
\hline 17 & $2.2 E+02$ & $8.5 E+01$ & -61 \\
\hline 18 & $7.0 E+02$ & $4.5 E+02$ & -33 \\
\hline 22 & $4.3 E+02$ & $3.8 \mathrm{E}+\mathrm{O} 2$ & -13 \\
\hline 26 & $2.1 E+02$ & $1.9 E+02$ & -10 \\
\hline 28 & $1.9 E+02$ & $2.3 E+02$ & 22 \\
\hline 29 & $6.7 E+01$ & $7.0 E+02$ & 4 \\
\hline 30 & $5.4 E+03$ & $4.5 E+03$ & -17 \\
\hline 31 & $4.2 E+02$ & $3.5 E+02$ & -18 \\
\hline 32 & $3.0 E+02$ & $2.8 E+02$ & -7 \\
\hline 34 & $9.6 E+03$ & $7.7 E+03$ & -17 \\
\hline 35 & $8.8 E+03$ & $7.6 E+03$ & -14 \\
\hline 37 & $3.3 E+02$ & $2.9 E+02$ & -11 \\
\hline 41 & $3.1 E+02$ & $2.8 E+02$ & -11 \\
\hline Average & $1.5 E+03$ & $1.3 E+03$ & -15 \\
\hline
\end{tabular}

\begin{tabular}{|c|c|}
\hline $\begin{array}{c}1301-\mathrm{N} \\
\text { site I.D. }\end{array}$ & $\begin{array}{c}97 \text { vs } 96 \\
\% \text { change }\end{array}$ \\
\hline 1 & -13 \\
\hline 2 & -13 \\
\hline 3 & -11 \\
\hline 4 & -29 \\
\hline 5 & -21 \\
\hline 6 & -13 \\
\hline 18 & -33 \\
\hline 22 & -13 \\
\hline 32 & -7 \\
\hline Average & -17 \\
\hline
\end{tabular}

\begin{tabular}{|c|c|}
\hline $\begin{array}{c}\text { 1325-N } \\
\text { site I.D. }\end{array}$ & $\begin{array}{c}97 \text { vs } 96 \\
\% \text { change }\end{array}$ \\
\hline 30 & -17 \\
\hline 34 & -17 \\
\hline 35 & -14 \\
\hline Average & -16 \\
\hline
\end{tabular}

\begin{tabular}{|c|c|}
\hline $\begin{array}{c}100 \mathrm{~K} \\
\text { site I.D. }\end{array}$ & $\begin{array}{c}97 \text { vs } 96 \\
\% \text { change }\end{array}$ \\
\hline 1 & -2 \\
\hline 2 & 0 \\
\hline 3 & -7 \\
\hline 4 & -21 \\
\hline 5 & -12 \\
\hline 6 & -2 \\
\hline 7 & -12 \\
\hline 8 & 94 \\
\hline 9 & 0 \\
\hline 10 & -20 \\
\hline 11 & -3 \\
\hline Average & -2 \\
\hline
\end{tabular}


Figure 5-1. Annual Average Dose Rate at N Springs.

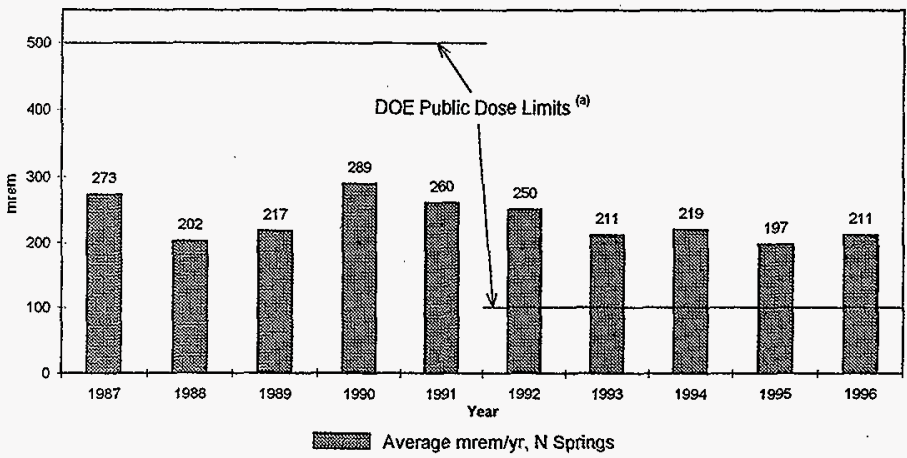

Note: DOE Limits were reduced from $500 \mathrm{mrem} / \mathrm{yr}$ in 1992 . The lower value was selected in recognition of the International Commission of Radiation Protection (ICRP) recommendation to limit the long-term average effective dose equivalence to 100 mrem (1 mSv) per year or less. (DOE Order 5400.5).

Figure 5-2. Comparison of Thermoluminescent Dosimeter Results at $100 \mathrm{~N}$ Area.

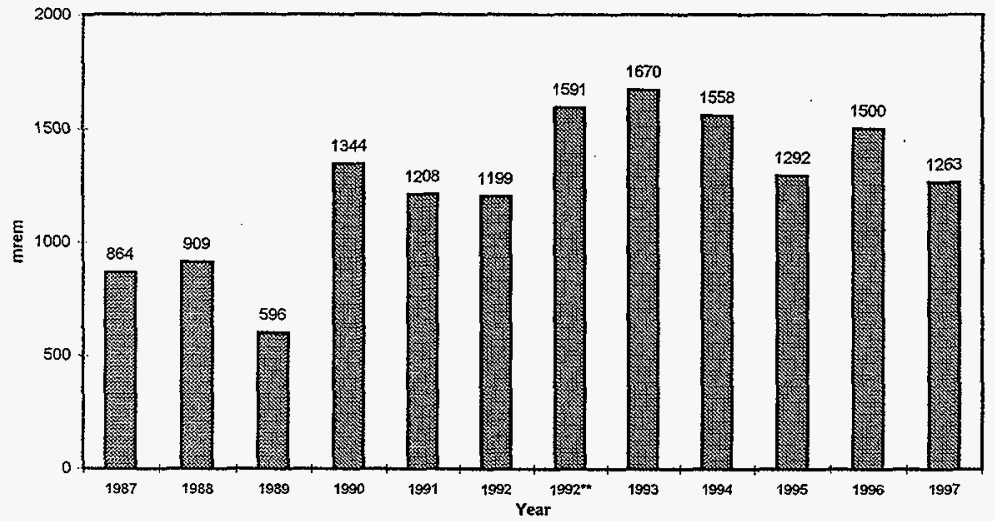




\subsubsection{Areas}

The 1997 TLD results for the 200 Areas indicated that the DOE exposure guidelines for nonradiation area workers $(100 \mathrm{mrem} / \mathrm{yr})$ were not exceeded in any nonradiologically controlled areas. Figure 5-3 shows the external radiation monitoring results for 1987 through 1997 .

Results indicated that operations in the 200 Areas did not contribute significantly to the external radiation dose rates of the general environment. The overall annual dose rate in the 200 Areas showed a $8 \%$ decrease from 1996 to 1997 . The exposure rate in the general 200 Areas environment was not significantly different from the exposure rate received offsite from natural radiation sources. As expected, external radiation levels were elevated at certain sites, radiological control areas, and facilities, reflecting the proximity to radioactive waste management activities. TLDs are positioned to measure facilities of higher impact as was the case in 1989 when the average annual dose rate increased from 106 to $241 \mathrm{mrem}$ per year. TLDs were removed from the grid configuration, where many were located in remote areas, to a configuration closer to operations facilities. Comparative data from 1997 and 1996 are provided in Table 5-3.

Figure 5-3. Comparison of Thermoluminescent Dosimeter Results at the 200 Areas.

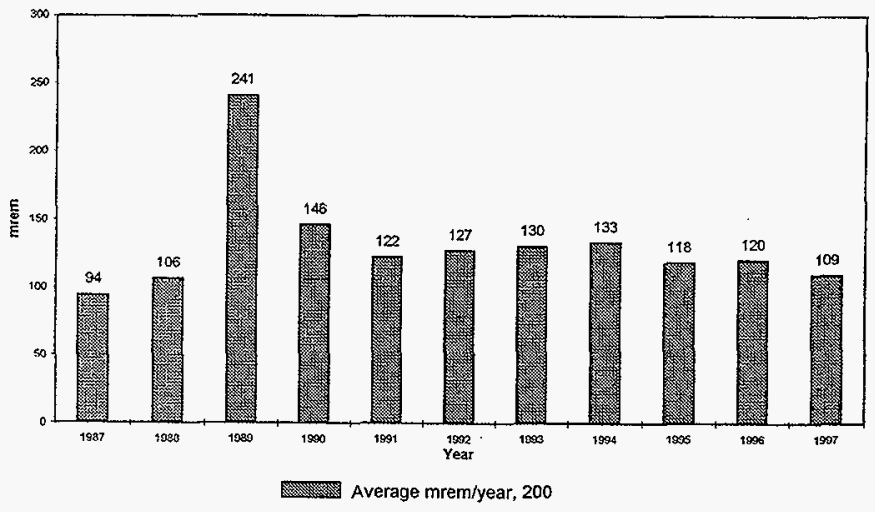


Table 5-3. 200 Areas Thermoluminescent Dosimeter Results (mrem/yr).

\begin{tabular}{|c|c|c|c|c|c|c|c|}
\hline Site I.D. & 1996 average & 1997 average & $\%$ change & Site I.D. & 1996 average & 1997 average & $\%$ change \\
\hline 202 & $9.0 \mathrm{E}+01$ & $9.1 E+01$ & 0 & 235 & $9.4 \mathrm{E}+01$ & $8.9 \mathrm{E}+01$ & 6 \\
\hline 203 & $1.0 E+02$ & $1.0 \mathrm{E}+\mathrm{O} 2$ & 3 & 236 & $9.8 E+01$ & $1.0 E+02$ & -5 \\
\hline 204 & $1.4 E+02$ & $1.4 E+02$ & 0 & 237 & $7.9 \mathrm{E}+01$ & $8.3 E+01$ & -4 \\
\hline 205 & $8.5 E+01$ & $8.5 E+01$ & 0 & 238 & $8.7 E+01$ & $9.5 E+01$ & -9 \\
\hline 206 & $1.1 E+02$ & $1.1 E+02$ & 1 & 239 & $9.0 E+01$ & $9.3 E+01$ & -2 \\
\hline 207 & $9.8 \mathrm{E}+01$ & $9.9 \mathrm{E}+01$ & -1 & 240 & $8.0 E+01$ & $8.2 E+01$ & -3 \\
\hline 208 & $1.3 E+02$ & $1.6 \mathrm{E}+02$ & -19 & 241 & $9.1 E+01$ & $9.3 E+01$ & -1 \\
\hline 209 & $8.8 E+01$ & $8.9 E+01$ & -1 & 242 & $8.7 E+01$ & $9.4 E+01$ & -7 \\
\hline 210 & $9.9 E+01$ & $1.0 \mathrm{E}+\mathrm{O} 2$ & -1 & 243 & $8.3 E+01$ & $8.5 E+07$ & -3 \\
\hline 211 & $9.5 E+01$ & $1.0 \mathrm{E}+02$ & -9 & 244 & $8.9 \mathrm{E}+01$ & $1.3 E+02$ & -30 \\
\hline 212 & $1.3 E+02$ & $1.2 E+02$ & 7 & 245 & $8.3 E+01$ & $8.4 E+01$ & -1 \\
\hline 213 & $1.9 E+02$ & $2.0 \mathrm{E}+02$ & -4 & 246 & $8.3 E+01$ & $8.5 E+01$ & -2 \\
\hline 214 & 8.0 E+01 & $8.2 E+01$ & -3 & 247 & $8.3 E+01$ & $8.5 E+01$ & -3 \\
\hline 215 & $9.2 E+01$ & $9.3 E+01$ & -2 & 249 & $1.1 E+02$ & $1.2 \mathrm{E}+\mathrm{O} 2$ & -10 \\
\hline 216 & $9.2 \mathrm{E}+01$ & $1.0 \mathrm{E}+\mathrm{O} 2$ & -9 & 250 & $2.3 E+02$ & $2.0 \mathrm{E}+\mathrm{O} 2$ & 15 \\
\hline 217 & $8.6 E+01$ & $8.6 \mathrm{E}+01$ & 0 & 251 & $1.4 E+02$ & $1.5 \mathrm{E}+02$ & -9 \\
\hline 218 & $7.7 E+01$ & $8.2 E+01$ & -6 & 252 & $1.0 E+02$ & $1.2 \mathrm{E}+02$ & -16 \\
\hline 219 & $9.2 E+01$ & $9.5 E+01$ & -3 & 253 & $1.0 E+02$ & $1.2 E+02$ & -17 \\
\hline 220 & $9.4 E+01$ & $9.6 E+01$ & -2 & 254 & $1.5 E+02$ & $2.0 E+02$ & -26 \\
\hline 221 & $8.9 E+01$ & $9.3 E+01$ & -4 & 255 & $1.3 E+02$ & $1.6 \mathrm{E}+\mathrm{O} 2$ & -15 \\
\hline 222 & $1.2 E+02$ & $1.2 \mathrm{E}+02$ & -1 & 256 & $3.0 \mathrm{E}+02$ & $3.6 E+02$ & -16 \\
\hline 223 & $1.4 E+02$ & $1.4 E+02$ & -3 & 258 & $3.5 E+02$ & $4.9 E+02$ & -30 \\
\hline 224 & $8.6 E+01$ & $9.0 E+01$ & -5 & 259 & $1.1 E+02$ & $1.8 \mathrm{E}+\mathrm{O} 2$ & -39 \\
\hline 226 & $8.8 \mathrm{E}+01$ & $8.9 E+01$ & -1 & 260 & $8.1 \mathrm{E}+01$ & $8.1 \mathrm{E}+01$ & 1 \\
\hline 227 & $8.4 E+01$ & $8.6 E+01$ & -2 & 261 & $9.5 E+01$ & $1.0 \mathrm{E}+\mathrm{O} 2$ & -8 \\
\hline 228 & $8.5 E+01$ & $8.6 E+01$ & -1 & 262 & $8.0 \mathrm{E}+01$ & $8.4 E+01$ & -5 \\
\hline 229 & $9.2 E+01$ & $9.3 E+01$ & -2 & 263 & $7.9 E+01$ & $8.1 E+01$ & -2 \\
\hline 230 & $1.2 E+02$ & $1.2 E+02$ & -5 & 264 & $8.7 E+01$ & $9.0 E+01$ & -4 \\
\hline 231 & $9.5 E+01$ & $9.4 E+01$ & 1 & 267 & $9.2 \mathrm{E}+01$ & $8.9 E+01$ & 4 \\
\hline 232 & $1.4 E+02$ & $1.5 \mathrm{E}+02$ & -7 & 268 & $9.4 \mathrm{E}+01$ & $1.2 \mathrm{E}+02$ & -20 \\
\hline 233 & $9.1 E+01$ & $9.1 E+01$ & 0 & 269 & $1.0 \mathrm{E}+02$ & $9.7 E+01$ & 6 \\
\hline 234 & $1.0 E+02$ & $1.1 E+02$ & -8 & All & $1.2 E+02$ & $1.1 E+02$ & -8 \\
\hline
\end{tabular}




\subsubsection{Tank Waste Remediation System Phase I Demonstration Project}

Ten new thermoluminescent dosimeter locations were established around the perimeter of the Tank Waste Remediation System (TWRS) Phase I Demonstration Project Site during the fourth quarter of 1997 to collect preoperational monitoring data. Because only 67 days of data were collected at these sites during 1997, the thermoluminescent dosimeter results were extrapolated to one year, resulting in an average of $78 \mathrm{mrem} / \mathrm{vr}$, which is comparable to offsite ambient background levels. These results are provided in Table 5-4.

Table 5-4. Tank Waste Remediation System Phase I Demonstration Project Thermoluminescent Dosimeter Results (mrem/yr).

\begin{tabular}{|l|l|c|}
\hline \multicolumn{1}{|c|}{ TWRS site I.D. } & \multicolumn{1}{|c|}{ Location description } & \multicolumn{1}{c|}{ mrem/yr } \\
\hline TWRS S 001 & South side of TWRS Phase I Site & $7.7 \mathrm{E}+01$ \\
\hline TWRS S 002 & South side of TWRS Phase I Site & $7.7 \mathrm{E}+01$ \\
\hline TWRS S 003 & South side of TWRS Phase I Site & $7.6 \mathrm{E}+01$ \\
\hline TWRS S 004 & South side of TWRS Phase I Site & $7.6 \mathrm{E}+01$ \\
\hline TWRS N 001 & North side of TWRS Phase I Site & $8.0 \mathrm{E}+01$ \\
\hline TWRS N 002 & North side of TWRS Phase I Site & $7.5 \mathrm{E}+01$ \\
\hline TWRS N 003 & North side of TWRS Phase I Site & $7.9 \mathrm{E}+01$ \\
\hline TWRS E 001 & East side of TWRS Phase I Site & $8.1 \mathrm{E}+01$ \\
\hline TWRS E 002 & East side of TWRS Phase I Site & $7.8 \mathrm{E}+01$ \\
\hline TWRS E 003 & East side of TWRS Phase I Site & $7.7 \mathrm{E}+01$ \\
\hline
\end{tabular}

TWRS = Tank Waste Remediation System.

\subsubsection{Environmental Restoration Disposal Facility}

This is the second year that thermoluminescent dosimeters have been placed in the ERDF to evaluate environmental restoration disposal activities. Dose rates measured at these locations were slightly lower than the results of 1996 TLD analyses with an average of $95 \mathrm{mrem} / \mathrm{year}$, which is comparable to offsite ambient background levels. Comparative data from 1997 and 1996 are provided in Table 5-5.

Table 5-5. Environmental Restoration Disposal Facility

Thermoluminescent Dosimeter Results (mrem/yr).

\begin{tabular}{|l|c|c|c|}
\hline \multicolumn{1}{|c|}{ ERDF site I.D. } & 1996 average & 1997 average & $\begin{array}{c}97 \text { vs } 96 \\
\% \text { change }\end{array}$ \\
\hline ERDF-1 & $1.0 \mathrm{E}+02$ & $1.0 \mathrm{E}+02$ & 0 \\
\hline ERDF-2 & $1.0 \mathrm{E}+02$ & $9.0 \mathrm{E}+01$ & -11 \\
\hline ERDF-3 & $\mathrm{NA}$ & $9.2 \mathrm{E}+01$ & $\mathrm{NA}$ \\
\hline Average & $1.0 \mathrm{E}+02$ & $9.7 \mathrm{E}+01^{\circ}$ & $-5^{\circ}$ \\
\hline
\end{tabular}

ERDF $=$ Environmental Restoration Disposal Facility.

"Only TLDs in service for both 1996 and 1997 were used for these calculations. 


\subsubsection{0/300 TEDF/400 Areas}

This was the fifth year of external radiation monitoring in the $300 / 400$ Areas and the fourth year of external radiation monitoring at the 300 TEDF. Figure 5-4 shows the external radiation monitoring results for 1991 through 1997. The 1997 TLD results for the 300/300 TEDF/400 Areas indicate that the DOE exposure guidelines were not exceeded in any nonradiologically controlled area.

Comparing the results of external monitoring for 1997 and 1996 indicates that the $300 / 400$ Areas did not contribute significantly to the external radiation dose rates of the general environment. Consequently, the dose rate in the general 300/300 TEDF/400 Areas environment was not significantly different from the dose rate received offsite from natural radiation sources. The overall annual dose rate in the $300 / 300$ TEDF/400 Areas showed decreases of $8 \%, 1 \%$, and $2 \%$, respectively. Comparative data from 1997 and 1996 are provided in Table 5-6.

Figure 5-4. Comparison of Thermoluminescent Dosimeter Results at the $300 / 300$ TEDF/400 Areas.

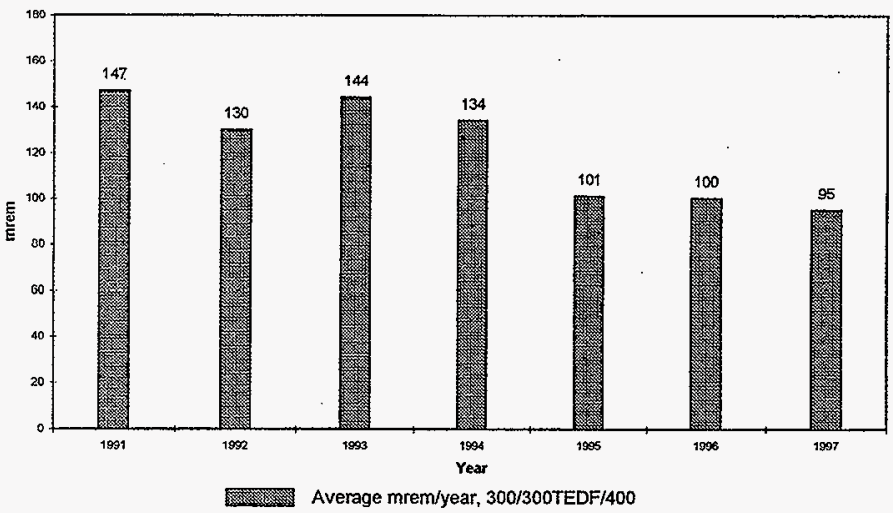


Table 5-6. 300/300 TEDF/400 Areas Thermoluminescent Dosimeter Results (mrem/yr).

\begin{tabular}{|c|c|c|c|c|}
\hline & Site I.D. & 1996 Average & 1997 Average & $\begin{array}{l}97 \text { vs } 96 \\
\% \text { Change }\end{array}$ \\
\hline \multirow{9}{*}{300 Area } & 1 & $8.5 E+01$ & $8.3 \mathrm{E}+01^{\circ}$ & $-2 \%$ \\
\hline & 2 & $9.1 E+01$ & $8.8 E+01$ & $-4 \%$ \\
\hline & 3 & $8.6 \mathrm{E}+01$ & $8.4 \mathrm{E}+01$ & $-2 \%$ \\
\hline & 4 & $2.4 E+02$ & $2.0 \mathrm{E}+\mathrm{O} 2$ & $-15 \%$ \\
\hline & 5 & $8.7 E+01$ & $8.6 E+01$ & $-1 \%$ \\
\hline & 6 & $1.0 \mathrm{E}+\mathrm{O} 2$ & $1.0 \mathrm{E}+02$ & $-2 \%$ \\
\hline & 7 & $2.1 E+02$ & $1.7 E+02$ & $-18 \%$ \\
\hline & 8 & $1.0 \mathrm{E}+02$ & $1.0 \mathrm{E}+02$ & $2 \%$ \\
\hline & All & $1.2 \mathrm{E}+\mathrm{O} 2$ & $1.1 E+02$ & $-8 \%$ \\
\hline \multirow{7}{*}{300 TEDF } & 301 & $8.5 \mathrm{E}+01$ & $8.8 E+01$ & $4 \%$ \\
\hline & 302 & 8.3E+01 & $8.4 E+01$ & $1 \%$ \\
\hline & 303 & $8.7 E+01$ & $8.8 E+01$ & $1 \%$ \\
\hline & 304 & $8.6 \mathrm{E}+01$ & $8.4 E+01$ & $-3 \%$ \\
\hline & 305 & $8.3 E+01$ & $8.2 E+01$ & $-2 \%$ \\
\hline & 306 & $8.6 \mathrm{E}+01$ & $8.8 E+01$ & $2 \%$ \\
\hline & All & $9.1 E+01$ & $9.0 E+01$ & $-1 \%$ \\
\hline \multirow{8}{*}{400 Area } & 1 & $8.3 E+01$ & $8.2 \mathrm{E}+01$ & $-1 \%$ \\
\hline & 2. & $7.9 E+01$ & $8.1 E+01$ & $2 \%$ \\
\hline & 3 & $9.2 E+01$ & $8.7 E+01$ & $-6 \%$ \\
\hline & 4 & $8.1 E+01$ & $7.9 \mathrm{E}+01$ & $-2 \%$ \\
\hline & 5 & $8.1 E+01$ & $7.9 \mathrm{E}+01$ & $-2 \%$ \\
\hline & 6 & $8.3 E+01$ & $8.3 E+01$ & $0 \%$ \\
\hline & 7 & $8.2 \mathrm{E}+01$ & $8.1 \mathrm{E}+01$ & $0 \%$ \\
\hline & A!I & $8.3 E+01$ & $8.2 \mathrm{E}+01$ & $-2 \%$ \\
\hline
\end{tabular}

TEDF = Treated Effluent Disposal Facility. 
HNF-EP-0573-6

This page intentionally left blank. 
HNF-EP-0573-6

\subsection{SURFACE WATER DISPOSAL UNITS AND SEEPAGES}

\subsection{INTRODUCTION}

Water, aquatic vegetation, and sediment samples were collected from the active ponds and ditches and the N Springs in 1997. Springs, ponds, and ditches in the 100,200, and $300 / 400$ Areas receive or have received waste water from the chemical and waste processing plants, reactors, and other facilities. All water was sampled at the point of discharge to ensure compliance with internal contractor standards and applicable DOE standards. As an additional operational check, water samples are collected from the ponds and ditches. Sampling locations and analytical results are shown in Appendix G.

One-liter water samples were collected weekly from the active ponds and ditches. The $\mathrm{pH}$ was determined each week, then the samples were composited and analyzed monthly for gamma-emitting radionuclides, ${ }^{238} \mathrm{Pu},{ }^{239},{ }^{240} \mathrm{Pu},{ }^{90} \mathrm{Sr}$, and uranium. In addition, a $1 \mathrm{~L}$ sample was collected quarterly from each site for nitrate and ${ }^{3} \mathrm{H}$ analyses. Samples of aquatic vegetation were collected from ponds and ditches yearly to determine root uptake of radionuclides from potentially contaminated sediments. Along with aquatic vegetation samples, surface sediment samples were collected at ponds and ditches to measure the accumulation of radionuclides. Surface sediment samples consisted of a composite of five plugs, each $10 \mathrm{~cm}$ (4 in.) in diameter by $2.5 \mathrm{~cm}$ $\left(1 \mathrm{in.}\right.$ ) deep. The vegetation and sediments were analyzed for gamma-emitting radionuclides, ${ }^{90} \mathrm{Sr}$, ${ }^{239}, 240 \mathrm{Pu}$, and uranium.

\subsection{RESULTS}

\subsubsection{Water}

6.2.1.1 100 Areas. Seepage weils along the $100 \mathrm{~N}$ Area shoreline (N Springs) are sampled annually to verify that the reported radionuclide releases to the Columbia River via the $\mathrm{N}$ Springs are credible. After characterizing the radionuclide concentrations in the seep wells, the results are compared to the radionuclide concentrations measured in the effluent monitoring weil (199-N-46) during the time period that included the seepage well sample collection. Historicaliy, the highest concentrations of radionuclides have been shown to be in the area of the effluent monitoring well. Release reporting uses the conservatively based radionuclide concentrations as measured in the effluent monitoring well, multiplied by the estimated groundwater flow into the river, to thereby provide conservatively high release values.

With the shutdown of $N$ Reactor in January 1987 and the resultant decreased effluent flow to the 1325-N LWDF, concentrations of most of the radionuclides that were typically present in the $\mathrm{N}$ Springs discharge during reactor operation have significantly decreased. The noteworthy exception to this has been and continues to be ${ }^{50} \mathrm{Sr}$.

The shoreline seepage well samples were collected using a bailer, carefully lowered into each weil water column to avoid sediment suspension. A $4 \mathrm{~L}$ sample was collected. Eleven samples were collected on November 14, 1997. At the time of sample collection, four sampling locations (Wells 9, 10, 12 and 13) were either immersed or partially immersed in, and therefore diluted by, the river. No samples were collected at these locations.

The ${ }^{90} \mathrm{Sr}$ concentration exceeded the DOE DCG value at seepage well $\mathrm{Y} 303$, the sample location nearest the effluent monitoring well. Tritium was detected in only one seepage well sample, location $Y 303$. The tritium concentration at seepage well $Y 303$ was well below the DCG value. 
The results obtained from the 1997 characterization indicated that the seepage wells with the highest concentrations of both tritium and ${ }^{90} \mathrm{Sr}$ were near the effluent monitoring well, signifying that this well continues to monitor $N$ Springs discharge in the region that provides conservatively high readings.

Tables 6-1 and 6-2 shows the historic concentrations for tritium and ${ }^{90} \mathrm{Sr}$ from samples collected from the N Springs shoreline wells, including effluent monitoring well 199-N-46. Because of river fluctuations and groundwater discharge rates, some wells may not have been sampled; results from those wells are not shown. Analytical results are shown in Appendix G, Table G-1.

Table 6-1. N Springs Shoreline Tritium Concentrations, 1987 to 1997 (pCi/L).

\begin{tabular}{|c|c|c|c|c|c|c|c|}
\hline Year & Site Y301 & Site $Y 302$ & $\begin{array}{c}\text { Effluent } \\
\text { monitoring well } \\
\end{array}$ & Site Y303 & Site Y304 & Site Y305 & Site Y306 \\
\hline 1987 & $6.8 E+04$ & $7.6 E+04$ & $9.5 E+04$ & $9.2 \mathrm{E}+04$ & $9.4 \mathrm{E}+04$ & $8.8 E+04$ & $7.9 E+04$ \\
\hline 1988 & $5.7 E+03$ & $2.8 E+04$ & $7.5 E+04$ & $6.9 \mathrm{E}+04$ & $7.4 \mathrm{E}+04$ & $\mathrm{NR}$ & NR \\
\hline 1989 & $2.5 E+04$ & $2.8 E+04$ & $3.9 \mathrm{E}+04$ & $3.6 \mathrm{E}+04$ & $5.0 \mathrm{E}+04$ & NR & $6.8 E+04$ \\
\hline 1990 & $2.9 \mathrm{E}+04$ & $3.2 \mathrm{E}+04$ & $3.8 E+04$ & $3.6 \mathrm{E}+04$ & NR & NR & $3.4 E+03$ \\
\hline 1991 & $2.2 \mathrm{E}+\mathrm{O} 2$ & $8.4 \mathrm{E}+01$ & 3.7 E+ 04 & $2.6 \mathrm{E}+03$ & $3.4 \mathrm{E}+04$ & NR & $4.0 \mathrm{E}+02$ \\
\hline 1992 & $7.2 \mathrm{E}+\mathrm{O} 2$ & NR & $5.0 E+04$ & $9.5 \mathrm{E}-01$ & NR & NR & $1.5 \mathrm{E}+\mathrm{O} 2$ \\
\hline 1993 & $2.8 E+02$ & $1.3 E+02$ & $2.7 E+04$ & $1.4 E+02$ & $5.6 E+02$ & $1.0 \mathrm{E}+02$ & $1.8 \mathrm{E}+02$ \\
\hline 1994 & NR & NR & $2.6 E+04$ & $4.0 E+01$ & NR & NR & $1.2 \mathrm{E}+02$ \\
\hline 1995 & NR & NR & $5.2 \mathrm{E}+03$ & $<-1.2$ & NR & NR & $<-4.4 E+01$ \\
\hline 1996 & $2.5 E+02$ & $8.5 E+02$ & $2.0 E+04$ & $1.6 \mathrm{E}+04$ & $4.2 E+03$ & $1.6 \mathrm{E}+02$ & $2.2 \mathrm{E}+02$ \\
\hline 1997 & ND & ND & $1.6 \mathrm{E}+04$ & $3.0 \mathrm{E}+03$ & ND & ND & ND \\
\hline Year & Site $Y 307$ & Site $Y 308$ & Site Y309 & Site $Y 310$ & Site Y311 & Site $Y 312$ & Site $Y 313$ \\
\hline 1987 & $7.3 \mathrm{E}+04$ & $4.6 \mathrm{E}+04$ & $7.5 E+04$ & $4.0 E+03$ & $5.8 E+04$ & $2.1 E+04$ & $1.3 E+03$ \\
\hline 1988 & $1.1 \mathrm{E}+04$ & $3.0 E+04$ & $1.0 \mathrm{E}+04$ & NR & $2.9 E+04$ & $1.9 E+04$ & $3.0 \mathrm{E}+03$ \\
\hline 1989 & NR & $7.7 E+04$ & $7.0 E+04$ & $3.5 E+04$ & $4.2 E+04$ & NR & NR \\
\hline 1990 & NR & $1.4 \mathrm{E}+04$ & $3.5 E+03$ & $9.7 E+03$ & $3.8 E+04$ & $2.0 E+04$ & $\mathrm{NR}$ \\
\hline 1991 & $8.1 E+02$ & $2.1 E+03$ & $6.5 \mathrm{E}+03$ & $7.9 E+02$ & $7.1 \mathrm{E}+\mathrm{O} 2$ & $2.4 E+03$ & $9.3 \mathrm{E}+00$ \\
\hline 1992 & NR & NR & $3.0 E+02$ & $4.3 \mathrm{E}+\mathrm{O} 2$ & $6.5 E+02$ & $1.7 E+02$ & NR \\
\hline 1993 & NR & NR & NR & NR & NR & NR & NR \\
\hline 1994 & $8.5 E+01$ & $1.3 E+02$ & $8.4 E+01$ & $4.0 E+02$ & $4.5 E+02$ & $2.9 E+02$ & NR \\
\hline 1995 & $<-2.1$ & $<-2.3$ & $<-3.1 E+07$ & $<-1.2$ & $3.2 \mathrm{E}+\mathrm{O} 2$ & $5.0 E+02$ & NR \\
\hline 1996 & $1.9 \mathrm{E}+\mathrm{O} 2$ & $2.4 \mathrm{E}+\mathrm{O} 2$ & NR & $\mathrm{NR}$ & $2.2 \mathrm{E}+\mathrm{O} 2$ & NR & NR \\
\hline 1997 & ND & ND & ND & ND & ND & $\mathrm{NR}$ & NR \\
\hline
\end{tabular}

NR $=$ Not reported (sample not collected or no analytical results provided).

ND $=$ Not detected (less than overall counting error, or no peak detected, or results less than zero).

$<=$ Analytical results were less than the overall counting error.

Negative value indicates results at or near background levels of radioactivity. 
Table 6-2. N Springs Shoreline ${ }^{90} \mathrm{Sr}$ Concentrations, 1987 to 1997 (pCi/L).

\begin{tabular}{|c|c|c|c|c|c|c|c|}
\hline Year & Site $Y 301$ & Site $Y_{302}$ & $\begin{array}{c}\text { Effluent } \\
\text { monitoring well }\end{array}$ & Site $Y 303$ & Site $Y 304$ & Site Y305 & Site Y306 \\
\hline 1987 & $1.7 E+03$ & $2.7 E+03$ & $6.1 E+03$ & $8.3 E+03$ & $4.1 \mathrm{E}+\mathrm{O} 3$ & $9.5 \mathrm{E}+02$ & $7.2 \mathrm{E}+02$ \\
\hline 1988 & $8.7 E+02$ & $3.0 \mathrm{E}+03$ & $7.9 E+03$ & $9.1 E+03$ & $3.5 E+03$ & NR & NR \\
\hline 1989 & $9.8 E+02$ & $2.1 \mathrm{E}+\mathrm{O} 3$ & $6.5 E+03$ & $5.4 E+03$ & $3.8 E+03$ & NR & $8.9 \mathrm{E}+02$ \\
\hline 1990 & $2.4 E+03$ & $2.9 \mathrm{E}+03$ & $4.9 E+03$ & $7.1 \mathrm{E}+03$ & NR & NR & $1.5 \mathrm{E}+\mathrm{O} 2$ \\
\hline 1991 & $1.6 \mathrm{E}+01$ & $2.4 E+01$ & $6.9 \mathrm{E}+03$ & $1.4 \mathrm{E}+03$ & $3.2 \mathrm{E}+03$ & NR & $8.6 E+01$ \\
\hline 1992 & NR & NR & $6.3 E+03$ & $1.5 \mathrm{E}+02$ & $\mathrm{NR}$ & NR & $9.6 \mathrm{E}+00$ \\
\hline 1993 & $1.2 \mathrm{E}+01$ & $8.3 E+01$ & $7.4 E+03$ & $1.2 \mathrm{E}+\mathrm{O} 2$ & $4.1 E+03$ & $4.1 E+01$ & $1.3 \mathrm{E}+01$ \\
\hline 1994 & $\mathrm{NR}$ & NR & $6.6 \mathrm{E}+03$ & $1.2 \mathrm{E}+\mathrm{O} 2$ & NR & NR & $6.4 E+00$ \\
\hline 1995 & NR & NR & $5.7 E+03$ & $3.0 E+02$ & NR & NR & $7.0 E+00$ \\
\hline 1996 & $5.8 \mathrm{E}+01$ & $2.6 \mathrm{E}+\mathrm{O} 2$ & $1.4 E+04$ & $5.8 E+03$ & $9.5 \mathrm{E}+\mathrm{O} 2$ & $3.7 \mathrm{E}+01$ & $1.6 E+01$ \\
\hline 1997 & $3.1 \mathrm{E}+01$ & $2.0 \mathrm{E}+02$ & $1.1 E+04$ & $3.2 \mathrm{E}+03$ & $1.7 E+02$ & $2.6 \mathrm{E}+01$ & $3.1 \mathrm{E}+00$ \\
\hline Year & Site Y307 & Site Y308 & Site Y309 & Site Y310 & Site Y311 & Site Y312 & Site Y313 \\
\hline 1987. & $1.3 E+01$ & $4.2 E+01$ & $2.4 \mathrm{E}+\mathrm{O} 2$ & $5.7 \mathrm{E}+0 ?$ & $6.6 \mathrm{E}+02$ & $5.8 \mathrm{E}+01$ & $5.0 \mathrm{E}+01$ \\
\hline 1988 & $1.5 \mathrm{E}+01$ & $3.2 \mathrm{E}+01$ & $4.1 E+01$ & NR & $3.4 E+02$ & $4.0 \mathrm{E}+01$ & $5.8 E+01$ \\
\hline 1989 & NR & $7.8 E+01$ & $2.9 E+02$ & $1.6 E+02$ & $9.5 E+02$ & NR & NR \\
\hline 1990 & NR & $9.0 E+01$ & 4.4E+01 & $3.1 E+01$ & $5.8 E+02$ & $5.4 E+01$ & NR \\
\hline 1991 & $1.4 E+01$ & $2.8 E+01$ & $1.0 \mathrm{E}+\mathrm{O} 2$ & $1.5 E+01$ & $4.0 \mathrm{E}+\mathrm{O} 2$ & $8.9 E+00$ & $8.1 E+00$ \\
\hline 1992 & NR & NR & $8.1 E+\infty O$ & $6.7 E+00$ & $1.1 E+02$ & $7.1 \mathrm{E}+00$ & NR \\
\hline 1993 & NR & NR & NR & NR & NR & NR & NR \\
\hline 1994 & $3.8 \mathrm{E}+00$ & $1.2 E+01$ & $3.4 E+00$ & $3.8 E+00$ & $5.1 \mathrm{E}+01$ & $1.8 E+01$ & NR \\
\hline 1995 & $3.8 E+00$ & $1.4 \mathrm{E}+01$ & $5.5 \mathrm{E}+00$ & $7.0 \mathrm{E}+00$ & $7.1 \mathrm{E}+01$ & $1.9 \mathrm{E}+01$ & NR \\
\hline 1996 & $6.5 \mathrm{E}+00$ & $2.2 \mathrm{E}+01$ & NR & NR & $1.7 E+02$ & NR & NR \\
\hline 1997 & ND & $1.1 E+01$ & $5.6 E+00$ & ND & $1.5 E+02$ & NR & $\mathrm{NR}$ \\
\hline
\end{tabular}

NR $=$ Not reported (sample not collected or no analytical results provided).

ND $=$ Not detected (less than overalf counting error, or no peak detected, or results less than zero).

6.2.1.2 200 Area. Water samples were collected from one active pond and one active ditch located in the 200 East Area. Sampling at the 216-B-3C Pond was discontinued in September 1997 when the discharge to this facility was terminated. The 200 East Area Powerhouse Ditch is the onfy remaining surface water discharge location in the 200 Areas. This pond and ditch in the 200 East Area received waste water from the waste processing plants and other facilities. All water is sampled at the point of discharge to ensure compliance with applicable DOE standards. Analytical results are shown in Appendix G, Table G-2.

6.2.1.3 300/400 Areas. No surface water was sampled in the 300 Area. The 400 Area Fast Flux Test Facility (FFTF) Process Pond is sampled by PNNL and the results are reported in the Hanford Site Environmental Report for Calendar Year 1997 (Dirkes and Hanf 1998). The data are shared with PHMC after they have been validated by PNNL. Total beta concentrations in the FFTF pond water were within the range observed in previous years. The concentrations of ${ }^{3} \mathrm{H}$ were 
HNF-EP-0573-6

comparable to those measured in the past. However, the tritium concentration in the July sample was $16,400 \mathrm{pCi} / \mathrm{L}$, which is much higher than that observed previously. During this time, backup water supply well 499-S0-7 was in use. Tritium levels in Well 499-SO-7 are typically above $20,000 \mathrm{pCi} / \mathrm{L}$. The use of backup water supply well 499-S0-7 is most likely responsible for the high levels of tritium observed. For a more detailed discussion, see Section 4.2, "Surface Water and Sediment Surveillance," Hanford Site Environmental Report for Calendar Year 1997 (Dirkes and Hanf 1998).

\subsubsection{Nonradiological Parameters}

6.2.2.1 100 Areas. No samples were analyzed for nonradioactive parameters in the 100 Areas during 1997.

6.2.2.2 200 Areas. Results of $\mathrm{pH}$ and nitrate determinations are summarized in Appendix $\mathrm{G}$, Table G-3. The $\mathrm{pH}$ annual averages ranged from neutral to slightly basic. The highest annual average $\mathrm{pH}$ of 8.8 was found at the 216-B-3C Pond. The results for $\mathrm{pH}$ were well within the Resource Conservation and Recovery Act (RCRA) corrosivity designation limits of 2.0 to 12.5. The 216-B-3C Pond exhibited maximum annual nitrate results of $0.22 \mathrm{mg} / \mathrm{L}$, while the maximum annual nitrate result for the 200 East Area Powerhouse Ditch was $0.24 \mathrm{mg} / \mathrm{L}$. All nitrate concentrations were below the drinking water standard (40 CFR 141) for nitrates $(45 \mathrm{mg} / \mathrm{L}$ ).

6.2.2.3 300/400 Area. Currently, no surface water is sampled in the 300 Area. The 400 Area Process Pond is sampled by PNNL and the 1997 results are reported in the Hanford Site Environmental Report for Calendar Year 1997 (Dirkes and Hanf 1998).

\subsubsection{Aquatic Vegetation}

6.2.3.1 100 Areas. No aquatic vegetation samples were collected in the 100 Areas during 1997.

6.2.3.2 200 Areas. Aquatic vegetation samples are collected from ponds and ditches that have growing aquatic vegetation. One vegetation sample each was collected from the 216-B-3C Pond and 200 East Area Powerhouse Ditch in 1997. Each sample consisted of growing stems and leaves from the predominant plant species at each location. The vegetation was analyzed for gamma-emitting radionuclides, as well as ${ }^{90} \mathrm{Sr},{ }^{239,}{ }^{240} \mathrm{Pu}$, and uranium. Analytical results are provided in Appendix G, Table G-4.

6.2.3.3 300/400 Area. No aquatic vegetation was sampled in the 300 and 400 Areas.

\subsubsection{Sediment (Surface Samples)}

6.2.4.1 100 Areas. No sediment samples were collected in the 100 Areas during 1997 .

6.2.4.2 200 Areas. The highest ${ }^{137} \mathrm{Cs}$ result was $11 \mathrm{pCi} / \mathrm{g}$ at the $216-\mathrm{B}-3 \mathrm{C}$ Pond near the 200 East Area. The highest plutonium result was $0.20 \mathrm{pCi} / \mathrm{g}$, found at the 200 East Area Powerhouse Pond. Both pond and ditch are within posted radiological control areas. Analytical results are provided in Appendix G, Table G-5.

6.2.4.3 $300 / 400$ Areas. Currently, no surface water sediment is sampled in the 300 or 400 Area. 


\subsection{CONCLUSIONS}

No significant changes in radioactivity were observed in surface water samples from ponds and ditches in 1997. All surface waters associated with separations-area operations were below the DOE DCG for all radionuclides. The analytical results of vegetation samples collected at the ponds and ditches revealed that, while some physiological uptake of radionuclides occurred, the amounts were relatively low. Sediment samples collected demonstrated elevated levels (above background) of mainly ${ }^{137} \mathrm{Cs}$ and plutonium.

Historical trending of the $100 \mathrm{~N}$ Area shoreline springs continue to indicate an overall decrease in radionuclide concentrations. However, as in past years, the seepage welis with the highest radionuclide concentrations were in the vicinity of the effluent monitoring well, indicating that this well continues to monitor N Springs discharges in the region of the highest concentrations. 
HNF-EP-0573-6

This page intentionally left blank. 
HNF-EP-0573-6

\subsection{RADIOLOGICAL SURVEYS}

\subsection{INTRODUCTION}

Radiological surveys are conducted to determine changes in the radiological status of the $100,200,300,400$, and 600 Areas outdoor waste sites. These sites include surface water disposal units, cribs, trenches, burial grounds, French drains, and reverse wells. The Routine Environmental Monitoring Schedule, Calendar Year 1997, WHC-SP-0098-8 (Markes and McKinney 1996), lists the waste sites and the survey frequency. Trends in radiation levels or radiological contamination may aid in assessing the adequacy of waste containment by detecting the movement of radioactive material away from radiological control areas, or detecting releases that might otherwise go unrecognized. The Quarterly Environmental Radiological Survey Summaries (McKinney and Markes 1997a, McKinney and Markes 1997b, McKinney and Markes 1997c, McKinney et al. 1998) contains a summary of the radiological surveys that were performed. The radiological survey locations are shown in Figures $\mathrm{H}-1$ through $\mathrm{H}-1 \mathrm{O}$ (Appendix $\mathrm{H}$ ). When activity is detected, the vehicle is stopped, and a thorough survey is performed using a portable count rate meter equipped with a thin-window, pancake-type probe. The appropriate managers are notified if contamination is identified and corrective actions are then initiated.

Activity in this report is shown in counts per minute (cpm) to accurately describe the contamination found in the field. The thin-window, pancake-type Geiger-Müeller probe is the principal instrument used in these surveys. The radiological survey records show the activity in disintegrations per minute $(\mathrm{dpm})$. The radiological control technicians use the Hanford Site standard correction factor of 10 to convert the field instrument readings taken in $\mathrm{cpm}$ to the $\mathrm{dpm}$ reported on the radiological survey record.

\subsection{ROADS AND RAILROADS}

\subsubsection{Roads}

Road surveys are performed using a vehicle equipped with sodium lodide detectors mounted on the undercarriage. The detector height is adjustable. The average survey height is $15 \mathrm{~cm}(6 \mathrm{in}$.) above the road or pavement. The vehicle is driven at approximately $3 \mathrm{~km} / \mathrm{h}(2 \mathrm{mi} / \mathrm{h})$.

Frequently traveled blacktop and improved roads and parking lots in and around the 200 Areas are surveyed bimonthly to detect the presence of radioactive material. Roads less frequently traveled or with low contamination potential are surveyed either quarteriy or semiannually.

Contaminated tumbleweed fragments, up to $40,000 \mathrm{cpm}$, were detected on the perimeter road inside the Liquid Effluent Retention Facility (LERF) and were removed for proper disposal.

Contaminated tumbleweed fragments, up to $5,000 \mathrm{cpm}$, were detected on 23rd Street inside 200 West and removed for proper disposal.

Gravel and fixed contamination in the asphalt, up to $9,000 \mathrm{cpm}$, were found at the corner of Alaska and Ginko Streets in the 300 Area and the area was posted.

Contaminated specks, up to $5,000 \mathrm{cpm}$, were detected on the BC Control Area access road and removed for proper disposal. 


\subsubsection{Railroads}

Radiological surveys of railroads are conducted with a vehicle equipped with "high railers" that allow the vehicle to travel on both roads and railroad tracks, and adjustable-height sodium iodide detectors. The average survey height is $15 \mathrm{~cm}(6 \mathrm{in}$.$) . The vehicle is driven at less than$ $8 \mathrm{~km} / \mathrm{h}(5 \mathrm{mi} / \mathrm{h})$. When activity is detected, the vehicle is stopped and portable hand-held survey instruments are used to thoroughly survey the area and identify the extent of the contamination. The appropriate managers are notified when contamination is identified. Corrective actions are then initiated.

The rail lines between the 300 Area and the 400 Area and inside the 200 East and West Areas, including spurs and sidings, are surveyed semiannually. All other railroad tracks outside the facilities are surveyed annually, including spurs and sidings and up to Columbia Center.

Contamination up to $36,000 \mathrm{cpm}$ was discovered in the soil in the railroad bed in the 200 West Area. The contaminated soil was located in the railroad bed west and north of the 2713-WB Green Hut. The contaminated soil and tumbleweed fragments were removed for proper disposal.

A contaminated soil particle, reading up to $40,000 \mathrm{cpm}$, was detected in the ballast in the railroad track north of 283-W Steam Plant. The soil particle was removed for proper disposal.

Contaminated mouse feces reading up to $5,000 \mathrm{cpm}$ were found in the railroad tracks east of 218-W-4B burial ground. The feces were removed and sent to the laboratory for analysis.

\subsection{SURFACE-WATER DISPOSAL UNITS}

Surface-water disposal units (pond and ditch banks) are routinely surveyed to identify potential contamination. Special survey plots are designated around the perimeters of these sites. They are marked with metal posts and numbered. The 216-B-3 Pond C Lobe and the 200 East Powerhouse Ditch are the only active surface-water disposal units remaining. The contamination levels remained at less than detectable values and no apparent changes were noted for 1997.

The east portion of the 216-B-3 Pond C Lobe was backfilled and down posted from a contamination/soil contamination (CA/SC) area to an underground radioactive materials (URM) area.

\subsection{SOLID WASTE DISPOSAL SITES}

The retired solid waste disposal sites are surveyed semiannually or annually to detect radiological changes, primarily via biological intrusion. Solid waste disposal sites are located in the 100 B, C, D, DR, F, K reactor areas; 200 East and 200 West; 300 Area; and the 600 Areas. (See Appendix $\mathrm{H}$, Figures $\mathrm{H}-1$ through $\mathrm{H}-10$.)

Contaminated vegetation and specks were identified at the 218-E-8, 218-E-12A, 218-E-12B, 218-W-3A, 218-W-4B, and 218-W-7 Burial Grounds in 1997 with a maximum contamination level of $36,000 \mathrm{cpm}$. The contamination, in all cases, was removed and/or backfilled shortly after it was identified.

The ERDF started receiving waste in July 1996. A milestone was reached in September 1997 with the ERDF having received 500,000 tons of contaminated waste. The waste came from remediation activities in the $100 \mathrm{~B} / \mathrm{C}, 100 \mathrm{D}$, and 300 Areas. The ERDF posting changes were added in 1997, with the URM area being reported as 1.9 ha (4.8 acres) and the CA/SC area being reported as 1.1 ha $(2.7$ acres). 
HNF-EP-0573-6

\subsection{AREAS LOW-LEVEL LIQUID WASTE DISPOSAL SITES}

Low-level liquid waste disposal sites, other than open ponds and ditches, consist of cribs, French drains, reverse or injection wells, trenches, covered ponds and ditches, and unplanned release sites. As with solid waste disposal sites, liquid waste sites are surveyed at least annually, and as often as quarterly, to detect changes in surface radiological conditions. The most significant survey results in 1997 are listed in Sections 7.5.1 through 7.5.11. Sites remediated or backfilled to meet radiological down-posting requirements are listed in Section 7.12.

The ERC and TWRS develop individual annual schedules for remediation of identified contamination. Sites are cleaned or stabilized as soon as possible, but, because of previously scheduled remediation and prioritization of the sites, contamination may not always be stabilized in the same year it is found.

\subsubsection{6-A Sites}

Contaminated tumbleweeds were found growing on the 216-A-30 crib with a maximum reading of $6,000 \mathrm{cpm}$. The area was posted and turned over to the landlord for disposition.

Fixed contamination was found on a riser in the $216-\mathrm{A}-8 \mathrm{crib}$. The riser was posted and operations were notified.

A contaminated speck reading $100,000 \mathrm{cpm}$ was found on the $216-\mathrm{A}-29 \mathrm{ditch}$. The speck was removed for proper disposal.

The surface soil contaminated section of the 216-A-25 (Gable Mountain Pond) extension discovered during the radiological mapping fly over in 1996, was stabilized and down posted to URM.

\subsubsection{6-B Sites}

Spotty contamination was discovered on the 2i6-B-2-1,2, and 3 covered ditches with the maximum reading of $7,000 \mathrm{cpm}$. One speck was removed for proper disposal and the other areas were posted.

A contaminated tumbleweed with a maximum reading of $95,000 \mathrm{cpm}$ was found on the 216-B-59 crib. The tumbleweed was picked up and placed in the low-level waste burial grounds. The remaining contaminated areas found on the 216-B-2-1,2, and 3 covered ditches were posted.

\subsubsection{6-C Sites}

Contaminated tumbleweed fragments with a maximum reading of $6,000 \mathrm{cpm}$ was found at the $216-\mathrm{C}-3$ crib. The contamination was removed and placed in the low-level burial grounds.

\subsubsection{6-N Sites}

No contamination was found during the routine radiological surveys of the $216-\mathrm{N}$ sites. 


\subsubsection{6-S Sites}

A contaminated soil particle reading up to $15,000 \mathrm{cpm}$ was discovered at the 216-S-25 crib. The soil particle was removed and placed in the low-level burial ground.

The remaining surface soil contaminated portion of UN-216-W-24, which was under the abandoned steam line, was scraped and placed into the 216-S-18 Vehicle Decontamination Trench and the trench was covered with clean soil. The area that was under the steam line was sampled and released and the decontamination trench was down posted from CA/SCA to URM.

\subsubsection{6-T Sites}

Contaminated coyote feces were found in the 216-T-4-2 ditch/pond area with readings up to $1,000 \mathrm{cpm}$. The contaminated feces were removed for proper disposal.

\subsubsection{6-U Sites}

Contaminated tumbleweeds and soil with readings of up to $2,000 \mathrm{cpm}$ were found in the 216-U-4B crib and the 216-U-14 ditch. The areas were posted CA/SC and the facility managers notified.

\subsubsection{6-Z Sites}

A contaminated ant mound was found at the $216-Z-9 \mathrm{crib}$. The ant mound was posted as $\mathrm{SC}$ and the facility manager notified.

\subsubsection{Unplanned Release Sites}

7.5.9.1 UN-216-E-13. Contaminated risers reading $8,500 \mathrm{cpm}$ were found at the R-13 utility pit and posted $C A$.

7.5.9.2 UN-216-E-41. Contaminated soil, tumbleweeds and a riser were found on the eastern section of UN-216-E-41. The contaminated soil and tumbleweeds were removed for proper disposal and the riser was posted CA.

7.5.9.3 UN-216-E-7. Contamination reading up to $70,000 \mathrm{cpm}$ was found in the soil at the UN-216-E-7 unplanned release site. The contaminated soil was removed for proper disposal.

7.5.9.4 UN-216-W-28. A contaminated paint chip reading $1,500 \mathrm{cpm}$ was found and removed for proper disposal.

7.5.9.5 UN-216-W-36. Contaminated tumbleweeds were found on this unplanned release site. The tumbleweeds were removed for proper disposal.

7.5.9.6 UN-216-W-37/UN-216-W-9. Contaminated tumbleweeds, reading greater than $100,000 \mathrm{cpm}$ were found on the UN-216-W-37 unplanned release site. The tumbleweeds were removed for proper disposal. 


\subsubsection{Underground Pipe Lines}

Contaminated tumbleweeds and rabbit brush reading $18,000 \mathrm{cpm}$ were found on the surface of the underground pipeline leading from 241-TX/TY tank farm to the 241-TR-152 diversion box south of 23 rd Street. The area was posted and the landlord notified.

\subsection{TANK FARM PERIMETERS}

Tank farm perimeters and associated facilities are surveyed semiannually to detect any migration of contamination. Tank farms and related facilities are sources of environmental contamination migration. Recontamination along the same fence lines from year to year appears to be associated with the prevailing wind direction and the contamination located within the tank farms and associated with tank farm activities.

Portions of the areas adjacent to and south of the 241-C tank farm and 271-CR vault, including a portion of 7th Street, found to be contaminated in 1994 and 1995 (up to $5,000 \mathrm{cpm}$ ) were cleaned up and down posted URM in 1997. The contamination appeared to be from animal intrusion and wind-borne activity both inside and outside the tank farm. Efforts to prevent further animal activity in these areas are ongoing.

Several contaminated tumbleweeds, with a maximum reading of $45,000 \mathrm{cpm}$, were discovered along the east fence line of the 241-A, AN, AX, AY, and AZ tank farms while performing the semiannual tank farm perimeter surveys. The tumbleweeds were removed for proper disposal.

A contaminated power pole reading $800 \mathrm{cpm}$ was found adjacent to the $241-\mathrm{BX} / \mathrm{BY}$ tank farm. The power pole was posted as being contaminated.

Contaminated tumbleweeds, soil and a mouse were discovered at the 241-S, SX, and SY tank farms while performing the semiannual tank farm perimeter surveys. The contaminated tumbleweeds and soil were removed for proper disposal and the mouse was sent to the laboratory for analysis.

Contaminated tumbleweed fragments and ant hills reading up to $12,000 \mathrm{cpm}$ were discovered at the 241-T tank farm and 241-TX-155 diversion box. The contaminated media was removed for proper disposal.

\subsection{BC CRIBS AND CONTROLLED AREA}

The $\mathrm{BC}$ cribs and trenches (Figure $\mathrm{H}-9$ ) are a series of liquid-waste disposal sites that were active in the mid-1950's. In 1958, animal intrusion was determined to have caused sadioactivity to be transported over an area estimated to exceed 1,000 ha $(2,500$ acres). In 1979 , special survey plots were established throughout the controlled area to monitor for contaminant migration. Data accumulated during the 10 -year period indicate that no significant additional migration of contamination away from the area has occurred. The cribs and trenches were surface stabilized in 1982.

In 1996, an investigative radiological survey was scheduled to specifically identify the boundary definition for the BC Control Area. Contamination was discovered outside the original posted $B C$ Control Area and the survey was continued over open land directly south of the 200 East Area. From the results of this survey, the BC Control Area (UN-216-E-11) was significantly enlarged to a size of approximately 3,849 ha $(9,511$ acres) and reclassified as a 600 Area site. 
Speck contamination was found along the $\mathrm{BC}$-controlled-area roads with readings up to $400 \mathrm{cpm}$. The specks were picked up at the time of survey, and disposed of in the low-level burial grounds.

\subsection{AREAS SITES}

In October 1990, contamination of the shoreline below $100 \mathrm{~N}$ Reactor was posted as a surface contamination area near the N Springs seeps. In 1991, the discovery of contaminated vegetation and mulberry trees resulted in the area being expanded. The contaminated trees were removed and a herbicide spray program was implemented in December 1991 and discontinued in 1997.

An area of speck contamination (e.g., a single grain of soil, rust particle, or piece of feces or vegetation) on the riverbank below the $107 \mathrm{KE}$ and $\mathrm{KW}$ Retention Basins remains posted as a contamination area. The area is approximately 8.0 ha $(2.0$ acres) with contamination levels ranging from 200 to $20,000 \mathrm{cpm}$.

A portion of the 107-H area that had become recontaminated and posted as CA/SC was stabilized and down posted to URM.

\subsection{N AREA RADIATION SURVEYS}

Direct radiation levels are measured annually along the $100 \mathrm{~N}$ Area portion of the Columbia River shoreline. Annual environmental radiation surveys historically conducted at intersecting points of survey grids established around the 1301-N and 1325-N LWDFs to monitor radiation levels associated with the disposal facilities were discontinued in 1995. Data obtained from the TLD stations located around the perimeters of these facilities provide adequate radiation assessment because neither facility is active.

A hand-held micro-rem meter was used to survey points along the $\mathrm{N}$ Springs area. In previous years, the instrument used for this survey was a micro- $\mathrm{A}$ meter. This instrument, which measures exposure rates, was known to "over respond" to low-energy gamma radiation. The micro-rem meter more accurately measures the true dose rate.

All readings were taken at a height of $1 \mathrm{~m}(3 \mathrm{ft})$ at $15 \mathrm{~m}(50 \mathrm{ft})$ intervals. The readings obtained for the survey reflect dose rates that are reiative only to each other.

\subsection{COLUMBIA RIVER SHORELINE SURVEY}

Relative dose rates were measured along the Columbia River shoreline starting near the $\mathrm{N}$ Area outfall (field location 5) and proceeding downstream past the $\mathrm{N}$ Springs. A graph of the shoreline survey data is presented in Figure 7-1. The locations of the $100 \mathrm{~N}$ Area facilities that potentially contribute to dose rates measured near the river shoreline are shown. At the time of the survey, N Reactor was shut down. The 1304-N Emergency Dump Tank was empty and there was no flow to the 1325-N LWDF. The 1310-N Radioactive Chemical Waste Storage Facility also was empty. 
Figure 7-1. $100 \mathrm{~N}$ Shoreline Survey Results.

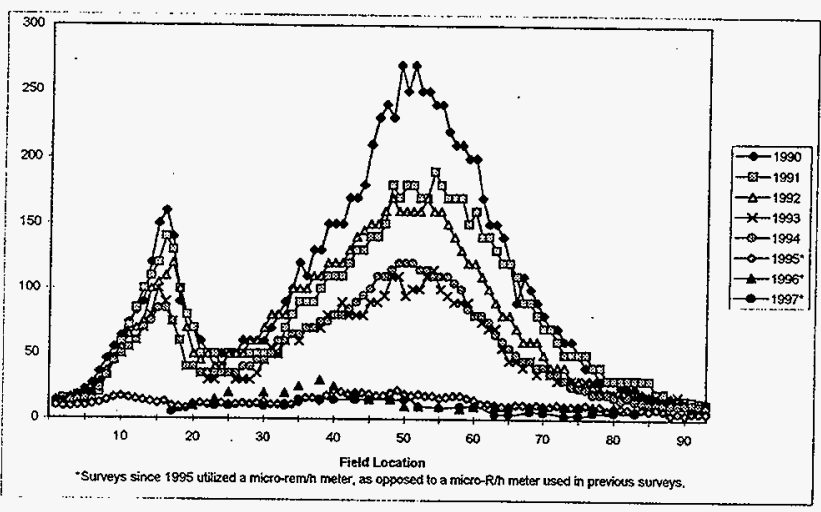

Columbia River

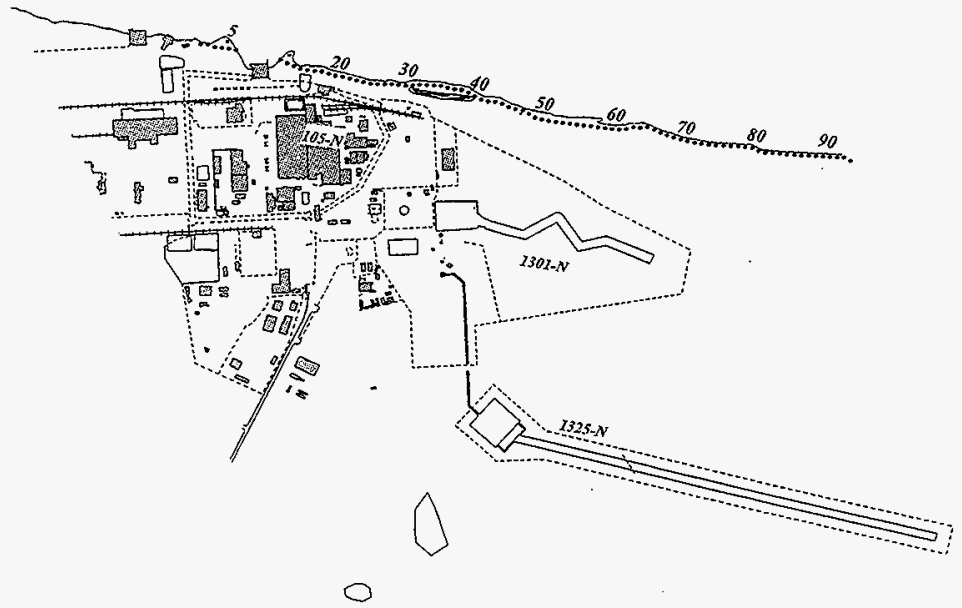


The overall shape of the curve shown on the graph (Figure 7-1) for 1997 indicates that the area along the $\mathrm{N}$ Springs shoreline with the highest exposure rate is, as in the past, along the area juxtapositional with the $1301-\mathrm{N}$ LWDF. The radiation levels measured this year are not directly comparable to the years prior to 1995 , though the overall shape of the curve derived by graphing the results shows the region of highest exposure to again agree with historic survey results.

Results of the shoreline survey are presented in Table $\mathrm{H}-1$ (Appendix $\mathrm{H})$.

\subsection{Retired 100 Area Waste Disposal Facilities}

Routine environmental radiological surveillance was performed annually on the retired 100 Area waste disposal facilities in 1997. The contamination levels remained at less than detectable vaiues except as listed in Table 7-1. The contaminated materials listed were either removed/disposed of or posted as indicated in Table 7-1. Contamination has been noted in previous radiation surveys in most of these areas.

\subsection{STABILIZATION AND SITE REMEDIATION}

In 1997, several contaminated sites were interim surface stabilized by scraping the contaminated top soil and consolidating the contamination into smaller areas. These smaller areas were covered with $15 \mathrm{~cm}$ ( 6 in.) of clean soil. Other sites were backfilled with $15 \mathrm{~cm}$ to $0.6 \mathrm{~m}$ $(6 \mathrm{in}$. to $2 \mathrm{ft})$ of clean soil. Both methods resulted in surface contamination area postings being reduced to underground radioactive material areas or being released from radiological control. The sites remediated and/or radiologically reposted in 1997 are shown in Table 7-2.

Table 7-1. Samples with Contamination Above Detectable Levels.

\begin{tabular}{|c|c|c|}
\hline Waste site/location & Activity (cpm) & Type \\
\hline 105-D/DR Exclusion Area & 33,800 & Speck \\
\hline 105-F Exclusion Area ${ }^{b}$ & 200 & Soil \\
\hline 105-B Exclusion Area ${ }^{a}$ & 500 & Soil \\
\hline $116-\mathrm{B}-10^{\mathrm{b}}$ & 1,200 & Soil \\
\hline 116-F-1 Lewis Canal ${ }^{\circ}$ & 30,000 & Specks \\
\hline 116-F-1 Lewis Canal ${ }^{b}$ & 30,000 & Speck \\
\hline $116-\mathrm{H}-7^{\mathrm{a}}$ & 30,000 & Speck \\
\hline $116-\mathrm{H}-7^{6}$ & 6,100 & Soil \\
\hline $118-\mathrm{B}-1^{\mathrm{a}}$ & 60,000 & Specks \\
\hline $118-\mathrm{H}-1^{2}$ & 68,000 & Speck \\
\hline $118-\mathrm{H}-5^{\mathrm{a}}$ & 300,000 & Speck \\
\hline
\end{tabular}

'Contaminated removed/disposed.

Site posted. 
Table 7-2. Sites Remediated or Radiologically Reposted in 1997 [approximate surface area in hectares (acres)].

\begin{tabular}{|l|l|l|}
\hline \multicolumn{1}{|c|}{ Site Designation } & \multicolumn{1}{|c|}{ Status change } & \multicolumn{1}{|c|}{$\begin{array}{c}\text { Area in } \\
\text { (acres) }\end{array}$} \\
\hline UN-216-W-24/216-S-18 Vehicle Decon Trench & CA/SC to URM & $0.04(0.1)$ \\
\hline East Portion 216-B-3 Pond C Lobe & CA/SC to URM & $5.2(12.9)$ \\
\hline GPS survey of the east side of 241-S/SX/SY Tank Farm & None to CA/SC & $0.65(1.6)$ \\
\hline $\begin{array}{l}\text { GPS survey of the 216-A-25 Gable Mountain Pond } \\
\text { boundaries }\end{array}$ & None to URM & $4.1(10.1)$ \\
\hline UN-216-W-35 around 207-U Basin & CA/SC to URM & $0.8(2.0)$ \\
\hline Portions of 216-U-14 Ditch south of 16th Street & CA/SC to URM & $1.0(2.4)$ \\
\hline Portions of 216-U-14 Ditch north of 16th Street & URM to CA/SC & $0.1(0.3)$ \\
\hline Slurry Line Corridor & CA/SC to URM & $0.4(1.0)$ \\
\hline 244-CR Vault perimeter & CA/SC to URM & $0.35(0.9)$ \\
\hline 216-A-25 Over Flow Portion & CA/SC to URM & $2.0(5.0)$ \\
\hline ERDF First Cell & None to URM & 1.9 (4.8) \\
\hline ERDF First Cell & None to CA/SC & $1.1(2.7)$ \\
\hline REDOX Railroad Cut & CA/SC to URM & $1.7(4.2)$ \\
\hline 107-H Basins & CA/SC to URM & $1.7(4.2)$ \\
\hline
\end{tabular}

$\mathrm{CA} / \mathrm{SC}=$ Contamination/soil contamination.

ERDF = Environmental Restoration Disposal Facility.

GPS $=$ Global Positioning System.

URM = Underground radioactive material. 
HNF-EP-0573-6

This page intentionally left blank. 


\subsection{INVESTIGATIVE SAMPLING}

\subsection{INTRODUCTION}

Investigative sampling was conducted in the operations areas to confirm the absence or presence of radioactive and/or hazardous contaminants. Investigative sampling took place near facilities such as storage and disposal sites for at least one of the following reasons:

- To follow-up radiological surface surveys that had indicated radioactive contamination was present.

- To conduct preoperational surveys that quantify the radiological/chemical conditions at a site before facility construction or operation.

- To quantify the radiological condition of a site before remediation.

- To determine if biotic intrusion (e.g., animal burrows or deep-rooted vegetation) had created a potential for contaminants to spread.

- To determine the integrity of waste containment systems.

Generally, the predominant radionuclides discovered during these efforts were activation products and ${ }^{90} \mathrm{Sr}$ in the 100 Areas, fission products in the 200 Areas, and uranium in the 300 Area. Hazardous chemicals generally have not been identified above background levels in preoperational environmental monitoring samples.

\subsection{COLLECTION AND ANALYSES OF INVESTIGATIVE SAMPLES}

Investigative samples collected in 1997 included sludge, soil, vegetation (e.g., grass, tumbleweeds, rabbitbrush, and sagebrush), insects (darkling beetles), reptiles (sagebrush lizards), a bird nest (starling), mammal feces (e.g., mouse, rabbit, and coyote), and mammals (e.g., deer mouse, Great Basin pocket mouse, and cottontail rabbit).

Methods for collecting or otherwise obtaining investigative samples are described in Environmental Monitoring (McKinney 1997). Field monitoring was conducted to detect radioactivity in samples before they were submitted for analysis. Field monitoring results are expressed as disintegrations per minute (dpm) when a Geiger-Müeller detector is used or as millirad per hour (mrads/h) when an ion chamber is used. To obtain the reported field instrument reading, the measured background radioactivity is subtracted from the Geiger-Müeller reading (in counts per minute) and converted to $\mathrm{dpm}$ by multiplying $\times 10$ (an average conversion), and further converted to $\mathrm{dpm}$ per $100 \mathrm{~cm}^{2}$ by multiplying $\times 6$ (approximate number of probe areas in $100 \mathrm{~cm}^{2}$ ). Laboratory sample analysis results are generally expressed in picocuries per gram (pCi/g), except for extremely small samples and then in $\mathrm{pCi}$ per sample (pCi/sample). Maximum concentrations, rather than averages, are presented in this subsection.

\subsection{RADIOLOGICAL RESULTS FOR INVESTIGATIVE SAMPLES}

Investigative samples were collected where known or suspected radioactive contamination was present, or to verify radiological conditions at project sites. In 1997, 30 samples were analyzed for radionuclides at the 222-S Laboratory and 27 showed measurable levels of contamination. Analytical results are provided in Appendix I, Table 1-1. Another 115 environmental 
contaminated samples were reported and disposed without isotopic analyses (although field instrument readings were recorded) during clean-up operations. These results are provided in Appendix 1 , Table $1-2$. Only those radionuclide concentrations above analytical detection limits are provided in this report.

\subsubsection{Sludge}

In 1997, two samples of dried sludge were collected from the contaminated C-5 Tank in the 200 East Area to determine if flaking and subsequent blowing might require the surface to be stabilized. Radioisotopic analysis detected only "less than" quantities of radionuclides.

\subsubsection{Soil}

In 1997, no investigative soil samples were collected for radioisotopic analysis. There were 51 incidents of contaminated soil or specks found during cleanup operations and disposed of in low-level burial grounds without analysis. External radioactivity ranged from slightly above background (approximately $1,000 \mathrm{dpm} / 100 \mathrm{~cm}^{2}$ ) to 38 milirads per hour (mrads/h). The contaminated areas were posted or cleaned up.

In 1997, the number of contamination incidents, the range of radiation dose levels, and radionuclide concentrations generally were within historical ranges. Areas of special soil sampling that were outside radiological control areas and had radiation levels greater than radiological control limits (FDH 1997) were posted as surface contamination areas.

\subsubsection{Vegetation}

In 1997, four tumbleweed samples, one rabbitbrush sample, and one grass sample were analyzed for radionuclide concentrations. The maximum radionuclide concentrations were in a tumbleweed sample from near $221-\mathrm{U}$ Building in the 200 West Area and consisted primarily of ${ }^{90} \mathrm{Sr}$ $(2.5 \mathrm{E}+05 \mathrm{pCi} / \mathrm{g})$ and ${ }^{137} \mathrm{Cs}(1.8 \mathrm{E}+06 \mathrm{pCi} / \mathrm{g})$. The rabbitbrush sample from near $241-\mathrm{T}$ Tank Farm in the 200 West Area contained primarily ${ }^{90} \mathrm{Sr}(1.1 \mathrm{E}+03 \mathrm{pCi} / \mathrm{g})$ and ${ }^{137} \mathrm{Cs}(3.1 \mathrm{E}+02 \mathrm{pCi} / \mathrm{g})$.

In addition, 40 instances of contaminated vegetation (e.g., grass, tumbleweeds, rabbitbrush, and sagebrush) were recorded in the operational areas in 1997. This vegetation was discovered during remedial operations, surveyed with field instruments, and disposed of in low-level burial grounds. The field instrument readings for the disposed vegetation samples ranged from approximately $2,500 \mathrm{dpm}$ per $100 \mathrm{~cm}^{2}$ to $1,000,000 \mathrm{dpm}$ per $100 \mathrm{~cm}^{2}(38 \mathrm{mrad} / \mathrm{h})$ and $<20 \mathrm{dpm}$ alpha per $100 \mathrm{~cm}^{2}$.

During 1997, the numbers of contaminated vegetation (both samples and those disposed without analysis) exceeded those of the previous year by a factor of four. This is believed to be primarily because of climatological conditions (i.e., increased frequency and quantity of precipitation) making the vegetation control program on the waste sites considerably less effective, and resulting in more tumbleweed growth. The radioactivity levels, and range of radionuclide concentrations were all within historical ranges (Johnson et al. 1994). Historically, the greatest number of contaminated vegetation samples (42) were submitted for analysis in 1978 (Johnson et al. 1994) but it is not recorded how many contaminated vegetation were disposed of without analysis that year. 


\subsubsection{Wildlife}

Wildife is collected either as part of an integrated pest management program designed to limit the exposure to and potential contamination of animais with radioactive material, or as a result of finding radiologically contaminated wildlife-related material (e.g., feces, nests, etc.) during a radioactivity surveillance. Animals were collected directly from, or near, facilities to identify potential problems with preventive measures designed to deter animal intrusion. Surveys were performed after collection to determine whether an animal was radioactively contaminated. If a live animai was found to be free of contamination, it was taken to an area of suitable habitat, still in a controlled area, and released. If an animal was contaminated, a decision was made based on the level of contamination, sampling facility, and frequency of occurrence either to collect the animal as a sample or to dispose of the animal in a low-level burial ground.

In 1997, 22 wildlife and wildlife-related samples were submitted for analysis. All 22 of the specia! animal samples analyzed showed detectable levels of contamination. This compares to 37 contaminated samples (of 41 collected) that were analyzed in 1996, 22 contaminated samples (of 25 collected) that were analyzed in 1995, and 16 contaminated samples (of 27 collected) in 1994 . The numbers of samples submitted depended on opportunity (i.e., resulting from the pest control activities at facilities) rather than exact numbers submitted from established sampling points. Contaminated animal samples, which were somewhat atypical for the special sample program, included darkling beetles found inside $272-\mathrm{S}$ in the 200 West Area with elevated ${ }^{90} \mathrm{Sr}(1.8 \mathrm{E}+02$ $\mathrm{pCi} / \mathrm{g})$; and 12 sagebrush lizards found dead in a cabinet outside PUREX in the 200 East Area with low levels of ${ }^{90} \mathrm{Sr}(6.8 \mathrm{E}+01 \mathrm{pCi} / \mathrm{g})$ and ${ }^{137} \mathrm{C} s(3.3 \mathrm{E}+01 \mathrm{pCi} / \mathrm{g})$. The maximum radionuciide concentrations in 1997 were in mouse feces collected along railroad tracks east of 218-W-4B Burial Ground in the 200 West Area. The concentrations included ${ }^{60} \mathrm{Co}(2.8 \mathrm{E}+04 \mathrm{pCi} / \mathrm{g}) ;{ }^{90} \mathrm{Sr}(1.7 \mathrm{E}+05$ $\mathrm{pCi} / \mathrm{g}) ;{ }^{137} \mathrm{Cs}(1.3 \mathrm{E}+05 \mathrm{pCi} / \mathrm{g}) ;{ }^{154} \mathrm{Eu}(2.6 \mathrm{E}+04 \mathrm{pCi} / \mathrm{g}) ;{ }^{155} \mathrm{Eu}(1.1 \mathrm{E}+04 \mathrm{pCi} / \mathrm{g}) ;{ }^{238} \mathrm{Pu}(8.1 \mathrm{E}+03$ $\mathrm{pCi} / \mathrm{g}) ;{ }^{239 / 240} \mathrm{Pu}(3.3 \mathrm{E}+04 \mathrm{pCi} / \mathrm{g})$, and total uranium $(1.6 \mathrm{E}+05 \mathrm{pCi} / \mathrm{g})$. A sample of three house mice from 244-AR Vault in the 200 East Area had $1.6 \mathrm{E}+05 \mathrm{pCi} / \mathrm{g}$ of ${ }^{90} \mathrm{Sr}$. The numbers of animals found to be contaminated with radioactivity, the radioactivity levels, and the range of radionuclide concentrations were within historical ranges \{Johnson et al. 1994).

There were eight cases of contaminated wildlife or related samples found during cleanup operations that were not analyzed. These samples included two ant hills, two mouse feces, one mouse urine, one rabbit feces, and two mice (one set of mouse bones in a raven nest). The field instrument readings for the disposed animal samples ranged from approximately $4,000 \mathrm{dpm} / 100$ $\mathrm{cm}^{2}$ to $400,000 \mathrm{dpm} / 100 \mathrm{~cm}^{2}$ beta/gamma and $<20 \mathrm{dpm}$ alpha.

\subsubsection{Special Characterization Sampling}

Special characterization projects conducted or completed in 1997 to verify the radiological, and in some cases, potential hazardous chemical status of operations included:

- Continued monitoring of ambient air to determine the levels of diffuse fugitive air emissions downwind of the Plutonium Finishing Plant (PFP) in 200 West Area. The preliminary analytical data and that from the nearby routine air monitor locations in 200 West Area indicated that emissions from the PFP associated facilities were below levels of regulatory concern. Results are presented in the air section.

- Completed preconstruction environmental monitoring in support of the Tank Waste Remediation System Phase I Privatization Site on the east side of the 200 East Area. issued the TWRS Phase / Privatization Site Preconstruction Characterization Report (Mitchell et al 1997). The analytical data did not identify any environmental concerns that would delay startup of the facilities. 
HNF-EP-0573-6

This page intentionally left blank. 


\subsection{REFERENCES}

10 CFR 835, 1997, "Occupational Radiation Protection," Code of Federal Regulations, as amended.

40 CFR 61, 1997, Subpart H, "National Emissions Standards for Hazardous Air Pollutants," Code of Federal Regulations, as amended.

40 CFR 141, 1997, "National Primary Drinking Water Regulations," Code of Federal Regulations, as amended.

AEC, 1974, Measurements of Radionuclides in the Environment, Sampling and Analysis of Plutonium in Soil, Nuclear Regulatory Guide 4.5, U.S. Atomic Energy Commission, Washington, D.C.

ANSI, 1975, Performance, Testing, and Procedural Specifications for Thermoluminescence Dosimetry: Environmental Application, ANSI-N545-1975, American National Standards institute, New York, New York.

Comprehensive Environmental Response, Compensation, and Liability Act of 1980, 42 USC 9601, et seq.

Dirkes, R. L., and R. W. Hanf, 1998, Hanford Site Environmental Report for Calendar Year 1997, PNNL-11795, Pacific Northwest National Laboratory, Richland, Washington.

Dirkes, R. L., and R. W. Hanf, 1995, Hanford Site Environmental Report for Calendar Year 1994, PNL10574, Pacific Northwest National Laboratory, Richland, Washington.

DOE, 1990, Radiation Protection of the Public and the Environment, DOE Order 5400.5, U.S. Department of Energy, Washington, D.C.

DOE, 1988a, General Environmental Protection Program, DOE Order 5400.1, U.S. Department of Energy, Washington, D.C.

DOE, 1988b, Radioactive Waste Management, DOE Order 5820.2A, U.S. Department of Energy, Washington, D.C.

Gilmore, T. J., D. R. Newcomer, S. K. Wurstner, and F. A. Spane, 1992, Calculation of Groundwater Discharge to the Columbia River in the 100 Area, PNL-0857, Pacific Northwest Laboratory, Richland, Washington.

Gleckler, B. P., 1998, Environmental Releases for Calendar Year 1997, HNF-EP-0527-06, Waste Management Federal Services of Hanford, Inc., Richland, Washington.

Hartman, M. J., and P. E. Dresel, 1997, Hanford Site Groundwater Monitoring for Fiscal Year 1997, PNNL-11793, Pacific Northwest National Laboratory, Richland, Washington.

HNF-PRO-454, Inactive Waste Sites, Fluor Daniel Hanford, Inc., Richland, Washington.

HSRCM-1, Hanford Site Radiological Control Manual, Pacific Northwest Laboratory, Richland, Washington.

Johnson, A. R., B. M. Markes, J. W. Schmidt, A. N. Shah, S. G. Weiss, and K. J. Wilson, 1994, Historical Records of Radioactive Contamination in Biota at the 200 Areas of the Hanford Site, WHC-MR-0418, Westinghouse Hanford Company, Richland, Washington. 
Markes, B. M., and S. M. McKinney, 1996, Routine Environmental Monitoring Schedule, Calendar Year 1997, WHC-SP-0098-8, Rust Federal Services, Inc., Richland, Washington.

McKinney, S. M., and B. M. Markes, 1997a, Quarterly Environmental Radiological Survey Summary, First Quarter 1997 100, 200, 300, and 600 Areas, HNF-SP-0665-24, Rust Federal Services, Inc., Northwest Operations, Richland, Washington, April 1997.

McKinney, S. M., and B. M. Markes, 1997b, Quarterly Environmental Radiological Survey Summary, Second Quarter 1997 100, 200, 300, and 600 Areas, HNF-SP-0665-25, Waste Management Federal Services, Inc., Northwest Operations, Richland, Washington, July 1997.

McKinney, S. M., and B. M. Markes, 1997c, Quarterly Environmental Radiological Survey Summary, Third Quarter 1997 100, 200, 300, and 600 Areas, HNF-SP-0665-26. Waste Management Federal Services, Inc., Northwest Operations, Richland, Washington, October 1997.

McKinney, S. M., B. M. Markes, and R. C. Roos, 1998, Quarterly Environmental Radiological Survey Summary, Fourth Quarter 1997 100, 200, 300, and 600 Areas, HNF-SP-0665-27, Waste Management Federal Services, Inc., Northwest Operations, Richland, Washington, January 1998.

Mitchell, R. M., K. A. Bergstrom, C. J. Chou, D. L. Edwards, D. G. Horton, V. G. Johnson, B. M. Markes, T. H. Mitchell, R. K. Price, S. P. Reidel, K. D. Reynolds, and W. R. Thackaberry, 1997, TWRS Phase 1 Privatization Site Preconstruction Characterization Report, HNF-2067, Fluor Daniel Hanford, Inc., Richland, Washington.

Resource Conservation and Recovery Act of 1976, 42 USC 6901, et seq.

RL, 1998, Radionuclide Air Emissions Report for the Hanford Site, Calendar Year 1997, DOE/RL-98-33, U.S. Department of Energy, Richland Operations Office, Richland, Washington.

RL, 1997, Radionuclide Air Emissions Report for the Hanford Site, Calendar Year 1996, DOE/RL-97-43, Draft 2.0, U.S. Department of Energy, Richland Operations Office, Richland, Washington.

RL, 1994a, Hanford Environmental Monitoring Plan, United States Department of Energy, Richland Operations Office, DOE/RL-91-50, Rev. 1, U.S. Department of Energy, Richland Operations Office, Richland, Washington.

RL, 1994b, Hanford Site Ground Water Protection Management Plan, DOE/RL-89-12, U.S. Department of Energy, Richland, Washington.

WAC 173-303, "Dangerous Waste Regulations," Washington Administrative Code, as amended.

WAC 246-247, "Radiation Protection--Air Emissions," Washington Administrative Code, as amended.

WMNW-CM-004, Operational Environmental Monitoring, Waste Management Federal Services, Inc., Northwest Operations, Richland, Washington. 
HNF-EP-0573-6

APPENDIX A

QUALITY ASSURANCE 
HNF-EP-0573-6

This page intentionally left blank. 


\section{QUALITY ASSURANCE}

Quality Assurance (OA) may be defined as the actions necessary to ensure the accuracy of a program. The near-facility environmental monitoring QA program consists of procedures and guides to demonstrate that environmental monitoring techniques and analyses are performed within established limits of acceptance. This is documented in the Near-Facility Environmental Monitoring QA Project Plan (McKinney 1997).

Written operating procedures are an integral part of near-facility environmental monitoring QA. Procedures for field operations are provided in an internal Waste Management Federal Services, Inc., Northwest Operations (WMNW) manual. This appendix briefly describes the essential components of the near-facility environmental monitoring QA program.

\section{DOCUMENTATION}

Record keeping is a vital part of any environmental monitoring program. Maintenance of environmental data is important from a QA standpoint, from a regulatory standpoint, and for trend analyses and optimization of environmental monitoring procedures. Each phase of near-facility environmental monitoring is documented. This documentation includes sampling logs, annual reports, and occurrence reports.

\section{SAMPLE REPLICATION}

Replicate sampling and subsequent analysis are the primary means of assessing sample variability. Duplicate samples of air, water, soil, sediment, and vegetation are collected.

\section{DATA ANALYSIS}

Environmental data are reviewed to determine compliance with applicable federal and company guides. The data are analyzed both graphically and by standard statistical tests to determine trends and impacts on the environment. Newly acquired data are compared with historical data and natural background levels. Routine environmental data are stored on both magnetic media (i.e., in a computer environment) and hard-copy printouts.

\section{TRAINING}

To ensure quality and consistency in sample collection and handling, all personnel performing such work received formal training. All radiological control technicians (RCT) are required to complete a certification program. In addition, those $\mathrm{RCTs}$ assigned to environmental monitoring receive special classroom orientation and on-the-job training by experienced personnel. WMNW Environmental Monitoring and Investigations personnel, in addition to their formal training received while obtaining professional degrees, received training in such courses as "Radiation in the Environment," taught through the Washington State University, courses taught at the Harvard School of Public Health, and various short courses.

\section{SAMPLE FREQUENCY}

1. Ambient air sample filters are collected biweekly. 
2. Water samples from active ponds and ditches are collected weekly.

3. Radiological surveys of roads are performed quarteriy, bimonthly, or annually, as stated in Section 7.0.

4. The thermoluminescent dosimeters (TLD) are exchanged quarterly.

5. Radiological surveys of waste sites are performed quarterly, semiannually, or annually depending on the operating status, condition, and history of the site.

6. Soil, vegetation, surface water, fecal samples and sediment and vegetation samples from active ponds and ditches also are collected annually.

\section{ANALYTICAL PROCEDURES}

Three laboratories provided analytical support to the near-facility environmental monitoring: Quanterra Environmental Services; the Waste Sampling and Characterization Facility; and the 222-S Analytical Laboratory. Samples are analyzed in accordance with prescribed procedures and quality control guides which are described briefly in the following paragraphs.

\section{Quanterra Environmental Services}

Environmental samples which require measurements at a very low detection limit and special samples in support of pre-operational environmental monitoring may be performed at Quanterra Environmental Services. The analytical laboratory may analyze soil, vegetation, animal feces, groundwater and air samples. Analyses are performed according to procedures and quality control guides described by the Environmental Measurements Laboratory (EML 1972), the U.S. Atomic Energy Commission (AEC 1974), and the National Council on Radiation Protection and Measurements (NCRPM 1976).

1. Soil Samples

a. Gamma energy analysis - Gamma-emitting radionuclides are measured using a Marinelli beaker and counting with a $\mathrm{Ge}(\mathrm{Li})$ detector equipped with a multichannel pulse-height analyzer.

b. Strontium-90 - The ${ }^{90} \mathrm{Sr}$ is removed from the soil sample by leaching the dried sample with nitric acid. The strontium in solution is converted to an oxalate followed by precipitation as strontium carbonate. The carbonate is deposited on a planchet and counted in the same manner as the ${ }^{90} \mathrm{Sr}$ water samples.

c. Technetium-99 - The ${ }^{99} \mathrm{Tc}$ is isolated from other elements using hydroxide carbonate coprecipitation, leaving it in solution as the pertechnethe ion $\left(\mathrm{TCO}_{4}{ }^{-}\right)$. Further purification is achieved by an anion-exchange column path, followed by liquid scintillation spectrometry.

2. Vegetation Samples

a. Gamma energy analysis - Gamma-emitting radionuclides are measured by direct counting of the sample with a $\mathrm{Ge}(\mathrm{Li})$ detector equipped with a multichannel pulse height analyzer. 
b. Strontium-90 - The ${ }^{90} \mathrm{Sr}$ is removed from the vegetation sample by leaching the dried sample with nitric acid. The strontium in solution is converted to an oxalate followed by precipitation as strontium carbonate. The carbonate is deposited on a planchet and counted in the same manner as the ${ }^{90} \mathrm{Sr}$ water samples.

c. Technetium-99 - The ${ }^{99} \mathrm{Tc}$ is isolated from other elements using hydroxide carbonate coprecipitation, leaving it in solution as the pertechnethe ion $\left(\mathrm{TcO}_{4}{ }^{-}\right)$. Further purification is achieved by an anion-exchange column path, followed by liquid scintillation spectrometry.

\section{Groundwater Samples}

a. Strontium-90 - The strontium is removed from the water sample by precipitating it as a nitrate using nitric acid. The sample is purified by repeated scavenging with barium chromate and precipitating with barium carbonate. The strontium carbonate then is counted with a low-background gas-flow proportional counter.

b. Gamma energy analysis - Gamma-emitting radionuclides are analyzed by directly counting the water sample with a germanium-lithium $\mathrm{Ge}(\mathrm{Li})$ detector equipped with a multi-channel pulse-height analyzer.

c. Tritium - Water samples are analyzed for tritium with a liquid scintillation spectrometer.

d. Total uranium - The water samples are analyzed for uranium first by treating them with sodium and lithium fluoride and then analyzing them with a fluorometer.

\section{Pacific Northwest National Laboratory Radiation Standards and Engineering}

Thermoluminescent Dosimeters. External radiation levels are measured using thermoluminescent dosimeters (TLDs). The Hanford Site uses the Harshaw 8807 dosimeter and the Harshaw 8800 reader. The TLDs are calibrated, packaged, and read by the Pacific Northwest National Laboratory (PNNL) Radiation Calibration Laboratory, Radiation Standards and Engineering Department. All TLD work is performed in accordance with the procedures and specific guides from the American National Standards Institute (ANSI 1975) and PNNL.

\section{2-S and Waste Sampling and Characterization Facility (WSCF) Analytical Laboratories}

The 222-S and Waste Sampling and Characterization Facility (WSCF) laboratories also provide analytical support to near-facility environmental monitoring. WSCF is used for the samples containing typical environmental levels of radioactivity. The 222-S Laboratory is normally used for samples containing higher than normal environmental levels of radioactivity. Analytical procedures and quality control guides are described by the Environmental Measurements Laboratory (EML 1972), the American Society for Testing and Materials (ASTM 1976), the American Public Health Association (APHA 1980), and the U.S. Environmental Protection Agency (EPA 1979). 
1. Air Samples

a. Gamma energy analysis - Gamma-emitting radionuclides are measured by direct counting of the air sample filter with a germanium detector. The gamma spectra are analyzed using a Nuclear Data 7700 software system.

b. Strontium - Airborne ${ }^{89} \mathrm{Sr}$ and ${ }^{90} \mathrm{Sr}$ are determined by leaching the composited air sample filters with nitric acid and initially precipitating them as a nitrate. The sample is purified by iron and barium scavenging. The final precipitate, strontium carbonate, then is counted for total beta (needed to set the ${ }^{89} \mathrm{Sr}$ value) with a low-background beta proportional counter. Both ${ }^{89} \mathrm{Sr}$ and ${ }^{90} \mathrm{Sr}$ are calculated from the resulting count data using a computer.

c. Plutonium - The various plutonium isotopes are leached from the air sample filter with nitric acid and passed through an ion-exchange resin. Further decontamination from lead, bismuth, and other transuranics by washing with nitric and hydrochloric acids is done. The plutonium then is eluted from the resin and electrodeposited on a stainless-steel disk, where it is counted using a surface barrier alpha spectrometer, and data are collected on a Nuclear Data system.

d. Uranium - The uranium is leached from the air sample filter and extracted as tetrapropyl ammonium uranyltrinitrate followed by back extraction into water. Following treatment with sodium and LiF, the aqueous sample is analyzed with a fluorometer to determine the mass of uranium.

\section{Pond and Ditch Water}

a. Total alpha and beta activity - An aliquot of the sample is evaporated to dryness and the total alpha and beta activities are measured by direct counting with a gas flow proportional counter.

b. Gamma energy analysis - The liquid sample is sealed inside a geometrically approved container. The gamma-emitting radionuclides are measured by direct counting with a $\mathrm{Ge}(\mathrm{Li})$ detector equipped with a multichannel analyzer.

c. Strontium-90 - The ${ }^{90} \mathrm{Sr}$ is removed from the aqueous sample by precipitating it out with barium carbonate. The strontium carbonate is purified by redissolving with nitric acid, precipitating as a nitrate, and precipitating again as a carbonate. The ${ }^{90} \mathrm{Sr}$ activity is determined by beta counting with a gas flow proportional counter.

d. Plutonium - Actinides are removed from the aqueous sample by precipitation with iron. The precipitate is redissolved in hydrochloric acid and the plutonium is separated from the other actinides by ion exchange. The plutonium is electrodeposited on a planchet and counted using alpha spectrometry. 
3. Groundwater Samples

a. Strontium-90 - The strontium is removed from the water sample by precipitating it as a nitrate using nitric acid. The sample is purified by repeated scavenging with barium chromate and precipitating with barium carbonate. The strontium carbonate then is counted with a low-background gas-flow proportional counter.

b. Gamma energy analvsis - Gamma-emitting radionuclides are analyzed by directly counting the water sample with a germanium-lithium $\mathrm{Ge}(\mathrm{Li})$ detector equipped with a multi-channel pulse-height analyzer.

c. Tritium - Water samples are analyzed for tritium with a liquid scintillation spectrometer.

d. Total uranium - The water samples are analyzed for uranium first by treating them with sodium and lithium fluoride and then analyzing them with a fluorometer.

4. Pond and Ditch Mud and Sediment

a. Gamma energy analysis - The gamma-emitting radionuclides are measured by direct counting of the dried sediment sample using a Ge(Li) detector equipped with a multichannel analyzer.

b. Soil leach - Strontium, plutonium, americium, and other elements are leached from the soil sample using a mixture of hydrochloric and nitric acids. The leachate is then analyzed for specific radionuclides.

5. Pond and Ditch Vegetation

a. Gamma energy analysis - The sample is sealed inside a geometrically approved container. The gamma-emitting radionuclides are measured by direct counting with a $\mathrm{Ge}(\mathrm{Li})$ detector equipped with a multichannel analyzer.

b. Vegetation leach - The vegetation samples are dry ashed in a furnace and then leached with a mixture of hydrochloric and nitric acids. The leachate is analyzed for specific radionuclides.

6. Soil Samples

a. Gamma energy analysis - Gamma-emitting radionuclides are measured using a Marinelli beaker and counting with a $\mathrm{Ge}(\mathrm{Li})$ detector equipped with a multichannel pulse-height analyzer.

b. Strontium-90 - The ${ }^{90} \mathrm{Sr}$ is removed from the soil sample by leaching the dried sample with nitric acid. The strontium in solution is converted to an oxalate followed by precipitation as strontium carbonate. The carbonate is deposited on a planchet and counted in the same manner as the ${ }^{90} \mathrm{Sr}$ water samples. 
c. Technetium-99 - The ${ }^{99} \mathrm{Tc}$ is isolated from other elements using hydroxide carbonate coprecipitation, leaving it in solution as the pertechnethe ion $\left.\left(\mathrm{TCO}_{4}\right)^{\top}\right)$. Further purification is achieved by an anion-exchange column path, followed by liquid scintillation spectrometry.

\section{Vegetation Samples}

a. Gamma eneray analysis - Gamma-emitting radionuclides are measured by direct counting of the sample with a Ge(Li) detector equipped with a multichannel pulse height analyzer.

b. Strontium-90 - The ${ }^{90} \mathrm{Sr}$ is removed from the vegetation by leaching the dried sample with nitric acid. The strontium in solution is converted to an oxalate followed by precipitation as strontium carbonate. The carbonate is deposited on a planchet and counted in the same manner as the ${ }^{90} \mathrm{Sr}$ water samples.

c. Technetium-99 - The ${ }^{99} \mathrm{Tc}$ is isolated from other elements using hydroxide carbonate coprecipitation, leaving it in solution as the pertechnethe ion $\left(\mathrm{TcO}_{4}{ }^{-}\right)$: Further purification is achieved by an anion-exchange column path, followed by liquid scintillation spectrometry.

\section{REFERENCES}

AEC, 1974, Measurements of Radionuclides in the Environment, Sampling and Analysis of Plutonium in Soil, Nuclear Regulatory Guide 4.5, U.S. Atomic Energy Commission, Washington, D.C.

ANSI, 1975, Performance, Testing, and Procedural Specifications for Thermoluminescence Dosimetry: Environmental Application, ANSI-N545-1975, American National Standards Institute, New York, New York.

APHA, 1980, Standard Methods for the Examination of Water and Waste Water, Fifteenth Edition, American Public Health Association, Washington, D.C.

ASTM, 1976, 1976 Annual Book of ASTM Standards, Water, Part 31, American Society for Testing and Materials, Philadelphia, Pennsyivania.

EML, 1972, HASL-300 Procedures, Environmental Measurements Laboratory, New York, New York.

EPA, 1979, EPA Methods for Chemical Analysis of Water and Wastes, EPA 600/4-79-020, Environmental Monitoring and Support Laboratory, U.S. Environmental Protection Agency, Cincinnati, Ohio.

McKinney, S. M., 1997, Near-Facility Environmental Monitoring QA Project Plan, HNF-EP-0538-3, Waste Management Federal Services, Inc., Northwest Operations, Richland, Washington.

NCRPM, 1976, Environmental Radiation Measurements, Report No. 50, National Council on Radiation Protection and Measurements, Washington, D.C., December 1976. 
HNF-EP-0573-6

APPENDIX B

GLOSSARY

B-i 


\section{HNF-EP-0573-6}

This page intentionally left blank. 


\section{DEFINITIONS}

Aboveground Tank - A tank situated so that its entire surface area is completely above the plane of the adjacent surrounding surface and the entire surface area of the tank (including the tank bottom) can be visually inspected (WAC 173-303-040).

Accessible Soils - Hanford soils that are not behind security fences must meet the Table 1 values found in the Fluor Daniel Hanford, Inc. Inactive Waste Sites, HNF-PRO-454. The values reflect a 10-mrem/yr effective dose equivalent limit from Hanford Site operations to the most exposed member of the public.

Administrative Control Value (ACV) - Operating contractor's internally imposed release limit for an airborne or liquid effluent. The ACV is usually expressed as a fraction or multiple of the derived concentration guide-public but may also be expressed as a total release value (curies per year for ${ }^{85} \mathrm{Kr}$ and tritium). In applying the ACV, the "Unity Rule" applies; the sum of the fractions of the $A C V$ in an effluent shall not exceed 1.

Airborne Radioactive Effluents - Radioactive particles, mists, vapors, fumes, and/or gases contained or entrained in airborne effluents.

Aquifer - A subsurface formation consisting of sufficient saturated permeable material to yield significant quantities of water.

Areal Contamination - Contamination generaliy confined to less than the first centimeter of soil. Numerically, the radioactivity content averaged over a suitable area.

As Low As Reasonably Achievable (ALARA) - The implementation of ALARA is described in HNF-PRO-159, ALARA Program Description. This concept applies to maintaining releases at or below prescribed regulatory limits.

Assure - To remove any doubt, to promise.

Audit - An announced examination of a facility or operation to determine compliance with the specific requirements.

Average Soil Contamination - Contamination generally dispersed through the soil. Numerically, the radioactivity content averaged over a suitable mass of soil.

Backfill Soil - The soil used as a plant growth medium between the depths of $30-120 \mathrm{~cm}$ as measured at the restabilization site.

Background Radiation - Refers to regional levels of radioactivity produced by sources other than those of specific interest (e.g., the nuclear activities at the Hanford Site).

Barrier or Engineered Barrier - At a disposal site, a manufactured addition designed to retard or preclude radionuclide or other contaminant transport and/or preserve the integrity of the disposal site.

Becquerel $(\mathrm{Bq})$ - The standard international unit of radioactivity. One Becquerel is one disintegration per second or: $\mathrm{Bq}=2.7 \mathrm{E}-11 \mathrm{Ci}$ 
Biological Transport - Means of biological transport may include one or more of the following processes:

- Movement of subsurface radioactivity to the surface by physiological vegetative processes.

- Dispersion of such vegetation by the wind.

- Contaminated urine and feces deposited by animais that have gained access to and ingested radioactive materials.

- Contaminated animals themselves that have ingested radioactive materials directly or ingested other contaminated animais or plants.

- Physical displacement of radioactive materials by burrowing animals.

- Nests built using contaminated materials.

Biota - The plant and animal life of a specific region.

Building - A structure created to shelter any form of human activity, such as a house, barn, church, hotel, or similar structure; it also may refer to a historically related complex, such as a courthouse and jail; or, on the Hanford Site, buildings to include: old reactor buildings, office buildings, e.g., 2750 Building, 222-S Laboratory Building, etc.

Burial Ground - A land area specifically designated to receive contaminated solid or solidified liquid waste packages and equipment. The contaminated articles are usually placed in trenches and covered with overburden.

Byproduct - A material that is not one of the primary products of a production process and is not solely or separately produced by the production process. Examples are process residues such as slags or distillation column bottoms. The term does not include a coproduct that is produced for the general public's use and is ordinarily used in the form in which it is produced by the process.

Calibration - Determining the deviation of an instrument from a standard traceable to the National Bureau of Standards or other recognized agency and reporting the deviations and/or eliminating them by adjustment.

Central Landfill - The U.S. Department of Energy-owned disposal site once used for the disposal of trash and other nonhazardous nonradioactive wastes. Landfill operations were discontinued in March 1996.

Chemical Processing - Chemical treatment of material to separate desired components selectively. At the Hanford Site, plutonium, uranium, and fission products were chemically separated from irradiated fuels.

Chemical Trench - The central landfill trench that was used for the disposal of chemicals, hazardous wastes, and hazardous substances.

Committed Dose Equivalent - The predicted total dose equivalent to a tissue or organ over a 50 year period after a known intake of a radionuclide into the body. It does not include contributions from external dose. Committed dose equivalent is expressed in units of rem (or sievert). 
Committed Effective Dose Equivalent - The sum of the committed dose equivalents to various tissues in the body, each multiplied by the appropriate weighing factor. Committed effective dose equivalent is expressed in units of rem (or sievert).

Composite Sample - A number of random samples initially collected from a waste and combined into a single sample; this sample is analyzed for the contaminants of concern.

Comprehensive Environmental Response, Compensation, and Liability Act of 1980 (CERCLA) Commonly known as "Superfund," CERCLA was enacted to respond to uncontrolled releases of hazardous substances to the environment, primarily at inactive sites that were not adequately addressed by the Resource Conservation and Recovery Act of 1976 (RCRA). CERCLA also applies to actively managed facilities and any onshore or offshore facility.

Confined Aquifer - A subsurface water-bearing region having defined and relatively impermeable upper and lower boundaries.

Conserve - Use methods and procedures necessary to protect any endangered or threatened species. Such methods and procedures include, but are not limited to, activities associated with scientific resources management such as research, census, law enforcement, habitat acquisition and maintenance, propagation, live trapping, and transplantation. In the extraordinary case where population pressures within a given ecosystem cannot be otherwise relieved, methods and procedures may include regulated taking.

Controlled Area - An area where access is controlled to protect individuals from exposure to radiation and/or radioactive materials.

Construction - Addition, enlargement, modification, replacement, alteration, or restart (after a period of five years of nonoperation) of any process or stationary source that may increase emissions or ambient air concentrations of any contaminant for which federal or state ambient or emission standards have been established.

Contamination Area - Any area where contamination levels are greater than the values specified in Chapter 2, Table 2-2, Hanford Site Radiological Control Manual, HSCRM-1, but less than or equal to 100 times those values.

Contamination Limit - That concentration limit or activity limit for radioactive materials below which posting restrictions and environmental controls to protect personnel or the environment are not necessary.

Crib - An underground structure designed to receive liquid waste that percolates into the soil directly or percolates into the soil after having traveled through a connected tile field.

Decommissioning - Actions taken to reduce the potential health and safety impacts of U.S. Department of Energy-controlled contaminated facilities. Actions could include stabilizing, reducing, or removing radioactivity or demolishing the contaminated facilities.

Decontamination - The removal of radioactive or hazardous contamination from facilities, equipment, or soils by washing, heating, chemical or electrochemical treating, mechanical cleaning, or other techniques. 
Demolition Waste - Solid waste, made up largely of inert waste resulting from the demolition or razing of buildings, roads, and other manufactured structures. Demolition waste consists of, but is not limited to, concrete, brick, bituminous concrete, wood and masonry, composition roofing and roofing paper, steel, and minor amounts of other metals such as copper. [Plaster (sheet rock or plaster board), asbestos, or any material other than wood that is likely to produce gases or a leachate during the decomposition process is not considered to be demolition waste.]

Derived Concentration Guide for Occupational Exposure (DCG-Occupational) - Those concentrations in air or water that an occupationally exposed individual would have to inhale/ingest in a year to result in 5,000 mrem $/ 50$ year dose commitment.

Derived Concentration Guide for Public Exposure (DCG-Public) - The concentration of radioactive materials in air or water specified in Appendix $\mathrm{J}$, "Derived Concentration Guides for Controlling Exposure to Members of the Public," of this manual. The DCG-Public are those concentrations of radionuclides in air or water that would result in a maximum effective committed dose equivalent of up to 100 mrem per year using ICRP-30 dose methodology under conditions of continuous exposure or use (i.e., continuously breathing or being in contact with contaminated air or exclusively drinking contaminated water).

Diffuse Source - A source or sources of radioactive or chemical contaminants released into the environment that do not have a defined point or origin of release (a nonpoint source). Such sources are also known as area sources.

Dispersible - Capable of being widely spread.

Disposal - Discharging, discarding, or abandoning dangerous waste, or the treatment, decontamination, or recycling of such waste once it has been discarded or abandoned. This includes the discharge of any dangerous waste into or on any land, air, or water (WAC 173-303-040). This definition also includes radioactive materials.

Disposal Facility - Any facility or part of a facility where hazardous and/or radioactive waste is intentionally placed or where any land or water waste will remain after closure.

District - A geographically definable area (urban or rural) with a significant concentration, linkage, or continuity of sites, buildings, structures, or objects united either by past events or aesthetically by planned physical development. A district may also be individual elements that are separated geographically but linked by association or history.

Disturbance - Includes, but is not limited to, the following: leveling; firebreak, road, or utility line construction; excavation of pits, foundations and trenches; quarrying and borrowing of rock and soils; seismic testing; and stockpiling of earth.

Ditch - An open surface site for transport of liquid wastes to a pond or trench structure designed for percolation.

Ecology - The Washington State Department of Ecology.

Effective Dose Equivalent (EDE) - The summation of the products of the dose equivalent received by specified tissues of the body and a tissue-specific weighing factor. This sum is a risk-equivalent value and can be used to estimate the health-effects risk of the exposed individual. The tissuespecific weighing factor represents the fraction of the total health risk resulting from uniform whole-body irradiation that would be contributed by that particular tissue. The EDE includes the committed EDE from internal deposition of radionuclides and the EDE caused by penetrating radiation from sources outside the body. EDE is expressed in units of rem (or sievert). 
Effluent - An airborne or liquid discharge from a facility after all engineered waste treatment and effluent controls have been performed. The term includes onsite discharges to the atmosphere, lagoons, ponds, cribs, injection wells, French drains, or ditches. The term does not include solid waste stored or removed for disposal or waste that is contained in retention basins or tanks before treatment and/or disposal.

Emissions Unit - Regarding air pollutant emissions, any part of a stationary source that emits or would have the potential to emit any pollutant subject to regulation.

Ensure - To make certain, make sure, make safe.

Environmental Monitoring Plan (EMP) - A two-part document prepared for each site, facility, or process that uses, generates, releases, or manages significant pollutants or hazardous materials.

Environmental Sites Database (ESD) - A database of environmental Sites which is administered by the Environmental Restoration Contractor.

Exhaust System - A stack, gaseous effluent system, building ventilation system, or any system that vents air or gaseous material to the atmosphere.

Expedited Response Action - An interim response action (IRA) or interim measure (IM) process. This includes any onsite response action (under CERCLA or RCRA authority) to mitigate site contamination conducted before initiation of remedial action or corrective action in accordance with final record of decision (ROD) or permit modifications.

Facility - A processing plant, tank farm, shop, laboratory, powerhouse, or laundry. Including all contiguous land and structures, other appurtenances, and improvements on land used for recycling, reusing, reclaiming, transferring, storing, treating of dangerous waste (including treatment, storage, and disposal sites as well as groundwater wells). (40 CFR 264, "Standards for Owners and Operators of Hazardous Waste Treatment Storage and Disposal Facilities," and WAC 173-303-040.)

Facility-Specific Environmental Monitoring - Routine environmental monitoring of all environmental media (air, biota, etc.) around facility parameters.

Field Blank - Aliquots of analyte-free water or solvents brought to the field in sealed containers and transported to the laboratory with the sample container. Field blanks include trip blanks and equipment blanks.

Field Duplicate - Field duplicates are collected at specified frequencies and are used to document precision. The field duplicate precision depends on the variance of waste composition, sampling techniques, and analytical technique.

Fish or Wildife - Any member of the animal kingdom including (without limitation) any mammal, fish, bird, amphibian, reptile, mollusk, crustacean, arthropod, or other invertebrate--including any part, product, egg, offspring, or the dead body or parts of the offspring.

French Drain - A covered or rock-filjed encasement with the bottom end open to allow liquids to seep into the ground.

Fugitive Emissions - Material that is generated incidental to an operation, process, or activity and that is released or dispersed into the open air. Fugitive emissions occur via pathways that do not allow routine measurement at the point of release.

Grab Sample - A single sample removed from a stream over a short time interval. 
Groundwater - Water that exists below the water table, also referred to as the zone of saturation. However, the capilary fringe directly above can be completely saturated if the sediment is fine enough. To avoid this ambiguous term, the use of phreatic water, which is water that enters freely into wells under both confined and unconfined conditions is suggested. Phreatic water is a term originally applied only to water that occurs in the upper part of the zone of saturation under water table conditions (unconfined groundwater or well water), but has come to be applied to all water in the zone of saturation, thus making it an exact synonym of groundwater. Above the water table is the vadose zone, where water pressures are less than atmospheric pressure. This zone still contains water, but the water is held to the soil particles or other groundwater material by capillary force. Thus, while this water still can move within the vadose zone, it cannot move out of the zone into a well or other places that are exposed to atmospheric pressure. The dividing line between water in the vadose zone and phreatic water is the atmospheric pressure between the two, with the pressure of vadose water being below atmospheric pressure and that of phreatic water (i.e., groundwater) above atmospheric pressure.

High-Efficiency Particulate Air (HEPA) Filter - To qualify as a HEPA filter, a filter must achieve an efficiency of $99.97 \%$ under laboratory conditions and $99.95 \%$ after installation for the removal of airborne particulates greater than $3 \mathrm{E}-0.5 \mathrm{~cm}$ (0.3 microns).

High-Level Nuclear Waste - Spent nuclear fuel or radioactive waste resulting directly from the dissolution and reprocessing of spent nuclear fuel. Secondary waste streams resulting from the dissolution and reprocessing of spent nuclear fuel are not considered high-level waste.

Immobile Radionuclides - All those radionuclides that are sorbed onto Hanford Site soils and usually would not migrate through the vadose zone or the groundwater below the future control zone.

Inaccessible Soils - Areas from which the general public is excluded (by fences, posting, patrols, or distance), but that are still subject to meteorological effects, are subject to a $10-\mathrm{mrem} / \mathrm{yr}$ operational EDE limit, as reflected in Table 1, Inactive Waste Sites, HNF-PRO-454.

Inactive Crib - A crib that has been designated as permanently out of service.

Inactive Radioactive Waste Site - Any waste site that is no longer needed for current operational programs and that is not currently an active waste disposal site.

Inactive Waste Sites - Inactive waste sites include units such as burial grounds, unplanned release sites, cribs, ditches, ponds, trenches, and basins, abandoned storage areas, drains, single-shell tank piping, transfer pits, and jumper boxes.

Injection Well - A well used for mixing liquid waste with groundwater.

Inert Waste - Noncombustible, nondangerous solid waste that is likely to retain its physical and chemical structure under expected conditions of disposal, including resistance to biological attack and chemical attack from acidic rainwater.

Insure - To cover by insurance against loss.

Less Than Detectable - An analytical term for a concentration in a sample that is lower than the minimum detection capabilities of that analytical equipment or process.

Low-Level Waste - Any gaseous, liquid, or solid radioactive waste not classified as high-level waste, transuranic waste, or spent nuclear fuel, as defined by U.S. Department of Energy Order 5820.2. 
Maximum Contaminant Level (MCL) - The drinking water standards specified in 40 CFR 141 , "National Primary Drinking Water Regulations." See Appendix C, "Maximum Contaminant Levels."

Minimum Detection Limit - See detection limit.

Mixed Waste - Dangerous waste that also contains enough radioactivity to be classified as radioactive waste.

Modification - Any physical or operational change to a stationary source that results in an increase in the rate of emission to the atmosphere of an air pollutant to which a standard applies.

Monitored Sample - A sample collected in a monitoring system and used to alert operations to nonroutine releases and to provide process control.

Monitoring System - Instrumentation that provides measurement of an airborne or liquid waste stream parameters. The system includes a detector and associated readout components. A continuous monitoring system measures the stream parameters on a near-real-time basis or as specified in applicable

Environmental Protection Agency regulations, 40 CFR 52, "Approval and Promulgation of Implementation Plans," Appendix E; 40 CFR 51, "Requirements for Preparation, Adoption, and Submittal of Implementation Plans," Appendix P, or as defined in applicable American National Standards Institute Standards. A radiation monitoring system is a system in which radiation or radioactivity is the measured parameter. An integrating monitoring system totals the instantaneously measured parameter over some time period. A sampling system does not measure or read out an instantaneous stream parameter.

Near Facility Environmental Monitoring - The collection and analysis of samples of air, water, soil, biota, and other media near nuclear facilities on U.S. Department of Energy sites and their environs and the measurement of external radiation to demonstrate compliance with applicable standards and assess radiation exposures to employees and members of the public, and the near-field environment.

Nonroutine Activities - Any actions on a large-scale $(>5$ acres), including stabilization, soil removal, fixative or sealant application, other surface treatments, or other activities that could affect future remediation activities in an inactive waste site.

Normally Contaminated Airborne or Liquid Effiuent - An airborne or liquid effluent from a facility or area that contains dispersible or unsealed radioactive materials in quantities sufficient to cause releases of radioactive materials in excess of $10 \%$ of the administrative control limit $(A C L)$ averaged over 1 year (i.e., the total activity present times an appropriate suspendable or immersible fraction civided by the total annual flow exceeds $10 \%$ of the $A C L)$.

Not Detected - A reporting term which describes any or all of the following: the overall analytical error was greater than the radionuclide concentration itself; or, after allowing for the subtraction of the background level of the radionuclide, the resulting concentration was less than zero; or, no radio analytical peak was detected during the analysis.

Operate - As used in this section with respect to the activities of the Project Hanford Management Contract, the term "operate" (and other forms of the term) will refer to the performance by the Project Hanford Management Contract of its day-to-day activities in connection with the tanks and tank systems. Such activities shall be subject to the overall management, control, and oversight by U.S. Department of Energy. 
Operations - In this report this term loosely refers to Project Hanford Management Contract activities including chemical processing, waste management, and decommissioning.

Operations - The contractor organization responsible for operating plants and facilities.

Overall Error - In this report, this term means the overall analytical error supplied by the laboratory performing the analysis.

Percolation - Downward movement of water through the interstices of unsaturated rock or soil because of gravity or hydrostatic pressure.

Pesticide - As defined in 40 CFR 162, the term pesticide covers all pest-control chemicals such as herbicides, rodenticides, and insecticides.

Plutonium Processing and Handling Facility - Any facility constructed primarily to process plutonium (including ${ }^{238} \mathrm{Pu}$ ) and that handles in-process plutonium.

Plutonium Storage Facility - Any facility constructed to store strategic (category I) quantities of plutonium.

Point of Release - The point at which a material is released to the environment such as the top of a stack or the end of a pipe.

Point Source - A single defined point (origin) of an airborne release, such as a vent or stack.

Pond - A surface impoundment used to contain or percolate low-level liquid radioactive waste, mixed waste, or hazardous waste.

Potentially Contaminated Airborne or Liquid Effluent - An airborne or liquid effluent from a facility or area that contains radioactive materials in quantities sufficient to cause releases of radioactive materials in excess of $10 \%$ of the ACV averaged over 1 year (the total activity present times a reasonable suspendable or immersible fraction divided by the annual flow equals more than $10 \%$ of the ACV).

Potentially Corrosive Liquid Waste Stream - Any liquid waste stream that has been identified as having the potential, during upset conditions, to discharge liquid with a $\mathrm{pH}$ value of less than or equal to 2, or greater than or equal to 12.5 .

Previously Disturbed Ground - Ground that has had its contours altered by leveling, filling, or excavation or that has been contaminated by radiation or dangerous chemicals to the extent that an individual cannot enter the area without wearing protective clothing. Disturbance is determined by observation, aerial photographs, and topographic maps.

Quality Assurance - A process designed to maintain the quality of the results of a program within established limits of acceptance.

Quality Assurance - All those planned and systematic actions necessary to provide adequate confidence that a structure, system, or component will perform satisfactorily in service.

Radiation Survey - Evaluation of an area or object with portable instruments to identify radioactive materials and radiation fields present. 
Radioactive Byproduct - Any radioactive material (except special nuclear material) yielded in or made radioactive by exposure to the radiation incident to the process of producing or using special nuclear material (Atomic Energy Act of 1954, as amended). The nonradioactive hazardous component of the waste material will be subject to regulation under the RCRA.

Radioactive Liquid Effluent - A liquid effluent that has a reasonable potential for containing radioactive materials in quantities such that the annual average concentration is equal to or greater than the MCL.

Radiological Control Area - An area where access is controlled to protect individuals from exposure to radiation and/or radioactive materials. Radiological control areas include, but are not limited to, areas posted as Radiation Area, Surface Contamination, and Underground Radioactive Materials, to describe the radiological condition of the area within.

Radiological Posting - Information in the form of signs and barriers to inform people of radiological conditions that warrant avoidance or special precautions for entry.

Release From Radiological Posting - Removal of signs and barriers when access to an area no longer needs to be restricted for radiological protection purposes.

Representative Sample - The average stream parameter being measured occurs in the sample in the same average proportion that it occurs in the environmental discharge.

Retired Waste Site - A waste site that is isolated and no longer available to receive waste in any form.

Reverse Well - Same as injection well.

Routine Activities - Any actions on a small-scale $(<5$ acres), including radioactive hot-spot removal, vegetation removal, fencing, posting, herbicide spraying, stabilization, or immediate spill response) in an inactive waste site. In general, these routine actions shall not interfere with RCRA/CERCLA response or site investigations.

Routine Emissions - Air emissions of pollutants or releases from accidents are not considered routine. However, releases of air pollutants to the atmosphere that result from normal operations and releases that are not routine, but are more likely than not to occur, are included as routine emissions.

Sampling System - Instrumentation and equipment that remove a part of a liquid or airborne waste stream for subsequent quantitative determination of stream parameters. The system generally employs such devices as filters, other sample collection media, or effluent traps of some kind. A continuous sampling system removes a part of the stream continuously except during sample change, maintenance, repair, or other necessary outages. A grab sampling system removes an instantaneous part of the stream or removes a part of the stream over a time period.

Sealed - A container having a cover bonded with the container or a cover strong enough to prevent contact with and dispersion of the contents of the container under conditions of use and wear for which it was designed.

Sediment Column - The sediment beneath a crib. It can mean either all the sediment beneath the bottom of the crib extending to the water table or all sediment beneath a crib contaminated by radioactive materials. 
Separations Area - The primary area on the Hanford Site where chemical processing and most waste management activities are performed. It includes the 200 Areas and nearby 600 Area sites.

Site - The location of a significant event, a prehistoric or historic occupation or activity, or a building or structure (whether standing, ruined, or vanished) where the location itself maintains historical or archeological value, regardless of the value of any existing structure.

Soll at depth - Soil below 36 in.

Soil Contamination - Contaminated soil not releasable in accordance with U.S. Department of Energy Order 5400.5.

Solid Waste - Any discarded material that is not excluded by WAC 173-303-017(2) or that is not excluded by a variance granted under WAC 173-303-017(5). Materials are solid waste if they are: (1) abandoned by being disposed of, burned, or incinerated, or (2) accumulated, stored, or treated (but not recycled) before (or in lieu of) being abandoned by being disposed of, burned, or incinerated. In addition, a solid waste includes any material considered to be inherently waste-like.

Speck Contamination - Single grains of soil, rust particles, feces, or pieces of vegetation.

Spent Materials - Used materials that are no longer usable without being regenerated, reclaimed, or reprocessed. Examples are spent solvents, spent activated carbon, spent catalysts, and spent acids. Does not include spent fuel from reactors.

Spot Contamination - A spot or quantity of contamination less than $1 \mathrm{~cm}^{3}$ in volume, or areal contamination less than $15 \mathrm{~cm}^{2}$ in area.

Stabilization - The process of covering surface contaminated areas with clean backfill or topsoil.

Standard - A specified set of rules or conditions concerned with the classification of components; delineation of procedures; definition of terms; designation of materials, performance, design, or operations; or measurements of quality in describing materials, products, systems, services, or practices. A standard is more general than a procedure or specification and more specific than a criterion.

Standard Error of the Mean - A measure of the uncertainty in the estimated averages of data because of random variations.

Stationary Source - Any building, structure, facility, or installation that emits or may emit any air pollutant.

Structure - A work constructed by humans that consists of interdependent and interrelated parts in a definite pattern of organization. It is often a large-scale engineering project.

Suitable Surface Area - Area no more than $15 \mathrm{~cm}^{2}$ over which spot contamination may be averaged in determining areal contamination.

Sump - Any stationary pit or reservoir, including those troughs/trenches connected to it, that serve to collect oil products, hazardous substances, low-level radioactive material, dangerous waste, mixed waste, high-level radioactive materials, or transuranic materials.

Surface Soil - Soil from $0 \mathrm{in.}$ to $2 \mathrm{in.}$ deep. 
Surface Stabilization - A remedial action program on waste disposal sites that includes the addition of at least 46 to $50 \mathrm{~cm}$ (18 to $20 \mathrm{in}$.) of clean soil followed by revegetation. It is designed to cover surface contamination and inhibit biological and wind transport.

Surplus Facilities - Surplus facilities include all facilities that have been accepted into a decommissioning program.

Survey - A method to detect the release, disposal, or presence of radioactive materials or hazardous substances under a specific set of conditions to determine actual or potential hazards. Such an evaluation may include, but is not limited to, tests, physical examinations, and measurements of radiation or concentrations of materials.

Suspect Waste Site - A site, believed to have been previously unknown or undocumented, that, because of characteristics present at the site or historical information about the site, is suspected of containing waste (i.e., non-dangerous, hazardous, dangerous, mixed, and radioactive).

Suspendable Fraction - The fraction of material that may become airborne.

Taking of Any Animal - To harass, harm, pursue, hunt, shoot, wound, kill, trap, capture, or collect any animal or attempt to engage in any such activity.

Tank - A stationary device designed to contain an accumulation of oil products, hazardous substances, low-level radioactive material, dangerous waste, mixed waste, high-level radioactive materials, or transuranic materials, that is constructed primarily of nonearthen materials (e.g., wood, concrete, steel, plastic) that provide structural support.

Tank Farm - An area of large underground tanks designed to store up to $1 \mathrm{Mgal}$ each of high-level liquid waste.

Technologically Enhanced Naturally Occurring Radioactivity - Refined or processed ores or other materials containing naturally occurring radioactive materials, such that radiation exposure or potential radiation exposure is increased.

Thermoluminescent Dosimeter - A chip or series of chips used for measuring external gamma radiation. It consists of a material capable of absorbing energy imparted by ionizing radiation, then emitting light as a result of thermal stimulation. A measure of that light is proportional to the radioactivity absorbed.

Topsoil - The soil used as a plant growth medium at the surface to a depth of $30 \mathrm{~cm}$ as measured at the restabilization site. Topsoil is added soil to support the stabilization of a retired disposal facility with the objective of controlling erosion, establishing the growth of perennial grasses, and preventing the growth of deep-rooted vegetation.

Transuranic (TRU) Radionuclide - Any radionuclide having an atomic number greater than 92 (U.S. Department of Energy Order 5820.2).

Transuranic Waste - Without regard to source or form, radioactive waste that at the end of institutional control periods is contaminated with alpha-emitting transuranium radionuclides with half-lives greater than 20 years and concentrations greater than $100 \mathrm{nCi} / \mathrm{g}(3700 \mathrm{~Bq} / \mathrm{g})$. The Waste Isolation Pilot Plant, high-level waste, and spent nuclear fuel as defined by U.S. Department of Energy Order 5820.2 are specifically excluded from this definition.

Trip Blank - A type of field blank used to accompany sample containers to and from the field and to detect contamination or cross-contamination that occurs during sample handling and transportation. 
Uncontaminated Soil - A soil or a land area that requires no controls or restrictions in any way for radiation protection purposes and/or meets the contamination limit specifications.

Underground Radioactive Material - A radiological posting status where subsurface radioactivity is present but where surface contamination does not exceed the soil standards.

Underground Storage Tank - Any one or any combination of tanks (including underground connection pipes) used to contain an accumulation of regulated substances. At least $10 \%$ of the volume of the tanks (including the volume of underground pipes) is beneath the surface of the ground (WAC 173-360-120). A tank situated in an underground area (such as a basement, cellar, etc.) is not considered to be underground if the tank is situated on or above the surface of the floor (WAC 173-360-110). Surface impoundments, pits, ponds, lagoons, storm water collection systems, or septic tanks are not considered to be underground storage tanks, and are not subject to the underground storage tank requirements (WAC 173-360-110).

Unity Rule - If more than one radionuclide is present, the sum of the fractions represented by each radionuclide concentration divided by its respective limiting concentration (ACV) shall not exceed unity. This rule could also apply to parameters other than radionuclide concentration.

Unplanned Release Site - An area that was contaminated by an unplanned release of radioactive contamination, making it a radiological control area.

Unrestricted Release - Values below which unrestricted release of soils will occur will be defined in an applicable record of decision.

Unsampled Liquid Disposal Site - A liquid waste disposal site that is currently receiving an effluent stream that does not require sampling.

U.S. Environmental Protection Agency (EPA) - The federal agency chartered with carrying out and monitoring the environmental regulations.

Vector - A vertebrate or invertebrate that can transmit toxic, radioactive, or infectious material from a disposal or storage site to uncontrolied areas. Vectors include flies, birds, and mice.

Waste Information Data System (WIDS) - A database that identifies waste management units on the Hanford Site. It is a subset of the ESD.

Waste Management - The activity involved with storing, disposing of, shipping, handling, and monitoring all radioactive waste.

Waste Sites - Any facility used for the planned disposal of hazardous, radioactive, toxic, or nonradioactive/nontoxic waste.

Water Table - The upper boundary of an unconfined aquifer below which saturated groundwater occurs. 
Table B-1. Radionuclide Nomenclature.

\begin{tabular}{|c|c|c|c|c|c|}
\hline Radionuclide & Symbol & Half-Life & Rádionuclide & Symbol & Half-Life \\
\hline tritium & ${ }^{3} \mathrm{H}$ & $12.3 \mathrm{yr}$ & cesium-137 & ${ }^{137} \mathrm{Cs}$ & $30.3 \mathrm{yr}$ \\
\hline beryllium-7 & ${ }^{7} \mathrm{Be}$ & $53.28 \mathrm{~d}$ & cerium-144 & ${ }^{144} \mathrm{Ce}$ & $284.6 \mathrm{~d}$ \\
\hline carbon-14 & ${ }^{14} \mathrm{C}$ & $5,715 \mathrm{yr}$ & promethium-147 & ${ }^{147} \mathrm{Pm}$ & $13.4 \mathrm{~m}$ \\
\hline sodium-22 & ${ }^{22} \mathrm{Na}$ & $2.6 \mathrm{yr}$ & europium-152 & ${ }^{152} \mathrm{Eu}$ & $13.5 \mathrm{y}$ \\
\hline potassium-40 & ${ }^{40} \mathrm{~K}$ & $1.26 \times 10^{9} \mathrm{yr}$ & europium-154 & ${ }^{154} \mathrm{Eu}$ & $8.6 y$ \\
\hline argon-41 & ${ }^{41} \mathrm{Ar}$ & $1.8 \mathrm{~h}$ & europium-155 & ${ }^{155} \mathrm{Eu}$ & $4.7 \mathrm{yr}$ \\
\hline chromium-51 & ${ }^{51} \mathrm{Cr}$ & $27.7 \mathrm{~d}$ & thallium-208 & ${ }^{208} \mathrm{Tl}$ & $3.1 \mathrm{~min}$ \\
\hline manganese- 54 & ${ }^{54} \mathrm{Mn}$ & $312 \mathrm{~d}$ & bismuth-212 & ${ }^{212} \mathrm{Bi}$ & $60.6 \mathrm{~min}$ \\
\hline cobalt -57 & ${ }^{57} \mathrm{Co}$ & $271.8 \mathrm{~d}$ & lead-212 & ${ }^{212} \mathrm{~Pb}$ & $10.6 \mathrm{~h}$ \\
\hline cobalt -60 & ${ }^{60} \mathrm{Co}$ & $5.3 \mathrm{yr}$ & polonium-212 & ${ }^{212} \mathrm{Po}$ & $0.3 \times 10^{-6} \mathrm{~s}$ \\
\hline nickel-63 & ${ }^{63} \mathrm{Ni}$ & $100 \mathrm{yr}$ & polonium-216 & ${ }^{216} \mathrm{Po}$ & $0.15 \mathrm{~s}$ \\
\hline zinc-65 & ${ }^{65} \mathrm{Zn}$ & $243.8 d$ & radon-220 & ${ }^{220} \mathrm{Rn}$ & $55.6 \mathrm{~s}$ \\
\hline krypton-85 & ${ }^{85} \mathrm{Kr}$ & $10.7 \mathrm{yr}$ & radium-226 & ${ }^{226} \mathrm{Ra}$ & $1,600 \mathrm{yr}$ \\
\hline strontium-89 & ${ }^{89} \mathrm{Sr}$ & $50.5 \mathrm{~d}$ & radium-228 & ${ }^{228} \mathrm{Ra}$ & $5.75 \mathrm{yr}$ \\
\hline strontium-90 & ${ }^{90} \mathrm{St}$ & $29.1 \mathrm{yr}$ & thorium-232 & ${ }^{232} \mathrm{Th}$ & $1.4 \times 10^{10} \mathrm{yr}$ \\
\hline niobium-95 & ${ }^{95} \mathrm{Nb}$ & $35.0 \mathrm{~d}$ & uranium total & $\begin{array}{l}U \text { or } \\
\text { uranium }\end{array}$ & - \\
\hline zirconium-95 & ${ }^{95} \mathrm{Zr}$ & $64.0 \mathrm{~d}$ & uranium-234 & ${ }^{234} \mathrm{U}$ & $2.4 \times 10^{5} \mathrm{yr}$ \\
\hline molybdenum-99 & ${ }^{99} \mathrm{Mo}$ & $2.7 \mathrm{~d}$ & uranium-235 & ${ }^{235} \mathrm{U}$ & $7 \times 10^{8} \mathrm{yr}$ \\
\hline technetium-99 & ${ }^{99} \mathrm{Tc}$ & $2.12 \times 10^{5} \mathrm{yr}$ & uranium-236 & ${ }^{236} \mathrm{U}$ & $2.3 \times 10^{7} \mathrm{yr}$ \\
\hline ruthenium-103 & ${ }^{103} \mathrm{Ru}$ & $39.4 d$ & uranium-238 & ${ }^{238} \mathrm{U}$ & $4.5 \times 10^{9} \mathrm{yr}$ \\
\hline ruthenium-106 & ${ }^{106} \mathrm{Ru}$ & $1.0 \mathrm{yr}$ & plutonium-238 & ${ }^{238} \mathrm{Pu}$ & $87.7 \mathrm{yr}$ \\
\hline $\operatorname{tin}-113$ & ${ }^{113} \mathrm{Sn}$ & $115 \mathrm{~d}$ & neptunium-239 & ${ }^{239} \mathrm{~Np}$ & $2.4 d$ \\
\hline antimony-125 & ${ }^{125} \mathrm{Sb}$ & $2.7 \mathrm{yr}$ & plutonium-239 & ${ }^{239} \mathrm{Pu}$ & $2.4 \times 10^{4} \mathrm{yr}$ \\
\hline iodine-129 & 129 & $1.7 \times 10^{7} \mathrm{yr}$ & plutonium-240 & ${ }^{240} \mathrm{Pu}$ & $6537 \mathrm{yr}$ \\
\hline iodine-131 & 1311 & $8.0 \mathrm{~d}$ & plutonium-241 & ${ }^{241} \mathrm{Pu}$ & $14.4 \mathrm{yr}$ \\
\hline barium-133 & ${ }^{133} \mathrm{Ba}$ & $10.53 \mathrm{yr}$ & americium-241 & ${ }^{241} \mathrm{Am}$ & $433 \mathrm{yr}$ \\
\hline cesium-134 & ${ }^{134} \mathrm{Cs}$ & $2.1 \mathrm{yr}$ & & & \\
\hline
\end{tabular}


HNF-EP-0573-6

This page intentionally left blank. 
HNF-EP-0573-6

APPENDIX C

AMBIENT AIR MONITORING FIGURES AND TABLES 
HNF-EP-0573-6

This page intentionally left blank. 
Figure C-1. The $100 \mathrm{~N}$ Reactor Area Air Sampler Locations.

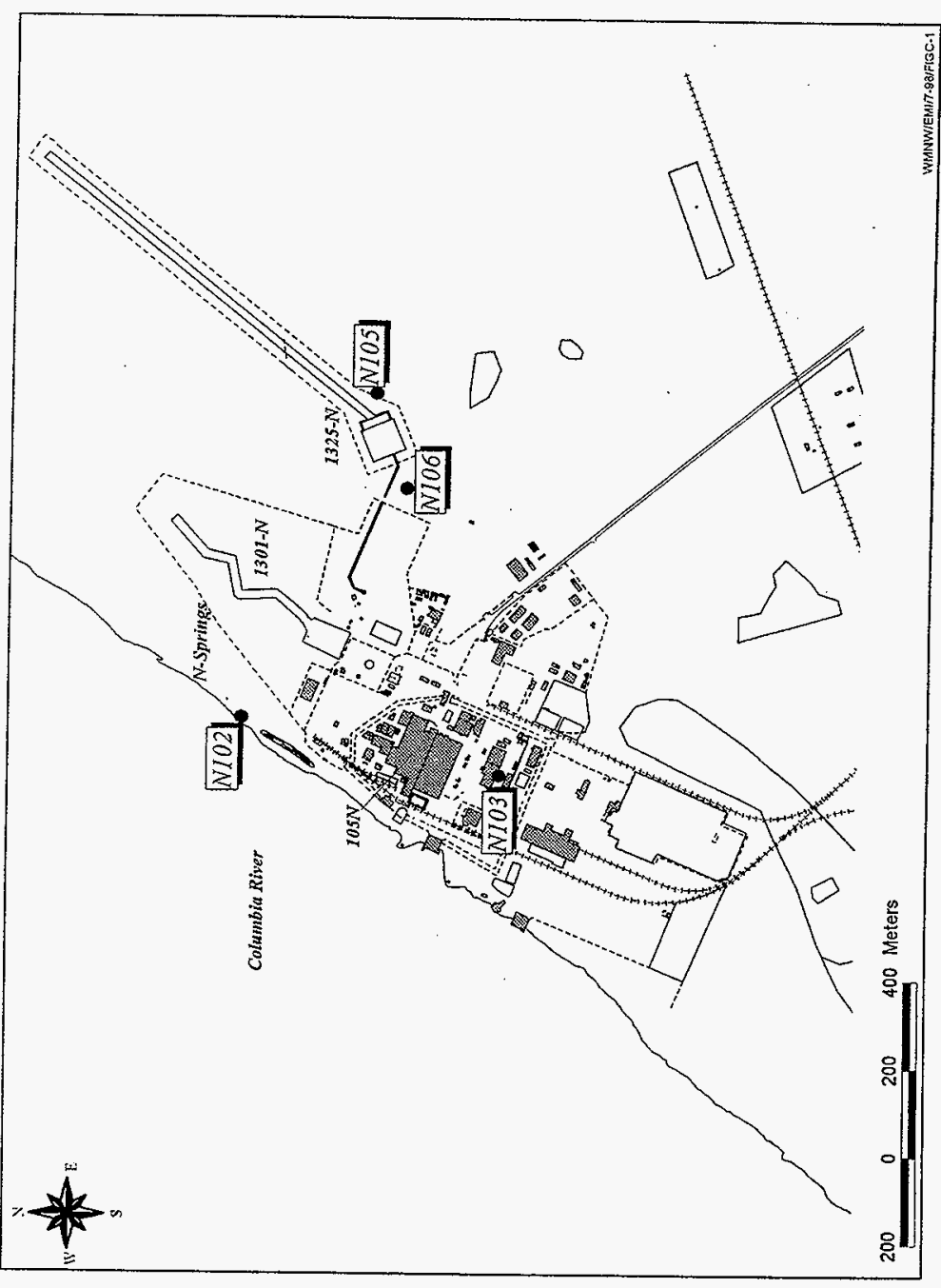


HNF-EP-0573-6

Figure C-2. The $100 \mathrm{KE} / \mathrm{KW}$ Reactors Area Air Sampler Locations.

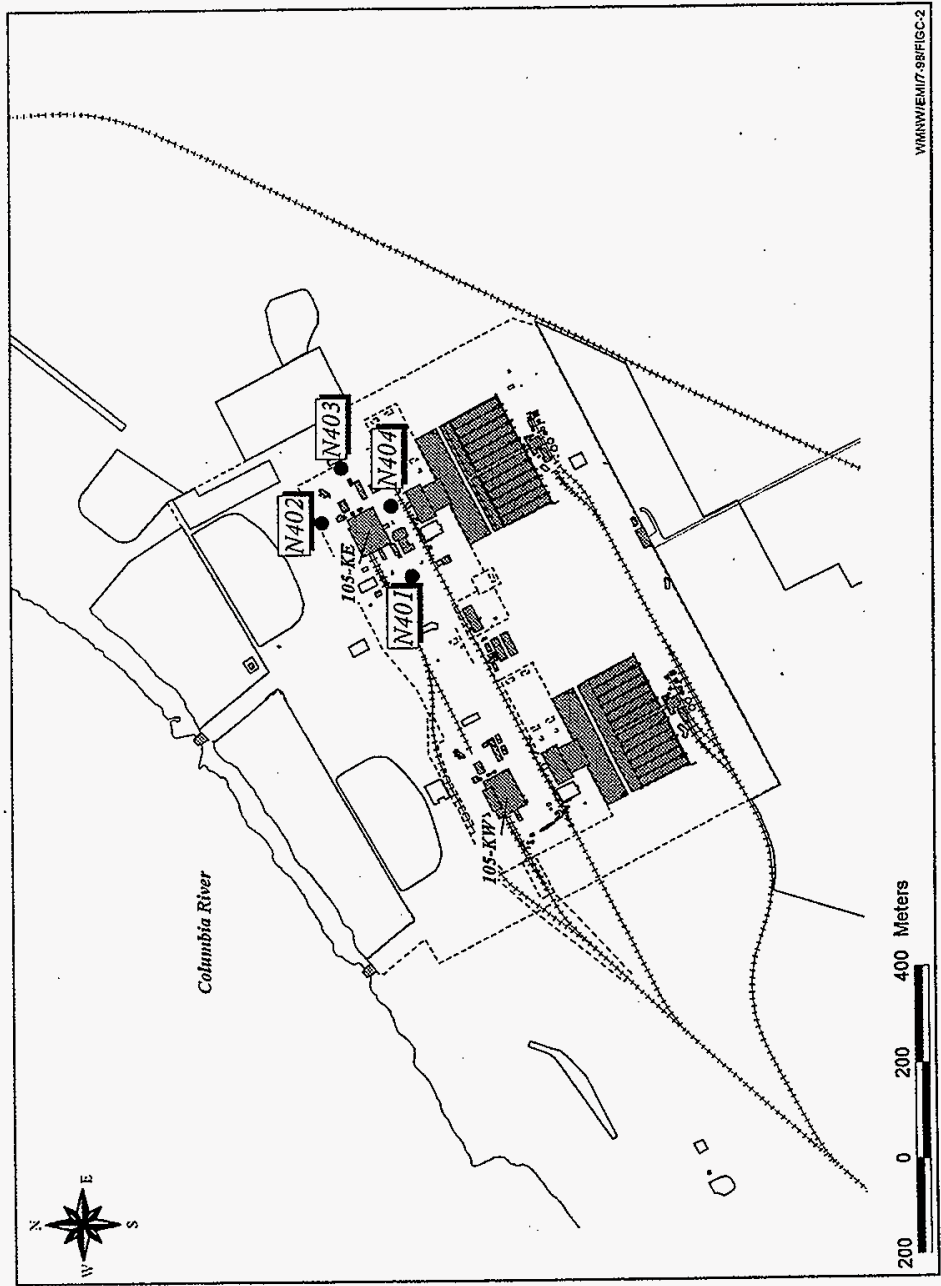




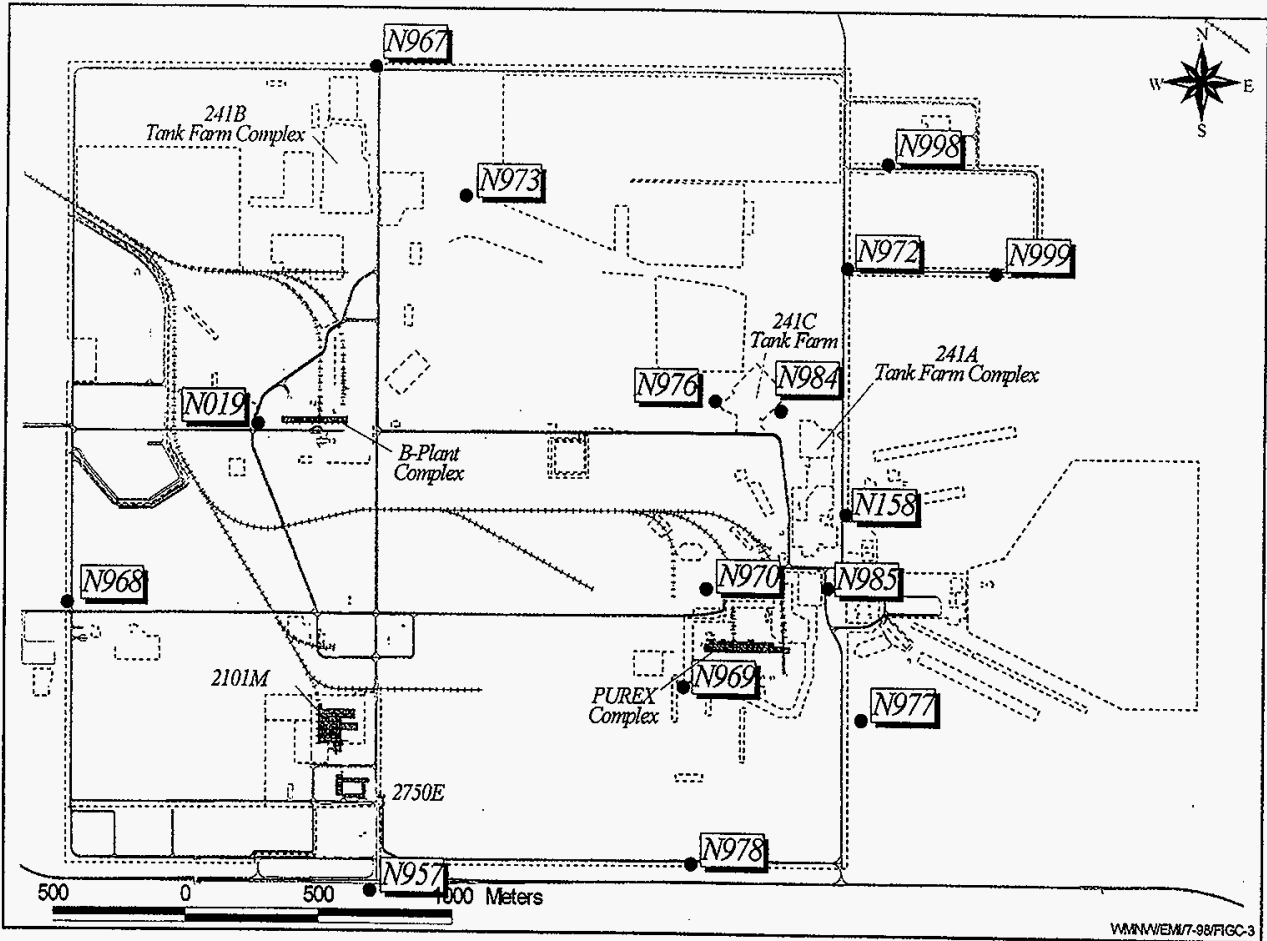


HNF-EP-0573-6

Figure C-4. The 200 West Area Air Sampler Locations.

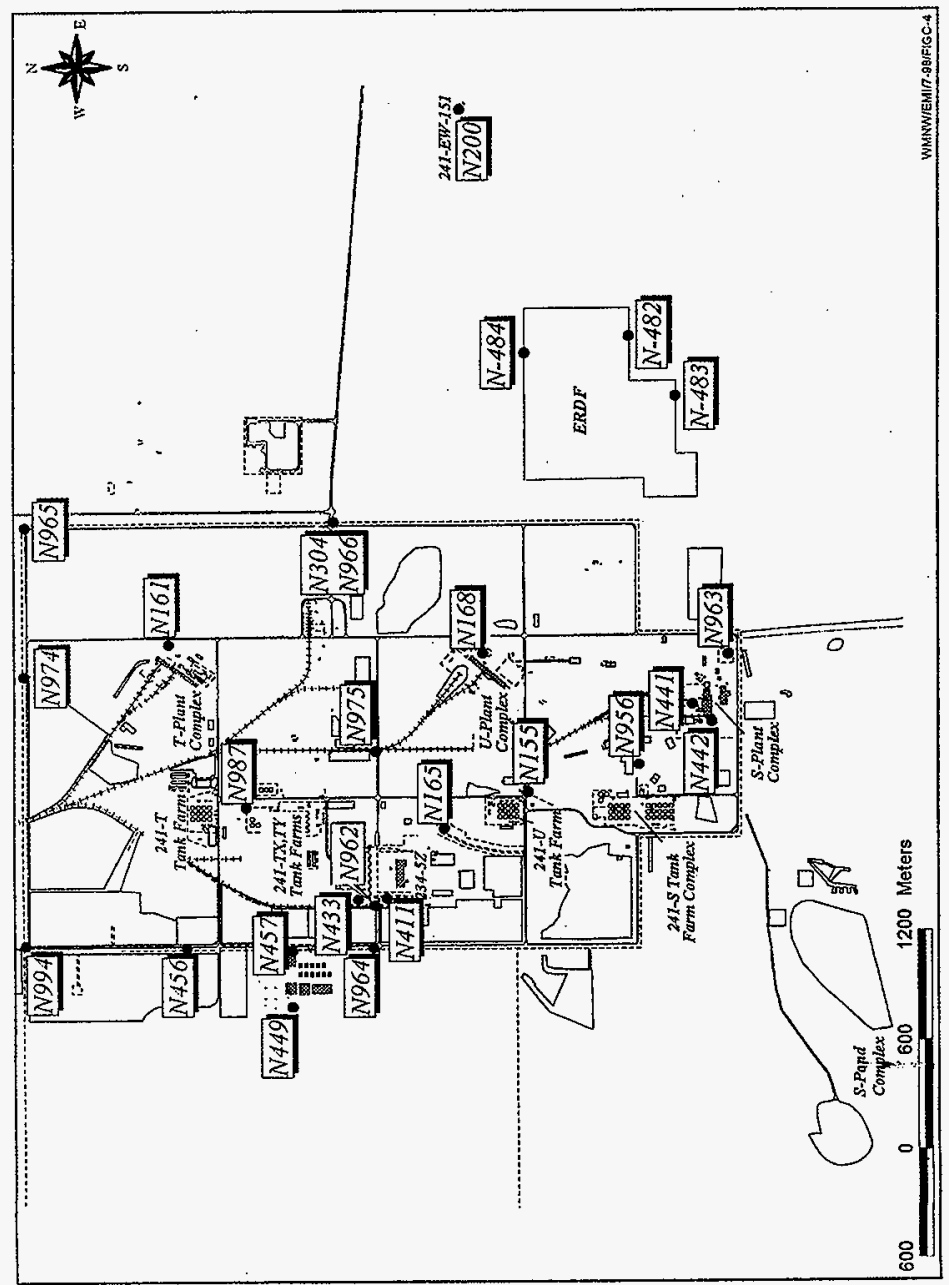


Figure C-5. The 300 Area Air Sampler Locations.

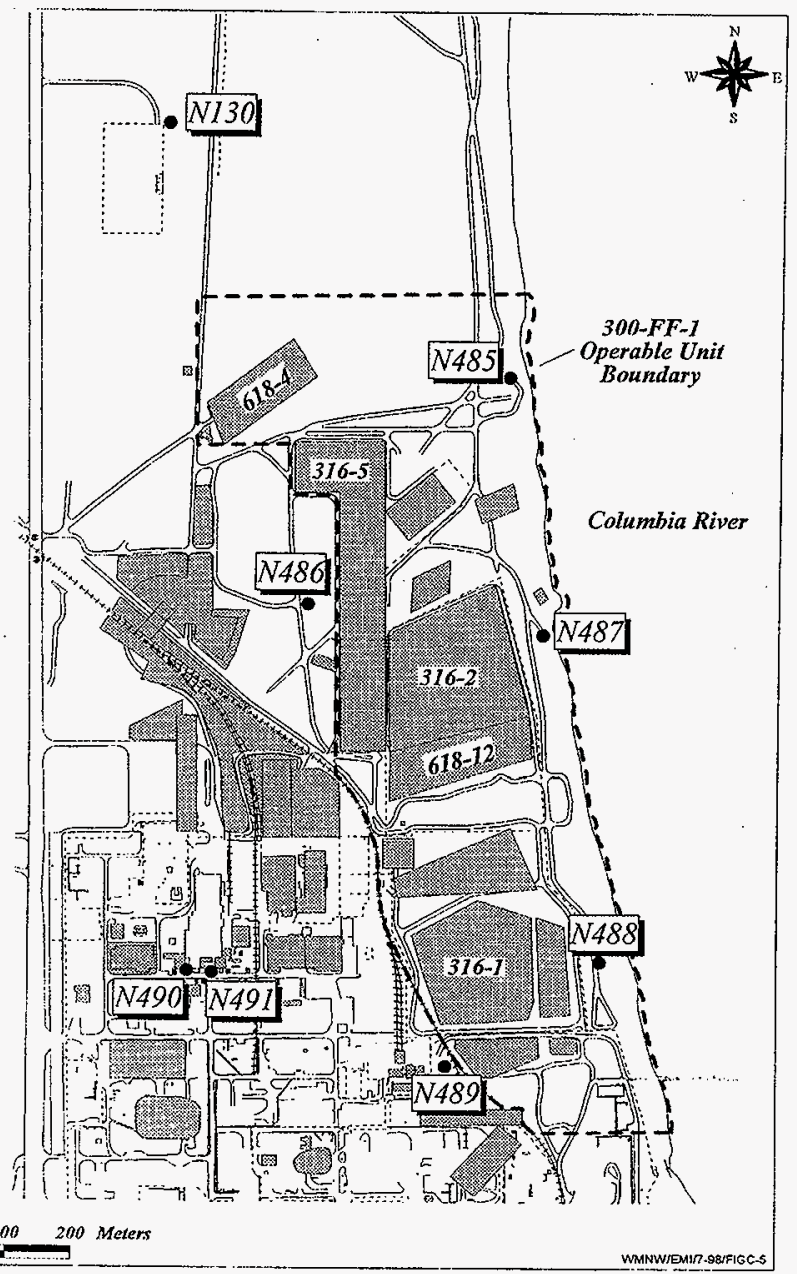


Figure C-6. The 600 Area Air Sampler Locations.

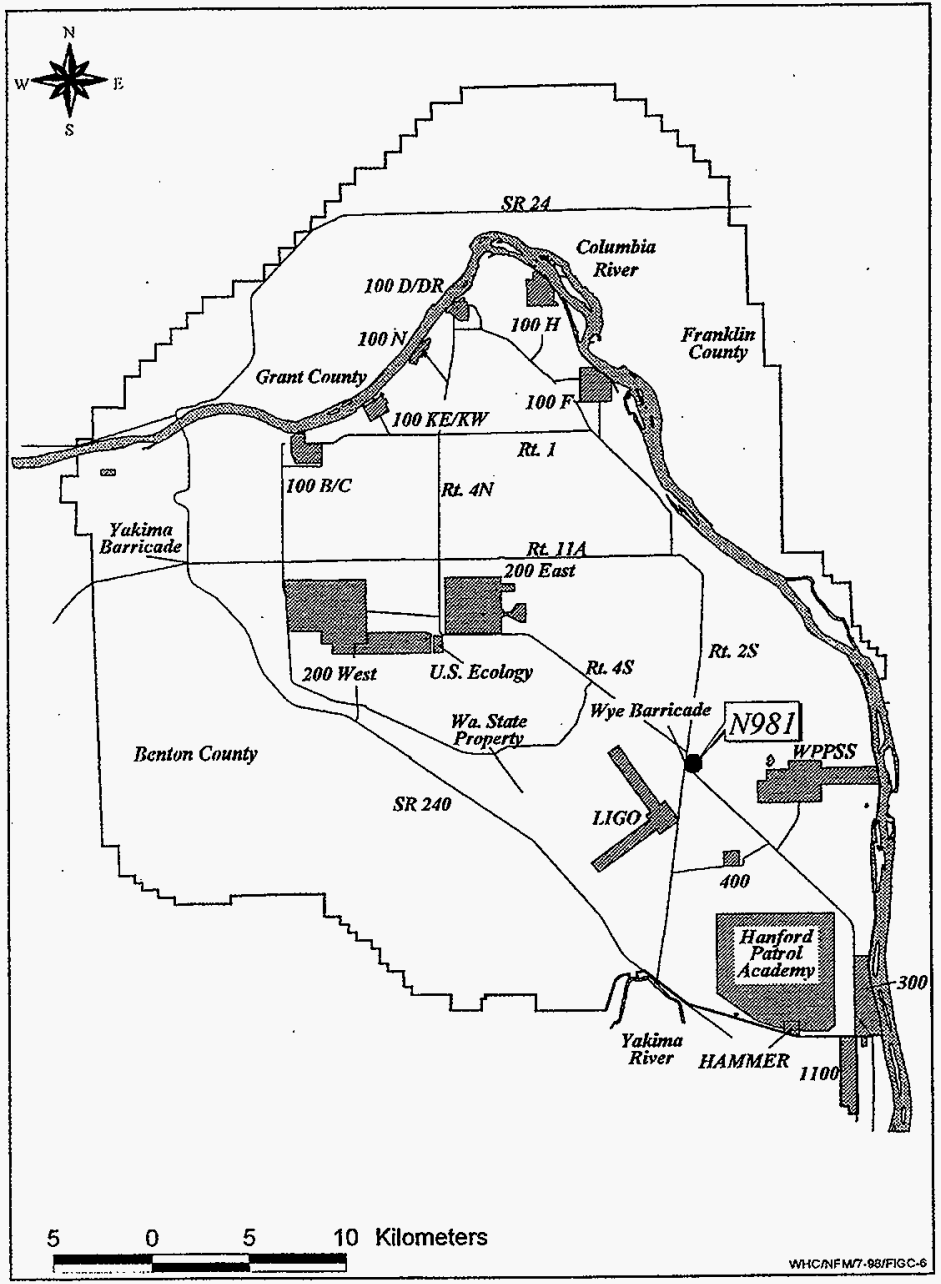


Figure C-7. The $100 \mathrm{~B} / \mathrm{C}$ Air Sampler Locations.

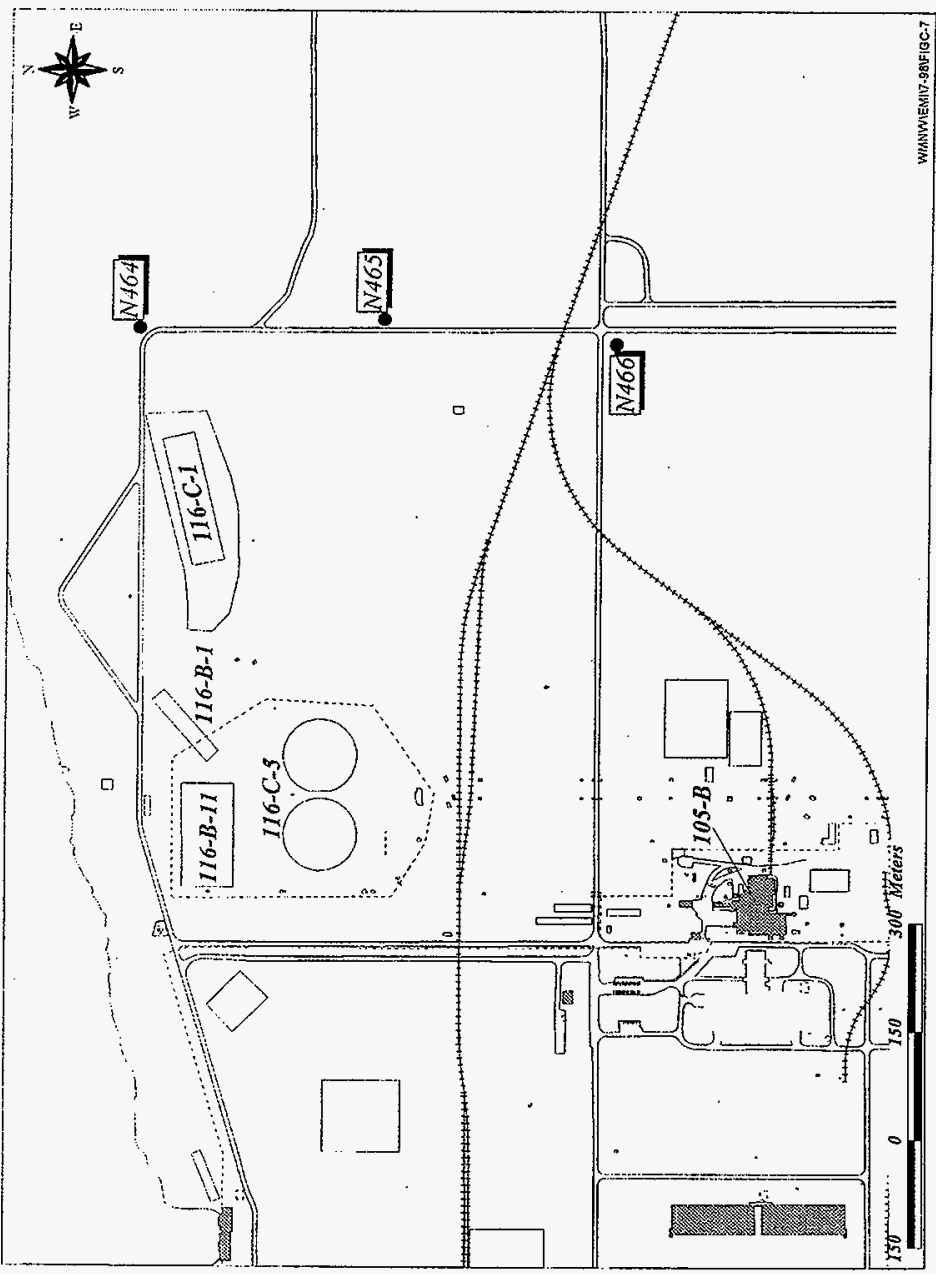


HNF-EP-0573-6

Figure C-8. The 100 D/DR Air Sampler Locations.

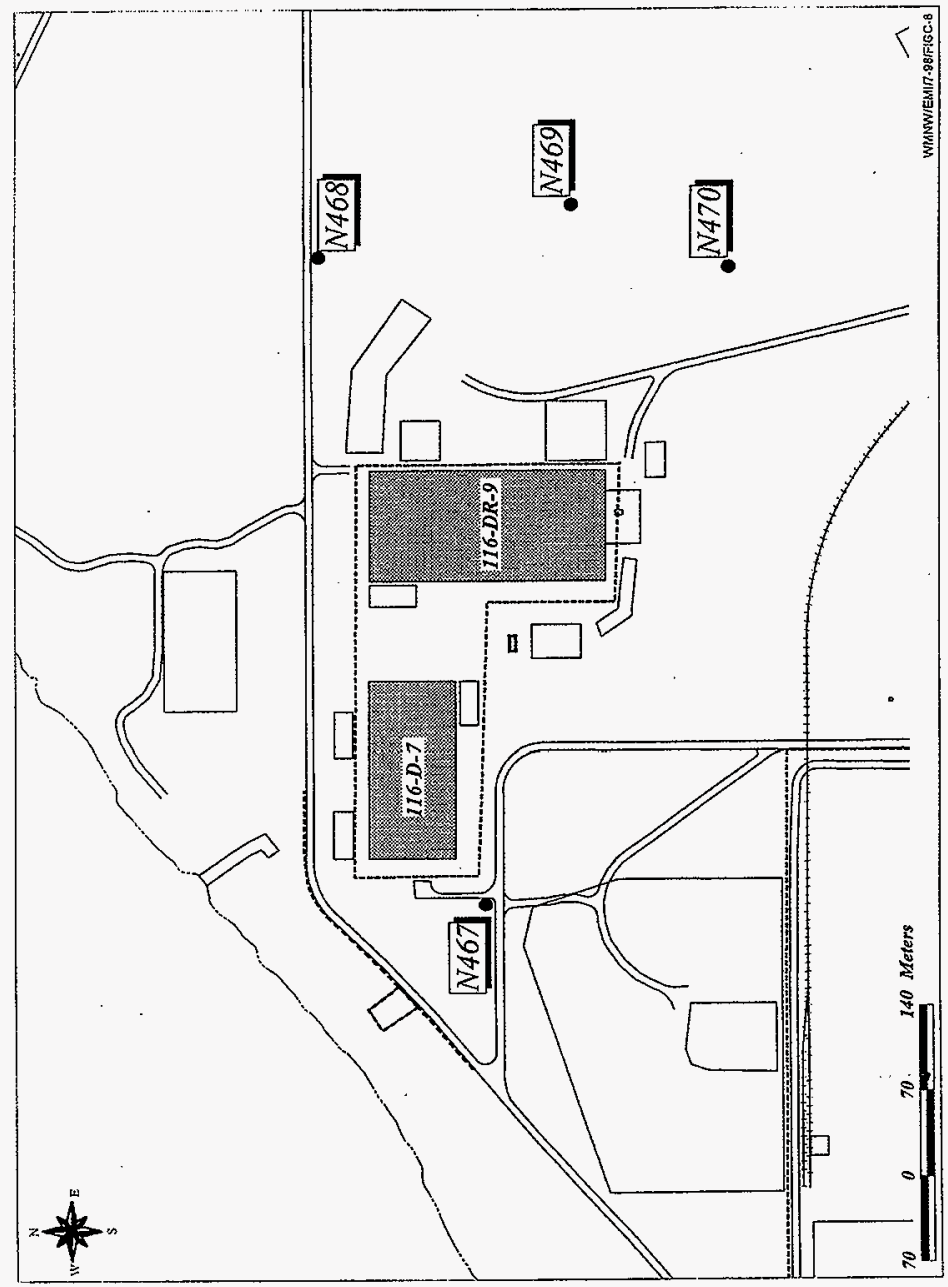


Figure C-9. The Environmental Restoration Disposal Facility Air Sampler Locations.

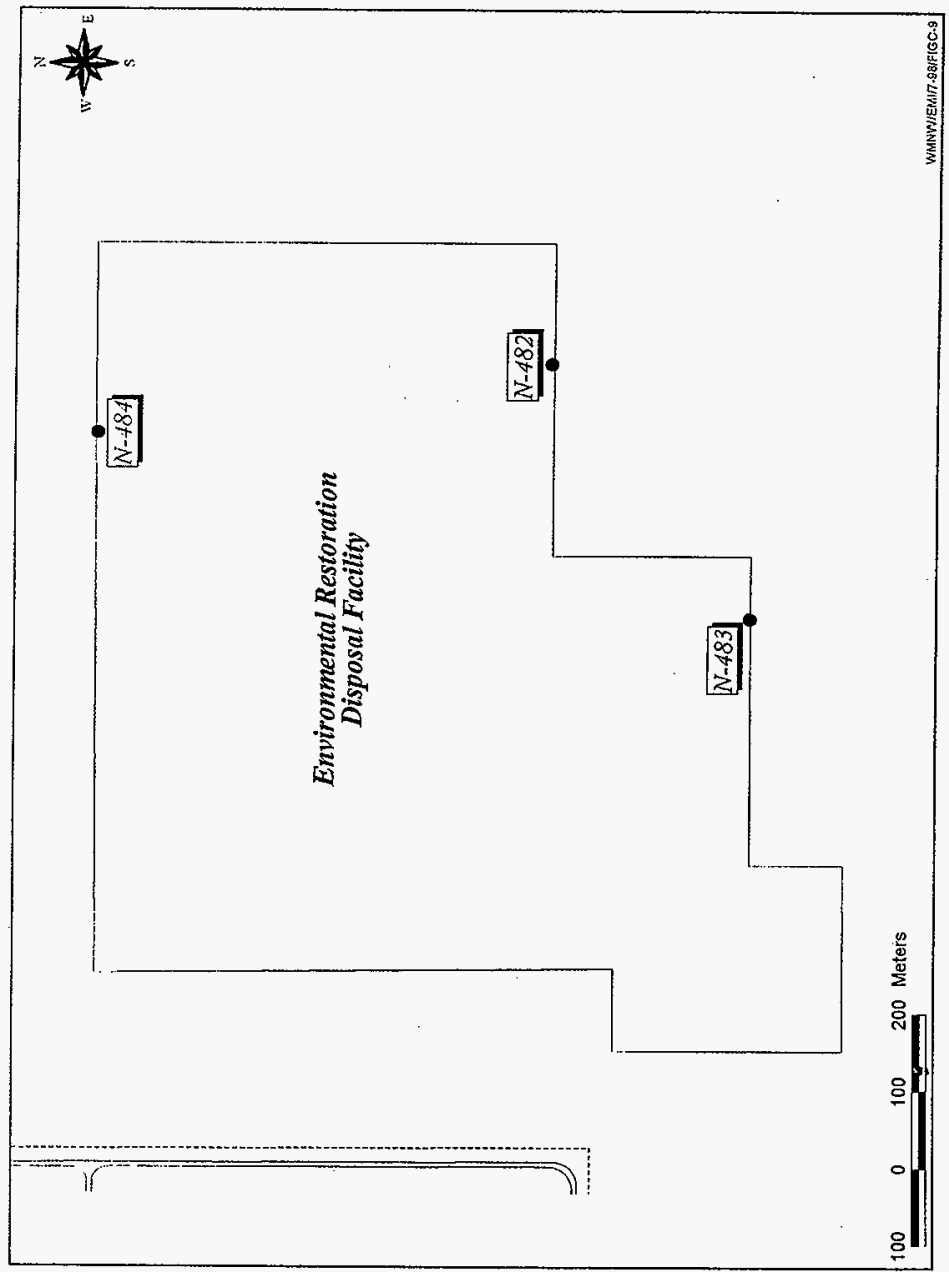


Table C-1: 1997 Air Sample Results, 100-N Area (pCi/m³).

\begin{tabular}{|c|c|c|c|c|}
\hline \multirow[b]{2}{*}{ Site } & \multirow{2}{*}{ Isotope } & $06 / 20 / 97$ & $11 / 06 / 97$ & Average \\
\hline & & Result \pm Uncertainty & Result \pm Uncertainty & Result \pm 2SEM \\
\hline \multirow{4}{*}{$\begin{array}{l}\text { N102 } \\
(100 \mathrm{~N})\end{array}$} & Sr-90 & $2.9 E-04 \pm 30.07$ & $\mathrm{ND}$ & $.2 .95-04$ \\
\hline & $\mathrm{U}-234$ & $1.8 \mathrm{E}-05 \pm 47.0 \%$ & WD & $1.8 \mathrm{E}-05$ \\
\hline & $\mathrm{U}-235$ & $1.2 \mathrm{E}-05 \pm 60.0 \mathrm{x}$ & AD & $1.2 \mathrm{E}=05$ \\
\hline & $u-238$ & $1.1 \mathrm{E}-05 \pm 60.0 \mathrm{x}$ & RD & $1.3 \mathrm{E}-05$ \\
\hline \multirow{7}{*}{$\begin{array}{c}\text { N103 } \\
(100 \mathrm{~N})\end{array}$} & Co-60 & $1.7 \pm-04 \pm 60.07$ & $\mathrm{ND}$ & $1.7 E-04$ \\
\hline & $5 x-90$ & $3.3 E-04 \pm 50.0 z$ & $\mathrm{ND}$ & $3.3 E-04$ \\
\hline & $\operatorname{cs}-137$ & $1.4 \mathrm{E}-04 \pm 86.0 \%$ & $9.9 E-05 \pm 56.07$ & $1.2 \mathrm{E}-04 \pm 34.37$ \\
\hline & $v-234$ & $2.7 \mathrm{E}-05 \pm 49.0 \mathrm{z}$ & $1.4 \mathrm{E}-05 \pm 59.07$ & $2.0 \mathrm{E}-05 \pm 63.4 \mathrm{x}$ \\
\hline & $u-235$ & $1.4 E-05 \pm 74.0 \%$ & $\mathrm{ND}$ & $2.4 E-05$ \\
\hline & $\mathrm{U}-238$ & $2.0 \mathrm{E}-05 \pm 58.0 \mathrm{x}$ & ND & $2.0 \mathrm{E}-0 \mathrm{~S}$ \\
\hline & Pu239/40 & ND & $1.3 \mathrm{E}-05 \pm 49.02$ & $1.3 E-05$ \\
\hline \multirow{7}{*}{$\begin{array}{l}\text { N105 } \\
\text { (100N) }\end{array}$} & $\cos -60$ & $3.0 \mathrm{E}-04 \pm 20.02$ & $5.2 \mathrm{E}-04 \pm 15.0 \%$ & $4.1 E-04 \pm 53.7 \%$ \\
\hline & $5 r-90$ & $3.2 E-04 \pm 40.02$ & ND & $3.2 \mathrm{E}-04$ \\
\hline & $C_{s}-137$ & $1.8 \mathrm{E}-04=45.0 \%$ & $\mathrm{ND}$ & $1.8 \mathrm{E}-04$ \\
\hline & $v-234$ & $1.5 E-05 \pm 45.0 \%$ & $2.1 \mathrm{E}-05 \pm 52.07$ & $1.8 \mathrm{E}-05 \pm 33.32$ \\
\hline & $\mathrm{U}-23 \mathrm{~s}$ & $\mathrm{ND}$ & $9.75-06 \pm 76.07$ & $9.7 \mathrm{E}-06$ \\
\hline & $\mathrm{u}-238$ & $1.4 E-0.5 \pm 48.07$ & $2.4 \mathrm{E}-05 \pm 46.03$ & $1.9 \mathrm{E}-05 \pm 52.67$ \\
\hline & Pu239/40 & $3.78-06 \pm 91.0 \%$ & ND & $3.78-06$ \\
\hline \multirow{5}{*}{$\begin{array}{l}N 106 \\
(100 \mathrm{~N})\end{array}$} & Co- 60 & $1.4 \mathrm{E}-04 \pm 41.07$ & $8.3 \mathrm{E}-04 \pm 11.0 \mathrm{z}$ & $4.9 E-04 \pm 142 Z$ \\
\hline & $5=-90$ & $3.9 E-04 \pm 30.07$ & SND & $3.9 E-04$ \\
\hline & $v-234$ & $1.2 E-04 \pm 24.0 \%$ & $6.6 \mathrm{E}-05 \pm 28.0 \mathrm{z}$ & $9.3 E-05 \pm 58.1 \%$ \\
\hline & $0-235$ & $1.0 E-04 \pm 26.07$ & WD & $1.0 \mathrm{E}-04$ \\
\hline & $\mathrm{u}-238$ & $6.15-05 \pm 30.07$ & $8.3 E-05 \pm 26.0 \%$ & $7.2 E-05 \pm 30.6 \%$ \\
\hline
\end{tabular}


Table C-2: 1997 Air Sample Results, $100 \cdot \mathrm{K}$ Area $\left(\mathrm{pCi} / \mathrm{m}^{3}\right)$.

\begin{tabular}{|c|c|c|c|c|}
\hline & & $06 / 20 / 97$ & $12 / 18 / 97$ & Average \\
\hline Site & Isotope & Result \pm Uncertainty & Result \pm Uncertainty & Result \pm 2 2SEM \\
\hline \multirow{6}{*}{$\begin{array}{l}\$ 401 \\
(100 \mathrm{~K})\end{array}$} & $5 x-90$ & $8.2 E-04 \pm 16.0 \%$ & $1.0 \Sigma-03 \pm 17.0 z$ & $9.1 \mathrm{E}-04 \pm 19.8 \%$ \\
\hline & Cs-137 & $3.5 \bar{E}-03 \pm 16.0 z$ & $9.08+03 \pm 14.07$ & $6.32-03 \pm 88.0 \%$ \\
\hline & $U-234$ & $1.8 \mathrm{E}-05 \pm 43.0 \mathrm{X}$ & $1.6 \mathrm{E}-05 \pm 41.07$ & $1.7 E-05 \pm 11.8 \%$ \\
\hline & $U-238$ & $9.5 \mathrm{E}-06 \pm 63.04$ & $9.6 \mathrm{E}-06 \pm 55.07$ & $9.5 E-06 \pm 1.07$ \\
\hline & Fu239/40 & No & $2.5 \mathrm{E}-05 \pm 74.0 \mathrm{z}$ & $2.5 E-05$ \\
\hline & Am-241 & $6.3 \mathrm{E}-05 \pm 31.0 \%$ & $4.4 \mathrm{E}-05 \pm 33.08$ & $5.3 E-05 \pm 35.5 x$ \\
\hline \multirow{6}{*}{$\begin{array}{c}\mathrm{N} 402 \\
(100 \mathrm{~K})\end{array}$} & $S x-90$ & $4.8 \mathrm{E}-04 \pm 20.07$ & $2.8 \mathrm{E}-04 \pm 40.07$ & $3.8 E-04 \pm 52.67$ \\
\hline & $C_{s-137}$ & $1.5 \mathrm{E}-04 \pm 40.0 \%$ & $1.1 E-04 \pm 57.0 \%$ & I. $3 \mathrm{E}-04 \pm 30.8 \mathrm{x}$ \\
\hline & $v-234$ & $9.5 \mathrm{E}-06 \pm 58.07$ & $1.2 \mathrm{E}-05 \pm 56.0 \mathrm{x}$ & $1.1 \mathrm{E}-05 \pm 23.37$ \\
\hline & $\mathrm{U}-235$ & $6.8 \mathrm{E}-06 \pm 71.02$ & $7.5 E-06 \pm 70.02$ & $7.1 E-06 \pm 9.8 Z$ \\
\hline & $\mathrm{u}-238$ & $1.1 \mathrm{E}-05 \pm 64.02$ & $1.6 \mathrm{E}-0.5 \pm 42.0 \mathrm{z}$ & $1.4 \mathrm{E}-05 \pm 37.07$ \\
\hline & $\operatorname{An}-241$ & $3.1 E-05 \pm 46.0 \%$ & $3.7 E-05 \pm 36.0 \%$ & $3.4 E-05 \pm 17.6 \%$ \\
\hline \multirow{6}{*}{$\begin{array}{c}\text { N403 } \\
(100 \mathrm{~K})\end{array}$} & $5 x-90$ & $4.3 E-04 \pm 25.0 \%$ & $3.2 \mathrm{E}-04 \pm 30.0 \mathrm{z}$ & $3.8 E-04 \pm 29.37$ \\
\hline & Cs -137 & $8.8 \mathrm{E}-04 \pm 18.07$ & $5.8 \mathrm{E}-04 \pm 24.0 \mathrm{z}$ & $7.3 \mathrm{E}-04 \pm 41.1 \mathrm{Z}$ \\
\hline & U-234 & $1.2 \mathrm{E}-05 \pm 53.0 \mathrm{z}$ & $1.4 \mathrm{E}-05 \pm 47.07$ & $1.3 \mathrm{E}-05 \pm 15.47$ \\
\hline & U-235 & $5.9 z-06 \pm 76.0 z$ & $4.6 \mathrm{E}-06 \pm 82.0 z$ & $5.2 E-06 \pm 24.8 \%$ \\
\hline & $\mathrm{W}-238$ & $8.2 E-06 \pm 61.0 \%$ & $1.8 \mathrm{E}-05 \pm 44.0 \mathrm{z}$ & $1.3 E-05 \pm 74.8 x$ \\
\hline & Am-241 & $2.0 \mathrm{E}-05, \pm 57.0 \%$ & $3.2 \mathrm{E}-05 \pm 46.0 \%$ & $2.6 \mathrm{E}-05 \pm 46.2 \mathrm{z}$ \\
\hline \multirow{6}{*}{$\begin{array}{l}1404 \\
(100 \mathrm{~K})\end{array}$} & $S x-90$ & $5.0 E-04 \pm 20.0 x$ & $1.6 \mathrm{E}-03 \pm 16.07$ & $1.1 E-03 \pm 104 \%$ \\
\hline & Cs-137 & $2.82-04 \pm 33.0 \%$ & $1.8 \mathrm{E}-04 \pm 38.0 \%$ & $1.8 \mathrm{E}-04 \pm 0.07$ \\
\hline & $\mathrm{U}-234$ & $1.8 \mathrm{E}-05 \pm 46.02$ & $1.7 z-05 \pm 40.02$ & $1.7 E-05 \pm 5.78$ \\
\hline & $\mathrm{u}-235$ & $5.5 E-06 \pm 82.07$ & $9.5 E-06 \pm 54.0 \%$ & $7.5 \mathrm{E}-06 \pm 53.37$ \\
\hline & $0-238$ & $2.0 \mathrm{E}-05 \pm 41.0 \%$ & $1.4 E-05 \pm 41.0 \%$ & $2.7 \mathrm{E}-05 \pm 35.37$ \\
\hline & $A m-241$ & $3.4 \mathrm{E}-05 \pm 40.0 x$ & $3.0 \mathrm{E}-05 \pm 42.07$ & $3.28-05 \pm 12.5 \%$ \\
\hline
\end{tabular}


Table C-3: 1997 Air Sample Resuits, 200 Areas $\left(\mathrm{pCi} / \mathrm{m}^{3}\right)$.

\begin{tabular}{|c|c|c|c|c|}
\hline & & $06 / 30 / 97$ & $12 / 31 / 97$ & Average \\
\hline Site & Isotope & Result \pm Uncertainty & Result \pm Uncertainty & Result \pm 2 SEM \\
\hline \multirow{6}{*}{$\begin{array}{l}\text { No19 } \\
(200 E)\end{array}$} & $5 x-90$ & $3.02-04 \pm 30.07$ & $9.8 \mathrm{E}-03 \pm 12.07$ & $5.1 E-03 \pm 1887$ \\
\hline & $\mathrm{Cs}-137$ & ND & $1.9 E-02 \pm 18.07$ & $1.9 E-02$ \\
\hline & $v-234$ & $2.12-05 \pm 35.0 \%$ & $1.7 \mathrm{E}-05 \pm 44.0 \%$ & $1.95-05 \pm 21.1 \%$ \\
\hline & $v-235$ & $1.5 E-05 \pm 43.07$ & $6.6 \mathrm{E}-06 \pm 68.0 \mathrm{z}$ & $1.1 \mathrm{E}-05 \pm 77.8 \%$ \\
\hline & $\mathrm{v}-238$ & $1.9 \mathrm{E}-05 \pm 35.0 \%$ & $1.3 \mathrm{E}-05 \pm 51.07$ & $1.6 \mathrm{E}-0.5 \pm 37.57$ \\
\hline & $P(239 / 40$ & WD & $4.3 E-06 \pm 83.0 x$ & $4.3 E-06$ \\
\hline \multirow{6}{*}{$\begin{array}{l}\text { N155 } \\
\text { (200\%) }\end{array}$} & $5 x-90$ & $\mathrm{ND}$ & $2.4 E-04 \pm 50.07$ & $2.4 E-04$ \\
\hline & $\operatorname{cs}-137$ & $9.9 \mathrm{E}-05 \pm 45.0 \%$ & SID & $9.9 E-05$ \\
\hline & $\mathrm{U}-234$ & $1.6 \mathrm{E}-0.5 \pm 39.07$ & $1.4 \mathrm{E}-0.5 \pm 48.0 \%$ & 1. $5 E-0.5 \pm 13.37$ \\
\hline & $\mathrm{u}-235$ & $1.0 \mathrm{E}-05 \pm 48.0 \%$ & $4.8 \mathrm{E}-06 \pm 83,0 \%$ & $7.4 \mathrm{E}-06 \pm 70.37$ \\
\hline & $\mathrm{t}-238$ & $1.0 \mathrm{E}-05 \pm 49.0 \mathrm{z}$ & $1.2 E-05 \pm 51.07$ & $1 . \pm E-05 \pm 18.27$ \\
\hline & $201239 / 40$ & $1.9 \mathrm{E}-05 \pm 44.0 \mathrm{z}$ & $1.4 \mathrm{E}-05 \pm 48.07$ & $1.7 E-05 \pm 30.37$ \\
\hline \multirow{5}{*}{$\begin{array}{l}\text { N158 } \\
(200 E)\end{array}$} & $5 x-90$ & ND & $1.8 \mathrm{E}-04 \pm 60.0 \%$ & $1.6 \mathrm{E}-04$ \\
\hline & $C s-137$ & ND & $2.8 \mathrm{E}-04 \pm 31.02$ & $2.8 \mathrm{E}-04$ \\
\hline & $\mathrm{v}-234$ & $1.7 \mathrm{E}-05 \pm 45.0 \mathrm{z}$ & $1.6 \mathrm{E}-0.5 \pm 48,0 z$ & $1.6 \mathrm{E}-05 \pm 6.1 \mathrm{z}$ \\
\hline & $\mathrm{v}-235$ & ND & $1.0 \bar{x}-05 \pm 62.0 \%$ & $1.0 E-05$ \\
\hline & $\mathrm{U}-238$ & $1.6 \mathrm{~g}-05 \pm 44.0 \%$ & $6.0 \mathrm{E}=06 \pm 77.07$ & $1.1 E-05 \pm 90.9 \%$ \\
\hline \multirow{5}{*}{$\begin{array}{c}N 161 \\
(200 W)\end{array}$} & $S r-90$ & $1.8 \mathrm{E}-04 \pm 60.0 \%$ & $1.8 \mathrm{E}-04 \pm 60.02$ & $1.8 \mathrm{E}-04 \pm 0.0 \mathrm{x}$ \\
\hline & $U-234$ & $8.4 \mathrm{E}-06 \pm 60.0 \mathrm{Y}$ & $1.6 \mathrm{E}-05 \pm 51.07$ & $1.2 E-05 \pm 62.3 \%$ \\
\hline & $u-235$ & $7.18-06 \pm 69.07$ & $9.6 \mathrm{E}-06 \pm 68.07$ & $8.32-06 \pm 29.92$ \\
\hline & $v-238$ & $1.2 F-05 \pm 49.0 \%$ & $1.7 E-05 \pm 50.0 \%$ & $1.4 E-05 \pm 34.5 \%$ \\
\hline & Pu239/40 & $2.9 \mathrm{E}-05 \pm 37.0 \mathrm{z}$ & $9.6 \mathrm{E}-06 \pm 71.0 \%$ & $1.9 \pm-05 \pm 1002$ \\
\hline \multirow{5}{*}{$\begin{array}{c}N 165 \\
(200 \mathrm{~W})\end{array}$} & $5 r-90$ & $3.7 \pm-04 \pm 30.07$ & $2.9 \mathrm{E}-04 \pm 40.0 \%$ & $3.3 E-04 \pm 24.2 z$ \\
\hline & $\mathrm{Cs}-137$ & $7.38-05 \pm 74.07$ & $7.1 \mathrm{E}-05 \pm 69.0 \mathrm{z}$ & $7.1 \mathrm{E}-05 \pm 0.0 \%$ \\
\hline & $u-234$ & $1.7 \mathrm{z}-05 \pm 46.0 \mathrm{x}$ & $1.18-05 \pm 52.0 \%$ & $1.4 \mathrm{E}-05 \pm 42.9 \%$ \\
\hline & $t-235$ & $9.6 \mathrm{E}-06 \pm 63.07$ & $1.0 \mathrm{E}-05 \pm 62.0 \%$ & $9.8 \mathrm{E}-06 \pm 4.1 \mathrm{z}$ \\
\hline & $U-238$ & $1.0 E-05 \pm 63.07$ & $8.8 E-0.6 \pm 60.07$ & $9.4 \mathrm{E}-06 \pm 12.87$ \\
\hline
\end{tabular}


Table C-3 (Cont.): 1997 Air Sample Results, 200 Areas $\left(\mathrm{pCi} / \mathrm{m}^{3}\right)$.

\begin{tabular}{|c|c|c|c|c|}
\hline & & $06 / 30 / 97$ & $12 / 30 / 97$ & Average \\
\hline Site & Isotope & Result \pm Uncertainty & Result \pm Uncertainty & Result \pm 2SEM \\
\hline \multirow{2}{*}{$\begin{array}{c}N 165 \\
(200 W)\end{array}$} & $\mathrm{Pu} 239 / 40$ & $1.3 E-04 \pm 32.0 z$ & $6.05-05 \pm 38.0 \%$ & $9.5 E-05 \pm 73.7 \%$ \\
\hline & $A n-241$ & $6.1 \mathrm{~F}-05 \pm 39.0 \%$ & $4.5 \mathrm{E}-05 \pm 42.0 z$ & $5.3 E-05 \pm 30.27$ \\
\hline \multirow{5}{*}{$\begin{array}{c}N 168 \\
(200 \mathrm{~W})\end{array}$} & $5 x-90$ & IND & $1.7 \pm-04 \pm 60.02$ & $1.7 \mathrm{E}-04$ \\
\hline & Cs -137 & $1.6 \mathrm{E}-04 \pm 53.0 \mathrm{z}$ & $1.5 E-04 \pm 51.07$ & $1.6 \mathrm{E}-04 \pm 6.5 \%$ \\
\hline & $U-234$ & $2.5 E-05 \pm 40.07$ & $2.8 \mathrm{E}-05 \pm 48.07$ & $2.7 \mathrm{E}-05 \pm 11.37$ \\
\hline & $v-238$ & $2.2 \mathrm{E}-0.5 \pm 43.0 z$ & $1.6 \mathrm{E}-05 \pm 49.0 \%$ & $1.9 \mathrm{E}-05 \pm 31.6 \%$ \\
\hline & Fu239/40 & $1.5 \mathrm{E}-05 \pm 48.0 \mathrm{z}$ & $4.0 \mathrm{E}-06 \pm 91.0 \%$ & $9.5 \mathrm{E}-06 \pm 1157$ \\
\hline \multirow{5}{*}{$\begin{array}{c}\mathrm{N} 200 \\
(200 \mathrm{E})\end{array}$} & $5 x-90$ & ND & $3.5 \mathrm{E}-04 \pm 30.0 \%$ & $3.5 E-04$ \\
\hline & $C_{s-137}$ & $1.5 \mathrm{E}-04 \pm 44.0 \%$ & $N D$ & $1.5 \mathrm{E}-04$ \\
\hline & $\mathrm{v}-234$ & $2.2 E-05 \pm 42.0 \%$ & $1.2 \mathrm{E}-05 \pm 61.0 \mathrm{Z}$ & $1.7 \mathrm{E}-05 \pm 58.8 \%$ \\
\hline & $u-235$ & $8.9 E-06 \pm 69.0 \%$ & $6.98-05 \pm 68^{\circ} .07$ & $7.9 E-06 \pm 25.37$ \\
\hline & $u-238$ & $2.15-05 \pm 42.0 \%$ & $1.8 \Sigma-05 \pm 44.0 z$ & $1.9 E-0.5 \pm 15.4 \%$ \\
\hline \multirow{5}{*}{$\begin{array}{c}N 304 \\
(200 W)\end{array}$} & $5 x-90$ & $\cdot \mathrm{ND}$ & $3.8 E-04 \pm 30.07$ & $3.8 \mathrm{E}-04$ \\
\hline & $U-234$ & $1.6 \mathrm{E}-05 \pm 49.07$ & $1.7 E-05 \pm 48.07$ & $1.6 \mathrm{E}-0.5 \pm 6.1 \%$ \\
\hline & $v-235$ & $6.3 \mathrm{E}-06 \pm 76.07$ & $6.18-06 \pm 81.07$ & $6.2 E-06 \pm 3.2 \%$ \\
\hline & $\mathrm{u}-238$ & $1.8 E-05 \pm 43.0 \%$ & ND & $1.8 \mathrm{E}-05$ \\
\hline & Pu239/40 & $1.2 \mathrm{E}-05 \pm 51.02$ & $9.4 \mathrm{E}-06 \pm 57.0 \mathrm{x}$ & $1.1 E \sim 05 \pm 24.37$ \\
\hline \multirow{6}{*}{$\begin{array}{c}1433 \\
(200 \mathrm{~W})\end{array}$} & $5 r-90$ & $5.6 E-04 \pm 20.07$ & $1.2 \mathrm{E}-03 \pm 25.0 \%$ & $8.8 \mathrm{E}-04 \pm 72.77$ \\
\hline & $\mathrm{Cs}-137$ & $6.5 E-05 \pm 74.0 \%$ & ND & $6.5 \mathrm{E}-05$ \\
\hline & $v-234$ & $1.3 E-05 \pm 46.02$ & $1.2 \mathrm{E}-05 \pm 45.07$ & $1.3 \mathrm{E}-05 \pm 8.07$ \\
\hline & $u-238$ & $6.2 E-06 \pm 67.07$ & $9.4 E-06 \pm 52.07$ & $7.8 \mathrm{E}-06 \pm 41.07$ \\
\hline & $P u 239 / 40$ & ND & $9.4 \mathrm{E}-06 \pm 83.07$ & $9.4 \mathrm{E}-06$ \\
\hline & Am-241 & $2.0 \mathrm{E}-05 \pm 50.0 \%$ & $1.8 \bar{z}-0.5 \pm 63.0 \%$ & $1.9 E-05 \pm 10.57$ \\
\hline \multirow{4}{*}{$\begin{array}{l}-N 441 \\
(200 \mathrm{~W})\end{array}$} & $5 x-90$ & $7.0 \mathrm{E}-04 \pm 25.0 \mathrm{z}$ & $2.1 E-04 \pm 50.0 z$ & $4.5 E-04 \pm 107 Z$ \\
\hline & Cs-137 & $1.1 E-04 \pm 51.08$ & ND & $1.1 E-04$ \\
\hline & $\mathrm{U}-234$ & $2.0 \mathrm{E}-05 \pm 42.0 \mathrm{x}$ & $1.5 E+05 \pm 52.0 \%$ & $1.8 E-05 \pm 28.6 \%$ \\
\hline & $U-235$ & $5.8 \mathrm{E}-06 \pm 82.0 z$ & $9.6 \mathrm{E}-06 \pm 62.07$ & $7.7 E-06 \pm 49.4 x$ \\
\hline
\end{tabular}


Table C-3 (Cont.): 1997 Air Sample Results, 200 Areas $\left(\mathrm{pCi} / \mathrm{m}^{3}\right)$.

\begin{tabular}{|c|c|c|c|c|}
\hline \multirow[b]{2}{*}{ Site } & \multirow[b]{2}{*}{ Isotope } & $06 / 30 / 97$ & $12 / 30 / 97$ & - Average \\
\hline & & Result I Uncertainty & Result \pm Uncertainty & Result \pm 2 SEM \\
\hline \multirow{2}{*}{$\begin{array}{c}N 441 \\
(200 W)\end{array}$} & $\mathrm{U}-238$ & $1.9 \mathrm{E}-05 \pm 44.0 \%$ & $1.7 E-05 \pm 50.0 \%$ & $1.8 \mathrm{E}-05 \pm 11.1 \mathrm{z}$ \\
\hline & Pu239/40 & $2.1 E-05 \pm 42.07$ & ND & $2.15-05$ \\
\hline \multirow{5}{*}{$\begin{array}{c}\text { N442 } \\
(200 W)\end{array}$} & $5 x-90$ & $3.7 E-04 \pm 40.07$ & $4.3 E-04 \pm 30.0 \%$ & $4.028-04 \pm 15.0 z$ \\
\hline & $C_{5}-137$ & ND & $2.4 \mathrm{E}-04 \pm 36.0 z$ & $2.4 E-04$ \\
\hline & $\mathrm{v}-234$ & $1.2 E-05 \pm 71.07$ & $1.6 \mathrm{E}-05 \pm 52.0 \%$ & $1.4 \mathrm{E}-05 \pm 28.6 \%$ \\
\hline & $\mathrm{U}-235$ & $8.3 \mathrm{E}-06 \pm 76.02$ & mo & 8.3E-06 \\
\hline & $\mathrm{y}-238$ & $7.6 \mathrm{E}-06 \pm 76.0 \mathrm{z}$ & $1.1 \mathrm{E}-05 \pm 58.07$ & $9.3 E-86 \pm 36.6 \%$ \\
\hline \multirow{4}{*}{$\begin{array}{c}N 449 \\
(200 W)\end{array}$} & $5 r-90$ & $8.2 \mathrm{E}-04 \pm 20.0 \mathrm{z}$ & ID & $8.2 E-04$ \\
\hline & $v-234$ & $8.9 \mathrm{E}-06 \pm 52.0 \mathrm{Z}$ & $1.6 \mathrm{E}-05 \pm 44.0 \%$ & $1.2 \mathrm{E}-05 \pm 57.07$ \\
\hline & $\mathrm{v}-235$ & $5.8 \mathrm{E}-06 \pm 75.0 \%$ & $1.6 \mathrm{E}-05 \pm 46.07$ & $1.18-05 \pm 93.67$ \\
\hline & $\mathrm{U}-238$ & $4.8 \mathrm{E}-06 \pm 67.0 \mathrm{z}$ & $1.0 \mathrm{E}-05 \pm 54.0 \mathrm{X}$ & $7.4 \mathrm{E}-06 \pm 70.37$ \\
\hline \multirow{4}{*}{$\begin{array}{c}1456 \\
\text { (200W) }\end{array}$} & $5 x-90$ & $6.75-04 \pm 20.0 \%$ & $.1 .3 \mathrm{E}-04 \pm 70.02$ & $4.0 \mathrm{E}-04 \pm 135 \%$ \\
\hline & $v-234$ & $1.5 z-05 \pm 40.0 \pi$ & $2.4 E-05 \pm 37.02$ & $2.05-05 \pm 46.2 x$ \\
\hline & $U-235$ & $4.7 \mathrm{E}-06 \pm 72.0 \mathrm{z}$ & $9.1 \mathrm{E}-06 \pm 60.0 \%$ & $6.9 \mathrm{E}-06 \pm 63.87$ \\
\hline & $\mathrm{u}-238$ & $6.9 \mathrm{E}-06 \pm 61.07$ & $2.0 \mathrm{E}-05 \pm 40.0 \mathrm{x}$ & $1.3 E-05 \pm 97.4 \%$ \\
\hline \multirow{6}{*}{$\begin{array}{c}\$ 457 \\
(200 \mathrm{~W})\end{array}$} & $\$ 5-90$ & $8.5 \mathrm{E}-04 \pm 20.02$ & $1.4 \mathrm{E}-03 \pm 40.0 \mathrm{x}$ & $1.1 E-03 \pm 48.97$ \\
\hline & $\mathrm{Cs}-137$ & ND & $1.1 \mathrm{E}-04 \pm 47.0 \%$ & $1.1 \mathrm{E}-04$ \\
\hline & $v-234$ & $2.2 \mathrm{E}-0.5 \pm 30.0 \mathrm{x}$ & $1.6 \mathrm{E}-05 \pm 45.07$ & $1.9 \mathrm{E}-05 \pm 31.6 \mathrm{x}$ \\
\hline & $\mathrm{v}-235$ & $1.2 E-05 \pm 40.0 \%$ & $6.6 \mathrm{E}-06 \pm 79.0 \%$ & $9.3 E-06 \pm 58.17$ \\
\hline & $v-238$ & $7.8 \bar{z}-06 \pm 46.07$ & $6.85-06 \pm 74.0 \%$ & $7.3 \mathrm{E}-06 \pm 13.7 \%$ \\
\hline & Fu239/40 & $4.2 \mathrm{E}-06 \pm 91.07$ & $9.5 E-06 \pm 63.0 \%$ & $6.8 \mathrm{E}-06 \pm 77.4 \%$ \\
\hline \multirow{6}{*}{$\begin{array}{l}\text { N956 } \\
\text { (200W) }\end{array}$} & $\mathrm{Sx}-90^{\circ}$ & $8.3 E-04 \pm 40.0 z$ & ND & $8.3 E-04$ \\
\hline & $\mathrm{Cs}-137$ & $2.4 \mathrm{E}-04 \pm 60.0 \%$ & $9.4 \mathrm{E}-04 \pm 21.0 \%$ & $5.9 \mathrm{E}-04 \pm 118 \mathrm{z}$ \\
\hline & $U-234$ & $3.4 \mathrm{E}-05 \pm 34.0 x$ & $2.7 E-05 \pm 32.0 x$ & $3.0 z-05 \pm 23.07$ \\
\hline & $\mathrm{U}-235$ & $1.0 \mathrm{E}-05 \pm 80.0 \%$ & $1.4 E-05 \pm 46.0 \%$ & $1.2 \mathrm{E}-05 \pm 33.37$ \\
\hline & $\mathrm{U}-238$ & $1.6 \mathrm{E}-05 \pm 50.0 \%$ & $2.4 E-05 \pm 34.0 \%$ & $2.0 E-05 \pm 40.0 z$ \\
\hline & Pu239/40 & $1.0 \mathrm{E}-05 \pm 72.07$ & $6.5 \mathrm{E}-0.6 \pm 74.0 \%$ & $8.3 E-06 \pm 42.4 Z$ \\
\hline
\end{tabular}


Table C-3 (Cont.): 1997 Air Sample Results, 200 Areas $\left(\mathrm{pCi} / \mathrm{m}^{3}\right)$.

\begin{tabular}{|c|c|c|c|c|}
\hline & & $06 / 30 / 97$ & $12 / 31 / 97$ & Average \\
\hline Site & Isotope & Result \pm Uncertainty & Result \pm Uncertainty & Result \pm 2SEM \\
\hline \multirow{3}{*}{$\begin{array}{c}\text { N957 } \\
(200 \mathrm{E})\end{array}$} & $5 x-90$ & ND & $1.5 E-04 \pm 60.08$ & $1.5 E-04$ \\
\hline & $v-234$ & $3.3 E-05 \pm 35.0 \%$ & $1.0 \mathrm{z}-05 \pm 67.07$ & $2.25-05 \pm 106 z$ \\
\hline & $U-238$ & $3.7 \mathrm{E}-0.5 \pm 32.08$ & ND & $3.7 E-05$ \\
\hline \multirow{7}{*}{$\begin{array}{l}\text { N963 } \\
\text { (200\%) }\end{array}$} & $5 x-90$ & ND & $2.8 \mathrm{E}-04 \pm 40.0 \mathrm{z}$ & $2.8 \varepsilon-04$ \\
\hline & $C s-137$ & $7.98-05 \pm 58.08$ & $\mathrm{ND}$ & $7.9 \mathrm{E}-05$ \\
\hline & $u-234$ & $2.8 \mathrm{E}-05 \pm 31.0 \%$ & $1.7 E-05 \pm 43.0 \%$ & $2.3 E-05 \pm 48.97$ \\
\hline & $\mathrm{v}-235$ & $1.6 \mathrm{E}-05 \pm 44.0 Z$ & $6.8 \mathrm{E}-06 \pm 71.0 \%$ & $1.1 E-0.5 \pm 80.7 x$ \\
\hline & $u-238$ & $1.6 E-05 \pm 41.07$ & $7.5 E-06 \pm 64.0 z$ & $1.3 \mathrm{E}-05 \pm 82.47$ \\
\hline & $P u-238$ & $1.4 E-05 \pm 51.08$ & ND & $1.4 \mathrm{E}-05$ \\
\hline & Pu239/40 & $8.2 \mathrm{E}-06 \pm 60.0 \mathrm{z}$ & $1.0 \mathrm{E}-05 \pm 55.0 \mathrm{x}$ & $9.1 z-06 \pm 19.87$ \\
\hline \multirow{7}{*}{$\begin{array}{c}\text { N964 } \\
(200 W)\end{array}$} & $5 x-90$ & $5.8 \mathrm{E}-04 \pm 25.0 \%$ & $6.1 E-04 \pm 25.07$ & $5.9 \mathrm{E}-04 \pm 5.0 \%$ \\
\hline & $\mathrm{Cs}-137$ & $1.6 \mathrm{E}-04 \pm 59.0 \%$ & ND & $1.6 E-04$ \\
\hline & $v-234$ & $8.7 \mathrm{E}-06 \pm 74.0 \mathrm{z}$ & $9.7 z-06 \pm 50.0 z$ & $9.2 E-06 \pm 10.92$ \\
\hline & $\mathrm{U}-235$ & $\mathrm{ND}$ & $4.1 \mathrm{E}-06 \pm 82.0 \mathrm{z}$ & $4.1 \mathrm{E} \sim 06$ \\
\hline & $\mathrm{U}-238$ & $8.7 \mathrm{E}-06 \pm 74.0 \mathrm{Z}$ & $1.4 \mathrm{E}-05 \pm 43.07$ & $1.18-05 \pm 46.77$ \\
\hline & Eu239/40 & ND & $1.0 \mathrm{E}-05 \pm 77.07$ & $1.0 \mathrm{E}-05$ \\
\hline & $A m-241$ & ND & $2.8 \mathrm{E}-05 \pm 46.0 \%$ & $2.8 E-05$ \\
\hline \multirow{5}{*}{$\begin{array}{l}\text { N965 } \\
\text { (200W) }\end{array}$} & $5 x-90$ & $1.5 \mathrm{E}-03 \pm 20.0 \mathrm{x}$ & $1.4 E-03 \pm 15.0 \%$ & $1.4 \mathrm{E}-03 \pm 6.97$ \\
\hline & $c s-137$ & ND & $9.0 \mathrm{E}-05 \pm 60.0 \%$ & $9.0 \mathrm{E}-05$ \\
\hline & $v-234$ & $1.2 E-05 \pm 49.0 x$ & $2.2 E-05 \pm 44.0 \mathrm{z}$ & $1.7 E-05 \pm 58.87$ \\
\hline & $\mathrm{tu}-235$ & $9.7 \mathrm{E}-06 \pm 60.07$ & ND & $9.7 E-06$ \\
\hline & $U-238$ & $9.08-06 \pm 64.0 \%$ & $1.2 E-05 \pm 56.07$ & $1.0 \mathrm{E}-05 \pm 28.6 \%$ \\
\hline \multirow{5}{*}{$\begin{array}{c}\text { N966 } \\
(200 W)\end{array}$} & $5 x-90$ & ND & $4.2 E-04 \pm 30.0 z$ & $4.2 E-04$ \\
\hline & $\mathrm{v}-234$ & $2.4 E-05 \pm 42.0 \%$ & $1.8 \mathrm{E}-05 \pm 43.0 \mathrm{z}$ & $2.1 E-05 \pm 28.67$ \\
\hline & $u-235$ & $8.6 E-06 \pm 71.07$ & $1.3 E-0 S \pm 50.0 z$ & $1.1 E+05 \pm 40.7 \%$ \\
\hline & $\mathrm{U}-238$ & $1.9 E-05 \pm 45.0 \%$ & $6.0 \mathrm{E}-06 \pm 79.0 \%$ & $1.2 \mathrm{E}-05 \pm 103 z$ \\
\hline & Fu239/40 & $1.1 E-05 \pm 64.0 \%$ & $5.8 \mathrm{E}-06 \pm 71.0 \mathrm{z}$ & $8.4 \mathrm{z}-06 \pm 61.97$ \\
\hline
\end{tabular}


Table C-3 (Cont.): 1997 Air Sample Results, 200 Areas $\left(\mathrm{pCi} / \mathrm{m}^{3}\right.$ ).

\begin{tabular}{|c|c|c|c|c|}
\hline \multirow[b]{2}{*}{ Site } & \multirow[b]{2}{*}{ Isotope } & $06 / 30 / 97$ & $12 / 31 / 97$ & - Average \\
\hline & & Result \pm Uncertainty & Result \pm Uncertainty & Result \pm 2 SEM \\
\hline \multirow{6}{*}{$\begin{array}{c}\text { N967 } \\
(200 E)\end{array}$} & $5 x-90$ & $6.7 \mathrm{E}-04 \neq 50.0 z$ & $5.2 \mathrm{E}-04 \pm 25.0 \%$ & $5.9 \mathrm{E}-04 \pm 25.27$ \\
\hline & $C s-137$ & $8.7 E-05 \pm 50.0 z$ & $2.5 \mathrm{E}-04 \pm 28.0 \%$ & $1.7 \mathrm{E}-04 \pm 96.7 \%$ \\
\hline & $U-234$ & $m$ & $1.4 E-05 \pm 49.0 z$ & $1.4 \mathrm{E}-0.5$ \\
\hline & $U-235$ & SD & $8.0 \mathrm{E}-06 \pm 70.0 \mathrm{z}$ & $8.0 \mathrm{E}-06$ \\
\hline & $v-238$ & ND. & $1.0 \mathrm{E}-05 \pm 58.0 z$ & $1.0 E-05$ \\
\hline & Pu239/40 & $4.75-06 \pm 77.07$ & WD & $.4 .75-06$ \\
\hline \multirow{6}{*}{$\begin{array}{l}\text { N968 } \\
(200 E)\end{array}$} & $5 x-90$ & $1.28-04 \pm 70.0 \%$ & $8.0 \mathrm{E}-04 \geq 20.0 \%$ & $4.6 \mathrm{E}-04 \pm 247 \%$ \\
\hline & Cs -137 & . ND & $1.7 E-04 \pm 46.08$ & $1.7 E-04$ \\
\hline & $v-234$ & $2.1 E-05 \pm 47.07$ & $2.1 z-05 \pm 48.07$ & $2.18-05 \pm 0.0 \%$ \\
\hline & $\mathrm{u}-235$ & $1.2 \mathrm{E}-05 \pm 70.0 \mathrm{z}$ & $2.2 \mathrm{E}-0.05 \pm 46.0 \%$ & $1.7 \mathrm{E}-05 \pm 58.87$ \\
\hline & $\mathrm{u}-238$ & $1.2 \mathrm{E}-05 \pm 58.0 \mathrm{z}$ & $2.3 \mathrm{E}-05 \pm 41.0 \%$ & $1.8 \mathrm{E}-05 \pm 62.9 \%$ \\
\hline & Fn239/40 & $4.6 \mathrm{E}-06 \pm 91.07$ & ND & $4.6 E-06$ \\
\hline \multirow{5}{*}{$\begin{array}{l}\text { N969 } \\
\text { (200E) }\end{array}$} & $5 x-90$ & WD & $4.3 \mathrm{E}-0 . \pm 30.0 \mathrm{x}$ & $4.3 E-04$ \\
\hline & $\mathrm{Cs}-137$ & ND & $1.4 \mathrm{E}-04 \pm 52.0 \%$ & $1.4 \mathrm{E}-04$ \\
\hline & $0-234$ & $1.3 E-05 \pm 52.0 \%$ & $1.4 \mathrm{E}-05 \pm 55.0 \mathrm{x}$ & $1.4 \mathrm{E}-0.5 \pm 7.4 \mathrm{z}$ \\
\hline & $\mathrm{U}-235$ & $6.1 \mathrm{E}-06 \pm 77.02$ & $5.2 E-06 \pm 77.07$ & $5.7 E-06 \pm 15.97$ \\
\hline & $U-238$ & $9.4 \mathrm{E}-06 \pm 60.07$ & $6.8 \mathrm{E}-06 \pm 65.0 \%$ & $8.2 \mathrm{E}-06 \pm 32.1 \mathrm{z}$ \\
\hline \multirow{5}{*}{$\begin{array}{l}\text { N970 } \\
(200 E)\end{array}$} & $5 x-90$ & $3.4 \mathrm{E}-04 \pm 30.07$ & $5.8 \mathrm{E}-04 \pm 25.0 \mathrm{z}$ & $4.6 \mathrm{E}-04 \pm 52.27$ \\
\hline & $v-234$ & $1.3 E-05 \pm 48.0 \%$ & $1.6 \mathrm{z}-05 \pm 50.0 \%$ & $1.4 E-05 \pm 20.7 z$ \\
\hline & $u-235$ & $3.7 E-06 \pm 91.0 x$ & $1.3 \bar{E}-05 \pm 52.0 \%$ & $8.3 E-06 \pm 111 x$ \\
\hline & $U-238$ & $1.1 E-05 \div 51.0 \%$ & $7.4 \mathrm{E}-06 \pm 72.0 \%$ & $9.2 E-06 \pm 39.1 \%$ \\
\hline & $F 12339 / 40$ & ND & $5.3 \mathrm{E}-06 \pm 77.0 \%$ & $5.3 E-06$ \\
\hline \multirow{5}{*}{$\begin{array}{c}\text { N972 } \\
(200 \mathrm{E})\end{array}$} & $S r-90$ & ND & $5.2 \mathrm{E}-04 \pm 40.07$ & $5.2 \mathrm{E}-04$ \\
\hline & $C s+137$ & $\mathrm{ND}$ & $1.2 \mathrm{E}-04 \pm 65.0 \%$ & $1.25-0.4$ \\
\hline & $v-234$ & $9.28=06 \pm 64.0 \%$ & $2.0 \mathrm{E}-05 \pm 46.0 \%$ & $1.5 E-05 \pm 74.07$ \\
\hline & $\mathrm{U}-235$ & $7.2 E-06 \pm 68.0 x$ & $1.38-05 \pm 56.02$ & $1.0 \mathrm{E}-05 \pm 57.4 \%$ \\
\hline & $0-238$ & $6.5 E-06 \pm 75.0 z$ & ND & $6.5 \mathrm{E}-06$ \\
\hline
\end{tabular}


Table C-3 (Cont.): 1997 Air Sample Results, 200 Areas $\left(p C i / m^{3}\right.$ ).

\begin{tabular}{|c|c|c|c|c|}
\hline & & $06 / 30 / 97$ & $12 / 31 / 97$ & Average \\
\hline Site & Isotope & Result \pm Uncertainty & Result \pm Uncertainty & Result \pm 2 SSM \\
\hline $\mathrm{N} 972$ & Pu239/40 & $3.9 E-06 \pm 91.07$ & $5.0 \mathrm{E}-06 \pm 91.0 \%$ & $4.4 E-06 \pm 24.7 \%$ \\
\hline \multirow{6}{*}{$\begin{array}{c}\text { N973 } \\
(200 E)\end{array}$} & $5 x-90$ & $\mathrm{ND}$ & $4.35-04 \pm 30.0 \%$ & $4.3 \mathrm{E}-04$ \\
\hline & Cs -137 & $1.1 E-04 \pm 65.0 \%$ & $6.4 \mathrm{E}-04 \pm 21.0 \%$ & $3.8 \mathrm{E}-04 \pm 141 \%$ \\
\hline & $v-234$ & $1.5 E-05 \pm 51.07$ & $2.1 \mathrm{E}-05 \pm 44.07$ & $1.8 \mathrm{E}-05 \pm 33.37$ \\
\hline & $U-235$ & $5.7 \mathrm{E}-06 \pm 77.03$ & $\mathrm{ND}$ & $5,7 \mathrm{E}-06$ \\
\hline & $\mathrm{u}-238$ & $1.0 \mathrm{E}-05 \pm 59.0 \%$ & $7.3 E-06 \pm 68.0 \%$ & $8.6 \mathrm{E}-06 \pm 31.27$ \\
\hline & Pu239/40 & $5.1 E \div 06 \pm 83.0 z$ & $N D$ & $5.1 E-06$ \\
\hline \multirow{5}{*}{$\begin{array}{c}N 974 \\
(200 \mathrm{~W})\end{array}$} & $S r-90$ & $\mathrm{SD}$ & $2.1 E-04 \pm 50.07$ & $2.1 E-04$ \\
\hline & $0-234$ & $2.62-05 \pm 35.0 z$ & $1.2 \mathrm{E}-05 \pm 50.0 \mathrm{x}$ & $1.95-05 \pm 73.77$ \\
\hline & $u-235$ & $1.0 \mathrm{E}-05 \pm 63.0 \mathrm{Z}$ & $1.3 \mathrm{E}-05 \pm 50.0 \mathrm{z}$ & $1.2 \mathrm{E}-05 \pm 26.17$ \\
\hline & $v-238$ & $1.0 \mathrm{E}-05 \pm 60.0 \%$ & $1.4 E-05 \pm 45.0 z$ & $1.28-05 \pm 33.37$ \\
\hline & Pu239/40 & $1.3 \mathrm{E}-05 \pm 56.0 \mathrm{z}$ & ND & $1.3 \Xi-05$ \\
\hline \multirow{6}{*}{$\begin{array}{c}\text { N975 } \\
\text { (200W) }\end{array}$} & $\mathrm{s} r-90$ & $3.6 \mathrm{E}-04 \pm 30.07$ & $6.2 \mathrm{E}-04 \pm 30.0 \mathrm{z}$ & $4.9 E-04 \pm 53,17$ \\
\hline & $v-234$ & $1.5 \mathrm{E}-05 \pm 49.0 \%$ & $1.8 E-05 \pm 44.02$ & $1.7 z-0.5 \pm 18.2 \%$ \\
\hline & $0-235$ & $8.0 \mathrm{E}-06 \pm 70.0 \%$ & $1.3 \mathrm{E}-05 \pm 57.0 \%$ & $1.08-05 \pm 47.6 \mathrm{z}$ \\
\hline & $U-238$ & $9.5 \mathrm{E}-06 \pm 56.0 \%$ & $1.2 \mathrm{E}-05 \pm 52.0 \mathrm{x}$ & $1.15-05 \pm 23.37$ \\
\hline & Pu239/40 & $6.9 E-05 \pm 36.0 \%$ & $3.3 E-05 \pm 56.0 \%$ & $5.1 E-05 \pm 70.67$ \\
\hline & $A m-241$ & $3.6 \mathrm{E}-05 \pm 47.07$ & $2.2 \mathrm{E}-05 \pm 61.0 \mathrm{z}$ & $2.9 \mathrm{E}-0.5 \pm 48.3 \mathrm{x}$ \\
\hline \multirow{6}{*}{$\begin{array}{c}\text { N976 } \\
\langle 200 E\rangle\end{array}$} & $\operatorname{sr}-90$ & ND & $2.95-04 \pm 40.02$ & $2.9 E-04$ \\
\hline & $C s-137$ & ND & $1.5 E-04 \pm 36.0 \%$ & $1.5 z-04$ \\
\hline & $\mathrm{v}-234$ & $1.8 \mathrm{E}-0.5 \pm 39.0 \mathrm{x}$ & $3.4 \dot{\mathrm{E}}-05 \pm 32.0 \mathrm{z}$ & $2.6 \mathrm{E}-05 \pm 61.5 \mathrm{z}$ \\
\hline & $\mathrm{U}-235$ & $9.5 \mathrm{E}-06^{\prime} \pm 60.0 \mathrm{X}$ & $1.0 \mathrm{E}-05 \pm 56.07$ & $9.7 \mathrm{E}-06 \pm 5.17$ \\
\hline & $U-238$ & $1.5 \mathrm{E}-05 \pm 42.0 \mathrm{z}$ & $2.2 \mathrm{E}-05 \pm 38.0 \mathrm{x}$ & $1.9 \mathrm{E}-05 \pm 37.8 \mathrm{z}$ \\
\hline & Eu239/40 & $4.9 \mathrm{E}-06 \pm 83.07$ & $\mathrm{ND}$ & $4.9 E-06$ \\
\hline \multirow{3}{*}{$\begin{array}{l}\text { N977 } \\
(200 E)\end{array}$} & $5 x-90$ & ND & $2.9 \bar{z}-04 \pm 40.07$ & $2.9 E-04$ \\
\hline & Cs -137 & $\mathrm{~m}$ & $1.4 E-04 \pm 46.0 z$ & $1.4 E-04$ \\
\hline & $\mathrm{i}-234$ & $1.2 \mathrm{E}-05 \pm 55.0 \mathrm{z}$ & $1.4 \bar{E}-05 \pm 46.0 \%$ & $1.3 \mathrm{E}-05 \pm 15.4 \%$ \\
\hline
\end{tabular}


Table C-3 (Cont.): 1997 Air Sample Results, 200 Areas $\left(\mathrm{pCi} / \mathrm{m}^{3}\right)$.

\begin{tabular}{|c|c|c|c|c|}
\hline \multirow[b]{2}{*}{ site } & \multirow{2}{*}{ Isotope } & $06 / 30 / 97$ & $12 / 31 / 97$ & Averaze \\
\hline & & Result \pm Uncertainty & Result \pm Uncertainty & Result $\pm 2 s$ M \\
\hline \multirow{2}{*}{$\begin{array}{l}\text { N977 } \\
(200 \mathrm{E})\end{array}$} & $v-235$ & $6.8 \mathrm{E}-06 \pm 68.0 \%$ & $8.8 \mathrm{E}-06 \pm 61.02$ & $7.8 \mathrm{E}-06 \pm 25.6 \mathrm{x}$ \\
\hline & $\mathrm{U}-238$ & $1.15-05 \neq 54.0 \%$ & ND & $1.1 E-05$ \\
\hline \multirow{5}{*}{$\begin{array}{c}\text { N978 } \\
\text { (200E) }\end{array}$} & $5 r-90$ & SD & $2.2 E-04 \pm 50.0 \%$ & $2.2 \mathrm{E}-04$ \\
\hline & $v-234$ & $1.2 E-05 \pm 56.07$ & $1.7 \mathrm{E}-05 \pm 45.07$ & $1.4 \mathrm{E}-05 \pm 34.5 \%$ \\
\hline & $u-235$ & $\mathrm{ND}$ & $1.05-05 \pm 60.0 x$ & 1. $0 E-05$ \\
\hline & $\mathrm{u}-238$ & $1.4 \mathrm{E}-05 \pm 48.07$ & $1.8 \mathrm{E}-05 \pm 44.0 \mathrm{x}$ & $1.6 \mathrm{E}-05 \pm 25.0 \mathrm{z}$ \\
\hline & Pu239/40 & $7.2 E-06 \pm 74.0 x$ & $5.8 \mathrm{E}-06 \pm 72.0 \%$ & $6.58-06 \pm 21.5 z$ \\
\hline \multirow{4}{*}{$\begin{array}{c}\text { N984 } \\
(200 E)\end{array}$} & $5 x-90$ & WD & $3.8 \mathrm{E}-04 \pm 30.02$ & $3.8 E-04$ \\
\hline & $C s-137$ & $2.2 E-04 \pm 31.0 \%$ & & $2.2 E-04$ \\
\hline & $\mathrm{U}-234$ & $2.4 \mathrm{E}-05 \pm 44.0 \%$ & $2.1 \mathrm{E}-05 \pm 36.07$ & $2.3 E-05 \pm 13.3 \mathrm{X}$ \\
\hline & $\mathrm{u}-238$ & $1.9 E-05 \pm 44.0 \%$ & $2.3 E-05 \pm 34.0 \%$ & $2.1 E-05 \pm 19.0 \%$ \\
\hline \multirow{6}{*}{$\begin{array}{c}\text { N985 } \\
(200 E)\end{array}$} & $5 x-90$ & ND & $4.1 E-04 \pm 30.0 \%$ & $4.12-04$ \\
\hline & $\mathrm{Cs}-137$ & $9.2 E-05 \pm 55.0 \%$ & & $9.25-0.5$ \\
\hline & $v-234$ & $2.3 \mathrm{E}-05 \pm 42.0 z$ & $1.9 E-05 \pm 40.0 \%$ & $2.1 E-05 \pm 19.0 \%$ \\
\hline & $t-235$ & $7.7 \mathrm{E}-06 \pm 76.0 \%$ & ND & $7.7 E-06$ \\
\hline & $\mathrm{d}-238$ & $2.0 \mathrm{E}-05 \pm 51.0 \mathrm{z}$ & $2.0 \mathrm{z}-05 \pm 40.0 \mathrm{z}$ & $2.0 E-05 \pm 0.07$ \\
\hline & Pu239/40 & $3.8 \mathrm{E}-06 \pm 90.0 \mathrm{z}$ & ND & $3.8 \mathrm{E}-06$ \\
\hline \multirow{7}{*}{$\begin{array}{c}\text { X987 } \\
(200 E)\end{array}$} & $5 x-90$ & $2.38-04 \pm 50.0 \%$ & $2.3 E-04 \pm 60.07$ & $2.3 E-04 \pm 0.02$ \\
\hline & $5 n-113$ & ND & $1.7 E-04 \pm 94.02$ & $1.7 \mathrm{E}-04$ \\
\hline & $C s-137$ & $\mathrm{ND}$ & $1.6 \mathrm{E}-04 \pm 81.07$ & $1.6 \mathrm{E}-04$ \\
\hline & $\mathrm{U}-234$ & $1.2 \mathrm{E}-05 \pm 60.02$ & $1.9 \mathrm{E}-05 \pm 49.0 \mathrm{x}$ & $1.5 \mathrm{E}-05 \pm 45.27$ \\
\hline & $\mathrm{u}-235$ & $7.8 E-06 \pm 71.02$ & $\mathrm{ND}$ & $7.8 E-06$ \\
\hline & $U-238$ & $9.9 \bar{E}-06 \pm 51.0 \%$ & $1.6 \mathrm{E}-05 \pm 53.0 \%$ & $1.3 \vec{E}-05 \pm 47.17$ \\
\hline & Fu239/40 & $1.1 \bar{z}-05 \pm 60.0 z$ & $6.4 \mathrm{E}-06 \pm 77.02$ & $8.7 \mathrm{E}-06 \pm 52.97$ \\
\hline \multirow{3}{*}{$\begin{array}{c}1994 \\
(200 W)\end{array}$} & $s x-90$ & $4.0 \mathrm{E}-04 \pm 30.07$ & $\mathrm{ND}$ & 4. $0 \mathrm{E}-04$ \\
\hline & $u=234$ & $1.8 \mathrm{E}-05 \pm 49.0 \%$ & $1.4 \bar{E}-05 \pm 49.0 z$ & $1.6 \mathrm{z}-05 \pm 25.0 \mathrm{z}$ \\
\hline & $\mathrm{v}-235$ & $8.2 E-06 \pm 71.07$ & $6.3 \mathrm{E}-06 \pm 71.07$ & $7.3 \mathrm{E}-06 \pm 26.2 \%$ \\
\hline
\end{tabular}


Table C-3 (Cont.): 1997 Air Sample Results, 200 Areas $\left(\mathrm{pCi} / \mathrm{m}^{3}\right.$ ).

\begin{tabular}{|c|c|c|c|c|}
\hline \multirow[b]{2}{*}{ Site } & \multirow{2}{*}{ Isotope } & $06 / 30 / 97$ & $12 / 30 / 97$ & Averago \\
\hline & & Result \pm Uncertainty & Result \pm Uncertainty & Result \pm 2 SEM \\
\hline N994 & $v-238$ & $7.6 \mathrm{E}-08 \pm 71.08$ & ND & $7.6 \mathrm{E}-06$ \\
\hline \multirow{5}{*}{$\begin{array}{l}\text { N998 } \\
\text { (200E) }\end{array}$} & $5 r-90$ & $4.6 \mathrm{E}-04 \pm 40.0 \%$ & ND & $4.6 \mathrm{E}-04$ \\
\hline & $C s-137$ & ND & $1.38-04 \pm 56.08$ & $1.3 \mathrm{E}-04$ \\
\hline & $\mathrm{w}-234$ & $7.2 E-05 \pm 31.07$ & $1.4 E-05 \pm 50.0 z$ & $4.3 E-05 \pm 1347$ \\
\hline & $v-235$ & $2.82-05 \pm 56.02$ & $8.1 E-06 \pm 67.0 \%$ & $1.8 \mathrm{E}-05 \pm 110 \mathrm{z}$ \\
\hline & $U-238$ & $7.4 \mathrm{E}-05 \pm 31.0 \mathrm{x}$ & $1.4 \mathrm{E}-05 \pm 50.02$ & $4.4 \mathrm{E}-05 \pm 136 \%$ \\
\hline \multirow{6}{*}{$\begin{array}{c}N 999 \\
(2005)\end{array}$} & $5 x-90$ & $5.3 E-04 \pm 25.0 \%$ & ND & $5.3 \mathrm{E}-0^{\circ} 4$ \\
\hline & Cs-137. & $2.5 E-04=32.0 \%$ & $1.0 \mathrm{E}-04 \pm 48.0 \%$ & $1.8 \mathrm{E}-04 \pm 85.7 \mathrm{z}$ \\
\hline & $\mathrm{U}-234$ & $2.3 E-05 \pm 44.07$ & $2.3 E-05 \pm 38.0 z$ & $2.3 E-0.5 \pm 0.0 z$ \\
\hline & $v-235$ & $\mathrm{ND}$ & $7.1 \mathrm{E}-06 \pm 74.0 \%$ & $7.1 \mathrm{E}-06$ \\
\hline & $\mathrm{U}-238$ & $1.7 E-05 \pm 52.0 z$ & $7.1 E-06 \pm 70.08$ & $1.2 \mathrm{E}-05 \pm 82.27$ \\
\hline & $\mathrm{Pu} 239 / 40$ & $9.8 E-06 \pm 62.08$ & ID & $9.8 \mathrm{E}-06$ \\
\hline
\end{tabular}


Table C-4: 1997 Air Sample Results, 300 Area $\left(p C i / \mathrm{m}^{3}\right)$.

\begin{tabular}{|c|c|c|c|c|}
\hline \multirow[b]{2}{*}{ Sițe } & \multirow[b]{2}{*}{ Isotope } & $06 / 20 / 97$ & $12 / 19 / 97$ & - Average \\
\hline & & Result \pm thcertainty & Result \pm Uncertainty & Result $\pm 2 S E M$ \\
\hline \multirow{5}{*}{$\begin{array}{c}\text { N130 } \\
300 \text { AREA }\end{array}$} & $5 x-90$ & ND & $3.98-04 \pm 30.0 z$ & $3.95-04$ \\
\hline & $C s-137$ & $8.38-05 \pm 72.0 \mathrm{z}$ & $1.1 \bar{E}-04 \pm 62.0 \%$ & $9.6 \mathrm{E}-05 \pm 28.0 \%$ \\
\hline & $t-234$ & $1.95-05 \pm 37.07$ & $1.8 E-05 \pm 45.0 Z$ & $1.8 \mathrm{E}-0.5 \pm 5.4 x$ \\
\hline & $0-235$ & $1.22-05 \pm 50.0 z$ & ND & $1.2 \mathrm{E}-05$ \\
\hline & $\mathrm{v}-238$ & $1.6 \mathrm{E}-05 \pm 41.07$ & $1.9 E-05 \pm 43.07$ & $1.78-05 \pm 17.17$ \\
\hline
\end{tabular}

Table C-5: 1997 Air Sample Results, 400 Area $\left(p \mathrm{Ci} / \mathrm{m}^{3}\right)$.

\begin{tabular}{|c|c|c|c|c|}
\hline & & $06 / 20 / 97$ & $12 / 19 / 97$ & Averago \\
\hline Sito & Isotope & Result \pm Uncertainty & Result \pm Uncertainty & Result \pm 2SRM \\
\hline \multirow{5}{*}{$\begin{array}{c}\text { N981 } \\
600 \text { AREA }\end{array}$} & $s x-90$ & WD & $4.2 \mathrm{E}-04 \pm 30.07$ & $4.25=04$ \\
\hline & $0-234$ & $1.9 E-05 \pm 42.0 \pi$ & $1.7 E-05 \pm 44.0 \mathrm{x}$ & $1.8 \mathrm{E}-05 \pm 11.1 \%$ \\
\hline & $\mathrm{v}-235$ & $6.3 \mathrm{E}-06 \pm 72.07$ & $6.7 E-06 \pm 68.02$ & $6.5 \mathrm{E}-06 \pm 6.2 \mathrm{z}$ \\
\hline & $\mathrm{U}-238$ & $7.4 E-06 \pm 76.0 z$ & $9.0 \mathrm{E}-06 \pm 58.0 \mathrm{z}$ & $8.2 \mathrm{E}-06 \pm 19.5 \%$ \\
\hline & Pu239/40 & $6.75-06 \pm 79.0 \%$ & ND & $6.78-06$ \\
\hline
\end{tabular}


Table C-6: 1997 Air Sample Results, 100-B/C Area (pCi/m³).

\begin{tabular}{|c|c|c|c|c|}
\hline \multirow[b]{2}{*}{ Site } & \multirow[b]{2}{*}{ Isotope } & $06 / 20 / 97$ & $12 / 18 / 97$ & Average \\
\hline & & Result \pm Uncertainty & Result \pm Uncertainty & Result $\pm 25 E M$ \\
\hline \multirow{6}{*}{$\begin{array}{c}\text { N464 } \\
100-B / C\end{array}$} & $5 r-90$ & $1.1 E-03 \pm 17.02$ & $1.4 \Sigma-04 \pm 80.0 x$ & $6.28-04 \pm 154 z$ \\
\hline & $C s-137$ & $-1.4 \mathrm{E}-04 \pm 71.0 \mathrm{z}$ & $1.18-04 \pm 87.0 z$ & $1.3 \mathrm{E}-04 \pm 24.0 \%$ \\
\hline & $\mathrm{U}-234$ & $1.6 \mathrm{E}-05 \pm 45.07$ & $1.1 \mathrm{E}-05 \pm 56.07$ & $1.4 \mathrm{E}-05 \pm 37.0 \%$ \\
\hline & $v-235$ & 1. $7 \mathrm{E}-05 \pm 46.0 \mathrm{z}$ & KD & $1.7 E-05$ \\
\hline & $v-238$ & $5.1 \mathrm{E}-06 \pm 77.08$ & $7.6 \mathrm{E}-06 \pm 65.0 \mathrm{z}$ & $6.3 \mathrm{E}-0 \mathrm{OE} \pm 39.4 \mathrm{Z}$ \\
\hline & Pun239/40 & $1.1 E-05 \pm 64.02$ & $4.4 \bar{z}-06 \pm 91.0 \%$ & $7.7 z-06 \pm 85.7 z$ \\
\hline \multirow{7}{*}{$\begin{array}{c}1465 \\
100-B / C\end{array}$} & $S x-90$ & $1.0 \mathrm{E}-03 \pm 19.07$ & ND & $1.0 \pm-0,3$ \\
\hline & $C s-137$ & $1.78-04 \pm 50.0 z$ & $8.9 E-05 \pm 56.0 \%$ & $1.3 E-04 \pm 62.57$ \\
\hline & $E u-152$ & $2.3 E-04 \pm 39.07$ & ID & $2.3 E-04$ \\
\hline & $\mathrm{U}-234$ & $5.4 \mathrm{E}-05 \pm 23.0 \mathrm{z}$ & $1.3 \mathrm{E}-05 \pm 50.08$ & $3.4 \mathrm{E}-05 \pm 122 z$ \\
\hline & $\mathrm{v}-235$ & $3.2 \mathrm{E}-05 \pm 28.0 \mathrm{x}$ & ND & $3.2 E-05$ \\
\hline & $\mathrm{U}-238$ & $1.3 \mathrm{E}-0.5 \pm 38.0 \mathrm{x}$ & $1.1 E-05 \pm 54.0 \%$ & $1.20-0.5 \pm 16.77$ \\
\hline & Pu239/40 & $1.4 \mathrm{E}-05 \pm 54.0 \mathrm{z}$ & $3.8 E-06 \pm 91.0 z$ & $8.9 E-06 \pm 1147$ \\
\hline \multirow{6}{*}{$\begin{array}{c}N 466 \\
100-B / C\end{array}$} & $5 z-90$ & $9.4 \bar{z}-04 \pm 20.02$ & MD & $9.4 E-04$ \\
\hline & $\operatorname{cs}-137$ & $3.7 E-04 \pm 25.08$ & $\mathrm{ND}$ & $3.7 E-04$ \\
\hline & $v-234$ & $1.0 E-05 \pm 49.0 \%$ & $1.7 \mathrm{E}-05 \pm 46.07$ & $1.3 \mathrm{E}-0 \mathrm{~S} \pm 51.9 \mathrm{X}$ \\
\hline & $\mathrm{U}-235$ & $7.2 \mathrm{E}-06 \pm 75.0 \%$ & $1.0 \mathrm{E}-05 \pm 60.0 \%$ & $8.6 E-06 \pm 32.67$ \\
\hline & $\mathrm{U}-238$ & $7.2 \mathrm{E}-06 \pm 58.0 \mathrm{z}$ & $1.4 \mathrm{E}-05 \pm 56.0 \%$ & $1.1 \mathrm{E}-05 \pm 64.2 \%$ \\
\hline & $20239 / 40$ & $8.7 \mathrm{E}-06 \pm 60.0 \mathrm{z}$ & $\mathrm{ND}$ & 8.7E-06 \\
\hline
\end{tabular}


Table C-7: 1997 Air Sample Results, 100-D/DR Area $\left(\mathrm{pCi} / \mathrm{m}^{3}\right)$.

\begin{tabular}{|c|c|c|c|c|}
\hline \multirow[b]{2}{*}{ Site } & \multirow[b]{2}{*}{ Isotope } & $06 / 20 / 97$ & $12 / 18 / 97$ & Average . \\
\hline & & Result \pm Uncertainty & Result \pm Uncertainty & Result \pm 2 2SM . \\
\hline \multirow{5}{*}{$\begin{array}{c}N 467 \\
100-D / D R\end{array}$} & $\mathrm{sx}-90$ & ND & $2.7 \mathrm{E}-04 \pm 40.0 \mathrm{z}$ & $2.7 E-04$ \\
\hline & $\mathrm{v}-234$ & $1.5 E-05 \pm 44.0 z$ & $1.4 \mathrm{E}-05 \pm 47.0 \%$ & $1.5 z-05 \pm 6.97$ \\
\hline & $\mathrm{v}-235$ & $1.4 E-05 \pm 47.0 \%$ & $9.4 \mathrm{E}-06 \pm 63.0 z$ & $1.25-05 \pm 39.37$ \\
\hline & $v-238$ & $9.3 \mathrm{E}-06 \pm 57.0 \%$ & $8.1 \mathrm{E}-06 \pm 66.07$ & $8.7 E-06 \pm 13.8 \%$ \\
\hline & Pu239/40 & $5.6 \mathrm{E}-06 \pm 72.0 \mathrm{X}$ & $8.7 \pm-06 \pm 60.0 \%$ & $7.1 E-06 \pm 43.4 z$ \\
\hline \multirow{5}{*}{$\begin{array}{c}N 468 \\
100-D / D R\end{array}$} & $5 r-90$ & $4.2 \mathrm{E}-04 \pm 40.07$ & ND & $4.2 E-04$ \\
\hline & Cs -137 & $1.1 E-04 \pm 61.07$ & $1.2 E-04 \pm 35.02$ & $1.2 E-04 \pm 8.7 x$ \\
\hline & $\mathrm{U}-234$ & $9.6 \mathrm{E}-06 \pm 37.02$ & $2.0 \mathrm{E}-05 \pm 47.0 \mathrm{z}$ & $1.5 \mathrm{E}-\dot{0} 5 \pm 70.3 \%$ \\
\hline & ษ 235 & $5.6 \mathrm{E}-06 \pm 55.0 \mathrm{z}$ & $8.8 \mathrm{E}-06 \pm 72.0 \mathrm{z}$ & $7.2 E-06 \pm 44.4 \%$ \\
\hline & $\mathrm{v}-238$ & $6.5 E-06 \pm 46.07$ & $1.0 \mathrm{E}-05 \pm 65.0 \mathrm{z}$ & $8.3 E-06 \pm 42.4 \pi$ \\
\hline \multirow{6}{*}{$\begin{array}{c}N 469 \\
100-D / D R\end{array}$} & $5 x-90$ & $\mathrm{SD}$ & $7.5 E-04 \pm 18.07$ & $7.5 E-04$ \\
\hline & $C s-137$ & $N D$ & $8.8 \mathrm{E}-05 \pm 53.07$ & $8.8 \mathrm{E}-05$ \\
\hline & $\mathrm{v}-234$ & $9.6 E-06 \pm 46.0 z$ & $\mathrm{ND}$ & $9.6 \mathrm{E}-06$ \\
\hline & $\mathrm{u}-235$ & $4.4 E-06 \pm 71.02$ & $\mathbb{N B}$ & $4.4 E-06$ \\
\hline & $\mathrm{v}-238$ & $6.8 \mathrm{E}-06 \pm 64.0 \%$ & $\mathrm{ND}$ & $6.8 \mathrm{E}-06$ \\
\hline & Fur239/40 & $8.2 E-06 \pm 68.0 z$ & ND & $8.2 E-06$ \\
\hline \multirow{4}{*}{$\begin{array}{c}N 470 \\
100-D / D R\end{array}$} & $5 x-90$ & $8.4 \mathrm{E}-04 \pm 25.07$ & $\mathrm{ND}$ & $8.4 E-04$ \\
\hline & $U-234$ & $9.1 E-06 \pm 58.0 \%$ & 2. DE-05 $\pm 51.0 z$ & $1.5 E-05 \pm 74.9 x$ \\
\hline & $\mathrm{U}-235$ & $8.4 \mathrm{E}-06 \pm 58.0 \mathrm{z}$ & $5.8 \mathrm{E}-06 \pm 83.0 \%$ & $7.1 E-06 \pm 36.67$ \\
\hline & $v-238$ & $6.9 E-06 \pm 61.07$ & $9.4 E-06 \pm 62.0 x$ & $8.2 E-06 \pm 30.7 x$ \\
\hline
\end{tabular}


Table C-8: 1997 Air Sample Results, ERDF $\left(\mathrm{pCi} / \mathrm{m}^{3}\right)$.

\begin{tabular}{|c|c|c|c|c|}
\hline \multirow[b]{2}{*}{ Site } & \multirow[b]{2}{*}{ Isotope } & $05 / 02 / 97$ & $12 / 19 / 97$ & Average \\
\hline & & Result \pm Uncertainty & Result \pm Uncertainty & Result \pm 2SBM \\
\hline \multirow{2}{*}{$\begin{array}{l}\text { N482 } \\
\text { (ERDF) }\end{array}$} & $S=-90$ & ND & $2.2 \mathrm{E}-04 \pm 61.0 \mathrm{Z}$ & $2.25-04$ \\
\hline & $\mathrm{v}-234$ & ND & $1.7 \mathrm{E}-0.5 \pm 57.0 \mathrm{x}$ & 1.7E-05 \\
\hline \multirow{4}{*}{$\begin{array}{l}N 483 \\
\text { (ERDF) }\end{array}$} & $5 r-90$ & $\mathrm{ND}$ & $2.1 \mathrm{E}-04 \pm 51.0 \%$ & 2. $2 E-04$ \\
\hline & $C s-137$ & $\mathrm{ND}$ & $1.0 \mathrm{E}-04 \pm 49.0 \mathrm{x}$ & $2.0 \mathrm{E}-04$ \\
\hline & $\mathrm{U}-234$ & ND & $1.5 E-05 \pm 46.07$ & $1.5 \mathrm{E}-05$ \\
\hline & $U-238$ & $N D$ & $1.5 E-05 \pm 47.07$ & $2.55-0.5$ \\
\hline \multirow{3}{*}{$\begin{array}{l}\text { N484 } \\
\text { (ERDF) }\end{array}$} & $v-234$ & $\mathrm{ND}$ & $1.5 \mathrm{E}-05 \pm 61.0 \mathrm{z}$ & $1.5 E-05$ \\
\hline & $\mathrm{v}-238$ & ND & $1: 6 \mathrm{E}-05 \pm 55.0 \mathrm{z}$ & $1.6 \mathrm{z}-05$ \\
\hline & Fu239/40 & & $1.5 E-05 \pm 51.07$ & $1.5 E-05$ \\
\hline
\end{tabular}

Table C-9: 1997 Air Sample Rẹsults, 300-FF-1 Project (pCi/m³).

\begin{tabular}{|c|c|c|c|c|}
\hline \multirow[b]{2}{*}{ Site } & \multirow[b]{2}{*}{ Isotope } & & $12 / 19 / 97$ & Average \\
\hline & & Result \pm Uncertainty & Resuit. \pm Uncertainty & Result \pm 2 SBS \\
\hline \multirow{3}{*}{$\begin{array}{r}1485 \\
(300)\end{array}$} & $v-234$ & . & $2.6 \mathrm{E}-0 \mathrm{~S} \pm 40.0 \mathrm{z}$ & $2.6 \mathrm{E}-0 \mathrm{~S}$ \\
\hline & $\mathrm{u}-235$ & & $1.1 \mathrm{E}-05 \pm 64.07$ & $1.18-05$ \\
\hline & $\mathrm{v}-238$ & & $2.8 \mathrm{E}-05 \pm 38.0 \mathrm{z}$ & $2.8 E-05$ \\
\hline \multirow{3}{*}{$\begin{array}{r}11486 \\
(300)\end{array}$} & $0-234$ & . & $5.8 \mathrm{E}-05 \pm 26.0 \mathrm{Z}$ & $5.8 \mathrm{E}-05$ \\
\hline & $\mathrm{u}-235$ & & $2.0 \mathrm{E}-05 \pm 41.07$ & $2.0 E-0 S$ \\
\hline & $\mathrm{U}-238$ & & $4.5 \mathrm{~F}-05 \pm 28.07$ & $4.5 \mathrm{E}-0.5$ \\
\hline \multirow{3}{*}{$\begin{array}{r}N 487 \\
(300) \\
-\end{array}$} & $U-234$ & & $6.5 \mathrm{z}-05 \pm 27.0 \mathrm{z}$ & $6.5 E-0.5$ \\
\hline & $\mathrm{U}-235$ & & $4.5 \mathrm{E}-06 \pm 91.0 \mathrm{x}$ & $4.5 E-06$ \\
\hline & $\mathrm{U}-238$ & & $6.6 \mathrm{E}-0.5 \pm 27.0 \mathrm{z}$ & $6.6 \mathrm{E}-05$ \\
\hline
\end{tabular}


HNF-EP-0573-6

This page intentionally left blank. 
HNF-EP-0573-6

APPENDIX D

GROUNDWATER MONITORING FIGURES AND TABLES 
HNF-EP-0573-6

This page intentionally left blank. 


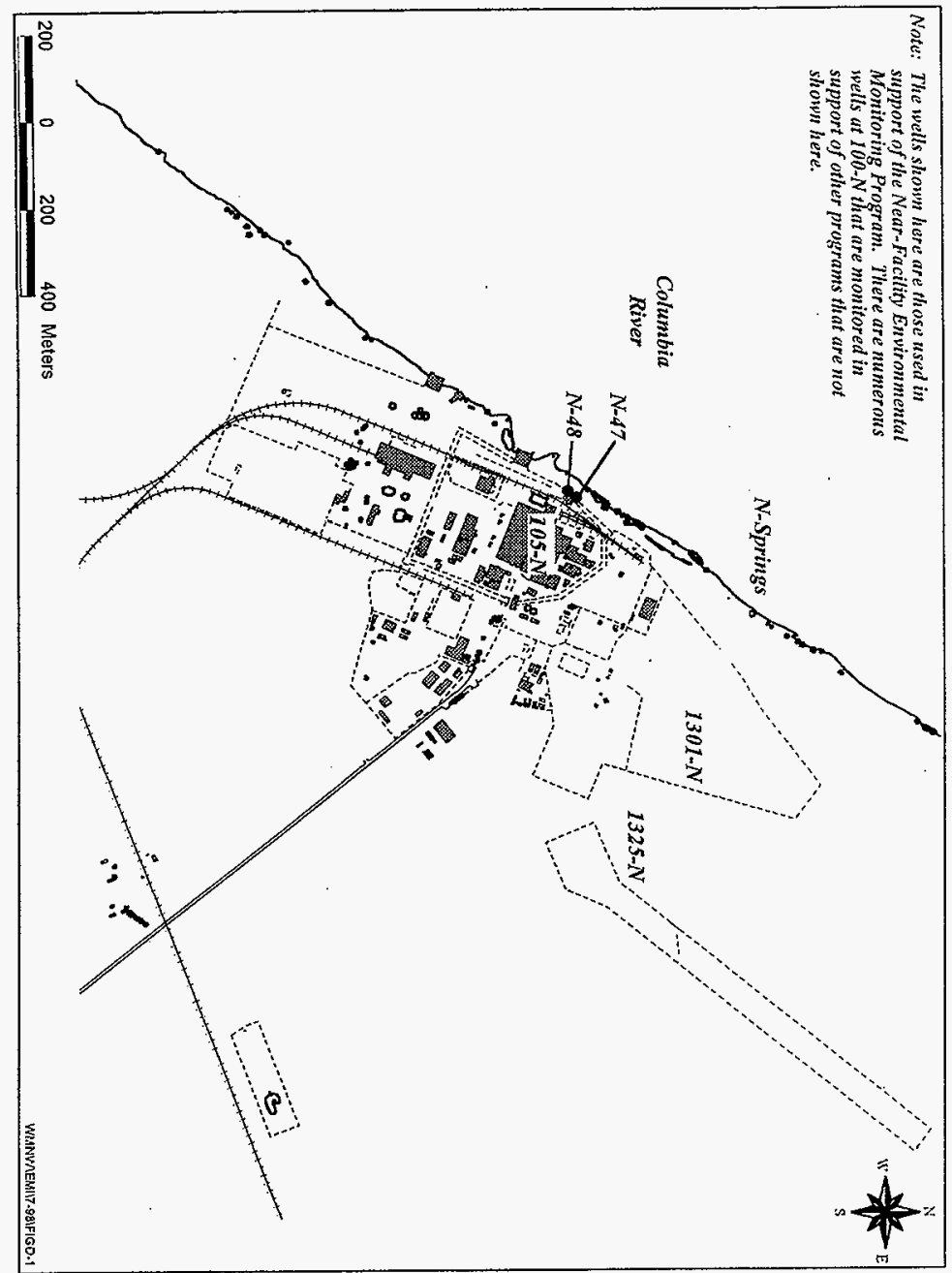

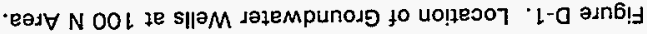


HNF-EP-0573-6

Table D-1

Radiological Results for Groundwater Samples (pCi/L) 100-N Area Groundwater Wells (197)

\begin{tabular}{|c|c|c|c|c|c|}
\hline EDP Code & $\mathrm{Co}-60$ & Cs-137 & $\mathrm{Eu}-152$ & $\mathrm{Eu}-154$ & Eu-155 \\
\hline \multirow[t]{11}{*}{ Y847 } & WD & ND & $\mathrm{ND}$ & ND & $\mathrm{ND}$ \\
\hline & & ND & $\mathrm{ND}$ & ND & $\mathrm{ND}$ \\
\hline & $5.8 E+00$ & $\mathrm{ND}$ & $\mathrm{ND}$ & ND & HD \\
\hline & & $N D$ & ND & ND & SD \\
\hline & ND & $7.4 E+00$ & ND & WD & $\mathrm{ND}$ \\
\hline & ND & KD & ND & RD & ND \\
\hline & HD & 5. $3 E+00$ & ND & & $\mathrm{ND}$ \\
\hline & KD & & $\mathrm{ND}$ & $\mathrm{WD}$ & $\mathrm{SD}$ \\
\hline & WD & WD & ND & $\mathrm{ND}$ & SD \\
\hline & KD & $\mathrm{WD}$ & ND & $\mathrm{ND}$ & ND \\
\hline & EDD & ND & ND & ND & ND \\
\hline Results & 1 & 2 & & & \\
\hline Average & $5.8 \mathrm{E}+00$ & $6.4 \mathrm{E}+00$ & & & \\
\hline Maximum & $5.8 E+00$ & $7.4 \mathrm{E}+00$ & & & \\
\hline Minimum & $5.8 \mathrm{E}+00$ & S. $3 E+00$ & & & \\
\hline $2 \operatorname{sen}$ & & $1.4 \mathrm{E}+00$ & & & \\
\hline
\end{tabular}


HNF-EP-0573-6

Table D-1

Radiological Results for Groundwater Samples (pCi/L) 100-N Area Groundwater Wells ('97)

\begin{tabular}{|c|c|c|c|c|c|}
\hline EDP Code & Co- 60 & Cs -137 & $E 12-152$ & $E u-154$ & Eu-155 \\
\hline \multirow[t]{11}{*}{$Y 848$} & ND & ND & ND & ND & ND \\
\hline & ND & ND & ND & ND & ND \\
\hline & $6.6 \mathrm{E}+00$ & 4. $4 E-01$ & ND & ND & SD \\
\hline & ND & ND & ND & $2 x B$ & $9.3 E+00$ \\
\hline & ND & ND & $N D$ & ND & ND \\
\hline & ND & ND & 1. $1 \mathrm{E}+01$ & ID & ND \\
\hline & ND & ND & ND & 1. $2 \mathrm{E}+01$ & ND \\
\hline & ND & ND & ND & wo & ND \\
\hline & ND & ND & ND & $\mathrm{mD}$ & XD \\
\hline & ND & SD & $N D$ & $\mathrm{MDO}$ & ND \\
\hline & ND & ND & $\mathbb{N D}$ & WD & ND \\
\hline HRosults & $I$ & 1 & 1 & 1 & 1 \\
\hline Average & $6.6 \mathrm{E}+00$ & 4. $4 E-01$ & 1. $1 E+01$ & 1. $2 \mathrm{E}+01$ & $9.3 E+00$ \\
\hline Maximum & $6.6 \mathrm{E}+00$ & $4,4 E-01$ & 1. $18+01$ & 1. $2 \mathrm{E}+01$ & $9.3 E+00$ \\
\hline Minimern & $6.6 \mathrm{E}+00$ & $4.4 E-01$ & 1. $1 \mathrm{E}+01$ & $1.2 \mathrm{E}+01$ & $9.3 \mathrm{E}+00$ \\
\hline 2SEMS & & & & & \\
\hline
\end{tabular}


HNF-EP-0573-6

This page intentionaliy left blank.

D-4 


\section{APPENDIX E}

SOIL AND VEGETATION MONITORING FIGURES AND TABLES 
HNF-EP-0573-6

This page intentionally left blank.

E-ii 
Figure E-1. Soil Sampling Locatons in the $100 \mathrm{~N}$ Area.

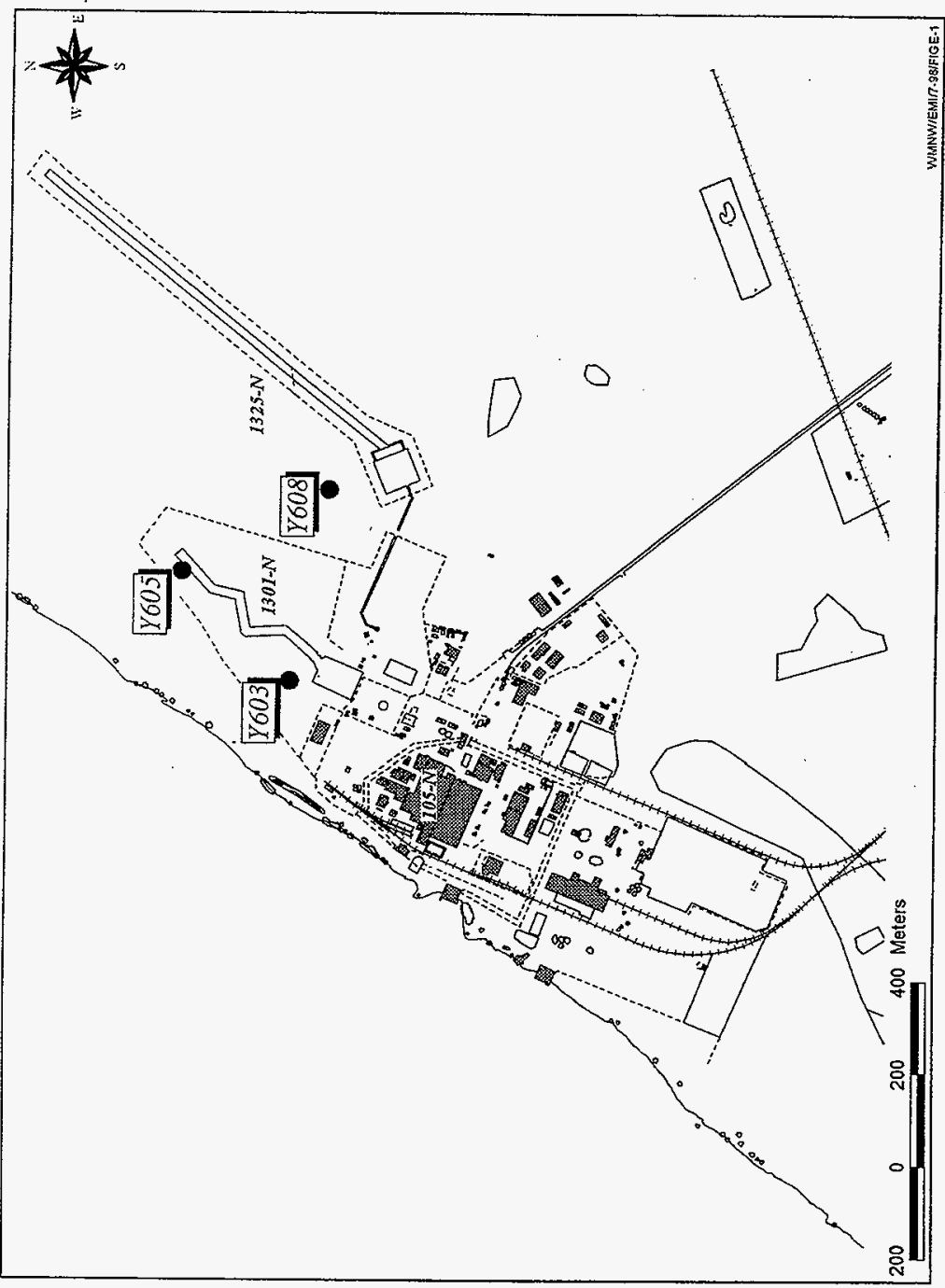




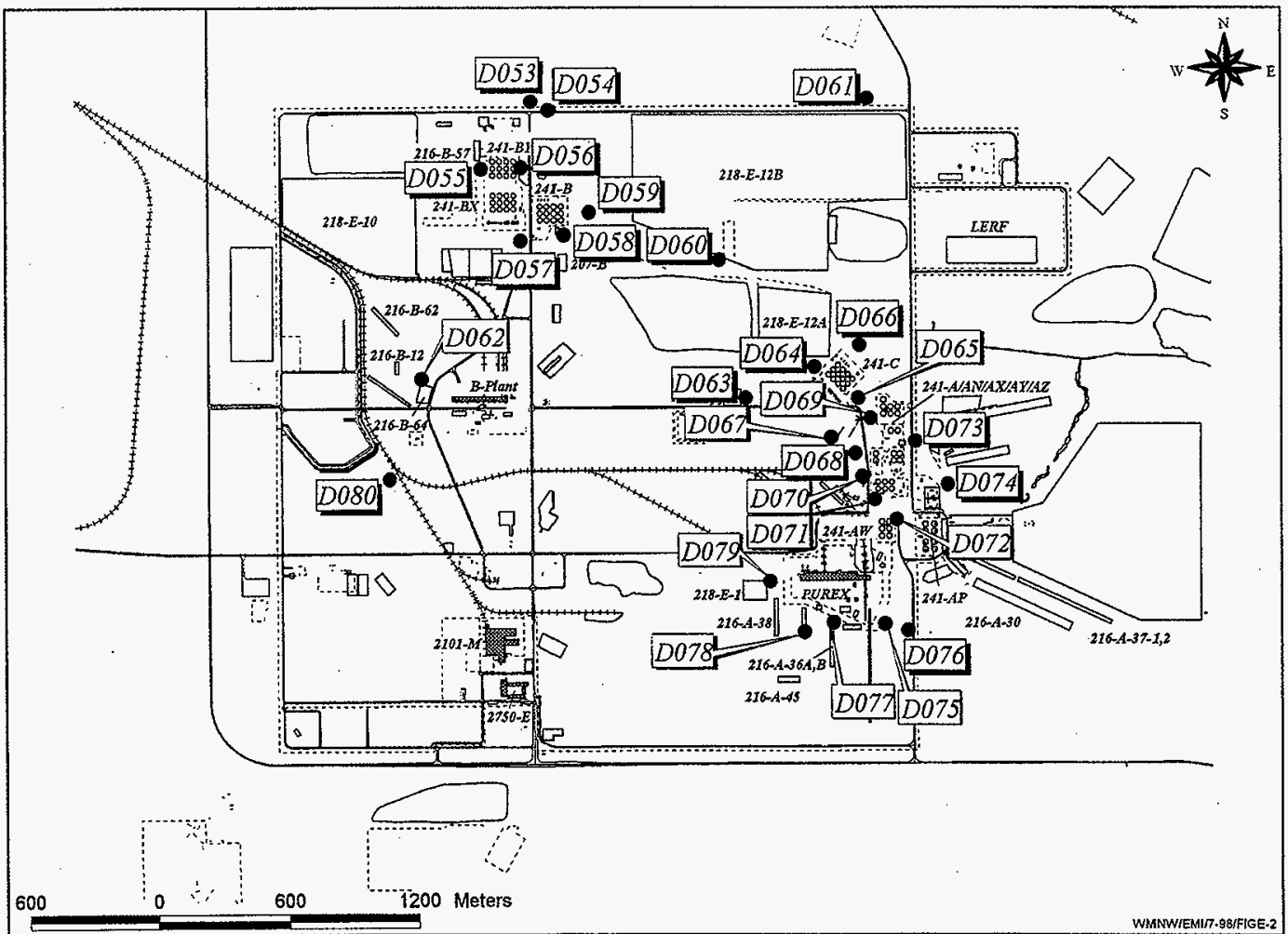

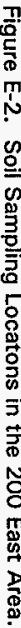


Figure E-3. Soil Sampling Locatons in the 200 West Area.

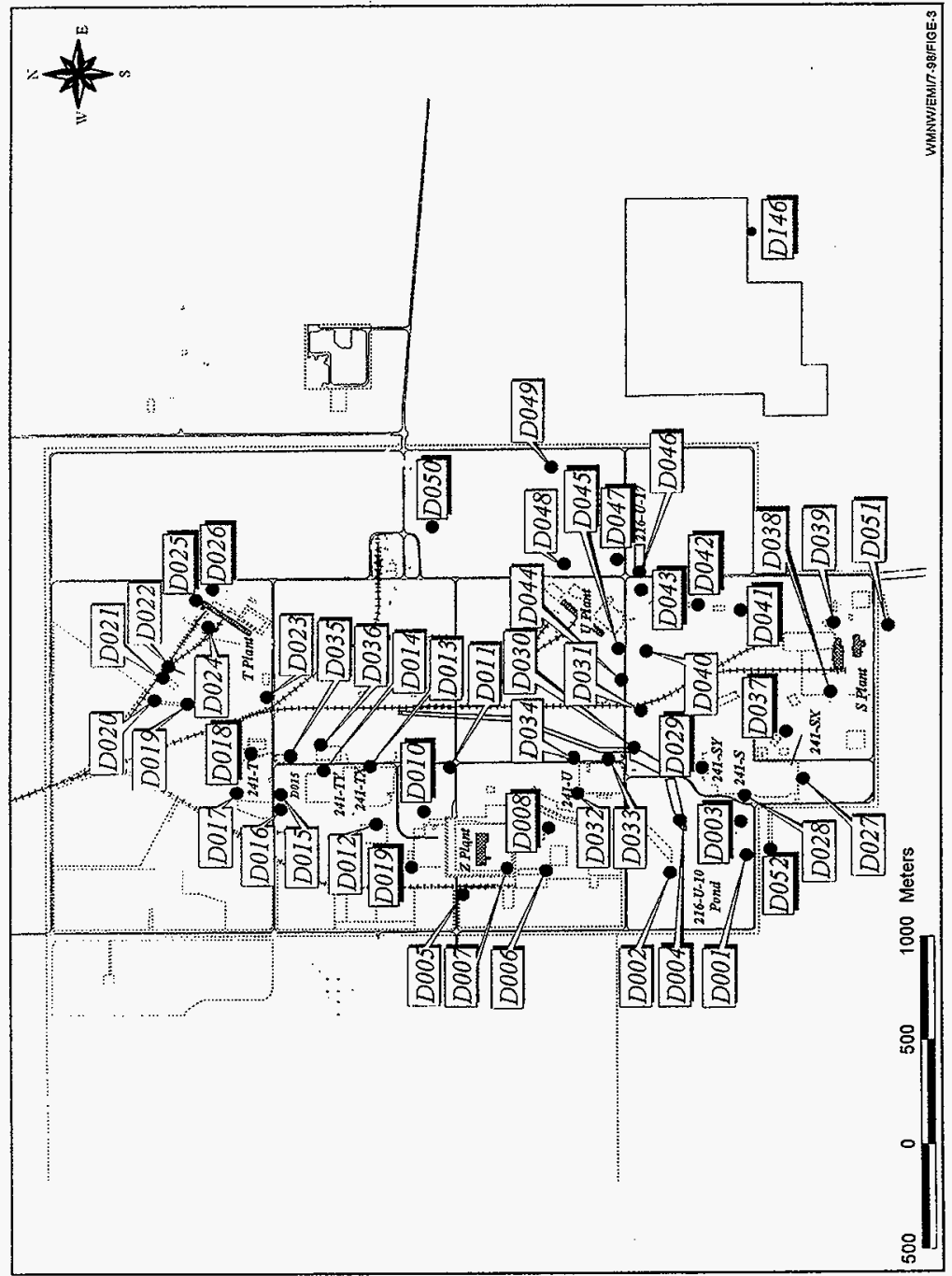




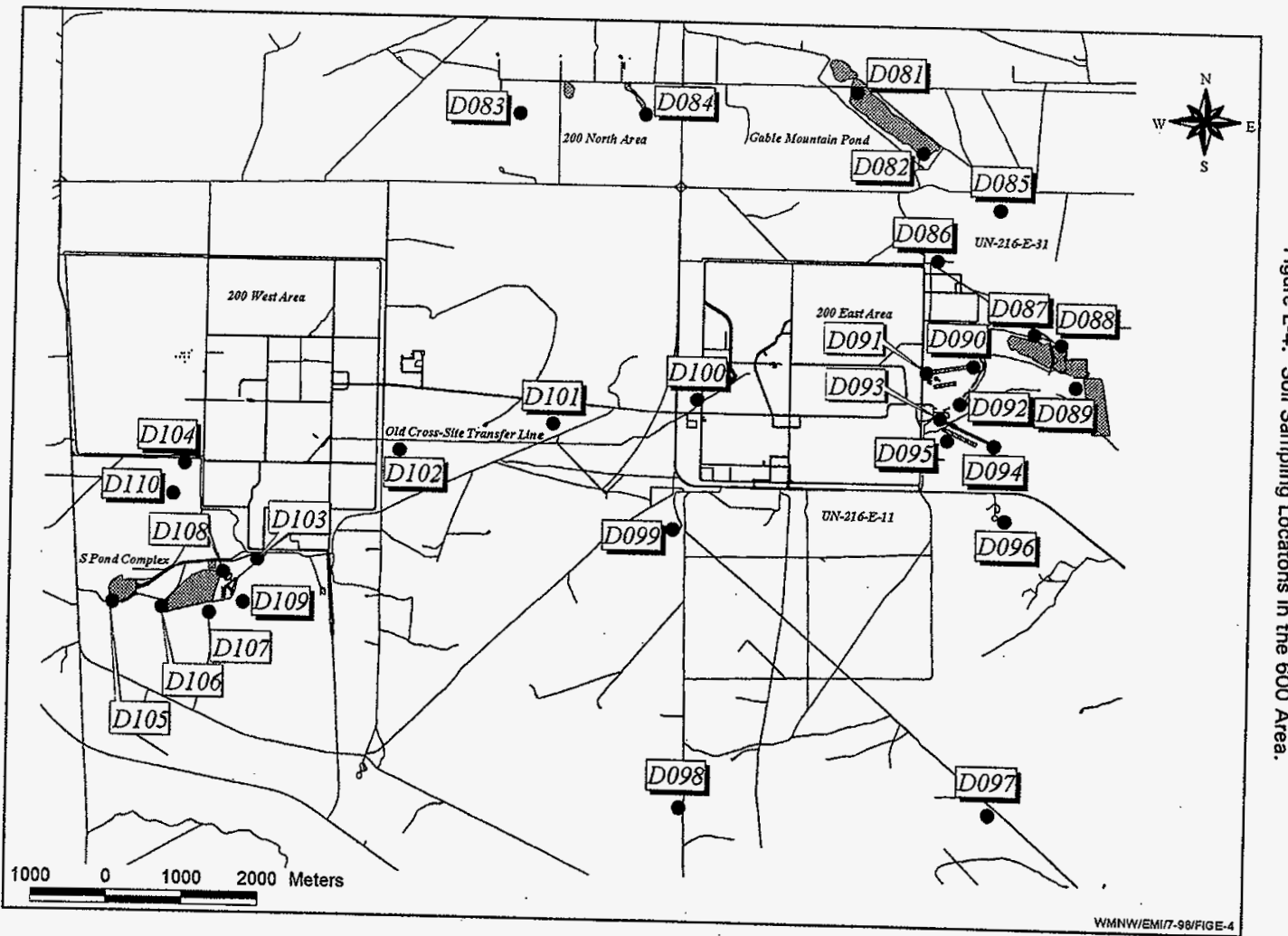




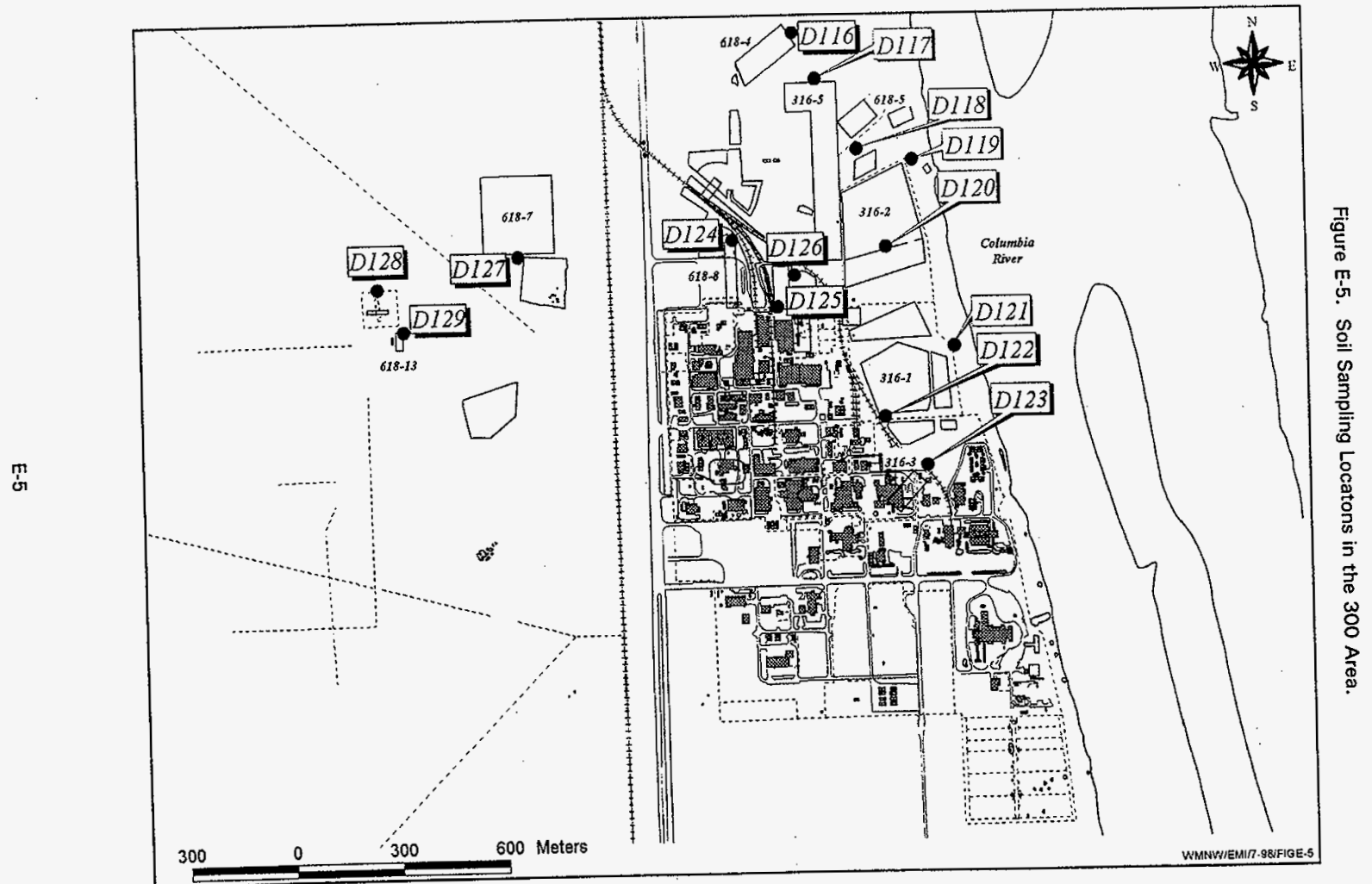


Figure E-6. Soil Sampling Locatons in the 400 Area.

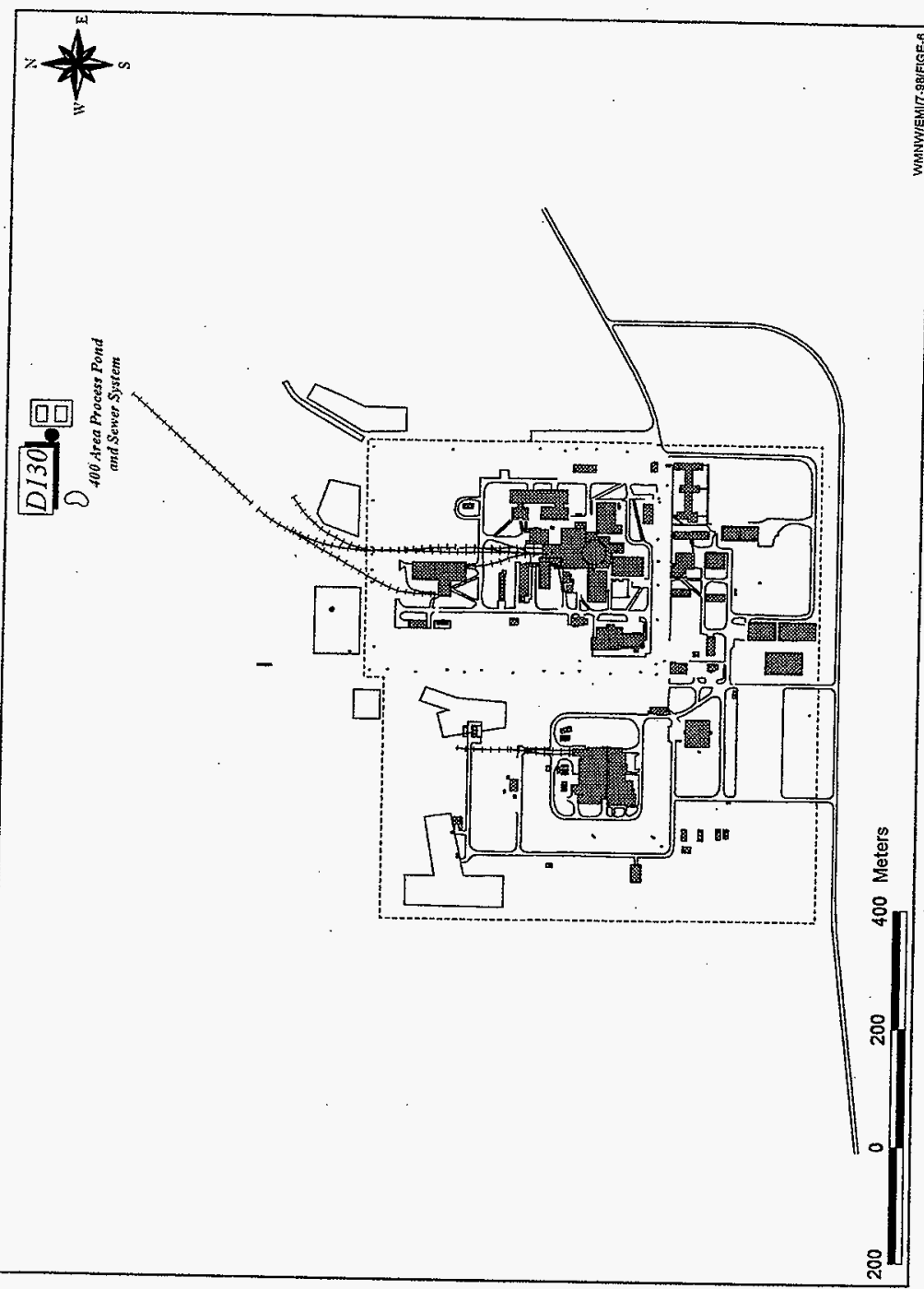


HNF-EP-0573-6

Figure E-7. Soil Sampling Locatons in the $100 \mathrm{~N}$ Area.

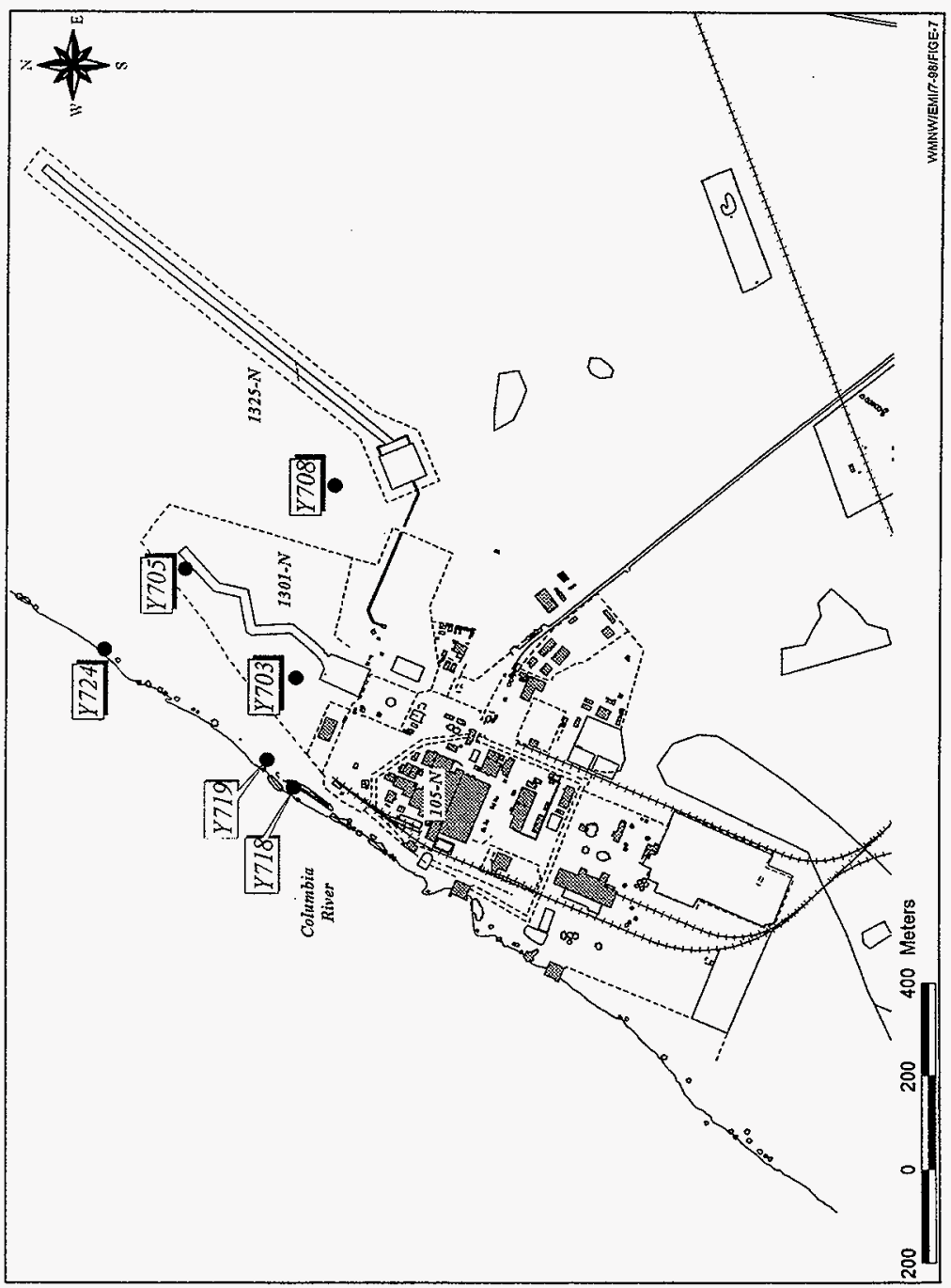


Figure E-8. Vegetation Sampling Locatons in the 200 East Area.

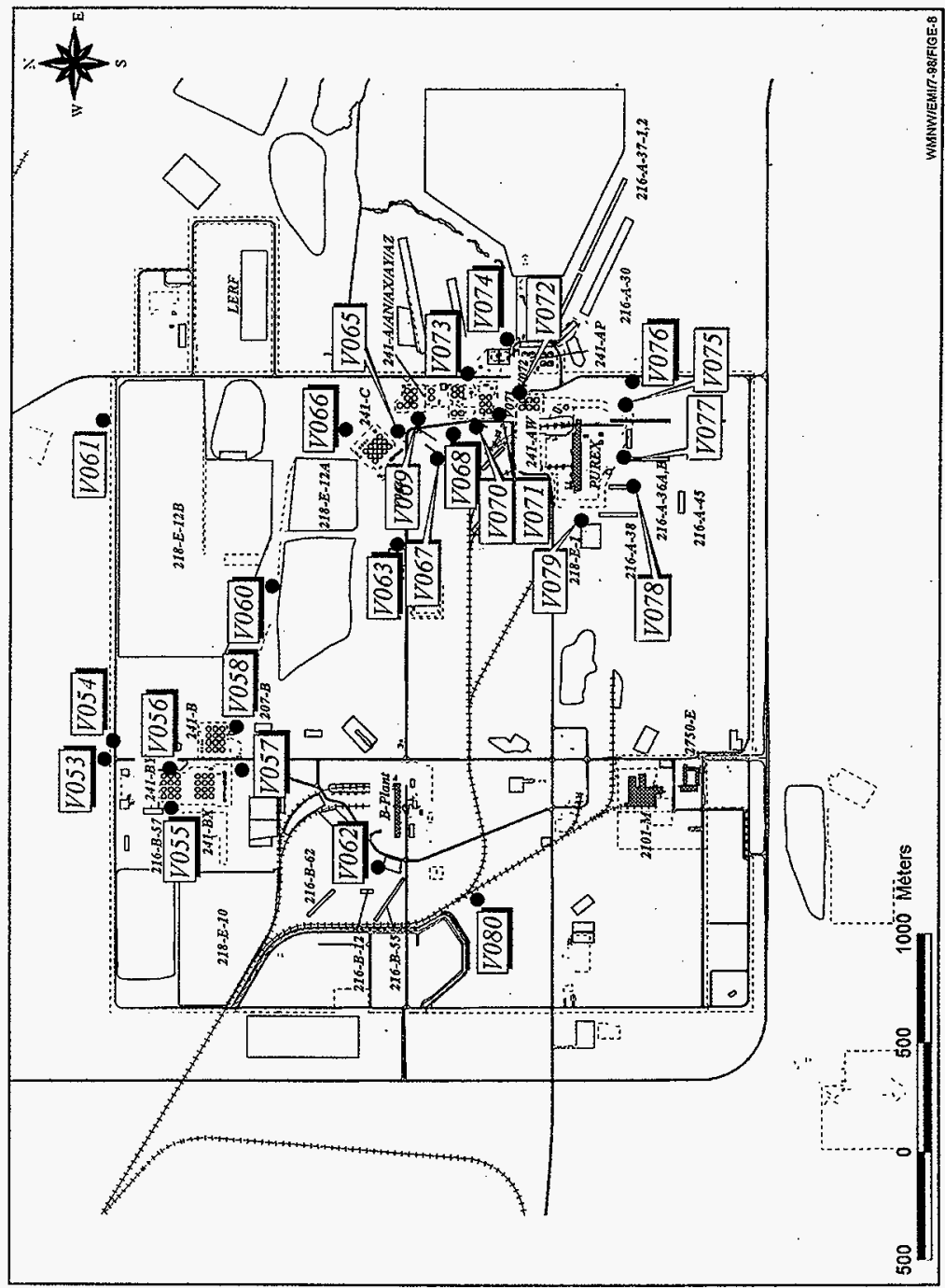




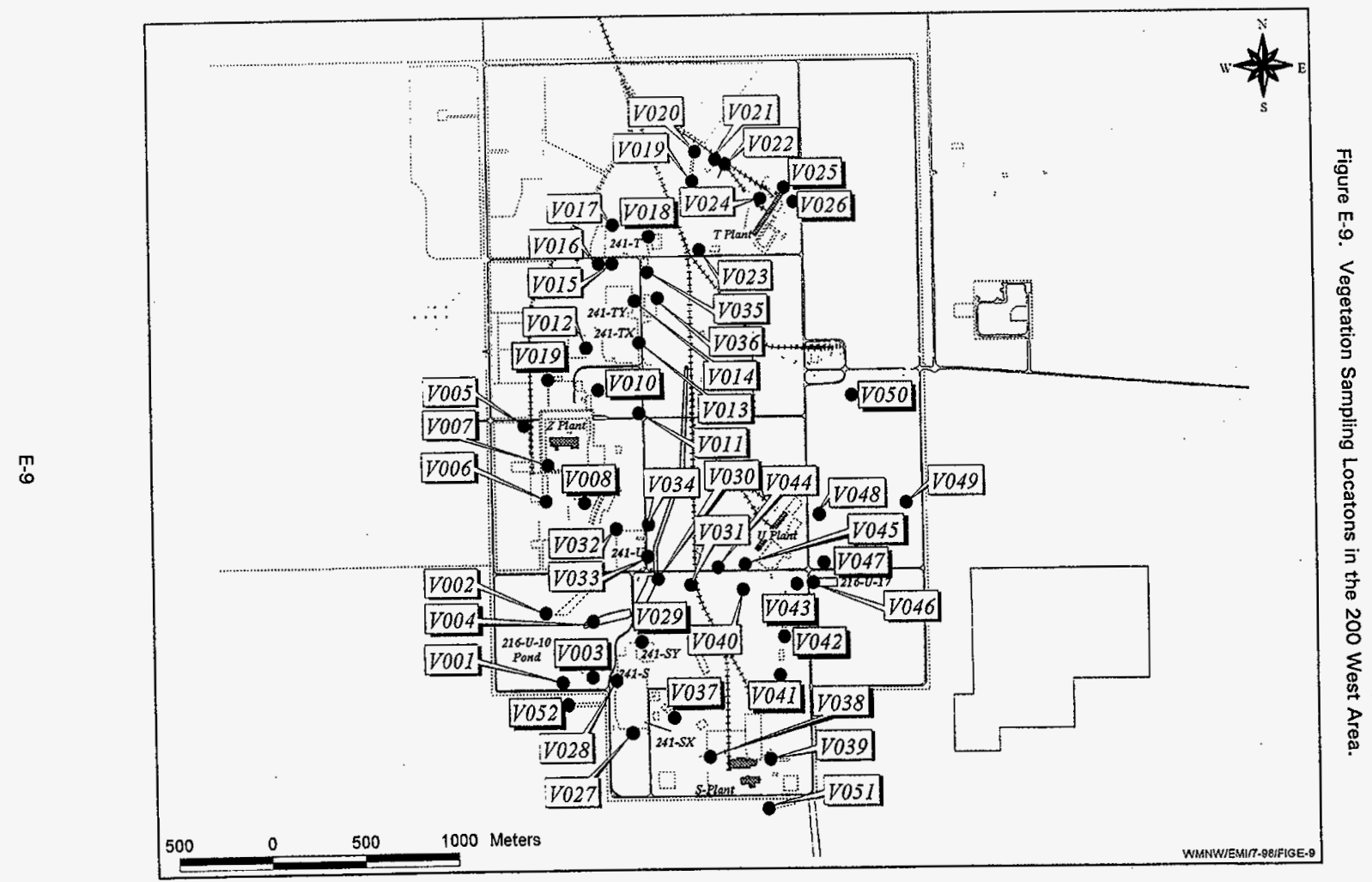


Figure E-10. Vegetation Sampling Locatons in the 600 Area.

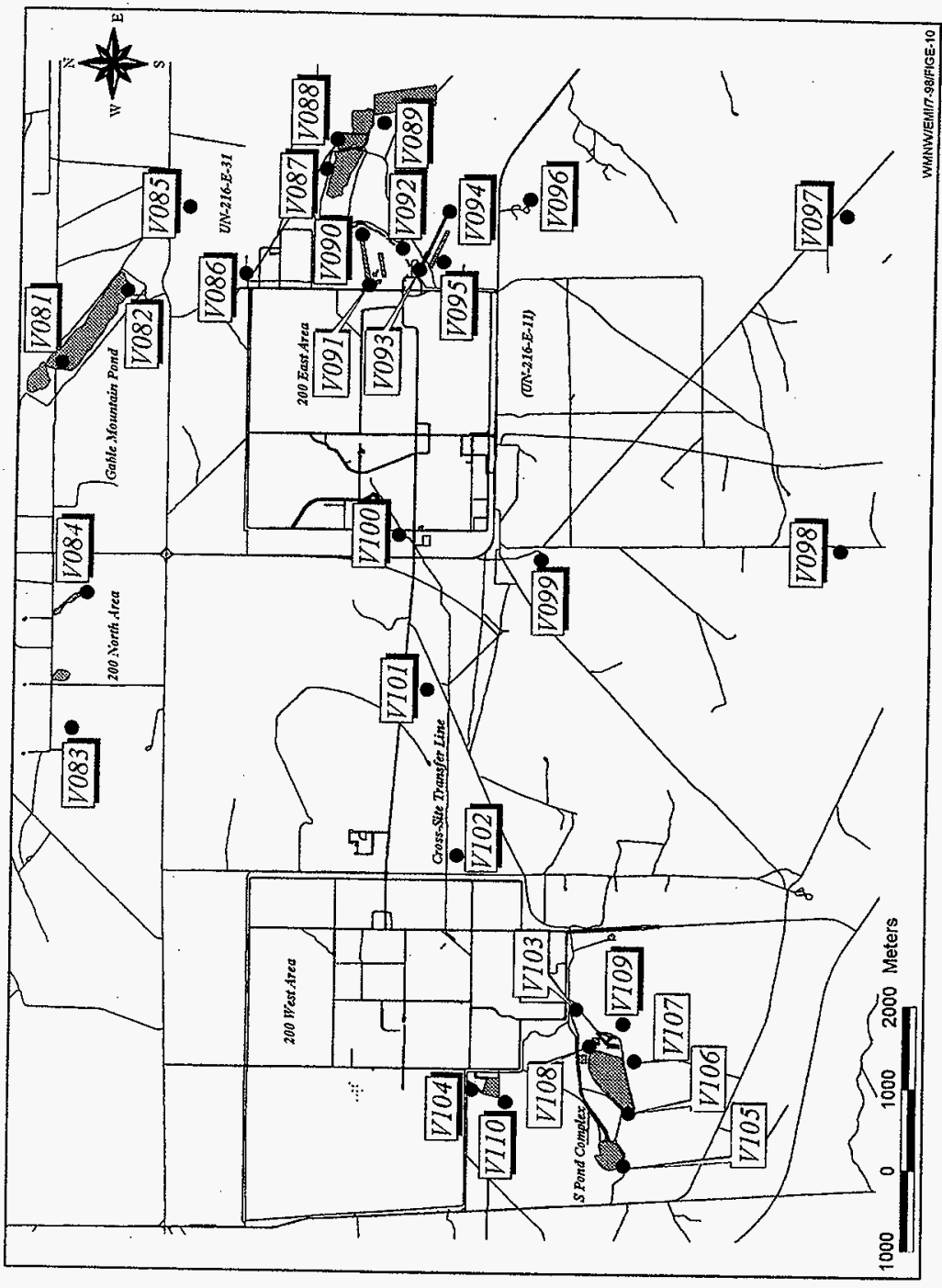


Figure E-11. Vegetation Sampling Locatons in the 300 Area.

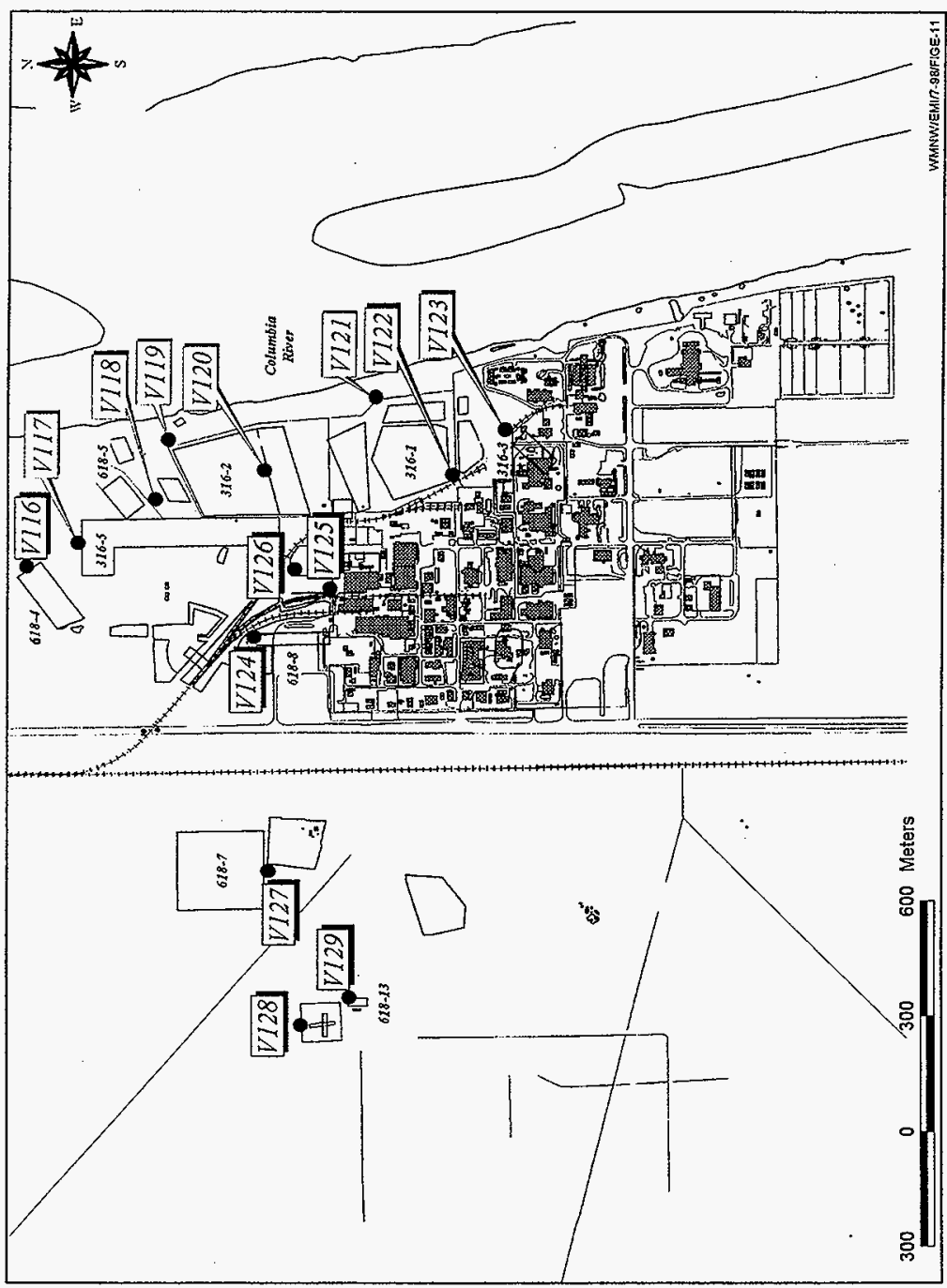


Figure E-12. Vegetation Sampling Locatons in the 400 Area.

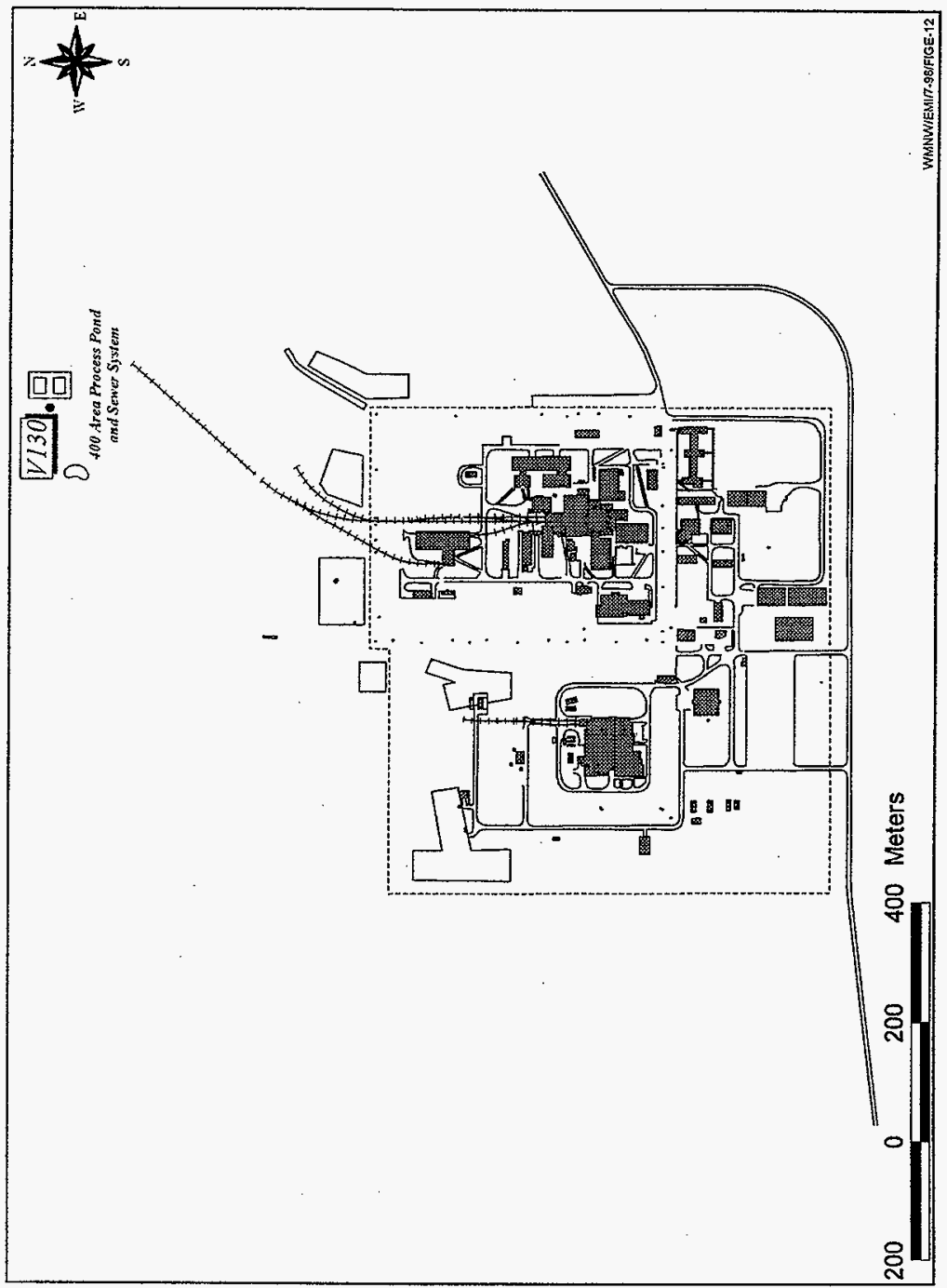


HNF-EP-0573-6

Figure E-13. Vegetation Sampling Locatons for the 100 D/DR Remediation Project.

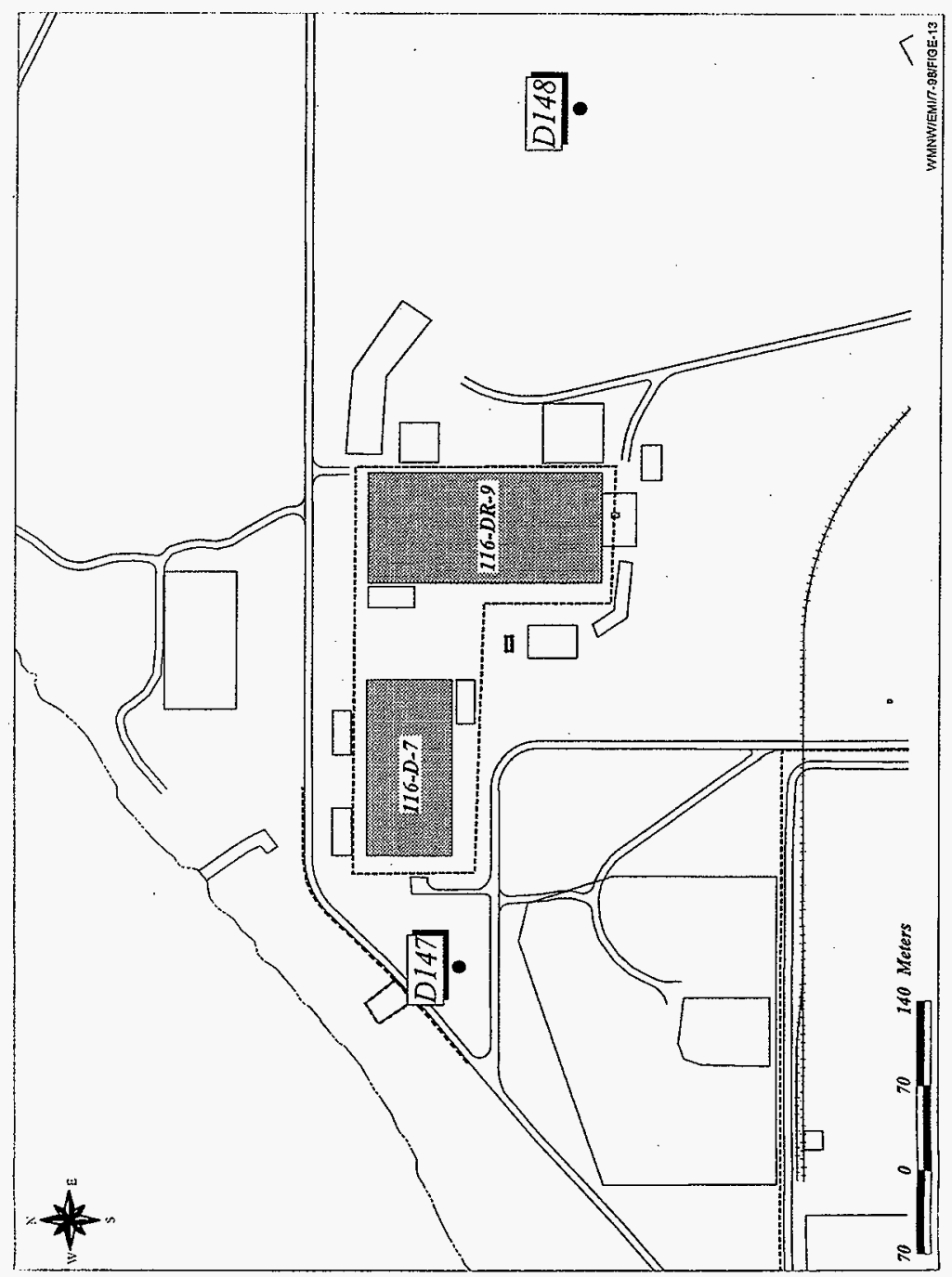


Table E-1: 1997 Soil Sample Results, 100-N Area (pCi/gm Dry Weight).

(Sheet 1 of 2)

\begin{tabular}{|c|c|c|c|c|c|}
\hline & Co-60 & $2 n-65$ & $\mathrm{~S} x-90$ & $\mathrm{Ru}-103$ & $R u-106$ \\
\hline Code & Result \pm Error & Result \pm Error & Result \pm Error & Result \pm Error & Result \pm Exror \\
\hline $\mathbf{7 6 0 2}$ & $1.2 \mathrm{E}+01 \pm 7.8 \%$ & ND & $2.2 E \div 01 \pm 14.0 \%$ & ND & ND \\
\hline Y603 & $2.2 \mathrm{E}+00 \pm 7.8 \mathrm{z}$ & ND & $5.0 \mathrm{E}-01 \pm 30.0 \%$ & ND & ND \\
\hline 9604 & $2.6 \mathrm{E}+00 \pm 7.8 \mathrm{z}$ & ND & $2.6 \mathrm{E}-01 \pm 50.07$ & Nं & $\mathrm{ND}$ \\
\hline$Y 605$ & $3.72-01 \pm 8.4 \%$ & $\mathrm{ND}$ & $2.42-01 \pm 50.07$ & SD & ND \\
\hline Y608 & $1.6 \mathrm{E}-01 \pm 9.7 \%$ & $\mathrm{ND}$ & $4.2 E-01 \pm 40.02$ & $\mathrm{MD}$ & ND \\
\hline$x 610$ & $4.1 E-02 \pm 20.0 x$ & ND & $2.7 \mathrm{E}-01 \pm 40.0 \mathrm{z}$ & $\mathrm{ND}$ & IND \\
\hline$Y 611$ & $2.6 \mathrm{E}-01 \pm 8.8 \mathrm{z}$ & XD & ND & ND & ND \\
\hline
\end{tabular}

\begin{tabular}{|c|c|c|c|c|c|}
\hline & $S n-113$ & $5 b-125$ & $C_{s-134}$ & Cs -137 & $\mathrm{Ce}-144$ \\
\hline Code & Result \pm Error & Result \pm Exror & Result \pm Exror & Result \pm Error & Result \pm Error \\
\hline Y602 & $\mathrm{ND}$ & $\mathrm{ND}$ & $\mathrm{ND}$ & $3.6 \mathrm{E}+00 \pm 13.0 \mathrm{x}$ & SDD \\
\hline Y603 & ND & $\mathrm{ND}$ & $2.4 \mathrm{E}-02 \pm 55.0 \mathrm{z}$ & $1.4 \mathrm{E}+00 \pm 13.0 \mathrm{x}$ & SD \\
\hline 1604 & $\mathrm{KD}$ & IND & $.3 .8 \mathrm{E}-0 \mathrm{Z} \pm 43.0 \mathrm{Z}$ & $6.9 E-01 \pm 13.02$ & ND \\
\hline Y605 & $\mathrm{ND}$ & $\mathrm{ND}$ & $4.28-02 \pm 31.07$ & $2.08-01 \pm 24.0 x$ & ND \\
\hline Y608 & ND & ND & $2.6 \mathrm{E}-02 \pm 41.0 \%$ & $6.7 \mathrm{E}-02 \pm 19.0 \mathrm{z}$ & ND \\
\hline Y610 & ND & SD & $4.8 E-02 \pm 26.07$ & $1.8 E-01 \pm 15.0 \%$ & NDD \\
\hline $\mathrm{Y} 611$ & ND & ND & $3.5 E-02 \pm 30.0 \%$ & $8.3 E-02 \pm 18.0 \%$ & ND \\
\hline
\end{tabular}


Table E-1: 1997 Soil Sample Results, 100-N Area (pCi/gm Dry Weight).

(Sheet 2 of 2 )

\begin{tabular}{|c|c|c|c|c|c|}
\hline & $\mathrm{Eu}-152$ & Eu-154 & $E t-15 s$ & $v-234$ & $U-235$ \\
\hline Code & Result \pm Error & Result \pm Error & Result \pm Exror & Result \pm Error & Result \pm Error \\
\hline $\mathrm{Y} 602$ & ND & $\mathrm{ND}$ & ND & $1.9 \mathrm{E}-01 \pm 22.02$ & $1.7 \mathrm{E}-02 \pm 56.07$ \\
\hline Y603 & $\mathrm{ND}$. & ND & $4.9 E-02 \pm 83.0 \%$ & $1.8 \mathrm{E}-01 \pm 22.0 z$ & $1.7 E-02 \pm 57.0 \%$ \\
\hline$Y 604$ & $\mathrm{ND}$ & KD & $5.6 E-02 \pm 73.0 \%$ & $2.0 \mathrm{E}-01 \pm 21.0 \mathrm{z}$ & 2. $2 E-02 \pm 48.0 \%$ \\
\hline 9605 & ND & $\mathrm{KD}$ & $\mathrm{ND}$ & $3.2 E=01 \pm 19.0 z$ & $2.5 E-02 \pm 47.0 z$ \\
\hline Y608 & WD & SDD & ND & $1.6 \mathrm{E}-01 \pm 23.0 \%$ & $1.9 \mathrm{E}-02 \pm 52.07$ \\
\hline Y610 & ND & ND & $\mathrm{ND}$ & $2.3 \mathrm{E}-01 \pm 20.03$ & $2.1 \mathrm{E}-02 \pm 47.02$ \\
\hline$\$ 611$ & $\mathrm{ND}$ & SD & $\mathrm{ND}$ & $1.9 E-01 \pm 21.0 \%$ & $2.0 \mathrm{E}-02 \pm 52.07$ \\
\hline
\end{tabular}

\begin{tabular}{|c|c|c|c|}
\hline & $\mathrm{U}-238$ & Pu-238 & $\operatorname{Ru} 239 / 40$ \\
\hline Code & Result \pm Error & Result \pm Error & Result \pm Error \\
\hline $\mathrm{Y} 602$ & $2.1 z-01 \pm 21.0 z$ & $7.8 \mathrm{E}-01 \pm 18.0 \mathrm{x}$ & $3.6 E+00 \pm 16.0 z$ \\
\hline 9603 & $1.7 \mathrm{E}-01 \pm 22.0 \%$ & ID & $3.1 E-02 \pm 39.0 \%$ \\
\hline Y 604 & $1.98-01 \pm 21.0 z$ & $\mathrm{ND}$ & SD \\
\hline Y605 & $3.0 E-01 \pm 20.0 z$ & No & $1.1 E-02 \pm 79.07$ \\
\hline Y608 & $1.6 \mathrm{E}-01 \pm 23.07$ & $\mathrm{ND}$ & ND \\
\hline $\mathrm{Y} 610$ & $2.3 \mathrm{E}-01 \pm 20.0 \%$ & FD & $4.85-03 \pm 90.07$ \\
\hline Y 611 & $1.9 \mathrm{E}-01 \pm 21.0 \mathrm{z}$ & ND & $\mathrm{ND}$ \\
\hline
\end{tabular}


Table E-2: 1997 Soil Sample Results, 200 Area (pCi/gm Dry Weight).

(Sheet 1 of 12)

\begin{tabular}{|c|c|c|c|c|c|}
\hline & Co-60 & $2 n-65$ & $5 x-90$ & $R \mathbf{R}-103$ & $\mathrm{Rut}-106$ \\
\hline Codo & Restult \pm Erxor & Result \pm Exror & Result \pm Error & Result \pm Error & Result \& Error \\
\hline D001 & SD & $\mathrm{ND}$ & ND & $\mathrm{KD}$ & ND \\
\hline Do03 & $\mathrm{MD}$ & $\mathrm{ND}$ & $2.4 \mathrm{E}-01 \pm 25.02$ & ND & ND \\
\hline .0005 & ND & ND & $S D$ & ND & $\mathrm{KD}$ \\
\hline D007 & $\mathrm{ND}$ & ND & $2.1 \mathrm{E}-01 \pm 25.07$ & ND & KDD \\
\hline D009 & $\mathrm{ND}$ & SDD & $1.5 z-01 \pm 30.0 \%$ & SD & ND \\
\hline D011 & ND & ND & $2.1 E-01 \pm 25.07$ & ND & SD \\
\hline D013 & $\mathrm{ND}$ & HD & $2.35+00 \pm 14.0 z$ & ND & ND \\
\hline D015 & ND & $\mathrm{ED}$ & $\mathrm{ND}$ & ND & KDD \\
\hline D017 & ND & $\mathrm{ND}^{-}$ & ND & $\mathrm{ND}$ & ND \\
\hline D019 & KD & $\mathrm{ND}$ & $1.6 E-01 \pm 90.07$ & ND & WD \\
\hline Do21 & $5.88-02 \pm 12.0 \%$ & सD & $2.1 \mathrm{E}-01 \pm 70.0 \mathrm{~K}$ & $\mathrm{ND}$ & ND \\
\hline D023 & SD & ND & KD & ND & ND \\
\hline D025 & $1.28-02 \pm 60.08$ & ND & $4.6 \mathrm{E}-01 \pm 16.0 z$ & $\mathrm{ND}$ & ND \\
\hline D027 & ND & ND & $1.9 E-01 \pm 25.0 z$ & $\mathrm{ND}$ & $\mathrm{SD}$ \\
\hline 0029 & ND & $1.2 \mathrm{E}-02 \pm 60.0 \mathrm{z}$ & $4.3 \bar{E}-01 \pm 15.07$ & ND & $S D$ \\
\hline D031 & ND & ND & $2.8 \mathrm{E}-01 \pm 70.07$ & ND & ND \\
\hline D033 & $\mathrm{ND}$ & ND & $9.1 \bar{E}-01 \pm 20.08$ & $\mathrm{ND}$ & $\mathrm{KD}$ \\
\hline D035 & ND & $\mathrm{ND}$ & $3.4 E+00 \pm 15.0 \%$ & $\mathrm{SD}$ & ND. \\
\hline D037 & $\mathrm{ND}$ & $\mathrm{ND}$ & $4.4 E-01 \pm 30.07$ & ND & $\mathrm{ND}$ \\
\hline D039 & $2.18-02 \pm 25.0 z$ & ND & $1.4 E+00 \pm 18.0 z$ & ND & ST \\
\hline D04i & ND & $\mathbb{N D}$ & STD & XND & $\mathrm{KD}$ \\
\hline D043 & $\mathrm{ND}$ & $\mathrm{ND}$ & $2.65-01 \pm 50.0 z$ & ND & $M$ \\
\hline D045 & $\mathrm{ND}$ & $\mathrm{ND}$ & $2.6 E+00 \pm 16.0 \%$ & ND & ND \\
\hline D047 & ND & ND & SD & $\mathrm{ND}$ & SD \\
\hline 0049 & $\mathrm{ND}$ & ND & $\mathrm{ND}$ & IND & ND \\
\hline D051 & ID & ND & ND & $\mathrm{SD}$ & $\mathrm{ND}$ \\
\hline D053 & ND & ND & SDD & $\mathrm{ND}$ & ND \\
\hline
\end{tabular}


Table E-2: 1997 Soil Sample Results, 200 Area (pCi/gm Dry Weight).

(Sheet 2 of 12)

\begin{tabular}{|c|c|c|c|c|c|}
\hline & $\mathrm{Co}-60$ & $2 n-65$ & $5 x-90$ & $R v_{2}-103$ & Rut-106 \\
\hline Code & Result \pm Exrox & Result \pm Error & Result \pm Error & Result \pm Errox & Result \pm Error \\
\hline Do55 & $\mathrm{ND}$ & ND & $M D$ & ND & $N D$ \\
\hline Do57 & ND & ND & $S D$ & ND & WD \\
\hline D059 & ND & ND & $1.8 \mathrm{E}-01 \pm 80.0 z$ & $\mathrm{SD}$ & N \\
\hline$D 062$ & $\mathrm{ND}$ & ND & $5.2 E-01 \pm 60.02^{\circ}$ & $\mathrm{ND}$ & ND \\
\hline D063 & ND & SD & $9.1 E-01 \pm 25.0 \%$ & KD & IND \\
\hline D065 & ND & ND & $6.65-01 \pm 40.0 \%$ & ND & WD \\
\hline$D 067$ & SND & $\mathrm{ID}$ & $6.1 E-01 \pm 40.0 x$ & ND & $\mathrm{ND}$ \\
\hline D069 & ND & $\mathrm{XD}$ & $5.3 E-01 \pm 50.0 \%$ & ND & ND \\
\hline D071 & $\mathrm{WD}$ & SD & $4.58-01 \pm 70.0 \%$ & $\mathrm{ND}$ & ND \\
\hline D073 & ND & ND & $5.4 E-01 \pm 50.0 z$ & ND & $\mathrm{SD}$ \\
\hline D075 & ND & $\mathrm{ND}$ & $8.4 \mathrm{E}-01 \pm 40.0 \%$ & ND & ND \\
\hline D077 & Mp & $\mathrm{ND}$ & $4.2 \mathrm{E}-01 \pm 60.0 \%$ & ND & ND \\
\hline D079 & WD & ND & ND & SD & ND \\
\hline Do81 & $\mathrm{ND}$ & ND & ND & $\mathrm{KD}$ & ND \\
\hline D083 & ND & NiD & $4.2 \mathrm{E}-01 \pm 70.02$ & $\mathrm{ND}$ & $\mathrm{ND}$ \\
\hline Do8s & $\mathrm{ND}$ & ND & ND & $\mathrm{ND}$ & ND \\
\hline 0087 & $\mathrm{ND}$ & ND & ND & NDD & ND \\
\hline D089 & ND & $\mathrm{ND}$ & $1.05+00 \pm 30.0 z$ & ND & WD \\
\hline D091 & ND & IND & $9.4 \mathrm{E}-01 \pm 30.0 z$ & ND & $\mathrm{ND}$ \\
\hline D093 & $\mathrm{ND}$ & MD & $4.0 \mathrm{E}-01 \pm 60.0 z$ & ND & $\mathrm{ND}$ \\
\hline D095 & $\mathrm{ND}$ & ID & $6.3 E-01 \pm 40.0 z$ & ND & $\mathrm{ND}$ \\
\hline D097 & $\mathrm{ND}$ & ND & $4.4 \bar{D}-01=60.0 z$ & ND & $\mathrm{ND}$ \\
\hline 0099 & $\mathrm{ND}$ & ND & $9.1 \mathrm{E}-01 \pm 40.0 \mathrm{z}$ & $\mathrm{ND}$ & $\mathrm{ND}$ \\
\hline D101 & ND & ND & $4.8 \mathrm{E}-0 \mathrm{I} \pm 30.0 \mathrm{z}$ & iND & $\mathrm{STD}$ \\
\hline D103 & IDD & $\mathrm{ND}$ & $2.9 \mathrm{E}-01 \pm 40.07$ & $\mathrm{ND}$ & ND \\
\hline D105 & ND & ND & $\mathrm{ND}$ & SND & ND \\
\hline D107 & $\mathrm{ND}$ & $\mathrm{ND}$ & $4.4 E-01 \pm 30.0 \%$ & SD & $\dot{\text { ND }}$ \\
\hline
\end{tabular}


HNF-EP-0573-6

Table E-2: 1997 Soil Sample Results, 200 Area (pCi/gm Dry Weight).

(Sheet 3 of 12)

\begin{tabular}{|l|c|c|c|c|c|}
\hline \multirow{2}{*}{$\begin{array}{l}\text { EDP } \\
\text { Code }\end{array}$} & Co-60 & Zn-65 & Sr-90 & Ru-103 & Ru-106 \\
\cline { 2 - 6 } & Result \pm Error & Result \pm Error & Result \pm Error & Result \pm Error & Result \pm Error \\
\hline D109 & SD & ND & $2.9 \mathrm{E}-01 \pm 50.0 \%$ & ND & ND \\
\hline
\end{tabular}


Table E-2: 1997 Soil Sample Results, 200 Area (pCi/gm Dry Weight).

(Sheet 4 of 12)

\begin{tabular}{|c|c|c|c|c|c|}
\hline & $5 n-113$ & $S b-125$ & Cs -134 & Cs -137 & $\mathrm{Ce}^{-144}$ \\
\hline $\operatorname{Cod} \theta$ & Result \pm Error & Result \pm Error & Result \pm Error & Result \pm Error & Result \pm Error \\
\hline DOOI & $\mathrm{KD}$ & ND & $2.9 E-02 \pm 18.0 z$ & $1.0 E+00 \pm 13.02$ & ND \\
\hline D003 & $\mathrm{ND}$ & HD & $3.6 \mathrm{E}-02 \pm 24.0 \%$ & $1.3 E+00 \pm 16.07$ & ND \\
\hline D005 & ND & ND & $2.5 E-02 \pm 25.02$ & $8.3 \mathrm{E}-02 \pm 16.07$ & ND \\
\hline D007 & SD & 203 & $3.2 E-02 \pm 18.0 \%$ & $7.4 E-02 \pm 14.07$ & ND \\
\hline Do09 & ND & ND & $2.4 \mathrm{E}-02 \pm 30.02$ & $1.3 E-01 \pm 16.07$ & SD \\
\hline D011 & ND & ND & $3.2 \mathrm{E}-02 \pm 25.0 z$ & $2.6 \mathrm{E}+00 \pm 14.07$ & ND \\
\hline D013 & ND & ND & $2.5 \mathrm{E}-02 \pm 29.0 z$ & $2.4 E+00 \pm 16.0 \%$ & $\mathrm{ND}$ \\
\hline D015 & ND & - ND & $3.2 \mathrm{E}-02 \pm 22.0 \%$ & $1.3 E+00 \pm 14.0 \%$ & ND \\
\hline p017 & ND & $\mathrm{ND}$ & $4.1 \mathrm{E}-02 \pm 23.0 \mathrm{z}$ & $1.48+00 \pm 15.0 \mathrm{x}$ & ND \\
\hline D019 & $\mathrm{ND}$ & ND & $3.1 \mathrm{E}-02 \pm 18.0 \mathrm{z}$ & $1.1 E+00 \pm 13.0 \%$ & $S D$ \\
\hline D021 & $\mathrm{ND}$ & ND & $3.0 \mathrm{E}-02 \pm 27.08$ & $1.6 \mathrm{E}+00 \pm 14.0 \mathrm{z}$ & ND \\
\hline $\mathrm{DO} 23$ & ND & $\mathrm{ND}$ & $2.6 \mathrm{E}-02 \pm 30.0 \mathrm{z}$ & $7.6 \mathrm{E}-01 \pm 16.0 \%$ & ND \\
\hline $\mathrm{D} 025$ & ND & ND & $3.4 E-02 \pm 29.02$ & $9.3 E+00 \pm 24.07$ & ND \\
\hline D0ट்7 & $\mathrm{ND}$ & $\mathrm{ND}$ & $3.1 E-02 \pm 26.02$ & $1.2 \mathrm{E}+00 \pm 15.0 \mathrm{x}$ & FD \\
\hline 0029 & ND & ND & $3.0 \mathrm{E}-02 \pm 18.0 \%$ & $5.7 E+00 \pm 13.0 x$ & $\mathrm{ND}$ \\
\hline D031 & ND & ND & $3.4 \mathrm{z}-02 \pm 26.07$ & $1.2 E+00 \pm 14.0 \%$ & $\mathrm{ND}$ \\
\hline D033 & $\mathrm{ND}$ & $\mathrm{KD}$ & $2.9 E-02 \pm 26.0 z$ & $1.2 E+01 \pm 16.02$ & RDD \\
\hline D035 & $\mathrm{ND}$ & ND & $2.4 \mathrm{E}-02 \pm 28.0 z$ & $5.7 \mathrm{E}+00 \pm 14.0 \mathrm{z}$ & $\mathrm{ND}$ \\
\hline D037 & ID & ID & $2.8 \mathrm{E}-02 \div 27.07$ & $1.4 \mathrm{E}+00 \pm 13.0 \%$ & ND \\
\hline 2039 & $\mathrm{AD}$ & $\mathrm{ND}$ & $2.1 \mathrm{E}-02 \pm 36.0 z$ & $7.0 \mathrm{E}+00 \pm 15.0 \%$ & $\mathrm{ND}$ \\
\hline D041 & $\mathrm{MD}$ & $\mathrm{ND}$ & $3.3 \mathrm{E}-02 \pm 21.07$ & $1.6 \mathrm{E}+00 \pm 14.0 \mathrm{z}$ & $\mathrm{ND}$ \\
\hline D043 & $\ldots N D$ & $\mathrm{ND}$. & $3.95-02 \pm 23.07$ & $2.95+00 \pm 26.07$ & in \\
\hline D045 & $\mathrm{ND}$ & $\mathrm{ND}$ & $2.8 \mathrm{E}-02 \pm 26.0 z$ & $6.9 E+00 \pm 14.0 z$ & $\mathrm{ND}$ \\
\hline D047 & $\mathrm{ND}$ & $\mathrm{ND}$ & $3.4 E-02 \pm 17.07$ & $3.0 E+00 \pm 13.0 x$ & $\mathrm{ND}$ \\
\hline D049 & $\mathrm{ND}$ & ND & $3.2 E-02 \pm 22.0 \%$ & $5.7 E-01 \pm 15.02$ & KD \\
\hline Dos1 & ND & ND & $2.9 \mathrm{E}-02 \pm 23.0 \mathrm{z}$ & $4.8 \mathrm{E}-01 \pm 14.0 \mathrm{Z}$ & ND \\
\hline 0053 & ND & WD & $3.0 E-02 \pm 30.02$ & $3.4 \mathrm{E}+00 \pm 14.0 \mathrm{x}$ & ND \\
\hline
\end{tabular}


Table E-2: 1997 Soil Sample Results, 200 Area (pCi/gm Dry Weight).

(Sheet 5 of 12)

\begin{tabular}{|c|c|c|c|c|c|}
\hline & $S n-113$ & $S b-125$ & $C_{s-134}$ & Cs-137 & $\mathrm{Ce}-144$ \\
\hline Code & Result \pm Errox & Result \pm Error & Result \pm EIzor & Result \pm Error & Result \pm Exror \\
\hline D055 & SD & $\mathrm{ND}$ & $3,2 \Sigma-02 \pm 25.07$ & $8.1 E-01 \pm 16.0 z$ & ND \\
\hline D057 & WD & ND & $3.4 \mathrm{E}-02 \pm 23.0 \mathrm{x}$ & $3.5 E+00 \pm 14.0 z$ & $\mathrm{ND}$ \\
\hline D059 & ND & ND & $3.6 \mathrm{E}-02 \pm 19.07$ & $6.2 \mathrm{E}-01 \pm 13.07$ & $\mathrm{ND}$ \\
\hline D06I & WD & $\mathrm{ND}$ & $3.0 \mathrm{E}-02 \pm 25.0 \%$ & $2.2 \mathrm{E}-01 \pm 26.0 \%$ & $\mathrm{ND}$ \\
\hline D063 & $\mathrm{ID}$ & ND & $3,3 \mathrm{E}-02 \pm 28.0 \%$ & $4.2 \mathrm{E}-01 \pm 14.07$ & ND \\
\hline D065 & SD & $6.4 \mathrm{E}-02 \pm 52.0 z$ & $3.0 \mathrm{E}-02 \pm 24.0 \%$ & $7.4 \mathrm{E}+00 \pm 15.0 \%$ & ND \\
\hline D067 & ND & ND & $3.1 E-02 \pm 25.0 \%$ & $1.6 \mathrm{E}-01 \pm 15.02$ & ND \\
\hline$D 069$ & KD & ND & $3.2 \mathrm{E}-02 \pm 24.0 \%$ & $3.8 \mathrm{E}-02 \pm 22.0 \mathrm{z}$ & ND \\
\hline 0071 & SD & $\mathrm{ND}$ & $2.5 \mathrm{E}-02 \pm 29.0 \%$ & $2.1 \mathrm{E}-01 \pm 14.0 \mathrm{z}$ & ND \\
\hline D073 & ND & $\mathrm{ND}$ & 4.3E-02 \pm 22.07 & $2.18+00 \pm 16.0 x$ & ND \\
\hline Do75 & ND & ND & $2.8 E-02 \pm 24.0 z$ & $1.9 E-01 \pm 15.07$ & ND \\
\hline D077 & ND & ND & $2.6 \mathrm{E}-02 \pm 17.0 z$ & $3.0 \mathrm{E}-01 \pm 13.07$ & ND \\
\hline D079 & ND & ND & $3.2 \mathrm{E}-02 \pm 26.0 z$ & $9.5 E-02 \pm 17.0 z$ & ND \\
\hline D08I & ND & ND & $3.6 \mathrm{E}-02 \pm 26.0 \mathrm{z}$ & WD. & ND \\
\hline D083 & SD & $\mathrm{ND}$ & $3.6 E-02 \pm 26.0 z$ & $1.1 E+00 \pm 16.0 \%$ & ND \\
\hline D085 & ND & ND & $3.2 E-02 \pm 23.07$ & $1.1 E+00 \pm 14.0 z$ & IND \\
\hline Do87 & ND & $\mathrm{ND}$ & $3.08-02 \pm 25.0 \%$ & $1.2 \mathrm{E}-01 \pm 16.0 \mathrm{z}$ & SD \\
\hline D089 & ND & $\mathrm{ND}$ & $3.1 \mathrm{E}-02 \pm 26.0 \mathrm{z}$ & $5.6 \mathrm{E}-02 \pm 18.0 \mathrm{z}$ & $\mathrm{ND}$ \\
\hline D091 & SD & ND & $3.2 E-02 \pm 25.0 z$ & $7.7 E-01 \pm 16.07$ & $\mathrm{KD}$ \\
\hline D093 & $\mathrm{ND}$ & ND & $2.2 \mathrm{E}-02 \pm 24.0 \%$ & $3.3 \mathrm{E}-01 \pm 14.02$ & ND \\
\hline D095 & ID & ND & $2.5 E-02 \pm 28.0 z$ & $5.1 E-01 \pm 15.07$ & $\mathrm{ID}$ \\
\hline D097 & $\ldots \mathrm{ID}$ & ND & $2.8 \mathrm{E}-02 \pm 19.0 \%$ & $6.65-02 \pm 14.07$ & $\mathrm{MD}$ \\
\hline D099 & $\mathrm{ND}$ & ND & $2.3 E-02 \pm 30.07$ & $4.6 E-02 \pm 20.02$ & $\mathrm{ND}$ \\
\hline D101 & $\mathrm{ND}$ & SD & $3.4 \mathrm{E}-02 \pm 24.0 z$ & $1.6 E+00 \pm 16.0 \%$ & $\mathrm{ND}$ \\
\hline D103 & IND & WD & $2.6 \mathrm{E}-02 \pm 22.0 z$ & $3.28-01 \pm 14.0 \%$ & ND \\
\hline$D 105$ & ND & $\mathrm{ND}$ & $3.2 E-.02 \pm 16.0 z$ & $1.2 E-01 \pm 13.0 z$ & ND \\
\hline$\$ 107$ & $\mathrm{ND}$ & ID & $2.5 \mathrm{E}-02 \pm 25.0 \%$ & $1.3 E-02 \pm 35.07$ & SD \\
\hline
\end{tabular}


Table E-2: 1997 Soil Sample Results, 200 Area (pCi/gm Dry Weight).

(Sheet 6 of 12)

\begin{tabular}{|c|c|c|c|c|c|}
\hline & $5 n-113$ & $\mathrm{Sb}-125$ & Cs -134 & $C s-137$ & $\mathrm{Ce}-144$ \\
\hline $\operatorname{Cod} \theta$ & Result \pm Error & Result \pm Error & Result \pm Error & Result \pm Error & Result \pm Exrox \\
\hline$D 109^{\circ}$ & IDD & KD & $3.2 \mathrm{E}-02 \pm 23.0 \mathrm{x}$ & $3.8 E-02 \pm 21.0 \mathrm{z}$ & ND \\
\hline
\end{tabular}


Table E-2: 1997 Soil Sample Results, 200 Area (pCi/gm Dry Weight).

(Sheet 7 of 12)

\begin{tabular}{|c|c|c|c|c|c|}
\hline & Eu-152 & Eu-154 & Eu-155 & $v-234$ & $\mathrm{U}-235$ \\
\hline Code & Result \pm Error & Result \pm Ezror & Result \pm Error & Result \pm Error & Result \pm Error \\
\hline D001 & $\mathbb{N D}$ & ND & SD & $2.3 E-01 . \pm 20.0 \%$ & $1.8 E-02 \pm 58.0 \%$ \\
\hline D003 & $\mathrm{ND}$ & ND & $4.2 \mathrm{E}-02 \pm 51.0 \mathrm{x}$ & $2.05-01 \pm 19.0 z$ & $1.3 \mathrm{E}-02 \pm 47.0 \%$ \\
\hline Do0s & $\mathrm{ND}$ & ND & $2.8 E-02 \pm 58.0 \%$ & $1.78-01 \pm 19.07$ & $1.4 \mathrm{E}-02 \pm 43.02$ \\
\hline D007 & ND & ND & $2.5 E-02 \pm 59.0 z$ & $2.0 \mathrm{E}-01 \pm 19.07$ & $1.4 \mathrm{E}-02 \pm 47.0 \%$ \\
\hline 0009 & ND & $\mathrm{ND}$ & ND & $1.58-01 \pm 20.0 z$ & $2.18-02 \pm 51.0 \%$ \\
\hline Do11 & $\mathrm{ND}$ & $\mathrm{ND}$ & No & $2.6 \mathrm{E}-01 \pm 18.0 \mathrm{x}$ & $1.5 E-02 \pm 47.07$ \\
\hline D013 & $\mathrm{ND}$ & $\mathrm{ND}$ & ND & $2.0 \mathrm{E}-01 \pm 12.0 \%$ & $1.0 \mathrm{E}-02 \pm 49.0 \mathrm{z}$ \\
\hline D015 & ND & $\mathrm{ND}$ & $4.9 E-02 \pm 51.0 \%$ & $1.8 \mathrm{E}-01 \pm 12.0 \mathrm{x}$ & $1.4 \mathrm{E}-02 \pm 40.02$ \\
\hline D017 & ND & $\mathrm{ND}$ & $3.8 \mathrm{E}-02 \pm 53.07$ & $1.6 \mathrm{E}-01 \pm 19.0 \mathrm{z}$ & $9.7 E-03 \pm 52.07$ \\
\hline D019 & . ND & $K D$ & $4.1 E-02 \pm 47.07$ & $1.6 E-01 \pm 20.0 \%$ & i. $28-02 \pm 50.0 z$ \\
\hline Do21 & $1.38-01 \pm 12.0 \%$ & $\mathrm{ND}$ & ND & $1.8 \mathrm{E}-01 \pm 19.0 \mathrm{z}$ & $9.2 \mathrm{E}-03 \pm 60.0 \%$ \\
\hline D023 & $\mathrm{ND}$ & $\mathrm{ND}$ & $3.6 \mathrm{E}-02 \pm 69.0 \%$ & $1.6 \mathrm{E}-01 \pm 19.0 \mathrm{z}$ & $1.5 E-02 \pm 44.0 z$ \\
\hline D025 & ND & ND & NSD & $1.9 \mathrm{E}-01 \pm 19.0 \mathrm{z}$ & $1.1 E-02 \pm 50.0 z$ \\
\hline D027 & $\mathrm{ND}$ & ND & $\mathrm{KD}$ & $1.8 \mathrm{E}-01 \pm 20.0 \%$ & $1.38-02 \pm 48.0 \%$ \\
\hline D029 & $\mathrm{ND}$ & ND & $N D$ & $1.6 \mathrm{E}-01 \pm 19.07$ & $6.9 \mathrm{E}-03 \pm 59.0 \%$ \\
\hline D031 & ND & WD & $4.7 \mathrm{E}-02 \pm 50.07$ & $1.7 E-01 \pm 19.0 \%$ & $1.2 E-02 \pm 49.0 z$ \\
\hline Do33 & $\mathrm{ND}$ & $\mathrm{MD}$ & ND & $2.4 \mathrm{E}-01 \pm 18.0 z$ & $1.3 E-02 \pm 46.0 z$ \\
\hline D035 & ND & $\mathrm{ND}$ & ND & $1.4 \mathrm{E}-01 \pm 20.0 z$ & $7.6 \mathrm{E}-03 \pm 58.0 \mathrm{z}$ \\
\hline D037 & ND & ND . & $2.7 \bar{E}-02 \pm 60.0 \%$ & $2.18-01 \pm 18.07$ & $9.6 \mathrm{E}-03 \pm 54.0 \mathrm{z}$ \\
\hline Do39 & $\mathrm{ND}$ & $3.2 E-02 \pm 31.0 \%$ & $5.7 E-02 \pm 60.07$ & $1.9 \mathrm{E}-01 \pm 19.0 \mathrm{x}$ & $1.3 E-02 \pm 46.02$ \\
\hline D041 & MD & $\mathbb{N D}$ & ND & $1.8 \mathrm{E}-01 \pm 19.07$ & $1.2 \mathrm{E}-02 \pm 47.07$ \\
\hline D043 & SND & $\$ 0$ & $6.38-02 \pm 46.0 \%$ & $2.9 E-01 \pm 12.07$ & $2.0 \mathrm{E}-0 \mathrm{Z} \pm 39.0 \mathrm{z}$ \\
\hline D045 & ND & $\mathrm{ND}$ & $\mathrm{ND}$ & $2.2 \mathrm{E}-01 \pm 13.07$ & $1.7 \mathrm{E}-02 \pm 40.02$ \\
\hline D047 & $\mathrm{ND}$ & SD & $3.5 \Sigma-02 \pm 41.0 \%$ & $3.8 \mathrm{E}-01 \pm 18.0 \%$ & $2.6 \mathrm{E}-02 \pm 38.0 \%$ \\
\hline D049 & ND & $\mathrm{ND}$ & ND & $2.3 E-01 \pm 22.0 x$ & $1.8 \mathrm{E}-02 \pm 58.02$ \\
\hline D051 & ND & ND & $4.4 \mathrm{E}-02 \pm 42.07$ & $3.3 \mathrm{E}-01 \pm 18.02$ & $2.6 \mathrm{E}-02 \pm 36.0 \%$ \\
\hline Dos3 & $\mathrm{ND}$ & ND & $\mathrm{ND}$ & $2.25-01 \pm 22.0 \%$ & ND \\
\hline
\end{tabular}


Table E-2: 1997 Soil Sample Results, 200 Area (pCi/gm Dry Weight).

(Sheet 8 of 12)

\begin{tabular}{|c|c|c|c|c|c|}
\hline & Eu-152 & $\mathrm{Eu}-154$ & Eu-155 & $U-234$ & $\mathrm{U}-235$ \\
\hline Code & Result \pm Ẽror & Result \pm Exror & Result \pm Error & Result \pm Error & Result \pm Error \\
\hline Dos5 & $\mathrm{ND}$ & WD & $\mathrm{ND}$ & $1.8 \mathrm{E}-01 \pm 22.0 \%$ & $1.8 \mathrm{E}-02 \pm 57.02$ \\
\hline DỌ57 & $\mathrm{ND}$ & $\mathrm{ND}$ & ND & $1.8 \mathrm{E}-01 \pm 17.0 \%$ & $1.2 \mathrm{E}-02 \pm 64.0 \%$ \\
\hline D059 & ND & ND & $3.2 \mathrm{E}-02 \pm 55.0 \mathrm{z}$ & $2.0 \mathrm{E}-01 \pm 12.0 \%$ & $1.2 \mathrm{E}-02 \pm 45.0 \mathrm{z}$ \\
\hline D062 & $\mathrm{ND}$ & $\mathrm{ND}$ & $4.4 \mathrm{E}-02 \pm 56.02$ & $1.5 E-01 \pm 17.0 \%$ & $1.18-02 \pm 61.0 \%$ \\
\hline D063 &. $\mathrm{MD}$ & ND & $\mathrm{ND}$ & $2.3 \mathrm{E}-01 \pm 21.02$ & $1.1 E-02 \pm 69.0 x$ \\
\hline Do65 & ND & $\mathrm{ND}$ & ND & $2.5 \mathrm{E}-01 \pm 21.0 \%$ & $1.5 \mathrm{E}-02 \pm 65.0 z$ \\
\hline$D 067$ & $\mathrm{ND}$ & $\mathrm{ND}$ & ND & $1.7 E-01 \pm 22.0 \%$ & $1.1 E-02 \pm 76.0 z$ \\
\hline D069 & $\mathrm{ND}$ & ND & ND & $1.6 \mathrm{E}-01 \pm 22.0 \%$ & $1.3 E-02 \pm 60.0 z$ \\
\hline D071 & $\mathrm{ND}$ & ND & $4.2 \bar{E}-02 \pm 53.0 z$ & $1.6 \mathrm{E}-01 \pm 22.0 \mathrm{z}$ & $1.6 \mathrm{E}-02 \pm 56.0 \%$ \\
\hline D073 & ND & $\mathrm{ND}$ & ND & $2.5 E-01 \pm 20.0 z$ & $1.5 \mathrm{E}-02 \pm 58.0 \%$ \\
\hline D075 & $\mathrm{ND}$ & ND & ND & $1.5 E-01 \pm 23.0 \%$ & $1.0 \mathrm{E}-02 \pm 75.07$ \\
\hline D077 & $\mathrm{ND}$ & NDD & $2.1 E-02 \pm 56.0 z$ & $1.3 E-01 \pm 24.0 z$ & $1.35-02 \pm 67.07$ \\
\hline Do79 & SD & ND & $\mathrm{ND}$ & $2.2 E-01 \pm 22.07$ & $2.1 E-02 \pm 54.0 \%$ \\
\hline D081 & ND & $\mathrm{ND}$ & ID & $2.7 E-01 \pm 13.0 \%$ & $1.5 \mathrm{E}-02 \pm 55.0 \mathrm{z}$ \\
\hline D083 & ND & ND & $3.5 E-02 \pm 68.0 \%$ & $1.4 \mathrm{E}-01 \pm 25.0 \%$ & ND \\
\hline D085 & 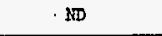 & $\mathrm{ND}$ & $\mathrm{ND}$ & $2.2 \mathrm{E}-01 \pm 22.0 z$ & ND \\
\hline D087 & $\mathrm{ND}$ & ND & $\mathrm{MD}$ & $2.3 E-01 \pm 20.07$ & $9.3 E-03 \pm 75.0 x$ \\
\hline D089 & ND & ND & $4.2 E-02 \pm 40.0 \%$ & $1.7 \mathrm{E}-01 \pm 21.0 \mathrm{z}$ & $1.6 \pm-02 \pm 52.0 x$ \\
\hline D091 & ND & ND & $3.2 E-02 \pm 48.07$ & $1.8 \mathrm{E}-01 \pm 21.0 \mathrm{Z}$ & $\mathrm{ND}$ \\
\hline D093 & $\mathrm{ND}$ & ND & $3.1 \mathrm{E}-02 \pm 55.07$ & $1.7 \mathrm{E}-01 \pm 22.0 \mathrm{Z}$ & $9.5 \mathrm{E}-03 \pm 75.07$ \\
\hline D095 & ND & $\mathrm{ND}$ & ND & $2.1 E-01 \pm 27.0 \%$ & $8.1 E-03 \pm 82.07$ \\
\hline D097 & $\ldots \quad \mathrm{KD}$ & 30 & $3.6 \mathrm{E}-02 \pm 41.02$ & $2.5 \mathrm{E}-01 \pm 15.0 \%$ & $1.9 E-02 \pm 58.0 z$ \\
\hline D099 & SD & ND & ND & $1.9 \mathrm{E}-01 \pm 16 . \dot{0} \mathrm{z}$ & $1.4 E-02 \pm 60.0 \%$ \\
\hline D101 & $\mathrm{ND}$ & WD & $\mathrm{ND}$ & $2.3 E-01 \pm 34.07$ & ND \\
\hline $\mathrm{D} 103$ & $\mathrm{ND}$ & $\mathrm{KD}$ & $3.5 \mathrm{E}-02 \pm 49.0 \%$ & $1.6 \mathrm{E}-01 \pm 17.0 \mathrm{z}$ & $1.3 \mathrm{E}-02 \pm 58.0 \mathrm{z}$ \\
\hline D105 & ND & $\mathrm{WD}$ & $\mathrm{ND}$ & $2.08-02 \pm 16.0 \%$ & $3.1 \mathrm{E}-02 \pm 39.0 \pi$ \\
\hline D107 & KD & ND & ND & $1.9 \mathrm{E}-01 \pm 16.0 \mathrm{x}$ & $1.6 E-02 \pm 58.0 \%$ \\
\hline
\end{tabular}




\section{HNF-EP-0573- 6}

Table E-2: 1997 Soil Sample Results, 200 Area (pCi/gm Dry Weight).

(Sheet 9 of 12)

\begin{tabular}{|l|c|c|c|c|c|}
\hline \multirow{2}{*}{$\begin{array}{l}\text { EDR } \\
\text { Code }\end{array}$} & Eu-152 & Eu-154 & Eu 155 & U-234 & $v-235$ \\
\cline { 2 - 6 } & Result \pm Error & .Result \pm Error & Result \pm Error & Result \pm Error & Result \pm Erro: \\
\hline D109 & ND & ND & $4.7 E-02 \pm 51.07$ & $2.8 E-01 \pm 13.07$ & $2.2 E-02 \pm 44.07$ \\
\hline
\end{tabular}


Table E-2: 1997 Soil Sample Results, 200 Area (pCi/gm Dry Weight).

(Sheet 10 of 12)

\begin{tabular}{|c|c|c|c|}
\hline & $\mathrm{v}-236$ & $\mathrm{Pu}-238$ & Pu239/40 \\
\hline Code. & Result \pm Error & Result \pm Error & Result \pm Error \\
\hline Do01 & $1.9 E-01 \pm 21.07$ & $6.1 E-03 \pm 91.07$ & $1.8 \mathrm{E}-02 \pm 54.0 z$ \\
\hline Do03 & $2.0 \mathrm{E}-01 \pm 19.0 \mathrm{z}$ & ND & $3.4 Z-02 \pm 29.07$ \\
\hline Doos & $1.7 \mathrm{E}-01 \pm 19.0 \mathrm{z}$ & ND & $9.2 \pm-03 \pm 54.0 \%$ \\
\hline D007 & $2.2 E-01 \pm 19.0 \%$ & ND & $2.4 \mathrm{E}-02 \pm 36.0 \%$ \\
\hline 0009 & $1.5 \mathrm{E}-01 \pm 20.0 \mathrm{z}$ & ND & $7.3 \mathrm{E}-03 \pm 68.07$ \\
\hline Do11 & $1.8 \mathrm{E}-01 \pm 20.0 z$ & ND & $9.5 E-02 \pm 33.02$ \\
\hline D013 & $1.9 \mathrm{E}-01 \pm 12.0 \mathrm{Z}$ & $\mathrm{KD}$ & $9.0 \mathrm{E}-02 \pm 21.0 \%$ \\
\hline D015 & $1.8 \mathrm{E}-01 \pm 12.0 \mathrm{z}$ & $\mathrm{ND}$ & $1.4 \mathrm{E}-02 \pm 41.0 \%$ \\
\hline D017 & $1.8 E-01 \pm 19.0 \%$ & ND & $2.95-02 \pm 36.07$ \\
\hline$D 019$ & $1.7 E-01 \pm 20.0 \%$ & ND & $1.8 \mathrm{E}-02 \pm 40.02$ \\
\hline$D 021$ & $1.6 \mathrm{E}-01 \pm 19.07$ & ND & $4.3 E-01 \pm 17.0 \%$ \\
\hline $\mathrm{D} 023$ & $1.7 E-01 \pm 19.08$ & ND & $2.8 \mathrm{E}-02 \pm 32.0 \mathrm{x}$ \\
\hline D025 & $2.3 E-01 \pm 18.0 \%$ & $3.2 \mathrm{E}-03 \pm 91.0 \mathrm{z}$ & $3.7 \mathrm{E}-0 \mathrm{I} \pm 18.0 \mathrm{z}$ \\
\hline Do27 & $1.6 \mathrm{E}-01 \pm 20.0 \mathrm{x}$ & ND & $2.7 E-02 \pm 33.07$ \\
\hline D029 & $1.7 E-01 \pm 18.0 \%$ & $\mathrm{ND}$ & $6.6 \mathrm{E}-02 \pm 24.07$ \\
\hline D031 & $1.6 \mathrm{E}-01 \pm 19.0 \mathrm{z}$ & ND & $2.6 \mathrm{E}-02 \pm 32.07$ \\
\hline Do33 & $2.4 \mathrm{E}-01 \pm 18.0 \mathrm{x}$ & ND & $1.3 \mathrm{E}-01 \pm 20.0 \mathrm{z}$ \\
\hline D035 & $1.3 \mathrm{E}-01 \pm 20.07$ & ND & $1.1 \mathrm{E}-02 \pm 50.0 z$ \\
\hline D037 & $2.1 E-01 \pm 18.0 \%$ & ND & $4.4 \mathrm{E}-03 \pm 65.07$ \\
\hline D039 & $2.0 \mathrm{E}-01 \pm 19.0 z$ & $5.0 E-02 \pm 26.0 z$ & $1.4 E+00 \pm 16.0 z$ \\
\hline D041 & $1.8 \mathrm{E}-01 \pm 19.0 \mathrm{z}$ & $4.3 \mathrm{E}-03 \pm 72.0 \%$ & $9.5 \mathrm{E}-02 \pm 22.07$ \\
\hline D043 & $2.7 \mathrm{E}-01 \pm 12.0 \mathrm{x}$ & ND & $3.6 \pm-02 \pm 33.07$ \\
\hline Do45 & $2.0 \mathrm{E}-01 \pm 13.0 \%$ & $4.8 E-03 \pm 77.07$ & $1.1 \mathrm{E}-01 \pm 22.0 \mathrm{z}$ \\
\hline D047 & $3.8 \mathrm{E}-01 \pm 18.02$ & $4.0 \mathrm{E}-03 \pm 72.0 x$ & $2.0 E-01 \pm 28.07$ \\
\hline D049 & $2.3 \mathrm{E}-01 \pm 22.0 \mathrm{z}$ & ND & $2.6 \mathrm{E}-02 \pm 46.07$ \\
\hline D051 & $3.2 E-01 \pm 18.0 \%$ & SND & $3.0 \mathrm{E}-02 \pm 38.0 z$ \\
\hline D053 & $2.0 \mathrm{E}-01 \pm 23.0 \mathrm{z}$ & ND & $N D$ \\
\hline
\end{tabular}


Table E-2: 1997 Soil Sample Results, 200 Area (pCi/gm Dry Weight).

(Sheet 11 of 12)

\begin{tabular}{|c|c|c|c|}
\hline & $\mathrm{u}-238$ & $\mathrm{Pu}-238$ & Pu239/40 \\
\hline Code & Result \pm Error & Result \pm Error & Result \pm Error \\
\hline p055 & $1.7 \mathrm{E}-01 \pm 22.0 z$ & ND & $\mathrm{ND}$ \\
\hline D057 & $1.6 \mathrm{E}-01 \pm 18.0 \%$ & $\mathrm{ND}$ & $1.4 \mathrm{E}-02 \pm 62.07$ \\
\hline D059 & $1.7 E-01 \pm 13.07$ & $1.0 E-02 \pm 48.0 z$ & $4.7 E-02 \pm 21.0 Z$ \\
\hline D061 & $1.6 \mathrm{E}-01 \pm 17.0 z$ & ND & $5.7 E-03 \pm 82.02$ \\
\hline D063 & $2.3 \mathrm{E}-01 \pm 21.0 \mathrm{x}$ & ND & 1. $7 \mathrm{E}-02 \pm 56.0 \mathrm{z}$ \\
\hline D065 & $2.2 \mathrm{E}-01 \pm 22.0 \%$ & KD & $\mathrm{ND}$ \\
\hline D067 & $1.75-01 \pm 22.0 \%$ & KD & $\mathrm{ND}$ \\
\hline D069 & $1.6 \mathrm{E}-01 \pm 22.0 \mathrm{z}$ & FD & $\mathrm{ND}$ \\
\hline Do71 & $1.8 \mathrm{E}-01 \pm 22.0 \mathrm{z}$ & $\mathrm{ID}$ & $\mathrm{ND}$ \\
\hline Do73 & $2.1 E-01 \pm 21.0 z$ & $N D$ & $\mathrm{ND}$ \\
\hline D075 & $2.1 E-01 \pm 21.0 \%$ & - $\quad \mathrm{ND}$ & $2.4 E-02 \pm 56.0 z$ \\
\hline D077 & $1.3 \mathrm{E}-01 \pm 24.07$ & $\mathrm{ND}$ & $2.0 \mathrm{E}-02 \pm 62.0 \mathrm{x}$ \\
\hline D079 & $2.5 \mathrm{E} \sim 01 \pm 21.0 \%$ & $\mathrm{ND}$ & $\mathrm{ND}$ \\
\hline Dos1 & $2.6 \mathrm{E}-01 \pm 13.0 \%$ & $\mathrm{KD}$ & $\mathrm{ND}$ \\
\hline D083 & $1.4 \mathrm{E}-01 \pm 26.0 \mathrm{z}$ & ND & $2.7 E-0 Z \pm 46.0 \%$ \\
\hline D085 & $1.75-01 \pm 23.0 \%$ & ND & $2.2 \mathrm{E}-02 \pm 50.0 \mathrm{z}$ \\
\hline D087 & $2.2 E-01 \pm 20.0 z$ & $2 \mathrm{WD}$ & ND \\
\hline D089 & $1.8 \mathrm{E}-01 \pm 21.02$ & SD & ND \\
\hline D091 & $1.8 \mathrm{E}-01 \pm 21.0 \mathrm{z}$ & WD & ND \\
\hline 0003 & $2,0 E-01 \pm 21.0 \%$ & No & $9.3 E-03 \pm 65.0 x$ \\
\hline D095 & $2.1 E-01 \pm 17.0 \%$ & $\mathrm{ND}$ & ND \\
\hline D097 & $2.4 \mathrm{E}-01 \pm 16.0 \%$ & ND & $\mathrm{ND}$ \\
\hline Do99 & $1.9 \mathrm{E}-01 \pm 16.0 \mathrm{z}$ & ND & KD \\
\hline D101 & $1.8 \mathrm{E}-01 \pm 39.0 z$ & $\mathrm{ND}$ & $6.2 \mathrm{E}-03 \pm 58.0 \mathrm{z}$ \\
\hline D103 & $2.1 \mathrm{E}-01 \pm 15.07$ & ND & $1.5 E-01 \pm 22.0 z$ \\
\hline D105 & $.2 .18-01 \pm 16.0 \%$ & $6.8 \mathrm{E}-03 \pm 72.0 \mathrm{z}$ & $2.4 E-01 \pm 19.0 z$ \\
\hline$D 107$ & $1.8 \mathrm{E}-01 \pm 16.0 \mathrm{z}$ & ND & MD \\
\hline
\end{tabular}


Table E-2: 1997 Soil Sample Results, 200 Area (pCi/gm Dry Weight).

(Sheet 12 of 12)

\begin{tabular}{|c|c|c|c|}
\hline \multirow{2}{*}{$\begin{array}{l}\text { EDP } \\
\text { Code }\end{array}$} & U-238 & Pu-238 & Pu239/40 \\
\cline { 2 - 4 } & Result \pm Error & Result \pm Error & Result \pm Error \\
\hline D109 & $2.6 \mathrm{E}-01 \pm 13.02$ & ND & ND \\
\hline
\end{tabular}


Table E-3: 1997 Soil Sample Results, 300/400 Area (pCi/gm Dry Weight).

(Sheet 1 of 4 )

\begin{tabular}{|c|c|c|c|c|c|}
\hline & $C_{0}-60$ & $2 n-65$ & $5 x-90$ & $R u-103$ & $\mathrm{Ru}-106$ \\
\hline Code & Result \pm Error & Result \pm Error & Result \pm Error & Result \pm Error & Result \pm Error \\
\hline D116 & $N D$ & SD & ND & $\mathrm{ND}$ & ND \\
\hline D117 & $\mathrm{ND}$ & ND & $3.0 E-01 \pm 50.0 \%$ & ND & ND \\
\hline D118 & ND & ND & $2.7 \mathrm{E}-01 \pm 50.0 \%$ & SD & ND \\
\hline D119 & ND & $\mathrm{ND}$ & $8.05-01 \pm 25.02$ & $X \mathbb{D}$ & ND \\
\hline D120 & $\mathrm{ID}$ & $\mathrm{ND}$ & $3.0 \mathrm{E}-01 \pm 50.0 \%$ & ND & $\mathrm{ND}$ \\
\hline$D 121$ & ND & SD & $\mathrm{ND}$ & IID & ND \\
\hline D122 & $\mathrm{BD}$ & ND & ND & ND & אס \\
\hline D123 & STD & SD & - ND & ND & $\mathrm{ND}$ \\
\hline D124 & ND & ND & ND & ND & ND \\
\hline D125 & $\mathrm{ND}$ & ND & $6.0 \mathrm{E}-01 \pm 40.0 \mathrm{x}$ & $\mathbb{N D}$ & $\mathrm{ND}$ \\
\hline D126 & $\mathrm{ND}$ & ND & ND & SD & ND \\
\hline D127 & $\mathrm{ND}$ & ND & ND & $\mathrm{ND}$ & ND \\
\hline D128 & ND & ND & ND & SD & ND \\
\hline D129 & ND & ND & $\mathrm{ND}$ & SD & ND \\
\hline Diso & $N D$ & ND & IND & $\mathrm{ND}$ & $\mathrm{ND}$ \\
\hline
\end{tabular}


Table E-3: 1997 Soil Sample Results, 300/400 Area (pCi/gm Dry Weight).

(Sheet 2 of 4)

\begin{tabular}{|c|c|c|c|c|c|}
\hline & $5 n-113$ & $5 b-125$ & $\mathrm{Cs}-134$ & $\mathrm{Cs}-137$ & $C e-144$ \\
\hline Code & Result \pm Error & Result \pm Error & Result \pm Error & Result \pm Error & Result \pm Error \\
\hline D116 & $\mathrm{ND}$ & $\mathrm{ND}$ & $2.6 \mathrm{E} \div 02 \pm 27.0 \mathrm{x}$ & $7.6 \mathrm{E}-03 \pm 62.02$ & ND \\
\hline D117 & $\mathrm{ND}$ & ND & $2.8 \mathrm{E}-02 \pm 27.07$ & $9.2 E-02 \pm 17.0 z$ & ND \\
\hline $\mathrm{D} 118$ & ND & ND & $1.9 E-02 \pm 28.0 z$ & $1.4 E-01 \pm 15.0 \%$ & ND \\
\hline D119 & ND & ND & $2.4 \mathrm{E}-02 \pm 24.0 \%$ & $2.0 E-01 \pm 15.0 \%$ & ND \\
\hline D120 & ID & NDD & $2.9 E-02 \pm 25.0 \%$ & $1.8 \mathrm{E}-02 \div 38.0 \%$ & ND \\
\hline D121 & ND & ND & $1.9 E-02 \pm 29.02$ & $8.0 \mathrm{E}-02 \pm 16.0 \mathrm{z}$ & $\mathrm{ND}$ \\
\hline D122 & SND & $\mathbb{S D}$ & $2.4 \mathrm{E}-02 \pm 27.07$ & $2.5 E-02 \pm 28.0 z$ & $\mathrm{ND}$ \\
\hline$D 123$ & ND & $\mathrm{SD}$ & $2.6 \mathrm{E}-02 \pm 34.0 \mathrm{x}$ & $1.8 \mathrm{E}-01 \pm 16.0 \%$ & $\mathrm{ND}$ \\
\hline$D 124$ & ND & $\mathrm{ND}$ & $2.4 E-02 \pm 29.0 z$ & $5.6 \mathrm{E}-02 \pm 18.0 \%$ & ND \\
\hline D125 & $\mathrm{ND}$ & ND & $2.5 E-02 \pm 21.0 z$ & $1.9 \mathrm{E}-02 \pm 22.0 \%$ & ND \\
\hline$D 126$ & SD & ND & $2.05-02 \pm 29.0 \%$ & $1.1 \mathrm{E}-02 \pm 40.0 z$ & ND \\
\hline D127 & ND & $\mathrm{ND}$ & $3.5 E-02 \pm 22.0 \%$ & $2.9 \mathrm{E}-02 \pm 26.02$ & ND \\
\hline D128 & ND & WD & $3.4 E \sim 02 \pm 24.07$ & $6.5 E-02 \pm 19.02$ & $\mathbb{N D}$ \\
\hline $\mathrm{D} 129$ & $\mathrm{IND}$ & $\mathrm{ND}$ & $2.6 \mathrm{E}-02 \pm 28.0 z$ & $8.9 E-02 \pm 17.0 x$ & ND \\
\hline D130 & $\mathrm{ND}$ & WD & $2.5 E-02 \pm 23.02$ & $1.08-02 \pm .47 .0 z$ & ND \\
\hline
\end{tabular}


Table E-3: 1997 Soil Sample Results, 300/400 Area (pCi/gri Dry Weight).

(Sheet 3 of 4)

\begin{tabular}{|c|c|c|c|c|c|}
\hline & Eu-152 & - Eu-154 & $\mathrm{Eu}-155$ & $v-234$ & $v-235$ \\
\hline Code & Result \pm Error & Result \pm Error & Result \pm Error & Result \pm Exror & Result \pm Error \\
\hline D116 & SD & WD & $\mathrm{KD}$ & $1.6 \mathrm{E}-01 \pm 21.0 \%$ & $1.4 \mathrm{E}-02 \pm 58.07$ \\
\hline D117 & ND & No & ND & $8.35-01 \pm 9.1 \%$ & $8.95-02 \pm 23.0 z$ \\
\hline 0118 & $S D$ & ND & WD & $9.5 \mathrm{E}-01 \pm 9.17$ & $5.6 \mathrm{E}-02 \pm 30.0 \mathrm{z}$ \\
\hline D119 & $\mathrm{ND}$ & ND & $3.55-02 \pm 63.0 \%$ & $7.95+00 \pm 8.12$ & $4.7 E-01 \pm 15.0 x$ \\
\hline D120 & SD & ND & $3.3 \mathrm{E}-02 \pm 52.0 x$ & $3.8 E-01 \pm 14.0 \%$ & $3.6 E-02 \pm 42.0 z$ \\
\hline D121 & ND & $\mathrm{ND}$ & $\mathrm{ND}$ & $4.3 E-02 \pm 13.0 z$ & $4.3 \mathrm{E}-02 \pm 40.0 \%$ \\
\hline D122 & SD & $\mathrm{MD}$ & $3.8 \mathrm{E}-02 \pm 38.0 \%$ & $4.8 \mathrm{E}-01 \pm 18.0 \%$ & $2.4 \mathrm{E}-02 \pm 54.0 \mathrm{z}$ \\
\hline$D 123$ & ND & $\mathrm{ND}$ & ND & $3.6 \mathrm{E}-01 \pm 19.0 \%$ & $2.8 \mathrm{E}-02 \pm 45.0 \mathrm{Z}$ \\
\hline D124 & ND & ND & ND & $2.2 E-01 \pm 21.0 x$ & $2.3 \mathrm{E}-02 \pm 54.0 \%$ \\
\hline$D 125$ & WD & ND & $1.8 \mathrm{E}-02 \pm 63.07$ & $3,4 \mathrm{E}-01 \pm 18,07$ & $3.5 \mathrm{E}-02 \pm 37.0 z$ \\
\hline D126 & ND & ND. & ND & $2.6 \mathrm{E}-01 \pm 22.0 z$ & $3.3 E-02 \pm 50.0 \%$ \\
\hline$D 127$ & ND & ND & $3.5 E-02 \pm 51.0 z$ & $3.9 E-01 \pm 19.0 Z$ & $2.3 E-02 \pm 48.0 x$ \\
\hline D128 & WD & ND & ND & $2.2 \mathrm{E}-01 \pm 21.0 \%$ & $1.4 E-02 \pm 67.0 \%$ \\
\hline D129 & $\mathrm{ND}$ & ND & $2.4 E-02 \pm 65.07$ & $3.9 \mathrm{E}-01 . \pm 18.0 z$ & $3.8 E-02 \pm 39.0 x$ \\
\hline D130 & ND & $N D$ & $4.1 \mathrm{E}-02 \pm 55.0 \mathrm{z}$ & $1.8 E-01 \pm 21.07$ & $9.8 \mathrm{E}-03 \pm 68.0 \%$ \\
\hline
\end{tabular}


Table E-3: 1997 Soil Sample Results, 300/400 Area (pCi/gm Dry Weight).

(Sheet 4 of 4)

\begin{tabular}{|c|c|c|c|}
\hline & $v-238$ & $\mathrm{Pu}-238$ & $20239 / 40$ \\
\hline Code & Result \pm Error & Result \pm Error & Result \pm Error \\
\hline D116 & $2.0 E-01 \pm 21.0 \%$ & ND & $\mathrm{ND}$ \\
\hline D117 & $7.2 \mathrm{E}-01 \pm 9.5 \%$ & $7.7 \mathrm{E}-03 \pm 72.07$ & ND \\
\hline D118 & $8.6 \mathrm{E}-01 \pm 9.37$ & $\mathrm{ND}$ & $6.5 E-03 \pm 91.07$ \\
\hline D119 & $7.8 \mathrm{E}+00 \pm 8.17$ & $7.6 \mathrm{E}-03 \pm 77.02$ & $1.7 \mathrm{E}-01 \pm 22.0 \mathrm{z}$ \\
\hline $\mathrm{D} 120$ & $3.4 \mathrm{E}-01 \pm 15.0 \mathrm{z}$ & $\mathrm{WD}$ & $6.0 \mathrm{E}-03 \pm 83.02$ \\
\hline D121 & $4.7 E-01 \pm 13.0 \%$ & WD & $1.3 E-02 \pm 64.07$ \\
\hline D122 & $5.1 E-01 \pm 18.0 z$ & ND & $2.5 \bar{E}-02 \pm 46.0 \%$ \\
\hline D123 & $3.3 \mathrm{E}-01 \pm 19.07$ & $\mathrm{KD}$ & ND. \\
\hline D124 & $2.0 \mathrm{E}-01 \pm 22.0 z$ & $\mathrm{IDD}$ & ND \\
\hline D125 & $2.8 \mathrm{E}-01 \pm 19.07$ & $\mathrm{ND}$ & ND \\
\hline D126 & $2.6 \mathrm{E}-01 \pm 22.0 z$ & ND & SID \\
\hline D127 & $3.6 \mathrm{E}-01 \pm 19.0 z$ & $\mathrm{SD}$ & ND \\
\hline $\mathrm{DI28}$ & $2.3 \mathrm{E}-01 \pm 21.0 \mathrm{z}$ & ND & ND \\
\hline D129 & $4.7 E-01 \pm 18.0 x$ & $N D$ & $\mathrm{ND}$ \\
\hline D130 & $1.7 \mathrm{E}-01 \pm 22.0 \%$ & $\mathrm{ND}$ & $5.6 \mathrm{E}-03 \pm 83.07$ \\
\hline
\end{tabular}


Table E-4: 1997 Soil Sample Results, ERC Sites (pCi/gm Dry Weight).

\begin{tabular}{|c|c|c|c|c|c|}
\hline & $\mathrm{Co}-60$ & $\mathrm{Zn}-65$ & st -90 & Ru-103 & Ru-106 \\
\hline Code & Result \pm Error & Result \pm Error & Result \pm Error & Result \pm Error & Result \pm Error \\
\hline D146 & WD & ND & KD & $\mathrm{ND}$ & $\mathrm{ND}$ \\
\hline D147 & NND & $\mathrm{ND}$ & $\mathrm{ND}$ & ND & $\mathrm{SD}$ \\
\hline$D 148$ & $W D$ & $\mathrm{ND}$ & ND & ND & $\mathrm{KD}$ \\
\hline
\end{tabular}

\begin{tabular}{|l|c|c|c|c|c|}
\hline \multirow{2}{*}{$\begin{array}{l}\text { EDP } \\
\text { Code }\end{array}$} & Sa-113 & Sb-125 & Cs-134 & Cs-137 & Ce-144 \\
\cline { 2 - 6 } & Result \pm Error & Result \pm Error & Result \pm Exzor & Resuit \pm Error & Result \pm Errox \\
\hline D146 & ND & ND & $3.1 E-02 \pm 33.0 \%$ & $2.9 E-01 \pm 14.0 \%$ & ND \\
\hline D147 & ND & ND & $2.7 E-02 \pm 27.0 \%$ & $1.1 E-01 \pm 18.02$ & ND \\
\hline D148 & ND & ND & $4.5 E-02 \pm 26.0 \%$ & $6.9 E-01 \pm 13.0 \%$ & ND \\
\hline
\end{tabular}

\begin{tabular}{|c|c|c|c|c|c|}
\hline & Eu-152 & Etu-154 & $\mathbf{E u}-155$ & $U-234$ & $\mathrm{U}-235$ \\
\hline Code & Result \pm Error & Result \pm Exfor & Result \pm Errox & Result \pm Error & Result \pm Exror \\
\hline D146 & ND & $\mathrm{MD}$ & ND & $2.2 \mathrm{E}-01 \pm 14.07$ & $2.2 E-02 \pm 44.0 z$ \\
\hline D147 & $\mathrm{ND}$ & WD & ND & $1.6 E-01 \pm 15.07$ & $2.1 E-02 \pm 42.07$ \\
\hline D148 & $2.8 \mathrm{E}-01 \pm 10.0 \mathrm{z}$ & ND & ND & $2.0 E-0.1 \pm 50.0 Z$ & $7.3 \mathrm{E}-02 \pm 29.0 \%$ \\
\hline
\end{tabular}

\begin{tabular}{|l|c|c|c|}
\hline \multirow{2}{*}{$\begin{array}{l}\text { EDP } \\
\text { Code }\end{array}$} & U $\sim 238$ & Pu-238 & Eu239/40 \\
\cline { 2 - 4 } & Result \pm Error & Result \pm Exror & Result \pm Error \\
\hline$D 146$ & $2.0 \mathrm{E}-01 \pm 15.0 \%$ & ND & $1.7 \mathrm{E}-02 \pm 63.0 \%$ \\
\hline$D 147$ & $1.6 \mathrm{E}-02 \pm 15.0 \%$ & ND & șD \\
\hline$D 148$ & $2.0 \mathrm{E}-01 \pm 21.0 \%$ & ND & $2.8 \mathrm{E}-02 \pm 58.0 \%$ \\
\hline
\end{tabular}


Table E-5: 1997 Vegetation Sample Results, 100-N Area (pCi/gm Dry Weight).

(Sheet 1 of 2)

\begin{tabular}{|c|c|c|c|c|c|}
\hline & Co- 60 & $2 x-65$ & $\mathbf{S r}-90$ & Ru-103 & Ru-106 \\
\hline Codo & Result \pm Error & Result \pm Error & Result \pm Errox & Result \pm Error & Result \pm Exror \\
\hline$Y 702$ & $4.2 \mathrm{E}-01 \pm 12.0 \%$ & ND. & $\mathrm{ND}$ & ND & ND \\
\hline$y 703$ & $3 D$ & ND & $\mathrm{ND}$ & $\mathrm{SD}$ & WD \\
\hline$Y 704$ & SND & ND & $4.9 E-01 \pm 30.0 z$ & $\mathrm{ND}$ & ND \\
\hline Y708 & ND & ND & $1.5 E-01 \pm 90.07$ & ND & ID \\
\hline$Y 710$ & ND & ND & $1.4 \mathrm{E}-01 \pm 90.07$ & ND & ND \\
\hline$¥ 711$ & ND & $\mathrm{MD}$ & $N D$ & ND & ND \\
\hline$Y 718$ & ND & ND & $2.3 E+00 \pm 16.0 z$ & SD & $\mathrm{IDD}$ \\
\hline Y719 & $\mathrm{KD}$ & ND & $8.2 E-01 \pm 25.02$ & SD & MD \\
\hline$Y 724$ & $\mathrm{KD}$ & 20 & $2.1 \Xi+01 \pm 11.0 \%$ & ND & $\mathrm{MD}$ \\
\hline$Y 725$ & ND & $\mathrm{ND}$ & $6.1 \mathrm{E}-01 \pm 30.07$ & $N D$ & $\mathrm{ND}$ \\
\hline
\end{tabular}

\begin{tabular}{|c|c|c|c|c|c|}
\hline & $5 n-113$ & $S b-125$ & $C=-134$ & $\mathrm{Cs}-137$ & $C \in-144$ \\
\hline Code & Result \pm Error & Result \pm Error & Result \pm Error & Result \pm Error & Result \pm Esfor \\
\hline$Y 702$ & ID & ND & $\mathrm{ND}$ & . $8.6 \mathrm{E}-02 \pm 41.0 \%$ & ND \\
\hline Y703 & ND & $\mathrm{KD}$ & $\operatorname{stD}$ & $1.7 \mathrm{E}-01 \pm 34.0 \%$ & ND \\
\hline$Y 704$ & ND & ND & ND & $1.7 \mathrm{E}-01 \pm 53.0 \%$ & ND \\
\hline 7708 & ND & ND & $\mathrm{ND}$ & . ND & ND \\
\hline$\$ 710$ & $\mathrm{ND}$ & SD & ND & IID & ND \\
\hline$Y 711$ & ND & ND & $\mathrm{KD}$ & SD & ND \\
\hline$Y 718$ & ND & ND & RD & $8.15-02 \pm 46.0 \%$ & SDD \\
\hline$Y 719$ & $\mathrm{ND}$ & $\mathrm{ND}$ & ND & ND & ND \\
\hline$\$ 724$ & $\mathrm{ND}$ & ND & IND & 1. $0 E-01 \pm 65.07$ & SD \\
\hline Y725 & AD & $\mathrm{ND}$ & ND & $3.5 \mathrm{E}-01 \pm 26.07$ & $\mathrm{MD}$ \\
\hline
\end{tabular}


Table E-5: 1997 Vegetation Sample Results, 100-N Area (pCi/gm Dry Weight).

(Sheet 2 of 2)

\begin{tabular}{|c|c|c|c|c|c|}
\hline & $2 u-152$ & $5 u-154$ & Eu-155 & $U-234$ & $v-235$ \\
\hline Code & Result \pm Errox & Result \pm Error & Result $\neq$ Exror & Result \pm Erxor & Result \pm Error \\
\hline Y702 & $N D$ & ND & ND & $8.3 E-03 \pm 48.0 \%$ & $5.0 \mathrm{E}-03 \pm 64.0 \%$ \\
\hline Y703 & ND & KD & $\mathrm{ND}$ & $9.9 \bar{E}-03 \pm 56.02$ & $\mathrm{ND}$ \\
\hline Y704 & ND & ND & WD & $1.6 \mathrm{E}-02 \pm 40.0 \mathrm{x}$ & $7.7 \mathrm{E}-03 \pm 56.0 \%$ \\
\hline Y708 & ND & ND & ND & $1.3 \bar{E}-02 . \pm 41.0 \%$ & ND \\
\hline 8710 & ND & ND & $\mathrm{MD}$ & $1.4 E-02 \pm 42.0 z$ & $2.98-03 \pm 91.0 \%$ \\
\hline Y711 & ND & ND & $\mathrm{ND}$ & $1.15-02 \pm 45.0 \%$ & $1.18-02 \pm 47.02$ \\
\hline Y718 & $N D$ & ND & ND & $1.1 E-02 \pm 50.0 z$ & $5.7 \mathrm{E}-03 \pm 79.07$ \\
\hline Y719 & $\mathbb{N D}$ & $S D$ & SD & $1.4 \mathrm{E}-02 \pm 42.0 \%$ & WD \\
\hline Y724 & ND & ND & ND & $8.2 \mathrm{E}-03 \pm 52.02$ & $4.8 \mathrm{E}-03 \pm 79.0 z$ \\
\hline 8725 & $2.6 \mathrm{E}-01 \pm 64.0 \mathrm{z}$ & ND & ND & $2.4 \mathrm{E}-02 \pm 30.07$ & ND \\
\hline
\end{tabular}

\begin{tabular}{|c|c|c|c|}
\hline \multirow{2}{*}{$\begin{array}{l}\text { EDP } \\
\text { Code }\end{array}$} & $v-238$ & $5 u-238$ & Pu239/40 \\
\hline & ResuIt \pm EIror & Result \pm Exror & Result \pm Error \\
\hline 7702 & $6.52-03 \pm 58.0 \%$ & ND & $\mathrm{ND}$ \\
\hline Y703 & ND & KD & WD \\
\hline Y704 & $1.0 z-02 \pm 47.0 z$ & ND & $\mathrm{SDD}$ \\
\hline Y708 & $7.0 \mathrm{E}-03 \pm 54.0 \mathrm{x}$ & $2 D$ & $\mathrm{ND}$ \\
\hline Y710 & $5.8 \mathrm{E}-03 \pm 62.02$ & $\mathrm{ND}$ & ND \\
\hline Y711 & $6.7 \mathrm{E}-03 \pm 58.07$ & ND & $\mathrm{ND}$ \\
\hline Y718 & $6.6 \mathrm{E}-03 \pm 64.0 z$ & $3.9 \mathrm{E}-03 \pm 80.0 \mathrm{z}$ & $\mathrm{ND}$ \\
\hline 8719 & $1.1 \mathrm{E}-02 \pm 45.0 \mathrm{z}$ & ND & ND \\
\hline$Y 724$ & $4.48-03 \pm 71.0 \%$ & $5.7 \mathrm{E}-03 \pm 67.0 \%$ & $\mathrm{SD}$ \\
\hline Y725 & $2.9 E-02 \pm 27.07$ & $\mathrm{KD}$ & ND \\
\hline
\end{tabular}


Table E-6: 1997 Vegetation Sample Results, 200/600 Areas (pCi/gm Dry Weight).

(Sheet 1 of 8 )

\begin{tabular}{|c|c|c|c|c|c|}
\hline & $\mathrm{Co}_{0}-60$ & $2 n-65$ & $5 x-90$ & $R u-103$ & Ru-106 \\
\hline Code & Result \pm Error & Result \pm Error & Result \pm Erxor & Result \pm Error & Result \pm Error \\
\hline Vool & ND & ND & $\mathrm{ND}$ & ND & ND \\
\hline vo03 & ND & ND & $2.6 \mathrm{E}-01 \pm 70.0 \%$ & IDD & SD \\
\hline voog. & ND & ND & $\mathrm{ND}$ & ND & ND \\
\hline V011 & ND & ND & $3.18-01 \pm 60.0 z$ & $2 D$ & $\mathrm{ND}$ \\
\hline v015 & ND & $\mathrm{ND}$ & $2.7 E+01 \pm 11.0 \%$ & $\mathrm{ND}-$. & ND \\
\hline v017 & ND & $6.6 \mathrm{E}-02 \pm 56.0 \mathrm{z}$ & $4.3 \mathrm{E}-01 \pm 30.0 z$ & IDD & ND. \\
\hline$\nabla 019$ & KD & ND & $1.6 \mathrm{E}-01 \pm 90.0 \mathrm{z}$ & ND & ND \\
\hline v023 & ND & SD & $2.0 E+00 \pm 13.0 x$ & ID & SD . \\
\hline v025 & ND & ND & $9.9 E-01 \pm 15.07$ & $\mathrm{ND}$ & ND \\
\hline 7027 & SDD & ND & $7.9 \mathrm{E}-01 \pm 16.0 \mathrm{x}$ & ND & WD \\
\hline v037 & $\mathrm{ND}$ & MD & $8.5 E-02 \pm 60.0 z$ & MD & $N D$ \\
\hline v033 & ND & ND & $1.38+01 \pm 12.0 z$ & ND & ND \\
\hline v035 & $\mathrm{ND}$ & $2 R D$ & $1.1 \mathrm{E}-01 \pm 50.0 \mathrm{x}$ & $\mathrm{MD}$ & SD \\
\hline v039 & ND & ND & HD & ND & ND \\
\hline v041 & $\mathrm{ND}$ & ND & ND & $\mathrm{ND}$ & $\mathrm{KD}$ \\
\hline v043 & $\mathrm{ND}$ & $\mathrm{ND}$ & $8.0 \mathrm{E}-01 \pm 30.0 \mathrm{x}$ & $\mathrm{ND}$ & ID \\
\hline vo45 & $\mathrm{ND}$ & SD & SD & $\mathrm{ND}$ & ND \\
\hline$\nabla 047$ & SD & NDD & WD & $\mathrm{ND}$ & ND \\
\hline Y049 & ND & SD & $3.0 E-01 \pm 60.0 z$ & ND & $\mathrm{SD}$ \\
\hline v0si & $\mathrm{ND}$ & $\mathrm{ND}$ & $4.7 \mathrm{E}-01 \pm 50.0 \mathrm{z}$ & ND & ND \\
\hline vo53 & ND & ND & $1.5 E+00 \pm 20.0 \%$ & ND & ND \\
\hline$\nabla 057$ & $-\quad$ ND & ND & $2.9 E-01 \pm 50.0 z$ & $\mathrm{ND}$ & ND \\
\hline vo59 & SD & ND & $7.0 \mathrm{E}-01 \pm 25.0 \%$ & IND & SD \\
\hline v061 & SD & ND & $1.5 \mathrm{E}+00 \pm 16.0 \mathrm{z}$ & $\mathrm{MD}$ & ND \\
\hline vo63 & 3D & ND & $1.8 \mathrm{E}+01 \pm 13.02$ & $N D$ & $\mathrm{ND}$ \\
\hline v077 & $\mathrm{ND}$ & ND & $3.92-02 \pm 40.0 z$ & ND & ND \\
\hline$\nabla 079$ & $N D$ & ND & $1.7 \mathrm{E}-01 \pm 90.0 z$ & ND & NDD \\
\hline
\end{tabular}


Table E-6: 1997 Vegetation Sample Results, 200/600 Areas (pCi/gm Dry Weight).

(Sheet 2 of 8 )

\begin{tabular}{|c|c|c|c|c|c|}
\hline & Co-60 & $2 n-65$ & $\mathrm{Sr}-90$ & $R u-103$ & $R u-106$ \\
\hline Code & Result \pm Error & Result \pm ExIor & Result \pm Error & Result \pm Error & Result \pm Error \\
\hline vos1 & $\mathrm{ND}$ & ND & ND & $\mathrm{ND}$ & ND \\
\hline v083 & $\mathbb{N D}$ & ND & ND & $\mathrm{ND}$ & ND \\
\hline v085 & $\mathrm{ND}$ & $\mathrm{ND}$ & ND & SD & ND \\
\hline v087 & ND & ND & $4.2 E-01 \pm 40.0 \%$ & $\mathrm{ND}$ & $\mathrm{ND}$ \\
\hline v089 & $\mathbb{N D}$ & ND & $1.9 \mathrm{E}-01 \pm 90.0 \%$ & SDD & $\mathrm{ND}$ \\
\hline vọ91 & ND & RD & $3.2 E+00 \pm 14.0 \%$ & ND. & ND \\
\hline vos 5 & ND & ND & ND & ND & ND \\
\hline vo97 & ND & $\mathrm{ID}$ & $\mathrm{ND}$ & ND & ND . \\
\hline v099 & ND & ND & ND & ND & ND \\
\hline V101 & $\mathrm{KD}$ & ND & $9.9 \mathrm{E}-01 \pm 15.07$ & $\mathrm{ND}$ & ND \\
\hline $\mathrm{V} 103$ & ND & ND & ND & ND & ND \\
\hline v105. & ND & ND & $\mathrm{ND}$ & ND & ND \\
\hline$\nabla 107$ & ND & ND & $1.8 \mathrm{E}-01 \pm 40.07$ & WD & ND \\
\hline$\nabla 109$ & ND & ND & ND & ND & ND \\
\hline
\end{tabular}


HNF-EP-0573-6

Table E-6: 1997 Vegetation Sample Results, 200/600 Areas (pCi/gm Dry Weight).

(Sheet 3 of 8 )

\begin{tabular}{|c|c|c|c|c|c|}
\hline & $5 n-113$ & $S b-125$ & $C_{s-134}$ & Cs -137 & $\mathrm{Ce}-144$ \\
\hline Code & Result \pm Error & Result \pm Error & Result \pm Error & Result \pm Errot & Result $\ddagger$ ErroI \\
\hline v001. & $\mathrm{ND}$ & ND & ND & $3.1 \mathrm{E}-02 \pm 68.07$ & אד \\
\hline v003 & $\mathrm{ND}$ & ND & $\mathrm{MD}$ & ND & ND \\
\hline v009 & $\mathrm{ND}$ & wo & ND & ND. & $\mathrm{ND}$ \\
\hline v011 & $\mathrm{ND}$ & ND & $\mathrm{MD}$ & $1.5 E-01 \pm 34.0 \%$ & ND \\
\hline v015 & ND & $\mathrm{ND}$ & ND & $2.3 \mathrm{E}-01 \pm 19.0 z$ & ND \\
\hline 8027 & ND & SD & ND & $1.3 \mathrm{E}-01 \pm 37.07$ & $\mathrm{ND}$ \\
\hline v019 & ND & SDD & ND & ND & ND \\
\hline vo23 & $\mathrm{ND}$ & ND & $\mathrm{ND}$ & $1.6 \mathrm{E}-01 \pm 47.07$ & $2 N D$ \\
\hline vo25 & $\mathbb{N D}$ & ND & ND & $2.08-01 \pm 34.07$ & ND \\
\hline v027 & ND & ND & ND & $9.0 \mathrm{E}-02 \pm 36.0 \%$ & $\mathrm{SD}$ \\
\hline v031 & $\mathrm{ND}$ & ND & ND & $5.9 \bar{z}-02 \pm 45.0 \%$ & $\mathrm{MD}$ \\
\hline v033 & ND & ND & $\mathbb{N D}$ & $5.9 \mathrm{E}-01 \pm 18.0 \mathrm{z}$ & ND \\
\hline vo35 & ND & ND & ND & $1.9 E-01 \pm 33.0 x$ & ND \\
\hline v039 & ND & $\mathrm{ND}$ & ND & $3.9 \Sigma-02 \pm 72.0 z$ & ND \\
\hline v041 & ND & $\mathrm{ND}$ & WD & ND & $\mathrm{ND}$ \\
\hline 7043 & ND & SDD & ND & $4.1 \dot{\mathrm{E}}-02 \pm 55.0 \%$ & $\mathbb{N D}$ \\
\hline v045 & ND & ND & ND & $\mathrm{ND}$ & ND \\
\hline 7047 & ND & ND & ND & 5. $1 E-02 \pm 61.0 x$ & $\mathrm{ND}$ \\
\hline V049 & ND & ND & ND & WD & ND \\
\hline vosi & ND & ND & ND & $7.8 E-02 \pm 30.0 z$ & ND \\
\hline vos3 & $\mathrm{ND}$ & ND & ND & $1.8 E-0 I \pm 24.0 \%$ & ND \\
\hline vos7 & ND & संD & $\mathrm{ND}$. & $6.2 \mathrm{E}-02 \pm 64.0 \mathrm{Z}$ & ND \\
\hline v0s9 & ND & SD & $\mathrm{ND}$ & $6.1 E-02 \pm 36.0 \%$ & $\mathrm{ND}$ \\
\hline v061 & ND & SDD & ND & SD & $\mathrm{ND}$ \\
\hline v063 & ND & ND & $\mathrm{KD}$ & ND & KD \\
\hline vor7 & ND & ND & ND & $\mathrm{ND}$ & ND \\
\hline v079 & $\mathrm{MD}$ & ND & ND & $\mathrm{ID}$ & $\mathrm{ND}$ \\
\hline
\end{tabular}


Table E-6: 1997 Vegetation Sample Results, 200/600 Areas (pCi/gm Dry Weight).

(Sheet 4 of 8 )

\begin{tabular}{|c|c|c|c|c|c|}
\hline & $\mathrm{Sn}-113$ & $\mathrm{Sb}-125$ & $C_{s}-134$ & $C_{s}-137$ & $C e-144$ \\
\hline Code & Result \pm Error & Result \pm Error & Result \pm Exror & Result \pm Error & Result \pm Error \\
\hline v081 & SD & ND & ND & FD & SD \\
\hline v083 & ND & $\mathrm{ND}$ & ND & ND & $\mathrm{ND}$ \\
\hline v085 & ND & IDD & ND & ND & $\mathrm{ND}$ \\
\hline V087 & ND. & NDD. & WD & SD . & $\mathrm{ND}$ \\
\hline$\nabla 089$ & $\mathrm{ND}$ & IDD & ND & ND. & $\mathrm{KD}$ \\
\hline v091 & ND & $\mathrm{ND}$ & ID & $1.1 E-01 \pm 34.07$ & $\mathrm{KD}$ \\
\hline v095 & ND - & ND & $\mathrm{ND}$ & $1.8 \mathrm{E}-02 \pm 59.0 \mathrm{x}$ & SD \\
\hline v097 & $\mathrm{ND}$ & ND & $\mathrm{IDD}$ & ND & ND \\
\hline v099 & $\mathrm{ND}$ & $\mathrm{ND}$ & $\mathrm{ND}$ & $\mathrm{ND}$ & $\mathrm{ND}$ \\
\hline V101 & ND & ND & $\mathrm{ND}$ & IND & $\mathrm{ND}$ \\
\hline v103 & $\mathrm{ND}$ & ND & ND & SD & $\mathrm{ND}$ \\
\hline $\mathrm{v} 105$ & ND & ND & ND & $\mathrm{SD}$ & ND \\
\hline $\mathrm{V} 107$ & ND & ND & ND & ND & $\mathrm{ND}$ \\
\hline v109 & IRD & ND & ND & ND & WD \\
\hline
\end{tabular}


HNF-EP-0573-6

Table E-6: 1997 Vegetation Sample Results, 200/600 Areas (pCi/gm Dry Weight).

(Sheet 5 of 8 )

\begin{tabular}{|c|c|c|c|c|c|}
\hline & Eu-152 & Eu- 154 & ELt 155 & $\mathrm{v}-234$ & $U-235$ \\
\hline Code & Result I Exror & Result \pm Error & Result \pm Error & Result \pm Exror & Result \pm Exror \\
\hline V001 & $\mathrm{ND}$ & ND & ND & $8.0 \mathrm{E}-03 \pm 60.0 \mathrm{z}$ & $N D$ \\
\hline v003 & $\mathrm{NDD}$ & $M D$ & ND & $6.8 \mathrm{E}-03 \pm 71.0 \%$ & $1.2 \mathrm{E}-02 \pm 52.0 \%$ \\
\hline voos & $\mathrm{MD}$ & ND & ND & $2.0 \mathrm{E}-02 \pm 36.0 \mathrm{z}$ & $6.2 \mathrm{E}-03 \pm 62.0 \mathrm{z}$ \\
\hline V011 & ND & ND & ND & $9.9 \mathrm{E}-03 \pm 48.0 \%$ & $4.08-03 \pm 77.0 \%$ \\
\hline v015 & $\mathrm{ND}$ & ND & $\mathrm{ND}$ & $1.4 \mathrm{E}-02 \pm 45.0 \%$ & KD \\
\hline v017 & ND & $\mathrm{ND}$ & $N D$ & $2.85-02 \pm 37.0 \%$ & ND \\
\hline 7019 & ND & ND & ND & $1.3 \mathrm{E}-02 \pm 46.0 \%$ & $8.0 \mathrm{z}-03 \pm 63.02$ \\
\hline$\nabla 023$ & $\mathrm{MD}$ & $\mathrm{ND}$ & ND & $1.5 E-02 \pm 56.0 \%$ & ND \\
\hline v025 & ND. & ND & ND. & $2.3 E-02 \pm 67.07$ & ND \\
\hline V027 & ND & ND & $\mathrm{ND}$ & $1.2 \mathrm{E}-02 \pm 61.0 \mathrm{~K}$ & ND \\
\hline v031 & MD & XD & ND & $9.4 \mathrm{E}-03 \pm 52.07$ & ND \\
\hline vo33 & $\mathrm{ND}$ & ND & $\mathrm{KD}$ & $1.7 \mathrm{E}-02 \pm 71.07$ & IND \\
\hline v035 & $\mathrm{ND}$ & $\mathrm{ND}$ & WD & $2.6 \mathrm{E}-02 \pm 33.0 z$ & $3.8 E-03 \pm 90.07$ \\
\hline v039 & $\mathrm{ND}$ & $\mathrm{ND}$ & NDD & $7.6 \mathrm{E}-03 \pm 58.07$ & $7.6 \mathrm{E}-03 \pm 61.0 \mathrm{z}$ \\
\hline vo41 & KD & NDD & $N D$ & $3.1 E-02 \pm 42.07$ & ND \\
\hline 7043 & KD & ND & ND & $1.2 \mathrm{E}-02 \pm 42.0 \%$ & $1.1 E-02 \pm 49.07$ \\
\hline v045 & ND & ND & $\mathrm{ND}$ & $2.1 \mathrm{E}-02 \pm 54.0 \mathrm{z}$ & $\mathrm{ND}$ \\
\hline v047 & $\mathrm{MD}$ & - ND & ND & $1.9 \mathrm{E}-02 \pm 49.0 \%$ & $\mathrm{MD}$ \\
\hline 7049 & ND & ND & $2 N D$ & $N D$ & $\mathrm{ND}$ \\
\hline vos: & $\mathrm{ND}$ & ND & ND & $2.9 \mathrm{E}-02 \pm 93.0 \mathrm{z}$ & ND \\
\hline$\nabla 0.53$ & $\mathrm{ND}$ & ND & $\mathrm{KD}$ & $2.5 E-02 \pm 67.07$ & ND \\
\hline$\nabla 057$ & ND & ND & ND & $9.2 \mathrm{E}-03 \pm 49.0 \%$ & ND \\
\hline v059 & ND & $\mathrm{ND}$ & $\mathrm{ND}$ & $3.75-03 \pm 76.07$ & ND \\
\hline v061 & सD & ND & $\mathrm{ND}$ & $9.8 \mathrm{E}-03 \pm 46.02$ & MD \\
\hline v063 & ND & KND & $\mathrm{ND}$ & $6.0 \mathrm{E}-03 \pm 66.0 \mathrm{x}$ & ND \\
\hline vo77 & ND & ND & $\mathrm{ND}$ & $1.4 \mathrm{E}-02 \pm 38.0 \pi$ & ND \\
\hline v079 & ND & ND & ND & $1.0 E-02 \pm 45.07$ & $5.6 E-03 \pm 63.07$ \\
\hline
\end{tabular}


Table E-6: 1997 Vegetation Sample Results, 200/600 Areas (pCi/gm Dry Weight).

(Sheet 6 of 8)

\begin{tabular}{|c|c|c|c|c|c|}
\hline & $E u-152$ & Eut-154 & Eu-15s & $v-234$ & $4-235$ \\
\hline Code & Result \pm Error & Result \pm Error & Result \pm Error & Result \pm Exror & Result \pm ErxoI \\
\hline vosi & $\mathrm{ND}$ & ND & $\mathrm{ND}$ & $4.12-03 \pm 76.07$ & $6.3 \mathrm{E}-03 \pm 64.0 \%$ \\
\hline 7083 & ND & ID & SD & $1.8 \mathrm{E}-02 \pm 44.07$ & $\mathrm{ND}$ \\
\hline v085 & WD & $\mathrm{ND}$ & ND & $1.6 \mathrm{E}-02 \pm 47.0 \%$ & $1.4 \mathrm{E}-02 \pm 53.0 \%$ \\
\hline v087 & SD & ND & ND & $2.3 \mathrm{E}-02 \pm 40.07$ & $1.6 \mathrm{E}-02 \pm 50.07$ \\
\hline vo89 & NDD & ND & ND & $2.5 E-02 \pm 33.0 z$ & $1.4 \mathrm{E}-02 \pm 47.0 \%$ \\
\hline vo91 & ND & $\mathrm{ND}$ & ND & $3.4 \mathrm{E}-02 \pm 26.0 \%$ & $1.9 \mathrm{E}-02 \pm 36.02$ \\
\hline v095 & ND & ND & KD & $1.5 E-02 \pm 45.0 \%$ & $1.3 E-02 \pm 61.0 \%$ \\
\hline v097 & IND & $\mathrm{ND}$ & $\mathrm{ND}$ & $1.58-02 \pm 43.08$ & $1.18-02 \pm 56.0 \%$ \\
\hline vogg & $\mathrm{ND}$ & $\mathrm{SD}$. & FD & $9.6 \mathrm{E}-03 \pm 52.0 z$ & $8.0 \mathrm{E}-03 \pm 60.0 z$ \\
\hline $\mathrm{V} 101$ & $\mathrm{ND}$ & ND & $\mathrm{KD}$ & $6.4 \mathrm{E}-03 \pm 60.0 \%$ & $7.58-03 \pm 58.0 \%$ \\
\hline V103 & ND & ND & ND & $1.4 \mathrm{E}-02 \pm 40.0 \mathrm{z}$ & WD \\
\hline$\nabla 105$ & $\mathrm{ND}$ & ND & BD & $\mathrm{ND}$ & ND \\
\hline v107 & ND & ND & ND & $1.3 E-02 \pm 48.0 \%$ & SD \\
\hline v109 & $\mathrm{ND}$ & ND & $\mathrm{ID}$ & $1.1 E-02 \pm 46.07$ & $7.9 \mathrm{E}-03 \pm 56.0 \mathrm{z}$ \\
\hline
\end{tabular}


Table E-6: 1997 Vegetation Sample Results, 200/600 Areas (pCi/gm Dry Weight).

(Sheet 7 of 8)

\begin{tabular}{|c|c|c|c|}
\hline & ปJ -238 & $P u-238$ & Pu239/40 \\
\hline Code & Result \pm Error & Result \pm Error & Result I Error \\
\hline v001 & $3.7 \mathrm{E}-03 \pm 82.0 \%$. & ND & ND \\
\hline vo03 & $8.9 E-03 \pm 62.07$ & $2.5 \mathrm{E}-03 \pm 91.0 \%$ & ND \\
\hline voog & $1.6 \mathrm{E}-02=39.0 z$ & KD & $3.0 \mathrm{E}-03 \pm 83.0 \mathrm{x}$ \\
\hline vo11 & 1. $3 \mathrm{E}-02 \pm 44.02$ & $\mathrm{ND}$ & $6.7 E-03 \pm 64.07$ \\
\hline vo15 & $9.1 \mathrm{E}-03 \pm 59.0 \%$ & $2.9 \mathrm{E}-03 \pm 83.0 \%$ & ND \\
\hline vo17 & $1.5 \mathrm{E}-02 \pm 39.0 z$ & $\mathrm{ND}$ & $4.7 E-03 \pm 75.0 \%$ \\
\hline V019 & $9.1 \mathrm{E}-03 \pm 52.02$ & $\mathrm{ND}$ & SD \\
\hline v023 & $2.15-02 \pm 49.0 \%$ & ND & ND \\
\hline vo2s & ND & ND & $9.7 E-03 \pm 52.07$ \\
\hline$\nabla 027$ & $1.3 \mathrm{E}-02 \pm 63.0 \%$ & SDD & ND \\
\hline v031 & $5.0 E-03 \pm 79.07$ & ND & ND \\
\hline v033 & $\mathrm{ND}$ & ND & $5.6 \varepsilon-03 \pm 77.0 \%$ \\
\hline vo35 & $2.5 E-02 \pm 34.0 z$ & MD & $8.7 \mathrm{E}-03 \pm 52.0 \%$ \\
\hline v039 & $7.0 \mathrm{E}-03 \pm 61.0 \%$ & ND & $\mathrm{ND}$ \\
\hline V041 & $2.4 E-02 \pm 48.0 x$ & $\mathrm{ND}$ & $7.2 E-03 \pm 62.0 \%$ \\
\hline vo43 & ND & $\mathrm{ND}$ & RD \\
\hline vo4s & ND & ND & $7.5 \mathrm{E}-03 \pm 56.07$ \\
\hline v047 & $1.0 \mathrm{E}-02 \pm 74.0 \mathrm{Z}$ & ND & ND \\
\hline $\mathrm{v049}$ & $\mathrm{ND}$ & ND & ND \\
\hline v051 & $\mathrm{ND}$ & ND & ND \\
\hline vos3 & ID & ND & ND \\
\hline vos7 & $5.9 \mathrm{E}-03 \pm 66.02$ & $\mathrm{ND}$ & $\mathrm{RD}$ \\
\hline v059 & $2.6 \mathrm{E}-03 \pm 90.0 \%$ & KD & ND \\
\hline v061 & $7.8 \mathrm{E}-03 \pm 52.07$ & $\mathrm{ND}$ & $5.6 \mathrm{E}-03 \pm 62.0 \%$ \\
\hline v063 & $1.0 \mathrm{E}-02 \pm 46.0 \%$ & ND & $\mathrm{ND}$ \\
\hline v077 & $2.2 \mathrm{E}-02 \pm 42.0 \%$ & $\mathrm{ND}$ & $\mathrm{ND}$ \\
\hline v079 & ND & ND & ND. \\
\hline
\end{tabular}


Table E-6: 1997 Vegetation Sample Results, 200/600 Areas (pCi/gm Dry Weight).

(Sheet 8 of 8 )

\begin{tabular}{|c|c|c|c|}
\hline & $U-238$ & $P x-238$ & $\mathrm{Pu} 239 / 40$ \\
\hline Code & Result \pm Exxor & Result \pm Error & Result \pm Error \\
\hline v081 & ND & सा & ND \\
\hline P083 & $4.3 \mathrm{E}-03 \pm 90.0 \%$ & SND & ND \\
\hline v085 & $1.3 \mathrm{E}-02 \pm 53.02$ & ND & ND \\
\hline V087 & $1.5 \mathrm{E}-02 \pm 52.07$ & SD & $N D$ \\
\hline vos9 & $1.1 \mathrm{E}-02 \pm 54.0 z$ & WD & $8.7 \mathrm{E}-03 \pm 59.0 \mathrm{x}$ \\
\hline 7091 & $1.8 \mathrm{E}-02 \pm 36.08$ & $\mathbb{N D}$ & $\mathrm{SD}$ \\
\hline v095 & $1.5 \mathrm{z}-02 \pm 46.0 \mathrm{z}$ & ND & ND \\
\hline v097 & $9.9 \mathrm{E}-03 \pm 50.0 \mathrm{z}$ & ID & ND \\
\hline vo99. & $5.1 E-03 \pm 74.0 x$ & $\mathrm{ND}$ & $4.6 \mathrm{E}-03 \pm 75.0 \%$ \\
\hline$\nabla 101$ & ND & ND & SD \\
\hline$\nabla 103$ & $1.2 \mathrm{E}-02 \pm 44.0 \%$ & ND & $8.95-03 \pm 62.07$ \\
\hline vios & ND & ND & $5.5 \mathrm{E}-03 \pm 75.02$ \\
\hline V107 & $7.28-03 \cdot \pm 70.0 \%$ & ND & ND \\
\hline V109 & $5.6 \mathrm{E}-03 \pm 63.0 \mathrm{z}$ & WD & $\mathrm{ND}$ \\
\hline
\end{tabular}


HNF-EP-0573-6

Table E-7: 1997 Vegetation Sample Results, 300/400 Areas (pCi/gm Dry Weight).

(Sheet 1 of 4)

\begin{tabular}{|c|c|c|c|c|c|}
\hline & $\mathrm{Co}_{0}-60$ & $2 n-65$ & $S r-90$ & Ru-103 & $R u-106$ \\
\hline Code & Result I Error & Result \pm Error & Result \pm Error & Result \pm Error & Result \pm Error \\
\hline$\nabla 116$ & - $\quad \mathrm{ND}$ & IDD & SD & ND & ND \\
\hline v117 & ND & SD & $1.9 \mathrm{E}-01 \pm 70.0 \mathrm{z}$ & ND & ND \\
\hline v118 & $\mathrm{ND}$ & ND & ND & ND & ND \\
\hline$v 119$ & $S D$ & $\mathrm{ND}$ & ND & ND & ND \\
\hline v120 & KD & ND & $\mathrm{ND}$ & ND & ND \\
\hline V121 & NDD & ND & WD & ND & ND \\
\hline $\mathrm{V} 122$ & ND & SND & $\mathrm{ND}$ & $\mathrm{SD}$ & $\mathrm{ND}$ \\
\hline$\nabla 123$ & ND & ND & $M D$ & WD & WD \\
\hline$\nabla 224$ & ND & ND & $\mathrm{ND}$ & $\mathrm{MD}$ & SD \\
\hline v125 & $\mathrm{ND}$ & ND & $8.4 \mathrm{E}-01 \pm 20.0 z$ & ND & $N D$ \\
\hline V126 & ND & ND & $\mathrm{ND}$ & $\mathrm{ND}$ & ND \\
\hline $\mathrm{v} 127$ & $\mathrm{ND}$ & ND & . $\mathrm{XD}$ & ND & $\mathrm{ND}$ \\
\hline V128 & ND & $\mathrm{ND}$ & ND & $\mathrm{ND}$ & SDD \\
\hline v129 & SD & $\mathrm{KD}$ & ND & ND & $\mathrm{ND}$ \\
\hline$\nabla 130$ & SR & $\mathrm{ND}$ & $9.6 \mathrm{E}-01 \pm 25.08$ & ND & ND \\
\hline
\end{tabular}


Table E-7: 1997 Vegetation Sample Results, 300/400 Areas (pCi/gm Dry Weight).

(Sheet 2 of 4)

\begin{tabular}{|c|c|c|c|c|c|}
\hline & $5 n-113$ & $\mathrm{Sb}-125$ & Cs -134 & Cs-137 & $\mathrm{Ce}_{e}-144$ \\
\hline Code & Result \pm Error & Result \pm Error & Result \pm Exror & Result \pm Exror & Result \pm Error \\
\hline v116 & $\mathrm{ND}$ & WD & $\mathrm{ND}$ & RD & ND \\
\hline v117 & ND & $\mathrm{ND}$ & ND & ND & ND \\
\hline V118 & IND & ND & ND & $\mathrm{ND}$ & $\mathrm{ND}$ \\
\hline v119 & ND & ND & IND & ND & ND \\
\hline v120 & $\mathrm{ND}$ & ND & IDD & ND & ND \\
\hline $\mathrm{v} 121$ & $\mathrm{KD}$ & ND & ND & $\mathrm{ND}$ & ND \\
\hline V122 & ND & SD & $\mathrm{MD}$ & ND & ND \\
\hline$\nabla 123$ & ND & $\mathrm{ND}$ & SD & ND & $\mathrm{ND}$ \\
\hline$\nabla 124$ & $\mathrm{ND}$ & SD & $N D$ & $\mathrm{KDD}$ & ND \\
\hline$\nabla 125$ & ND & $\mathrm{ND}$ & ND & $S D$ & $\mathrm{ND}$ \\
\hline v126 & ND & $\mathrm{ND}$ & ND & ND & ND \\
\hline v127 & ND & ND & ND & SD & $\mathrm{AD}$ \\
\hline v128 & ND & WD & ND & SD & ND \\
\hline$\nabla 129$ & WD & ND & ND & D & ND \\
\hline$\nabla 130$ & ND & WD & ND & ND & ND \\
\hline
\end{tabular}


Table E-7: 1997 Vegetation Sample Results, 300/400 Areas (pCi/gm Dry Weight).

(Sheet 3 of 4)

\begin{tabular}{|c|c|c|c|c|c|}
\hline & Eu-152 & Eu-154 & $E u-155$ & $v-234$ & $\mathrm{v}-235$ \\
\hline Code & Result \pm Error & Result \pm Errox & Result \pm Error & Result \pm Error & Result \pm Error \\
\hline$\nabla 116$ & ND & ND & ND & $1.5 \mathrm{E}-02 \pm 39.07$ & ND \\
\hline$\nabla 117$ & ND & SD & ND & $2.0 \mathrm{E}-02 \pm 43.07$ & $1.1 E-02 \pm 61.0 x$ \\
\hline v118 & ND & ND & $\mathrm{ND}$ & $5.1 \mathrm{E}-02 \pm 21.07$ & $6.3 \mathrm{E}-03 \pm 61.07$ \\
\hline V119 & DD & ND & STD & $1.3 E-01 \pm 15.07$ & $2.2 \mathrm{E}-02 \pm 36.02$ \\
\hline v120 & ND & ND & ND & $3.62-01 \pm 9.82$ & $1.8 \mathrm{E}-02 \pm 38.07$ \\
\hline$v 121$ & $\mathrm{ND}$ & $\mathrm{ND}$ & ND & $6.4 \mathrm{E}-02 \pm 20.02$ & $9.5 \mathrm{E}-03 \pm 52.07$ \\
\hline$\nabla 122$ & SD & $\mathrm{ND}$ & ND & $2.0 \mathrm{E}-01 \pm 12.07$ & $2.2 E-02 \pm 33.0 \%$ \\
\hline$\nabla 123$ & $\mathrm{ND}$ & ND & SD & $3.4 E-02 \pm 27.0 z$ & $5.0 \mathrm{E}-03 \pm 79.0 z$ \\
\hline$\nabla 124$ & ND & ND & ND & $2.0 \mathrm{E}-02 \pm 34.0 \%$ & $2.3 E-02 \pm 44.08$ \\
\hline V125 & SDD & ND & IDD & $2.4 \mathrm{E}-02 \pm 34.07$ & $7.2 E-03 \pm 66.02$ \\
\hline $\mathrm{V} 126$ & ND & ND & ND. & $5.12-02 \pm 21.0 \%$ & $6.58-03 \pm 63.0 \%$ \\
\hline v127 & KD & ND & $\mathrm{ND}$ & $1.5 \mathrm{E}-02 \pm 42.02$ & $5.4 \mathrm{E}-03 \pm 67.02$ \\
\hline V128 & $\mathrm{ND}$ & ISD & SD & $1.4 \mathrm{E}-02 \pm 38.07$ & $4.2 E-03 \pm 71.0 \%$ \\
\hline $\mathrm{V} 129$ & ID & MD & ND & $9.4 \mathrm{E}-03 \pm 50.02$ & $4.6 \mathrm{~B}-03 \pm 79.0 \%$ \\
\hline$\nabla 130$ & SD & $\mathrm{ND}$ & $\mathrm{sD}$ & $2.2 \mathrm{E}-02 \pm 31.02$ & $5.1 E-03 \pm 67.07$ \\
\hline
\end{tabular}


Table E-7: 1997 Vegetation Sample Resuits, 300/400 Areas (pCi/gm Dry Weight).

(Sheet 4 of 4)

\begin{tabular}{|c|c|c|c|}
\hline & $v-238$ & Iu-238 & Fu239/40 \\
\hline Code & Result \pm Error & Result \pm Error & Resw2t $\neq$ Error \\
\hline V116 & $7.9 \mathrm{E}-03 \pm 50.0 \mathrm{z}$ & ID & $\mathrm{ND}$ \\
\hline$\nabla 117$ & $1.1 \mathrm{E}-02 \pm 58.0 z$ & ND & $\mathrm{ND}$ \\
\hline V118 & $4.5 \mathrm{E}-02 \pm 23.0 \mathrm{x}$ & ND & ND \\
\hline v119 & $1.25-01 \pm 16.0 \%$ & ND & $\mathrm{ND}$ \\
\hline$\nabla 120$ & $3.3 \mathrm{E}-0 \mathrm{I} \pm 10.0 \mathrm{z}$ & $\mathrm{ND}$ & ND \\
\hline$\nabla 121$ & $6.15-02 \pm 21.0 z$ & ND & ND \\
\hline v122 & $2.0 \mathrm{E}-01 \pm 12.0 \mathrm{Z}$ & $N D$ & ND \\
\hline v123 & $2.35-02 \pm 32.0 z$ & ND & $4.4 \mathrm{E}-03 \pm 65.07$ \\
\hline$\nabla 124$ & $1.9 \mathrm{E}-02 \pm 35.0 \%$ & $\mathrm{ND}$ & ND \\
\hline v125 & $1.62-02 \pm 39.0 x$ & $\mathrm{ND}$ & ND \\
\hline $\mathrm{V} 126$ & $4.8 \mathrm{E}-02 \pm 21.0 \%$ & ND & ND \\
\hline v127 & $1.0 \mathrm{E}-02 \pm 49.02$ & WD & ND \\
\hline$\nabla 128$ & $9.2 \mathrm{E}-0 \mathrm{~s} \pm 46.0 \mathrm{x}$ & ND & ND \\
\hline v129 & $1.58-02 \pm 38.0 z$ & WD & in \\
\hline$\nabla 130$ & $1.4 \mathrm{z}-02 \pm 40.0 z$ & ND & $\mathrm{ND}$ \\
\hline
\end{tabular}




\section{APPENDIX F}

EXTERNAL RADIATION MONITORING FIGURES AND TABLES 


\section{HNF-EP-0573-6}

This page intentionaliy left blank. 
Figure F-1. Thermoluminescent Dosimeter Locations in the $100 \mathrm{~B} / \mathrm{C}$ Area.

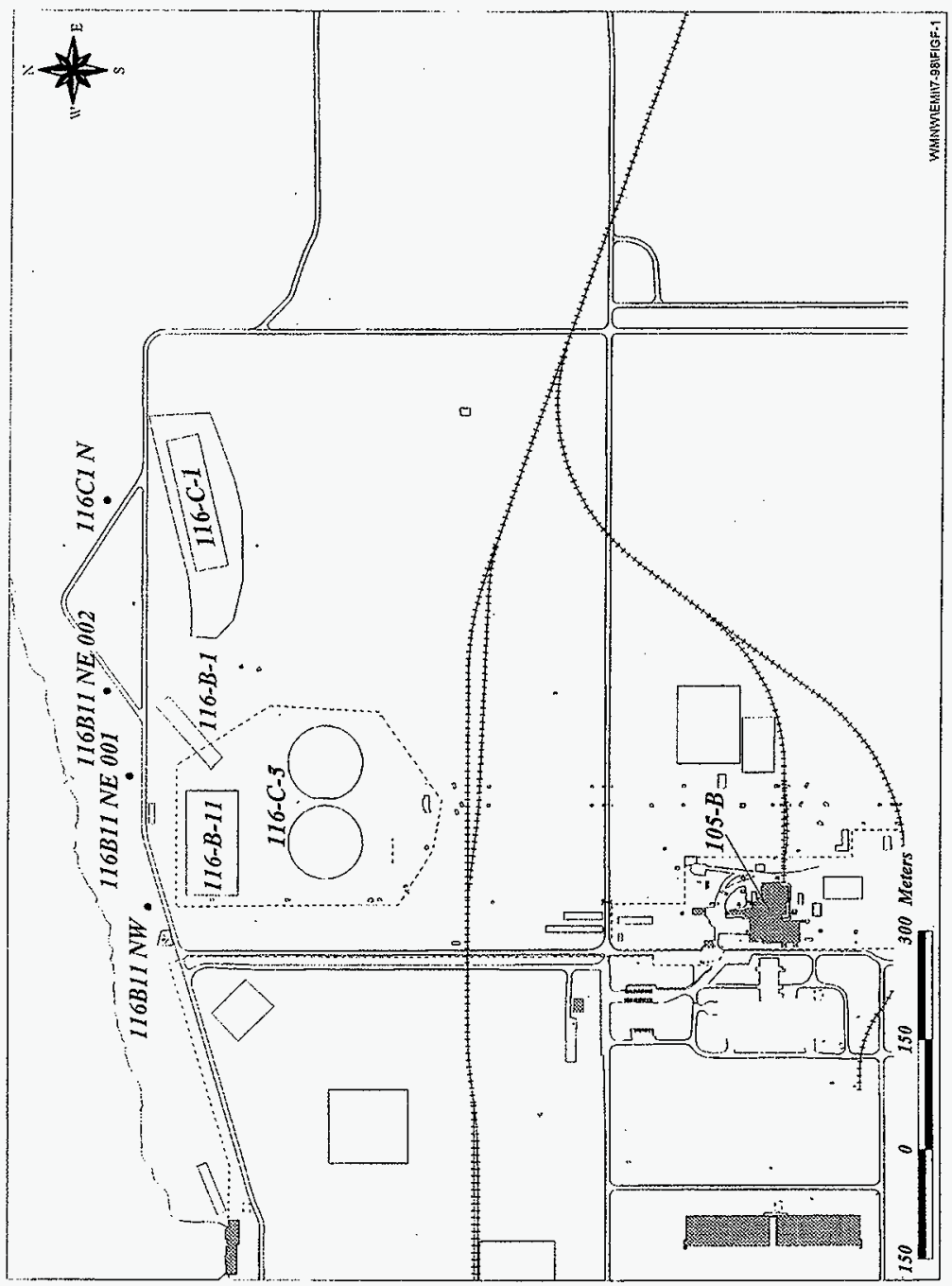


Figure F-2. Thermoluminescent Dosimeter Locations in the 100 D/DR Area.

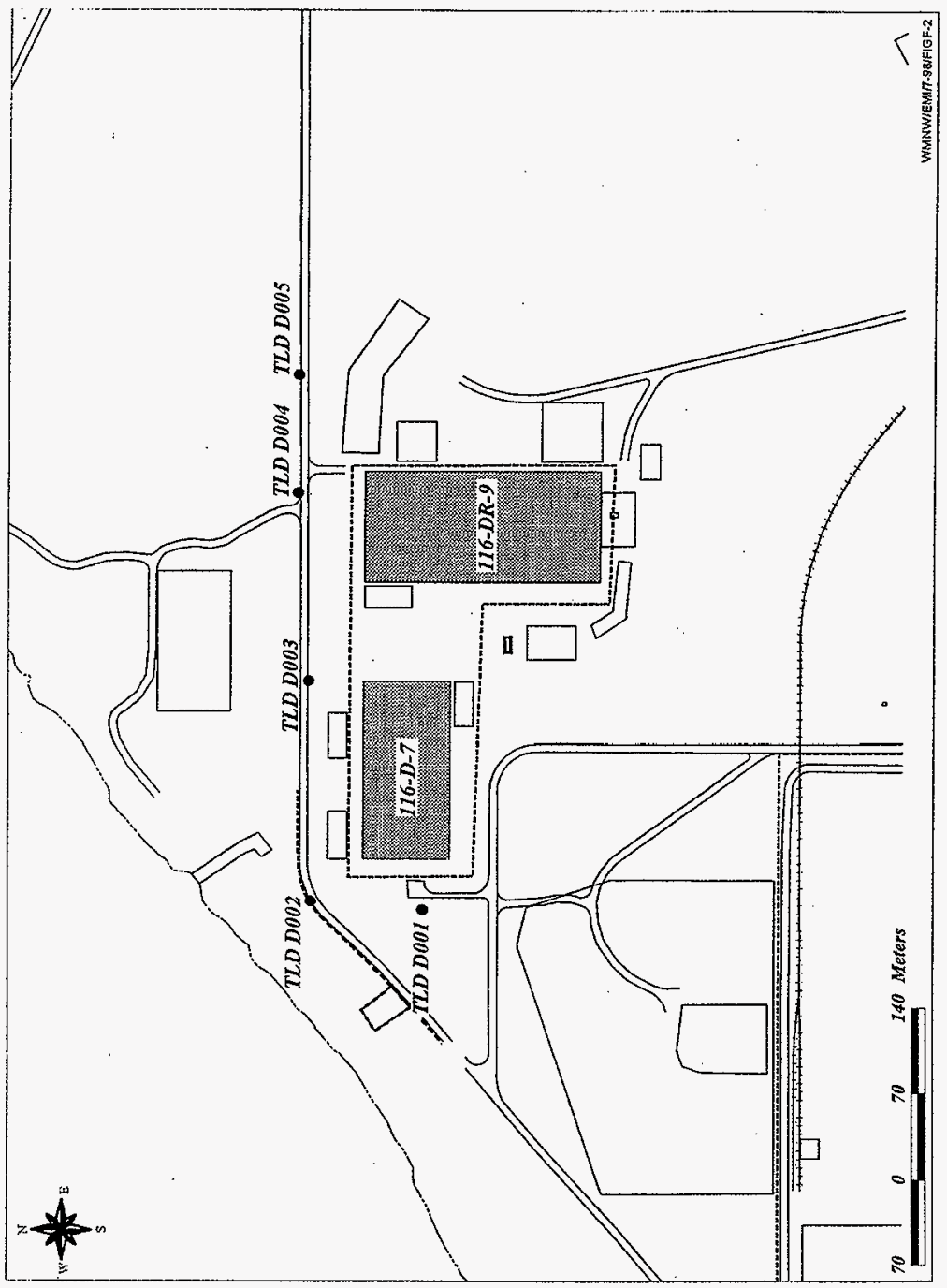


HNF-EP-0573-6

Figure F-3. Thermoluminescent Dosimeter Locations in the $100 \mathrm{KE} / \mathrm{KW}$ Area.

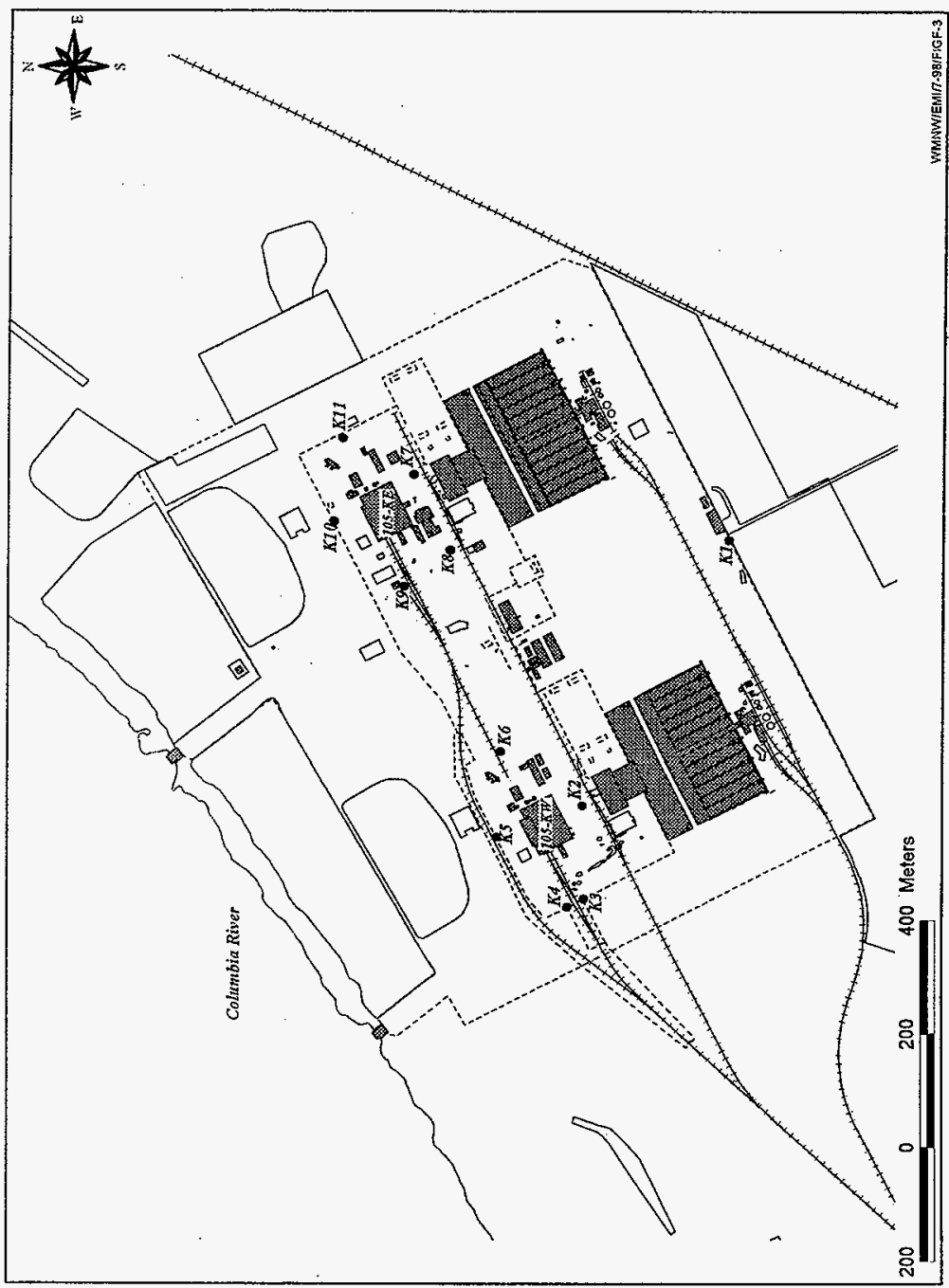


HNF-EP-0573-6

Figure F-4. Thermoluminescent Dosimeter Locations in the $100 \mathrm{~N}$ Area.

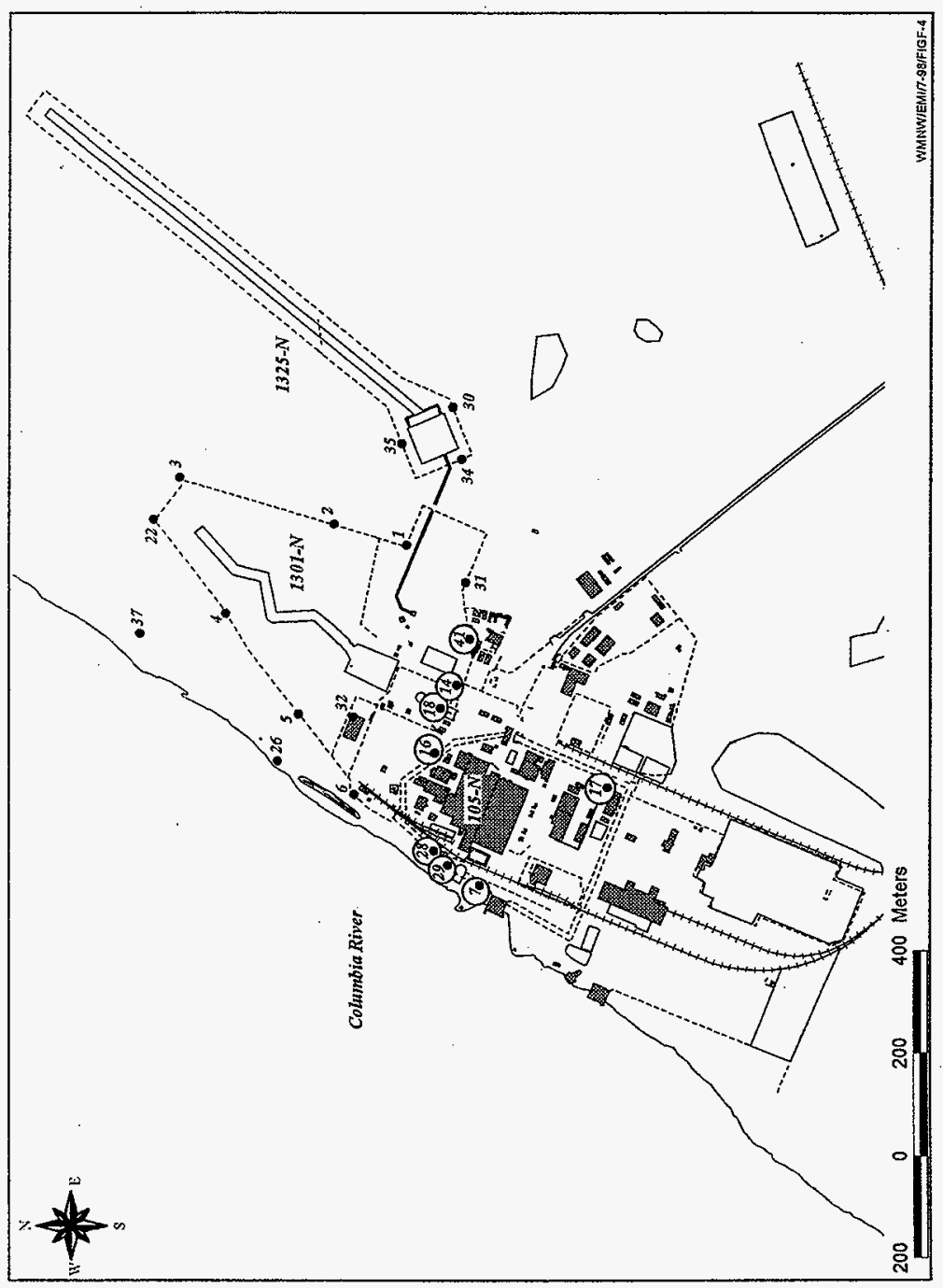

F-4 
HNF-EP-0573-6

Figure F-5. Thermoluminescent Dosimeter Locations in the 200 East Area.

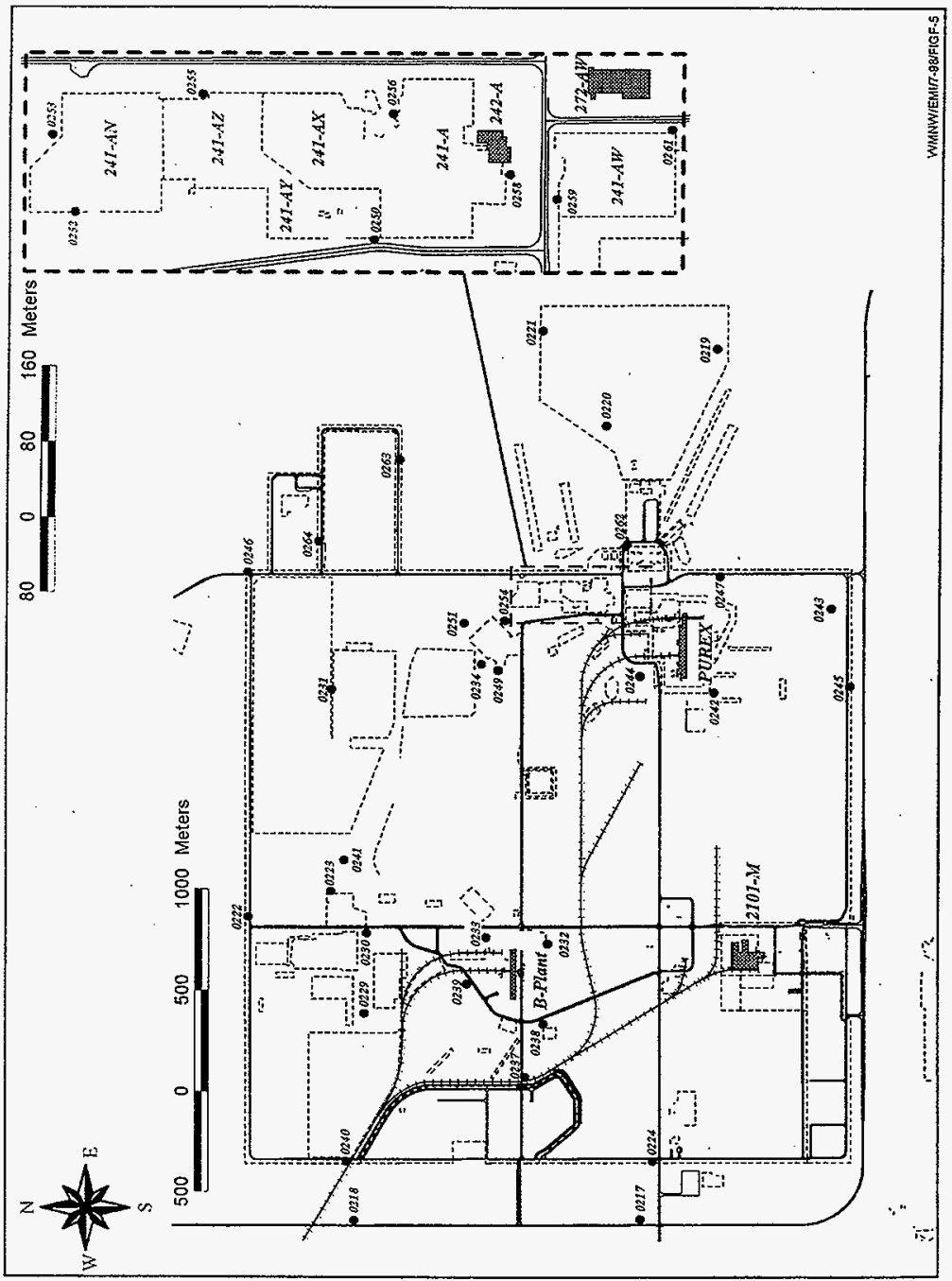


Figure F-6. Thermoluminescent Dosimeter Locations in the 200 West Area.

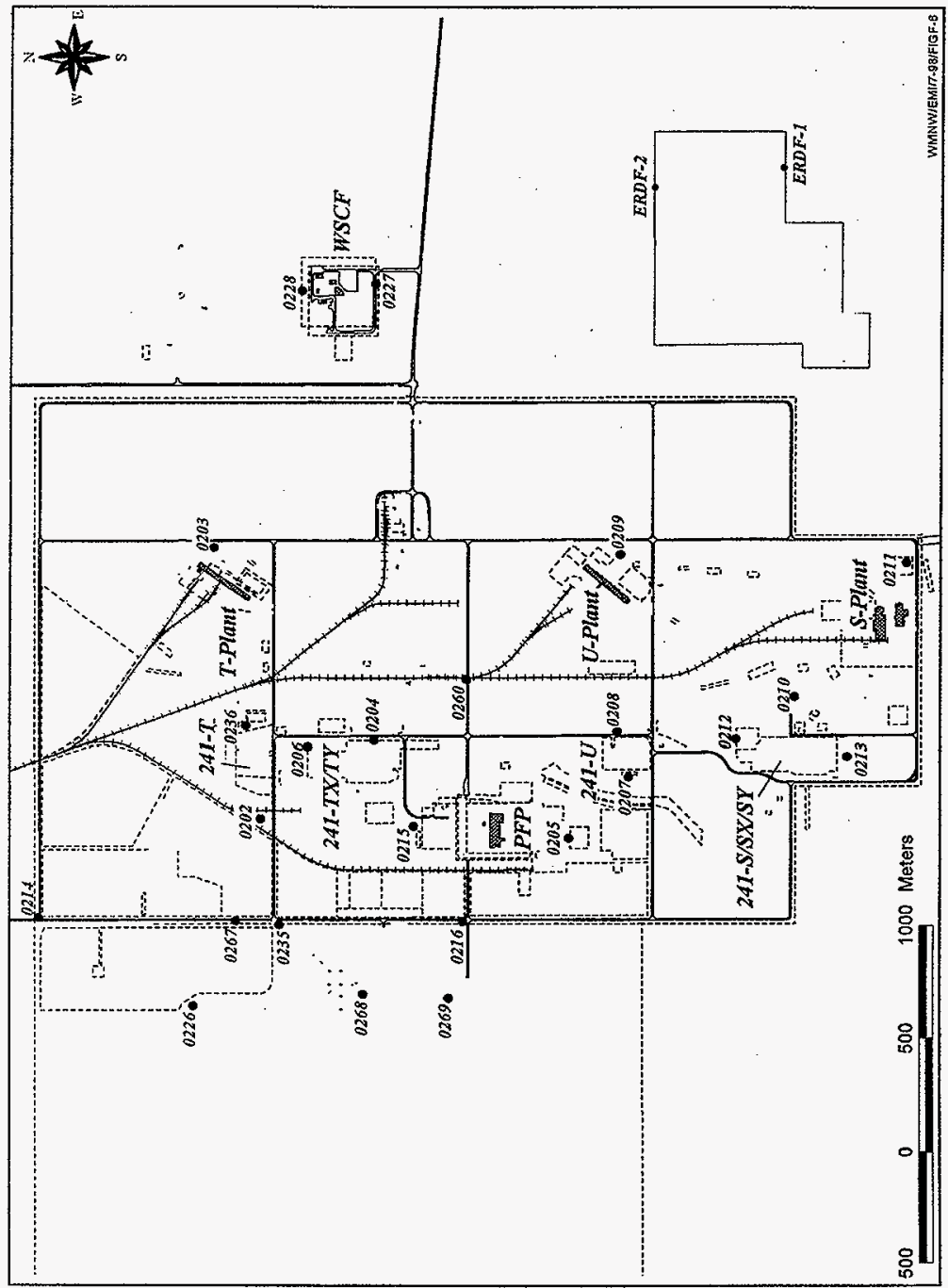




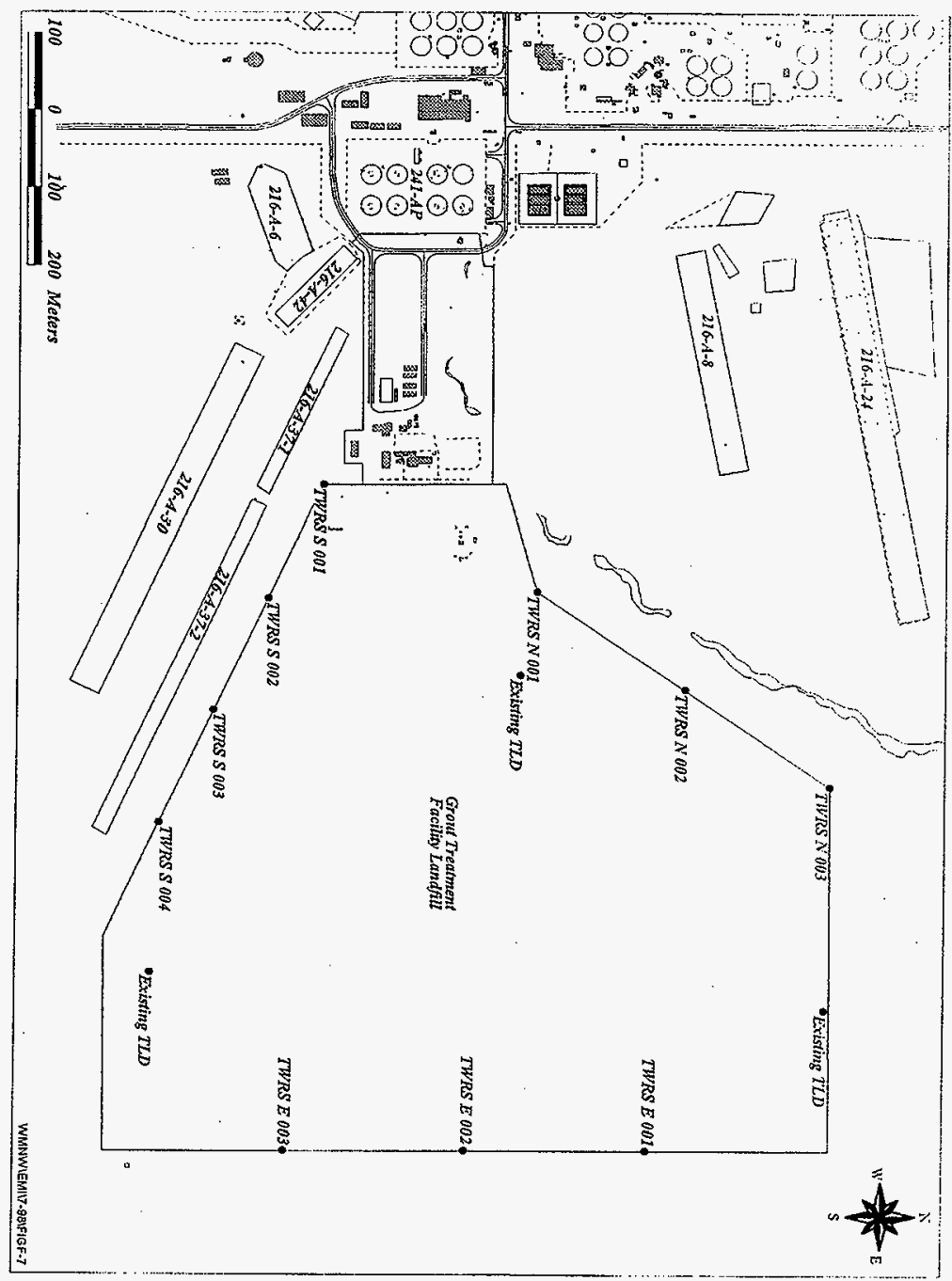

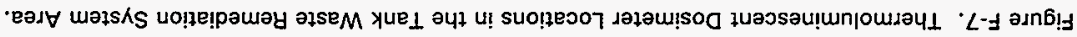


Figure F-8. Thermoluminescent Dosimeter Locations in the Environmental Restoration Disposal Facility Area.

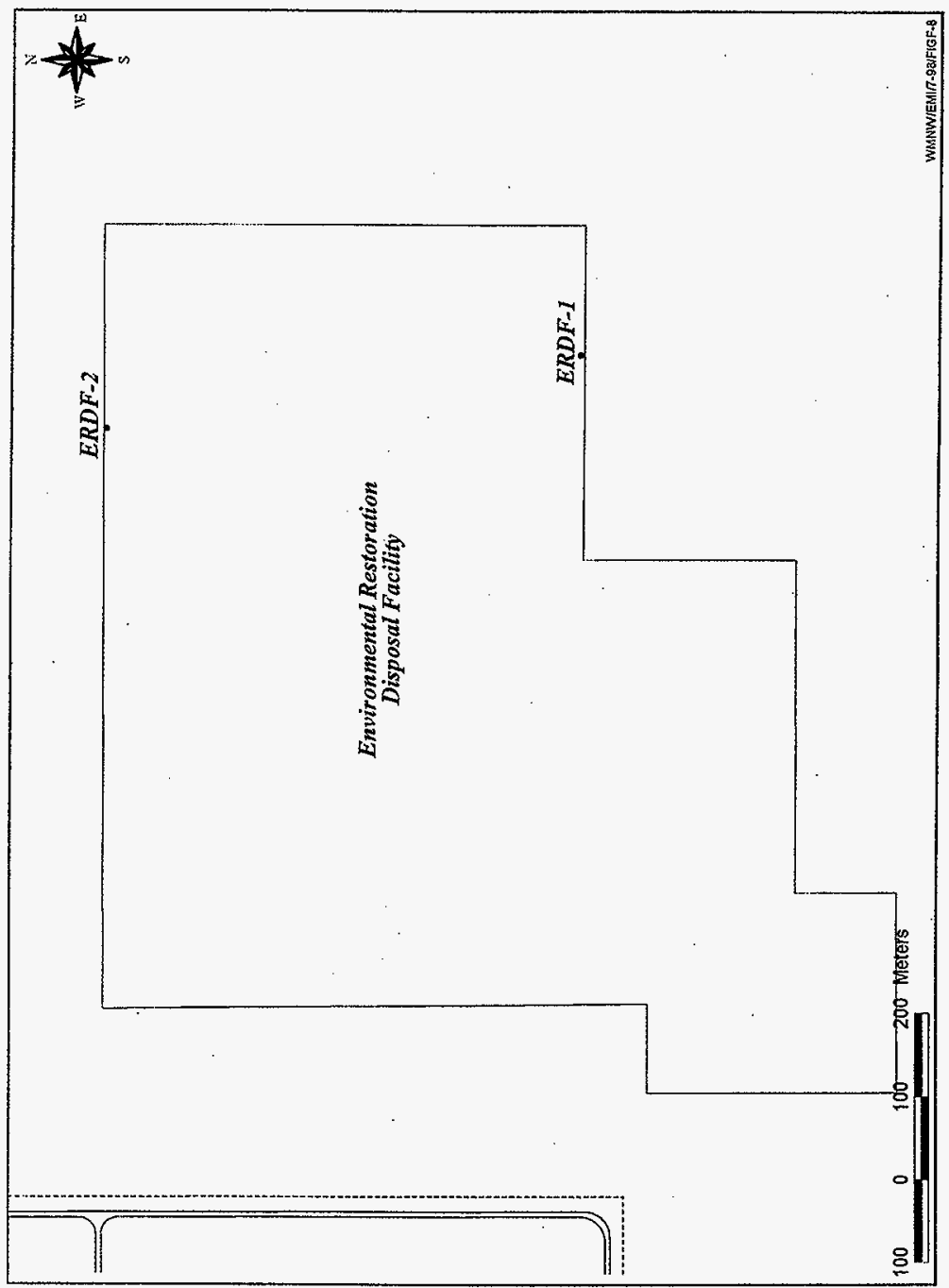


Figure F-9. Thermoluminescent Dosimeter Locations in the 300 Area.

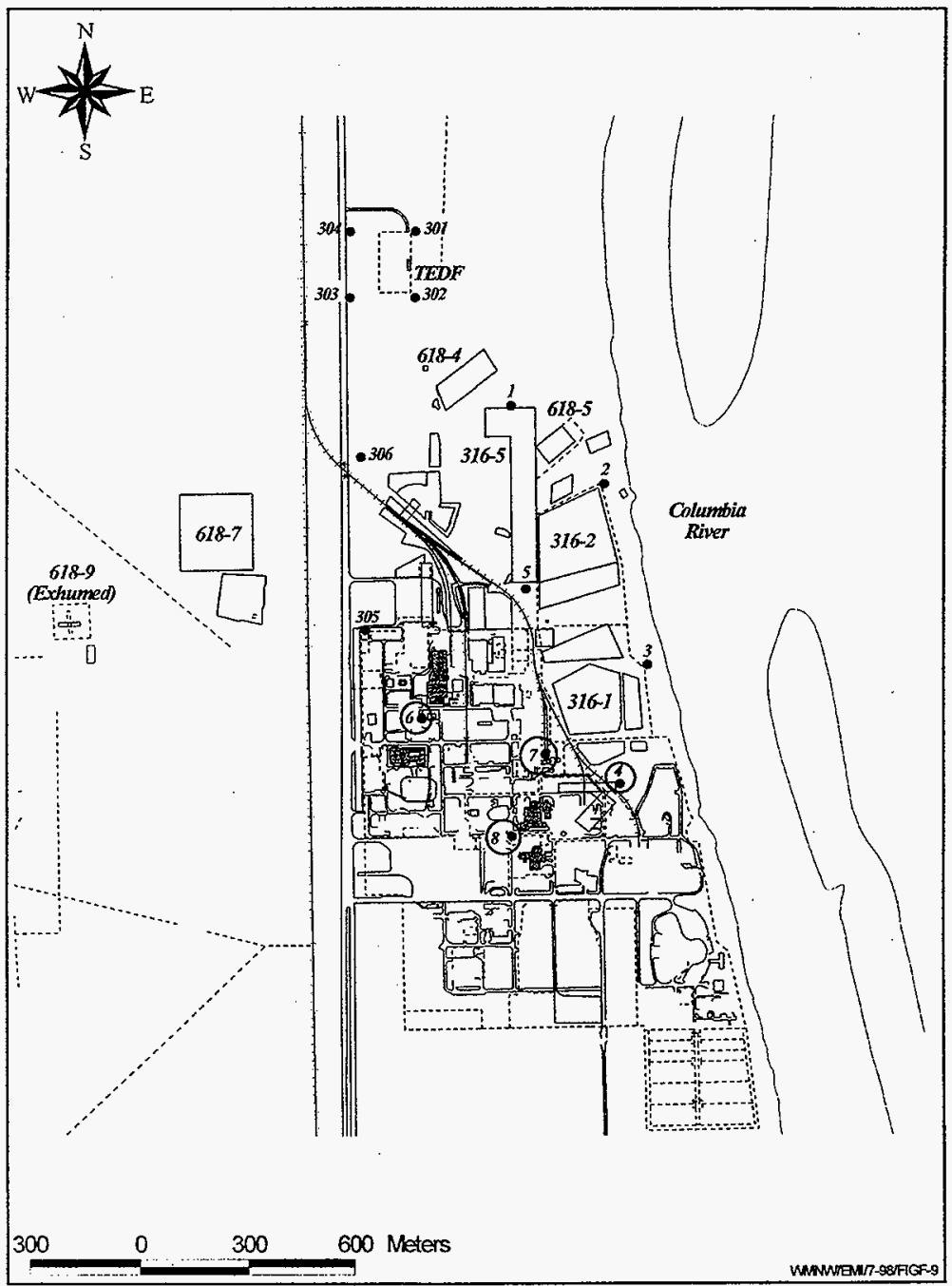


HNF-EP-0573-6

Figure F-10. Thermoluminescent Dosimeter Locations in the 400 Area.

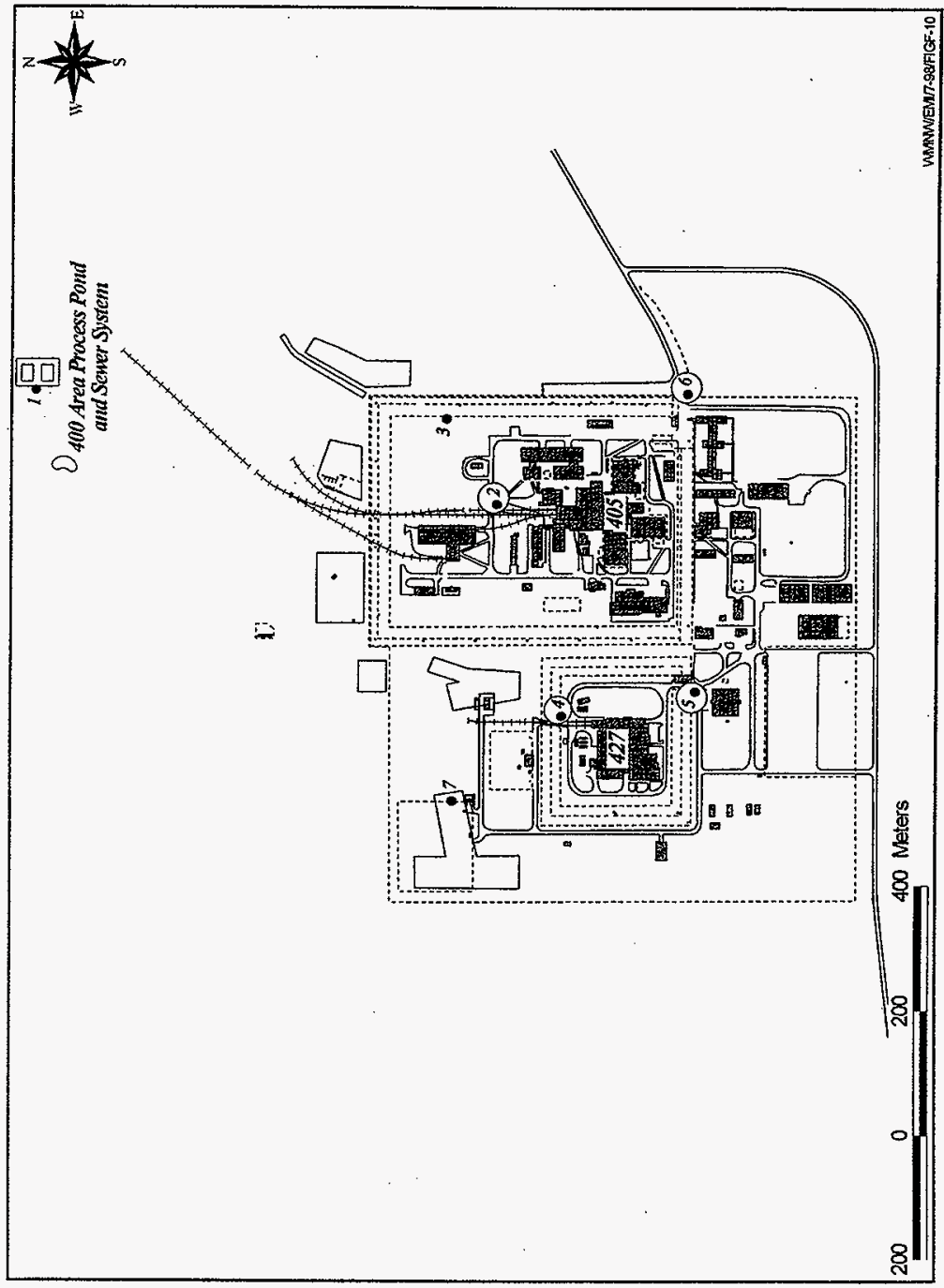


Table F-1. 100-B/C Thermoluminescent Dosimeter Exposure Results, Calendar Year 1997.

\begin{tabular}{|c|c|c|}
\hline \multirow{2}{*}{ Site I.D. } & \multicolumn{2}{|c|}{ 4th Quarter } \\
\cline { 2 - 3 } & $\mathrm{mrem} / \mathrm{h}$ & $\mathrm{mrem} / \mathrm{yr}$ \\
\hline 1 & $1.1 \mathrm{E}-02$ & $9.6 \mathrm{E}+01$ \\
\hline 2 & $1.0 \mathrm{E}-02$ & $9.1 \mathrm{E}+01$ \\
\hline 3 & $1.1 \mathrm{E}-02$ & $9.6 \mathrm{E}+01$ \\
\hline 4 & $1.0 \mathrm{E}-02$ & $8.9 \mathrm{E}+01$ \\
\hline
\end{tabular}

Table F-2. 100-D/DR Thermoluminescent Dosimeter Exposure Results, Calendar Year 1997.

\begin{tabular}{|c|c|c|c|c|c|c|c|c|c|c|c|}
\hline \multirow{2}{*}{$\begin{array}{c}\text { Site } \\
\text { i.D. }\end{array}$} & \multicolumn{2}{|c|}{$1 \mathrm{st}$ Quarter } & \multicolumn{2}{c|}{ 2nd Quarter } & \multicolumn{2}{c|}{ 3rd Quarter } & \multicolumn{2}{c|}{ 4th Quarter } & \multicolumn{3}{c|}{ Annual Resuits \{mrem/yri } \\
\hline & mrem/h & mrem/yr & mrem/h & mrem/yr & mrem/h & mrem/yr & mrem/h & mrem/yr & Maximum & Minimum & Average \\
\hline 1 & $9.4 \mathrm{E}-03$ & $8.3 \mathrm{E}+01$ & $9.0 \mathrm{E}-03$ & $7.9 \mathrm{E}+01$ & $1.0 \mathrm{E}-02$ & $8.8 \mathrm{E}+01$ & $1.1 \mathrm{E}-02$ & $9.7 \mathrm{E}+01$ & $9.7 \mathrm{E}+01$ & $7.9 \mathrm{E}+01$ & $8.7 \mathrm{E}+01$ \\
\hline 2 & $9.1 \mathrm{E}-03$ & $8.0 \mathrm{E}+01$ & $8.5 \mathrm{E}-03$ & $7.4 \mathrm{E}+01$ & $9.0 \mathrm{E}-03$ & $7.9 \mathrm{E}+01$ & $1.0 \mathrm{E}-02$ & $9.1 \mathrm{E}+01$ & $9.1 \mathrm{E}+01$ & $7.4 \mathrm{E}+01$ & $8.1 \mathrm{E}+01$ \\
\hline 3 & $9.3 \mathrm{E}-03$ & $8.2 \mathrm{E}+01$ & $9.5 \mathrm{E}-03$ & $8.3 \mathrm{E}+01$ & $1.1 \mathrm{E}-02$ & $9.5 \mathrm{E}+01$ & $1.2 \mathrm{E}-02$ & $1.1 \mathrm{E}+02$ & $1.1 \mathrm{E}+02$ & $8.2 \mathrm{E}+01$ & $9.2 \mathrm{E}+01$ \\
\hline 4 & $9.8 \mathrm{E}-03$ & $8.5 \mathrm{E}+01$ & $9.5 \mathrm{E}-03$ & $8.4 \mathrm{E}+01$ & $1.1 \mathrm{E}-02$ & $9.3 \mathrm{E}+01$ & $1.2 \mathrm{E}-02$ & $1.0 \mathrm{E}+02$ & $1.0 \mathrm{E}+02$ & $8.4 \mathrm{E}+01$ & $9.1 \mathrm{E}+01$ \\
\hline 5 & $9.7 \mathrm{E}-03$ & $8.5 \mathrm{E}+01$ & $1.0 \mathrm{E}-02$ & $8.7 \mathrm{E}+01$ & $1.0 \mathrm{E}-02$ & $9.0 \mathrm{E}+01$ & $1.1 \mathrm{E}-02$ & $9.9 \mathrm{E}+01$ & $9.9 \mathrm{E}+01$ & $8.5 \mathrm{E}+01$ & $9.0 \mathrm{E}+01$ \\
\hline
\end{tabular}

Table F-3. $100 \mathrm{~K}$ Thermoluminescent Dosimeter Exposure Results, Calendar Year 1997.

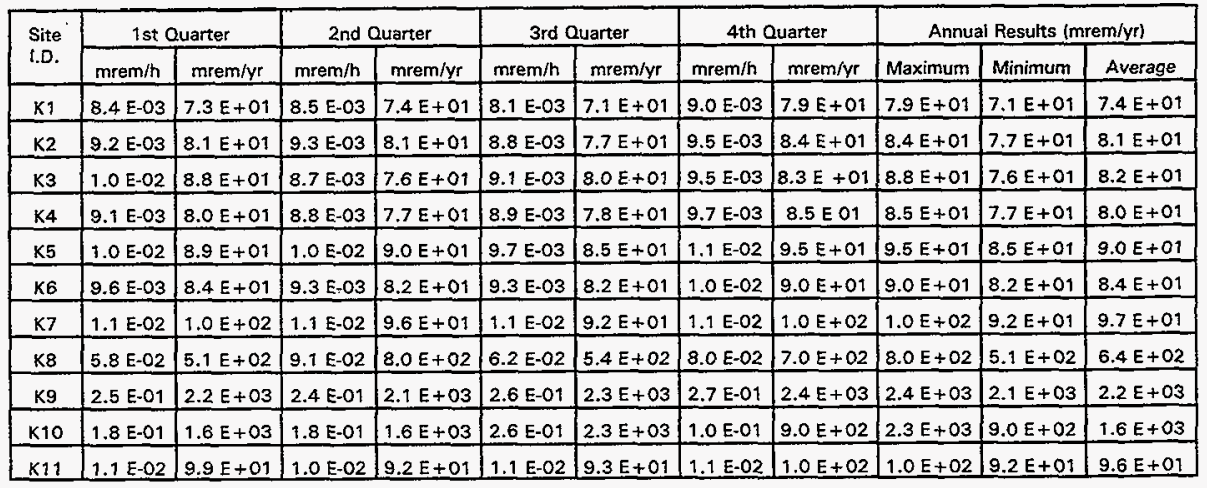


Table F-4. 100-N Thermoluminescent Dosimeter Exposure Results, Calendar Year 1997.

\begin{tabular}{|c|c|c|c|c|c|c|c|c|c|c|c|}
\hline \multirow[t]{2}{*}{ Site I.D. } & \multicolumn{2}{|c|}{ 1st Quarter } & \multicolumn{2}{|c|}{ 2nd Quarter } & \multicolumn{2}{|c|}{ 3rd Quarter } & \multicolumn{2}{|c|}{ 4th Quarter } & \multicolumn{3}{|c|}{ Annual Results (mrem/yr) } \\
\hline & mrem/hr & mrem/yr & $\mathrm{rem} / \mathrm{hr}$ & mrem/yr & mrem/hr & mrem/yr & mrem $/ \mathrm{hr}$ & mrem/yr & Maximum & Minimum & Average \\
\hline 1 & $8.3 \mathrm{E}-02$ & $7.3 \mathrm{E}+02$ & $8.3 E-02$ & $7.2 \mathrm{E}+02$ & $7.8 \mathrm{E}-02$ & $6.9 \mathrm{E}+02$ & $8.7 \mathrm{E}-02$ & $7.6 \mathrm{E}+02$ & $7.6 \mathrm{E}+02$ & $6.9 \mathrm{E}+02$ & $7.2 E+02$ \\
\hline 2 & 8.4 E-02 & $7.4 \overline{E+02}$ & $8.3 \mathrm{E}-02$ & $7.3 \mathrm{E}+02$ & $7.8 \mathrm{E}-02$ & $6.8 E+02$ & $8.5 \mathrm{E}-02$ & $7.4 \mathrm{E}+02$ & $7.4 \mathrm{E}+02$ & $6.8 \mathrm{E}+02$ & $7.2 \mathrm{E}+02$ \\
\hline 3 & $3.8 E-02$ & $3.3 E+02$ & $3.8 \mathrm{E}-02$ & $3.3 E+02$ & $3.6 \mathrm{E}-02$ & $3.2 E+02$ & $3.8 \mathrm{E}-02$ & $3.3 \mathrm{E}+02$ & $3.3 E+02$ & $3.2 \mathrm{E}+02$ & $3.3 E+02$ \\
\hline 4 & $1.5 \mathrm{E}-01$ & $1.3 E+03$ & $1.4 E-01$ & $1.3 E+03$ & $1.3 \mathrm{E}-01$ & $1.1 \mathrm{E}+03$ & $1.4 E-01$ & $1.2 \mathrm{E}+03$ & $1.3 E+03$ & $1.1 E+03$ & $1.2 \mathrm{E}+03$ \\
\hline 5 & 5.0 E-02 & $4.4 E+02$ & $5.3 \mathrm{E}-02$ & $4.6 \mathrm{E}+02$ & $4.8 \mathrm{E}-02$ & $4.2 \mathrm{E}+02$ & $5.0 \mathrm{E}-02$ & $4.4 E+02$ & $4.6 \mathrm{E}+02$ & $4.2 \mathrm{E}+02$ & $4.4 E+02$ \\
\hline 6 & $2.2 \mathrm{E}-02$ & $1.9 \mathrm{E}+02$ & 2.3 E-02 & $2.0 \mathrm{E}+02$ & $2.3 E-02$ & $2.0 \mathrm{E}+02$ & $2.4 \mathrm{E}-02$ & $2.1 \mathrm{E}+02$ & $2.1 \mathrm{E}+02$ & $1.9 \mathrm{E}+02$ & $2.0 \mathrm{E}+02$ \\
\hline 7 & $4.0 \mathrm{E}-02$ & $3.5 \mathrm{E}+02$ & $4.0 E-02$ & $3.5 E+02$ & $3.7 \mathrm{E}-02$ & $3.2 \mathrm{E}+02$ & $3.8 \mathrm{E}-02$ & $3.3 E+02$ & $3.5 E+02$ & $3.2 \mathrm{E}+02$ & $3.4 \mathrm{E}+02$ \\
\hline 14 & $3.5 \mathrm{E}-02$ & $3.1 \mathrm{E}+02$ & $3.6 E-02$ & $3.1 E+02$ & $3.6 \mathrm{E}-02$ & $3.2 \mathrm{E}+02$ & $1.5 E-01$ & $1.3 \mathrm{E}+03$ & $1.3 E+03$ & $3.1 \mathrm{E}+02$ & $5 \mathrm{E}+02$ \\
\hline 16 & $2.9 \mathrm{E}-02$ & $2.5 \mathrm{E}+02$ & $3.5 \mathrm{E}-02$ & $3.1 \mathrm{E}+02$ & $3.3 \mathrm{E}-02$ & $2.9 \mathrm{E}+02$ & $3.8 E-02$ & $3.3 E+02$ & $3.3 \mathrm{E}+02$ & $2.5 E+02$ & $3.0 E+02$ \\
\hline 17 & $9.9 \mathrm{E}-03$ & $8.7 \mathrm{E}+01$ & 9.1 E-03 & $8.0 \mathrm{E}+01$ & $1.0 \mathrm{E}-02$ & $8.8 \mathrm{E}+01$ & $1.0 \mathrm{E}-02$ & $8.8 \mathrm{E}+01$ & $8.8 \mathrm{E}+01$ & $8.0 \mathrm{E}+01$ & $8.5 E+01$ \\
\hline 18 & $4.3 \mathrm{E}-02$ & $3.8 \mathrm{E}+02$ & $4.4 \mathrm{E}-02$ & $3.8 \mathrm{E}+02$ & $4.8 \mathrm{E}-02$ & $4.2 E+02$ & $7.2 \mathrm{E}-02$ & $6.3 E+02$ & $6.3 E+02$ & $3.8 E+02$ & $4.5 \mathrm{E}+02$ \\
\hline 22 & $4.4 \mathrm{E}-02$ & $3.8 \mathrm{E}+02$ & $4.3 \mathrm{E}-02$ & $3.7 E+02$ & $4.0 \mathrm{E}-02$ & $3.5 \mathrm{E}+02$ & $4.5 E-02$ & $3.9 \mathrm{E}+02$ & $3.9 \mathrm{E}+02$ & $3.5 \mathrm{E}+02$ & $3.8 \mathrm{E}+02$ \\
\hline 26 & $2.4 \mathrm{E}-02$ & $1.8 E+02$ & $2.1 \mathrm{E}-02$ & $1.9 \mathrm{E}+02$ & $2.2 \mathrm{E}-02$ & $1.9 E+02$ & $2.3 E-02$ & $2.0 \mathrm{E}+02$ & $2.0 \mathrm{E}+02$ & $1.8 E+02$ & $1.9 \mathrm{E}+02$ \\
\hline 28 & $1.8 \mathrm{E}-02$ & $1.6 \mathrm{E}+02$ & $2.0 \mathrm{E}-02$ & $1.7 \mathrm{E}+02$ & $2.8 \mathrm{E}-02$ & $2.4 \mathrm{E}+02$ & $4.0 \mathrm{E}-02$ & $3.5 \mathrm{E}+02$ & $3.5 \mathrm{E}+02$ & $1.6 \mathrm{E}+02$ & $2.3 E+02$ \\
\hline 29 & $7.0 \mathrm{E}-02$ & $6.1 E+02$ & NR & NR & NR & NR & $8.9 \mathrm{E}-02$ & $7.8 \mathrm{E}+02$ & $7.8 E+02$ & $6.1 \mathrm{E}+02$ & $7.0 \mathrm{E}+02$ \\
\hline 30 & $5.4 \mathrm{E}-01$ & $4.7 E+03$ & $5.3 \mathrm{E}-01$ & $4.6 \mathrm{E}+03$ & 4.4 E-0.1 & $3.9 \mathrm{E}+03$ & $5.5 \mathrm{E}-01$ & $4.8 \mathrm{E}+03$ & $4.8 \mathrm{E}+03$ & $3.9 \mathrm{E}+03$ & $4.5 E+03$ \\
\hline 31 & $3.8 \mathrm{E}-02$ & $3.3 E+02$ & $3.9 \mathrm{E}-02$ & $3.4 \mathrm{E}+02$ & $3.9 \mathrm{E}-02$ & $3.4 \mathrm{E}+02$ & $4.2 \mathrm{E}-02$ & $3.7 E+02$ & $3.7 \mathrm{E}+02$ & $3.3 E+02$ & $3.5 \mathrm{E}+02$ \\
\hline 32 & $3.0 \mathrm{E}-02$ & $2.6 \mathrm{E}+02$ & $2.9 \mathrm{E}-02$ & $2.6 E+02$ & $3.3 \mathrm{E}-02$ & $2.9 E+02$ & $3.5 \mathrm{E}-02$ & $3.0 E+02$ & $3.0 \mathrm{E}+02$ & $2.6 \mathrm{E}+02$ & $2.8 E+02$ \\
\hline 34 & $9.2 \mathrm{E}-01$ & $8.0 \mathrm{E}+03$ & $9.0 E-01$ & $7.9 E+03$ & $7.4 \mathrm{E}-01$ & $6.5 \mathrm{E}+03$ & $9.4 \mathrm{E}-01$ & $8.2 E \div 03$ & $8.2 \mathrm{E}+03$ & $6.5 \mathrm{E}+03$ & $7.7 E+03$ \\
\hline 35 & $8.8 E-01$ & $7.7 E+03$ & 8.8 E-01 & $7.7 \mathrm{E}+03$ & $7.7 \mathrm{E}-01$ & $6.7 \mathrm{E}+03$ & $9.3 E-01$ & $8.2 E+03$ & $8.2 E+03$ & $6.7 \mathrm{E}+03$ & $7.6 \mathrm{E}+03$ \\
\hline 37 & $3.3 \mathrm{E}-02$ & $2.9 E+02$ & $3.3 E-02$ & $2.9 \mathrm{E}+02$ & $3.2 \mathrm{E}-02$ & $2.8 \mathrm{E}+02$ & $3.5 \mathrm{E}-02$ & $3.1 E+02$ & $3.1 \mathrm{E}+02$ & $2.8 \mathrm{E}+02$ & $2.9 \mathrm{E}+02$ \\
\hline 41 & $3.0 \mathrm{E}-02$ & $2.6 \mathrm{E}+02$ & $3.1 E-02$ & $2.7 E+02$ & $3.0 \mathrm{E}-02$ & $2.7 E+02$ & $3.5 \mathrm{E}-02$ & $3.1 \mathrm{E}+02$ & $3.1 E+02$ & $2.6 \mathrm{E}+02$ & $2.8 E+02$ \\
\hline
\end{tabular}

Table F-5. 200/600 Areas Thermoluminescent Dosimeter Exposure Results, Calendar Year 1997.

\begin{tabular}{|c|c|c|c|c|c|c|c|c|c|c|c|}
\hline \multirow{2}{*}{$\begin{array}{l}\text { Site } \\
\text { I.D. }\end{array}$} & \multicolumn{2}{|c|}{ 1st Quarter } & \multicolumn{2}{|c|}{ 2nd Quarter } & \multicolumn{2}{|c|}{ 3rd Quarter } & \multicolumn{2}{|c|}{ 4th Quarter } & \multicolumn{3}{|c|}{ Annual Results mrem/yr } \\
\hline & mrem $/ \mathrm{h}$ & $\mathrm{mrem} / \mathrm{vr}$ & mrem $/ \mathrm{h}$ & $\mathrm{mrem} / \mathrm{yr}$ & $\mathrm{mrem} / \mathrm{h}$ & $\mathrm{mrem} / \mathrm{yr}$ & $\mathrm{mrem} / \mathrm{h}$ & mrem/yr & Maximum & Minimum & Average \\
\hline 202 & $1.0 \mathrm{E}-02$ & $9.1 \mathrm{E}+01$ & $9.6 \mathrm{E}-03$ & $8.4 \mathrm{E}+01$ & $1.0 \mathrm{E}-02$ & $9.1 E+01$ & $1.1 \mathrm{E}-02$ & $9.5 E+01$ & $9.5 \mathrm{E}+01$ & $8.4 E+01$ & $9.0 \mathrm{E}+01$ \\
\hline 203 & $2 \mathrm{E}-02$ & $1.1 \mathrm{E}+02$ & $1.1 \varepsilon-02$ & $9.3 \varepsilon+01$ & $1.2 \mathrm{E}-02$ & 1.0E+02 & $1.2 \mathrm{E}-02$ & $1.1 E+02$ & $1.1 E+02$ & $9.3 E+01$ & $1.0 E+02$ \\
\hline 204 & $1.7 \mathrm{E}-02$ & $1.4 E+02$ & $1.5 \mathrm{E}-02$ & $E+02$ & $1.5 \mathrm{E}-02$ & $1.4 \mathrm{E}+02$ & $1.8 \mathrm{E}-02$ & $1.5 E+02$ & $1.5 E+02$ & $1.3 E+02$ & $1.4 E+02$ \\
\hline 205 & 9.9 & $8.7 E+01$ & $8.9 \mathrm{E}-03$ & $7.8 \mathrm{E}+01$ & $9.6 \mathrm{E}-03$ & $8.4 \mathrm{E}+01$ & $1.0 \mathrm{E}-02$ & $9.1 E+01$ & $9.1 E+01$ & $7.8 \mathrm{E}+01$ & $8.5 \mathrm{E}+01$ \\
\hline 206 & $1.3 \mathrm{E}-02$ & $1.2 E+02$ & $1.2 \mathrm{E}-02$ & $1.1 \mathrm{E}+02$ & $1.3 \mathrm{E}-02$ & $1.2 \mathrm{E}+02$ & $1.4 \mathrm{E}-02$ & $1.2 \mathrm{E}+02$ & $1.2 \mathrm{E}+02$ & $1.1 E+02$ & $1.1 \mathrm{E}+02$ \\
\hline 207 & $1.1 \mathrm{E}-02$ & $9.8 E+01$ & $1.1 \mathrm{E}-02$ & $9.3 \mathrm{E}+01$ & $1.1 \mathrm{E}-\mathrm{O} 2$ & $9.7 E+01$ & 22 & +02 & $1.0 E+02$ & $9.3 E+01$ & $9.8 E+01$ \\
\hline 208 & $E-02$ & $1.8 E+02$ & $1.5 \mathrm{E}-02$ & $1.3 E+02$ & 1.2 & $1.1 E+02$ & $1.3 \mathrm{E}-02$ & $1.2 \mathrm{E}+02$ & $1.8 E+02$ & $1.1 \mathrm{E}+02$ & $1.3 E+02$ \\
\hline 209 & $1 . c$ & $8.9 E+01$ & $9.4 \mathrm{E}-03$ & $8.2 \mathrm{E}+01$ & $9.9 \mathrm{E}-03$ & $8.7 E+01$ & $1.1 \mathrm{E}-02$ & $9.5 \mathrm{E}+01$ & $9.5 E+01$ & $8.2 \mathrm{E}+01$ & $8.8 E+01$ \\
\hline 210 & 02 & $1.0 \mathrm{E}+02$ & $1.0 \mathrm{E}-02$ & $9.2 \mathrm{E}+0 \mathrm{~T}$ & $1.1 \mathrm{E}-\mathrm{O} 2$ & $9.7 E+01$ & $1.2 E-02$ & $1.1 \varepsilon+02$ & $1.1 E+02$ & $9.2 \mathrm{E}+01$ & $9.9 \mathrm{E}+01$ \\
\hline 211 & $1.2 \mathrm{E}-02$ & 1.1 $E+02$ & $9.7 \mathrm{E}-03$ & $8.5 \mathrm{E}+01$ & $1.1 \mathrm{E}-02$ & $9.2 E+01$ & NR & NR & $1.1 \mathrm{E}+02$ & $8.5 E+01$ & $9.5 E+01$ \\
\hline 212 & $1.8 \mathrm{E}-02$ & $1.6 E+02$ & $1.5 \mathrm{E}-02$ & $1.3 E+02$ & $1.3 \varepsilon-02$ & $1.2 E+02$ & $1.4 \mathrm{E}-02$ & $1.2 E+02$ & $1.6 E+02$ & $1.2 \mathrm{E}+02$ & $1.3 E+02$ \\
\hline 213 & $2.3 \mathrm{E}-02$ & $2.0 E+02$ & $2.2 E-02$ & $1.9 E+02$ & $2.1 \mathrm{E}-02$ & $1.8 \mathrm{E}+02$ & $2.2 \mathrm{E}-02$ & $1.9 E+02$ & $2.0 \mathrm{E}+02$ & $1.8 E+02$ & $1.9 E+02$ \\
\hline 214 & $9.9 \mathrm{E}-03$ & $8.7 \varepsilon+01$ & $8.5 \mathrm{E}-03$ & $7.4 \mathrm{E}+01$ & $8.8 \mathrm{E}-03$ & $7.7 \mathrm{E}+01$ & $9.3 E-03$ & $8.2 \mathrm{E}+01$ & $8.7 \mathrm{E}+0 \mathrm{t}$ & $7.4 \mathrm{E}+01$ & $8.0 \mathrm{E}+01$ \\
\hline 215 & $1.7 \mathrm{E}-02$ & $9.5 \mathrm{E}+01$ & $9.8 \mathrm{E}-03$ & $8.5 E+01$ & $1.0 \mathrm{E}-02$ & $8.9 \mathrm{E}+01$ & $1.1 \mathrm{E}-02$ & $9.6 \mathrm{E}+01$ & $9.6 \mathrm{E}+01$ & $8.5 \mathrm{E}+03$ & $9.2 E+01$ \\
\hline 216 & $1.1 \mathrm{E}-02$ & $9.9 \mathrm{E}+01$ & $1.0 \mathrm{E}-02$ & $8.8 E+01$ & $1.0 \mathrm{E}-02$ & $8.8 E+01$ & $1.0 \mathrm{E}-02$ & $9.2 E+01$ & $9.9 E+01$ & $8.8 E+01$ & $9.2 E+01$ \\
\hline 217 & $1.0 \mathrm{E}-02$ & $8.8 E+01$ & $9.4 \mathrm{E}-03$ & $8.2 E+01$ & $9.5 \mathrm{E}-03$ & $8.4 E+01$ & $1.0 \mathrm{E}-02$ & $9.0 E+01$ & $9.0 E+01$ & $8.2 \mathrm{E}+01$ & $8.6 \mathrm{E}+01$ \\
\hline 218 & $9.0 \mathrm{E}-03$ & $7.9 E+01$ & 8.4 E-03 & $7.3 E+01$ & $8.6 \mathrm{E}-03$ & $7.6 \mathrm{E}+01$ & $9.1 \mathrm{E}-03$ & $8.0 E+01$ & $8.0 \mathrm{E}+01$ & $7.3 \mathrm{E}+01$ & $7.7 E+01$ \\
\hline 219 & $1.1 \mathrm{E}-02$ & $9.5 E+01$ & $1.0 \mathrm{E}-02$ & $8.9 E+01$ & $1.0 \mathrm{E}-02$ & $9.1 E+01$ & $1.1 \mathrm{E}-02$ & $9.4 E+01$ & $9.5 \mathrm{E}+01$ & $8.9 E+01$ & $9.2 E+01$ \\
\hline
\end{tabular}


Table F-5. 200/600 Areas Thermoluminescent Dosimeter Exposure Results, Calendar Year 1997.

\begin{tabular}{|c|c|c|c|c|c|c|c|c|c|c|c|}
\hline \multirow{2}{*}{$\begin{array}{l}\text { Site } \\
\text { I.D. }\end{array}$} & \multicolumn{2}{|c|}{ 1st Quarter } & \multicolumn{2}{|c|}{ 2nd Quarter } & \multicolumn{2}{|c|}{ 3rd Quarter } & \multicolumn{2}{|c|}{ 4th Quarter } & \multicolumn{3}{|c|}{ Annual Results mrem/yr } \\
\hline & mrem/h & mrem/yr & $\boldsymbol{n}$ & $\mathrm{yr}$ & $n$ & mrem/yr & Vh & vem/yr & um & linimum & verage \\
\hline 220 & & & & & & & & & & & \\
\hline 221 & & +01 & & +01 & & & & $E+01$ & & & \\
\hline 222 & 02 & $2 E+02$ & 02 & $E+02$ & & .02 & $E-02$ & $2 \mathrm{E}+02$ & $2 \mathrm{E}+02$ & +02 & +02 \\
\hline & & & & +02 & & & & $E+02$ & $E+02$ & & \\
\hline 224 & & & & $7.9 \mathrm{E}+01$ & & & $1.0 \mathrm{E}-02$ & $8.9 \mathrm{E}+01$ & $E+01$ & & \\
\hline 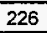 & & $E+01$ & .03 & $8.0 E+01$ & & & $1.1 \mathrm{E}-02$ & $E+01$ & $3 E+01$ & $E+01$ & $E+01$ \\
\hline 227 & & +01 & & +01 & & & & $E+01$ & $E+01$ & +01 & \\
\hline 228. & & & & +01 & & & & $E+01$ & & & \\
\hline 229 & & +01 & & +01 & & & & $=+01$ & $9.6 \mathrm{E}+01$ & & $= \pm 01$ \\
\hline 230 & & 02 & & +02 & & & -02 & +02 & 2 & +02 & +02 \\
\hline & & & & & & & & & & & \\
\hline 23 & & & & & & & & +02 & & & $E+02$ \\
\hline 233 & & & & 01 & & & & +01 & 1 & 01 & 01 \\
\hline & & & & & & & & & & & \\
\hline & & & & & & & & 102 & & & \\
\hline 236 & & & & & & & & 02 & 2 & 01 & +01 \\
\hline & & & & & & & & & & & \\
\hline & & & & & & & & & & & \\
\hline 23. & & & & & & & & \pm+01 & & & \\
\hline & & & & & & & & & & & \\
\hline & & & & & & & & & & & \\
\hline 24 & & & & & & & & & & & \\
\hline 24 & & & & & & & & & & & 01 \\
\hline 24 & & & & & & & & & & & \\
\hline 24 & & & & & & & & & & & \\
\hline 24 & & & & & & & & & & 01 & \\
\hline & & & & & & & & & & & 01 \\
\hline & & & & & & & & & & & \\
\hline 25 & & & & & & & & & & & +02 \\
\hline 20 & & & & & & & & & & & +02 \\
\hline & & & & & & & & & & & \\
\hline & & & & & & & & & & & \\
\hline 254 & & & & & & & & & & & \\
\hline 25 & & & & 02 & & & & & & 02 & \\
\hline 25 & & & & & & & & & & & +02 \\
\hline 25 & & & & & & & & & & & \\
\hline 25 & & & & & & & & & & & \\
\hline 260 & & & & +01 & & +01 & & +01 & & $E+01$ & $E+01$ \\
\hline & & & & & & & & & & & +01 \\
\hline-5 & & & & & & & & & & & \\
\hline $26:$ & & & & & & & & & & & \\
\hline 264 & & & & $8.0 \mathrm{E}+01$ & & & & & & & $7 \mathrm{E}+01$ \\
\hline 26 & & & & & & & & +01 & & $=+01$ & $2 \mathrm{E}+01$ \\
\hline & & & & & & & & & & & \\
\hline & Was & Liva & & $9.0[+01$ & & 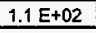 & & & & 每 & \\
\hline
\end{tabular}


HNF-EP-0573-6

Table F-6. Tank Waste Remediation System

Thermoluminescent Dosimeter Exposure

Results, CalendarYear 1997.

\begin{tabular}{|c|c|c|}
\hline \multirow{2}{*}{ Site I.D. } & \multicolumn{2}{|c|}{ 4th Quarter } \\
\cline { 2 - 3 } & $\mathrm{mrem} / \mathrm{h}$ & $\mathrm{mrem} / \mathrm{yr}$ \\
\hline 300 & $8.8 \mathrm{E}-03$ & $7.7 \mathrm{E}+01$ \\
\hline 301 & $8.8 \mathrm{E}-03$ & $7.7 \mathrm{E}+01$ \\
\hline 302 & $8.7 \mathrm{E}-03$ & $7.6 \mathrm{E}+01$ \\
\hline 303 & $8.6 \mathrm{E}-03$ & $7.6 \mathrm{E}+01$ \\
\hline 304 & $9.1 \mathrm{E}-03$ & $8.0 \mathrm{E}+01$ \\
\hline 305 & $8.6 \mathrm{E}-03$ & $7.5 \mathrm{E}+01$ \\
\hline 306 & $9.0 \mathrm{E}-03$ & $7.9 \mathrm{E}+01$ \\
\hline 307 & $9.3 \mathrm{E}-03$ & $8.1 \mathrm{E}+01$ \\
\hline 308 & $8.9 \mathrm{E}-03$ & $7.8 \mathrm{E}+01$ \\
\hline 309 & $8.8 \mathrm{E}-03$ & $7.7 \mathrm{E}+01$ \\
\hline
\end{tabular}

Table F-7. Environmental Restoration Disposal Facility Thermoluminescent Dosimeter Exposure Results, Calendar Year 1997.

\begin{tabular}{|c|c|c|c|c|c|c|c|c|c|c|c|}
\hline Site 1.D. & \multicolumn{2}{|c|}{ 1st Quarter } & \multicolumn{2}{|c|}{ 2nd Quarter } & \multicolumn{2}{|c|}{ 3rd Quarter } & \multicolumn{2}{|c|}{ 4th Quarter } & \multicolumn{3}{|c|}{ Annual Results (mrem/yr) } \\
\hline ERDF-1 & $1.5 \mathrm{E}-02$ & $1.3 \mathrm{E}+02$ & 9.5 E.03 & $8.3 E+01$ & 9.9 E-03 & $8.7 E+01$ & $1.3 \mathrm{E}-02$ & $1.2 \mathrm{E}+02$ & $1.3 E+02$ & $8.3 E+01$ & $1.0 \mathrm{E}+02$ \\
\hline ERDF-2 & $1.0 \mathrm{E}-02$ & $8.9 E+01$ & $9.3 \mathrm{E}-03$ & $8.1 E+01$ & $9.6 \mathrm{E}-03$ & $8.4 E+01$ & $1.2 \mathrm{E}-\mathrm{O} 2$ & $1.1 \mathrm{E}+\mathrm{O} 2$ & $1.1 E+02$ & $8.1 E+01$ & $9.0 \mathrm{E}+01$ \\
\hline ERDF-3 & $9.8 \mathrm{E}-03$ & $8.5 E+01$ & 9.6 E-O3 & $8.4 E+01$ & 9.8 E-03 & $8.6 E+01$ & $1.3 \mathrm{E}-02$ & $1.1 \mathrm{E}+02$ & $1.1 E+02$ & $8.4 E+01$ & $9.2 E+01$ \\
\hline
\end{tabular}


Table F-8. 300/300 TEDF/400 Areas Thermoluminescent Dosimeter Exposure Results, Calendar Year 1996.

\begin{tabular}{|c|c|c|c|c|c|c|c|c|c|c|c|}
\hline \multirow{2}{*}{$\begin{array}{l}\text { Site I.D. } \\
300 \text { Area }\end{array}$} & \multicolumn{2}{|c|}{ 1st Quarter } & \multicolumn{2}{|c|}{ 2nd Quarter } & \multicolumn{2}{|c|}{ 3rd Quarter } & \multicolumn{2}{|c|}{ 4th Quarter } & \multicolumn{3}{|c|}{ Annual Results (mrem/yr) } \\
\hline & mrem $/ \mathrm{h}$ & $\mathrm{mrem} / \mathrm{yr}$ & $\mathrm{rem} / \mathrm{h}$ & mrem/yr & mrem $/ \mathrm{h}$ & mrem/yr & mrem $/ \mathrm{h}$ & $\mathrm{mrem} / \mathrm{yr}$ & Maximum & Minimum & Average \\
\hline 1 & 3 & $8.5 \mathrm{E}+01$ & 9.1 E-03 & 1 & 3 & 1 & 3 & 01 & $E+01$ & $8.0 E+01$ & $8.3 E+01$ \\
\hline 2 & $.0 \mathrm{E}-02$ & +01 & E-0 & $8.5 E+01$ & $1.0 \mathrm{E}-02$ & $8.8 E+01$ & $9.9 E-03$ & $8.7 E+01$ & $9.1 \mathrm{E}+01$ & $8.5 E+01$ & $8.8 E+01$ \\
\hline 3 & $9.7 \mathrm{E}-\mathrm{O}$ & $8.5 E+01$ & $9.5 \mathrm{E}-03$ & $8.4 E+01$ & $9.8 \mathrm{E}-03$ & $8.6 E+01$ & $9.5 \mathrm{E}-03$ & $8.4 E+01$ & $8.6 E+01$ & 01 & -01 \\
\hline 4 & $2.4 \mathrm{E}-02$ & $2.1 \mathrm{E}+02$ & 02 & $1.9 E+02$ & $2.3 \mathrm{E}-02$ & 22 & $\mathrm{E}-\mathrm{02}$ & $2.1 \mathrm{E}+02$ & $2.1 \mathrm{E}+02$ & $1.9 E+02$ & $2.0 \mathrm{E}+02$ \\
\hline 5 & $O E-02$ & $.8 \mathrm{E}+01$ & $9.3 \mathrm{E}-03$ & $8.2 \mathrm{E}+01$ & $9.8 \mathrm{E}-03$ & $8.5 E+01$ & $1.0 \mathrm{E}-02$ & $8.9 E+01$ & $8.9 \mathrm{E}+01$ & $8.2 \mathrm{E}+01$ & $8.6 E+01$ \\
\hline 6 & $1.2 \mathrm{E}-02$ & $1.0 \mathrm{E}+02$ & $1.1 \mathrm{E}-02$ & $9.6 \mathrm{E}+01$ & $1.2 \mathrm{E}-02$ & $1.1 E+02$ & $1.2 \mathrm{E}-02$ & $1.0 \mathrm{E}+02$ & $1.1 E+02$ & $9.6 \mathrm{E}+01$ & $1.0 E+02$ \\
\hline 7 & $2.0 \mathrm{E}-02$ & $1.8 E+02$ & $1.9 \mathrm{E}-02$ & $1.7 E+02$ & $1.9 \mathrm{E}-02$ & $1.6 \mathrm{E}+02$ & $2.0 \mathrm{E}-02$ & $1.8 \mathrm{E}+02$ & $1.8 \mathrm{E}+02$ & $1.6 \mathrm{E}+02$ & $1.7 E+02$ \\
\hline 8 & $.4 \mathrm{E}-02$ & $1.2 \mathrm{E}+\mathrm{O} 2$ & 1.2 E-02 & $1.0 \mathrm{E}+02$ & $1.1 \mathrm{E}-02$ & $9.3 \mathrm{E}+01$ & $1.0 \mathrm{E}-02$ & $9.1 E+01$ & $1.2 E+02$ & $9.1 E+01$ & $1.0 E+02$ \\
\hline
\end{tabular}

\begin{tabular}{|c|c|c|c|c|c|c|c|c|c|c|c|}
\hline \multirow{2}{*}{$\begin{array}{c}\text { Site I.D. } \\
300 \\
\text { TEDF }\end{array}$} & \multicolumn{2}{|c|}{ 1st Quarter } & \multicolumn{2}{|c|}{ 2nd Quarter } & \multicolumn{2}{|c|}{ 3rd Quarter } & \multicolumn{2}{|c|}{ 4th Quarter } & \multicolumn{3}{|c|}{ Annual Results (mrem/yr) } \\
\hline & mrem/h & mrem/yr & $\mathrm{mrem} / \mathrm{h}$ & $\mathrm{mrem} / \mathrm{yr}$ & $\mathrm{mrem} / \mathrm{h}$ & mrem/yr & mrem $/ \mathrm{h}$ & mrem/yr & Maximum & Minimum & Average \\
\hline 401 & 1.0.E-02 & $9.1 E+01$ & $9.2 \mathrm{E}-03$ & $8.0 \mathrm{E}+01$ & $9.9 \mathrm{E}-03$ & 8.7 $E+01$ & $1.1 \mathrm{E}-02$ & $9.5 E+01$ & $9.5 E+01$ & $8.0 E+01$ & $8.8 E+01$ \\
\hline 402 & $9.7 \mathrm{E}-03$ & $8.5 E+01$ & $9.3 \mathrm{E}-03$ & $8.1 E+01$ & $9.6 \mathrm{E}-03$ & $8.4 \mathrm{E}+01$ & $9.9 \mathrm{E}-03$ & $8.7 E+01$ & $8.7 E+01$ & $8.1 E+01$ & $8.4 \varepsilon+01$ \\
\hline 403 & 1.1. E-02 & $9.3 E+01$ & $9.3 \mathrm{E}-03$ & $8.2 E+01$ & 9.9 E -03 & $8.7 E+01$ & $1.0 \mathrm{E}-02$ & $9.0 E+01$ & $9.3 E+01$ & $8.2 E+01$ & $8.8 \dot{E}+01$ \\
\hline 404 & 9.9 E-03 & $8.7 E+01$ & 9.4 E-O3 & $8.2 E+01$ & 9.3 E-03 & $8.1 E+01$ & $9.9 \mathrm{E}-03$ & $8.7 E+01$ & 8.7E+01 & $8.1 E+01$ & $8.4 E+01$ \\
\hline 405 & $9.7 \mathrm{E}-03$ & $8.5 E+01$ & 9.1 E-03 & 8.0 E+01 & 9.0 E-03 & $7.8 \mathrm{E}+01$ & $9.5 \mathrm{E}-03$ & $8.3 E+01$ & $8.5 E+01$ & $7.8 E+01$ & $8.2 E+01$ \\
\hline 406 & $9.5 \mathrm{E}-03$ & $8.4 \varepsilon+01$ & $9.3 \mathrm{E}-03$ & $8.1 E+01$ & $1.0 \mathrm{E}-02$ & $9.0 E+01$ & $1.1 \mathrm{E}-02$ & $9.5 E+01$ & $9.5 E+01$ & $8.1 E+01$ & $8.8 E+01$ \\
\hline
\end{tabular}

\begin{tabular}{|c|c|c|c|c|c|c|c|c|c|c|c|}
\hline \multirow{2}{*}{$\begin{array}{l}\text { Site I.D. } \\
400 \text { Area }\end{array}$} & \multicolumn{2}{|c|}{ 1st Quarter } & \multicolumn{2}{|c|}{ 2nd Quarter } & \multicolumn{2}{|c|}{ 3rd Quarter } & \multicolumn{2}{|c|}{ 4th Quarter } & \multicolumn{3}{|c|}{ Annual Results (mrem/yr) } \\
\hline & mrem/h & mrem/yr & mrem $/ \mathrm{h}$ & mrem/yr & mrem $/ \mathrm{h}$ & mrem/yr & mrem/h & mrem/yr & Maximum & Minimum & Average \\
\hline 1 & $9.6 \mathrm{E}-03$ & $4 \mathrm{E}+01$ & -03 & $7.8 \mathrm{E}+01$ & 9.4 E-03 & $8.2 \mathrm{E}+01$ & $9.5 \mathrm{E}-03$ & $8.3 E+01$ & $8.4 \mathrm{E}+01$ & $7.8 E+01$ & $8.2 E+01$ \\
\hline 2 & $9.8 E-03$ & $8.6 E+01$ & $8.8 \mathrm{E}-03$ & $7.7 E+01$ & 9.1 E-03 & $8.0 E+01$ & $9.4 E-03$ & $8.2 E+01$ & $8.6 E+01$ & $7.7 \mathrm{E}+01$ & $8.1 \mathrm{E}+01$ \\
\hline 3 & $1.0 \mathrm{E}-02$ & $8.9 \mathrm{E}+01$ & 9.8 E-03 & $8.5 \mathrm{E}+01$ & $1.0 \mathrm{E}-02$ & $8.8 \mathrm{E}+01$ & $9.8 \mathrm{E} .03$ & $8.5 E+01$ & $8.9 \mathrm{E}+01$ & $8.5 E+01$ & $8.7 E+01$ \\
\hline 4 & 9.4 E-03 & $8.2 \mathrm{E}+01$ & $8.9 \mathrm{E}-03$ & $7.8 \mathrm{E}+01$ & 9.1 E-03 & $8.0 \mathrm{E}+01$ & $8.8 \mathrm{E}-03$ & $7.7 \mathrm{E}+01$ & $2 \mathrm{E}+01$ & $7.7 \mathrm{E}+01$ & $7.9 E+01$ \\
\hline 5 & $9.3 E-03$ & $8.1 E+01$ & $8.8 \mathrm{E}-03$ & $7.7 \mathrm{E}+01$ & $9.1 \mathrm{E}-03$ & $8.0 \mathrm{E}+01$ & $8.8 E-03$ & $7.7 E+01$ & $8.1 E+01$ & $7.7 \mathrm{E}+01$ & $7.9 \mathrm{E}+01$ \\
\hline 6 & $9.8 \mathrm{E}-03$ & $8.5 \mathrm{E}+01$ & $9.0 \mathrm{E}-03$ & $7.9 \mathrm{E}+01$ & $9.6 \mathrm{E}-03$ & $8.4 \mathrm{E}+01$ & 9.4 E-03 & $8.2 \mathrm{E}+01$ & $8.5 E+01$ & $7.9 \mathrm{E}+01$ & $8.3 E \div 01$ \\
\hline 7 & $9.9 \mathrm{E}-03$ & $8.7 \mathrm{E}+01$ & $8.8 E-03$ & $7.7 \mathrm{E}+01$ & 9.3 E-03 & $8.2 E+01$ & $9.2 \mathrm{E}-03$ & $8.1 \mathrm{E}+01$ & $8.7 E+01$ & $7.7 \mathrm{E}+01$ & $8.1 E+01$ \\
\hline
\end{tabular}


HNF-EP-0573-6

This page intentionally left blank. 
HNF-EP-0573-6

APPENDIX G

SURFACE WATER DISPOSAL UNIT (POND AND DITCH) MONITORING FIGURES AND TABLES 
HNF-EP-0573-6

This page intentionally left blank.

G-ji 
Figure G-1. N Springs Sample Locations.

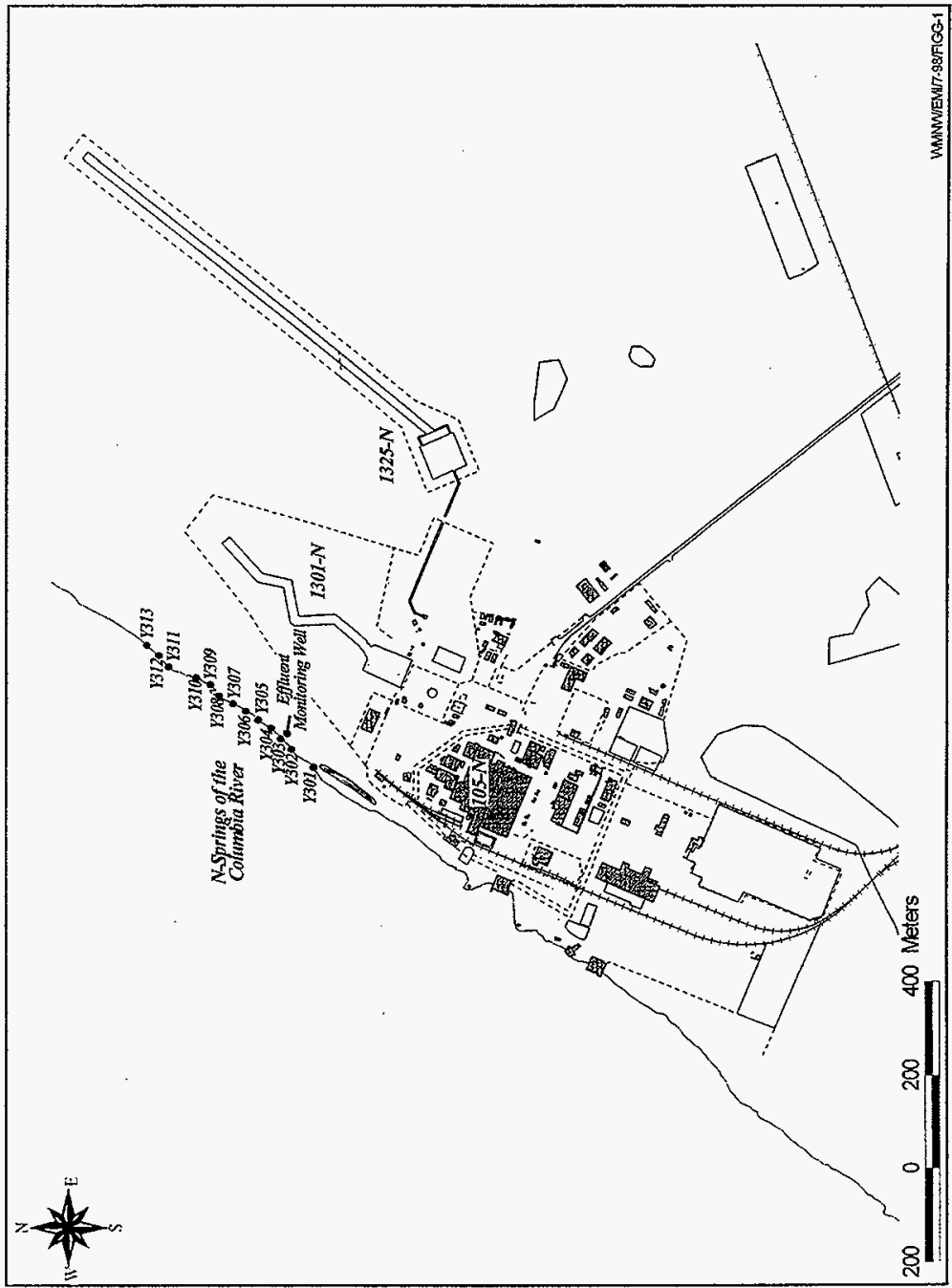


HNF-EP-0573-6

Figure G-2. 200 East Area Liquid Waste Disposal Facilities.

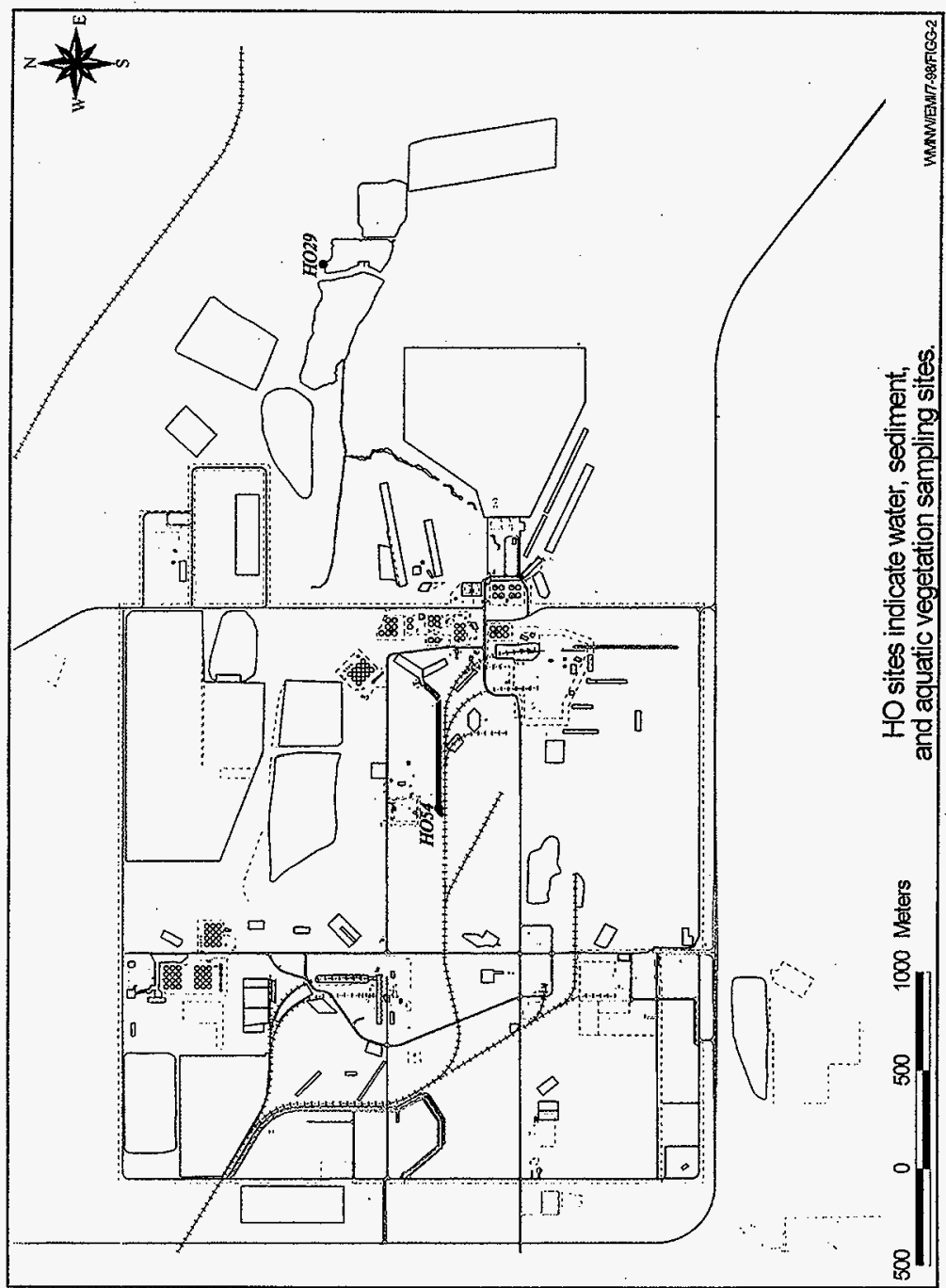


Table G-1. 1997 Radioiogical Results for N Springs Samples (pCi/l)

\begin{tabular}{|c|c|c|c|c|}
\hline Site & H-3 & Co-60 & Sr-90 & Cs-137 \\
\hline$Y 301$ & ND & ND & $3.1 E+01$ & ND \\
\hline Y302 & ND & ND & $2.0 E+02$ & ND \\
\hline$Y 303$ & $3.0 E+03$ & ND & $3.2 E+03$ & ND \\
\hline$Y 304$ & ND & ND & $1.7 E+02$ & ND \\
\hline$Y 305$ & ND & ND & $2.6 E+01$ & ND \\
\hline$Y 306$ & ND & ND & $3.1 E+00$ & ND \\
\hline$Y 307$ & ND & ND & ND & ND \\
\hline$Y 308$ & ND & $9.1 E+00$ & $1.1 E+01$ & ND \\
\hline$Y 309$ & ND & ND & $5.6 E+00$ & ND \\
\hline$Y 310$ & ND & ND & ND & ND \\
\hline$Y 311$ & ND & ND & $1.5 E+02$ & ND \\
\hline
\end{tabular}

Table G-2. Radiological Results for Liquid Samples from Surface Water Disposal Units, 200 Areas, 1997 (pCi/L).

\begin{tabular}{|c|c|c|c|c|c|c|c|c|}
\hline Sample Location & $\begin{array}{c}\text { Number } \\
\text { of } \\
\text { Samples }\end{array}$ & & ${ }^{238} \mathrm{Pu}$ & ${ }^{239,}{ }^{240} \mathrm{Pu}$ & rotait & ${ }^{137} \mathrm{Cs}$ & ${ }^{90} \mathrm{Sr}$ & ${ }^{3} H^{(3)}$ \\
\hline $\begin{array}{l}200 \text { East } \\
\text { Powerhouse Ditch }\end{array}$ & 12 & $\begin{array}{l}\text { Mean } \\
\text { Max } \\
\text { Min }\end{array}$ & $\begin{array}{c}6.4 \mathrm{E}-06 \\
3.6 \mathrm{E}-05 \\
\text { ND }\end{array}$ & $\begin{array}{c}1.4 \mathrm{E}-05 \\
7.3 \mathrm{E}-05 \\
\text { ND }\end{array}$ & $\begin{array}{l}4.7 \mathrm{E}-\mathrm{O} 4 \\
5.8 \mathrm{E}-04 \\
3.1 \mathrm{E}-\mathrm{O} 4\end{array}$ & $\begin{array}{l}\text { ND } \\
\text { ND } \\
\text { ND }\end{array}$ & $\begin{array}{c}\text { 3.OE-O4 } \\
3.0 \mathrm{E}-03 \\
\mathrm{ND}\end{array}$ & $\begin{array}{l}\text { ND } \\
\text { ND } \\
\text { ND }\end{array}$ \\
\hline 216-B-3C Pond & 9 & $\begin{array}{l}\text { Mean } \\
\text { Max } \\
\text { Min }\end{array}$ & $\begin{array}{l}\text { ND } \\
\text { ND } \\
\text { ND }\end{array}$ & $\begin{array}{l}\text { ND } \\
\text { ND } \\
\text { ND }\end{array}$ & $\begin{array}{l}6.5 \mathrm{E}-04 \\
9.9 \mathrm{E}-04 \\
3.6 \mathrm{E}-04\end{array}$ & $\begin{array}{l}\text { ND } \\
\text { ND } \\
\text { ND }\end{array}$ & $\begin{array}{l}\text { ND } \\
\text { ND } \\
\text { ND }\end{array}$ & $\begin{array}{l}\text { ND } \\
\text { ND } \\
\text { ND }\end{array}$ \\
\hline
\end{tabular}

ND $=$ Not Detected

a ${ }^{3} \mathrm{H}$ Analyses are performed quarterly

Tabie G-3. Nonradiological Results for Liquid Samples from Surface Water Disposal Units, 200 Areas, 1996.

\begin{tabular}{|l|c|c|c|c|c|c|c|c|}
\hline \multirow{2}{*}{ Sample Locations } & \multicolumn{3}{|c|}{$\mathrm{pH}$} & \multicolumn{3}{c|}{ Nitrate (NO $\mathrm{Ng}_{3 / \mathrm{L})}$} \\
\cline { 2 - 9 } & $\begin{array}{c}\text { Number of } \\
\text { Samples }\end{array}$ & Mean & Max & Min & $\begin{array}{c}\text { Number } \\
\text { of } \\
\text { Samples }\end{array}$ & Mean & Max & Min \\
\hline $216-$-B-3C Pond & 52 & 8.8 & 9.3 & 7.8 & 4 & 0.17 & 0.22 & 0.10 \\
\hline $\begin{array}{l}200 \text { East Area } \\
\text { Powerhouse Pond }\end{array}$ & 46 & 8.4 & 9.0 & 8.0 & 4 & 0.22 & 0.24 & 0.10 \\
\hline
\end{tabular}


Table G-4. Radiological Results for Aquatic Vegetation from Surface Water Disposal Units, 200 Area, 1996 (pCi/g dry weight).

\begin{tabular}{|l|c|c|c|c|c|c|}
\hline \multicolumn{1}{|c|}{ Sample Locations } & ${ }^{137} \mathrm{Cs}$ & ${ }^{90} \mathrm{Sr}$ & ${ }^{239,}{ }^{240} \mathrm{Pu}$ & ${ }^{234} \mathrm{U}$ & ${ }^{235} \mathrm{U}$ & ${ }^{238} \mathrm{U}$ \\
\hline $216-\mathrm{B}-3 \mathrm{C}$ Pond & $3.8 \mathrm{E}-01$ & $3.4 \mathrm{E}-01$ & $3.8 \mathrm{E}-03$ & $1.7 \mathrm{E}-02$ & $8.0 \mathrm{E}-03$ & $8.5 \mathrm{E}-03$ \\
\hline 200 East Area Powerhouse Pond & ND & $3.7 \mathrm{E}-01$ & $\mathrm{ND}$ & $1.3 \mathrm{E}-02$ & $9.9 \mathrm{E}-03$ & $7.8 \mathrm{E}-03$ \\
\hline
\end{tabular}

ND $=$ Not Detected

Table G-5. Radiological Results for Sediment (Surface) from Surface Water Disposal Units, 200 Area, 1996 (pCi/g dry weight).

\begin{tabular}{|l|c|c|c|c|c|c|}
\hline \multicolumn{1}{|c|}{ Sample Locations } & ${ }^{137} \mathrm{Cs}$ & ${ }^{90} \mathrm{Sr}$ & ${ }^{239 .}{ }^{240} \mathrm{Pu}$ & ${ }^{234} \mathrm{U}$ & ${ }^{235} \mathrm{U}$ & ${ }^{238} \mathrm{U}$ \\
\hline $216-\mathrm{B}-3 \mathrm{C}$ Pond & $1.1 \mathrm{E}+1$ & $7.1 \mathrm{E}-01$ & $2.0 \mathrm{E}-01$ & $3.3 \mathrm{E}-01$ & $6.0 \mathrm{E}-02$ & $2.9 \mathrm{E}-01$ \\
\hline 200 East Area Powerhouse Pond & $\mathrm{ND}$ & $\mathrm{ND}$ & $4.9 \mathrm{E}-03$ & $3.8 \mathrm{E}-01$ & $2.2 \mathrm{E}-02$ & $3.9 \mathrm{E}-01$ \\
\hline
\end{tabular}


HNF-EP-0573-6

APPENDIX H

100 N AREA SHORELINE RADIATION SURVEYS 
HNF-EP-0573-6

This page intentionally left blank. 
Figure $\mathrm{H}-1$. Radiological Survey Locations in the $100 \mathrm{~B} / \mathrm{C}$ Area.

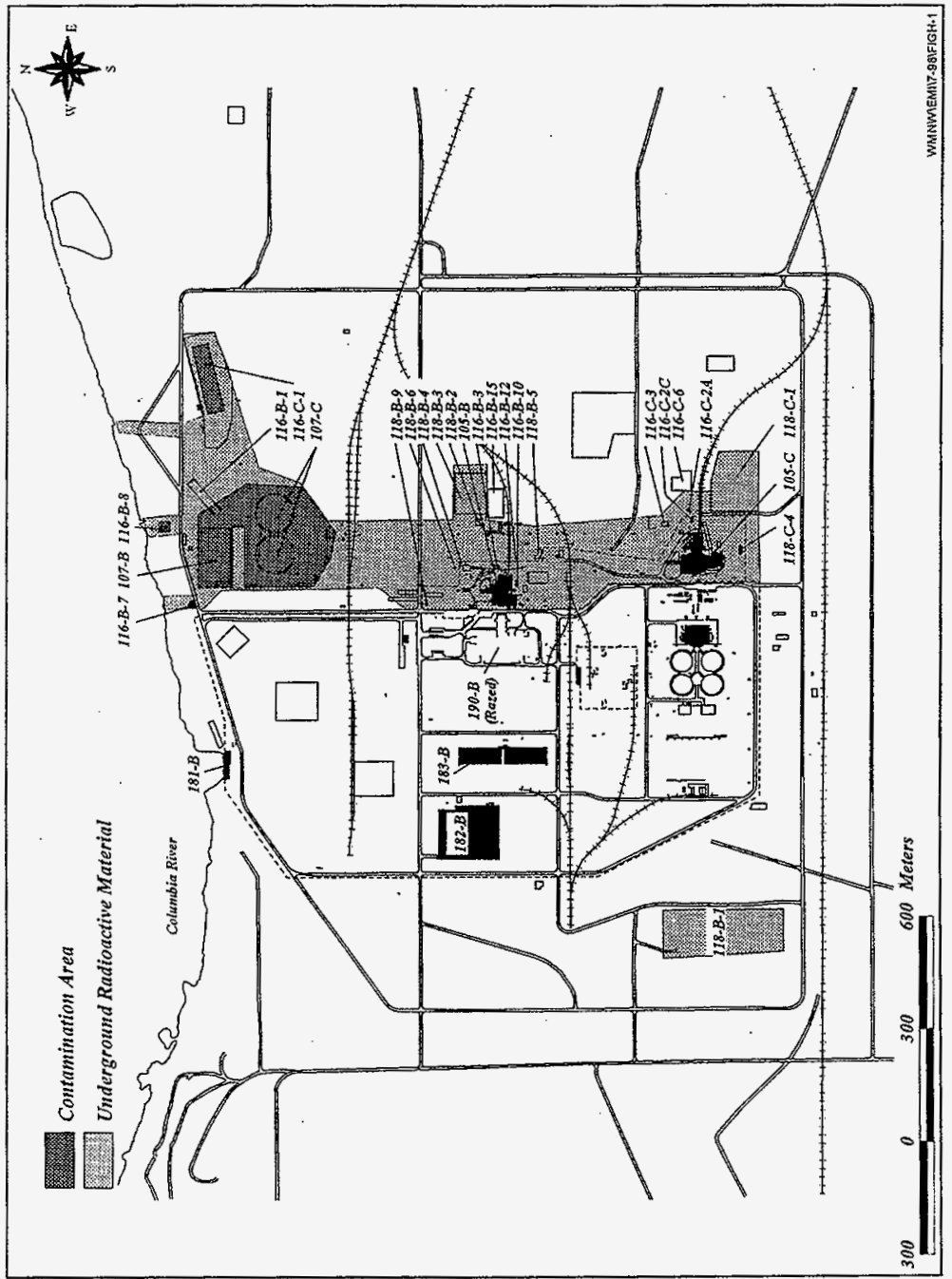


HNF-EP-0573-6

Figure H-2. Radiological Survey Locations in the $100 \mathrm{D} / \mathrm{DR}$ Area.

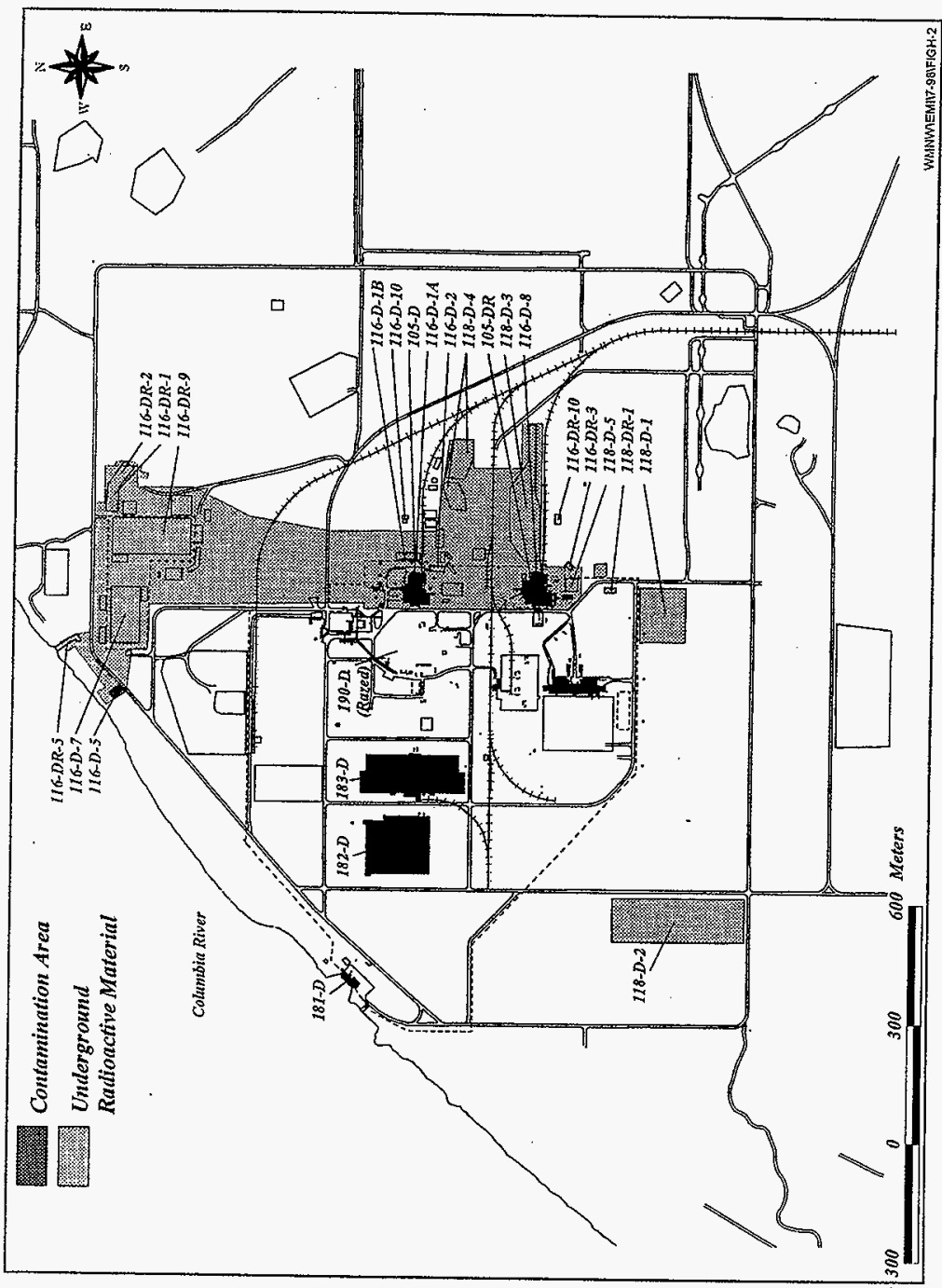




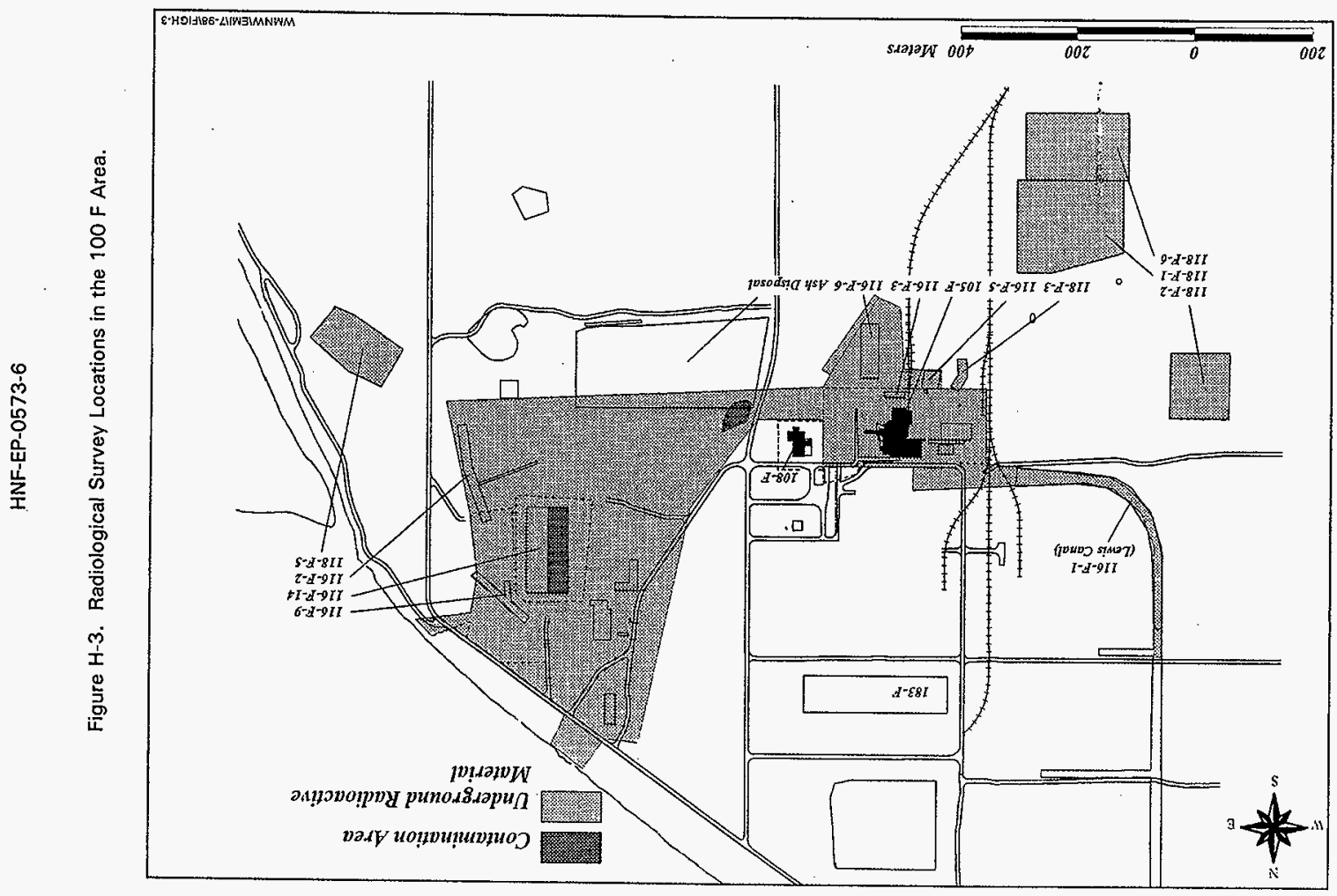


Figure H-4. Radiological Survey Locations in the $100 \mathrm{H}$ Area.

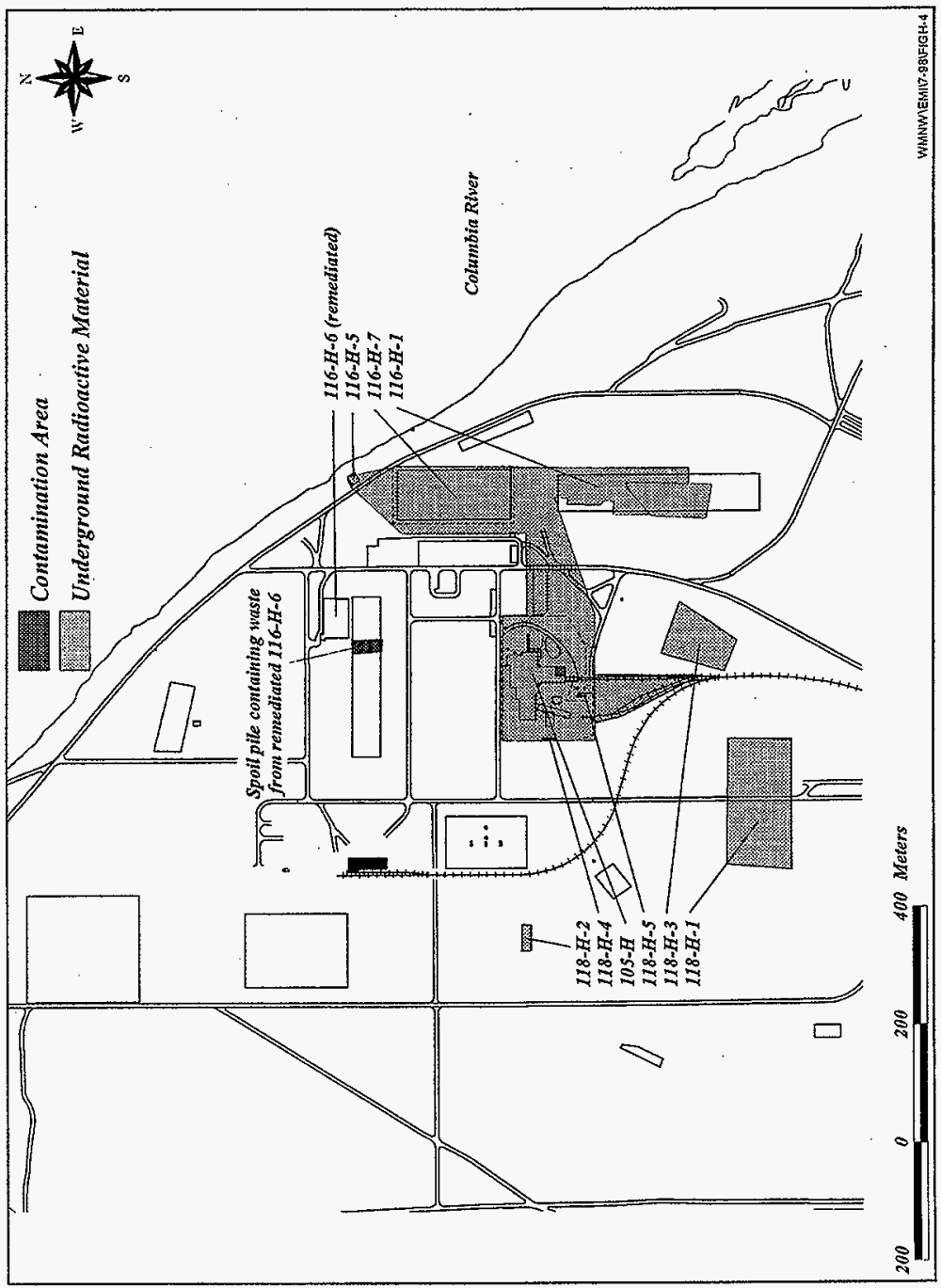


Figure $\mathrm{H}-5$. Radiological Survey Locations in the $100 \mathrm{KE} / \mathrm{KW}$ Area.

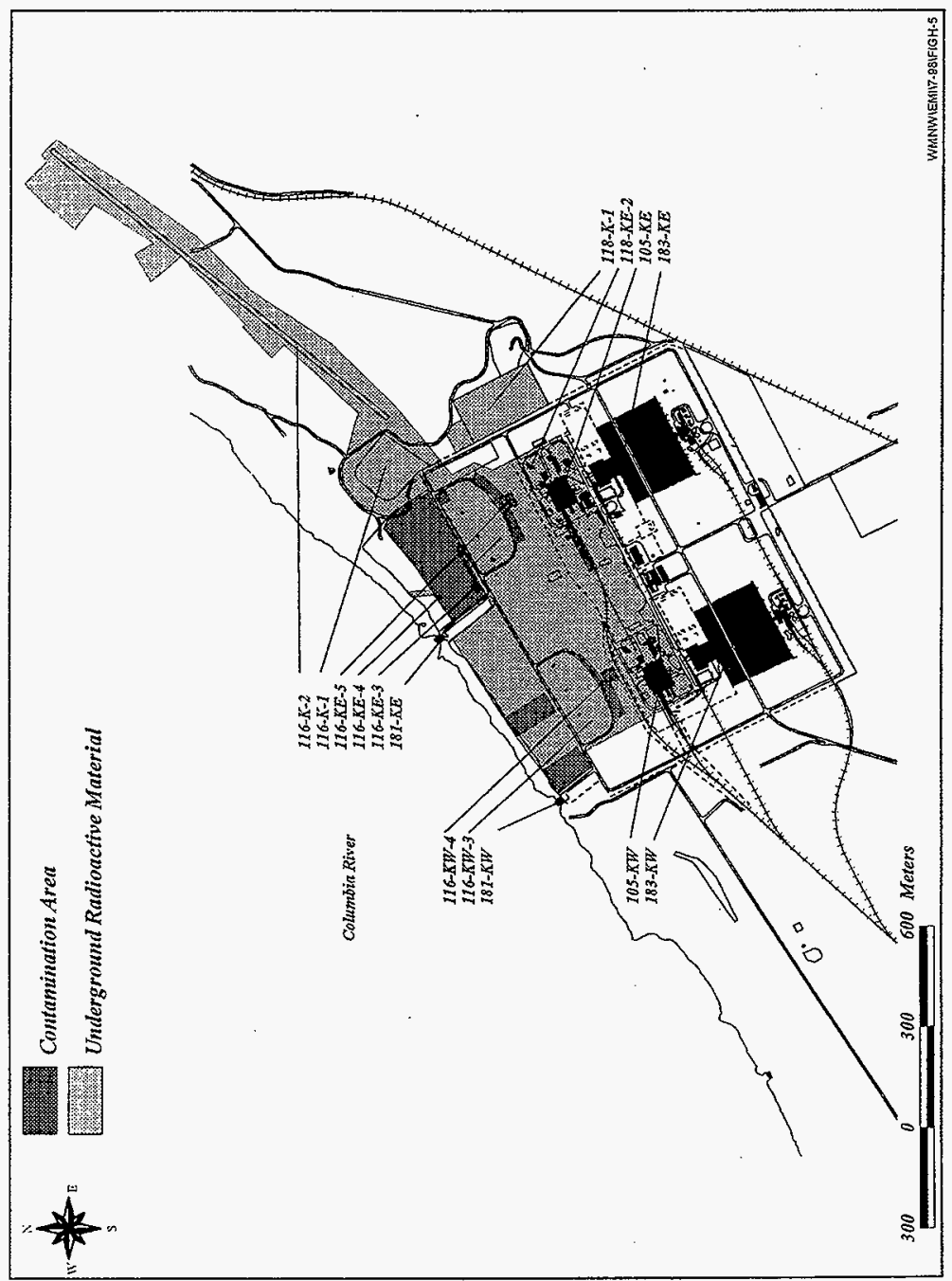


Figure H-6. Radiological Survey Locations in the $100 \mathrm{~N}$ Area.

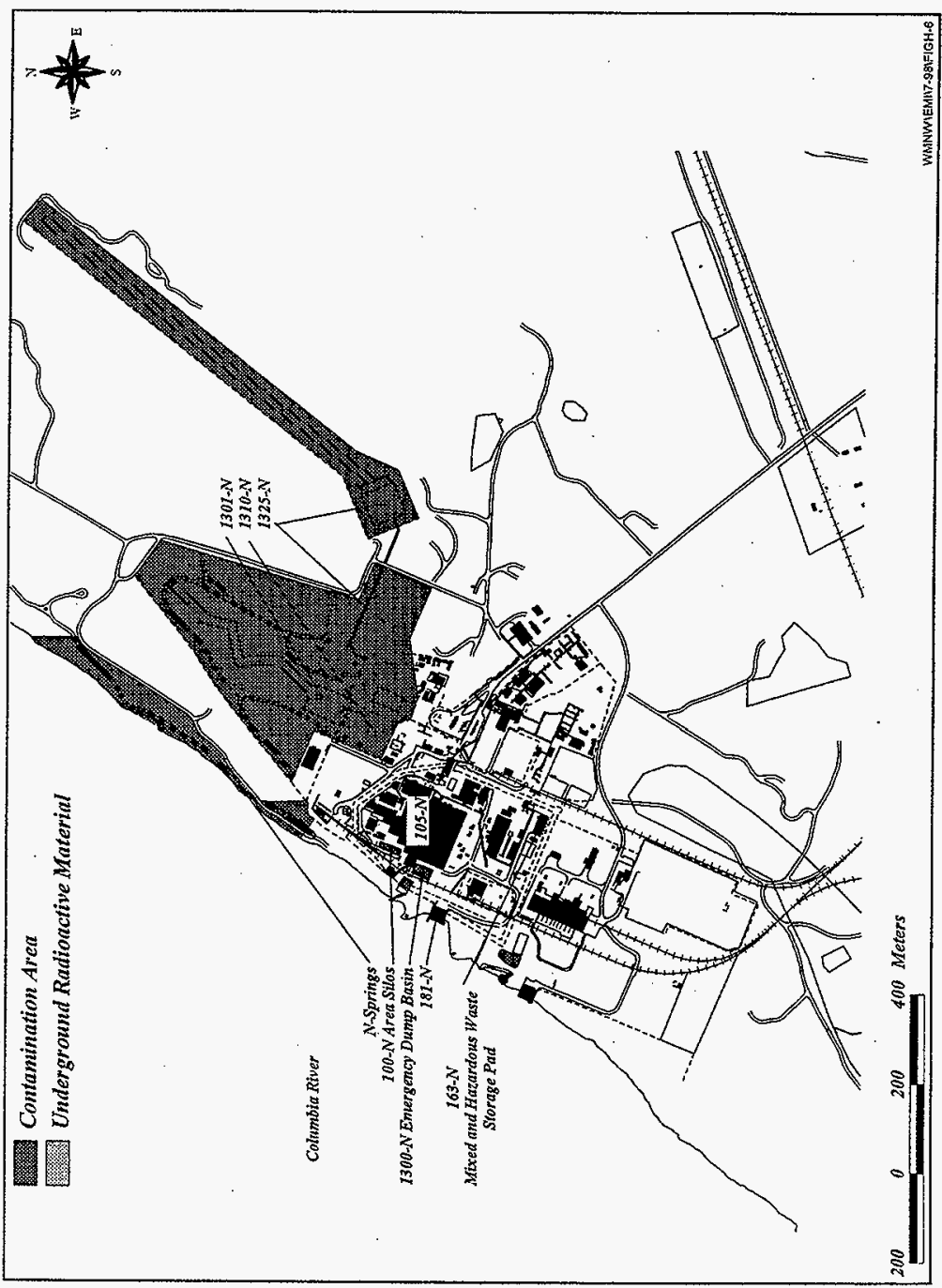


HNF-EP-0573-6

Figure H-7. Radiological Survey Locations in the 200 East Area.

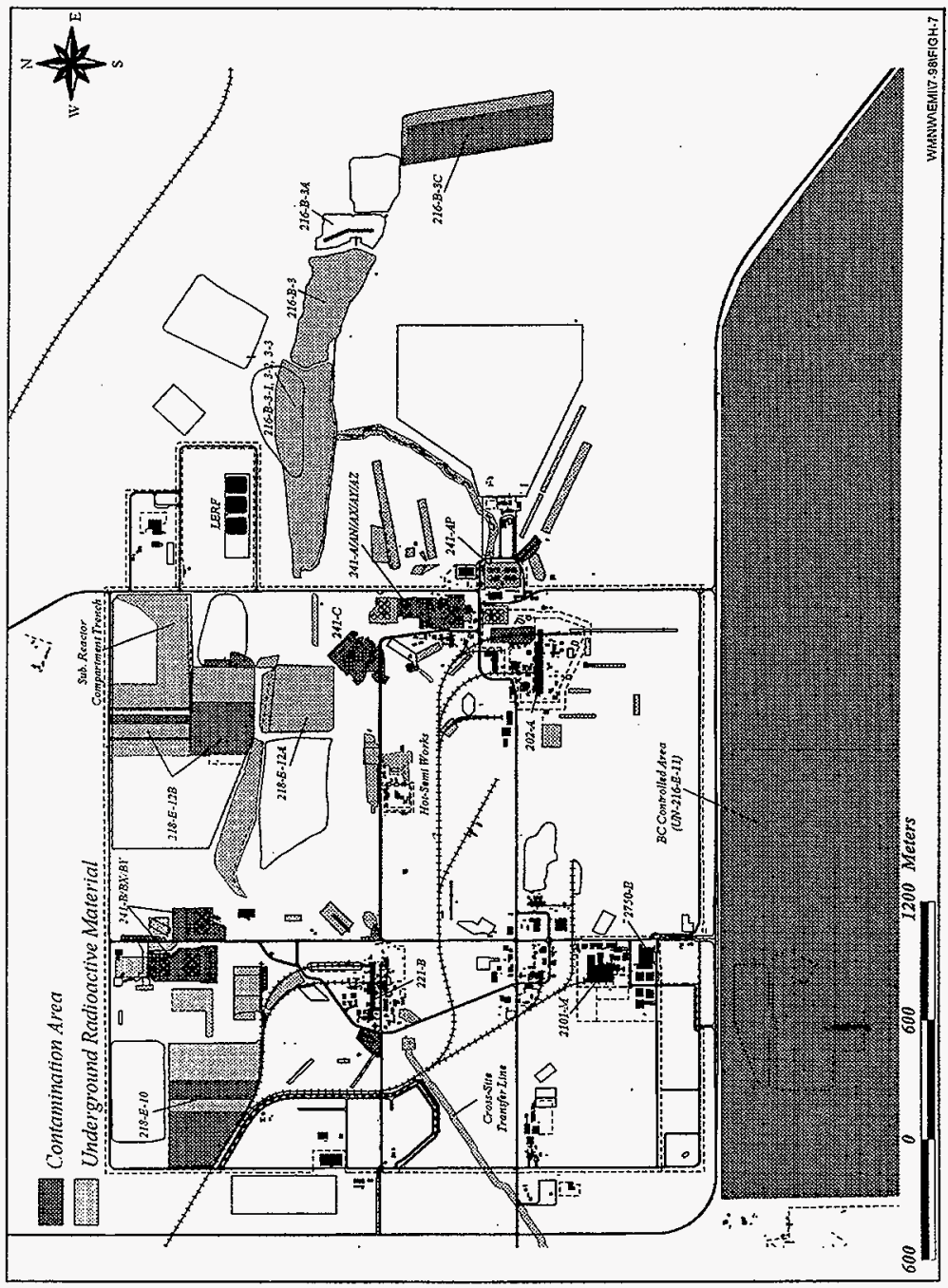


HNF-EP-0573-6

Figure H-8. Radiological Survey Locations in the 200 West Area.

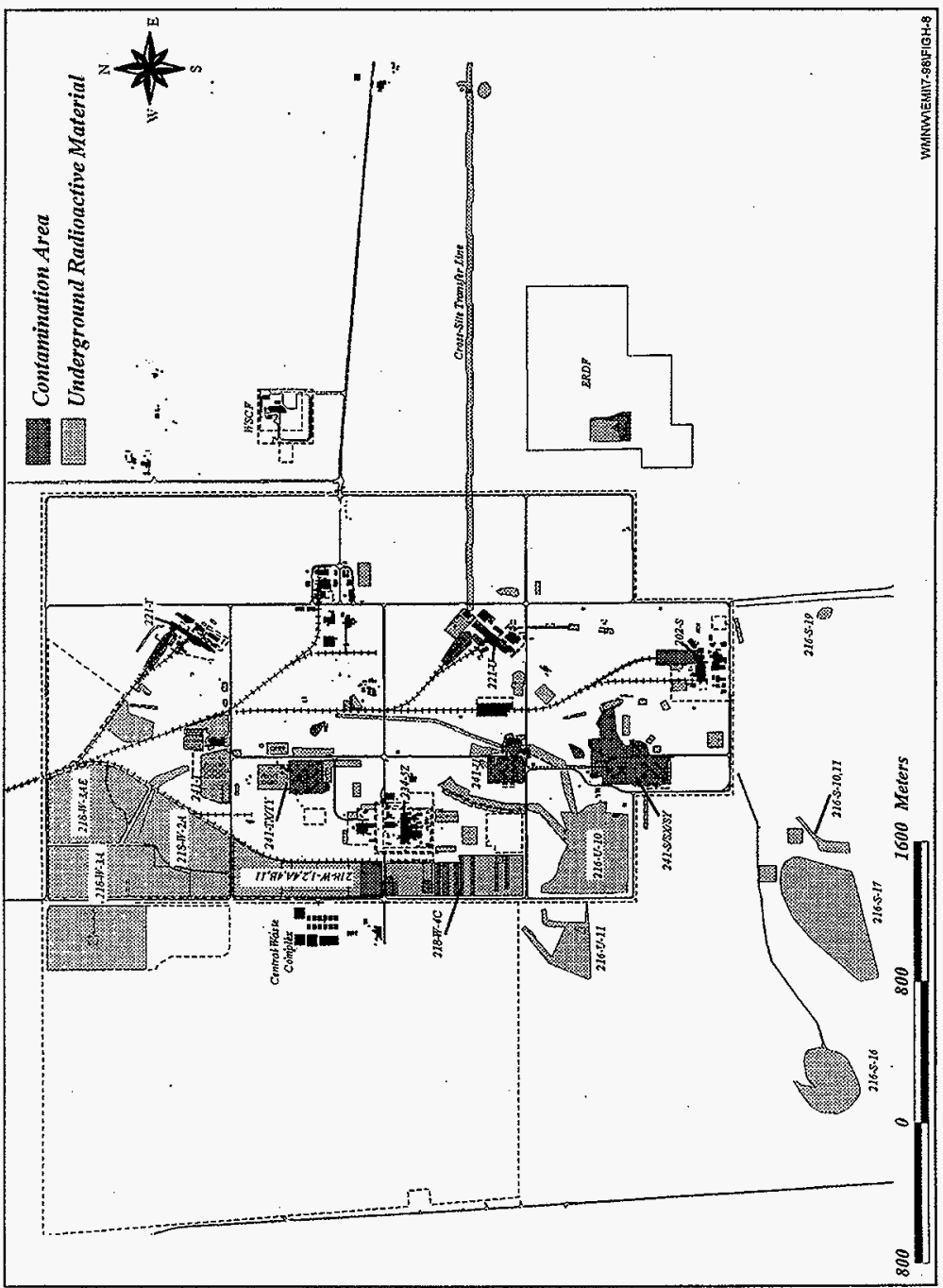




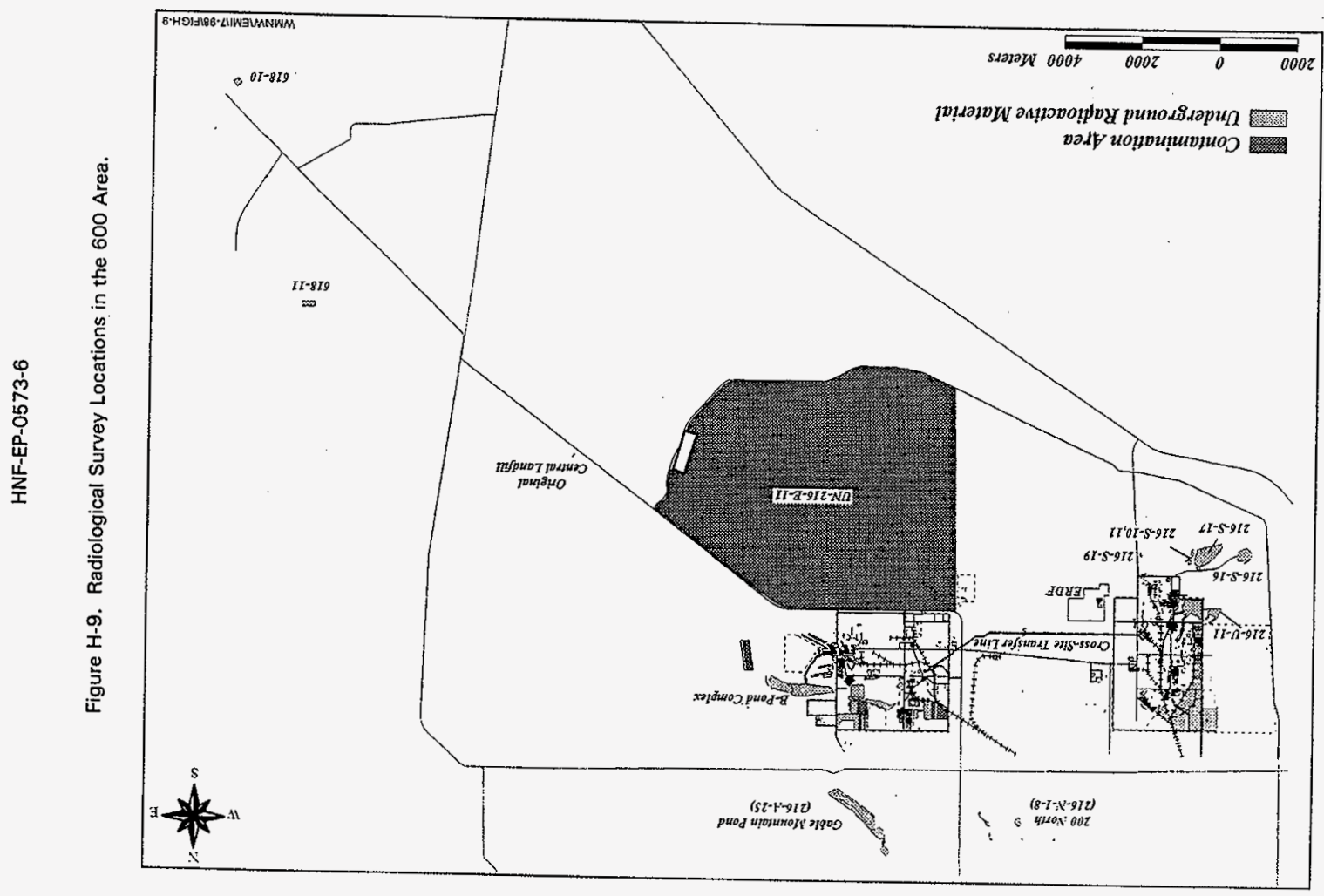


Figure H-10. Radiological Survey Locations in the 300 Area.

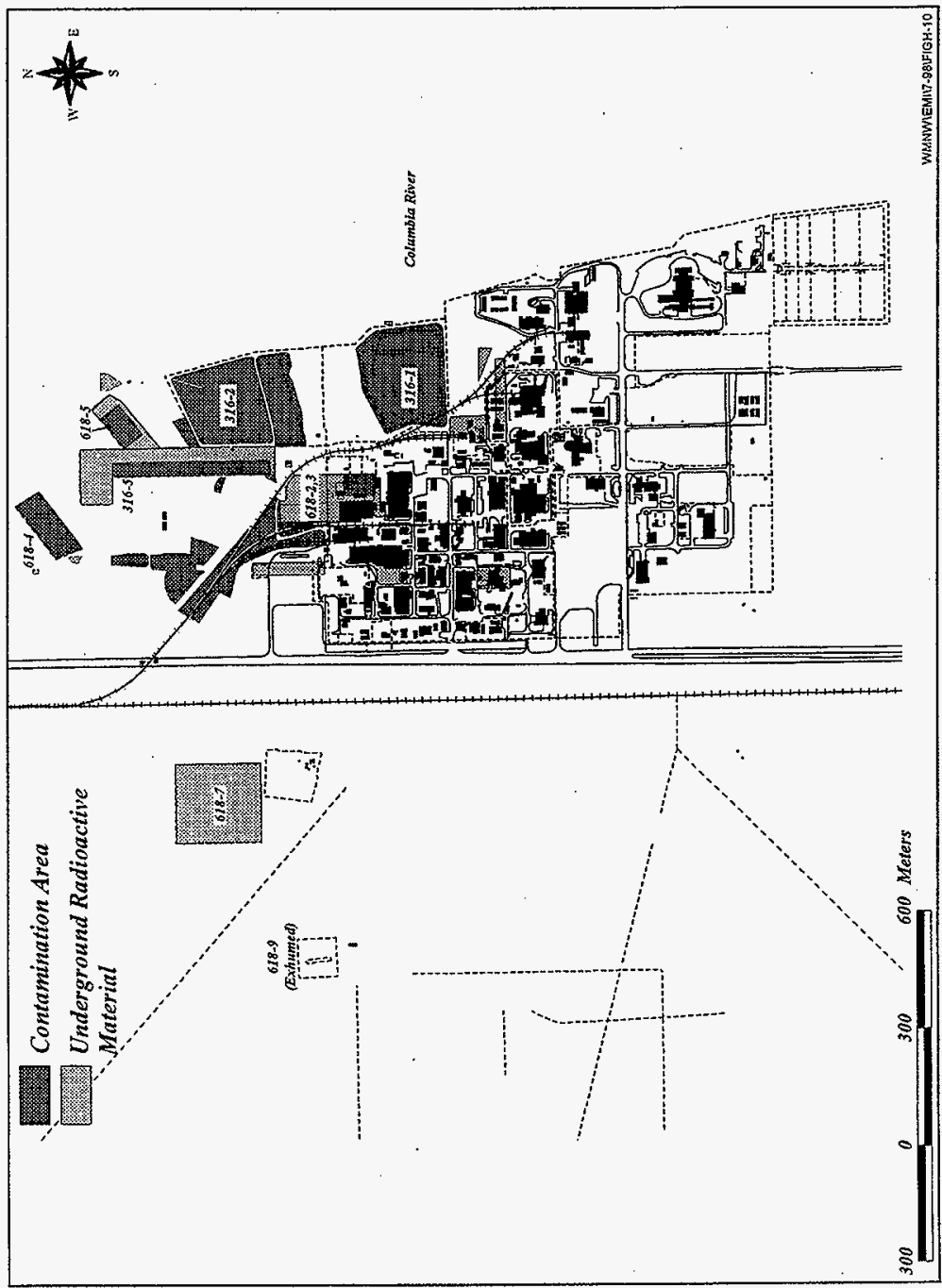


Table H-1. $100 \mathrm{~N}$ Area Shoreline Radiation Surveys $(\mu / \mathrm{h})$. (3 sheets total)

\begin{tabular}{|c|c|c|c|c|c|c|}
\hline Location & 1992 & 1993 & 1994 & $1995^{*}$ & $1996^{*}$ & $1997^{*}$ \\
\hline 1 & 12 & 11 & 10 & 10 & & \\
\hline & 13 & 13 & 11 & 9 & & \\
\hline & 14 & 14 & 13 & 9 & & \\
\hline & 18 & 17 & 14 & 10 & & \\
\hline & 18 & 18 & 15 & 10 & & \\
\hline & 20 & 20 & 14 & 11 & & \\
\hline & 28 & 27 & 23 & 12 & & \\
\hline & 37 & 35 & 33 & 14 & & \\
\hline & 46 & 45 & 44 & 16 & & \\
\hline 10 & 55 & 50 & 50 & 17 & & \\
\hline & 65 & 55 & 55 & 16 & & \\
\hline & 70 & 60 & 60. & 15 & & \\
\hline & 75 & 70 & 70 & 14 & & \\
\hline & 100 & 80 & 75 & 13 & & \\
\hline & 105 & 100 & 85 & 12 & & \\
\hline & 110 & 90 & 85 & 13 & & \\
\hline & 120 & 75 & 75 & 10 & & \\
\hline & 100 & 60 & 60 & 9 & & 5 \\
\hline & 70 & 40 & 40 & 8 & & \\
\hline 20 & 50 & 40 & 40 & 11 & & \\
\hline & 45 & 35 & 35 & 12 & 10 & 10 \\
\hline & 50 & 30 & 35 & 11 & & \\
\hline & 40 & 30 & 35 & 11 & & \\
\hline & 40 & 35 & 35 & 12 & 15 & 10 \\
\hline . & 50 & 35 & 35 & 11 & - & \\
\hline & 50 & 30 & 35 & 11 & 20 & 10 \\
\hline & 50 & 30 & 40 & 12 & & \\
\hline & 60 & 30 & 40 & 11 & & \\
\hline & 60 & 35 & 45 & 12 & & \\
\hline 30 & 70 & 45 & 45 & 13 & & \\
\hline & 80 & 55 & 50 & 11 & 20 & 10 \\
\hline & 80 & 50 & 60 & 11 & & \\
\hline & 80 & 60 & 60 & 12. & & \\
\hline & 100 & 65 & 65 & 11 & 20 & 10 \\
\hline & 100 & 60 & 65 & 13 & & \\
\hline & 100 & 70 & 70 & 16 & 25 & 15 \\
\hline
\end{tabular}


HNF-EP-0573-6

Table H-1. $100 \mathrm{~N}$ Area Shoreline Radiation Surveys $(\mu / \mathrm{h})$. (3 sheets total)

\begin{tabular}{|c|c|c|c|c|c|c|}
\hline Location & 1992 & 1993 & 1994 & $1995 *$ & $1996 *$ & $1997^{*}$ \\
\hline & 110 & 70 & 70 & 16 & & \\
\hline & 110 & 70 & 75 & 14 & & \\
\hline & 120 & 80 & 75 & 18 & 30 & 15 \\
\hline \multirow[t]{10}{*}{40} & 120 & 80 & 80 & 22 & & \\
\hline & 120 & 90 & 80 & 21 & 25 & 15 \\
\hline & 130 & 80 & 85 & 19 & & \\
\hline & 140 & 80 & 90 & 20 & & \\
\hline & 145 & 80 & 95 & 20 & 20 & 15 \\
\hline & 150 & 90 & 100 & 19 & & \\
\hline & 150 & 90 & 110 & 18 & 15 & 15 \\
\hline & 160 & 95 & 110 & 18 & & \\
\hline & 370 & 110 & 115 & 20 & & . \\
\hline & 160 & 110 & 120 & 22 & 15 & 15 \\
\hline \multirow[t]{10}{*}{50} & 160 & 95 & 120 & 19 & & \\
\hline & 160 & 100 & 120 & 18 & 10 & 15 \\
\hline & 160 & 100 & 115 & 19 & & \\
\hline & 170 & 110 & 115 & 18 & 10 & 10 \\
\hline & 160 & 115 & 110 & 18 & & \\
\hline & 160 & 100 & 110 & 16 & & \\
\hline & 150 & 95 & 110 & 17 & 10 & 9 \\
\hline & 140 & 90 & 105 & 18 & & \\
\hline & 130 & 90 & 100 & 18 & & \\
\hline & 120 & 90 & 85 & 16 & 8 & 10 \\
\hline \multirow[t]{10}{*}{60} & 120 & 80 & 80 & 15 & & \\
\hline & 110 & 75 & 80 & 12 & 10 & 10 \\
\hline & 100 & 70 & 75 & 12 & & \\
\hline & 90 & 70 & 65 & 1.1 & & \\
\hline & 80 & 55 & 60 & 10 & 10 & 4 \\
\hline & 80 & 45 & 55 & 10 & & \\
\hline & 70 & 45 & 50 & 11 & 10 & 4 \\
\hline & 60 & 40 & 45 & 11 & & \\
\hline & 60 & 45 & 45 & 10 & & \\
\hline & 60 & 35 & 40 & 11 & 10 & 6 \\
\hline \multirow[t]{2}{*}{70} & 50 & 40 & 40 & 10 & & \\
\hline & 40 & 35 & 35 & 10 & 10 & 5 \\
\hline
\end{tabular}


Table H-1. $100 \mathrm{~N}$ Area Shoreline Radiation Surveys $(\mu / \mathrm{h})$. (3 sheets total)

\begin{tabular}{|c|c|c|c|c|c|c|}
\hline Location & 1992 & 1993 & 1994 & $1995^{*}$ & 1996* & $1997^{*}$ \\
\hline & 40 & 30 & 35. & 11 & & \\
\hline & 40 & 30 & 30 & 10 & & \\
\hline & 30 & 30 & 25 & 10 & 10 & 3 \\
\hline & 30 & 25 & 24 & 10 & & \\
\hline & 27 & 30 & 23 & 11 & 10 & 3 \\
\hline & 26 & 30 & 19 & 8 & & \\
\hline & 22 & 25 & 19 & 10 & 10 & 5 \\
\hline & 21 & 20 & 19 & 7) & & \\
\hline 80 & 20 & 20 & 17 & 9 & & \\
\hline & 19 & 25 & 15 & 9 & 10 & 5 \\
\hline & 17 & 20 & 17 & 8 & & \\
\hline & 17 & 18 & 14 & 7 & & \\
\hline & 16 & 18 & 13 & 8 & & 5 \\
\hline & 14 & 18 & 12 & 7 & & \\
\hline & 13 & 18 & 12 & 8 & . & \\
\hline & 14. & 15 & 11 & 7 & & \\
\hline & 14 & 15 & 11. & 4 & & \\
\hline & 12 & 18 & 11 & 5 & & \\
\hline 90 & 12 & 15 & 11 & 4 & & \\
\hline & 11 & 10 & 12. & 5 & & \\
\hline & 10 & 10. & 11 & 5 & & \\
\hline & 9 & 12 & 10 & 5 & & \\
\hline
\end{tabular}

*This survey used an alternate field instrument. 
HNF-EP-0573-6

This page intentionally left blank. 
HNF-EP-0573-6

APPENDIX 1

INVESTIGATIVE SPECIAL SAMPLE TABLES 
HNF-EP-0573-6

This page intentionally left blank. 
HNF-EP-0573-6

Table 1-1. Investigative Samples Collected from the Operational Areas, 1997. (2 sheets total)

\begin{tabular}{|l|l|c|c|}
\hline \multicolumn{1}{|c|}{ Sample type } & \multicolumn{1}{|c|}{$\begin{array}{c}\text { Collection area } \\
\text { (number of samples) }\end{array}$} & Radionuclides & $\begin{array}{c}\text { Maximum concentration, } \\
\text { pCi/g }\end{array}$ \\
\hline Great Basin pocket mouse & 200 East Area (2) & $\begin{array}{c}{ }^{90} \mathrm{Sr} \\
{ }^{137} \mathrm{Cs}\end{array}$ & $\begin{array}{c}1.9 \mathrm{E}+04 \\
2.6 \mathrm{E}+02\end{array}$ \\
\hline Great Basin pocket mouse & 600 Area (1) & ${ }^{90} \mathrm{Sr}$ & $2.2 \mathrm{E}+02$ \\
& & ${ }^{137} \mathrm{Cs}$ & $1.7 \mathrm{E}+01$ \\
\hline Cottontail rabbit feces & 200 West Area (1) & ${ }^{90} \mathrm{Sr}$ & $4.4 \mathrm{E}+03$ \\
& & ${ }^{137} \mathrm{Cs}$ & $1.2 \mathrm{E}+02$ \\
\hline Cottontail rabbit & 200 East Area (1) & ${ }^{90} \mathrm{Sr}$ & $7.2 \mathrm{E}+03$ \\
& & ${ }^{137} \mathrm{Cs}$ & $2.6 \mathrm{E}+03$ \\
& & ${ }^{\mathrm{T} t{ }^{13} \mathrm{U}}$ & $1.2 \mathrm{E}+03$ \\
\hline Cottontail rabbit & 200 West Area (2) & ${ }^{90} \mathrm{Sr}$ & $5.0 \mathrm{E}+03$ \\
& & ${ }^{137} \mathrm{Cs}$ & $1.9 \mathrm{E}+02$ \\
\hline Coyote feces & 200 West Area (1) & ${ }^{90} \mathrm{Sr}$ & $4.5 \mathrm{E}+01$ \\
& & ${ }^{137} \mathrm{Cs}$ & $7.1 \mathrm{E}+02$ \\
\hline
\end{tabular}


Table 1-1. Investigative Samples Collected from the Operational Areas, 1997. (2 sheets total)

\begin{tabular}{|c|c|c|c|}
\hline Sample type & $\begin{array}{c}\text { Coliection area } \\
\text { (number of samples) }\end{array}$ & Radionuclides & $\begin{array}{c}\text { Maximum concentration, } \\
\mathrm{pCi} / \mathrm{g} \\
\end{array}$ \\
\hline Dried sludge & 200 East Area (2) & $\begin{array}{c}{ }^{60} \mathrm{Co} \\
{ }^{90} \mathrm{Sr} \\
{ }^{137} \mathrm{Cs} \\
{ }^{152} \mathrm{Eu} \\
{ }^{154} \mathrm{Eu} \\
{ }^{155} \mathrm{Eu} \\
{ }^{238} \mathrm{Pu} \\
{ }^{239 / 240} \mathrm{Pu} \\
{ }^{\text {Total }} \mathrm{U}\end{array}$ & Not detected \\
\hline Grass & 200 East Area (1) & $\begin{array}{c}{ }^{60} \mathrm{Co} \\
{ }^{90} \mathrm{Sr} \\
{ }^{137} \mathrm{Cs} \\
{ }^{152} \mathrm{Eu} \\
{ }^{154} \mathrm{Eu} \\
{ }^{155} \mathrm{Eu} \\
{ }^{238} \mathrm{Pu} \\
{ }^{239 / 240} \mathrm{Pu} \\
{ }^{\text {Total }} \mathrm{U}\end{array}$ & Not detected \\
\hline Rabbitbrush & 200 West Area & $\begin{array}{r}90 \mathrm{St} \\
{ }^{137} \mathrm{Cs} \\
\end{array}$ & $\begin{array}{l}1.1 E+03 \\
3.1 E+02 \\
\end{array}$ \\
\hline Tumbleweeds & 200 East Area (2) & $\begin{array}{l}{ }^{90} \mathrm{Sr} \\
{ }^{137} \mathrm{Cs}\end{array}$ & $\begin{array}{l}2.8 \mathrm{E}+00 \\
1.5 \mathrm{E}+02 \\
\end{array}$ \\
\hline Tumbleweeds & 200 West Area (2) & ${ }^{90} \mathrm{Sr}$ & $\begin{array}{l}2.5 E+05 \\
1.8 E+06\end{array}$ \\
\hline Darkling beetles & 200 West Area (1) & ${ }^{90} \mathrm{Sr}$ & $1.8 \mathrm{E}+02$ \\
\hline Sagebrush lizards & 200 East Area (1) & $\begin{array}{l}{ }^{90} \mathrm{Sr} \\
{ }^{137} \mathrm{Cs} \\
\end{array}$ & $\begin{array}{l}6.8 E+01 \\
3.3 E+01 \\
\end{array}$ \\
\hline Bird nest & 200 West Area $\{1\}$ & $\begin{array}{l}{ }^{90} \mathrm{Sr} \\
{ }^{137} \mathrm{Cs} \\
\end{array}$ & $\begin{array}{l}2.9 E+01 \\
3.7 E+02 \\
\end{array}$ \\
\hline Mouse feces & 200 West Area (1) & $\begin{array}{c}{ }^{60} \mathrm{Co} \\
{ }^{90} \mathrm{Sr} \\
{ }^{137} \mathrm{Cs} \\
{ }^{154} \mathrm{Eu} \\
{ }^{155} \mathrm{Eu} \\
{ }^{238} \mathrm{Pu} \\
{ }^{2391240} \mathrm{Pu} \\
{ }^{\text {Total }} \mathrm{U} \\
\end{array}$ & $\begin{array}{l}2.8 E+05 \\
1.7 E+05 \\
1.3 E+05 \\
2.6 E+05 \\
1.1 E+05 \\
8.1 E+03 \\
3.3 E+04 \\
1.6 E+05 \\
\end{array}$ \\
\hline Mouse nest & 200 East Area (2) & ${ }^{90} \mathrm{Sr}$ & $\begin{array}{l}9.2 E+03 \\
8.6 E+02 \\
\end{array}$ \\
\hline House mouse & 200 East Area (1) & $\begin{array}{l}{ }^{90} \mathrm{Sr} \\
{ }^{137} \mathrm{Cs}\end{array}$ & $\begin{array}{l}1.6 \mathrm{E}+05 \\
4.6 \mathrm{E}+03 \\
\end{array}$ \\
\hline Deer mouse & 200 East Area (3) & $\begin{array}{l}{ }^{50} \mathrm{Sr} \\
{ }^{137} \mathrm{Cs} \\
\end{array}$ & $\begin{array}{l}5.9 E+02 \\
1.0 E+03 \\
\end{array}$ \\
\hline Deer mouse & 200 West Area (3) & $\begin{array}{l}{ }^{90} \mathrm{Sr} \\
{ }^{137} \mathrm{Cs} \\
\end{array}$ & $\begin{array}{l}9.2 E+04 \\
1.2 E+04 \\
\end{array}$ \\
\hline Great Basin pocket mouse & $1000 \mathrm{~N}$ Area (1) & $\begin{array}{r}{ }^{60} \mathrm{Co} \\
{ }^{90} \mathrm{Sr} \\
{ }^{137} \mathrm{Cs} \\
\end{array}$ & $\begin{array}{l}4.3 E+01 \\
1.5 E+02 \\
1.0 E+01 \\
\end{array}$ \\
\hline
\end{tabular}


Table 1-2. Radioactive Contaminated Samples Disposed of from the Operations Areas in 1997. (3 sheets total)

\begin{tabular}{|c|c|c|}
\hline Sample type & Location & $\begin{array}{l}\text { External radioactivity } \\
\left(\mathrm{dpm} / 100 \mathrm{~cm}^{2}\right)\end{array}$ \\
\hline Vegetation & Corner of Baltimore \& 12 th Street/200 East Area & $2.5 E+03$ \\
\hline Sagebrush & Southeast of $209-E / 200$ East Area & $8.0 \mathrm{E}+03$ \\
\hline Soil & East of 241-B Tank Farm/200 East Area & $5.0 \mathrm{E}+03$ \\
\hline Soil & 100-B Excavation Site & $4.0 E+03$ \\
\hline Rabbit brush & Pipeline between B-Plant \& 241-C/200 East Area & $2.5 E+04$ \\
\hline Soil & Pipeline between B-Plant \& 241-C/200 East Area & $1.0 E+05$ \\
\hline Specks & North of Baltimore \& 7 th Street $/ 200$ East Area & $8.0 \mathrm{E}+05$ \\
\hline Tumbleweed fragments & 218-W-3A Burial Ground Trench $26 / 200$ West Area & $6.0 \mathrm{E}+04$ \\
\hline Mouse feces & 217-B/200 East Area & $4.0 \mathrm{E}+05$ \\
\hline Tape & 241-BY Tank Farm Perimeter Fence/200 East Area & $1.4 E+05$ \\
\hline Specks & South of 241-TX-151 Diversion Box/200 West Area & $1.7 E+05$ \\
\hline Ant Hill & East of 241-TX-155 Diversion Box/200 West Area & $4.8 \mathrm{E}+04$ \\
\hline Specks & Southwest of 241-TX-155 Diversion Box/200 West Area & $3.0 \mathrm{E}+05$ \\
\hline Tumbleweed fragments & 216-C-3/200 East Area & $6.0 E+04$ \\
\hline Tumbleweed & 241-AX Tank Farm Perimeter/200 East Area & $1.0 \mathrm{E}+06$ \\
\hline Tumbleweeds (2) & Cross-site Transfer Line/600 Area & $2.4 E+04$ \\
\hline Soil & Cross-site Transfer Line/600 Area & $1.5 E+05$ \\
\hline Specks & UN-216-E-7/200 East Area & $7.0 E+05$ \\
\hline Soil & West of 3734 Building/300 Area & $7.5 E+03$ \\
\hline Speck & 216-A-29 Ditch/200 East Area & $\begin{array}{c}1.0 \mathrm{E}+06 \\
\{38 \mathrm{mrad} / \mathrm{h})\end{array}$ \\
\hline Risers & UN-216-E-13/200 East Area & $8.5 \mathrm{E}+04$ \\
\hline Speck & 276-S-18 Crib/200 West Area & $7.5 E+04$ \\
\hline Specks & 216-BC Control Area Road/600 Area & $5.0 \mathrm{E}+04$ \\
\hline Soil & UN-216-E-7/200 East Area & $2.0 \mathrm{E}+05$ \\
\hline Tumbleweed & 216-B-1 \& 2 Ditch/200 East Area & $3.5 \mathrm{E}+0.5$ \\
\hline Pipe elbow & 216-A-8 Crib/200 East Area & $5.0 E+03$ \\
\hline Tumbleweed & 216-B-1 \& 2 Ditch/200 East Area & $3.5 E+05$ \\
\hline Speck & 241.AX Tank Farm/200 East Area & $1.5 E+05$ \\
\hline Tumbleweed fragments & SW Perimeter of $241-C$ Tank Farm/200 East Area & $1.0 E+05$ \\
\hline Well Pump & 299-W19-37 Well/200 West Area & $1.5 \mathrm{E}+04$ \\
\hline Soil and alpha caisson & 218-W-4B Burial Ground/200 West Area & $2.1 E+04$ \\
\hline Tumbleweed fragments & East of 241-A Tank Farm/200 East Area & $3.0 \mathrm{E}+05$ \\
\hline Tumbleweed fragments & 218-E-12B Burial Ground Trench $42 / 200$ East Area & $1.5 \mathrm{E}+05$ \\
\hline Speck & 218-W-4B Burial Ground/200 West Area & $4.0 \mathrm{E}+05$ \\
\hline
\end{tabular}


Table 1-2. Radioactive Contaminated Samples Disposed of from the Operations Areas in 1997. (3 sheets total)

\begin{tabular}{|c|c|c|}
\hline Sample type & Location & $\begin{array}{c}\text { External radioactivity } \\
\left\{\text { dpm } / 100 \mathrm{~cm}^{2}\right\}\end{array}$ \\
\hline Soil & South of $221-B / 200$ East Area & $9.0 \mathrm{E}+04$ \\
\hline Grass & 241-B Tank Farm/200 East Area & $1.0 \mathrm{E}+06$ \\
\hline Tumbleweed fragments & 241-S/SX Tank Farm West Perimeter/200 West Area & $1.0 \mathrm{E}+05$ \\
\hline Speck & 241-8 Tank Farm/200 East Area & $4.0 E+04$ \\
\hline Tumbleweed & 200 West NW Perimeter $/ 200$ West Area & $6.0 \mathrm{E}+04$ \\
\hline Railcar & Excess Yard/1100 Area & $4.0 \mathrm{E}+04$ \\
\hline Specks & 241-U-151 \& -152 Diversion Boxes/200 West Area & $2.5 E+05$ \\
\hline Tumbleweed fragments & 200 East NE Perimeter $/ 200$ East Area & $3.0 E+04$ \\
\hline Vegetation & 241-SY Tank Farm East Perimeter/200 West Area & $3.0 \mathrm{E}+05$ \\
\hline Rabbit and mouse feces & Steam Line South of 7th Street/200 East Area & $2.5 E+5$ \\
\hline Soil & Railroad Tracks West of $2713-$ WB $/ 200$ West Area & $2.5 E+05$ \\
\hline Soil specks $(10)$ & Raiiroad Tracks West of $2713-$ WB/200 West Area & $3.0 \mathrm{E}+04$ \\
\hline Speck & 2706-T/200 West Area & $5.5 E+04$ \\
\hline Speck & Railroad Tracks North of 283-W/200 West Area & $4.0 \mathrm{E}+05$ \\
\hline Speck & 241-UX-154 Diversion Box/200 West Area & $4.5 \mathrm{E}+05$ \\
\hline Speck & 216-S-25/200 West Area & $1.5 E+05$ \\
\hline Tumbleweed fragments & UN-216-E-20 \& -21/200 East Area & $3.5 E+05$ \\
\hline Tumbleweed fragments & LERF Loop Road/200 East Area & $4.0 \mathrm{E}+05$ \\
\hline Tumbleweed & 241-AX/AZ Tank Farm Perimter $/ 200$ East Area & $6.0 E+05$ \\
\hline Railcar & Excess Yard/1100 Area & $1.1 E+04$ \\
\hline Ant hill & NW of $216-Z-9 B / 200$ West Area & $4.0 E+04$ \\
\hline Mouse & 100-K Basin/100 K Area & $4.0 \mathrm{E}+03$ \\
\hline Tumbleweed fragments & 23rd Street/200 West Area & $5.0 E+04$ \\
\hline Flatbed trailer & Excess Yard/1100 Area & $3.1 E+05$ \\
\hline Tumbleweed & 241-AZ Tank Farm East Perimeter/200 East Area & $5.0 E+04$ \\
\hline Speck & 241-C Tank Farm/200 East Area & $5.0 E+04$ \\
\hline Vegetation & 218-E-12B Burial Ground Trench 42/200 East Area & $1.0 \mathrm{E}+05$ \\
\hline Asphalt & Intersection of Alaska \& Ginko/300 Area & $9.0 E+04$ \\
\hline Vegetation & $1301-\mathrm{N} / 100 \mathrm{~N}$ Area & $3.5 E+04$ \\
\hline Soil & 296-B-10 Stack Base/200 East Area & $7.5 E+04$ \\
\hline Gravel & Intersection of Alaska \& Ginko/300 Area & $2.0 \mathrm{E}+04$ \\
\hline Flatbed trailer & Excess Yard/1100 Area & $1.0 E+06$ \\
\hline Soil & B Plant Sanitary Tile Field/200 East Area & $7.5 E+04$ \\
\hline Paint chip & UN-216-W-28 Outside 241-U Tank Farm / 200 West Area & $1.5 E+04$ \\
\hline
\end{tabular}


Table 1-2. Radioactive Contaminated Samples Disposed of from the Operations Areas in 1997. ( 3 sheets total)

\begin{tabular}{|c|c|c|}
\hline Sample type & Location & $\begin{array}{c}\text { External radioactivity } \\
\left(\mathrm{dpm} / 100 \mathrm{~cm}^{2}\right)\end{array}$ \\
\hline Soil specks & 241-TX-152 Diversion Box/200 West Area & $1.5 \mathrm{E}+0.5$ \\
\hline Speck & 307 Basins/300 Area & $7.5 E+05$ \\
\hline Mouse urine & Underneath CONEX Box/200 West Area & $9.5 E+04$ \\
\hline Tumbleweed fragments & 216-E-12B Burial Ground Trench $94 / 200$ East Area & $2.5 \mathrm{E}+0.5$ \\
\hline Mouse feces & Inside Vehicle at 2711 -E Garage/200 East Area & $5.5 E+04$ \\
\hline Soil & 222-S Laboratory/200 West Area & $8.5 E+04$ \\
\hline Tumbleweeds & 216-A-30 Crib/200 East Area & $4.6 \mathrm{E}+04$ \\
\hline Tumbleweed & 200 East NE Perimeter/200 East Area & $4.8 E \div 05$ \\
\hline Specks & UN-216-W-19/200 West Area & $4.0 \mathrm{E}+05$ \\
\hline Tumbleweed & 241-C Tank Farm/200 East Area & $7.0 \mathrm{E}+04$ \\
\hline $\begin{array}{l}\text { Mouse bones in raven } \\
\text { nest }\end{array}$ & 221-S Water Tower/200 West Area & $1.5 E+04$ \\
\hline Soil and grass & Pipeline East of $24 \uparrow-T Y$ Tank Farm/200 West Area & $2.4 E \div 05$ \\
\hline Metal cart & Excess Yard/1100 Area & $2.5 \mathrm{E}+05$ \\
\hline Soil and tumbleweeds & South of $241-U$ Tank Farm/200 West Area & $\begin{array}{l}1.0 \mathrm{E}+06 \\
(8 \mathrm{mrad} / \mathrm{h}) \\
\end{array}$ \\
\hline Tumbleweeds & UN-216-W-36 by $216-T-30 / 200$ West Area & $2.0 E+05$ \\
\hline $\begin{array}{l}\text { Personal protective } \\
\text { clothing (rubber shoe) }\end{array}$ & Richland Landfill & $6.0 \mathrm{E}+04$ \\
\hline Tumbleweeds & 216-U-14 Ditche/200 West Area & $1.0 E+04$ \\
\hline Steam line stanchion & Near 244-AR/200 East Area & $6.0 E+04$ \\
\hline Truck bed & 105-N/100 N Area & $5.0 E+04$ \\
\hline Tumbleweeds & 218-E-12B Burial Ground/200 East Area & $1.5 E+05$ \\
\hline Electrical conduit & 2727 Sodium Laydown Yard/200 West Area & $6.0 E+04$ \\
\hline Tumbleweed & 216-B-59/200 East Area & $9.5 E+04$ \\
\hline Specks (6) & WESF Truck Port/200 East Area & $1.0 \mathrm{E}+05$ \\
\hline Tumbleweed & 216-A-42 Basins/200 East Area & 12,000 \\
\hline Speck & 244-A Lift Station/200 East Area & $2.0 \mathrm{E}+05$ \\
\hline Pipe gasket & 200 East North Perimeter Fence/200 East Area & $1.0 \mathrm{E}+\mathrm{OB}$ \\
\hline Tumbleweed & 200 East North Perimeter Fence/200 East Area & $1.2 \mathrm{E}+05$ \\
\hline Soil & 2706-T/200 West Area & $7.5 \mathrm{E}+04$ \\
\hline Tumbleweed fragments & 218-E-12B Burial Ground Trench 16/200 East Area & $1.0 E+05$ \\
\hline
\end{tabular}


HNF-EP-0573-6

This page intentionally left blank. 
HNF-EP-0573-6

This page intentionally left blank. 
HNF-EP-0573-6

APPENDIX $\mathrm{J}$

CONCENTRATION GUIDES 
Table J-1. Airborne Derived Concentration Guides.

\begin{tabular}{|l|c|}
\hline \multicolumn{1}{|c|}{ Radionuclide } & $\begin{array}{c}\text { DCG } \\
(\mathrm{pCi} / \mathrm{m} 3)\end{array}$ \\
\hline${ }^{90} \mathrm{Sr}$ & 9 \\
\hline${ }^{137} \mathrm{Cs}$ & 400 \\
\hline${ }^{106} \mathrm{Ru}$ & 30 \\
\hline${ }^{234} \mathrm{U}$ & 0.09 \\
\hline${ }^{235} \mathrm{U}$ & 0.10 \\
\hline${ }^{238} \mathrm{U}$ & 0.10 \\
\hline${ }^{239.240} \mathrm{Pu}$ & 0.02 \\
\hline
\end{tabular}

$\mathrm{DCG}=$ Derived concentration guide .

Table J-2. Derived Concentration Guides for Water ( $\mathrm{pCi} / \mathrm{L})$.

\begin{tabular}{|l|c|}
\hline \multicolumn{1}{|c|}{ Radionuclide } & DCG \\
\hline${ }^{3} \mathrm{H}$ & $2.0 \mathrm{E}+05$ \\
\hline${ }^{60} \mathrm{Co}$ & $5.0 \mathrm{E}+03$ \\
\hline${ }^{90} \mathrm{Sr}$ & $1.0 \mathrm{E}+03$ \\
\hline${ }^{99} \mathrm{Tc}$ & $1.0 \mathrm{E}+05$ \\
\hline${ }^{106} \mathrm{Ru}$ & $6.0 \mathrm{E}+03$ \\
\hline${ }^{129} \mathrm{I}$ & $5.0 \mathrm{E}+02$ \\
\hline${ }^{137} \mathrm{Cs}$ & $3.0 \mathrm{E}+03$ \\
\hline${ }^{234} \mathrm{U}$ & $5.0 \mathrm{E}+02$ \\
\hline${ }^{235} \mathrm{U},{ }^{238} \mathrm{U}$ & $6.0 \mathrm{E}+02$ \\
\hline${ }^{238} \mathrm{Pu}$ & $4.0 \mathrm{E}+01$ \\
\hline${ }^{239} \mathrm{Pu},{ }^{240} \mathrm{Pu}$ & $3.0 \mathrm{E}+01$ \\
\hline
\end{tabular}

DCG $=$ Derived concentration guide. 
Table J-3. Inaccessible Soil Concentration Limits, $\mathrm{pCi} / \mathrm{g}$.

\begin{tabular}{|c|c|c|c|c|c|c|}
\hline Radionuclide & $100 \mathrm{BDKN}$ & $100 \mathrm{~F}, \mathrm{H}$ & $200 \mathrm{~W}$ & $200 \mathrm{E}$ & 300 Area & 400 Area \\
\hline${ }^{3} \mathrm{H}$ & $1.4 E+08$ & $7.4 E+07$ & $3.7 E+08$ & $2.0 \mathrm{E}+08$ & $9.5 E+06$ & $1.4 E+07$ \\
\hline${ }^{14} \mathrm{C}$ & $6.2 \mathrm{E}+05$ & $6.2 E+05$ & $6.2 E+05$ & $6.2 \mathrm{E}+05$ & $6.2 E+05$ & $6.2 E+05$ \\
\hline${ }^{55} \mathrm{Fe}$ & $9.7 E+06$ & $9.7 E+06$ & $3.6 E+10$ & $1.9 \mathrm{E}+10$ & $1.0 \mathrm{E}+07$ & $1.4 E+09$ \\
\hline${ }^{58} \mathrm{Co}$ & $9.8 E+06$ & $9.8 E+06$ & $8.1 E+09$ & $4.3 E+09$ & $1.2 \mathrm{E}+07$ & $3.1 E+08$ \\
\hline${ }^{60} \mathrm{Co}$ & $9.9 E+0.5$ & $9.9 E+05$ & $5.7 E+08$ & $3.0 E+08$ & $1.0 \mathrm{E}+06$ & $9.9 E+06$ \\
\hline${ }^{63} \mathrm{Ni}$ & $1.5 E+08$ & $1.5 E+08$ & $6.9 E+09$ & $6.9 \mathrm{E}+09$ & $1.5 E+08$ & $2.2 E+08$ \\
\hline${ }^{90} \mathrm{Sr}^{*}$ & $8.3 E+05$ & $8.3 E+05$ & $2.2 E+08$ & $1.2 E+08$ & $8.3 E+05$ & $8.4 E+06$ \\
\hline${ }^{99} \mathrm{TC}$ & $1.3 E+07$ & $1.3 E+07$ & $1.3 E+07$ & $1.3 E+07$ & $1.3 E+07$ & $1.3 E+07$ \\
\hline${ }^{106} \mathrm{Ru}^{*}$ & $2.0 E+07$ & $2.0 E+07$ & $5.7 E+08$ & $3.0 E+08$ & $1.5 E+07$ & $2.2 E+07$ \\
\hline${ }^{125} \mathrm{Sb}^{*}$ & $9.1 E+06$ & $9.1 E+06$ & $5.7 \mathrm{E}+09$ & $3.0 E+09$ & $9.2 E+06$ & $1.1 E+08$ \\
\hline 129 & $2.8 E+05$ & $2.8 E+05$ & $2.8 \mathrm{E}+0.5$ & $2.8 \mathrm{E}+05$ & $2.2 E+05$ & $2.8 E+05$ \\
\hline${ }^{134} \mathrm{Cs}$ & $1.7 E+04$ & $1.7 E+04$ & $2.5 E+08$ & $1.4 E+08$ & $2.4 E+04$ & $9.7 E+06$ \\
\hline${ }^{237} \mathrm{Ce}^{*}$ & $3.7 E+04$ & $1.7 E+04$ & $3.5 E+08$ & $1.8 E \div 08$ & $1.7 E+04$ & $1.3 E+07$ \\
\hline${ }^{144} \mathrm{Cs}^{*}$ & $1.4 \mathrm{E}+06$ & $1.4 \mathrm{E}+06$ & $7.4 E+08$ & $4.0 E+08$ & $1.9 \mathrm{E}+06$ & $2.8 \mathrm{E}+07$ \\
\hline${ }^{147} \mathrm{Pm}$ & $3.4 \mathrm{E}+07$ & $3.4 \mathrm{E}+07$ & $7.4 E+09$ & $4.0 \mathrm{E}+09$ & $3.5 \mathrm{E}+07$ & $2.8 \mathrm{E}+08$ \\
\hline${ }^{152} \mathrm{Eu}$ & $4.5 E+06$ & $4.5 E+06$ & $1.2 E+09$ & $6.2 \mathrm{E}+08$ & $4.6 \mathrm{E}+06$ & $4.5 E+07$ \\
\hline${ }^{154} \mathrm{Eu}$ & $3.3 E+06$ & $3.3 E+06$ & $8.8 E+08$ & $4.7 E+08$ & $3.3 E+06$ & $3.4 E+07$ \\
\hline${ }^{155} \mathrm{Eu}$ & $2.3 \mathrm{E}+07$ & $2.3 E+07$ & $6.9 \mathrm{E}+09$ & $3.7 E+09$ & $2.4 E+07$ & $2.6 E+08$ \\
\hline${ }^{226}{ }^{R a *}$ & $1.3 E+05$ & $1.3 E+05$ & $2.1 E+05$ & $2.1 E+05$ & $1.3 E+05$ & $1.4 E+05$ \\
\hline${ }^{227} \mathrm{AC} *$ & $2.4 E+03$ & $2.4 \mathrm{E}+03$ & $5.4 \mathrm{E}+04$ & $2.9 E+04$ & $1.4 E+03$ & $2.1 E+03$ \\
\hline${ }^{232} \mathrm{Th}^{*}$ & $2.0 \mathrm{E}+04$ & $2.0 \mathrm{E}+04$ & $2.0 \mathrm{E}+04$ & $2.0 E+04$ & $4.7 \mathrm{E}+03$ & $7.1 E+03$ \\
\hline${ }^{232} \mathrm{U}^{*}$ & $5.5 E+04$ & $5.5 E+04$ & $1.4 \mathrm{E}+0.5$ & $1.4 E+05$ & $9.9 E+03$ & $1.5 E+04$ \\
\hline${ }^{233} \mathrm{U}$ & 4.5E+O5 & $4.5 E+05$ & $4.5 \mathrm{E}+05$ & $4.5 E+0.5$ & $6.7 E+04$ & $1.0 \mathrm{E}+05$ \\
\hline${ }^{234} \mathrm{U}$ & $4.6 E+0.5$ & $4.6 E+05$ & $4.6 \mathrm{E}+05$ & $4.6 E+0.5$ & $6.9 \mathrm{E}+04$ & $1.0 E+05$ \\
\hline${ }^{235} \mathrm{U}^{*}$ & $4.9 E+05$ & $4.9 E+05$ & $4.9 E+05$ & $4.9 E+05$ & $7.3 \mathrm{E}+0$. & $1.1 E+05$ \\
\hline${ }^{236} \mathrm{U}$ & $4.9 E+05$ & $4.9 E+05$ & $4.9 E+05$ & $4.9 E+05$ & $7.1 E+04$ & $1.1 \mathrm{E}+05$ \\
\hline${ }^{238} \mathrm{U}^{*}$ & $4.7 E+05$ & $4.7 E+0.5$ & $4.7 E+05$ & $4.7 E+05$ & $7.7 \mathrm{E}+04$ & $1.2 E+05$ \\
\hline${ }^{237} \mathrm{~Np}^{*}$ & $8.9 E+02$ & $8.9 E+02$ & $8.9 E+02$ & $8.9 E+02$ & $8.9 E+02$ & $8.9 E+02$ \\
\hline${ }^{238} \mathrm{Pu}$ & $1.3 \mathrm{E}+04$ & $1.3 E+04$ & $8.8 E+05$ & $4.7 E+05$ & $1.3 E \div 04$ & $3.4 \mathrm{E}+04$ \\
\hline${ }^{239} \mathrm{Pu}$ & $1.2 \mathrm{E}+04$ & $1.2 \mathrm{E}+04$ & $1.2 E+04$ & $1.2 \mathrm{E}+04$ & $1.2 \mathrm{E}+04$ & $1.2 E \div 04$ \\
\hline${ }^{240} \mathrm{Pu}$ & $1.2 \mathrm{E}+04$ & $1.2 \varepsilon+04$ & $1.4 E+04$ & $1.4 \mathrm{E}+04$ & $1.2 \mathrm{E}+04$ & $1.2 \mathrm{E}+04$ \\
\hline${ }^{241} \mathrm{Pu}$ & $6.1 E+05$ & $6.1 E+05$ & $4.2 \mathrm{E}+07$ & $2.2 \mathrm{E}+07$ & $6.1 E+05$ & $1.2 \mathrm{E}+06$ \\
\hline${ }^{241} \mathrm{Am}$ & $2.5 E+04$ & $2.5 E+04$ & $7.4 \mathrm{E}+05$ & $4.0 E+05$ & $1.9 E+04$ & $2.8 E+04$ \\
\hline
\end{tabular}

Note: Asterisks mark nuclides with progeny that are assumed to be present in equilibrium amounts.

Note: Asterisks mark nuclides with progeny that are assumed to be present in
However, ${ }^{234} \mathrm{U}$ was not included in the ${ }^{238} \mathrm{U}$ limits. For supporting references see WHC-SD-EN-TI-070. 
Table J-4. Accessible Soil Concentration Limits, pCi/g.

\begin{tabular}{|c|c|c|c|c|c|c|}
\hline Radionuclide & $100 \mathrm{BDKN}$ & $100 \mathrm{~F}, \mathrm{H}$ & $200 \mathrm{~W}$ & $200 \mathrm{E}$ & 300 Area & 400 Area \\
\hline${ }^{3} \mathrm{H}$ & $1.4 E+08$ & $7.4 \mathrm{E}+07$ & $3.7 E+08$ & $2.0 E+08$ & $9.5 E+06$ & $1.4 E+07$ \\
\hline${ }^{14} \mathrm{C}$ & $6.2 E+05$ & $6.2 \mathrm{E}+05$ & $6.2 E+05$ & $6.2 E+05$ & $6.2 \mathrm{E}+0.5$ & $6.2 \mathrm{E}+05$ \\
\hline${ }^{55} \mathrm{Fe}$ & $5.3 \mathrm{E}+05$ & $5.3 E+05$ & $5.3 \mathrm{E}+0.5$ & $5.3 E+05$ & $5.3 E+05$ & $5.3 E+05$ \\
\hline${ }^{58} \mathrm{Co}$ & $1.8 E+01$ & $1.8 E+01$ & $1.8 E+01$ & $1.8 E+01$ & $1.8 E+01$ & $1.8 \mathrm{E}+01$ \\
\hline${ }^{60} \mathrm{Co}$ & $7.1 E+00$ & $7.1 E+00$ & $7.1 E+\infty$ & $7.1 \mathrm{E}+\mathrm{OO}$ & $7.1 \mathrm{E}+00$ & $7.1 \mathrm{E}+00$ \\
\hline${ }^{63} \mathrm{Ni}$ & $2.5 E+07$ & $2.5 \mathrm{E}+07$ & $2.5 \mathrm{E}+07$ & $2.5 E+07$ & $2.5 E+07$ & $2.5 E+07$ \\
\hline${ }^{90} \mathrm{Sr}^{*}$ & $2.8 E+03$ & $2.8 E+03$ & $2.8 \mathrm{E}+03$ & $2.8 \mathrm{E}+03$ & $2.8 E+03$ & $2.8 \mathrm{E}+03$ \\
\hline${ }^{99} \mathrm{Tc}$ & $1.0 E+06$ & $1.0 \mathrm{E}+06$ & 1.0E+OG & $1.0 E+06$ & $1.0 \mathrm{E}+06$ & $1.0 \mathrm{E}+06$ \\
\hline${ }^{106} \mathrm{Ru}^{*}$ & $7.7 E+01$ & $7.7 E+01$ & $7.7 E+01$ & $7.7 E+01$ & $7.7 E+01$ & $7.7 \mathrm{E} \div 01$ \\
\hline${ }^{125} \mathrm{Sb}^{*}$ & $3.7 E+0$ ? & $3.7 \mathrm{E}+01$ & $3.7 E+01$ & $3.7 \mathrm{E}+01$ & $3.7 E+01$ & $3.7 E+01$ \\
\hline${ }^{129} \mid$ & $1.0 \mathrm{E}+04$ & $1.0 \mathrm{E}+04$ & $1.0 \mathrm{E}+04$ & $1.0 \mathrm{E}+04$ & $1.0 E+04$ & $1.0 \mathrm{E}+04$ \\
\hline${ }^{134} \mathrm{Cs}$ & $1.0 \mathrm{E}+01$ & $1.0 \mathrm{E}+01$ & $1.0 \mathrm{E}+01$ & $1.0 \mathrm{E}+01$ & $1.0 \mathrm{E}+01$ & $1.0 \mathrm{E}+01$ \\
\hline${ }^{137} \mathrm{Cs}^{*}$ & $3.0 \mathrm{E}+01$ & $3.0 \mathrm{E}+01$ & $3.0 \mathrm{E}+01$ & $3.0 E+01$ & $3.0 \mathrm{E}+01$ & $3.0 E+01$ \\
\hline${ }^{144} \mathrm{Ce}^{*}$ & $3.3 E+02$ & $3.3 \mathrm{E}+02$ & $3.3 E+02$ & $3.3 E+02$ & $3.3 E+02$ & $3.3 E+02$ \\
\hline${ }^{147} \mathrm{Pm}$ & $1.1 \mathrm{E}+06$ & $1.1 E+06$ & $1.1 E+06$ & $1.1 E+06$ & $1.1 E+06$ & $1.1 E+06$ \\
\hline${ }^{152} \mathrm{Eu}$ & $1.5 E+01$ & $1.5 \mathrm{E}+01$ & $1.5 \mathrm{E}+01$ & $1.5 E+.01$ & $1.5 E+01$ & $1.5 E+01$ \\
\hline${ }^{154} \mathrm{Eu}$ & $1.4 \mathrm{E}+01$ & $1.4 E+01$ & $1.4 \mathrm{E}+01$ & $1.4 \mathrm{E}+01$ & $1.4 E+01$ & $1.4 \mathrm{E}+01$ \\
\hline${ }^{155} \mathrm{Eu}$ & $6.3 E+02$ & $6.3 \mathrm{E}+02$ & $6.3 \mathrm{E}+\mathrm{O} 2$ & $6.3 E+02$ & $6.3 E+02$ & $6.3 \mathrm{E}+\mathrm{O} 2$ \\
\hline${ }^{226} \mathrm{Ra}^{*}$ & $1.0 \mathrm{E}+01$ & $1.0 E+01$ & $1.0 \mathrm{E}+01$ & $1.0 \mathrm{E}+01$ & $1.0 \mathrm{E}+01$ & $1.0 \mathrm{E}+01$ \\
\hline${ }^{227} \mathrm{Ac} *$ & $1.0 E+01$ & $1.0 \mathrm{E}+01$ & $1.0 \mathrm{E}+01$ & $1.0 \mathrm{E}+01$ & $1.0 \mathrm{E}+01$ & $1.0 \mathrm{E}+01$ \\
\hline${ }^{232} \mathrm{Th}^{*}$ & $5.9 \mathrm{E}+00$ & $5.9 E+\infty 0$ & $5.9 E+00$ & $5.9 \mathrm{E} \div 00$ & $5.9 \mathrm{E}+00$ & $5.9 E+\infty$ \\
\hline${ }^{232} \mathrm{U}^{*}$ & $1.0 \mathrm{E}+01$ & $1.0 E+01$ & $1.0 \mathrm{E}+01$ & $1.0 \mathrm{E}+01$ & $1.0 \mathrm{E}+01$ & $1.0 \mathrm{E}+01$ \\
\hline${ }^{233} \mathrm{U}$ & $6.3 E+02$ & $6.3 \mathrm{E}+02$ & $6.3 E+02$ & $6.3 E+02$ & $6.3 E+02$ & $6.3 \mathrm{E}+02$ \\
\hline${ }^{234} \mathrm{U}$ & $6.3 E+02$ & $6.3 E+02$ & $6.3 \mathrm{E}+\mathrm{O} 2$ & $6.3 E+02$ & $6.3 E+02$ & $6.3 \mathrm{E}+02$ \\
\hline${ }^{235} \mathrm{U}^{*}$ & $1.7 E+02$ & $1.7 E+O 2$ & $1.7 E+02$ & $1.7 E+02$ & 1.7 $E+02$ & $1.7 \mathrm{E}+02$ \\
\hline${ }^{236} \mathrm{U}$ & $6.7 \mathrm{E}+02$ & $6.7 E+02$ & $6.7 \mathrm{E}+\mathrm{O} 2$ & $6.7 \mathrm{E} \div 02$ & $6.7 \mathrm{E}+02$ & $6.7 \mathrm{E}+\mathrm{O} 2$ \\
\hline${ }^{238} \mathrm{U}^{*}$ & $3.7 \mathrm{E}+02$ & $3.7 E+02$ & $3.7 E+02$ & $3.7 E+02$ & $3.7 E+02$ & $3.7 \mathrm{E} \div \mathrm{O} 2$ \\
\hline${ }^{237} \mathrm{~Np}^{*}$ & $4.8 \mathrm{E}+01$ & $4.8 E+01$ & $4.8 \mathrm{E}+01$ & $4.8 E+01$ & $4.8 \mathrm{E}+01$ & $4.8 E+01$ \\
\hline${ }^{238} \mathrm{Pu}$ & $2.1 \mathrm{E}+\mathrm{O} 2$ & $2.1 E+02$ & $2.1 \mathrm{E}+\mathrm{O} 2$ & $2.1 E+02$ & $2.1 E+02$ & $2.1 \mathrm{E}+02$ \\
\hline${ }^{239} \mathrm{Pu}$ & $1.9 \mathrm{E}+02$ & $1.9 \mathrm{E}+02$ & $1.9 \mathrm{E}+\mathrm{O} 2$ & $1.9 E+02$ & $1.9 E+02$ & $1.9 E+02$ \\
\hline${ }^{240} \mathrm{Pu}$ & $1.9 E+02$ & $1.9 \mathrm{E}+02$ & $1.9 \mathrm{E}+02$ & $1.9 E+02$ & $1.9 E+02$ & $1.9 \mathrm{E}+02$ \\
\hline${ }^{241} \mathrm{Pu}$ & $1.0 \mathrm{E}+04$ & $1.0 E+04$ & $1.0 \mathrm{E}+04$ & $1.0 \mathrm{E}+04$ & $1.0 E+04$ & $1.0 \mathrm{E}+04$ \\
\hline${ }^{241} \mathrm{Am}$ & $1.8 E+02$ & $1.8 E+02$ & $1.8 \mathrm{E}+\mathrm{O} 2$ & $1.8 \mathrm{E}+\mathrm{O} 2$ & $1.8 E+02$ & $1.8 E+02$ \\
\hline
\end{tabular}

Note: Asterisks mark nuclides with progeny that are assumed to be present in equilibrium amounts. However, ${ }^{234} \mathrm{U}$ was not included in the ${ }^{238} \mathrm{U}$ limits. For supporting references see WHC-SD-EN-TI-070. 
HNF-EP-0573-6

This page intentionally left blank. 
HNF-EP-0573-6

\section{APPENDIX K}

\section{DATA SUMMARY METHODS}


Measuring any physical quantity has some degree of inherent uncertainty. This uncertainty results from the combination of all possible inaccuracies in the measurements process, including such factors as the reading of the result, the calibration of the measuring device, and numerical rounding errors. In this report, individual radioactive measurements are accompanied by a plus or minus $( \pm)$ value, which is the uncertainty term known as a two-sigma counting error.

The two-sigma counting error gives information on what the measurement might be if the same sample were counted again under identical conditions. The two-sigma counting error implies that approximately $95 \%$ of the time, a recount of the same sample would give a value within plus or minus the two-sigma counting error at the value reported.

Values in the tables that are less than the minimum detectable activity indicate that the reported result might have come from a sample with no radioactivity. Such values are considered below the detection limits of the measuring instrument. Also note that each radioactive measurement must have the random background radioactivity of the measuring instrument subtracted; therefore, negative results are possible, especially when the sample has very little radioactivity.

Reported averages aiso are accompanied by two standard errors of the mean. If the data fluctuate randomly, the standard error is a measure of the uncertainty in the estimated average of the data because of this randomness. If trends of periodic fluctuation are present, the standard error is primarily a measure of the variability in the trends and fluctuations about the average of the data, rather than a measure of the uncertainty of the estimated average because of random fluctuations in the data.

The mean, $X$, is computed as:

$$
X=\frac{1}{n} \sum_{i=1}^{n} x_{i}
$$

where $X_{i}$ is the ith measurement and $n$ is the number of measurements.

The standard error of the mean was computed as:

$$
S E=\sqrt{\frac{S^{2}}{n}}
$$

where $S^{2}$, the variance of the $n$ measurements, was computed as:

$$
S_{M}^{2}=\frac{1}{n-1} \sum_{i=1}^{n}\left(x_{i}-x\right)^{2}
$$

This estimator, $\mathrm{S}^{2}$, includes the variance among the samples and the counting variance. The estimated $\mathrm{S}^{2}$ occasionally may be less than the average counting variance. 
HNF-EP-0573-6

This page intentionally left blank. 


\section{DISTRIBUTION}

Number of copies

\section{OFFSITE}

Confederated Tribes of the Umatilla Indian Reservation

P.O. Box 638

Pendleton, Oregon 97801

J. R. Wilkinson

1

Nez Perce Tribe

P.O. Box 305

Lapwai, Idaho 83540

Donna Powaukee

U.S. Department of Energy-Headquarters

R. W. Barber (2)

1

RAPIC

Building $\mathrm{K} 1210$

Mail Stop 7256

P.O. Box 2003

Oak Ridge, Tennessee 37831-7256

L. Estes

1

U.S. Department of Interior

Fish and Wildlife Service

Fish and Wildlife Enhancement

Moses Lake, Field Office

P.O. Box 1157

Moses Lake, Washington 98837

L. Block

1

U.S. Department of Interior

Fish and Wildlife Service

Columbia National Wildife Refuge

P.O. Box Drawer F

Othello, Washington 99344

D. Goeke

1

U.S. Ecology, Inc.

P.O. Box 638

Richland, Washington 99352

A. Palmer

Distr-1 


\section{DISTRIBUTION (cont)}

Number of copies

OFFSITE

2

U.S. Environmental Protection Agency, Region 10 1200 Sixth Avenue

Seattle, Washington 98081

G. M. Leitch

AT-081

R. W. Poeton

AT-081

1

Washington Public Power Supply System

P.O. Box 968

Richland, Washington 99352

J. E. McDonald

MD 1020

8

Washington State Department of Health

Division of Radiation Protection

Airdustrial Center, Building 5

P.O. Box 47827

Olympia, Washington 98504-0095

A. W. Conklin

MS-7827

J. C. Berkey

MS-7827

R. A. Danielson

MS-7827

J. L. Erickson

MS-7827

P. J. Martell

MS-7827

D. McBaugh

MS-7827

G. Robertson

MS-7827

J. W. Schmidt

MS-7827

1

Washington State Department of Wildlife

Lower River Road

Benton City, Washington 99320

L. Fitzner

1

Yakima Indian Nation

Environmental Restoration Waste

P.O. Box 151

Toppenish, Washington 98948

Jim Russell, Manager 


\section{DISTRIBUTION (cont)}

Number of copies

\section{ONSITE}

E. M. Bowers

S. E. Clarke

E. B. Dagan

P. F. Dunigan, Jr.

M. J. Furman

J. B. Hali

R. D. Hildebrand

R. G. McLeod

R. O. Puthoff

H. M. Rodriguez

K. M. Thompson

D. C. Ward

Public Reading Room (4)

Bechtel Hanford, Inc.

R. L. Biggerstaff

R. A. Carison

E. T. Coenenburg

D. B. Erb

R. G. Egge

K. R. Fecht

M. J. Galgoul

K. A. Gano

W. M. Hayward

G. C. Henckel

R. P. Henckel

R. J. Landon

W. L. Osborne

W. H. Price

F. V. Roeck

J. E. Rugg

K. A. Smith

C. D. Wade

S. G. Weiss

T. M. Wintczak

C. D. Wittreich

J. G. Woolard

J. P. Zoric

Environmental Resource Center
S7-55

A5-15

S7-55

A5-15

HO-12

A5-15

$\mathrm{H}$ O-12

HO-12

A7-80

A5-15

HO-12

A5-15

H2-53

$\mathrm{HO}-02$

L6-06

$\mathrm{H} 9-03$

$\mathrm{H} 9-03$

S3-21

$\mathrm{HO}-02$

LO-2O

$\mathrm{HO}-\mathrm{O} 2$

T7-05

HO-O9

S3-21

$\mathrm{HO}-\mathrm{O} 2$

T7-05

$\mathrm{HO}-2 \mathrm{O}$

$\mathrm{HO}-17$

S3-21

$\mathrm{X} 0-23$

T7-15

H9-03

HO-21

H9-03

$\mathrm{HO}-\mathrm{O} 2$

$\times 5-53$

HO-09 
DISTRIBUTION (cont)

Number of copies

\section{ONSITE}

8

B\&W Hanford Company

S. L. Bump

T. A. Dillhoff

R. E. Heineman

D. L. Johnson

S. E. Killoy

D. E. Rasmussen

S. W. Scott

D. R. Speer
L5-65

N2-57

K8.60

L1-05

S6-60

L1-04

N2-57

S4-49

3

DE\&S Hanford, Inc.

R. G. Gant

$\times 3-79$

G. M. Stevens

$\times 3-79$

D. J. Watson

$\times 3-79$

1

Environmenta! Management, Inc.

R. C. Roos

H1-12

4

DynCorp Tri-Cities Services, Inc.

B. J. Dixon

G3-26

A. D. Poor

R1-51

G. B. Carlson

G2-06

D. A. Marsh

G3-07

6

Fluor Daniel Hanford, Inc.

W. D. Adair

H6-21

G. T. Berlin

K9-46

D. L. Mitchel]

H6-23

S. M. Price

H6-23

D. G. Ranade

H6-23

F. A. Ruck

H6-23

Hanford Environmental Health Foundation

J. K. Samuels

$\mathrm{H1}-78$ 
DISTRIBUTION (cont)

Number of copies

\section{ONSITE}

6

Lockheed Martin Hanford Corporation

M. L. Dexter

R1-51

C. L. Caldwell

S5-07

R. E. Elder

S7-07

B. G. Erlandson

R1 -51

O. R. Rasmussen

R1-51

W. E. Ross

S7-84

2

Lockheed Martin Services, Inc.

Central Files

Document Processing Center

1

Numatec Hanford_Corporation

D. S. Dutt

H5-25

14

Pacific Northwest National Laboratory

E. J. Antonio

K3-54

R. W. Bryce

$\mathrm{K} 6-75$

C. J. Chou

K6-81

R. L. Dirkes

K6-75

B. M. Gillespie

K6-96

R. W. Hanf

K6-75

V. G. Johnson

K6-96

S. P. Luttrell

K6-96

G. W. Patton

K6-75

T. M. Poston

K6-75

K. Rhoads

K3-54

H. T. Tilden

P7-79

J. P. Schmidt

K7:80

Technical Library

P8-55

2

Washington State Department of Ecology

R. J. Julian

B5-18

P. R. Staats

B5-18 
DISTRIBUTION (cont)

Number of copies

\section{ONSITE}

Waste Management Federal Services of Hanford. Inc.
H. C. Boynton
T4-52
A. K. Dasgupta
S3-28
L. P. Diediker (5)
H6-36
R. H. Engeimann
D. L. Flyckt
H6-26
D. W. Fritz
S6-71
C. K. Girres
H6-36
B. P. Gleckler
T3-01
E. M. Greager
H6-36
P. R. Gunter
H6-36
D. W. Lindsey
S6-71
J. J. Luke
T3-01
K. M. McDonald
H6-25
J. K. Perry
H6-06
J. R. Prilucik
H6-25
L. W. Roberts
T6-14
J. 0 . Skolrud
T4-51
J. C. Sonnichsen
H6-2O
H6-26
R. W. Szelmeczka
L.6-05
M. T. York
T3-06

46

Waste Management Federal Serivces, Inc. Northwest Operations

P. K. Brockman

H1-11

J. J. Dorian

H1-13

L. M. Hay

H1-15

A. R. Johnson (5)

$\mathrm{H} 1-13$

B. M. Markes

$\mathrm{H} 1-13$

S. M. McKinney (10)

H1-12

R. M. Mitchell (5)

H1-13

D. J. Moak

H1-11

S. E. Myers

H1-12

C. J. Perkins (20)

H1-12

Yakima Indian Nation

D. Dogsleep

G1-02 